\title{
Water-Quality and Lake-Stage Data for Wisconsin Lakes, Water Year 200I
}

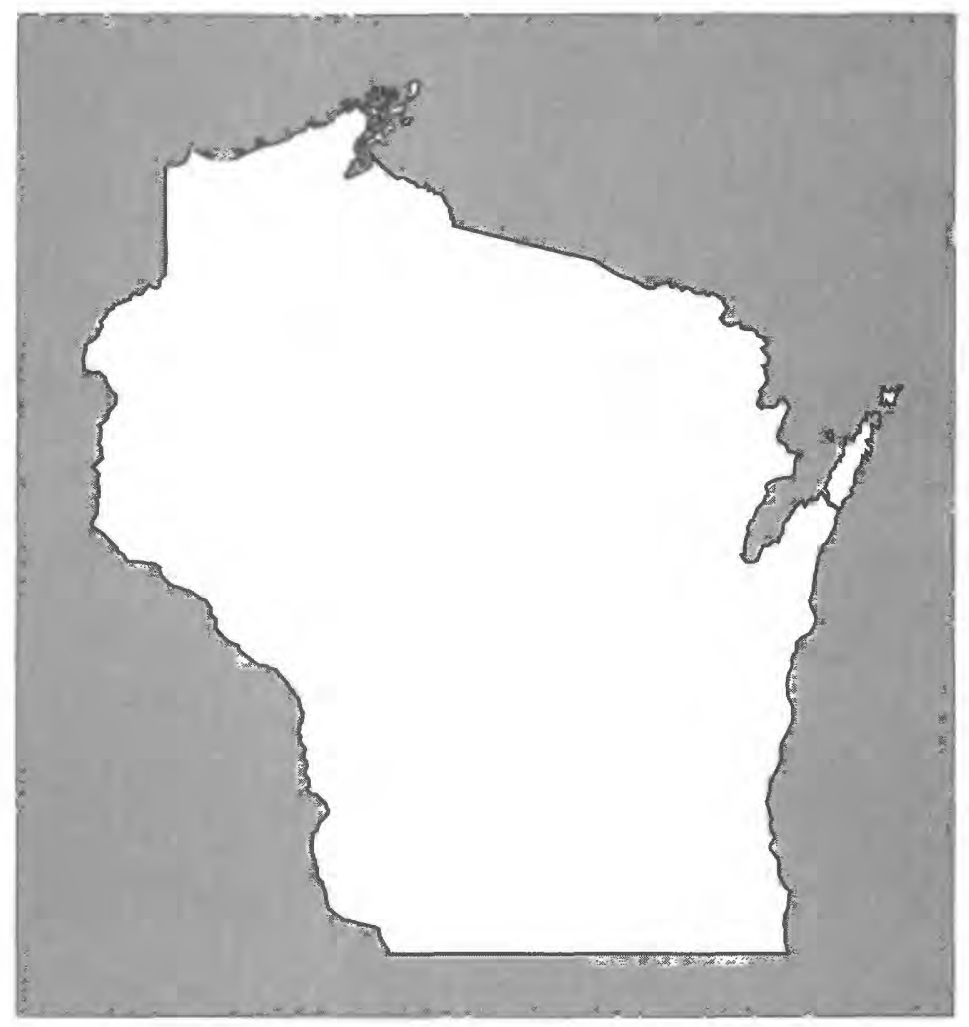

U.S. GEOLOGICAL SURVEY Open-File Report 02-135

Prepared in cooperation with the State of Wisconsin and local agencies 



\section{WATER-QUALITY AND LAKE-STAGE DATA FOR WISCONSIN LAKES, WATER YEAR 2001}

\section{By Wisconsin District Lake-Studies Team}

U.S. GEOLOGICAL SURVEY

Open-File Report 02-135

A report by the Wisconsin District Lake-Studies TeamW.J. Rose (team leader), J.F. Elder, H.S. Garn, G.L. Goddard E.A. Mergener, D.L. Olson, and D.M. Robertson

Prepared in cooperation with

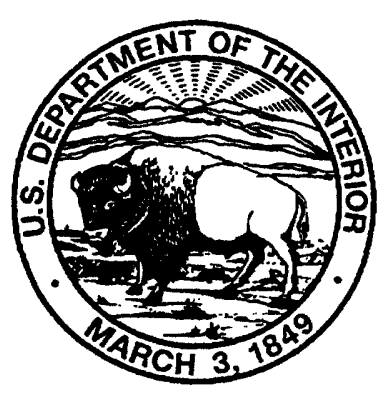

THE STATE OF WISCONSIN AND OTHER AGENCIES

Middleton, Wisconsin 2002 


\title{
U.S. DEPARTMENT OF THE INTERIOR GALE A. NORTON, Secretary
}

\author{
U.S. GEOLOGICAL SURVEY \\ Charles G. Groat, Director
}

For additional information write to:

District Chief

U.S. Geological Survey

8505 Research Way

Middleton, WI 53562
Copies of this report can be purchased from:

U.S. Geological Survey

Earth Science Information Center

Open-File Reports Section

Box 25286, MS 517

Denver Federal Center

Denver, CO 80225 


\section{CONTENTS}

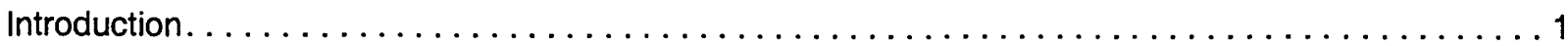

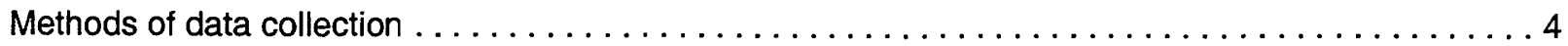

Explanation of physical and chemical characteristics of lakes $\ldots \ldots \ldots \ldots \ldots \ldots \ldots \ldots \ldots \ldots$

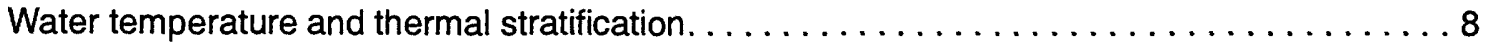

Specific conductance. . . . . . . . . . . . . . . . . . . . . . . . . . . . . . . 10

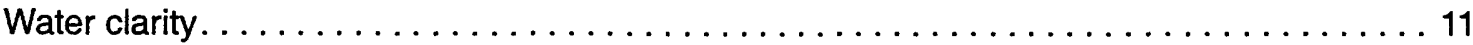

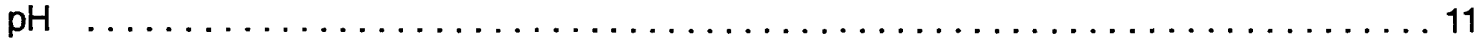

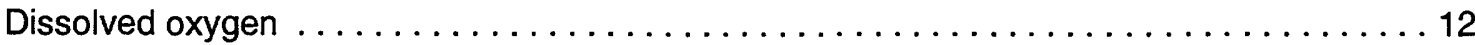

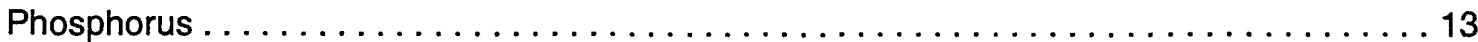

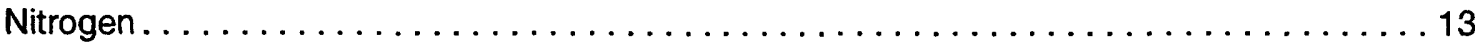

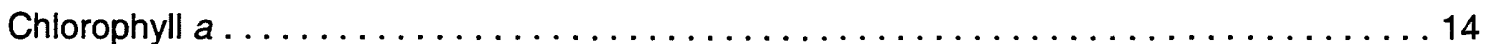

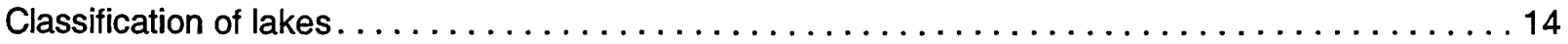

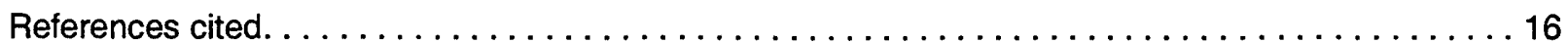

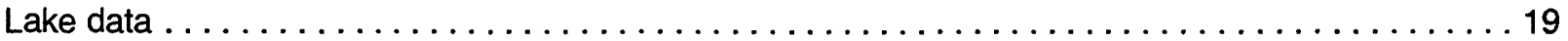

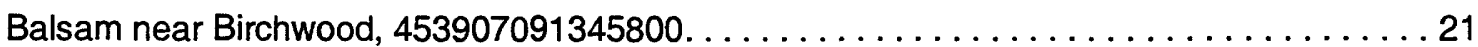

Big Cedar

North Site, near West Bend, 432409088151600 . . . . . . . . . . . . . . . . . . . . . 24

South Site, near West Bend, $432224088154900 \ldots \ldots \ldots \ldots \ldots$. . . . . . . . . . . . . 26

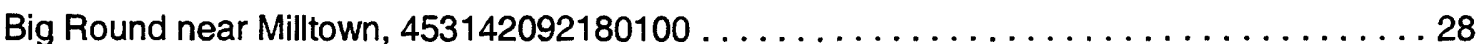

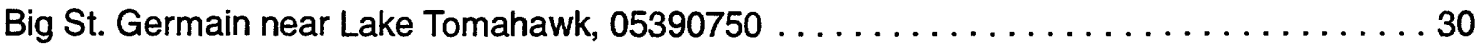

Big Sand

Deep Hole, near Hertel, 454910092134000 . . . . . . . . . . . . . . . . . . . . . . . . 31

East Site, near Hertel, 454921092124300 . . . . . . . . . . . . . . . . . . . . . . . . . . . 33

Big Sissabagama

near Stone Lake, 454724091303600 . . . . . . . . . . . . . . . . . . . . . . . . . . . . . . . 35

North Site, near Stone Lake, $454800091312900 \ldots \ldots \ldots \ldots \ldots \ldots \ldots \ldots$. . . . . . . 38

Booth near East Troy, 424800088254800 . . . . . . . . . . . . . . . . . . . . . . . . . . . . . 40

Buffalo

Center Site, at Packwaukee, 434558089260600 . . . . . . . . . . . . . . . . . . . . . . . 42

East End, at Montello, $434720089201600 \ldots \ldots \ldots \ldots \ldots \ldots \ldots \ldots$. . . . . . . . . 44

West End, near Endeavor, $434414089282400 \ldots \ldots \ldots \ldots \ldots \ldots \ldots$. . . . . . . . . . . . . 46

Delavan

near Delavan, $423706088363400 \ldots \ldots \ldots \ldots \ldots \ldots \ldots \ldots$. . . . . . . . . . . . . . . . 48

at Center near Delavan Lake, 423556088365001 . . . . . . . . . . . . . . . . . . . . . . . 49

at North End near Lake Lawn, $423659088354401 \ldots \ldots \ldots \ldots \ldots \ldots \ldots \ldots \ldots$

at SW End near Delavan Lake, $423526088380101 \ldots \ldots \ldots \ldots \ldots \ldots \ldots \ldots$

Devils near Baraboo, 05404500 . . . . . . . . . . . . . . . . . . . . . . . . . . . . . . . 55

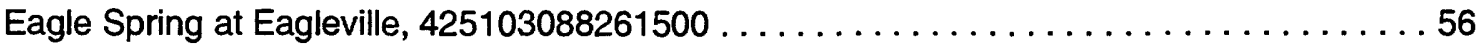

Fish near Sauk City, $05406050 \ldots \ldots \ldots$. . . . . . . . . . . . . . . . . . . . . . . . . . . . 58

Geneva

at Lake Geneva, $423525088260400 \ldots \ldots$. . . . . . . . . . . . . . . . . . . . . . . . . . 59

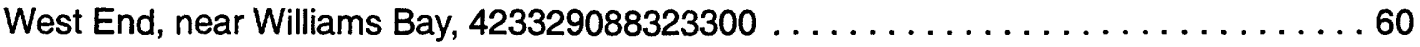




\section{CONTENTS--Continued}

Green at County Trunk Highway A near Green Lake, 434928088553601 . . . . . . . . . 64

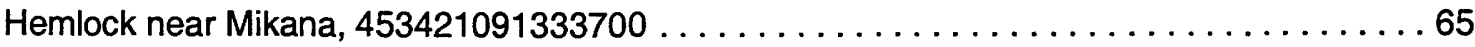

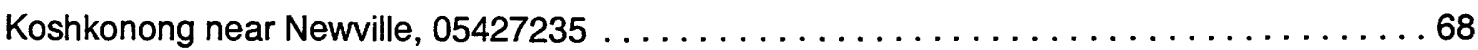

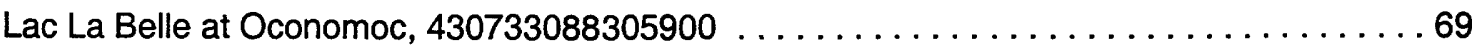

Lauderdale

Middle at Lauderdale, $424621088335500 \ldots \ldots \ldots \ldots \ldots \ldots \ldots \ldots \ldots \ldots \ldots \ldots$

Little Arbor Vitae near Woodruff, $455446089370300 \ldots \ldots \ldots \ldots \ldots \ldots \ldots \ldots \ldots$

Little Green, at Center, near Markesan, $43441208859070079 \ldots \ldots \ldots \ldots \ldots \ldots \ldots \ldots 74$

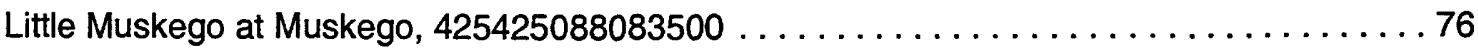

Little St. Germain

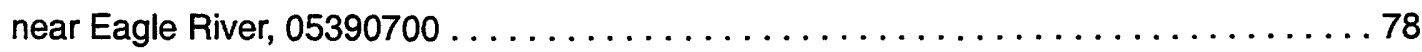

Upper East Bay, at St. Germain, 4553208925390 . . . . . . . . . . . . . . . . . 79

Northeast Bay, near St. Germain, $455545089262500 \ldots \ldots \ldots \ldots \ldots \ldots \ldots \ldots$. . . . . 82

South Bay, near St. Germain, $455437089270800 \ldots \ldots \ldots \ldots \ldots \ldots \ldots \ldots \ldots$

West Bay, at St. Germain, $455428089282400 \ldots \ldots \ldots \ldots \ldots \ldots \ldots \ldots$. . . . . . . 88

Little Sand--Site Number 2--near Mole Lake, 452826088544101 . . . . . . . . . . . . . . . 91

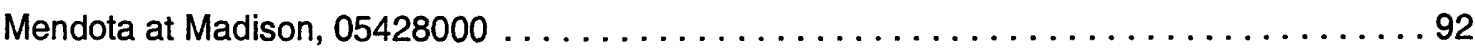

Middle Genesee near Oconomowoc, $430309088284800 \ldots \ldots \ldots \ldots \ldots \ldots \ldots \ldots$

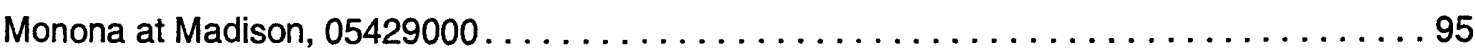

Muskego (Big Muskego)

Bass Bay, near Muskego, $425344088070100 \ldots \ldots \ldots \ldots \ldots \ldots \ldots \ldots$

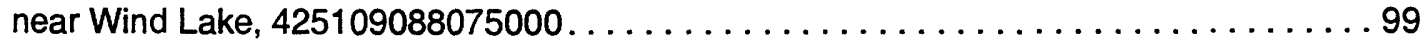

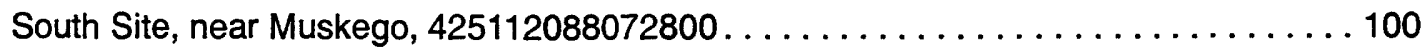

Muskellunge, near Lake Outlet near Eagle River, 455706089232400 . . . . . . . . . . . 102

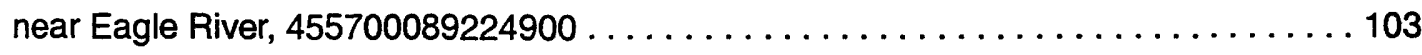

Oconomowoc

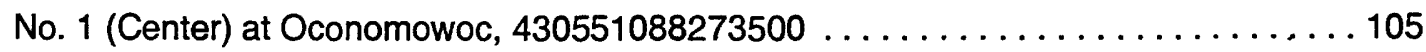

No. 2 (off Hewitt Point) at Oconomowoc, $430609088262200 \ldots \ldots \ldots \ldots \ldots \ldots$. . . . . . 107

Okauchee

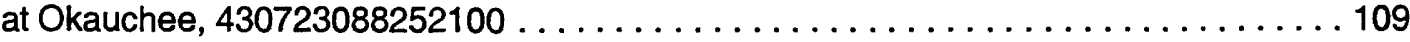

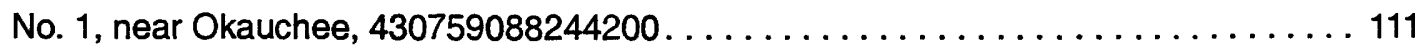

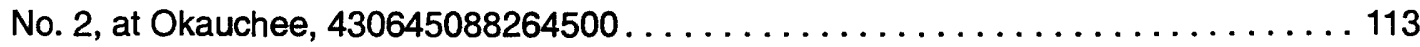

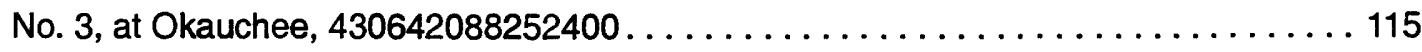

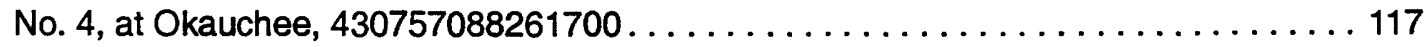

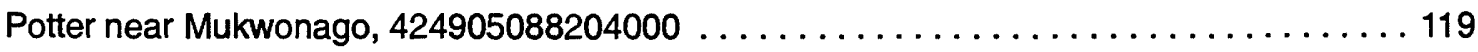

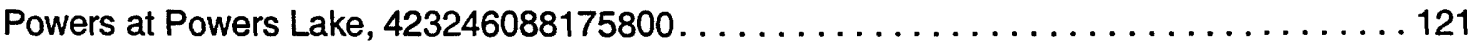

Red Cedar, at Mikana, $453522091360600 \ldots \ldots \ldots \ldots \ldots \ldots \ldots \ldots \ldots \ldots \ldots$

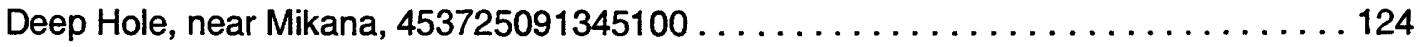

South End, at Mikana, $453519091352500 \ldots \ldots \ldots \ldots \ldots \ldots \ldots \ldots \ldots \ldots$

Whitefish

North Basin, near Gordon, $461321091520900 \ldots \ldots \ldots \ldots \ldots \ldots \ldots$. . . . . . . . . 130

South Basin, near Gordon, $461212091523200 \ldots \ldots \ldots \ldots \ldots \ldots \ldots \ldots \ldots \ldots$ 


\section{CONTENTS--Continued}

Whitewater near Whitewater, $424608088414800 \ldots \ldots \ldots \ldots \ldots \ldots \ldots \ldots \ldots \ldots$

Wind, at Outlet at Wind Lake, 424848088083100138

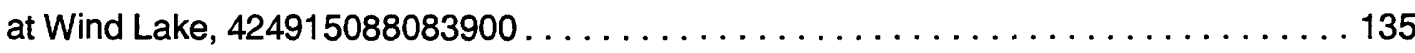

Winnebago

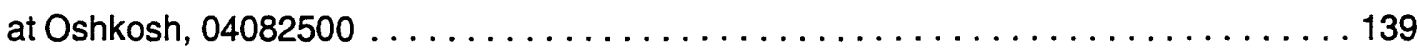

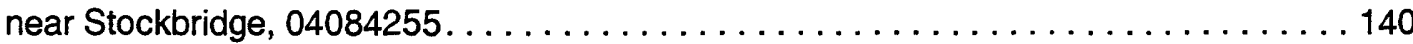

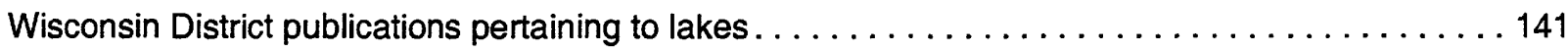

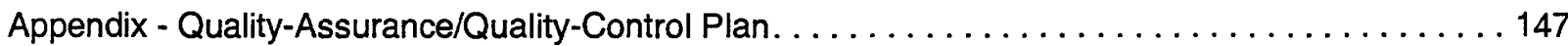

\section{FIGURE}

Figure 1. Map showing location of lake water-quality and lake-stage stations in Wisconsin $\ldots \ldots \ldots 2$

\section{TABLES}

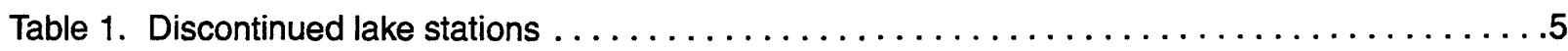

2. Parameter identification numbers and laboratory reporting levels (LRL) for chemical parameters commonly measured in lakes, and analyzed at the National

Water-Quality Laboratory or the Wisconsin State Laboratory of Hygiene 


\begin{tabular}{rcl}
\hline Multiply & By & To Obtain \\
\hline mile (mi) & 1.609 & kilometer \\
pound (lb) & 453.6 & gram \\
acre & 0.4048 & hectare \\
foot (ft) & 0.3048 & meter \\
gallon $(\mathrm{gal})$ & 3.785 & liter \\
square mile $\left(\mathrm{mi}^{2}\right)$ & 2.590 & square kilometer
\end{tabular}

Temperature, in degrees Celsius $\left({ }^{\circ} \mathrm{C}\right)$ can be converted to degrees Fahrenheit $\left({ }^{\circ} \mathrm{F}\right)$ by use of the following equation:

$$
{ }^{\circ} \mathrm{F}=1.8\left({ }^{\circ} \mathrm{C}\right)+32 .
$$

Sea level: In this report "sea level" refers to the National Geodetic Vertical Datum of 1929 (NGVD of 1929)-a geodetic datum derived from a general adjustment of the first-order level nets of both the United States and Canada, formerly called Sea Level Datum of 1929.

\begin{abstract}
Abbreviated water-quality units: Chemical concentrations and water temperature are given in metric units. Chemical concentration is given in milligrams per liter $(\mathrm{mg} / \mathrm{L})$ or micrograms per liter $(\mu \mathrm{g} / \mathrm{L})$. Milligrams per liter is a unit expressing the concentration of chemical constituents in solution as weight (milligrams) of solute per unit volume (liter) of water. One thousand micrograms per liter is equivalent to one milligram per liter. For water with dissolved-solids concentrations less than $7,000 \mathrm{mg} / \mathrm{L}$, the numerical values for concentrations expressed as $\mathrm{mg} / \mathrm{L}$ and $\mu \mathrm{g} / \mathrm{L}$ are the same as for concentrations in parts per million and parts per billion, respectively.
\end{abstract}

Specific conductance of water is expressed in microsiemens per centimeter at 25 degrees Celsius $(\mu \mathrm{S} / \mathrm{cm})$. This unit is equivalent to micromhos per centimeter at 25 degrees Celsius $(\mu \mathrm{mho} / \mathrm{cm})$, formerly used by the U.S. Geological Survey. 


\title{
WATER-QUALITY AND LAKE-STAGE DATA FOR WISCONSIN LAKES, WATER YEAR 2001
}

\author{
By Wisconsin District Lake-Studies Team
}

\section{INTRODUCTION}

The U.S. Geological Survey (USGS), in cooperation with local and other agencies, collects data at selected lakes throughout Wisconsin. These data, accumulated over many years, provide a data base for developing an improved understanding of the water quality of lakes. To make these data available to interested parties outside the USGS, the data are published annually in this report series. The locations of water-quality and lake-stage stations in Wisconsin for water year 2001 are shown in figure 1. A water year is the 12-month period from October 1 through September 30. It is designated by the calendar year in which it ends. Thus, the period October 1 , 2000 through September 30, 2001 is called "water year 2001."

The purpose of this report is to provide information about the chemical and physical characteristics of Wisconsin lakes. Data that have been collected at specific lakes, and information to aid in the interpretation of those data, are included in this report. Data collected include measurements of in-lake water quality and lake stage. Time series of Secchi depths, surface total phosphorus and chlorophyll a concentrations collected during non-frozen periods are included for all lakes. Graphs of vertical profiles of temperature, dissolved oxygen, $\mathrm{pH}$, and specific conductance are included for sites where these parameters were measured. Descriptive information for each lake includes: location of the lake, area of the lake's watershed, period for which data are available, revisions to previously published records, and pertinent remarks. Additional data, such as streamflow and water quality in tributary and outlet streams of some of the lakes, are published in another volume: "Water Resources Data-Wisconsin, 2001."

Water-resources data, including stage and discharge data at most streamflow-gaging stations, are available throught the World Wide Web on the Internet. The Wisconsin District's home page is at http://wi.water.usgs.gov/. Information on the Wisconsin District's Lakes Program is found at wi.water.usgs.gov/lake/index.html. 


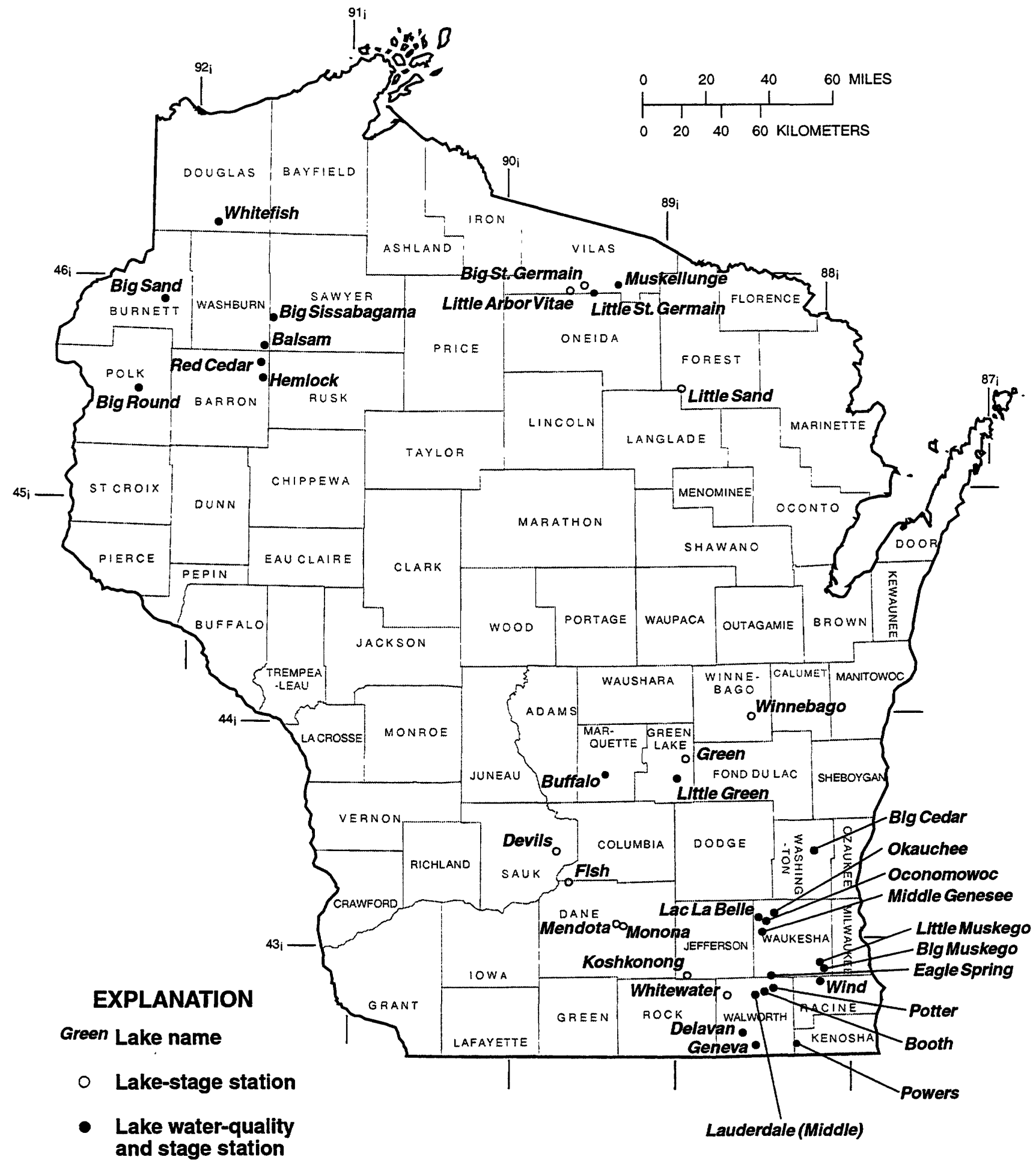

Note: at some lakes more than one site may be monitored.

Figure 1. Location of lake water-quality and lake-stage stations in Wisconsin. 
The USGS has done cooperative lake monitoring with local and other agencies since 1983. Cooperators in 2001 included:

Big Cedar Lake Protection and Rehabilitation District

Big Muskego Lake District

Booth Lake Management District

Buffalo Lake District

City of Muskego

Dane County Department of Public Works

Eagle Spring Lake Management District

Geneva Lake Environmental Agency

Green Lake Sanitary District

Lac La Belle Management District

Lauderdale Lakes Lake District

Little Green Lake Protection and Rehabilitation District

Little Muskego Lake Protection and Rehabilitation District

Little St. Germain Lake Protection and Rehabilitation District (Muskellunge Lake Association)

Middle Genesee Lake District

Okauchee Lake Management District

Potters Lake Protection and Rehabilitation District

Powers Lake District

Rock County Public Works Department

St. Croix Tribe

Town of Cedar Lake (Red Cedar Lake Association)

Town of Delavan (Delavan Lake)

Town of Sand Lake (Big Sissabagama Lake Association)

Town of Wascott (Whitefish Lake Association)

U.S. Army Corps of Engineers

Village of Oconomowoc Lake

Whitewater Lake Management District

Wind Lake Management District

Wisconsin Department of Natural Resources 
Lake data-collection sites are identified by a unique identification number. Lake waterquality sites are identified by a 15-digit number that is a concatenation of the site's latitude, longitude, and a two-digit sequence number. The sequence number is used to distinguish between sites located at the same latitude-longitude designation. The site identification number is permanently assigned to the site; actual latitude and longitude of the site are subject to update and are stored separately. For some lakes, which have historical records of lake stage, an eightto-ten digit number is assigned according to downstream order. Gaps are left in the numerical series to allow for new stations; hence, the numbers are not consecutive. The first two digits of the complete eight-to-ten digit number, such as 04087000 or 054310157 , designate the major river basin. For example, "04" designates the St. Lawrence River Basin and "05" designates the Upper Mississippi River Basin.

The water-quality lake stations that were discontinued prior to water year 2001 are listed in table 1. Discontinued lake-stage stations are not included in this table.

This report is the culmination of a concerted effort by a number of people who collected, compiled, analyzed, verified, and organized the data, and who typed, edited, and assembled the report. The authors had primary responsibility for assuring that the information contained herein is accurate, complete, and adheres to USGS policy and established guidelines. Technicians in charge of the field offices are: T.J. Popowski (Rice Lake), J.J. Hanig (Merrill), and J. Habale (Middleton). The data were collected and processed by S.M. Berg, G.L. Goddard, J.J. Hanig, D.E. Housner, E.A. Mergener, D.L. Olson, R.L. Rewey, E.D. Roerish, and J.G. Schuler. P.A. Stark assembled, edited, and formatted the report. Additional assistance in preparation of the report was provided by M.M. Greenwood.

\section{METHODS OF DATA COLLECTION}

Depth profiles of water temperature, dissolved oxygen, $\mathrm{pH}$, and specific conductance were collected using multi-parameter meters. Prior to measurements, the meters were calibrated using standards for $\mathrm{pH}$ and conductance, and dissolved oxygen was calibrated using the air calibration method. Generally, field measurements in profiles were made at $0.5-\mathrm{m}$ intervals if the maximum depth of the lake was $5 \mathrm{~m}$ or less and at 1.0-m intervals if the maximum depth was greater than $5 \mathrm{~m}$. 
Table 1. Discontinued lake stations

\begin{tabular}{lr}
\hline \multicolumn{1}{c}{ Station name } & Site identification numb \\
\hline Alma Lake near St. Germain & 455426089254700 \\
& \\
Balsam Lake, off Cedar Island, at Balsam Lake & 452755092264600 \\
off Little Narrows, near Balsam Lake & 452858092265300 \\
off Rock Island, near Balsam Lake & 452754092234300 \\
Bass Lake near Shawano & 445215088300300 \\
Bear Lake at Deep Hole near Haugen & 453754091490900 \\
Beaver Dam Lake, South end, at Beaver Dam & 432814088515000 \\
North end, near Beaver Dam & 433122088545700 \\
Benedict Lake near Powers Lake & 423201088180800 \\
Big Blacksmith Lake near Keshena & 445401088334500 \\
Big Hills (Hills) Lake near Wild Rose & 440912089092000
\end{tabular}

Big Muskego Lake, at North Site, near Muskego

425301088061300

Research Base, near Muskego

Big St. Germain Lake near St. Germain

Denoon Lake at Wind Lake

Druid Lake near Hartford

Eagle Lake near Kansasville

Eagle Lake, at Deep Hole, near Kansasville

Elizabeth Lake near Twin Lakes

Forest Lake near Dundee

Fowler Lake, Center, at Oconomowoc

Fox Lake Deep Hole at Fox Lake

Geneva Lake

Geneva Bay, at Lake Geneva

Williams Bay, at Williams Bay

Center, near Lake Geneva

East End, near Lake Geneva

Hooker Lake at Salem

Kirby Lake near Cumberland

(Site 1) near Cumberland

(Site 2) near Cumberland

(Site 3) near Cumberland

(Site 4) near Cumberland

(Site 5) near Cumberland

(Site 6) near Cumberland

Lac La Belle, NW, at Oconomowoc

SE at Oconomowoc
425235088075300

455557089311000

425044088100300

431643088243300

05544500

424207088072400

423051088155300

433632088100200

433458088560600

423455088263800

423420088320500

423402088301400

423421088272300

423335088060300

453554092042101

453608092035801

453601092035301

453612092034901

453603092035701

453608092041201

453555092040901

430809088313900

430707088301400
430653088294601
Period of record

Oct. 1984-Sept. 1990,

May 1992-Sept.1996

Feb. 1991-Aug. 1994

May 1991-Aug. 1994

May 1991-Aug. 1994

Feb. 1990-Aug. 1992

Mar. 1992-Aug. 1993

June-Oct. 1991

June-Oct. 1991

May 1998-Aug. 2000

Feb. 1990-Aug. 1992

June 1983-Aug. 1984,

Feb.-Aug. 1987,

Feb.-Aug. 1990,

Feb.-Aug. 1993,

Feb.-Aug. 1996,

Feb.-Aug. 1999

Feb.-Aug. 1988

May-June 1994

Feb. 1992-Aug. 1996

Feb. 1991-Aug. 1996

Feb. 1991-Sept. 1996

1936-64, 1975-77, 1979,

Feb. 1993-Sept. 1996

Feb. 1993-Aug. 1996

Feb. 1995-Sept. 1997

Mar. 1994-Aug. 1996

Jan.-Dec. 1984,

Oct. 1986-Sept. 1996

June 1991-Mar. 1993

Apr. 1997-Feb. 1999

Apr. 1997-Feb. 1999

Apr. 1997-Mar. 1999

Apr. 1997-May 2000

Feb. 1992-Aug. 1993

Nov. 1995-Oct. 1996

Nov. 1995-Nov. 1996

Nov. 1995-Nov. 1996

Nov. 1995-Nov. 1996

Nov. 1995-Nov. 1996

Nov. 1995-Nov. 1996

Nov. 1995-Nov. 1996

Feb. 1984-Aug. 1985

Feb. 1984-Aug. 1985 
Table 1. Discontinued lake stations

\begin{tabular}{|c|c|c|}
\hline Station name & Site identification number & Period of record \\
\hline Lake Blass at Lake Delton & 433545089482400 & Mar. 1989-Aug. 1990 \\
\hline Lake Keesus, East Bay, near Merton & 430957088183400 & Apr. 1991-Aug. 1995 \\
\hline North Bay, near Merton & 431006088191000 & Apr. 1991-Aug. 1995 \\
\hline Lake Morris at Mount Morris & 440654089120500 & Jun. 1983-Sept. 1989 \\
\hline Lake Nebagamon, Northeast Bay, at Lake Nebagamon & 463050091412300 & May 1992-Aug. 1995 \\
\hline Southeast Bay, at Lake Nebagamon & 462928091413500 & Mar. 1992-Sept. 1995 \\
\hline West Bay, at Lake Nebagamon & 463034091425300 & May 1992-Aug. 1995 \\
\hline Lake Noquebay near Crivitz & 451511087550900 & $\begin{array}{l}\text { Feb. 1987-Aug. } 1988 \text {, } \\
\text { Apr. 1991-Aug. } 1994\end{array}$ \\
\hline East End, near Crivitz & 451540087525700 & Apr. 1991-Aug. 1994 \\
\hline Lamotte Lake near Shawano & 445305088361200 & Feb. 1990-Aug. 1992 \\
\hline \multicolumn{3}{|l|}{ Lauderdale Lakes } \\
\hline at Lauderdale & 424554088332700 & Oct. $1993-O c t .1994$ \\
\hline Green near Lauderdale & 424652088341500 & Nov. 1993-Nov. 1994 \\
\hline Green, Auxiliary, Number 1, near Lauderdale & 424640088341900 & June 1999-Sept. 2000 \\
\hline Mill at Lauderdale & 424555088335700 & Nov. 1993-Nov. 1994 \\
\hline Legend Lake (site 1) near Shawano & 445342088312700 & Feb. 1990-Feb. 1992 \\
\hline \multicolumn{3}{|l|}{ Little Cedar Lake } \\
\hline North Site, near West Bend & 432255088134700 & Feb. 1997-Aug. 1999 \\
\hline South Site, near West Bend & 432249088134500 & Feb. 1997-Aug. 1999 \\
\hline Little Rock Lake near Woodruff & 455946089415702 & Oct. 1983-Sept. 1996 \\
\hline Long (Kee Nong Go-Mong) Lake at Wind Lake & 424937088103400 & $\begin{array}{l}\text { Feb. 1988-Aug. } 1989, \\
\text { Feb. 1991-Aug. } 1996\end{array}$ \\
\hline Loon Lake near Shawano & 445009088303700 & Feb. 1991-Aug. 1993 \\
\hline Lost Lake near Beaver Dam & 432640088580500 & June-Oct. 1991 \\
\hline \multicolumn{3}{|l|}{ McKenzie Lakes } \\
\hline \multicolumn{3}{|l|}{ McKenzie (Big McKenzie) } \\
\hline Deep Hole, near Spooner & 455507092013500 & Feb. 1987-Aug. 1998 \\
\hline Northern Site, near Spooner & 455540092022000 & June 1997-Aug. 1998 \\
\hline South Site, near Spooner & 455437092022300 & June 1997-Aug. 1998 \\
\hline Lower McKenzie, near Webb Lake & 455902092011900 & June 1997-Aug. 1998 \\
\hline Middle McKenzie, near Spooner & 455635092021800 & June 1997-Aug. 1998 \\
\hline Mary (Marie) Lake at Twin Lakes & 423128088151200 & Feb. 1995-Aug. 1997 \\
\hline Max Lake near Woodruff & 460128089423501 & Mar. 1988-Dec. 1996 \\
\hline Mead Lake, East Bay near Willard & 444720090445000 & Apr. 1991-Aug. 1995 \\
\hline West Bay near Willard & 444733090460100 & Feb. 1991-Sept. 1995 \\
\hline Montello Lake at Montello & 434748089195800 & Feb. 1995-Aug. 1998 \\
\hline Moon Lake near St. Germain & 455504089260500 & Feb. 1992-Aug. 1996 \\
\hline Morgan Lake near Fence & 454622088324801 & Oct. 1987-Sept. 1998. \\
\hline Moshawquit Lake near Shawano & 445352088295800 & Feb. 1990-Aug. 1992 \\
\hline \multicolumn{3}{|l|}{ Muskego (Big Muskego) } \\
\hline & & Jan. 1991-Sept. 2000 \\
\hline Auxiliary Number 1, near Muskego & 425329088054000 & June 1996-Aug. 2000 \\
\hline
\end{tabular}


Table 1. Discontinued lake stations

\begin{tabular}{|c|c|c|}
\hline Station name & Site identification number & Period of record \\
\hline \multicolumn{3}{|l|}{ Namekagon Lakes } \\
\hline Garden, near Cable & 461224091033200 & Mar. 1998-Aug. 1999 \\
\hline Jackson, near Cable & 461457091065900 & Mar. 1998-Aug. 1999 \\
\hline \multicolumn{3}{|l|}{ Namekagon } \\
\hline Deep Hole, near Cable & 461308091065100 & Mar. 1998-Aug. 1999 \\
\hline East Basin, near Cable & 461228091044300 & Mar. 1998-Aug. 1999 \\
\hline Northeast Basin, near Cable & 461410091050700 & Mar. 1998-Aug. 1999 \\
\hline Park Lake (site 1) at Pardeeville & 433239089175800 & $\begin{array}{l}\text { Feb. } 1986-\text { Aug. } 1987 \\
\text { May-Nov. } 1993\end{array}$ \\
\hline (site 2) at Pardeeville & 433226089175500 & May-Nov. 1993 \\
\hline (site 3) at Pardeeville & 433245089173000 & May-Nov. 1993 \\
\hline (site 4) at Pardeeville & 433257089165100 & May-Nov. 1993 \\
\hline Pike Lake near Hartford & 431835088200600 & Dec. 1998-Nov. 2000 \\
\hline Pretty Lake, at Deep Hole, near Dousman & 425722088295000 & Feb. 1993-Aug. 1997 \\
\hline Rice Lake at Deep Hole near Whitewater & 424629088415700 & Apr.-Nov. 1991 \\
\hline Round Lake near Shawano & 445328088335000 & Feb. 1990-Aug. 1992 \\
\hline Sand Lake (Deep Hole) near Keshena & 445321088323101 & June-Aug. 1992 \\
\hline Shell Lake at Shell Lake & 05334000 & Aug. 1936-Sept. 1999 \\
\hline Silver Lake near Oconomowoc & 430436088293300 & Apr. 1992-Aug. 1996 \\
\hline Silver Lake near West Bend & 432322088125000 & Feb. 1996-Aug. 1997 \\
\hline Sinissippi Lake, off Anthony Is., at Hustisford & 432113088361100 & Feb. 1991-Aug. 1993 \\
\hline off Butternut Is., near Hustisford & 432240088363900 & Apr. 1991-Aug. 1993 \\
\hline off Sam Point, near Hustisford & 432300088374200 & Apr. 1991-Aug. 1993 \\
\hline Spirit Lake near Keshena & 445400088320100 & Apr.-Aug. 1992 \\
\hline Stewart Lake at Mt. Horeb & 430117089442701 & May 1992-Sept. 1993 \\
\hline Tichigan Lake near Waterford & 424854088123300 & Mar. 1994-Aug. 1996 \\
\hline Tombeau Lake near Powers Lake & 423153088184800 & May 1998-Aug. 2000 \\
\hline Upper Nemahbin Lake, Center, near Delafield & 430400088254900 & June 1993-Aug. 1995 \\
\hline South Site, near Delafield & 430339088254800 & June 1993-Aug. 1995 \\
\hline Outlet near Delafield & 430334088255400 & June 1993-Aug. 1995 \\
\hline Vandercook Lake near Woodruff & 455909089405602 & Nov. 1980-Aug. 1998 \\
\hline Watosah-skice Lake near Keshena & 445330088361400 & Feb. 1990-Aug. 1992 \\
\hline Waubeesee Lake at Wind Lake & 424857088101500 & $\begin{array}{l}\text { Feb. 1988-Aug. 1989, } \\
\text { Feb. 1991-Aug. } 1996\end{array}$ \\
\hline Wazee Lake near Black River Falls & 441721090431700 & Nov. 1999-Aug. 2000 \\
\hline Whitewater Lake, off Heart Prairie, near Whitewater & 424533088420100 & Apr.-Nov. 1991 \\
\hline near Whitewater & 424608088414800 & Apr.-Oct. 1991 \\
\hline North Bay, near Whitewater & 424625088405500 & Apr.-Nov. 1991 \\
\hline South Bay, near Whitewater & 424501088422300 & Apr.-Nov. 1991 \\
\hline Wind Lake, Northeast Basin, at Wind Lake & 424938088080800 & Feb. 1997-Aug. 1998 \\
\hline Wolf Lake near Mt. Calvary & 435152088123100 & $\begin{array}{l}\text { Nov. } 1983-S e p t .1986, \\
\text { Nov. 1992-Sept. } 1997 \\
\end{array}$ \\
\hline
\end{tabular}


In most lakes, water samples were collected at two depths - near the surface and near the bottom. Chemical analyses of water samples were performed using standard analytical methods by either the USGS National Water Quality Laboratory (Wershaw and others, 1987; Fishman and Friedman, 1989; Fishman, 1993) or the Wisconsin State Laboratory of Hygiene (Wisconsin State Laboratory of Hygiene, 1993). Analyses for dissolved constituents were performed on samples that were filtered in the field through a $0.45-\mu \mathrm{m}$ (micrometer) pore-size filter. Total or total recoverable constituents were determined by analyzing unfiltered water samples. Preservation and shipment of samples followed standard protocols established by the laboratories. Water-quality data were archived in the Water Quality Data Base (QWDATA) of the National Water Information System (NWIS). Additional descriptive information about water-quality data is available in the data report: "Water Resources Data - Wisconsin, 2001". NWIS parameter codes and minimum laboratory reporting levels for chemical constituents are given in table 2.

Records of lake stage are considered complete when one or more manual or automatic measurements were obtained per day. Partial records of lake stage result when measurements were less frequent than daily. A complete description of manual or automatic measurements of lake stage is described by Rantz and others (1982).

\section{EXPLANATION OF PHYSICAL AND CHEMICAL CHARACTERISTICS OF LAKES}

Following are brief, generalized explanations of some of the common measurements of water quality and some of the physical processes occurring in lakes that influence these measures of water quality. More detailed explanations of water-quality data and lake processes are given by Wetzel (1983), Hem (1985), and Shaw and others (1993).

\section{Water Temperature and Thermal Stratification}

Water temperature in lakes is important because of its role in stratification and because of the temperature dependence of many chemical reactions and life processes of aquatic organisms. The extent of thermal stratification in lakes depends on the interaction between the lake's shape, water clarity, solar heating, and wind-driven mixing. Complete mixing of the lake is usually inhibited by thermal stratification in summer and by ice cover in winter. Thermal stratification affects water quality and the distribution of organisms in the lake. Summer thermal stratification can occur in any lake, but in Wisconsin it commonly occurs in lakes deeper than about $6 \mathrm{~m}$ (Shaw and others, 1993). 
Table 2. Parameter identification numbers and laboratory reporting levels (LRL) for chemical parameters commonly measured in lakes, and analyzed at the National Water Quality Laboratory (NWQL) or the Wisconsin State Laboratory of Hygiene (WSLH)

\begin{tabular}{|c|c|c|c|c|c|c|c|c|c|}
\hline \multirow[b]{3}{*}{ Parameter Name } & \multirow[b]{3}{*}{ Units } & \multirow[b]{3}{*}{$\begin{array}{c}\text { CAS } \\
\text { Number } \\
\text { (1) }\end{array}$} & \multirow[b]{3}{*}{$\begin{array}{l}\text { Parameter } \\
\text { Code } \\
\text { (2) }\end{array}$} & \multicolumn{4}{|c|}{ (NWQL) } & \multicolumn{2}{|c|}{ (WSLH) } \\
\hline & & & & \multicolumn{2}{|c|}{ Standard Analysis } & \multicolumn{2}{|c|}{ Low-Level Analysis } & \multirow[b]{2}{*}{ LRL } & \multirow[b]{2}{*}{$\begin{array}{l}\text { Test } \\
\text { Code }\end{array}$} \\
\hline & & & & LRL & $\begin{array}{l}\text { Lab } \\
\text { Code }\end{array}$ & LRL & $\begin{array}{l}\text { Lab } \\
\text { Code }\end{array}$ & & \\
\hline Calcium, diss. (Ca) & $\mathrm{mg} / \mathrm{L}$ & $7440-70-2$ & 00915 & 0.020 & 659 & 0.002 & 1895 & 0.02 & I23OIUD \\
\hline Magnesium, diss. (Mg) & $\mathrm{mg} / \mathrm{L}$ & $7439-95-4$ & 00925 & 0.004 & 663 & 0.001 & 1897 & 0.02 & I390IUD \\
\hline Sodium, diss. (Na) & $\mathrm{mg} / \mathrm{L}$ & $7440-23-5$ & 00930 & 0.09 & 675 & 0.025 & 1898 & 0.09 & I80IUD \\
\hline Potassium, diss. (K) & $\mathrm{mg} / \mathrm{L}$ & $7440-09-7$ & 00935 & 0.24 & 54 & 0.01 & 833 & 0.3 & I540IUD \\
\hline Sulfate, diss. $\left(\mathrm{SO}_{4}\right)$ & $\mathrm{mg} / \mathrm{L}$ & $14808-79-8$ & 00945 & 0.31 & 1572 & 0.01 & 1263 & 1.0 & I600DLD \\
\hline Chloride, diss. (Cl) & $\mathrm{mg} / \mathrm{L}$ & $16887-00-6$ & 00940 & 0.29 & 1571 & 0.01 & 1259 & 0.1 & 1240ELD \\
\hline Fluoride, diss. (F) & $\mathrm{mg} / \mathrm{L}$ & $16984-48-8$ & 00950 & 0.100 & 31 & 0.01 & 1260 & 0.03 & I330FLD \\
\hline Iron; diss. (Fe) & $\mu g / L$ & $7439-89-6$ & 01046 & 10 & 645 & 3 & 1896 & 10 & I370IUD \\
\hline Manganese, diss. (Mn) & $\mu \mathrm{g} / \mathrm{L}$ & $7439-96-5$ & 01056 & 2.2 & 648 & 1 & 1793 & 0.4 & I400IUD \\
\hline Silica, diss. $\left(\mathrm{SiO}_{2}\right)$ & $\mathrm{mg} / \mathrm{L}$ & $7631-86-9$ & 00955 & 0.1 & 56 & 0.02 & 1899 & 0.008 & I560LLD \\
\hline Nitrogen, $\mathrm{NO}_{2}+\mathrm{NO}_{3}$, diss. & $\mathrm{mg} / \mathrm{L}$ & & 00631 & 0.05 & 1975 & 0.005 & 1979 & 0.01 & I460MLD \\
\hline Nitrogen, ammonia, diss. & $\mathrm{mg} / \mathrm{L}$ & $7664-41-7$ & 00608 & 0.02 & 1976 & 0.002 & 1980 & 0.013 & 1440NLD \\
\hline Nitrogen, organic, total (3) & $\mathrm{mg} / \mathrm{L}$ & & & & & & & & \\
\hline Nitrogen, amm.+org., total (4) & $\mathrm{mg} / \mathrm{L}$ & $17778-88-0$ & 00625 & 0.100 & 1985 & & & 0.2 & I470BLT \\
\hline Nitrogen, total (5) & $\mathrm{mg} / \mathrm{L}$ & & & & & & & & \\
\hline Phosphorus, total & $\mathrm{mg} / \mathrm{L}$ & $7723-14-0$ & 00665 & 0.05 & 1984 & 0.001 & 837 & 0.005 & I520PLT \\
\hline Phosphorus, ortho, diss. & $\mathrm{mg} / \mathrm{L}$ & $14265-44-2$ & 00671 & 0.01 & 1262 & 0.001 & 1978 & 0.002 & 1530CLD \\
\hline Chlorophyll a, phytoplankton & $\mu \mathrm{g} / \mathrm{L}$ & $479-61-8$ & 70953 & 0.1 & 586 & & & & \\
\hline Chlorophyll a, phytoplankton & $\mu g / L$ & $479-61-8$ & 32210 & & & & & 1 & I250UNF \\
\hline
\end{tabular}

Footnotes:

1: CAS (Chemical Abstracting Services) number = unique identification for each constituent

2: Parameter Code - unique number for storage of data in database

3: Calculated as difference between total ammonia + organic nitrogen and ammonia nitrogen

4: Also known as Total Kjeldahl Nitrogen (TKN)

5: Calculated as sum of TKN + Nitrogen as $\left(\mathrm{NO}_{2}+\mathrm{NO}_{3}\right)$ 
The density of water increases with decreasing temperature down to a temperature of $4^{\circ} \mathrm{C}$, then decreases with decreasing temperature between $4^{\circ} \mathrm{C}$ and the freezing point of water $\left(0^{\circ} \mathrm{C}\right)$. For a brief period in the spring after the ice is out, water temperature is usually uniform through the entire water column and wind action causes the lake to mix completely. This process is known as "spring turnover." As the lake absorbs the sun's energy, the surface water becomes warmer and its density decreases, making it more resistant to complete mixing. The difference in density caused by different water temperatures can prevent warm and cold water from mixing. In most lakes, therefore, a density "barrier" forms between the warmer surface water (epilimnion) and the underlying colder water (hypolimnion). This barrier is often marked by a sharp temperature gradient known as the "thermocline (metalimnion)." During the stratified summer period, these three distinct layers of lake water are often present. As the temperature difference between surface and deep water increases, this "stratified" condition stabilizes and can persist until surface temperatures decrease in the fall, which decreases the stability of the stratification. The mixing of the lake water in the fall is known as "fall turnover."

Thermal stratification may also occur under ice cover in the winter. In the winter, the coldest water (near $0^{\circ} \mathrm{C}$ ) under the ice at the surface of the lake is less dense than water deeper in the lake with warmer temperatures.

\section{Specific Conductance}

Specific conductance is a measure of the ability of water to conduct an electrical current and is an indicator of the concentration of dissolved solids in the water. Because conductance is temperature related, reported values are normalized at $25^{\circ} \mathrm{C}$ and are termed specific conductance. As the concentration of dissolved minerals increases, specific conductance increases. During winter and summer thermal stratification, concentrations of dissolved constituents near the lake bottom increase due to the decomposition of materials settling from the epilimnion, or release of dissolved materials (such as iron, manganese, and phosphorus) from the bottom sediments during anoxic periods. Therefore, differences in specific conductance with depth indicate differences in concentrations of dissolved solids. 


\section{Water Clarity}

Water clarity, or transparency, is commonly measured using a Secchi disc. The range of depths within which photosynthetic activity occurs depends largely on depth of light penetration, which is influenced by water clarity. A Secchi disc, most commonly an 20-cm.-diameter disc with alternating black-and-white quadrants, is lowered to a depth at which it is no longer visible. This depth is referred to as the Secchi depth. Clarity can be reduced by algae, zooplankton, water color, and suspended sediment. Algae are often the most dominant influence on clarity in lakes and, therefore, Secchi depth is usually correlated with the algal abundance. Secchi depths are generally the least during summer when algal populations are largest.

\section{pH}

The $\mathrm{pH}$ is a measure of the acidity of the water. It is defined as the negative logarithm of hydrogen-ion concentration and varies over a 14-unit log scale, with a pH of 7 being neutral. Values less than 7 indicate acidic conditions; the lower the value, the stronger the acidity. Values greater than 7 indicate alkaline conditions. The $\mathrm{pH}$ of water is influenced in part by photosynthesis and respiration of planktonic algae and aquatic plants. It is important because it affects the solubility of many chemical constituents, and because aquatic organisms have limited $\mathrm{pH}$ tolerances. Planktonic algae and aquatic plants produce oxygen and consume carbon dioxide as they photosynthesize during daytime; they consume oxygen and produce carbon dioxide when they respire at night. Carbon dioxide combines with the water molecule to form carbonic acid; therefore respiration causes a decrease in $\mathrm{pH}$ at night and photosynthesis during the day causes an increase in $\mathrm{pH}$. The result is a daily cycle in $\mathrm{pH}$. Because phytoplankton are usually concentrated in the near-surface water, changes in $\mathrm{pH}$ in the epilimnion are more extreme than in the hypolimnion, where less photosynthesis usually occurs.

Lakes having good fish populations and productivity generally have a pH between 6.7 and 8.2. Values of $\mathrm{pH}$ greater than 8.5 have been shown to cause the release of phosphorus from lake sediments (James and Barko, 1991). 


\section{Dissolved Oxygen}

Dissolved oxygen is one of the most critical factors affecting a lake ecosystem because it is essential to most aquatic organisms, and it is involved in many chemical reactions. Very low dissolved oxygen concentrations can control some types of chemical reactions. The solubility of oxygen in water is inversely related to temperature-that is, oxygen solubility decreases as water temperature increases. This relation is important because at warmer temperatures the metabolic rate of organisms increases but less oxygen is available for respiration. The primary sources of dissolved oxygen are from the air and from photosynthesis. The minimum dissolved oxygen concentration specified in national water-quality criteria for early life stages of warmwater aquatic life is 5.0 $\mathrm{mg} / \mathrm{L}$ (U.S. Environmental Protection Agency, 1986).

In early summer, if thermal stratification develops, the metalimnion restricts the surface supply of dissolved oxygen to the hypolimnion. The hypolimnion can become isolated from the atmosphere. Thus, as summer progresses, the dissolved oxygen concentration can decrease in response to decomposition of dead algae that settle from the epilimnion and in response to the biological and chemical oxygen demand of the sediments. The oxygen demand from these processes may completely deplete the oxygen (anoxia) in the water near the lake bottom. The oxygen depletion then progresses upward but usually is confined to the hypolimnion.

Anoxia in the hypolimnion is common in stratified eutrophic (nutrient-rich) lakes in Wisconsin. Complete anoxia, however, is often not detected because of meter constraints. During anoxic conditions, many aquatic organisms cannot survive, but many other species (primarily bacteria) actually function only in such conditions. Therefore, a shift from oxic to anoxic conditions produces a rapid and dramatic change in the biological community and chemical environment. Anoxia also can cause release of phosphorus from the bottom sediments. This phosphorus then mixes throughout the water column during spring and fall turnover. 


\section{Phosphorus}

Phosphorus is one of the essential nutrients for plant growth. High phosphorus concentrations can cause dense algal populations (blooms) and can therefore be a major cause of eutrophication in lakes. When phosphorus concentrations exceed $0.025 \mathrm{mg} / \mathrm{L}$ at the time of spring overturn in lakes and reservoirs, these water bodies may occasionally experience excess or nuisance growth of algae or other aquatic plants (U.S. Environmental Protection Agency, 1986). In many regions of the country, including the upper Midwest, other nutrients, particularly nitrogen, tend to be in abundant supply. Phosphorus is often the nutrient in shortest supply, therefore limiting or controlling plant growth. About 90 percent of the lakes in Wisconsin are limited by phosphorus (Shaw and others, 1993). In water, dissolved orthophosphate is that part of total phosphorus that is most readily available for use by algae.

Internal phosphorus recycling occurs in many lakes. Phosphorus used by algae, aquatic plants, fish, and zooplankton is stored within these organisms. As these organisms die and decompose, this phosphorus is returned to the lake water and sediments. Anoxia in the hypolimnion makes phosphorus more soluble, adding further to the release of phosphorus from the falling particles and the lake sediments. During spring and fall turnover the phosphorus, which was released from the bottom sediments into the hypolimnion during anoxia, is mixed throughout the lake. The phosphorus is then available for algal growth. These phenomena are part of the internal-recycling processes of lakes.

\section{Nitrogen}

Nitrogen, like phosphorus, is an essential nutrient for plant and algal growth. Usually in Wisconsin lakes, nitrogen is in abundant supply from the atmosphere and other sources. If phosphorus is abundant relative to algal needs, nitrogen can become the limiting nutrient. In that case, algal blooms are more likely to be triggered by increases in nitrogen than by increases in phosphorus. Some bluegreen algal species can fix nitrogen from the atmosphere (Wetzel, 1983). Therefore, in situations where other types of algae are excluded because of a shortage of nitrogen, the nitrogen-fixing bluegreen algae have a competitive advantage and may be present in abundance. 
Lakes with a nitrogen to phosphorus ratio larger than 15 to 1 near the surface may generally $b$ onsidered phosphorus limited; a ratio from 10 to 1 to 15 to 1 indicates a transition situation; a a ratio smaller than 10 to 1 generally indicates nitrogen limitation. Total nitrogen is the sum or ammonia, organic nitrogen, and nitrate-plus-nitrite nitrogen. The near-surface concentration is commonly used to compute the total nitrogen to phosphorus ratio because most algal species grow near the lake surface.

\section{Chlorophyll a}

Chlorophyll $a$ is a photosynthetic pigment found in algae (Wetzel, 1983) and other green plants. Its concentration, therefore, is commonly used as a measure of the density of the algal population in a lake. Chlorophyll a concentrations are generally highest during summer when algal populations are highest. Moderate populations of desirable algae are important in the food chain; however, excessive populations or algal blooms are undesirable. Algal blooms can cause taste and odor problems, and limit light penetration needed to support growth of submerged aquatic plants. Certain species of bluegreen algae can produce toxins (Rapavich and others, 1987).

\section{CLASSIFICATION OF LAKES}

Two methods are commonly used to classify and evaluate Wisconsin lakes according to their water quality or trophic state: Lillie and Mason's (1983) water-quality index and a modification of Carlson's (1977) Trophic State Index (TSI) by Lillie and others (1993). Three waterquality measures are used in these classification systems: near-surface concentrations of total phosphorus and chlorophyll $a$, and water clarity as indicated by the Secchi depth.

Lillie and Mason's (1983) water-quality indices for Wisconsin lakes were developed based on random summer measurements of total phosphorus and chlorophyll a concentrations, and Secchi depth to classify the lakes' water quality as shown below:

\begin{tabular}{lccc}
\hline $\begin{array}{c}\text { Water-quality } \\
\text { index }\end{array}$ & $\begin{array}{c}\text { Total phosphorus range } \\
(\mathrm{mg} / \mathrm{L})\end{array}$ & $\begin{array}{c}\text { Chlorophyll a range } \\
(\mu \mathrm{g} / \mathrm{L})\end{array}$ & $\begin{array}{c}\text { Water clarity range } \\
(\text { Secchi depth, in meters })\end{array}$ \\
\hline "Excellent" & $<0.001$ & $<1.0$ & $>6.0$ \\
"Very good" & $.001-.009$ & $1.0-4.9$ & $3.0-6.0$ \\
"Good" & $.010-.029$ & $5.0-9.9$ & $2.0-2.9$ \\
"Fair" & $.030-.049$ & $10.0-14.9$ & $1.5-1.9$ \\
"Poor" & $.050-.149$ & $15.0-30.0$ & $1.0-1.4$ \\
"Very poor" & $>.150$ & $>30.0$ & $<1.0$ \\
\hline
\end{tabular}


The TSI approach to lake classification assigns numerical ranges to the three trophic conditions generally used to describe the wide range of lake water-quality conditions. Oligotrophic lakes are typically clear, algal populations and phosphorus concentrations are low, and the deepest water is likely to contain oxygen throughout the year. Mesotrophic lakes typically have a moderate supply of nutrients, experience moderate algal blooms, and have occasional oxygen depletions at depth. Eutrophic lakes are nutrient rich with relatively severe water-quality problems, such as frequent seasonal algal blooms, oxygen depletion in lower parts of the lakes, and poor clarity. When eutrophic conditions are very severe, the lake is considered hypereutrophic.

The WDNR modified the lakes classification scheme developed by Carlson (1977) to apply specifically to Wisconsin lakes. The WDNR system (Lillie and others, 1993) uses surface total phosphorus and chlorophyll a concentrations, and Secchi depth for ice-free periods to calculate values for TSl's. The WDNR has adopted the following TSI ranges to classify Wisconsin lakes: indices of less than 40 define oligotrophic conditions, 40 to 50 define mesotrophic conditions, greater than 50 to define eutrophic conditions, and greater than 70 define hypereutrophic conditions (Wisconsin Department of Natural Resources, 1992). These ranges are used to make relative comparisons in Wisconsin lake trophic-state evaluations by the WDNR and others.

The TSI for a lake can be calculated using the following equations (Lillie and others, 1993):

$\mathrm{TSI}_{\text {Secchi }}=60.0-33.2 \times\left(\log _{10}\right.$ Secchi depth $)$

$\mathrm{TSI}_{\text {chlorophyll a }}=34.82+\left(17.41 \times\left(\log _{10}\right.\right.$ chlorophyll a concentration $\left.)\right)$

$\mathrm{TSI}_{\text {total phosphorus }}=28.24+\left(17.81 \times\left(\log _{10}\right.\right.$ total phosphorus concentration $\left.\left.\times 1,000\right)\right)$

where: Secchi depth is in meters, chlorophyll $a$ is in micrograms per liter, and total phosphorus is in milligrams per liter.

The three trophic conditions are defined with the following boundaries for total phosphorus, Secchi disc, and chlorophyll a:

\begin{tabular}{lcccc}
\hline $\begin{array}{l}\text { Trophic } \\
\text { Level }\end{array}$ & $\begin{array}{c}\text { Trophic State } \\
\text { Index }\end{array}$ & $\begin{array}{c}\text { Total phosphorus } \\
(\mathrm{mg} / \mathrm{L})\end{array}$ & $\begin{array}{c}\text { Secchi depth } \\
(\mathrm{m})\end{array}$ & $\begin{array}{c}\text { Chlorophyll a } \\
(\mu \mathrm{g} / \mathrm{L})\end{array}$ \\
\hline
\end{tabular}

Eutrophic $-50$ 0.017 $-2.0$ $-7.4$

Mesotrophic 40 0.005 4.0 $-2.0$

Oligotrophic 


\section{REFERENCES CITED}

Carlson, R.E., 1977, A trophic state index for lakes: Limnology and Oceanography, March, v. 22, no. 2, p. 361-369.

Fishman, M.J., ed., 1993, Methods of analysis by the U.S. Geological Survey National Water Quality Laboratory-Determination of inorganic and organic constituents in water and fluvial sediments: U.S. Geological Survey Open-File Report 93-125, 217 p.

Fishman, M.J., and Friedman, L.C., eds., 1989, Methods for determination of inorganic substances in water and fluvial sediments ( $3 d$ ed.): U.S. Geological Survey Techniques of Water-Resources Investigations, book 5, chap. A1, 545 p.

Hem, J.D., 1985, Study and interpretation of the chemical characteristics of natural water (3rd ed.): U.S. Geological Survey Water-Supply Paper 2254, 263 p.

James, W.F., and Barko, J.W., 1991, Littoral-pelagic phosphorus dynamics during nighttime convective circulation: Limnology and Oceanography, v. 36, no. 5, p. 946-960.

Lillie, R.A., Graham, S., and Rasmussen, P., 1993, Trophic-State Index equations and regional predictive equations for Wisconsin lakes: Wisconsin Department of Natural Resources Research Management Findings No. 35, 4 p.

Lillie, R.A., and Mason, J.W., 1983, Limnological characteristics of Wisconsin lakes: Wisconsin Department of Natural Resources Technical Bulletin No. 138, 116 p.

Rantz, S.E., and others, 1982, Measurement and computation of streamflow: U.S. Geological Survey Water-Supply Paper 2175, $631 \mathrm{p}$.

Rapavich, W.M., Sonzogni, W.C., Standridge, J.H., Vennie J.G., and Wedepohl, R.E., 1987, Incidence of algal toxins in Wisconsin water experiencing blue-green algae blooms: Wisconsin State Laboratory of Hygiene and Wisconsin Department of Natural Resources, Informational Paper, 8 p.

Shaw, B., Mechenich, C., and Klessig, L., 1993, Understanding Lake Data: G3582: University of Wisconsin Extension, Madison, Wis., $19 \mathrm{p}$.

U.S. Environmental Protection Agency, 1986, Quality Criteria for Water 1986: U.S. Environmental Protection Agency publication, EPA 440/5-86-001 [variously paged].

Wershaw, R.L., Fishman, M.J., Grabbe, R.R., and Lowe, L.E., eds., 1987, Methods for the determination of organic substances in water and fluvial sediments: U.S. Geological Survey Techniques of Water-Resources Investigations, book 5, chap. A3, 80 p.

Wetzel, R.G., 1983, Limnology (2d ed.): New York, W.B. Saunders, 767 p. 
Wisconsin Department of Natural Resources, 1992, Wisconsin water quality assessment-Report to Congress, 1992: Wisconsin Department of Natural Resources Publ-WR254-92-REV, $220 \mathrm{p}$.

Wisconsin State Laboratory of Hygiene, Environmental Sciences Section, 1993, Manual of analytical methods, inorganic chemistry unit: Wisconsin State Laboratory of Hygiene, revised November 1993 [variously paged]. 
LAKE DATA 
LOCATION.--Lat 45 39'07", long 91 $34^{\prime} 58^{\prime \prime}$, in NE 1/4 NE 1/4 sec.34, T.37 N., R.10 W., Washburn County, Hydrologic Unit 07050007, $1.2 \mathrm{mi}$ southwest of Birchwood.

PERIOD OF RECORD.--March 1993 to August 1994, March 1996 to August 1997, and March to September 2001.

REMARKS.--Lake sampled near southern end at a depth of about $14 \mathrm{~m}$. Lake ice-covered during March sampling. Water-quality analyses by Wisconsin State Laboratory of Hygiene.

WATER-QUALITY DATA, MARCH 1 TO JUNE 12, 2001

(Milligrams per liter unless otherwise indicated)

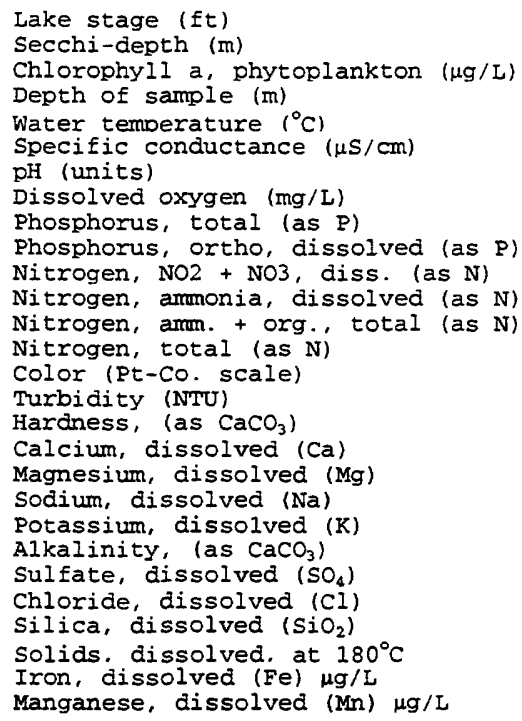

\begin{tabular}{|c|c|c|c|c|}
\hline \multicolumn{2}{|c|}{$\operatorname{Mar}-1$} & May-2 & \multicolumn{2}{|c|}{ Jun -12} \\
\hline \multicolumn{2}{|c|}{$\begin{array}{l}--- \\
--- \\
---\end{array}$} & $\begin{array}{c}10.94 \\
2.1 \\
8\end{array}$ & \multicolumn{2}{|c|}{$\begin{array}{c}10.66 \\
3.6 \\
6\end{array}$} \\
\hline $\begin{array}{c}0.5 \\
0.1 \\
162 \\
7.3 \\
11.8 \\
0.040\end{array}$ & $\begin{array}{c}12.0 \\
4.6 \\
243 \\
7.0 \\
0.2 \\
0.596\end{array}$ & $\begin{array}{c}0.5 \\
12.0 \\
136 \\
7.7 \\
11.7 \\
0.037\end{array}$ & $\begin{array}{c}0.5 \\
19.8 \\
137 \\
8.2 \\
11.3 \\
0.022\end{array}$ & $\begin{array}{c}12.0 \\
7.2 \\
179 \\
6.9 \\
0.3 \\
0.101\end{array}$ \\
\hline--- & --- & 0.005 & --- & --- \\
\hline-- & $\cdots$ & 0.217 & $\cdots$ & --- \\
\hline--- & -- & 0.024 & --- & --- \\
\hline$m$ & -- & 0.57 & -- & -- \\
\hline--- & $-\cdots$ & 0.787 & --- & -- \\
\hline--- & -- & 20 & -- & --- \\
\hline--- & $-\infty$ & 2.1 & $\cdots$ & --- \\
\hline--- & --- & 68.8 & $\cdots$ & $\cdots$ \\
\hline$-\infty$ & --- & 18 & --- & $\ldots$ \\
\hline$\cdots$ & --- & 5.8 & $-\cdots$ & $\cdots$ \\
\hline-- & $\cdots$ & 2.8 & $\cdots$ & -- \\
\hline--- & -- & 0.7 & $-\cdots$ & $-\infty$ \\
\hline--- & -- & 64 & $\cdots$ & $\cdots$ \\
\hline$\cdots$ & -- & $<4.5$ & --- & --- \\
\hline-- & $\cdots$ & 2.7 & --- & --- \\
\hline$\cdots$ & --- & 14.2 & --- & $-\cdots$ \\
\hline-- & -- & 96 & --- & --- \\
\hline$\ldots-$ & $\cdots$ & 30 & --- & -- \\
\hline$\cdots$ & -- & 2.5 & --- & --- \\
\hline
\end{tabular}
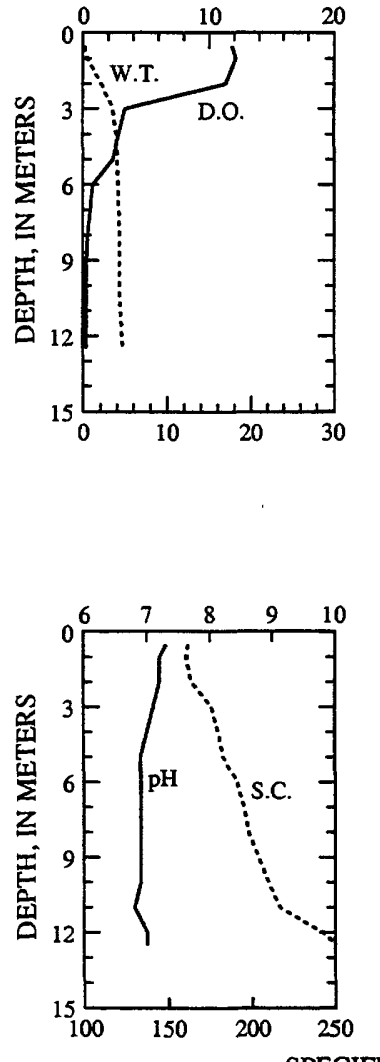

DISSOLVED OXYGEN (D.O.), IN MILLIGRAMS PER LITER

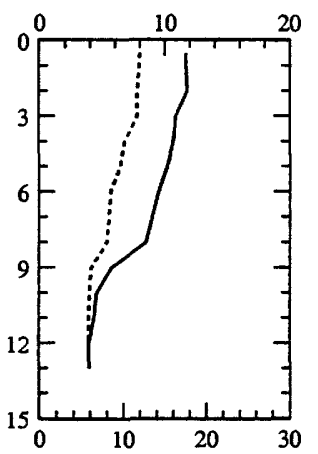

WATER TEMPERATURE (W.T.), IN DEGREES CELSIUS
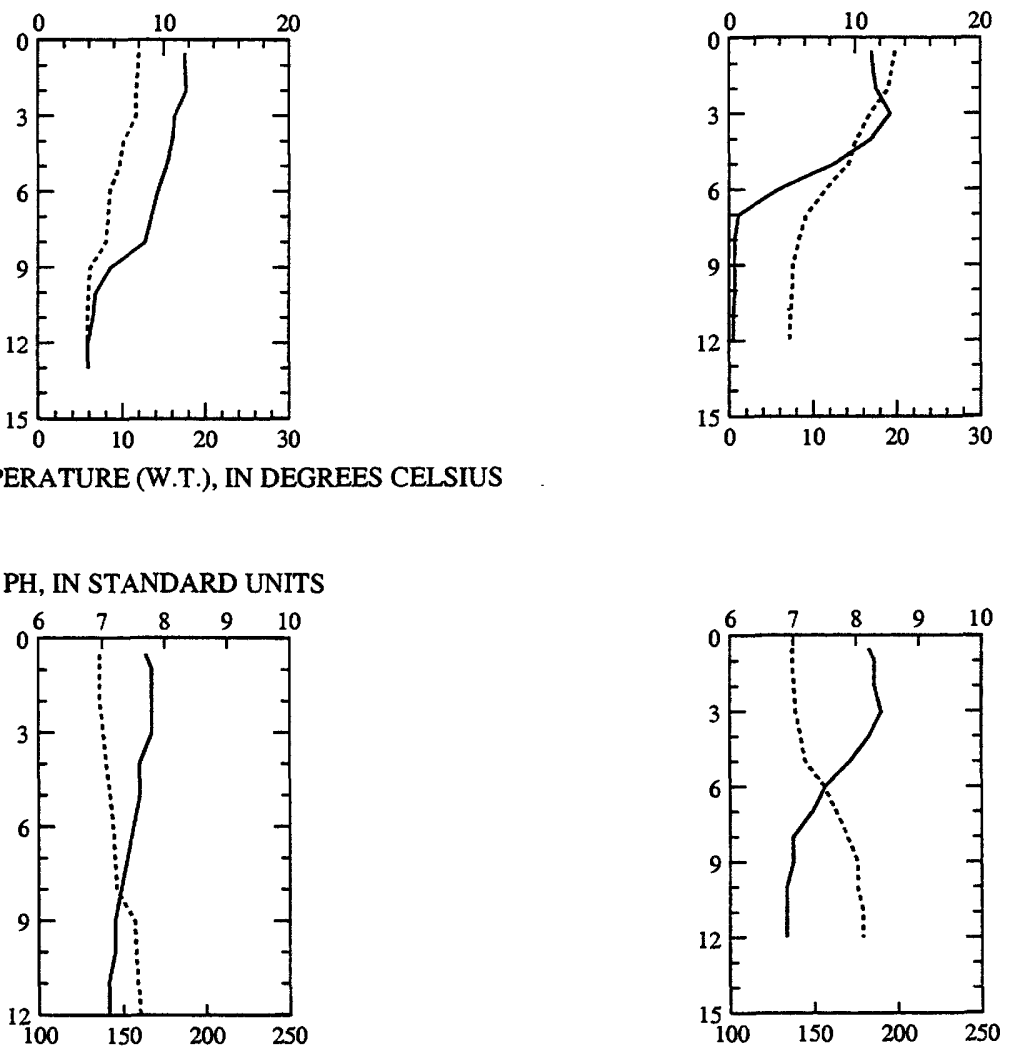

SPECIFC CONDUCTANCE (S.C.), IN MICROSIEMENS PER CENTIMETER AT 25 DEGREES CELSIUS 
WATER-QUALITY DATA, JULY 10 TO SEPTEMBER 20, 2001

(Milligrams per liter unless otherwise indicated)

Lake stage ( $f t$ )

Secchi-depth (m)

Chlorophyll a, phytoplankton $(\mu \mathrm{g} / \mathrm{L})$

Depth of sample (m)

water temperature $\left({ }^{\circ} \mathrm{C}\right)$

Specific conductance $(\mu \mathrm{S} / \mathrm{cm})$

pH (units)

Dissolved oxygen (mg/L)

Phosphorus, total (as P)

\begin{tabular}{cc} 
Jul 10 \\
\multicolumn{2}{c}{10.72} \\
2.6 \\
0.5 & 12.0 \\
24.6 & 7.6 \\
143 & 193 \\
8.6 & 7.1 \\
10.3 & 0.3 \\
-- & 0.287
\end{tabular}

0.5
25.0
147
8.7
10.5
0.022

Aug-13

10.67

1.7

11

4.5

17.4

158

7.2

$\begin{array}{ll}0.4 & 0.2 \\ 0.032 & 0.40\end{array}$

$\begin{array}{ccc} & \text { Sep-20 } \\ & 10.74 \\ & 2.6 \\ & 17 & \\ 0.5 & 7.0 & 12.0 \\ 18.4 & 11.6 & 8.1 \\ 160 & 187 & 220 \\ 8.3 & 7.1 & 7.0 \\ 10.3 & 0.6 & 0.3 \\ 0.025 & 0.040 & 0.512\end{array}$

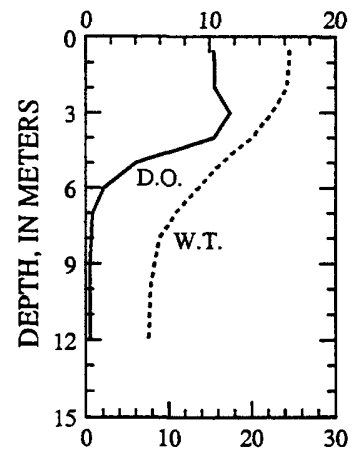

DISSOLVED OXYGEN (D.O.), IN MILLIGRAMS PER LITER
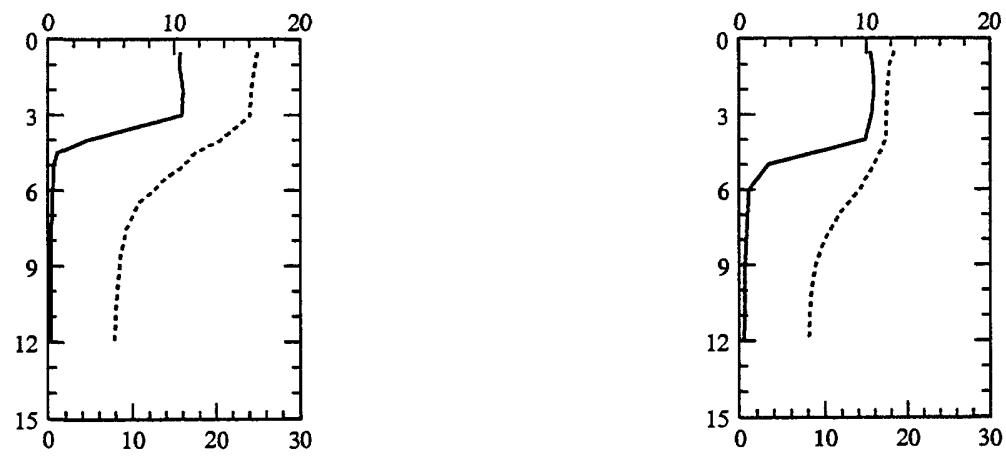

WATER TEMPERATURE (W.T.), IN DEGREES CELSIUS

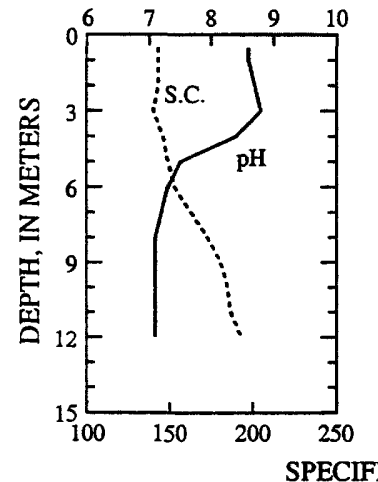

PH, IN STANDARD UNITS
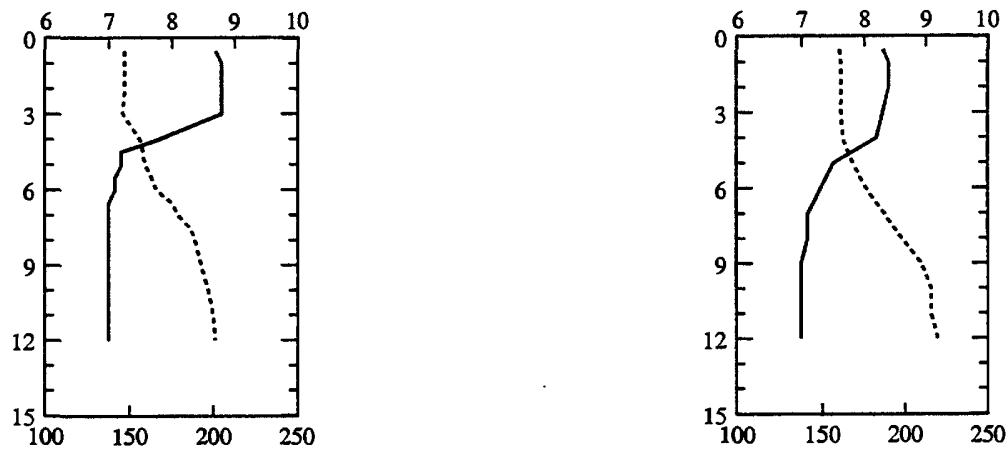

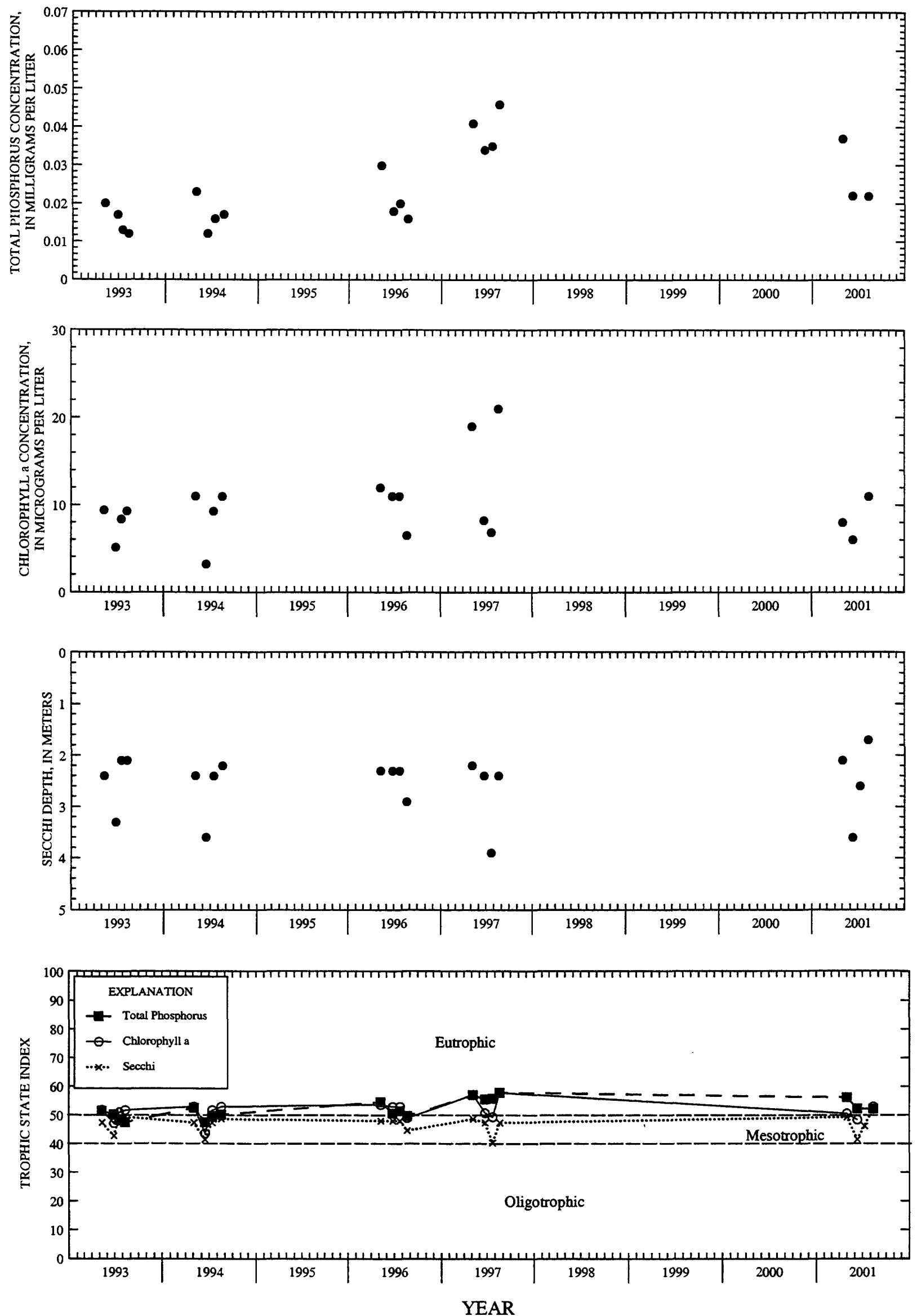

Surface total phosphorus, chlorophyll a concentrations, Secchi depths, and TSI data for Balsam Lake, near Birchwood, Wisconsin. 
LOCATION.--Lat $43^{\circ} 24^{\prime} 09^{\prime \prime}$, long $88^{\circ} 15^{\prime} 16^{\prime \prime}$, in NE $1 / 4$ sw 1/4 sec.20, T.11 N., R.19 E., Washington County, Hydrologic Unit 04040003, near West Bend.

PERIOD OF RECORD.--February 2000 to to current year.

REMARKS.--Lake sampled on north side at a depth of $12 \mathrm{~m}$. Lake ice-covered during February sampling. Water-quality analyses by Wisconsin State Laboratory of Hygiene.

WATER-QUALITY DATA, FEBRUARY 14 TO AUGUST 21, 2001

(Milligrams per liter unless otherwise indicated)

Feb-14

\begin{tabular}{cccc} 
& \multicolumn{2}{c}{ Apr-30 } \\
& \multicolumn{3}{c}{2.2} \\
0.5 & 11.0 & 0.5 & 4.7 \\
1.6 & 3.6 & 13.9 & 11.5 \\
577 & 721 & 523 & 526 \\
7.9 & 7.5 & 7.8 & 7.5 \\
9.0 & 1.2 & 11.0 & 5.6 \\
0.012 & 0.021 & 0.017 & 0.015 \\
-- & --- &.-- & -- \\
-- & -- & -- & -- \\
-- & -- & $-\cdots$ & $-\cdots$
\end{tabular}

Jun-21

3.4

$0.5^{2.6} 11.5$

$22.5 \quad 14.8$

$514 \quad 541$

$\begin{array}{ll}8.3 & 7.6 \\ 8.9 & 1.6\end{array}$

$0.016 \quad 0.017$

---

$--$

$--$

$--$

\begin{tabular}{cccc}
\multicolumn{4}{c}{ Aug-21 } \\
\multicolumn{4}{c}{1.9} \\
0.5 & 7.0 & 9.0 & 11.0 \\
22.9 & 18.2 & 16.0 & 14.7 \\
503 & 547 & 553 & 567 \\
8.4 & 7.4 & 7.3 & 7.2 \\
8.8 & 0.5 & 0.3 & 0.2 \\
0.022 & 0.021 & 0.027 & 0.041 \\
0.005 & $-\ldots$ &.-- &.-- \\
0.01 &.-- &.-- &.-- \\
$<0.013$ & --- & -- & --
\end{tabular}

2-14- 01

4-30-01

6-21-01

$8-21-01$

DISSOLVED OXYGEN (D.O.), IN MILLIGRAMS PER LITER
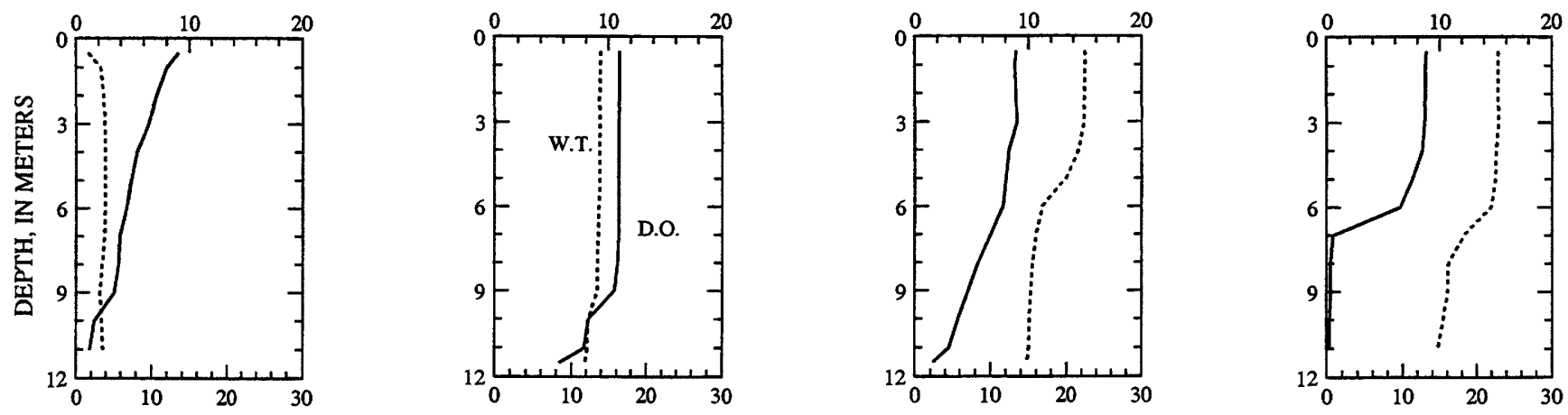

WATER TEMPERATURE (W.T.), IN DEGREES CELSIUS

PH, IN STANDARD UNITS
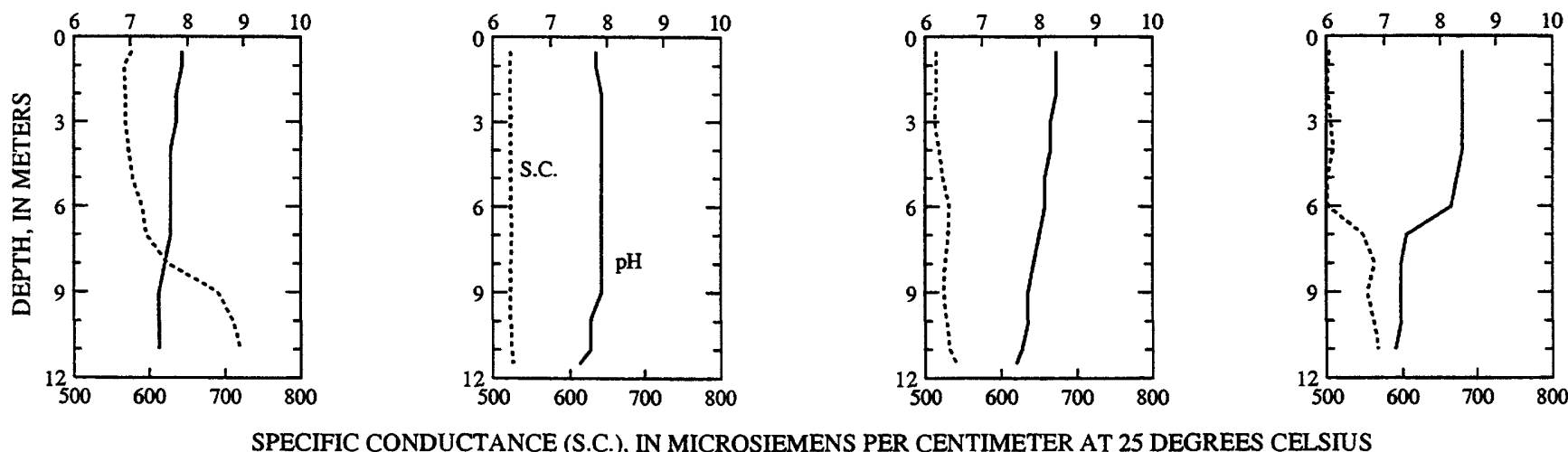

PECIFIC CONDUCTANCE (S.C.), IN MICROSIEMENS PER CENTIMETER AT 25 DEGREES CELSIUS 

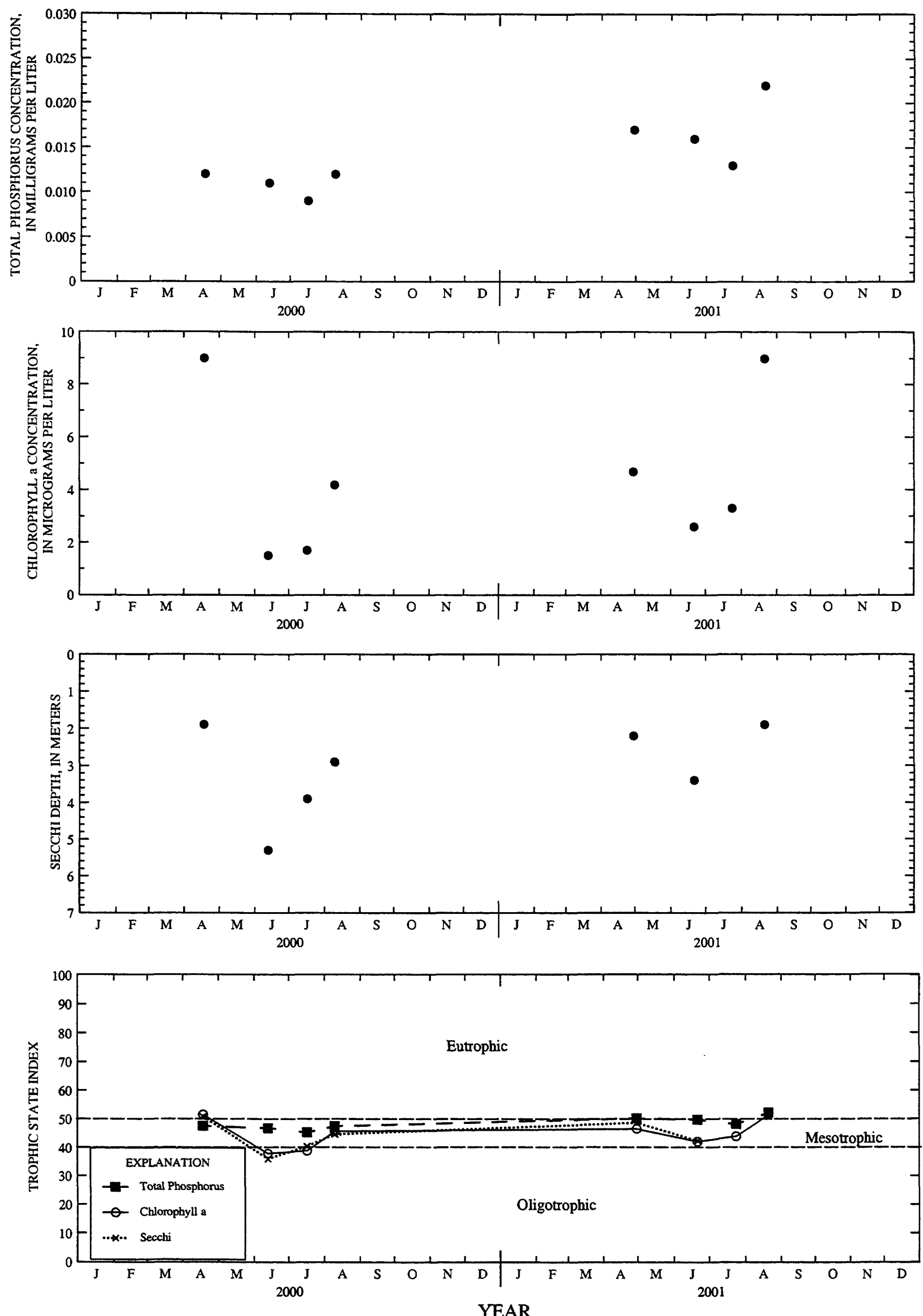

Surface total phosphorus, chlorophyll a concentrations, Secchi depths, and TSI data for Big Cedar Lake, North Site, near West Bend, Wisconsin. 
LOCATION.--Lat $43^{\circ} 22^{\prime} 24^{\prime \prime}$, long $88^{\circ} 15^{\prime} 49^{\prime \prime}$, in NE 1/4 SE $1 / 4$ sec.31, T.11 N., R.19 E., Washington County, Hydrologic Unit 04040003, near West Bend.

PERIOD OF RECORD.--February 2000 to current year.

REMARKS.--Lake sampled on south side at deep hole. Lake ice-covered during February sampling. Water-quality analyses by Wisconsin State Laboratory of Hygiene.

WATER-QUALITY DATA, FEBRUARY 14 TO AUGUST 21, 2001

(Milligrams per liter unless otherwise indicated)

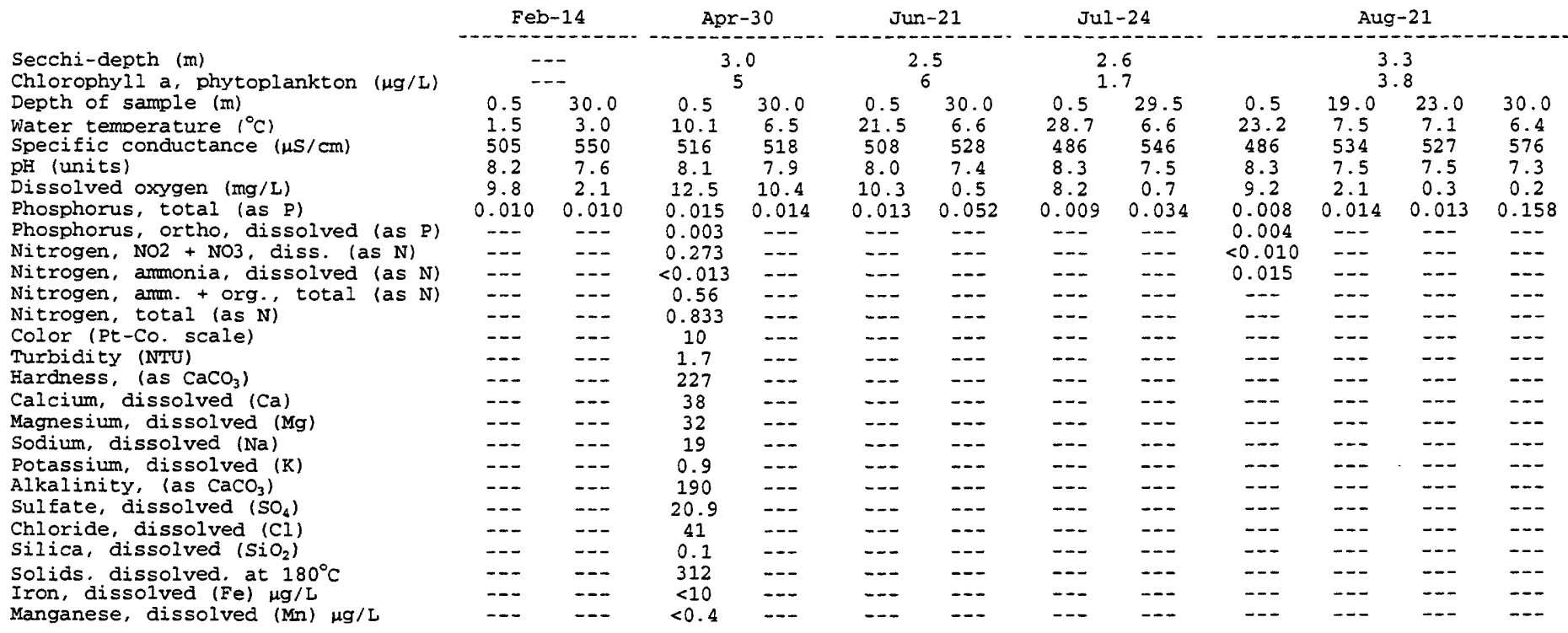
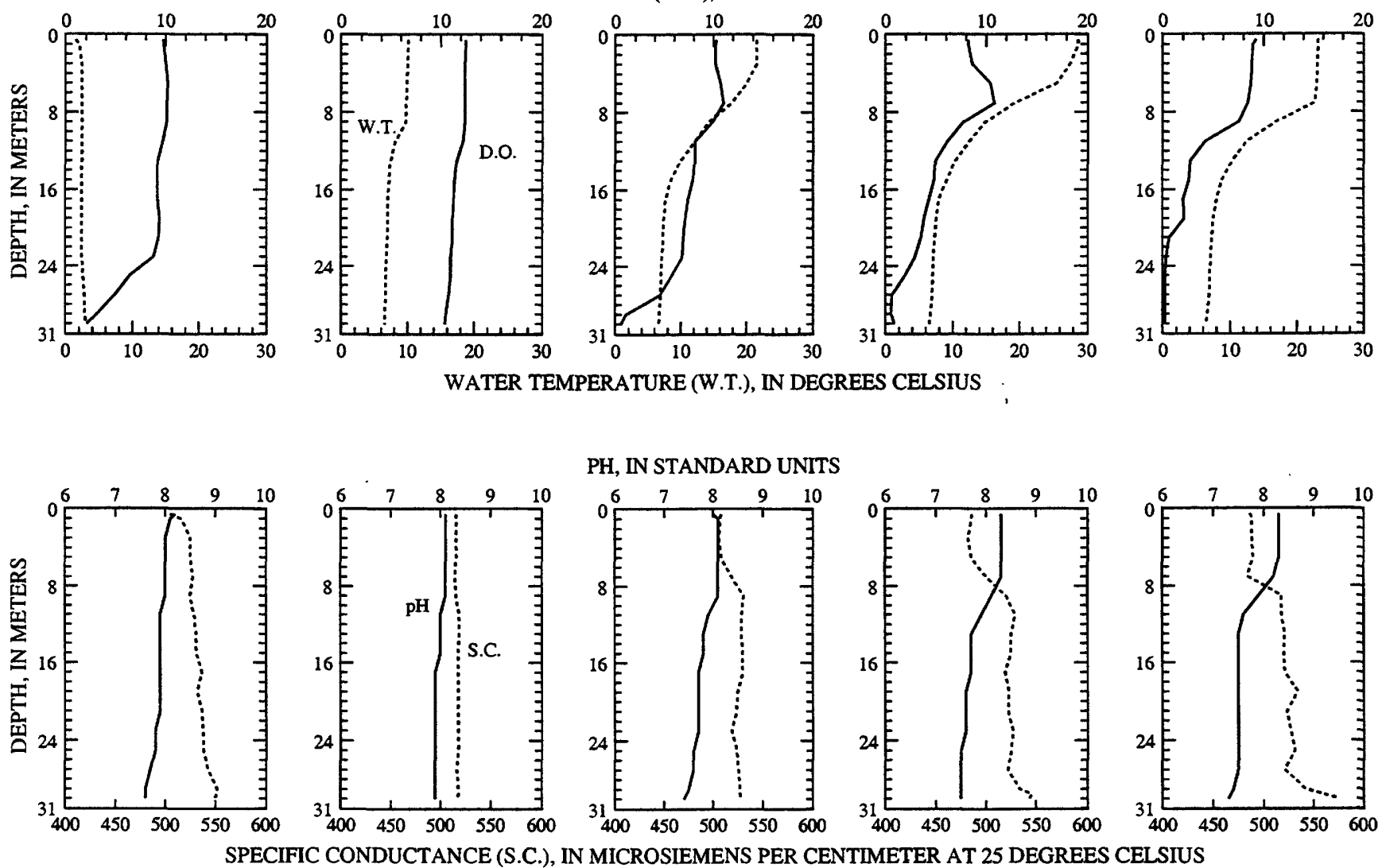

PH, IN STANDARD UNITS
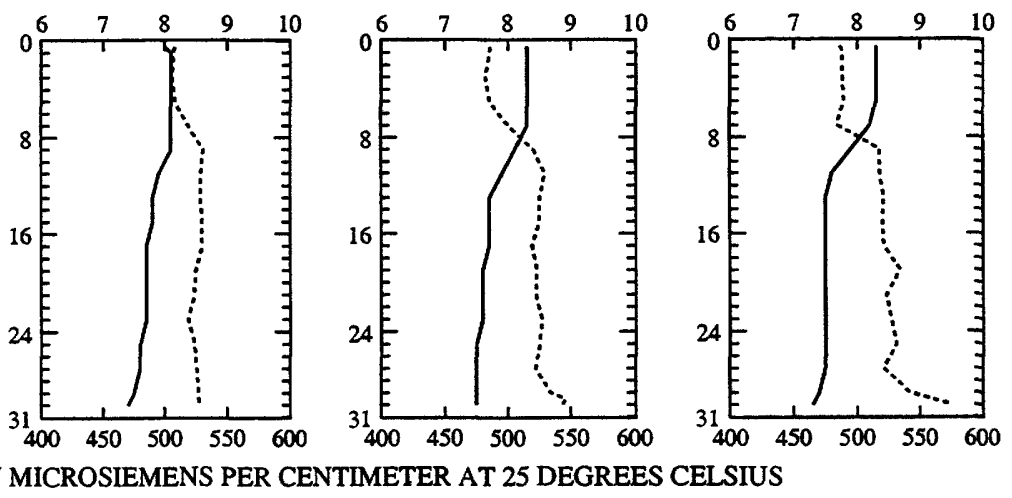

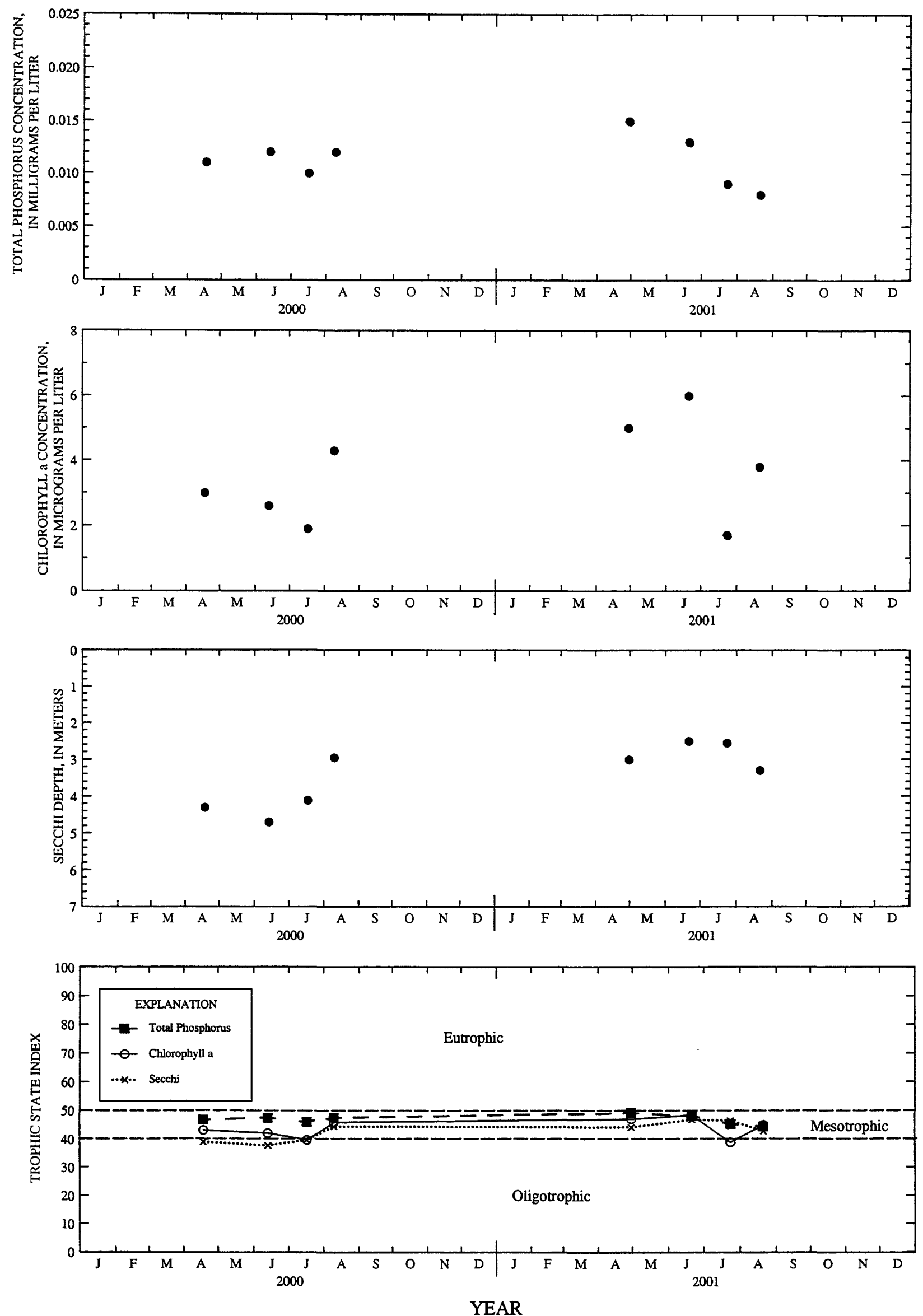

Surface total phosphorus, chlorophyll a concentrations, Secchi depths, and TSI data for Big Cedar Lake, South Site, near West Bend, Wisconsin. 
LOCATION.--Lat $45^{\circ} 31^{\prime} 42^{\prime \prime}$, long $92^{\circ} 18^{\prime} 01^{\prime \prime}$, in NE 1/4 SW 1/4 SW 1/4 sec.12, T.35 N., R.16 W., Polk County, Hydrologic Unit 07030005, near Millton.

PERIOD OF RECORD.--February to September 2001.

REMARKS.--Lake sampled near center of lake at a depth of $4.0 \mathrm{~m}$. Water-quality analyses done by Wisconsin State Laboratory of Hygiene.

WATER-QUALITY DATA, MARCH 09 TO AUGUST 14, 2001

(Milligrams per liter unless otherwise indicated)

Lake stage ( $f t$ )

Secchi-depth (m)

Chlorophyll a, phytoplankton ( $\mu \mathrm{g} / \mathrm{L})$

Depth of sample (m)

water temperature $\left({ }^{\circ} \mathrm{C}\right)$

Specific conductance $(\mu \mathrm{S} / \mathrm{cm})$

$\mathrm{pH}$ (units)

Dissolved oxygen (mg/L)

Phosphorus, total (as P)

Phosphorus, ortho, dissolved (as P)

Nitrogen, NO2 + NO3, diss. (as N)

Nitrogen, ammonia, dissolved (as N)

Nitrogen, amm. + org., total (as N)

Nitrogen, total (as $\mathrm{N}$ )

Color (Pt-Co. scale)

Turbidity (NTU)

Hardness, (as $\mathrm{CaCO}_{3}$ )

Calcium, dissolved (Ca)

Magnesium, dissolved (Mg)

Sodium, dissolved (Na)

Potassium, dissolved (K)

Alkalinity, (as $\mathrm{CaCO}_{3}$ )

Sulfate, dissolved $\left(\mathrm{SO}_{4}\right)$

Chloride, dissolved (Cl)

Silica, dissolved $\left(\mathrm{SiO}_{2}\right)$

Solids. dissolved, at $180^{\circ} \mathrm{C}$

Iron, dissolved (Fe) $\mu \mathrm{g} / \mathrm{L}$

Manganese, dissolved (Mn) $\mu \mathrm{g} / \mathrm{L}$

\begin{tabular}{|c|c|}
\hline \multicolumn{2}{|c|}{$\operatorname{Mar}-9$} \\
\hline \multicolumn{2}{|c|}{$-\cdots$} \\
\hline \multicolumn{2}{|c|}{---} \\
\hline & \\
\hline 0.5 & 4. 0 \\
\hline 0.1 & 4.3 \\
\hline 242 & 253 \\
\hline 7.0 & 7.0 \\
\hline 9.4 & 1.2 \\
\hline 0.027 & 0.053 \\
\hline-- & --- \\
\hline--- & -- \\
\hline--- & $\cdots$ \\
\hline$\cdots$ & --- \\
\hline$-\cdots$ & $\cdots$ \\
\hline$\cdots$ & $\cdots$ \\
\hline$\cdots$ & $\cdots$ \\
\hline$--\infty$ & --- \\
\hline--- & --- \\
\hline--- & --- \\
\hline-- & --- \\
\hline-- & -- \\
\hline-- & -- \\
\hline--- & -- \\
\hline$\ldots$ & $\ldots$ \\
\hline-- & --- \\
\hline--- & -- \\
\hline--- & --- \\
\hline
\end{tabular}

4-30-01

6-11-01
Jun-11

4.31

2.6

15

$\begin{array}{cc}0.5 & 15.0 \\ 12.9 & 12.9 \\ 193 & 193\end{array}$

$193 \quad 193$

$8.3 \quad 8.3$

$0.030 \quad 0.033$

0.030

0.003
$<0.010$

$<0.013$

0.58

- 20

0.3

99.1

26

$3^{8.3}$

0.7

$<4.5$

3.1
18.5

18.5

126
$<10$

3-9-01

DISSOLVED OXYGEN (D.O.), IN MILLIGRAMS PER LITER
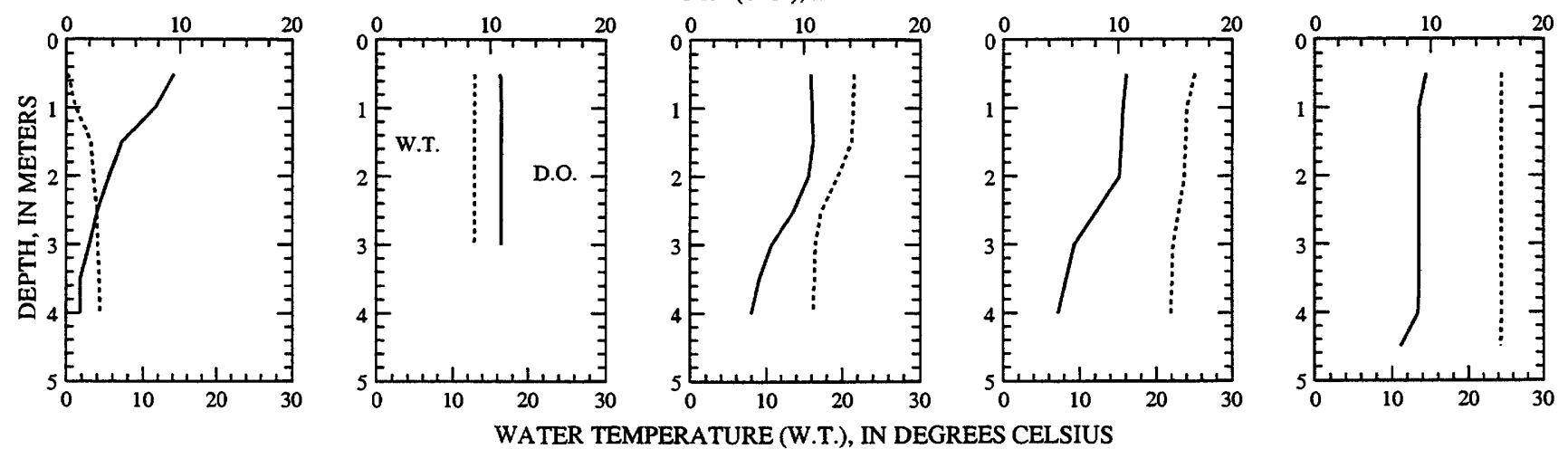

PH, IN STANDARD UNITS
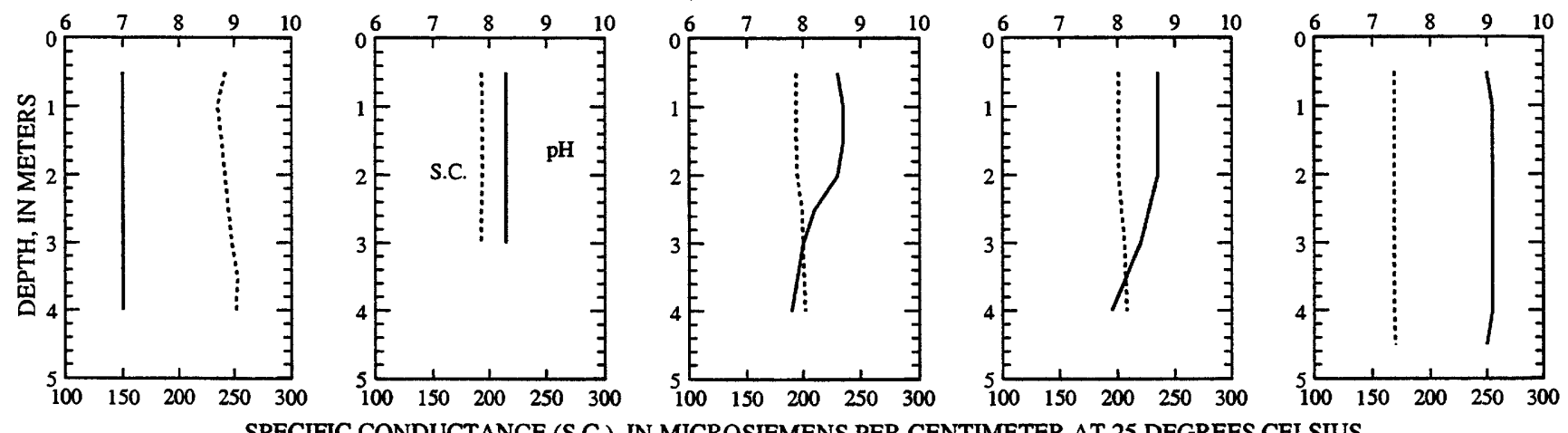

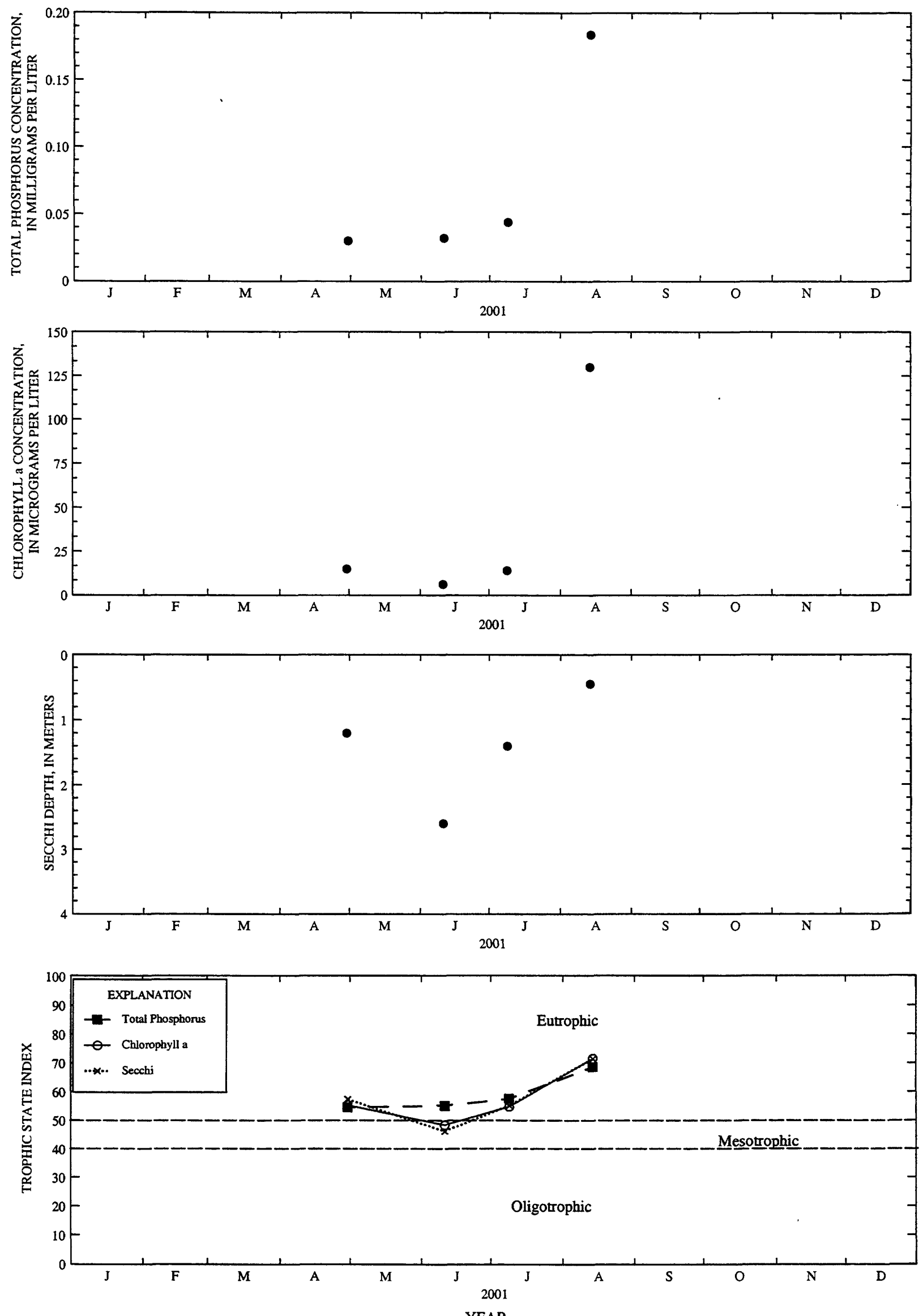

Surface total phosphorus, chlorophyll a concentrations, Secchi depths, and TSI data for Big Round Lake, near Milltown, Wisconsin. 
LOCATION.--Lat $45^{\circ} 55^{\prime} 00^{\prime \prime}$, long 89 $31^{\prime} 55^{\prime \prime}$ in NE 1/4 SE 1/4 sec.30, T.40 N., R.8 E., Vilas County, Hydrologic Unit 07070001, at dam outlet, 7.7 mi northeast of Lake Tomahawk.

DRAINAGE AREA.--73.1 $\mathrm{mi}^{2}$.

PERIOD OF RECORD.--October 1991 to current year. Lake stages for previous years were recorded by Wisconsin Valley Improvement Company.

GAGE.--Nonrecording gage. Datum of gage is $1,580 \mathrm{ft}$, above sea level.

COOPERATION.--Lake stages provided by Wisconsin Valley Improvement Company.

EXTREMES FOR PERIOD OF RECORD.--Maximum gage height observed, $10.98 \mathrm{ft}$, May 9, 1999; minimum observed, $8.16 \mathrm{ft}$, Jan. 26 , 1996, and Mar. 11, 2001.

EXTREMES FOR CURRENT YEAR.--Maximum gage height observed, $10.78 \mathrm{ft}$, June 19, 20; minimum observed, $8.16 \mathrm{ft}$, Mar. 11.

GAGE HEIGHT, FEET, WATER YEAR OCTOBER 2000 TO SEPTEMBER 2001

DAILY MEAN VALUES

\begin{tabular}{|c|c|c|c|c|c|c|c|c|c|c|c|c|}
\hline DAY & OCT & NOV & $\mathrm{DEC}$ & JAN & $F E B$ & MAR & APR & MAY & $\pi N$ & JUL & AUG & SEP \\
\hline 1 & 10.56 & --- & 9.78 & $\ldots$ & --- & 8.18 & 8.48 & 10.68 & 10.56 & 10.48 & 10.60 & 10.50 \\
\hline 2 & 10.54 & --- & $-\infty$ & 8.66 & 8.34 & 8.18 & 8.56 & 10.70 & 10.54 & 10.48 & 10.76 & 10.48 \\
\hline 3 & 10.54 & 10.10 & 9.74 & -- & --- & -- & 8.66 & 10.76 & 10.54 & 10.46 & 10.76 & 10.48 \\
\hline 4 & 10.54 & --- & $-\cdots$ & --- & 8.34 & 8.22 & 8.74 & 10.72 & 10.52 & 10.46 & 10.76 & 10.48 \\
\hline 5 & 10.54 & 10.08 & 9.70 & 8.62 & -- & -- & 8.82 & 10.68 & 10.52 & 10.46 & 10.76 & 10.46 \\
\hline 6 & 10.52 & --- & --- & --- & 8.34 & 8.20 & 8.92 & 10.66 & 10.52 & 10.46 & 10.76 & 10.46 \\
\hline 7 & 10.52 & 10.04 & -- & 8.58 & --- & --- & 9.00 & 10.64 & 10.52 & 10.46 & 10.68 & 10.54 \\
\hline 8 & 10.52 & --- & 9.62 & $-\cdots$ & --- & --- & 9.12 & 10.62 & 10.50 & 10.44 & 10.64 & 10.66 \\
\hline 9 & --- & --- & -- & 8.52 & 8.40 & 8.20 & 9.18 & 10.60 & 10.50 & 10.46 & 10.60 & 10.68 \\
\hline 10 & 10.52 & 10.00 & 9.58 & --- & --- & --- & 9.30 & 10.64 & 10.50 & 10.46 & 10.56 & 10.64 \\
\hline 11 & -- & -- & -- & -- & 8.42 & 8.16 & 9.42 & 10.66 & 10.54 & 10.46 & 10.54 & 10.62 \\
\hline 12 & --- & 9.96 & 9.50 & 8.42 & --- & -- & 9.56 & 10.64 & 10.58 & 10.48 & 10.50 & 10.60 \\
\hline 13 & 10.52 & --- & --- & -- & 8.42 & 8.30 & 9.68 & 10.64 & 10.66 & 10.48 & 10.52 & 10.58 \\
\hline 14 & --- & 9.94 & --- & 8.46 & -- & -- & 9.74 & 10.64 & 10.66 & 10.48 & 10.52 & 10.60 \\
\hline 15 & 10.52 & $\cdots$ & 9.28 & --- & --- & --- & 9.84 & 10.62 & 10.64 & 10.46 & 10.54 & 10.60 \\
\hline 16 & $\cdots$ & --- & --- & 8.46 & 8.42 & 8.40 & 9.92 & 10.60 & 10.64 & 10.50 & 10.54 & 10.58 \\
\hline 17 & 10.52 & 9.90 & 9.10 & --- & --- & -- & 10.02 & 10.58 & 10.62 & 10.48 & 10.52 & 10.58 \\
\hline 18 & --- & --- & -- & --- & --- & 8.44 & 10.08 & 10.54 & 10.64 & 10.48 & 10.54 & 10.58 \\
\hline 19 & --- & 9.90 & 9.08 & 8.45 & --- & -- & 10.16 & 10.50 & 10.78 & 10.46 & 10.58 & 10.60 \\
\hline 20 & 10.48 & -- & --- & $-\infty$ & 8.40 & 8.46 & 10.22 & 10.48 & 10.78 & 10.58 & 10.58 & 10.60 \\
\hline 21 & --- & 9.88 & --- & 8.45 & --- & $-\infty$ & 10.28 & 10.48 & 10.76 & 10.66 & 10.58 & 10.60 \\
\hline 22 & 10.44 & -- & 8.98 & -- & $\ldots$ & 8.46 & 10.40 & 10.48 & 10.72 & 10.66 & 10.56 & 10.60 \\
\hline 23 & --- & --- & --- & 8.42 & 8.38 & 8.46 & 10.50 & 10.50 & 10.68 & 10.64 & 10.56 & 10.66 \\
\hline 24 & 10.38 & 9.86 & 8.88 & --- & -- & 8.46 & 10.60 & 10.52 & 10.62 & 10.64 & 10.56 & 10.62 \\
\hline 25 & --- & --- & --- & --- & 8.38 & 8.46 & 10.62 & 10.54 & 10.60 & 10.64 & 10.56 & 10.62 \\
\hline 26 & --- & 9.84 & 8.76 & 8.40 & --- & 8.46 & 10.68 & 10.54 & 10.58 & 10.62 & 10.56 & 10.58 \\
\hline 27 & --- & --- & --- & --- & 8.28 & 8.46 & 10.68 & 10.56 & 10.56 & 10.62 & 10.56 & 10.56 \\
\hline 28 & $-\cdots$ & 9.82 & --- & 8.38 & $\ldots$ & 8.46 & 10.68 & 10.56 & 10.52 & 10.62 & 10.54 & 10.54 \\
\hline 29 & 10.18 & -- & 8.77 & -- & --- & 8.46 & 10.70 & 10.56 & 10.50 & 10.61 & 10.54 & 10.54 \\
\hline 30 & --- & --- & --- & 8.36 & --- & 8.47 & 10.68 & 10.56 & 10.50 & 10.62 & 10.52 & 10.54 \\
\hline 31 & 10.16 & --- & 8.68 & 8.36 & -- & 8.47 & --- & 10.56 & --- & 10.62 & 10.50 & --- \\
\hline MEAN & --- & --- & --- & --- & --- & --- & 9.77 & 10.60 & 10.59 & 10.53 & 10.59 & 10.57 \\
\hline $\operatorname{MAX}$ & --- & --- & --- & --- & --- & -- & 10.70 & 10.76 & 10.78 & 10.66 & 10.76 & 10.68 \\
\hline MIN & --- & $-\cdots$ & $\rightarrow-$ & -- & -- & -- & 8.48 & 10.48 & 10.50 & 10.44 & 10.50 & 10.46 \\
\hline
\end{tabular}




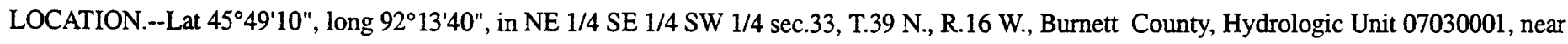
Hertel.

PERIOD OF RECORD.--February to September 2001.

REMARKS.--Lake sampled on west side of lake at a depth of $16.0 \mathrm{~m}$. Water-quality analyses by Wisconsin State Laboratory of Hygiene.

WATER-QUALITY DATA, MARCH 02 TO AUGUST 14, 2001

(Milligrams per liter unless otherwise indicated)

Lake stage (ft)

Secchi-depth (m)

Chlorophyll a, phytoplankton ( $\mu \mathrm{g} / \mathrm{L})$

Depth of sample (m)

Water temperature $\left({ }^{\circ} \mathrm{C}\right)$

Specific conductance $(\mu \mathrm{S} / \mathrm{cm})$

$\mathrm{pH}$ (units)

Dissolved oxygen (mg/L)

Phosphorus, total (as P)

Phosphorus, ortho, dissolved (as P)

Nitrogen, NO2 + NO3, diss. (as N)

Nitrogen, ammonia, dissolved (as $N$ )

Nitrogen, anm. + org., total (as N)

Nitrogen, total (as $\mathrm{N}$ )

Color (Pt-Co. scale)

Turbidity (NTU)

Hardness, (as $\mathrm{CaCO}_{3}$ )

Calcium, dissolved ( $\mathrm{Ca}$ )

Magnesium, dissolved (Mg)

Sodium, dissolved (Na)

Potassium, dissolved (K)

Alkalinity, (as $\mathrm{CaCO}_{3}$ )

Sulfate, dissolved $\left(\mathrm{SO}_{4}\right)$

Chloride, dissolved (Cl)

Silica, dissolved $\left(\mathrm{SiO}_{2}\right)$

Solids, dissolved, at $180^{\circ} \mathrm{C}$

Iron, dissolved ( $\mathrm{Fe}$ ) $\mu \mathrm{g} / \mathrm{L}$

Manganese, dissolved (Mn) $\mu \mathrm{g} / \mathrm{L}$

\begin{tabular}{|c|c|c|c|}
\hline \multicolumn{2}{|c|}{$\operatorname{Mar}-2$} & \multicolumn{2}{|c|}{ May-1 } \\
\hline \multicolumn{2}{|c|}{$\cdots$} & \multicolumn{2}{|c|}{$\begin{array}{c}4.92 \\
3.1 \\
5.2\end{array}$} \\
\hline $\begin{array}{c}0.5 \\
0.2 \\
121 \\
7.7 \\
11.2 \\
0.013\end{array}$ & $\begin{array}{c}16.0 \\
4.7 \\
121 \\
6.8 \\
0.5 \\
0.018\end{array}$ & $\begin{array}{c}0.5 \\
13.0 \\
90 \\
8.1 \\
11.2 \\
0.012\end{array}$ & $\begin{array}{c}16.5 \\
8.1 \\
90 \\
7.5 \\
11.0 \\
0.016\end{array}$ \\
\hline-- & -- & 0.002 & --- \\
\hline-- & --- & $<0.010$ & --- \\
\hline$\cdots$ & $-\cdots$ & 0.019 & --- \\
\hline--- & $\cdots$ & 0.54 & $\cdots$ \\
\hline-- & --- & $-\cdots$ & --- \\
\hline-- & --- & 15 & $\ldots$ \\
\hline-- & $-\cdots$ & 4.6 & --- \\
\hline$\cdots$ & $-\cdots$ & 44 & --- \\
\hline-- & $-\cdots$ & 12 & --- \\
\hline$-\cdots$ & --- & 3.4 & --- \\
\hline--- & $\cdots$ & 2.1 & -- \\
\hline--- & -- & 0.6 & --- \\
\hline-- & --- & 41 & --- \\
\hline--- & --- & $<4.5$ & -- \\
\hline--- & --- & 2 & $\cdots$ \\
\hline-- & $-\cdots$ & 0.9 & --- \\
\hline-- & $-\infty$ & 56 & --- \\
\hline-- & --- & 120 & --- \\
\hline-- & $\cdots$ & 1.3 & -- \\
\hline
\end{tabular}

\begin{tabular}{|c|c|c|c|c|c|c|}
\hline \multicolumn{2}{|c|}{ Jun-11 } & \multicolumn{2}{|c|}{ Ju1-11 } & \multicolumn{3}{|c|}{ Aug-14 } \\
\hline \multicolumn{2}{|c|}{$\begin{array}{l}4.70 \\
3.6 \\
3.2\end{array}$} & \multicolumn{2}{|c|}{$\begin{array}{l}4.30 \\
3.2 \\
1.8\end{array}$} & & $\begin{array}{c}3.70 \\
2.50 \\
3.5\end{array}$ & \\
\hline $\begin{array}{c}0.5 \\
21.4 \\
88 \\
8.6 \\
10.0 \\
0.012\end{array}$ & $\begin{array}{c}16.0 \\
14.1 \\
89 \\
7.6 \\
5.6 \\
0.011\end{array}$ & $\begin{array}{c}0.5 \\
24.8 \\
88 \\
8.8 \\
9.6 \\
0.015\end{array}$ & $\begin{array}{c}16.0 \\
16.5 \\
99 \\
7.2 \\
0.2 \\
0.024\end{array}$ & $\begin{array}{c}0.5 \\
23.9 \\
86 \\
7.4 \\
7.7 \\
0.011\end{array}$ & $\begin{array}{c}10.0 \\
20.2 \\
104 \\
6.9 \\
0.4 \\
0.017\end{array}$ & $\begin{array}{c}14.0 \\
18.1 \\
117 \\
7.1 \\
0.2 \\
0.033\end{array}$ \\
\hline$\ldots$ & $\ldots$ & --- & $\ldots$ & $\ldots$ & -- & $\ldots$ \\
\hline-- & --- & --- & $\cdots$ & -- & --- & $\cdots$ \\
\hline$\cdots$ & $\ldots$ & $\ldots$ & $\ldots$ & $\ldots$ & -- & $\cdots$ \\
\hline$-\cdots$ & -- & -- & $\ldots$ & -- & -- & -- \\
\hline$-\cdots$ & $-\infty$ & $\sim-$ & $\cdots$ & $\cdots$ & --- & -- \\
\hline-- & --- & --- & --- & -- & --- & --- \\
\hline--- & --- & --- & --- & --- & --- & $-\cdots$ \\
\hline--- & --- & --- & $-\infty$ & -- & -- & $\cdots$ \\
\hline-- & --- & $-\cdots$ & $\ldots$ & $\cdots$ & $\cdots$ & $\cdots$ \\
\hline-- & $\ldots$ & --- & -- & $\ldots$ & --- & $-\cdots$ \\
\hline--- & --- & --- & --- & --- & --- & --- \\
\hline-- & -- & --- & --- & -- & --- & --- \\
\hline-- & --- & --- & --- & --- & -- & --- \\
\hline--- & --- & --- & -- & --- & --- & --- \\
\hline-- & $-\infty$ & $-\infty$ & $-\infty$ & --- & --- & $\cdots$ \\
\hline--- & --- & --- & -- & -- & --- & --- \\
\hline--- & --- & $-\cdots$ & -- & --- & --- & --- \\
\hline--- & --- & --- & --- & $-\infty$ & --- & -- \\
\hline--- & --- & --- & --- & --- & --- & --- \\
\hline
\end{tabular}

DISSOLVED OXYGEN (D.O.), IN MILLIGRAMS PER LITER
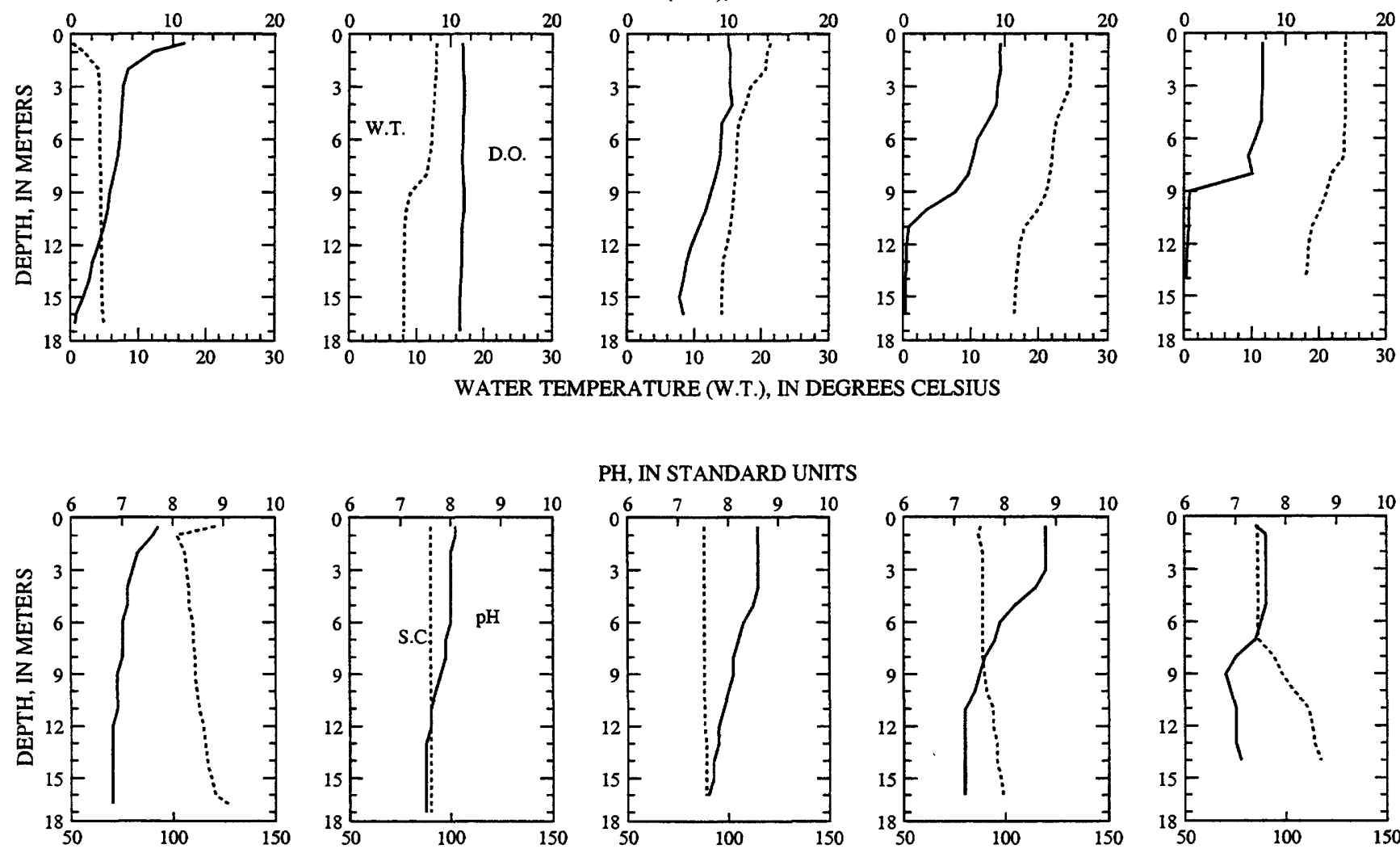

PH, IN STANDARD UNITS
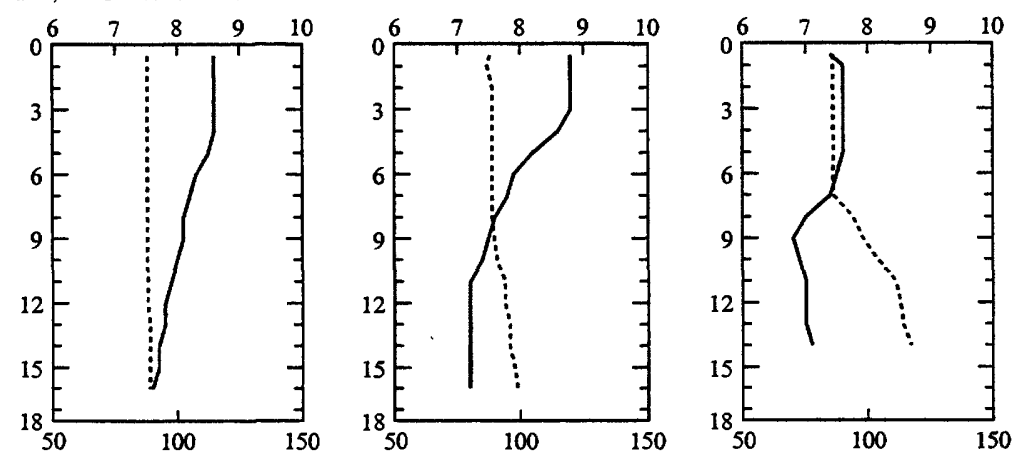

SPECIFIC CONDUCTANCE (S.C.), IN MICROSIEMENS PER CENTIMETER AT 25 DEGREES CELSIUS 

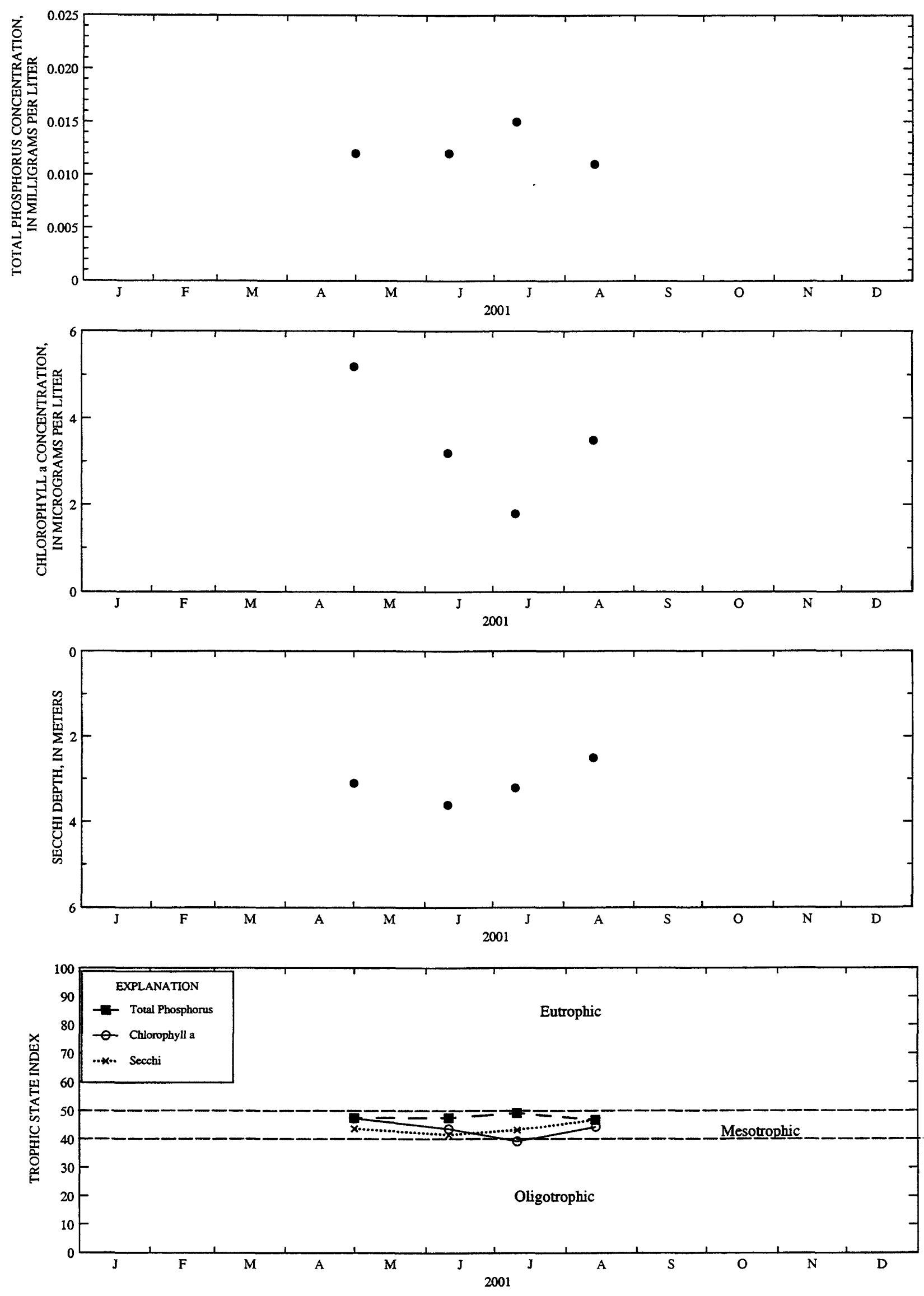

YEAR

Surface total phosphorus, chlorophyll a concentrations, Secchi depths, and TSI data for Big Sand Lake, Deep Hole, near Hertel, Wisconsin. 
LOCATION.--Lat $45^{\circ} 49^{\prime} 21^{\prime \prime}$, long $92^{\circ} 12^{\prime} 43^{\prime \prime}$, in NE 1/4 NW 1/4 SW $1 / 4$ sec.34, T.39 N., R.15 W., Burnett County, Hydrologic Unit 07030001 , near Hertel.

PERIOD OF RECORD.--February to September 2001.

REMARKS.--Lake sampled on east side of lake at a depth of $9.0 \mathrm{~m}$. Water-quality analyses by Wisconsin State Laboratory of Hygiene.

WATER-QUALITY DATA, MARCH 02 TO AUGUST 14, 2001

(Milligrams per liter unless otherwise indicated)

Lake stage (ft)

Secchi-depth (m)

Chlorophyll a phytoplankton $(\mu g / t)$

Depth of sample (m)

water temoerature $\left({ }^{\circ} \mathrm{C}\right)$

Specific conductance ( $\mu \mathrm{S} / \mathrm{cm})$

$\mathrm{pH}$ (units)

Dissolved oxygen (mg/L)

Phosphorus, total (as P)
Mar-2

$+-+$

$-\cdots$

$--\frac{1}{2}$

0.5

103

7.7

12.4

0.016

$\mathrm{Ma}$
4.
3
0
13
8
10

May-1

4.92

3.2

0.5

13.2

8.0

10.8

0.010

Jun-
4.70
4.
1.
0.
21.9
8
8.5
9

4.70
4.1
1.7
0.5
21.9
88
8.5
9.1
0.010

Jul-11

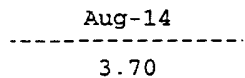

3. 3

2.1

2.1
0.5

$\begin{array}{ccc}89 & 24.2 & 19.6 \\ 8 & 86 & 138\end{array}$

8.6

9.5

0.010

8.2

8.5

0.013

7.3

0.2

3-2-01

5-1-01

6-11-01

7-11-01

8-14-01

DISSOLVED OXYGEN (D.O.), IN MILLIGRAMS PER LITER
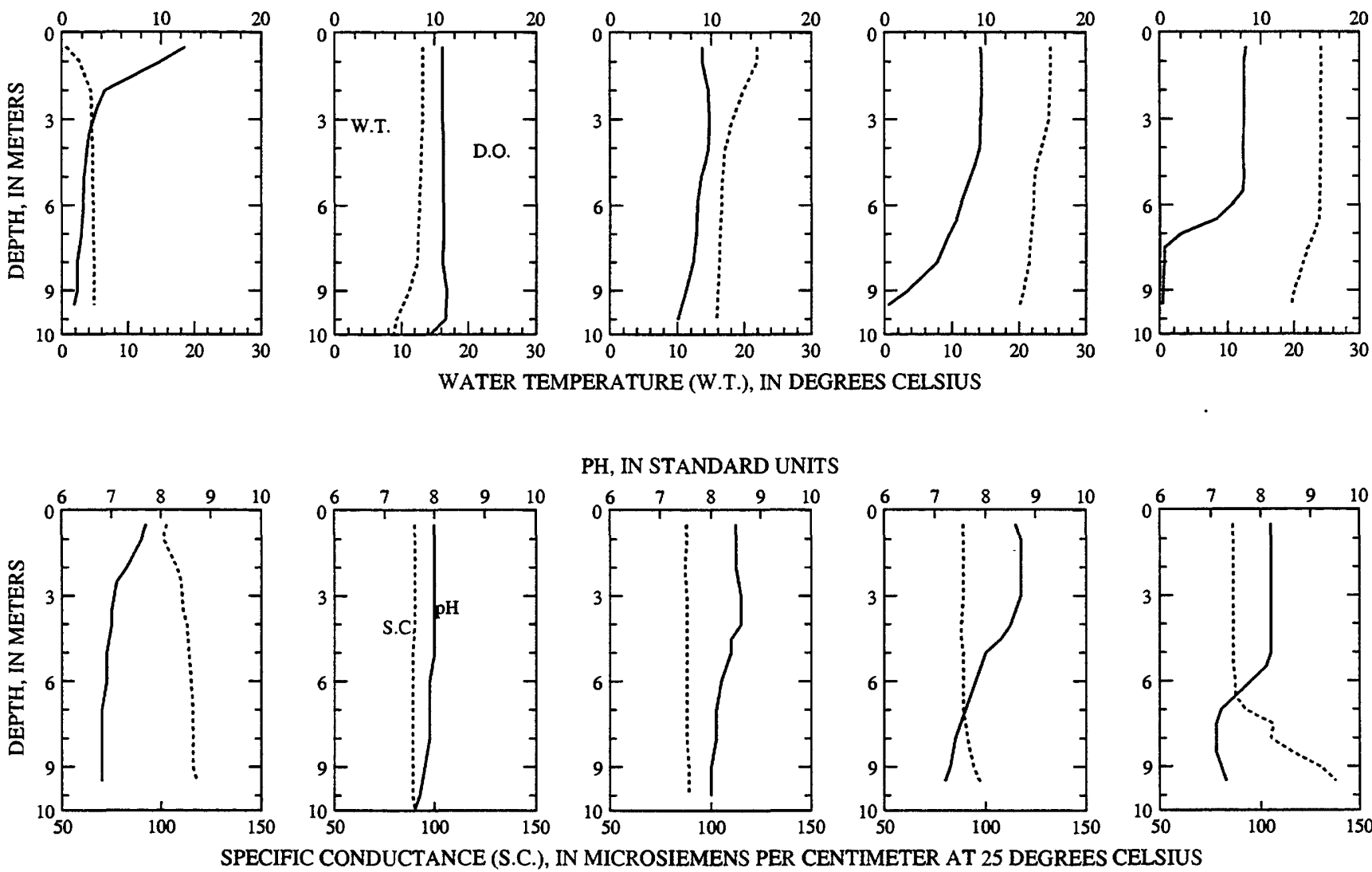

PH, IN STANDARD UNITS
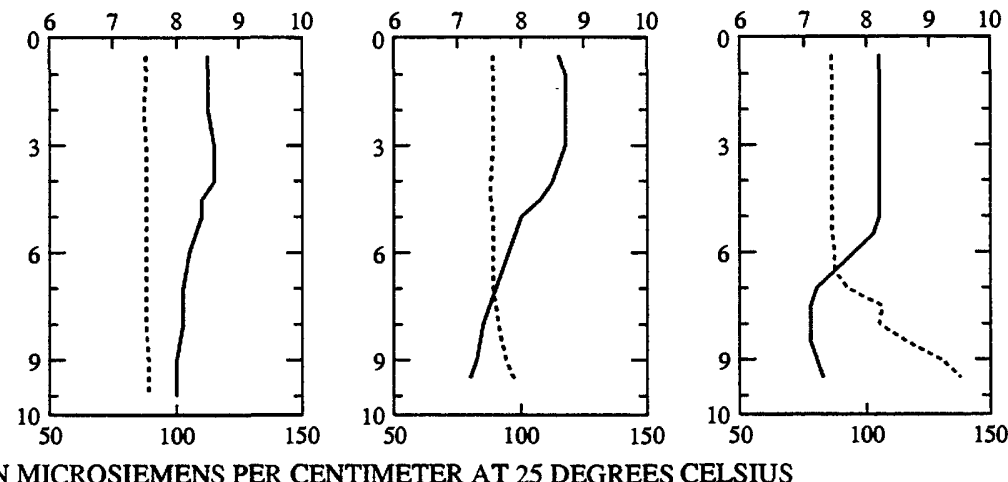

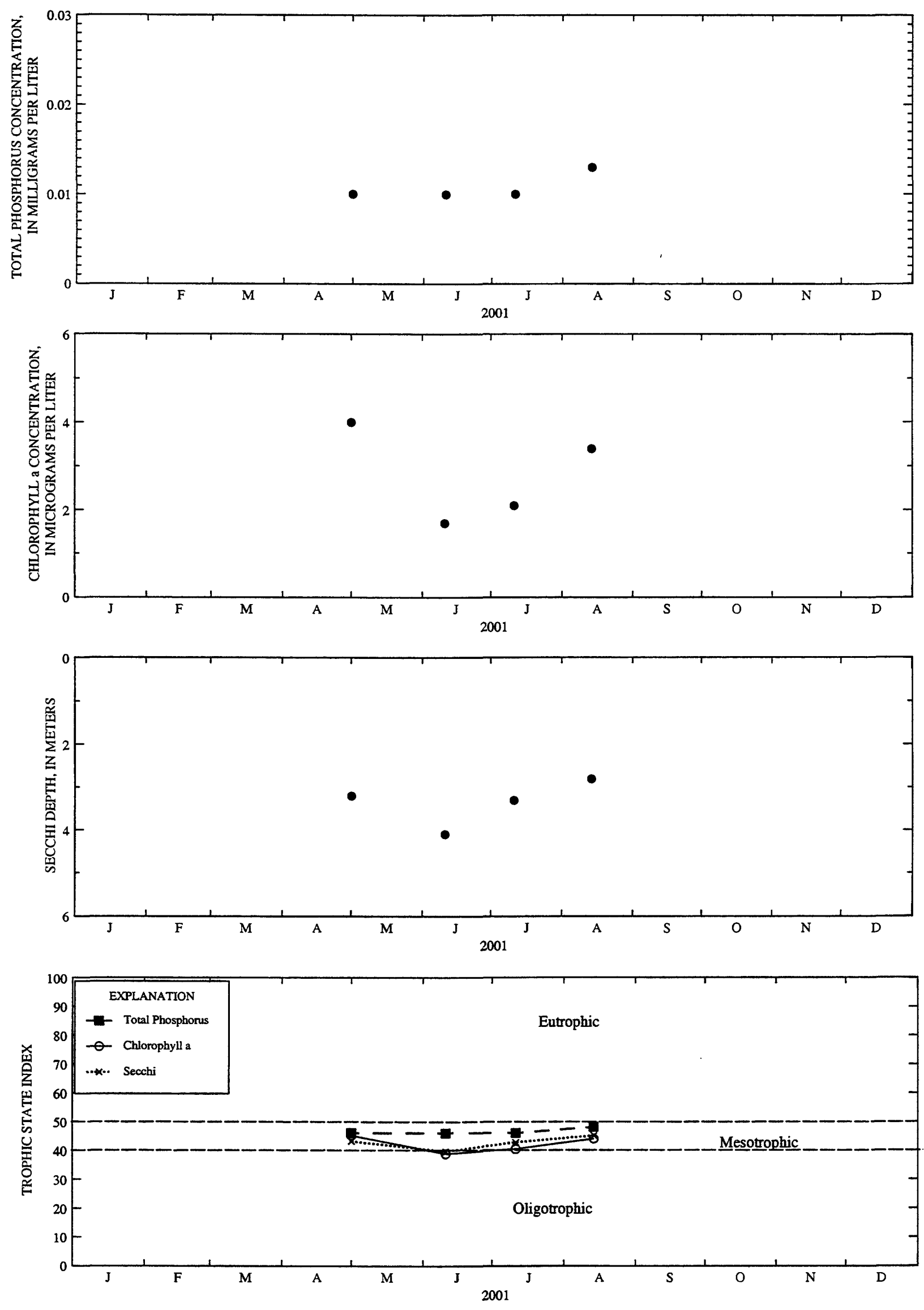

YEAR

Surface total phosphorus, chlorophyll a concentrations, Secchi depths, and TSI data for Big Sand Lake, East Site, near Hertel, Wisconsin. 
LOCATION.--Lat 4547'24", long 91'30'36", in NW 1/4 SE 1/4 sec.6, T.38 N., R.9 W., Sawyer County, Hydrologic Unit 07050001, near Stone Lake.

DRAINAGE AREA.--9.47 $\mathrm{mi}^{2}$.

\section{LAKE-STAGE RECORDS}

PERIOD OF RECORD.--April 1986 to September 1996, and October 1997 to current year, during open-water periods.

GAGE.--Water surface measured from reference point near lake outlet. Measurements were made by Richard Roehrich and James Eary.

EXTREMES FOR PERIOD OF RECORD: Maximum gage height observed, $6.39 \mathrm{ft}$, July 9, 2000; minimum observed, $4.78 \mathrm{ft}$, Sept. 15 , 16 , 1988.

EXTREMES FOR CURRENT YEAR.--Maximum gage height observed, $6.36 \mathrm{ft}$, Apr. 25; minimum observed, less than $5.38 \mathrm{ft}$, Oct. 8 -12.

GAGE HEIGHT, FEET, WATER YEAR OCTOBER 2000 TO SEPTEMBER 2001

DAILY MEAN VALUES

\begin{tabular}{|c|c|c|c|c|c|c|c|c|c|c|c|c|}
\hline DAY & OCT & NOV & $\mathrm{DEC}$ & JAN & FEB & MAR & APR & MAY & JUN & JUL & AUG & SEP \\
\hline $\begin{array}{l}1 \\
2 \\
3 \\
4 \\
5\end{array}$ & $\begin{array}{l}5.55 \\
5.54 \\
5.51 \\
5.48 \\
5.46\end{array}$ & $\begin{array}{l}5.53 \\
5.74 \\
5.80 \\
5.80 \\
5.80\end{array}$ & $\begin{array}{l}--- \\
-- \\
--- \\
---\end{array}$ & $\begin{array}{l}--- \\
--- \\
--- \\
---\end{array}$ & $\begin{array}{l}\cdots \\
-\cdots \\
-\cdots \\
---\end{array}$ & $\begin{array}{l}--- \\
--- \\
--- \\
---\end{array}$ & $\begin{array}{l}--- \\
--- \\
--- \\
---\end{array}$ & $\begin{array}{l}6.20 \\
6.15 \\
6.11 \\
6.08 \\
6.06\end{array}$ & $\begin{array}{l}5.86 \\
5.84 \\
5.80 \\
5.80 \\
5.78\end{array}$ & $\begin{array}{l}5.84 \\
5.81 \\
5.79 \\
5.76 \\
5.74\end{array}$ & $\begin{array}{l}5.85 \\
5.84 \\
5.84 \\
5.82 \\
5.82\end{array}$ & $\begin{array}{l}5.57 \\
5.55 \\
5.54 \\
5.54 \\
5.53\end{array}$ \\
\hline $\begin{array}{r}6 \\
7 \\
8 \\
9 \\
10\end{array}$ & $\begin{array}{l}5.43 \\
5.40 \\
5.38 \\
5.38 \\
5.38\end{array}$ & $\begin{array}{l}5.84 \\
5.90 \\
5.89 \\
5.88 \\
5.85\end{array}$ & $\begin{array}{l}--- \\
--- \\
-- \\
--\end{array}$ & \begin{tabular}{l}
--- \\
\hdashline- \\
--- \\
---
\end{tabular} & $\begin{array}{l}-- \\
\cdots- \\
-\cdots \\
-\cdots\end{array}$ & $\begin{array}{l}--- \\
--- \\
--- \\
---\end{array}$ & $\begin{array}{l}--- \\
--- \\
--- \\
---\end{array}$ & $\begin{array}{l}6.05 \\
6.04 \\
6.02 \\
6.00 \\
6.08\end{array}$ & $\begin{array}{l}5.76 \\
5.80 \\
5.76 \\
5.78 \\
5.83\end{array}$ & $\begin{array}{l}5.73 \\
5.71 \\
5.70 \\
5.70 \\
5.69\end{array}$ & $\begin{array}{l}5.81 \\
5.80 \\
5.73 \\
5.75 \\
5.70\end{array}$ & $\begin{array}{l}5.53 \\
5.60 \\
5.63 \\
5.64 \\
5.62\end{array}$ \\
\hline $\begin{array}{l}11 \\
12 \\
13 \\
14 \\
15\end{array}$ & $\begin{array}{l}5.38 \\
5.38 \\
5.39 \\
5.40 \\
5.41\end{array}$ & $\begin{array}{l}5.84 \\
5.86 \\
5.89 \\
5.90 \\
5.90\end{array}$ & $\begin{array}{l}-- \\
-- \\
-- \\
--\end{array}$ & $\begin{array}{l}--- \\
--- \\
--- \\
--- \\
---\end{array}$ & $\begin{array}{l}--- \\
--- \\
--- \\
---\end{array}$ & $\begin{array}{l}--- \\
-- \\
-- \\
-- \\
--\end{array}$ & \begin{tabular}{l}
--- \\
--- \\
\hdashline- \\
-- \\
--
\end{tabular} & $\begin{array}{l}6.05 \\
6.04 \\
6.03 \\
6.15 \\
6.12\end{array}$ & $\begin{array}{r}--- \\
--98 \\
6.00 \\
5.98\end{array}$ & $\begin{array}{r}5.68 \\
5.67 \\
5.63\end{array}$ & $\begin{array}{l}5.69 \\
5.68 \\
5.66 \\
5.65 \\
5.66\end{array}$ & $\begin{array}{l}5.62 \\
5.61 \\
5.60 \\
5.55 \\
5.55\end{array}$ \\
\hline $\begin{array}{l}16 \\
17 \\
18 \\
19 \\
20\end{array}$ & $\begin{array}{l}5.41 \\
5.41 \\
5.41 \\
5.41 \\
5.42\end{array}$ & $\begin{array}{l}5.90 \\
5.89 \\
5.88 \\
5.87 \\
5.87\end{array}$ & $\begin{array}{l}--- \\
-- \\
--- \\
--- \\
--\end{array}$ & $\begin{array}{l}--- \\
-- \\
--- \\
---\end{array}$ & $\begin{array}{l}-- \\
-- \\
-- \\
-- \\
--\end{array}$ & $\begin{array}{l}--- \\
--- \\
--- \\
---\end{array}$ & $\begin{array}{l}--- \\
--- \\
--- \\
---\end{array}$ & $\begin{array}{r}6.10 \\
6.06 \\
-0.00 \\
5.96\end{array}$ & $\begin{array}{l}5.99 \\
5.97 \\
6.05 \\
6.10 \\
6.10\end{array}$ & $\begin{array}{r}5.62 \\
5.63 \\
5.68 \\
5.66 \\
-.-\end{array}$ & $\begin{array}{l}5.67 \\
5.66 \\
5.67 \\
5.66 \\
5.65\end{array}$ & $\begin{array}{l}5.55 \\
5.55 \\
5.55 \\
5.55 \\
5.55\end{array}$ \\
\hline $\begin{array}{l}21 \\
22 \\
23 \\
24 \\
25\end{array}$ & $\begin{array}{l}5.42 \\
5.42 \\
5.43 \\
5.44 \\
5.45\end{array}$ & $\begin{array}{l}5.87 \\
5.86 \\
5.86 \\
5.85 \\
5.84\end{array}$ & $\begin{array}{l}-- \\
\cdots \\
-\cdots \\
--\end{array}$ & $\begin{array}{c}--- \\
-- \\
\cdots- \\
--- \\
--\end{array}$ & $\begin{array}{l}--- \\
--- \\
--- \\
---\end{array}$ & $\begin{array}{l}-- \\
-- \\
--- \\
---\end{array}$ & $\begin{array}{c}--- \\
-\cdots \\
-36 \\
6.36\end{array}$ & $\begin{array}{l}5.98 \\
6.00 \\
5.98 \\
5.95 \\
6.00\end{array}$ & $\begin{array}{r}6.08 \\
6.05 \\
-.0 \\
6.00 \\
5.98\end{array}$ & $\begin{array}{l}5.75 \\
5.75 \\
5.73 \\
5.70\end{array}$ & $\begin{array}{l}5.65 \\
5.64 \\
5.63 \\
5.61 \\
5.60\end{array}$ & $\begin{array}{l}5.55 \\
5.56 \\
5.56 \\
5.55 \\
5.52\end{array}$ \\
\hline $\begin{array}{l}26 \\
27 \\
28 \\
29 \\
30 \\
31\end{array}$ & $\begin{array}{l}5.46 \\
5.48 \\
5.46 \\
5.48 \\
5.50 \\
5.51\end{array}$ & $\begin{array}{r}5.84 \\
5.84 \\
5.84 \\
5.84 \\
5.84 \\
-\end{array}$ & $\begin{array}{c}--- \\
--- \\
--- \\
--- \\
---\end{array}$ & $\begin{array}{l}--. \\
--- \\
-- \\
-- \\
-\cdots \\
--\end{array}$ & $\begin{array}{l}-\cdots \\
-\cdots \\
--- \\
-\cdots \\
-\cdots\end{array}$ & $\begin{array}{l}--- \\
--- \\
-- \\
--- \\
---\end{array}$ & $\begin{array}{r}6.31 \\
6.25 \\
6.23 \\
6.22 \\
6.21 \\
-.-\end{array}$ & $\begin{array}{l}5.98 \\
5.96 \\
5.95 \\
5.94 \\
5.90 \\
5.88\end{array}$ & $\begin{array}{r}5.95 \\
5.93 \\
5.91 \\
5.89 \\
5.85 \\
-\end{array}$ & $\begin{array}{l}5.69 \\
5.67 \\
5.70 \\
5.69 \\
5.69 \\
5.69\end{array}$ & $\begin{array}{l}5.60 \\
5.60 \\
5.59 \\
5.59 \\
5.60 \\
5.59\end{array}$ & $\begin{array}{r}5.55 \\
5.50 \\
5.46 \\
5.45 \\
5.42 \\
---\end{array}$ \\
\hline $\begin{array}{l}\text { MEAN } \\
\text { MAX } \\
\text { MIN }\end{array}$ & $\begin{array}{l}5.44 \\
5.55 \\
5.38\end{array}$ & $\begin{array}{l}5.84 \\
5.90 \\
5.53\end{array}$ & $=-$ & $\overline{--}$ & $\begin{array}{l}--- \\
---\end{array}$ & $=-$ & $\begin{array}{l}-\cdots \\
--\end{array}$ & $\begin{array}{l}--- \\
--- \\
---\end{array}$ & $\begin{array}{l}--- \\
--- \\
---\end{array}$ & $=-$ & $\begin{array}{l}5.69 \\
5.85 \\
5.59\end{array}$ & $\begin{array}{l}5.55 \\
5.64 \\
5.42\end{array}$ \\
\hline
\end{tabular}




\section{WATER-QUALITY RECORDS}

PERIOD OF RECORD.--1986 to 1996, and March 1998 to current year.

REMARKS.--Lake sampled near center at the deep hole. Lake ice-covered during March sampling. Water-quality analyses done by Wisconsin State Laboratory of Hygiene.

WATER-QUALITY DATA, MARCH 01 TO AUGUST 13, 2001
(Milligrams per liter unless otherwise indicated)

Lake stage (ft)

Secchi-depth (m)

Chlorophy11 a, phytoplankton ( $\mu \mathrm{g} / \mathrm{L})$

Depth of sample (m)

Water temperature $\left({ }^{\circ} \mathrm{C}\right)$

Specific conductance $(\mu \mathrm{S} / \mathrm{cm})$

$\mathrm{pH}$ (units)

Dissolved oxygen ( $\mathrm{mg} / \mathrm{L}$ )

Phosphorus, total (as P)

Phosphorus, ortho, dissolved (as P)

Nitrogen, NO2 + NO3, diss. (as N)

Nitrogen, ammonia, dissolved (as $\mathrm{N}$ )

Nitrogen, amm. + org., total (as N)

Nitrogen, total (as $\mathrm{N}$ )

Color (Pt-Co. scale)

Turbidity (NTU)

Hardness, (as $\mathrm{CaCO}_{3}$ )

Calcium, dissolved (Ca)

Magnesium, dissolved (Mg)

Sodium, dissolved (Na)

Potassium, dissolved (K)

Alkalinity, (as $\mathrm{CaCO}_{3}$ )

Sulfate, dissolved $\left(\mathrm{SO}_{4}\right)$

Chloride, dissolved (C1)

Silica, dissolved ( $\mathrm{SiO}_{2}$ )

Solids. dissolved. at $180^{\circ} \mathrm{C}$

Iron, dissolved (Fe) $\mu \mathrm{g} / \mathrm{L}$

Manganese, dissolved (Mn) $\mu \mathrm{g} / \mathrm{L}$

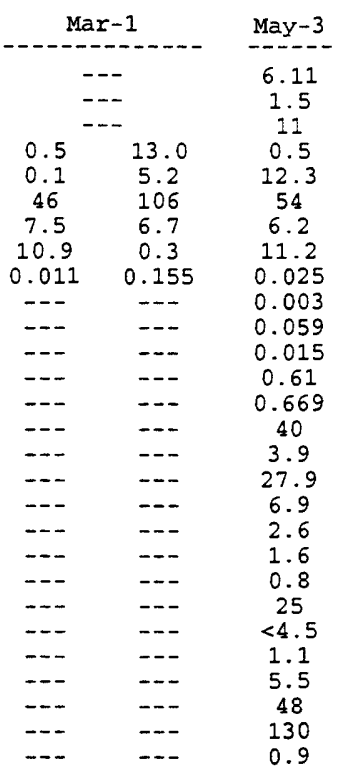

\begin{tabular}{|c|c|}
\hline \multicolumn{2}{|c|}{ Jun-12 } \\
\hline \multicolumn{2}{|c|}{$\begin{array}{c}5.88 \\
2.4 \\
5\end{array}$} \\
\hline $\begin{array}{c}0.5 \\
22.1 \\
56 \\
7.7 \\
10.3 \\
0.019\end{array}$ & $\begin{array}{c}14.0 \\
12.5 \\
68 \\
6.8 \\
0.7 \\
0.096\end{array}$ \\
\hline-- & $\ldots$ \\
\hline--- & $-\infty$ \\
\hline-- & $\cdots$ \\
\hline-- & $\cdots$ \\
\hline$\cdots$ & $\cdots$ \\
\hline$-\cdots$ & -- \\
\hline-- & $\cdots$ \\
\hline-- & $\cdots$ \\
\hline$\cdots$ & -- \\
\hline--- & $-\infty$ \\
\hline--- & $-\infty$ \\
\hline-- & $\cdots$ \\
\hline--- & $\cdots$ \\
\hline-- & $\cdots$ \\
\hline--- & --- \\
\hline-- & --- \\
\hline--- & -- \\
\hline- & $\ldots$ \\
\hline
\end{tabular}

6-12-01

\begin{tabular}{|c|c|}
\hline \multicolumn{2}{|c|}{ JuI-11 } \\
\hline \multicolumn{2}{|c|}{$\begin{array}{c}5.68 \\
2.3\end{array}$} \\
\hline & \\
\hline $\begin{array}{c}0.5 \\
20.3\end{array}$ & $\begin{array}{l}14.0 \\
13.1\end{array}$ \\
\hline 55 & 90 \\
\hline $\begin{array}{c}8.0 \\
10.5\end{array}$ & $\begin{array}{l}7.2 \\
0.2\end{array}$ \\
\hline$\ldots$ & 0.099 \\
\hline--- & --- \\
\hline$\ldots$ & $\ldots$ \\
\hline--- & --- \\
\hline$\ldots$ & --- \\
\hline--- & --- \\
\hline-- & --- \\
\hline--- & --- \\
\hline--- & --- \\
\hline-- & --- \\
\hline-- & --- \\
\hline$\cdots$ & --- \\
\hline$\cdots$ & --- \\
\hline--- & $\cdots$ \\
\hline--- & --- \\
\hline--- & --- \\
\hline-- & --- \\
\hline-- & --- \\
\hline--- & --- \\
\hline- & -- \\
\hline
\end{tabular}

7-11-01

8-13-01

DISSOLVED OXYGEN (D.O.), IN MILLIGRAMS PER LITER
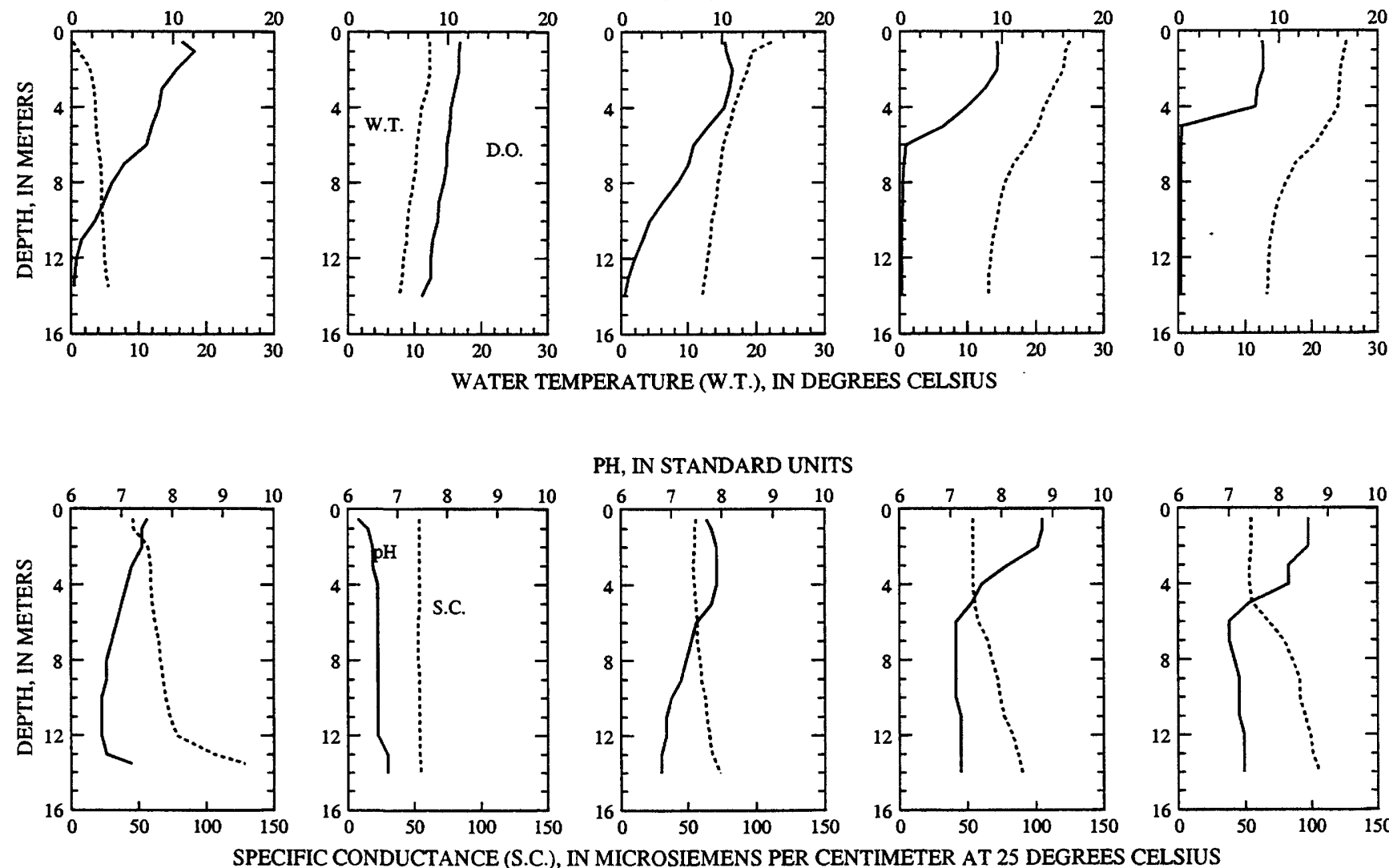

PH, IN STANDARD UNITS
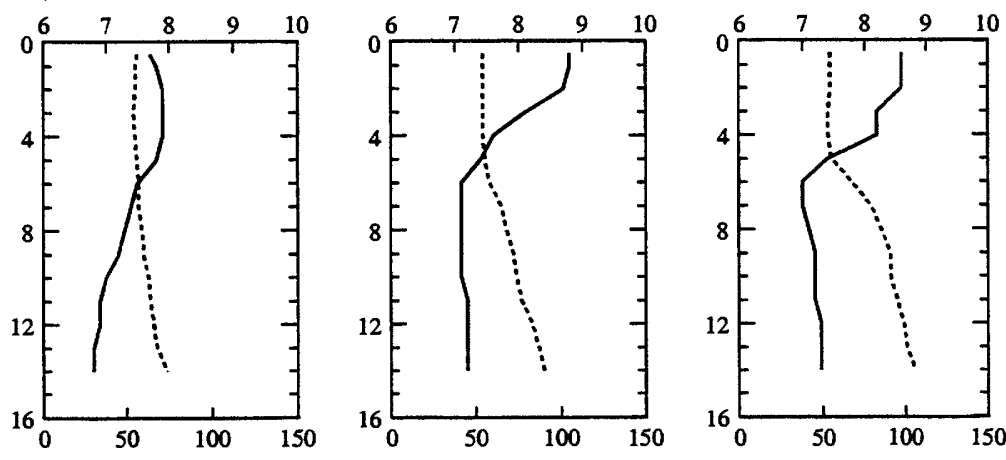

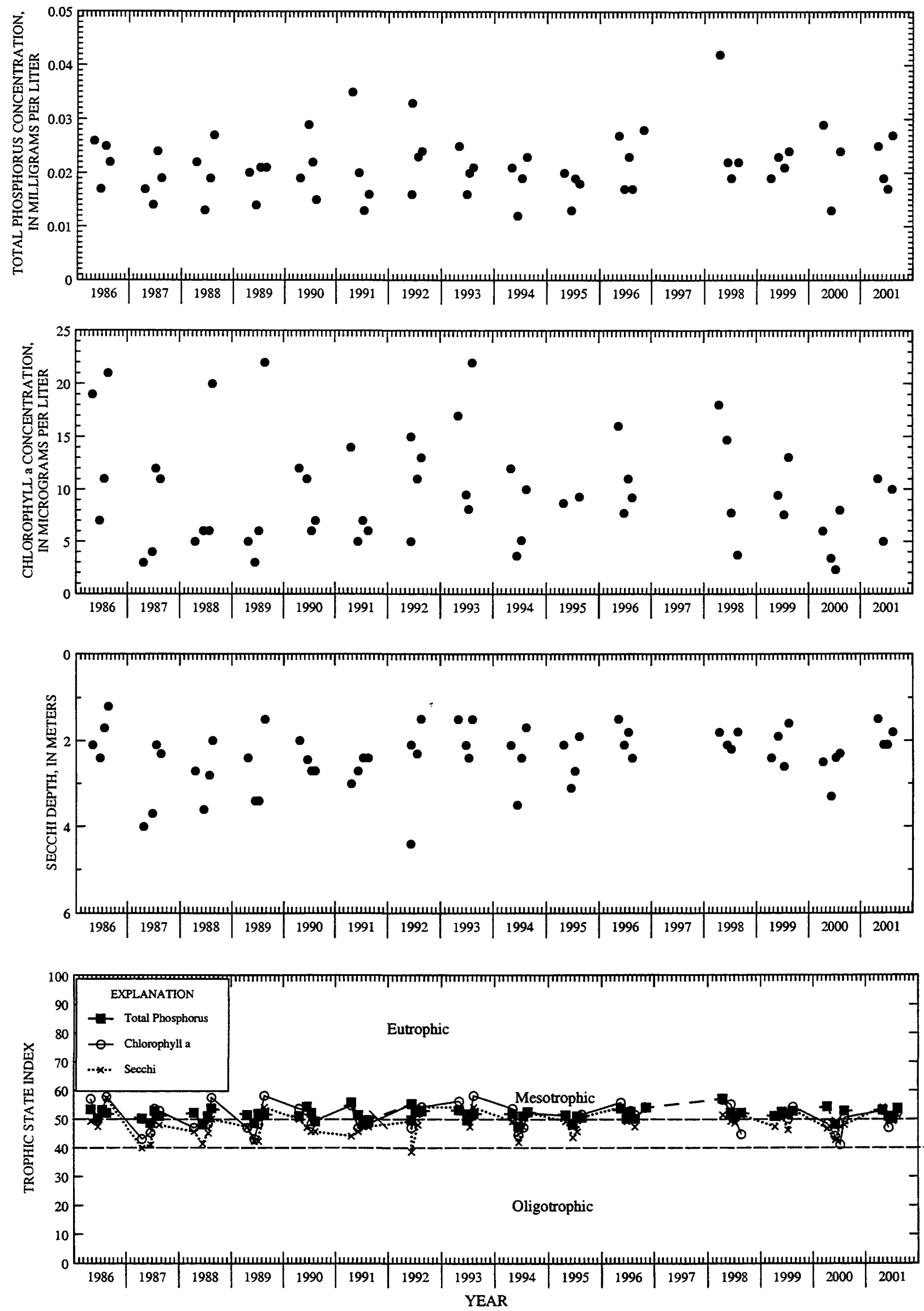

Surface total phosphorus, chlorophyll a concentrations, Secchi depths, and TSI data for Big Sissabagma Lake, near Stone Lake, Wisconsin. 
LOCATION.--Lat $42^{\circ} 48^{\prime} 00^{\prime \prime}$, long 91 $31^{\prime} 29^{\prime \prime}$, in NE 1/4 SE 1/4 sec.6, T.38 N., R.9 W., Sawyer County, Hydrologic Unit 07050001, near Stone Lake.

DRAINAGE AREA.--9.47 $\mathrm{mi}^{2}$.

PERIOD OF RECORD.--March 1998 to current year.

REMARKS.--Lake sampled near the deepest part of the North Bay. Lake ice-covered during March sampling. Water-quality analyses done by Wisconsin State Laboratory of Hygiene.

WATER-QUALITY DATA, MARCH 01 TO AUGUST 13, 2001

(Milligrams per liter unless otherwise indicated)

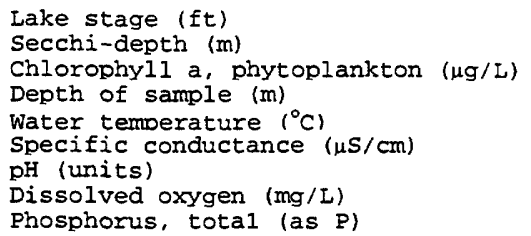

\begin{tabular}{cccccc} 
Mar-1 & May-3 & Jun-12 & Jul-11 & \multicolumn{2}{c}{ Aug-13 } \\
\hdashline-- & 6.11 & 5.88 & 5.68 & \multicolumn{2}{c}{5.66} \\
$\cdots$ & 1.6 & 2.3 & 2.1 & \multicolumn{2}{c}{1.7} \\
-- & 11 & 4.2 & 3.5 & \multicolumn{2}{c}{10} \\
0.5 & 0.5 & 0.5 & 0.5 & 0.5 & 6.5 \\
0.1 & 13.0 & 20.3 & 24.8 & 25.0 & 15.9 \\
56 & 55 & 55 & 54 & 54 & 113 \\
7.8 & 7.5 & 8.0 & 8.4 & 8.5 & 7.2 \\
11.9 & 10.8 & 10.5 & 9.4 & 8.7 & 0.2 \\
0.012 & 0.020 & 0.019 & 0.020 & 0.021 & 0.041
\end{tabular}
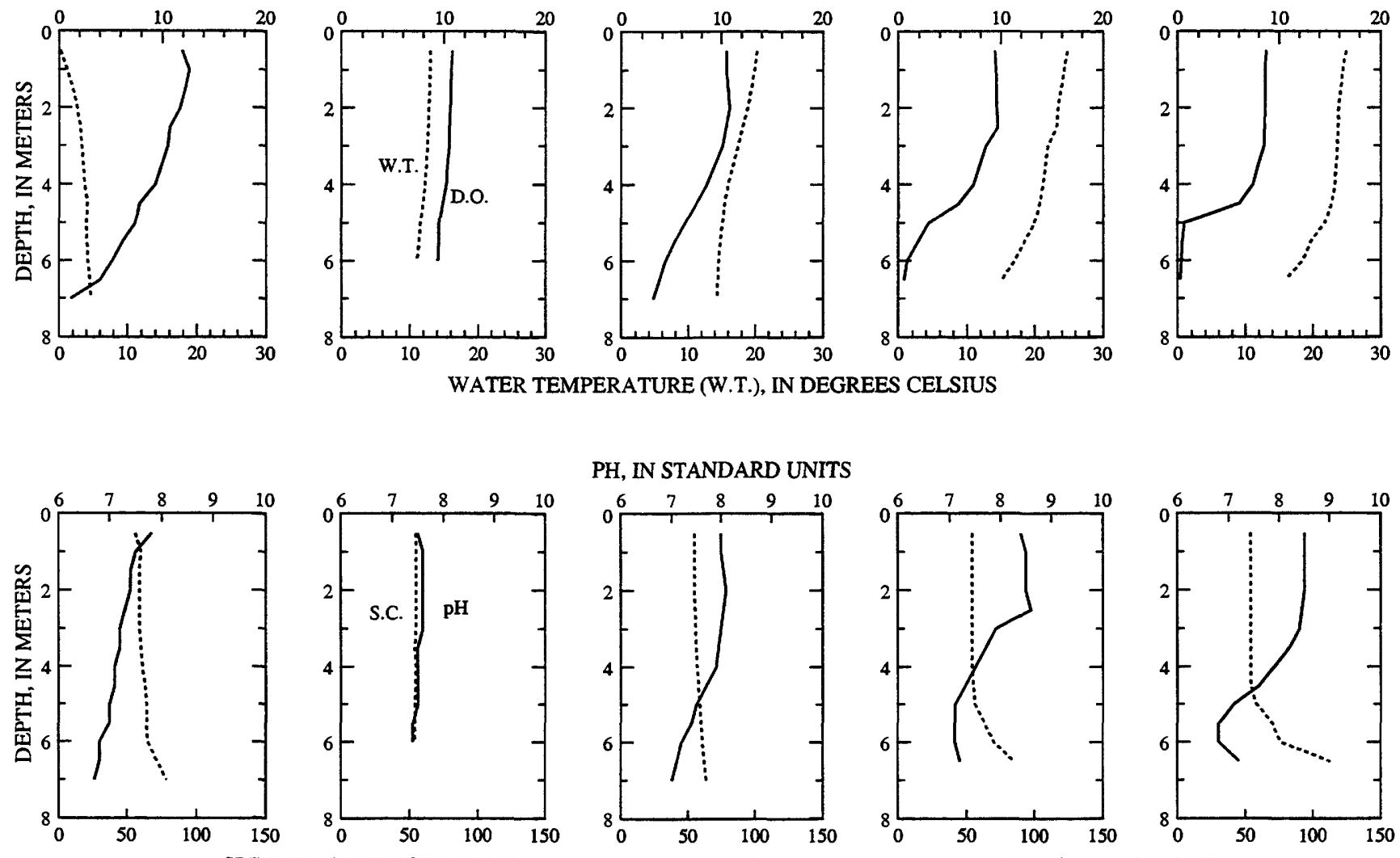

PH, IN STANDARD UNITS
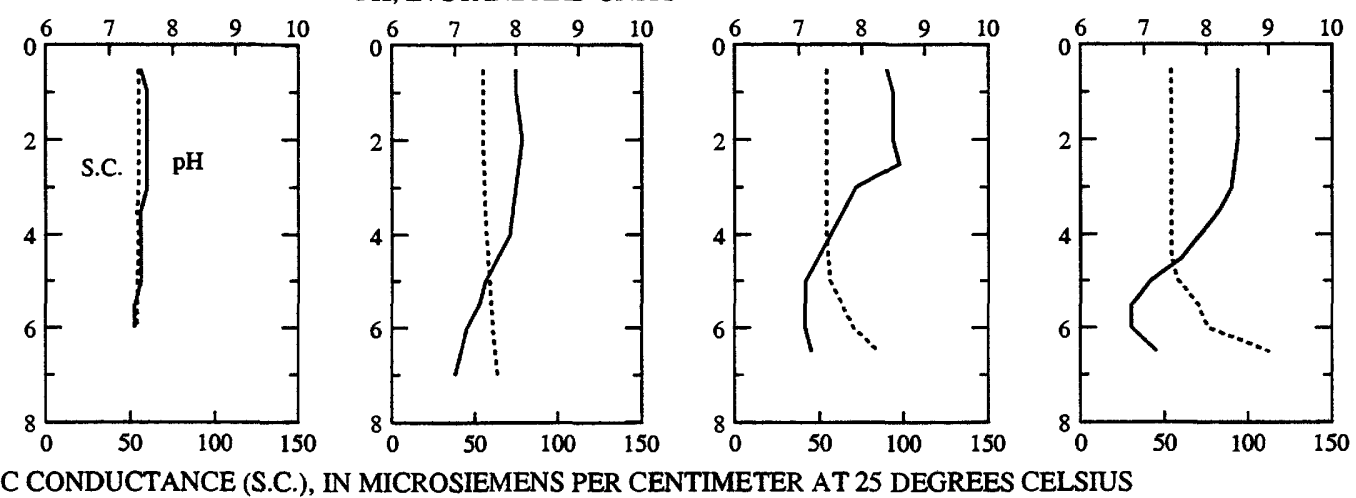

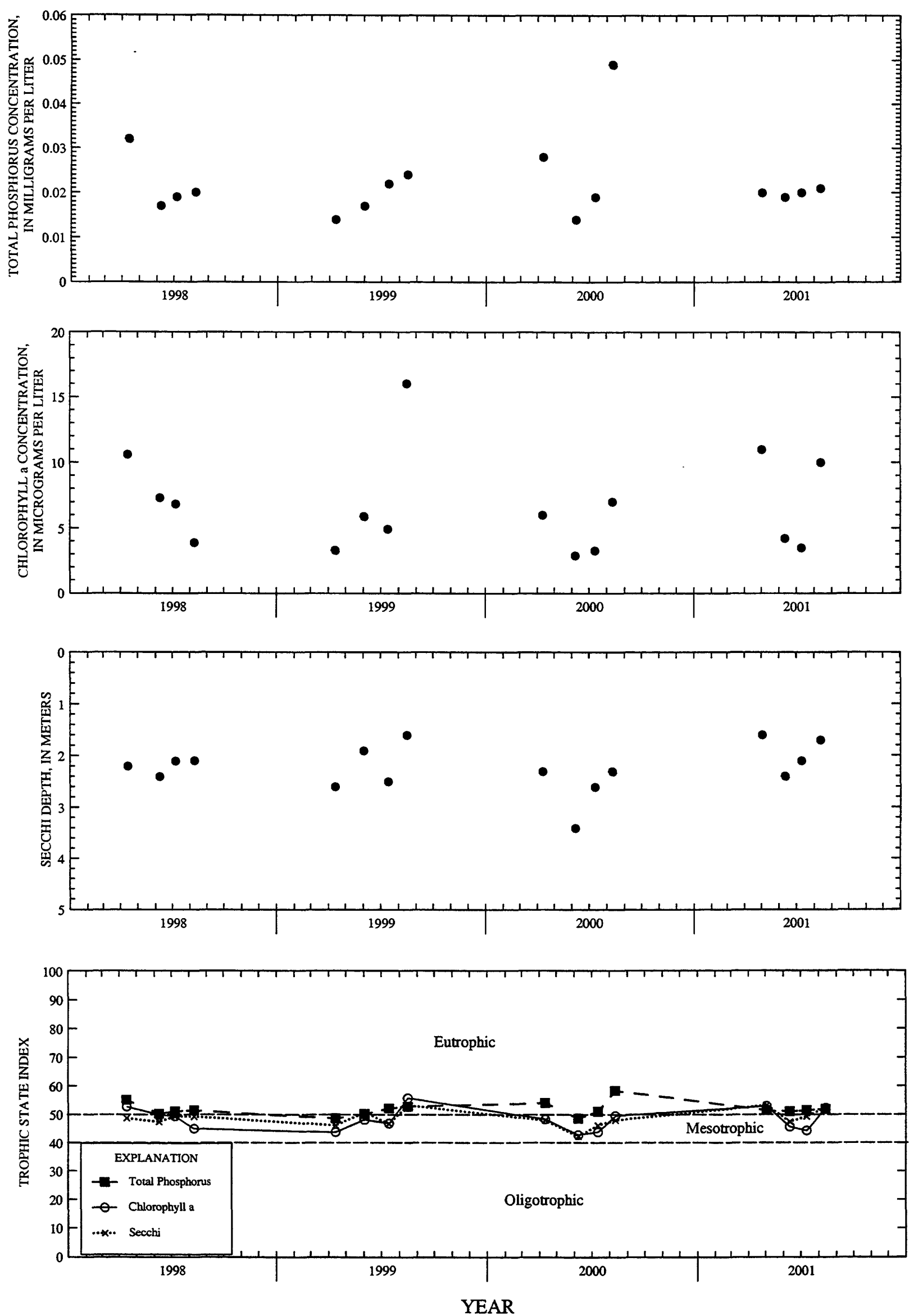

Surface total phosphorus, chlorophyll a concentrations, Secchi depths, and TSI data for Big Sissabagma Lake, North Site, near Stone Lake, Wisconsin. 
LOCATION.--Lat $42^{\circ} 48^{\prime} 00^{\prime \prime}$, long $88^{\circ} 25^{\prime} 48^{\prime \prime}$, in SW 1/4 SE 1/4 sec.13, T.4 N., R.17 E., Walworth County, Hydrologic Unit 07120006, 1.6 mi northwest of East Troy.

PERIOD OF RECORD.--February 1992 to August 1994, February to August 2001.

REMARKS.--Lake sampled near center of lake at the deep hole. Lake ice-covered during February sampling. Water-quality analyses done by Wisconsin State Laboratory of Hygiene.

WATER-QUALITY DATA, FEBRUARY 12 TO AUGUST 14, 2001

(Milligrams per liter unless otherwise indicated)

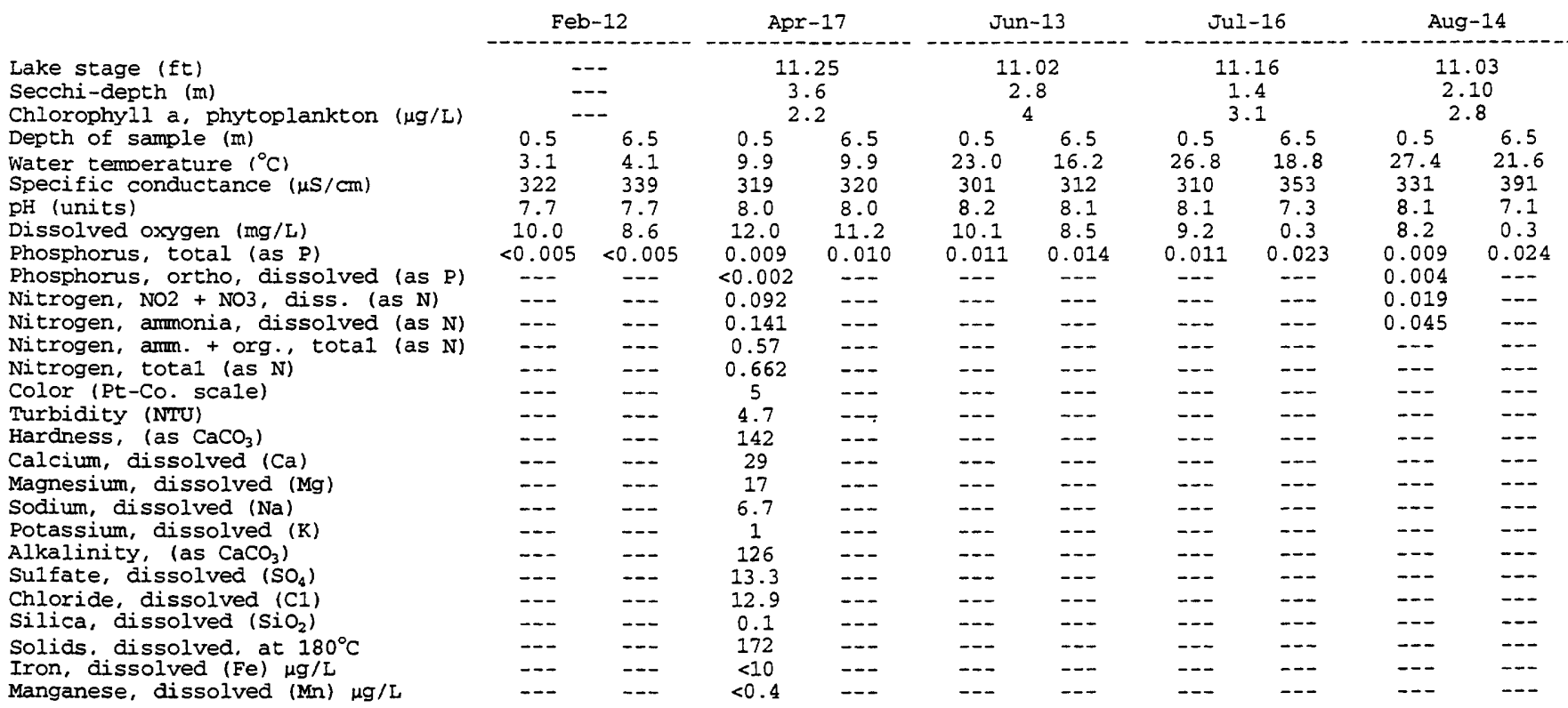

DISSOLVED OXYGEN (D.O.), IN MILLIGRAMS PER LITER
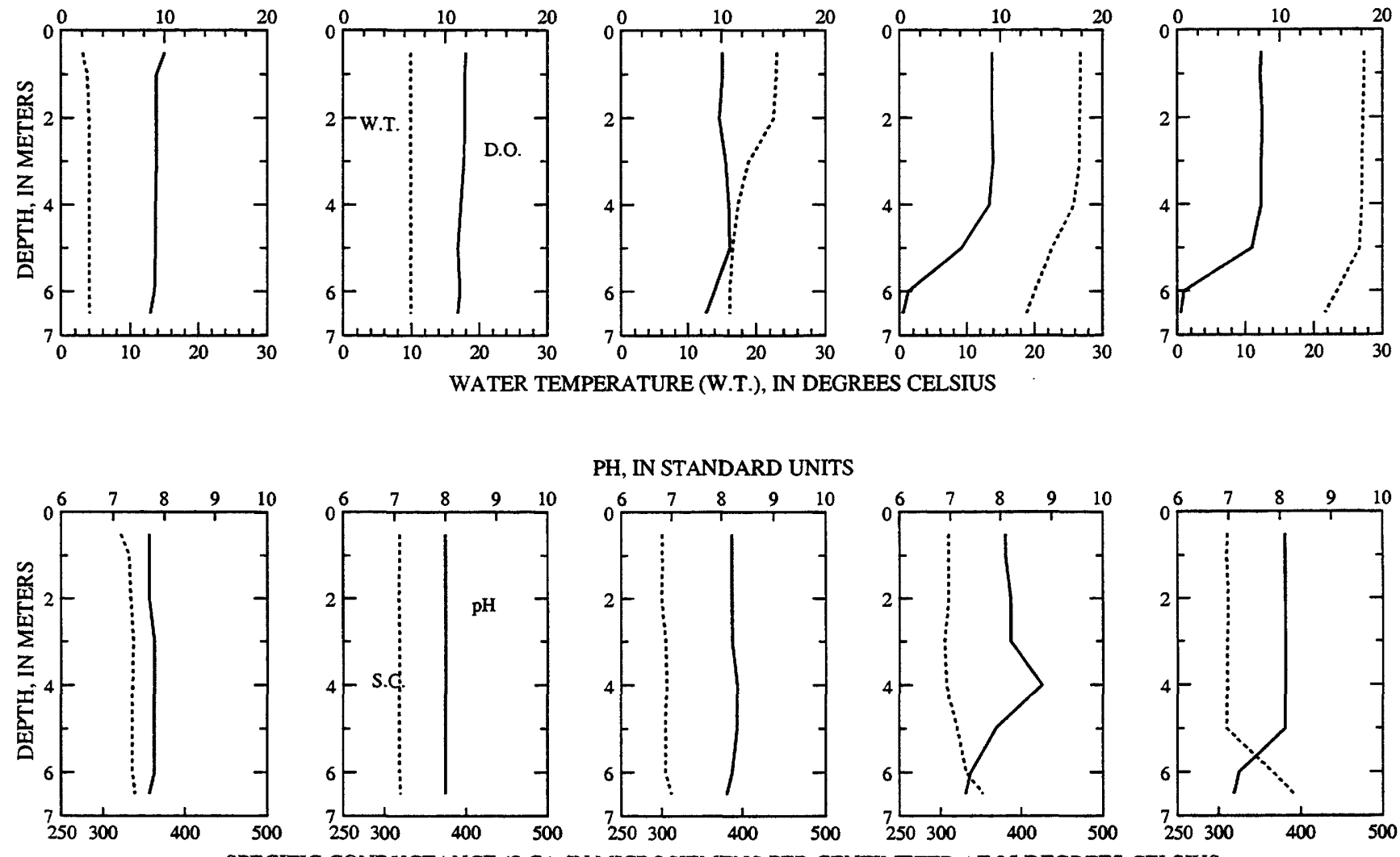

PH, IN STANDARD UNITS
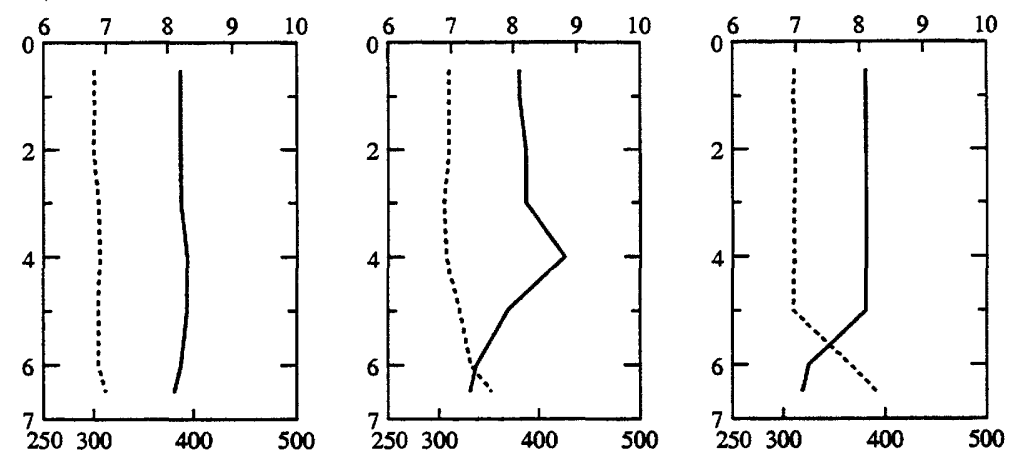

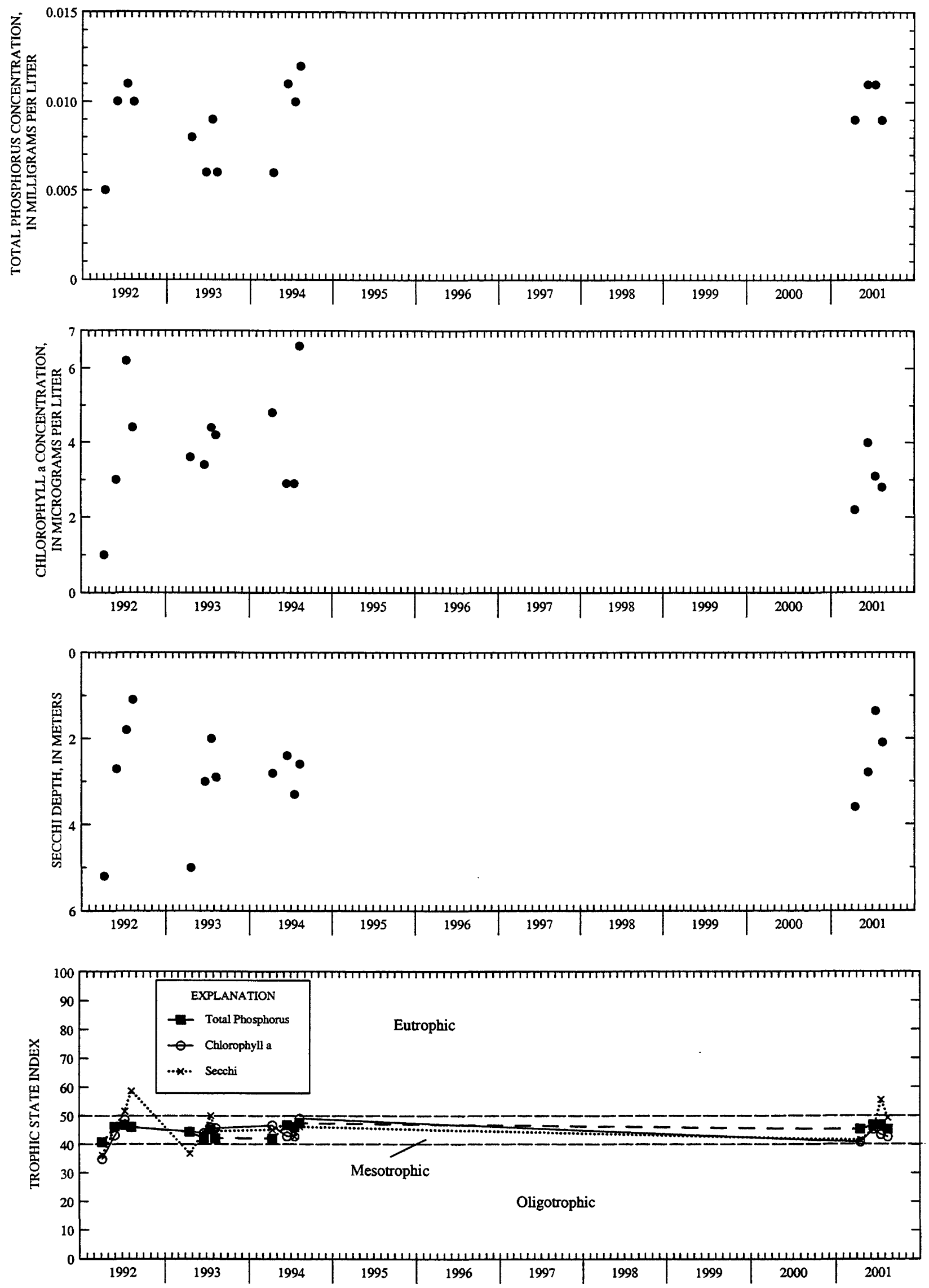

Surface total phosphorus, chlorophyll a concentrations, Secchi depths, and TSI data for Booth Lake, near East Troy, Wisconsin. 


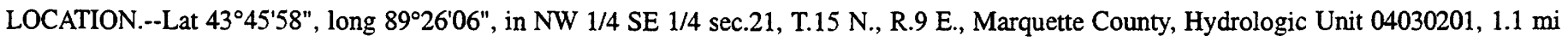
northeast of Pacl: zukee.

PERIOD OF RECOR, , --May 1998 to current year. Data collected 1991-94 by Wisconsin Department of Natural Resources are available.

REMARKS.--Site sampled near center of lake. Lake ice-covered during February sampling. Water-quality analyses done by Wisconsin State Laboratory of Hygiene.

WATER-QUALITY DATA, FEBRUARY 15 TO AUGUST 22, 2001

(Milligrams per liter unless otherwise indicated)

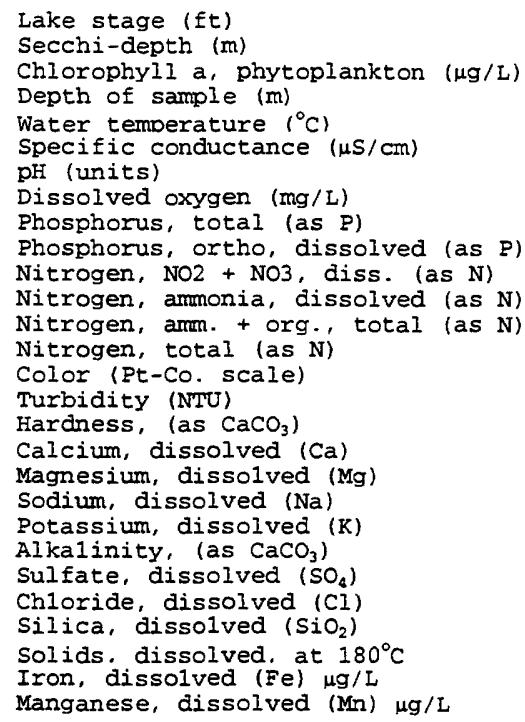

$2-15-01$

$$
\text { 4-25-01 }
$$

\begin{tabular}{|c|c|c|c|c|}
\hline Feb-15 & Apr -25 & Jun-21 & Jul-24 & Aug-22 \\
\hline 1.80 & 1.68 & 2.29 & 1.37 & 1.36 \\
\hline-- & 0.5 & 1.0 & 0.7 & 1.0 \\
\hline$\ldots$ & 81 & 8.5 & 14 & 16.3 \\
\hline 0.5 & 0.5 & 0.25 & 0.5 & 0.5 \\
\hline 1.0 & 14.2 & 24.7 & 28.1 & 23.0 \\
\hline 479 & 382 & 333 & 375 & 351 \\
\hline 7.6 & 8.4 & 7.1 & 7.8 & 8.0 \\
\hline 10.2 & 14.2 & 6.2 & 6.6 & 8.2 \\
\hline 0.034 & $<0.005$ & 0.138 & 0.276 & 0.091 \\
\hline-- & 0.004 & -- & -- & -- \\
\hline$\ldots$ & 0.841 & --- & $\ldots$ &.-- \\
\hline--- & $<0.013$ & $\ldots$ & --- & $-\cdots$ \\
\hline-- & 1.7 & -- & --- & -- \\
\hline$\cdots$ & 2.53 & --- & -- & --- \\
\hline$-\cdots$ & 50 & --- & $\ldots$ & $\ldots$ \\
\hline--- & 12 & $\ldots$ & $\ldots$ & --- \\
\hline$\ldots$ & 196 & $\ldots$ & --- & $\ldots$ \\
\hline--- & 42 & -- & $\ldots$ & -- \\
\hline-- & 22 & -- & -- & -- \\
\hline--- & 5.4 & -- & -- & --- \\
\hline-- & 1.5 & -- & -- & -- \\
\hline$\ldots$ & 167 & $\ldots$ & --- & $-\cdots$ \\
\hline$\ldots$ & 14.3 & $\ldots$ & $\ldots$ & $\ldots$ \\
\hline--- & 10.9 & -- & $\ldots$ & $\cdots$ \\
\hline-- & 3.9 & -- & -- & $\cdots$ \\
\hline--- & 230 & -- & -- & $--\infty$ \\
\hline-- & 100 & -- & $-\infty-$ & --- \\
\hline--- & 1.6 & -- & --- & $-\cdots$ \\
\hline
\end{tabular}

6-21-01

DISSOLVED OXYGEN (D.O.), IN MILLIGRAMS PER LITER
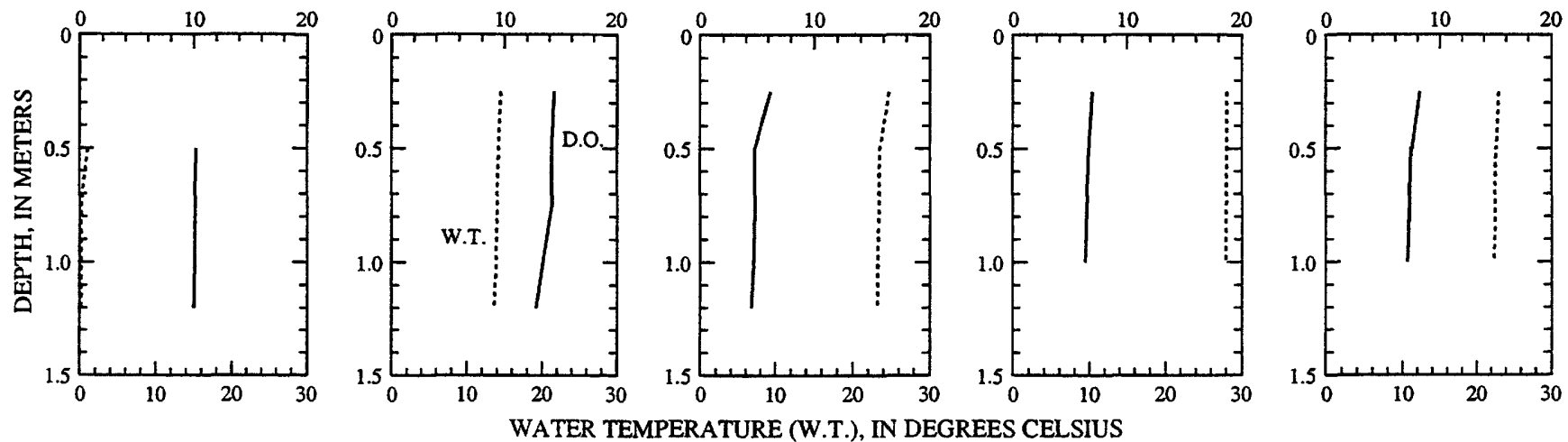

PH, IN STANDARD UNITS
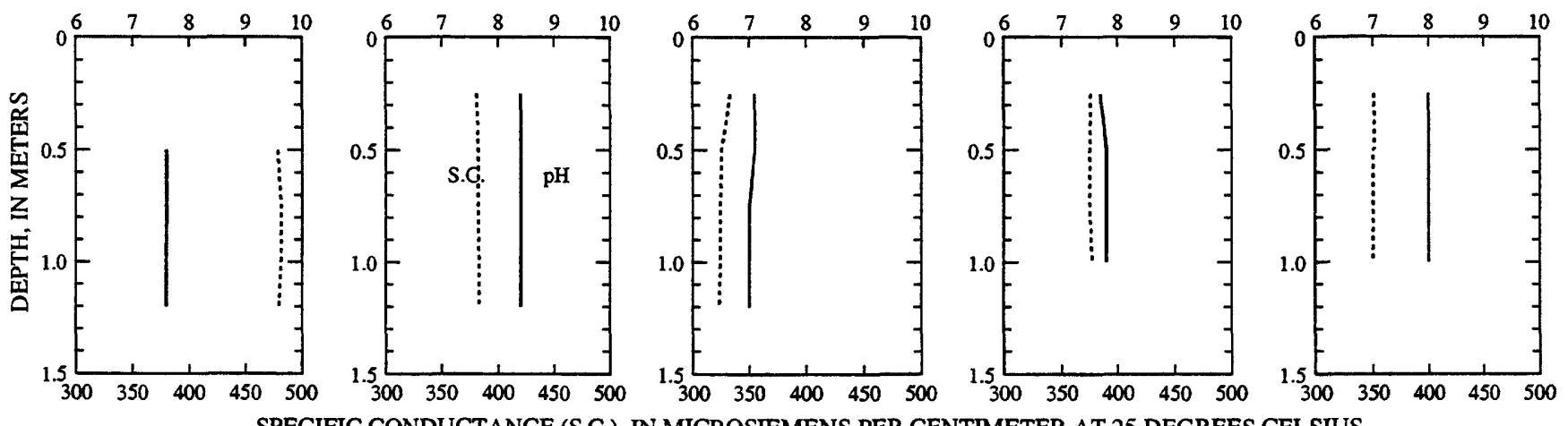

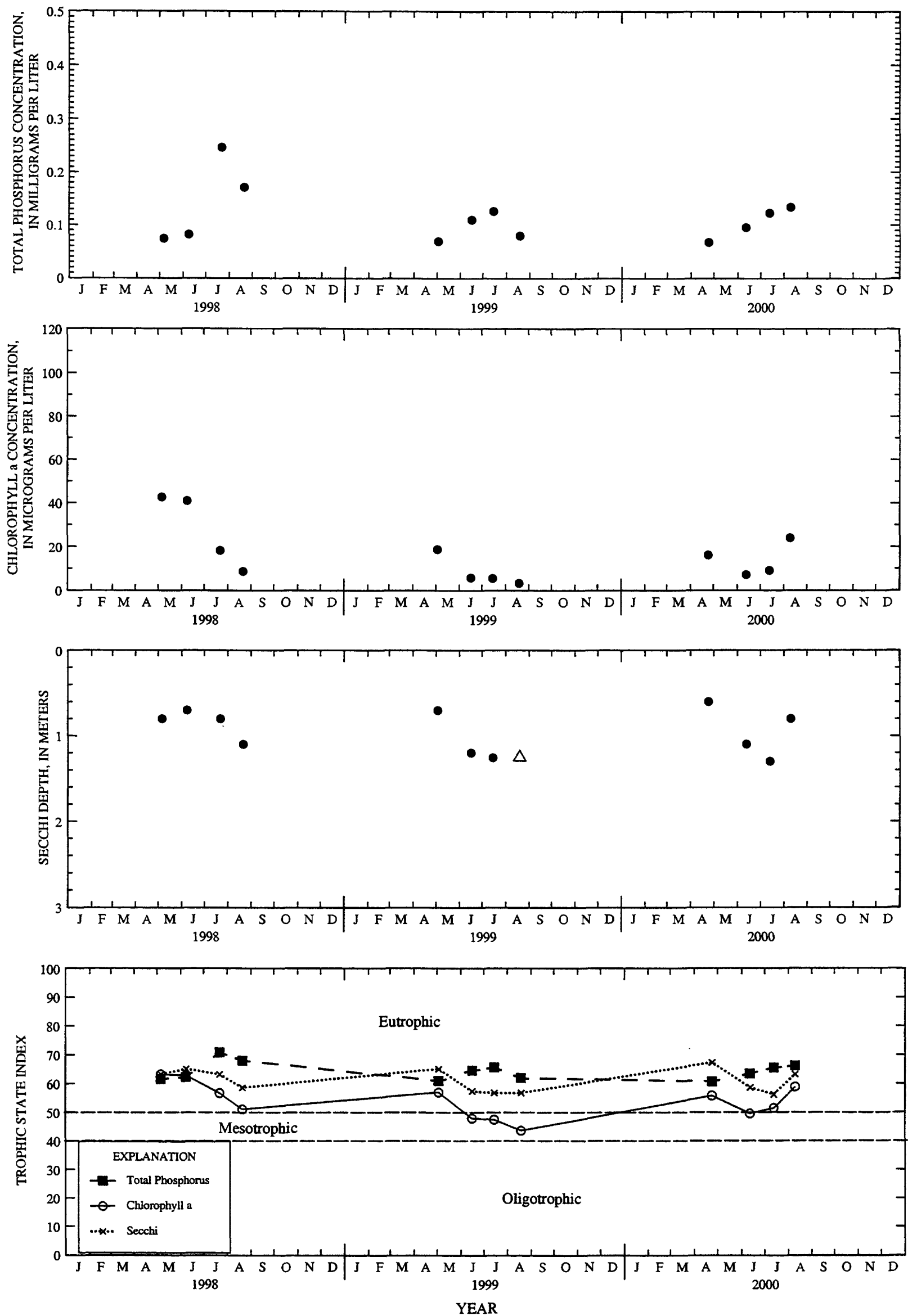

Surface total phosphorus, chlorophyll a concentrations, Secchi depths, and TSI data for Buffalo Lake, Center Site, near Packwaukee, Wisconsin.

(Triangles indicate maximum depth at sampling site. Actual Secchi depth on these days was greater than plotted values) 


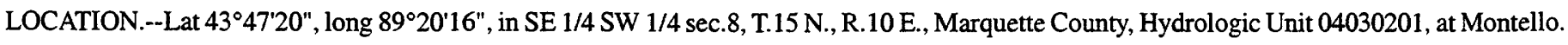
PERIOD OF RECORD.--May 1998 to current year. Data collected 1991-94 by Wisconsin Department of Natural Resources are available.

REMARKS.--Site sampled at east end of lake. Lake ice-covered during February sampling. Water-quality analyses done by Wisconsin State Laboratory of Hygiene.

WATER-QUALITY DATA, FEBRUARY 15 TO AUGUST 22, 2001

(Milligrams per liter unless otherwise indicated)

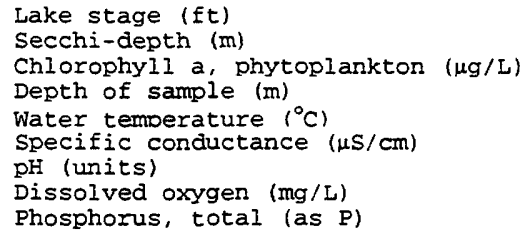

\begin{tabular}{ccccc} 
Feb-15 & Apr-25 & Jun-21 & Jul-24 & Aug-22 \\
\hline 1.80 & 1.68 & 2.29 & 1.37 & 1.36 \\
\hdashline- & 0.7 & 1.2 & --- & 0.8 \\
\hline-5 & 57 & 13 & 32 & 41 \\
0.5 & 0.5 & 0.25 & 0.5 & 0.25 \\
0.3 & 14.5 & 24.2 & 27.7 & 23.2 \\
409 & 366 & 306 & 363 & 351 \\
7.8 & 8.5 & 8.1 & 7.9 & 8.1 \\
11.6 & 14.4 & 9.7 & 6.7 & 8.9 \\
0.012 & 0.086 & 0.116 & 0.225 & 0.139
\end{tabular}

2-15-01

4-25-01

6-21-01

7-24-01

8-22-01

DISSOLVED OXYGEN (D.O.), IN MILLIGRAMS PER LITER
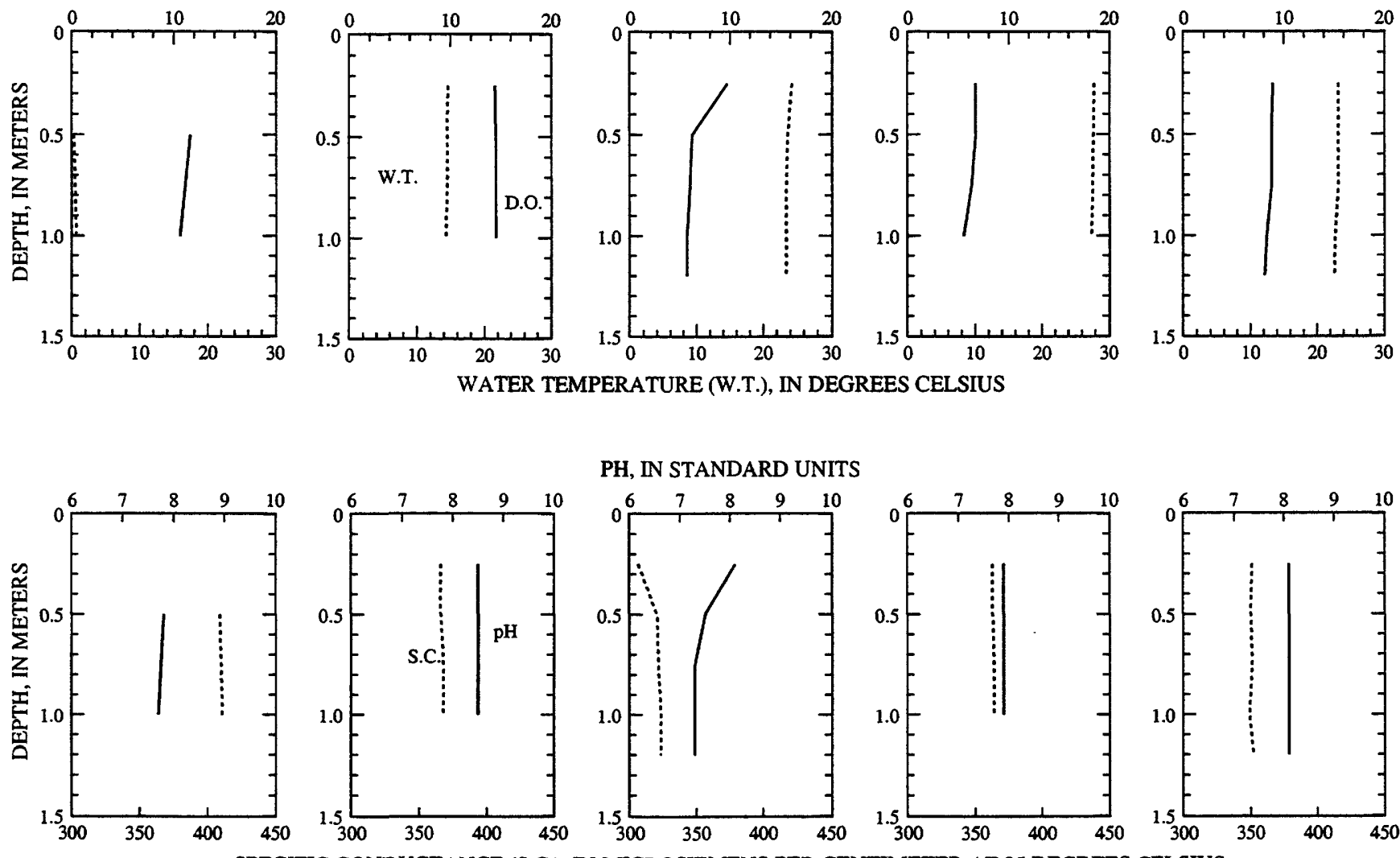

PH, IN STANDARD UNITS
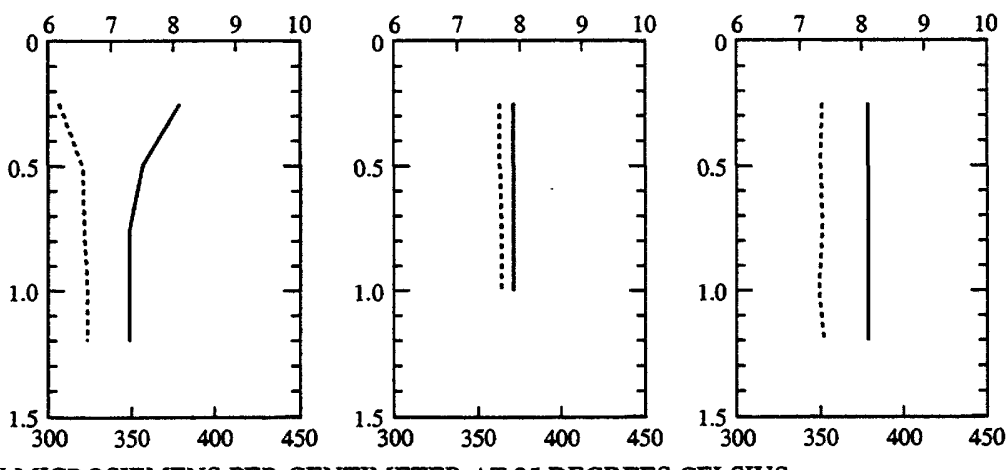

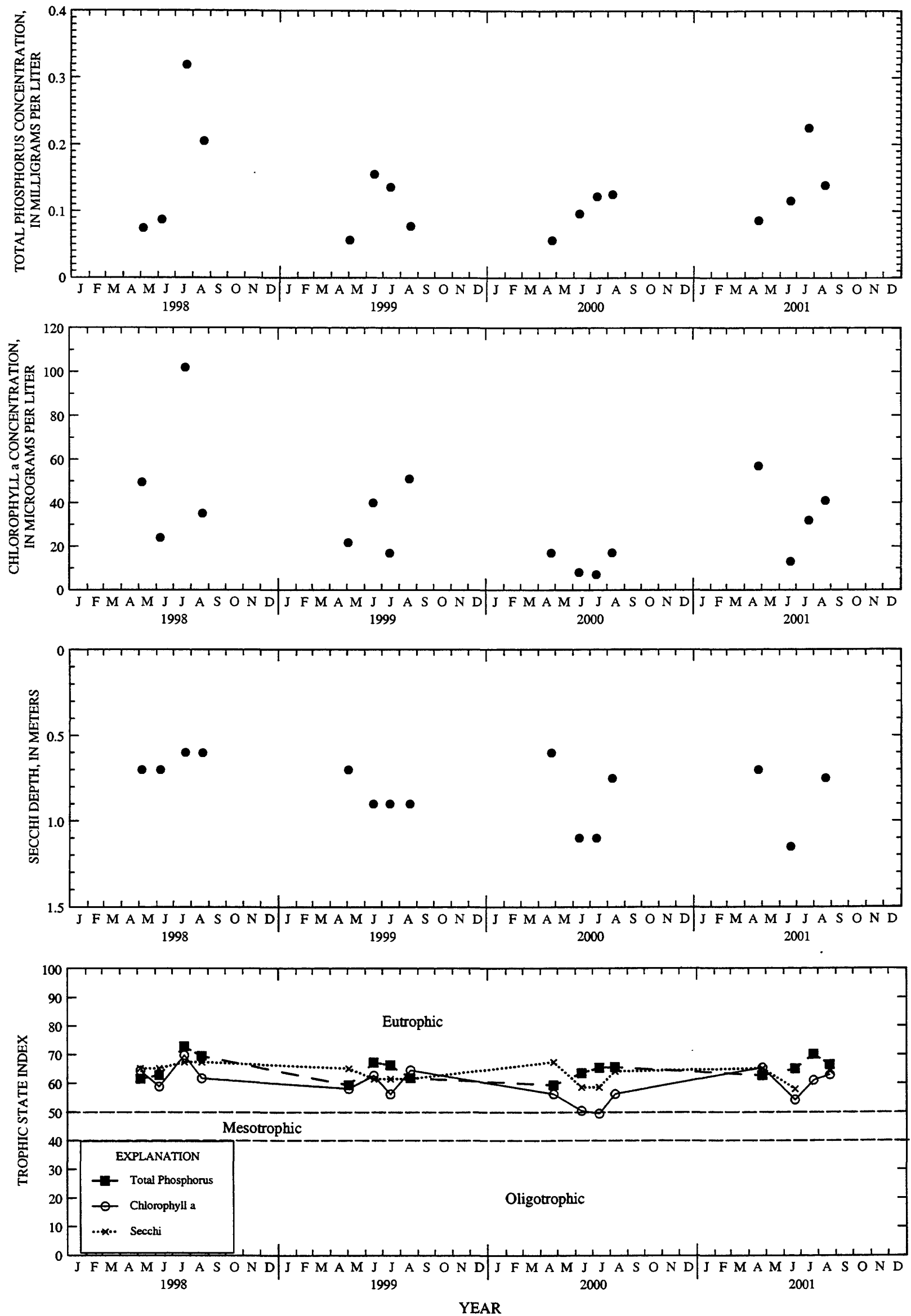

Surface total phosphorus, chlorophyll a concentrations, Secchi depths, and TSI data for Buffalo Lake, East Site, at Montello, Wisconsin. 
LOCATION.--Lat $43^{\circ} 44^{\prime} 14^{\prime \prime}$, long $89^{\circ} 28^{\prime} 24^{\prime \prime}$, in NW 1/4 SE 1/4 sec.31, T.15 N., R.9 E., Marquette County, Hydrologic Unit 04030201 , 1.5 mi north of Endeavor.

PERIOD OF RECORD.--May 1998 to current year. Data collected 1991-94 by Wisconsin Department of Natural Resources are available.

REMARKS.--Site sampled near west end of lake. Lake ice-covered during February sampling. Water-quality analyses done by Wisconsin State Laboratory of Hygiene.

WATER-QUALITY DATA, FEBRUARY 15 TO AUGUST 22, 2001

(Milligrams per liter unless otherwise indicated)

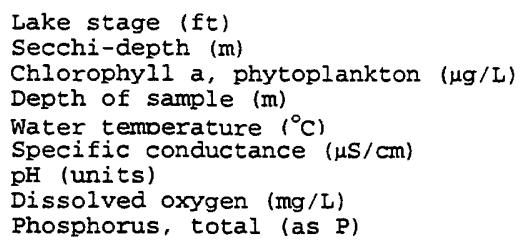



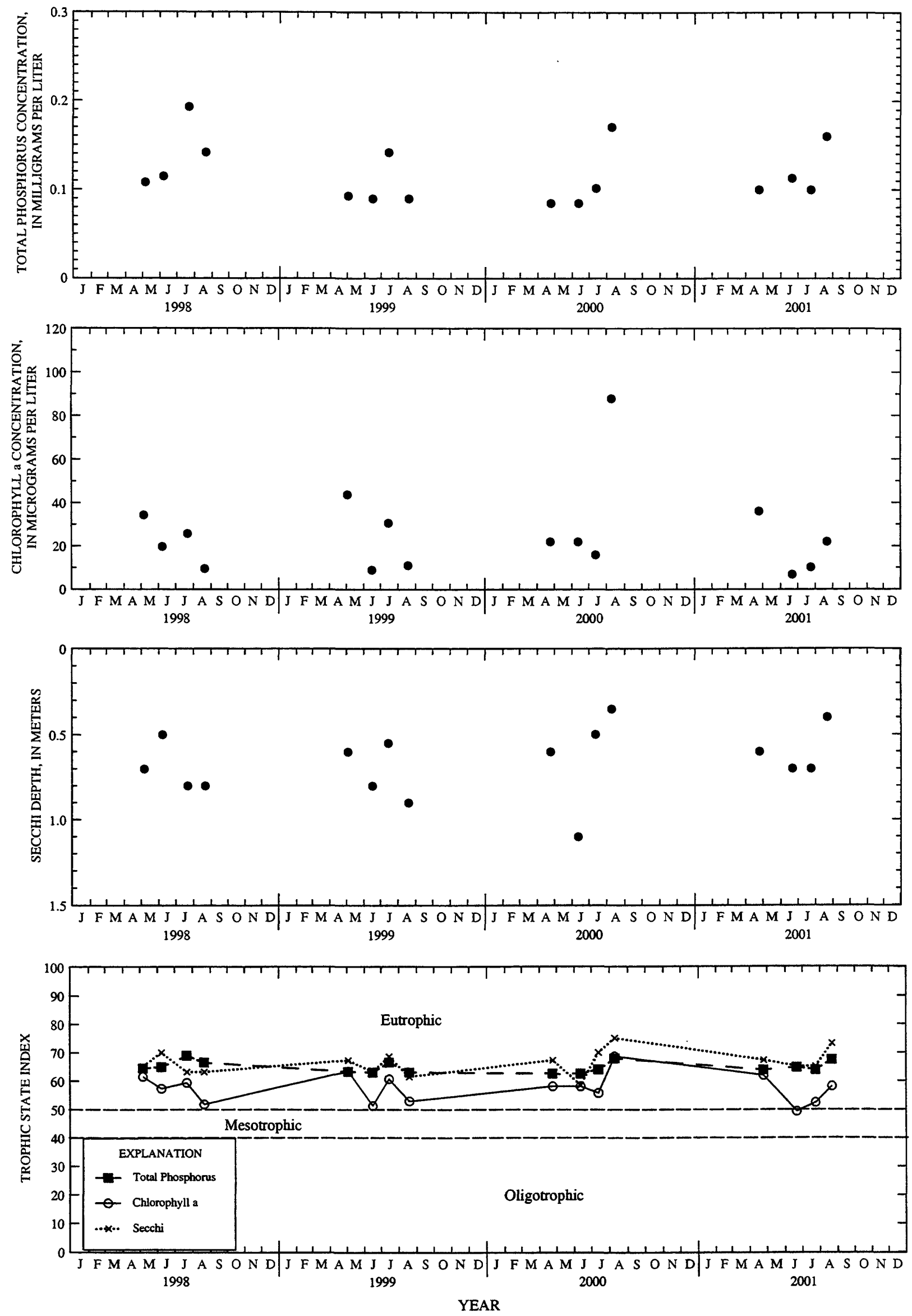

Surface total phosphorus, chlorophyll a concentrations, Secchi depths, and TSI data for Buffalo Lake, West Site, near Endeavor, Wisconsin. 
LOCATION.--Lat $42^{\circ} 36^{\prime} 27^{\prime \prime}$, long $88^{\circ} 36^{\prime} 19^{\prime \prime}$, in SW 1/4 NE 1/4 sec.28, T.2 N., R.16 E., Walworth County, Hydrologic Unit 07090001, at Delavan Lake Sanitary District Lift Station No. 2 at Delavan Lake Yacht Club, 1.0 mi southeast of outlet, and $2.7 \mathrm{mi}$ southeast of Delavan.

DRAINAGE AREA.--41.4 mi ${ }^{2}$, of which $2.3 \mathrm{mi}^{2}$ is non-contributing. Area of Delavan Lake, 2,072 acres.

PERIOD OF RECORD.--October 1983 to current year. October 1983 to September 1985 data published in Water Resources Investigation series report "Water Quality and Hydrology of Delavan Lake in Southeastern Wisconsin" by S. J. Field and M. D. Duerk (1988).

GAGE.--Water-stage recorder. Datum of gage is $922.92 \mathrm{ft}$ above sea level. Prior to Sept. 5, 1989, staff gage at bridge on North Shore Drive at same datum.

REMARKS.--Lake was ice covered from Dec. 13 to Apr. 7. Lake levels controlled by Delavan Lake Sanitary District. Gage-height telemeter at station.

EXTREMES FOR PERIOD OF RECORD.--Maximum gage height observed, $6.19 \mathrm{ft}$, Feb. 21, 1994; minimum daily, -4.44 ft, Nov. 6 , 1989 (lake drawn down for lake rehabilitation program).

EXTREMES FOR CURRENT YEAR.--Maximum gage height, 5.57 ft, Feb. 11, 12; minimum, $4.74 \mathrm{ft}$, July 15-17.

GAGE HEIGHT, FEET, WATER YEAR OCTOBER 2000 TO SEPTEMBER 2001

DAILY MEAN VALUES

\begin{tabular}{|c|c|c|c|c|c|c|c|c|c|c|c|c|}
\hline DAY & OCT & NOV & DEC & JAN & EEB & MAR & APR & MAY & $\pi \mathrm{N}$ & JUL & AUG & SEP \\
\hline $\begin{array}{l}1 \\
2 \\
3 \\
4 \\
5\end{array}$ & $\begin{array}{l}4.99 \\
4.99 \\
5.00 \\
5.03 \\
5.02\end{array}$ & $\begin{array}{l}5.00 \\
5.02 \\
5.02 \\
5.01 \\
5.00\end{array}$ & $\begin{array}{l}5.06 \\
5.05 \\
5.04 \\
5.02 \\
5.00\end{array}$ & $\begin{array}{l}5.00 \\
4.99 \\
4.98 \\
4.97 \\
4.96\end{array}$ & $\begin{array}{l}5.07 \\
5.04 \\
5.01 \\
4.99 \\
4.99\end{array}$ & $\begin{array}{l}5.12 \\
5.06 \\
5.03 \\
5.07 \\
5.09\end{array}$ & $\begin{array}{l}4.98 \\
4.98 \\
4.98 \\
4.98 \\
4.98\end{array}$ & $\begin{array}{l}4.96 \\
4.96 \\
4.97 \\
5.00 \\
4.99\end{array}$ & $\begin{array}{l}5.07 \\
5.13 \\
5.10 \\
5.07 \\
5.15\end{array}$ & $\begin{array}{l}4.92 \\
4.89 \\
4.88 \\
4.87 \\
4.86\end{array}$ & $\begin{array}{l}4.80 \\
4.88 \\
4.91 \\
4.91 \\
4.90\end{array}$ & $\begin{array}{l}4.99 \\
4.98 \\
4.98 \\
5.01 \\
5.01\end{array}$ \\
\hline $\begin{array}{r}6 \\
7 \\
8 \\
9 \\
10\end{array}$ & $\begin{array}{l}5.02 \\
5.00 \\
4.99 \\
4.99 \\
4.99\end{array}$ & $\begin{array}{l}5.00 \\
5.08 \\
5.07 \\
5.07 \\
5.06\end{array}$ & $\begin{array}{l}4.99 \\
5.00 \\
5.01 \\
5.02 \\
5.03\end{array}$ & $\begin{array}{l}4.95 \\
4.94 \\
4.95 \\
4.95 \\
4.95\end{array}$ & $\begin{array}{l}4.99 \\
4.99 \\
5.02 \\
5.25 \\
5.49\end{array}$ & $\begin{array}{l}5.09 \\
5.07 \\
5.06 \\
5.04 \\
5.05\end{array}$ & $\begin{array}{l}5.01 \\
5.02 \\
5.03 \\
5.06 \\
5.07\end{array}$ & $\begin{array}{l}4.98 \\
4.99 \\
4.98 \\
4.98 \\
5.00\end{array}$ & $\begin{array}{l}5.25 \\
5.19 \\
5.09 \\
5.01 \\
4.95\end{array}$ & $\begin{array}{l}4.85 \\
4.84 \\
4.84 \\
4.85 \\
4.84\end{array}$ & $\begin{array}{l}4.89 \\
4.88 \\
4.88 \\
4.87 \\
4.88\end{array}$ & $\begin{array}{l}5.02 \\
5.17 \\
5.31 \\
5.33 \\
5.35\end{array}$ \\
\hline $\begin{array}{l}11 \\
12 \\
13 \\
14 \\
15\end{array}$ & $\begin{array}{l}4.99 \\
4.99 \\
4.98 \\
4.98 \\
4.99\end{array}$ & $\begin{array}{l}5.04 \\
5.02 \\
5.00 \\
5.00 \\
4.99\end{array}$ & $\begin{array}{l}5.06 \\
5.09 \\
5.09 \\
5.10 \\
5.10\end{array}$ & $\begin{array}{l}4.96 \\
4.97 \\
4.97 \\
4.99 \\
5.01\end{array}$ & $\begin{array}{l}5.56 \\
5.55 \\
5.51 \\
5.47 \\
5.41\end{array}$ & $\begin{array}{l}5.07 \\
5.08 \\
5.05 \\
5.00 \\
4.99\end{array}$ & $\begin{array}{l}5.10 \\
5.18 \\
5.18 \\
5.14 \\
5.10\end{array}$ & $\begin{array}{l}5.09 \\
5.11 \\
5.10 \\
5.09 \\
5.07\end{array}$ & $\begin{array}{l}4.94 \\
5.24 \\
5.39 \\
5.26 \\
5.14\end{array}$ & $\begin{array}{l}4.82 \\
4.80 \\
4.79 \\
4.78 \\
4.77\end{array}$ & $\begin{array}{l}4.86 \\
4.84 \\
4.82 \\
4.81 \\
4.79\end{array}$ & $\begin{array}{l}5.28 \\
5.18 \\
5.09 \\
4.99 \\
4.95\end{array}$ \\
\hline $\begin{array}{l}16 \\
17 \\
18 \\
19 \\
20\end{array}$ & $\begin{array}{l}5.00 \\
5.00 \\
5.00 \\
5.00 \\
5.00\end{array}$ & $\begin{array}{l}5.00 \\
5.01 \\
5.01 \\
5.00 \\
5.00\end{array}$ & $\begin{array}{l}5.10 \\
5.08 \\
5.07 \\
5.08 \\
5.07\end{array}$ & $\begin{array}{l}5.02 \\
5.02 \\
5.03 \\
5.03 \\
5.03\end{array}$ & $\begin{array}{l}5.35 \\
5.28 \\
5.20 \\
5.12 \\
5.04\end{array}$ & $\begin{array}{l}5.01 \\
4.97 \\
4.92 \\
4.89 \\
4.90\end{array}$ & $\begin{array}{l}5.05 \\
5.01 \\
4.98 \\
4.97 \\
5.01\end{array}$ & $\begin{array}{l}5.06 \\
5.03 \\
5.02 \\
5.00 \\
5.00\end{array}$ & $\begin{array}{l}5.12 \\
5.06 \\
5.02 \\
4.98 \\
4.99\end{array}$ & $\begin{array}{l}4.75 \\
4.77 \\
4.79 \\
4.79 \\
4.79\end{array}$ & $\begin{array}{l}4.88 \\
4.89 \\
4.90 \\
4.96 \\
4.95\end{array}$ & $\begin{array}{l}4.94 \\
4.93 \\
4.93 \\
5.01 \\
5.02\end{array}$ \\
\hline $\begin{array}{l}21 \\
22 \\
23 \\
24 \\
25\end{array}$ & $\begin{array}{l}5.00 \\
5.00 \\
5.01 \\
5.03 \\
5.04\end{array}$ & $\begin{array}{l}5.00 \\
4.99 \\
4.99 \\
4.99 \\
4.99\end{array}$ & $\begin{array}{l}5.07 \\
5.05 \\
5.04 \\
5.03 \\
5.01\end{array}$ & $\begin{array}{l}5.03 \\
5.02 \\
5.02 \\
5.02 \\
5.01\end{array}$ & $\begin{array}{l}4.97 \\
4.90 \\
4.85 \\
4.84 \\
4.99\end{array}$ & $\begin{array}{l}4.92 \\
4.94 \\
4.96 \\
4.97 \\
4.97\end{array}$ & $\begin{array}{l}5.06 \\
5.09 \\
5.08 \\
5.07 \\
5.04\end{array}$ & $\begin{array}{l}5.01 \\
5.02 \\
5.02 \\
5.03 \\
5.09\end{array}$ & $\begin{array}{l}4.99 \\
4.99 \\
4.98 \\
4.97 \\
4.96\end{array}$ & $\begin{array}{l}4.79 \\
4.81 \\
4.85 \\
4.87 \\
4.86\end{array}$ & $\begin{array}{l}4.95 \\
4.99 \\
5.01 \\
5.01 \\
5.02\end{array}$ & $\begin{array}{l}5.06 \\
5.06 \\
5.11 \\
5.13 \\
5.09\end{array}$ \\
\hline $\begin{array}{l}26 \\
27 \\
28 \\
29 \\
30 \\
31\end{array}$ & $\begin{array}{l}5.04 \\
5.05 \\
5.04 \\
5.03 \\
5.02 \\
5.01\end{array}$ & $\begin{array}{l}5.02 \\
5.03 \\
5.03 \\
5.05 \\
5.06 \\
\ldots--\end{array}$ & $\begin{array}{r}4.99 \\
4.98 \\
4.98 \\
\mathrm{e} 4.99 \\
\mathrm{e} 5.01 \\
5.01\end{array}$ & $\begin{array}{l}5.01 \\
5.01 \\
5.00 \\
5.03 \\
5.09 \\
5.09\end{array}$ & $\begin{array}{r}5.17 \\
5.20 \\
5.17 \\
--- \\
--- \\
---\end{array}$ & $\begin{array}{l}4.97 \\
4.96 \\
4.96 \\
4.96 \\
4.96 \\
4.96\end{array}$ & $\begin{array}{l}5.00 \\
4.98 \\
4.97 \\
4.96 \\
4.96 \\
---\end{array}$ & $\begin{array}{l}5.12 \\
5.11 \\
5.10 \\
5.09 \\
5.06 \\
5.05\end{array}$ & $\begin{array}{l}4.96 \\
4.95 \\
4.95 \\
4.94 \\
4.93 \\
-\end{array}$ & $\begin{array}{l}4.84 \\
4.82 \\
4.81 \\
4.81 \\
4.80 \\
4.80\end{array}$ & $\begin{array}{l}5.04 \\
5.04 \\
5.03 \\
5.02 \\
5.01 \\
5.00\end{array}$ & $\begin{array}{l}5.04 \\
5.02 \\
5.00 \\
5.00 \\
5.00 \\
\ldots\end{array}$ \\
\hline $\begin{array}{l}\text { MEAN } \\
\text { MAX } \\
\text { MIN }\end{array}$ & $\begin{array}{l}5.01 \\
5.05 \\
4.98\end{array}$ & $\begin{array}{l}5.02 \\
5.08 \\
4.99\end{array}$ & $\begin{array}{l}5.04 \\
5.10 \\
4.98\end{array}$ & $\begin{array}{l}5.00 \\
5.09 \\
4.94\end{array}$ & $\begin{array}{l}5.16 \\
5.56 \\
4.84\end{array}$ & $\begin{array}{l}5.00 \\
5.12 \\
4.89\end{array}$ & $\begin{array}{l}5.03 \\
5.18 \\
4.96\end{array}$ & $\begin{array}{l}5.03 \\
5.12 \\
4.96\end{array}$ & $\begin{array}{l}5.06 \\
5.39 \\
4.93\end{array}$ & $\begin{array}{l}4.82 \\
4.92 \\
4.75\end{array}$ & $\begin{array}{l}4.92 \\
5.04 \\
4.79\end{array}$ & $\begin{array}{l}5.07 \\
5.35 \\
4.93\end{array}$ \\
\hline
\end{tabular}

e Estimated

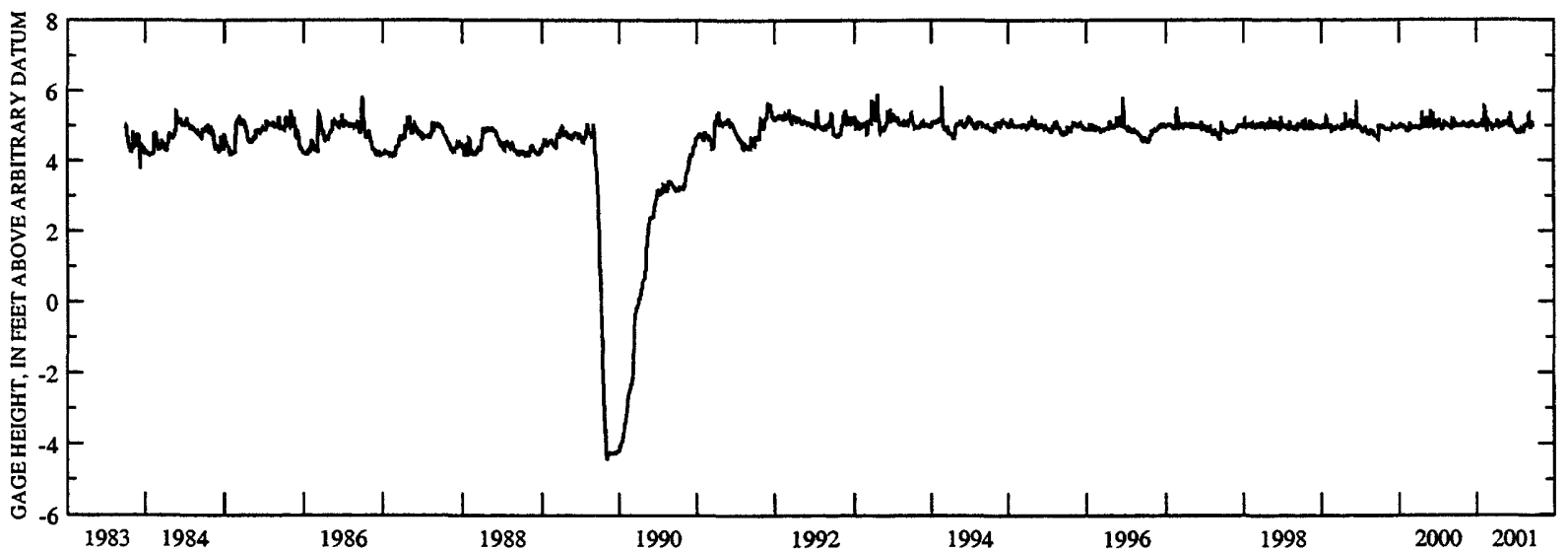


LOCATION.--Lat $42^{\circ} 35^{\prime} 56^{\prime \prime}$, long $88^{\circ} 36^{\prime} 50^{\prime \prime}$, in SE 1/4 SW 1/4 sec.28, T.2 N., R.16 E., Walworth County, Hydrologic Unit 07090001, 2.6 mi southeast of Delavan.

DRAINAGE AREA.--41.4 $\mathrm{mi}^{2}$, of which $2.3 \mathrm{mi}^{2}$ is non-contributing. Area of Delavan Lake, 2,072 acres.

PERIOD OF RECORD.--October 1983 to current year.

REMARKS.--Lake ice-covered during February measurements. Water-quality analyses done by the U.S. Geological Survey National Water Quality Laboratory. Samples for determination of chlorophyll-a concentration are collected from the top $1.5 \mathrm{ft}$ of the lake.

WATER-QUALITY DATA, NOVEMBER 15, 2000 TO APRIL 18, 2001

(Milligrams per liter unless otherwise indicated)

Lake stage (ft)

Secchi-depth (m)

Chlorophyll a, phytoplankton ( $\mu \mathrm{g} / \mathrm{L})$

Depth of sample (m)

Water temperature $\left({ }^{\circ} \mathrm{C}\right)$

specific conductance ( $\mu \mathrm{S} / \mathrm{cm})$

$\mathrm{pH}$ (units)

Dissolved oxygen (mg/L)

Phosphorus, total (as P)

Phosphorus, ortho, dissolved (as P)

Nitrogen, $\mathrm{NO} 2+\mathrm{NO} 3$, diss. (as N)

Nitrogen, ammonia, dissolved (as $\mathrm{N}$ )

Nitrogen, amm. + org., total (as $\mathrm{N}$ )

Nitrogen, total (as $\mathrm{N}$ )

Color (Pt-Co. scale)

Turbidity (NTU)

Hardness, (as $\mathrm{CaCO}_{3}$ )

Calcium, dissolved ( $\mathrm{Ca}$

Magnesium, dissolved (Mg)

Sodium, dissolved (Na)

Potassium, dissolved (K)

Alkalinity, (as $\mathrm{CaCO}_{3}$ )

sulfate, dissolved $\left(\mathrm{SO}_{4}\right)$

Chloride, dissolved $(\mathrm{Cl}$

Silica, dissolved $\left(\mathrm{SiO}_{2}\right)$

Solids, dissolved, at $180^{\circ} \mathrm{C}$

Iron, dissolved $(\mathrm{Fe}) \mu \mathrm{g} / \mathrm{L}$

Manganese, dissolved (Mn) $\mu \mathrm{g} / \mathrm{L}$

\begin{tabular}{|c|c|}
\hline \multicolumn{2}{|c|}{ Nov-15 } \\
\hline \multicolumn{2}{|c|}{$\begin{array}{c}4.99 \\
4.6 \\
2.1\end{array}$} \\
\hline $\begin{array}{l}0.5 \\
9.0 \\
549 \\
7.8 \\
9.2\end{array}$ & $\begin{array}{c}16.0 \\
9.0 \\
550 \\
7.9 \\
9.1\end{array}$ \\
\hline 0.117 & 0.116 \\
\hline 0.093 & 0.093 \\
\hline 0.157 & $\cdots$ \\
\hline $\begin{array}{l}0.141 \\
0.64\end{array}$ & --- \\
\hline 0.8 & --- \\
\hline-- & -- \\
\hline-- & $-\ldots$ \\
\hline-- & -- \\
\hline--- & --- \\
\hline$\cdots-$ & $\cdots$ \\
\hline$\ldots$ & - \\
\hline-- & --- \\
\hline--- & -- \\
\hline- & --- \\
\hline- & -.- \\
\hline & \\
\hline
\end{tabular}

2-20-01

\begin{tabular}{|c|c|c|}
\hline & Feb-20 & \\
\hline & $\begin{array}{l}5.04 \\
1.4 \\
6.7\end{array}$ & \\
\hline $\begin{array}{l}0.5 \\
0.6\end{array}$ & $\begin{array}{c}13.0 \\
3.7\end{array}$ & $\begin{array}{c}16 . \\
4 .\end{array}$ \\
\hline 531 & 617 & 93 \\
\hline 8.2 & 7.5 & 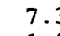 \\
\hline 14.8 & 3.4 & \\
\hline 0.089 & 0.142 & 0.1 \\
\hline 0.061 & 0.115 & 0.1 \\
\hline 0.318 & $\cdots$ & - \\
\hline 0.071 & --- & - \\
\hline 0.57 & $\cdots$ & . \\
\hline 0.88 & $\cdots$ & \\
\hline-- & -- & \\
\hline-- & $\cdots$ & \\
\hline--- & -- & \\
\hline--- & -- & \\
\hline--- & -- & \\
\hline$-\ldots$ & $\ldots$ & \\
\hline-- & -- & \\
\hline$\cdots$ & - & \\
\hline-- & $\ldots$ & \\
\hline-- & $-\cdots$ & \\
\hline & -.- & \\
\hline$=-$ & $\cdots$ & \\
\hline
\end{tabular}

\begin{tabular}{|c|c|c|}
\hline & & 18 \\
\hline & & \\
\hline .0 & 0.5 & 16.0 \\
\hline .4 & 7.6 & 7.1 \\
\hline 935 & $\begin{array}{r}578 \\
83\end{array}$ & $\begin{array}{l}583 \\
81\end{array}$ \\
\hline & $\begin{array}{l}8.3 \\
12.8\end{array}$ & 10.2 \\
\hline 97 & 0.087 & 0.083 \\
\hline 14 & 0.018 & 0.038 \\
\hline & 0.395 & 0.408 \\
\hline & 0.019 & 0.056 \\
\hline & 0.77 & 0.53 \\
\hline & $\begin{array}{l}1.2 \\
10\end{array}$ & $\begin{array}{c}0.94 \\
2\end{array}$ \\
\hline & 1.6 & 3.6 \\
\hline & 240 & 240 \\
\hline & 44.2 & 44 \\
\hline & 22.9 & 22.5 \\
\hline & 2.96 & 2.98 \\
\hline & 193 & 191 \\
\hline & 26.2 & 26.6 \\
\hline & 54.3 & 53.9 \\
\hline & 2.7 & 3.3 \\
\hline & 318 & 319 \\
\hline & $<10$ & $<10$ \\
\hline & $<3.2$ & $<3.2$ \\
\hline
\end{tabular}

$11-15-00$

DISSOLVED OXYGEN (D.O.), IN MILLIGRAMS PER LITER
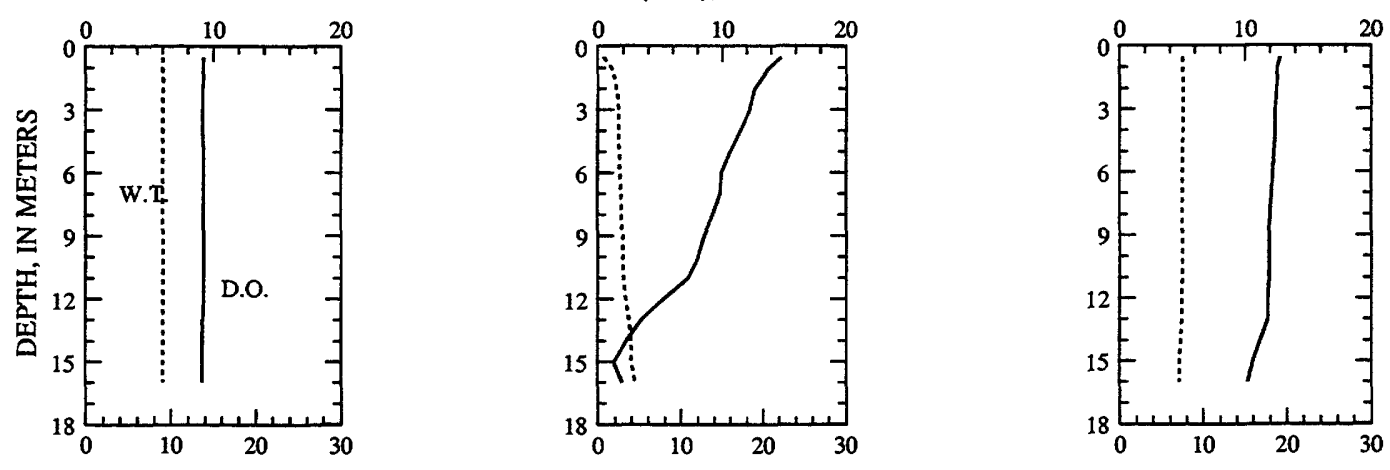

WATER TEMPERATURE (W.T.), IN DEGREES CELSIUS

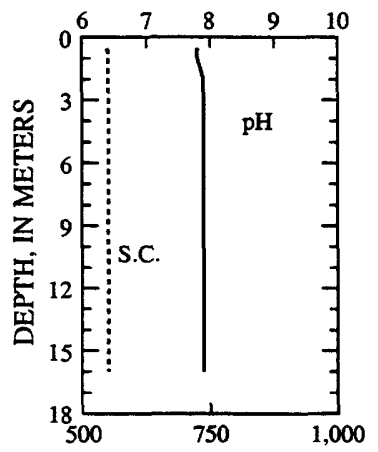

PH, IN STANDARD UNITS
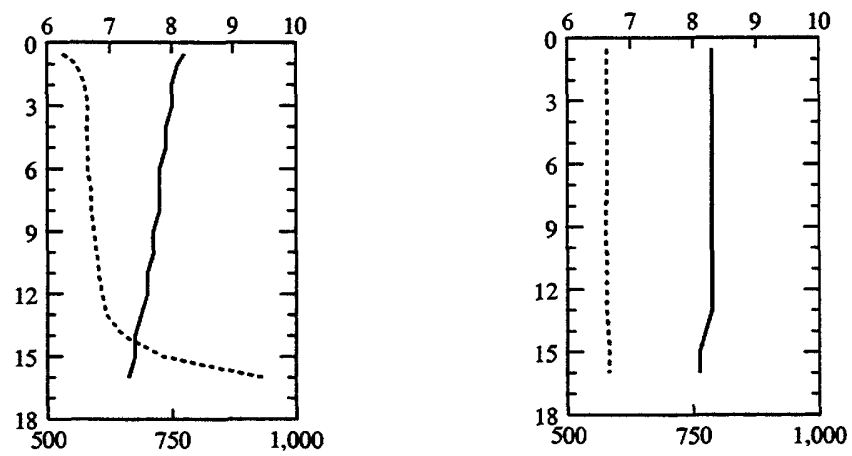

SPECIFIC CONDUCTANCE (S.C.), IN MICROSIEMENS PER CENTIMETER AT 25 DEGREES CELSIUS 
WATER-QUALITY DATA, MAY 16 TO JULY 16, 2001

(Milligrams per liter unless otherwise indicated)

Lake stage (ft)

Secchi-depth (m)

Chlorophyll a, phytoplankton ( $\mu \mathrm{g} / \mathrm{L})$

Depth of sample (m)

Water temoerature $\left({ }^{\circ} \mathrm{C}\right)$

Specific conductance $(\mu \mathrm{S} / \mathrm{cm})$

pH (units)

Dissolved oxygen (mg/L)

Phosphorus, total (as P)

Phosphorus, ortho, dissolved (as P)

Nitrogen, $\mathrm{NO} 2+\mathrm{NO} 3$, diss. (as N)

Nitrogen, ammonia, dissolved (as N)

Nitrogen, amm. + org., total (as N)

Nitrogen, total (as N)

May -16

Jun -20

Jul-16

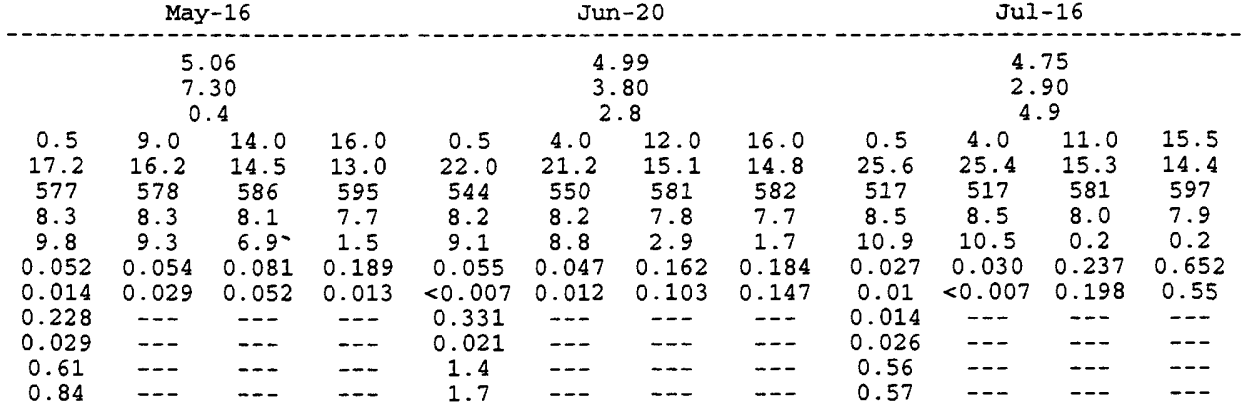

$5-16-01$

6-20-01

7-16-01

DISSOLVED OXYGEN (D.O.), IN MILLIGRAMS PER LITER
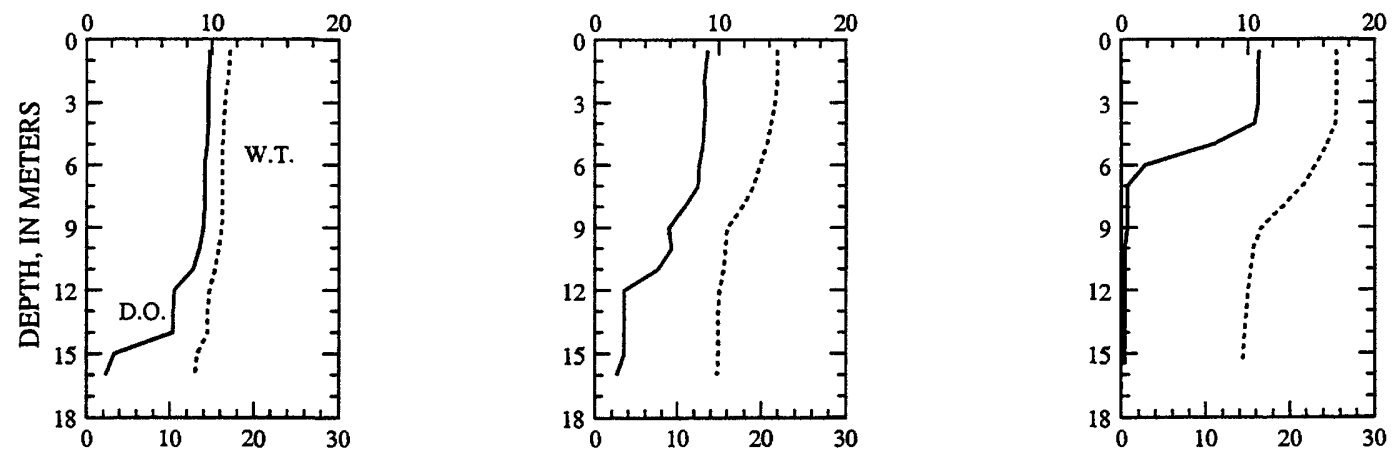

WATER TEMPERATURE (W.T.), IN DEGREES CELSIUS

PH, IN STANDARD UNITS
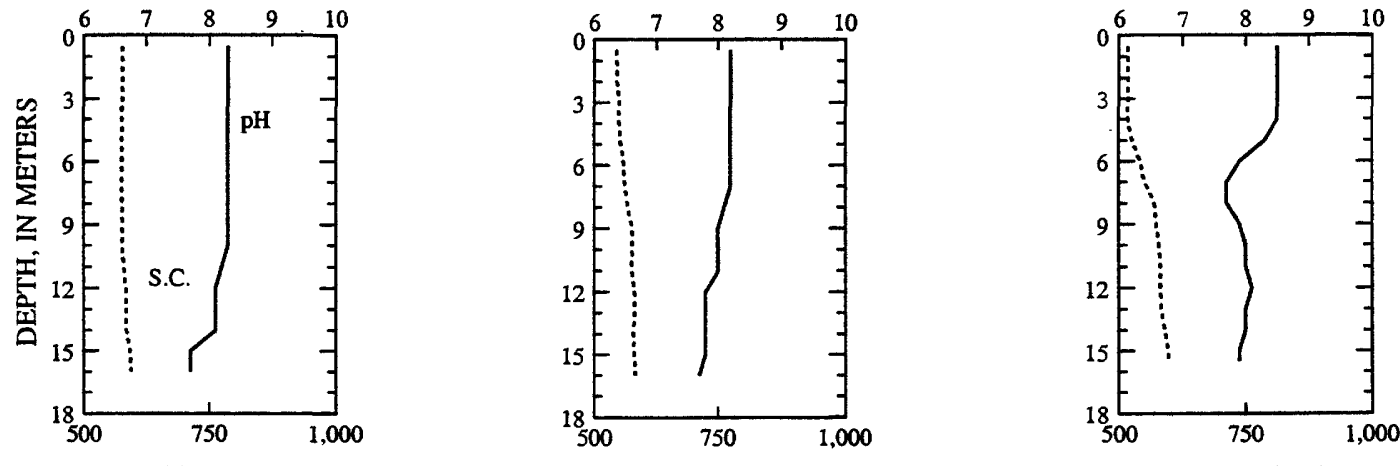

SPECIFIC CONDUCTANCE (S.C.), IN MICROSIEMENS PER CENTIMETER AT 25 DEGREES CELSIUS 
WATER-QUALITY DATA, AUGUST 20 TO SEPTEMBER 13, 2001

(Milligrams per liter unless otherwise indicated)

Lake stage (ft)

Secchi-depth (m)

Chlorophy11 a, phytoplankton ( $\mu \mathrm{g} / \mathrm{L})$

Depth of sample (m)

Water temperature $\left({ }^{\circ} \mathrm{C}\right)$

Specific conductance $(\mu \mathrm{S} / \mathrm{cm})$

$\mathrm{pH}$ (units)

Dissolved oxygen (mg/L)

Phosphorus, total (as P)

Phosphorus, ortho, dissolved (as P)

Nitrogen, NO2 + NO3, diss. (as N)

Nitrogen, ammonia, dissolved (as $N$ )

Nitrogen, amm. + org. total (as N)

Nitrogen, total (as $\mathrm{N}$ )
Aug-20

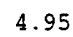

4.95
2.90

$\begin{array}{llllllllll}0.5 & 4.0 & 8.0 & 10.0^{6.8} & 12.0 & 14.0 & 15.0 & 16.0 & 0.5 & 10.0^{0.2}\end{array}$

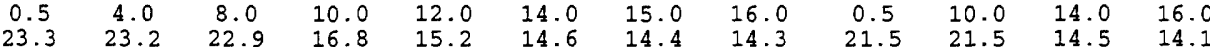

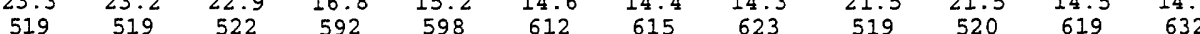

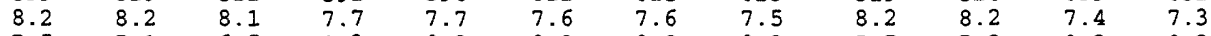

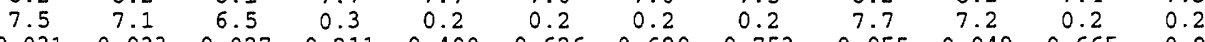

$\begin{array}{llllllllllll}0.031 & 0.033 & 0.027 & 0.211 & 0.400 & 0.626 & 0.690 & 0.753 & 0.055 & 0.049 & 0.665 & 0.9\end{array}$

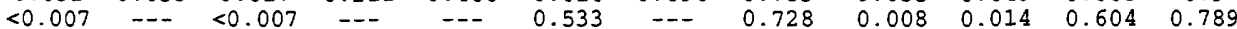

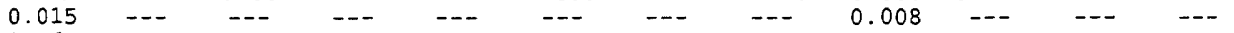

0.067 -

0.67 -

DISSOLVED OXYGEN (D.O.), IN MILLIGRAMS PER LITER
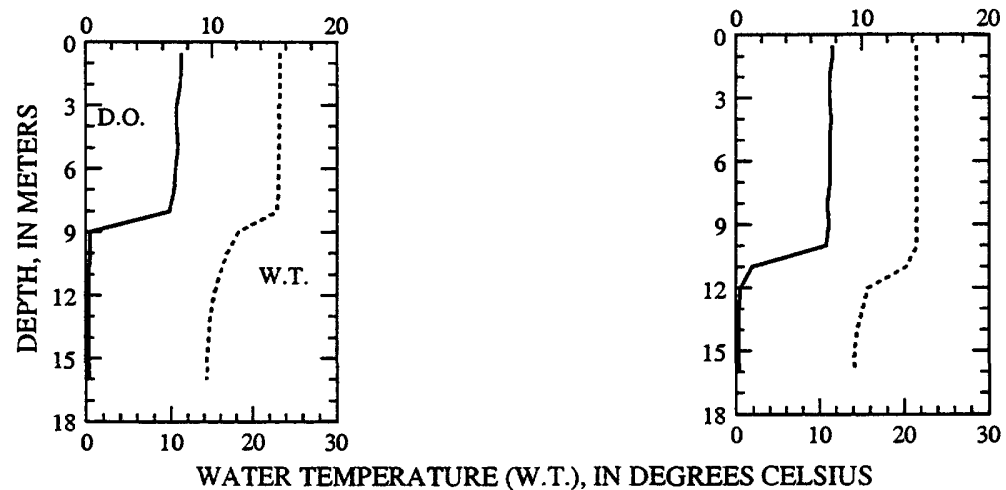

PH, IN STANDARD UNITS
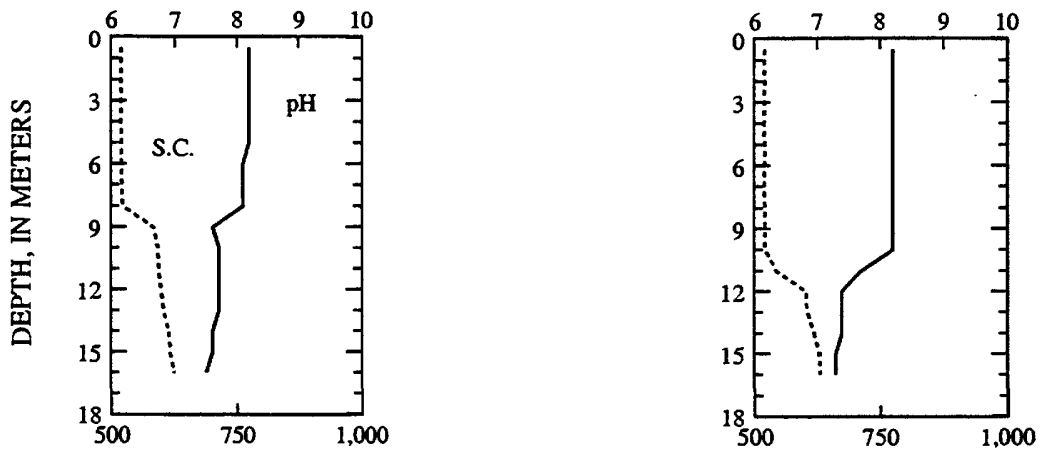

SPECIFIC CONDUCTANCE (S.C.), IN MICROSIEMENS PER CENTIMETER AT 25 DEGREES CELSIUS 

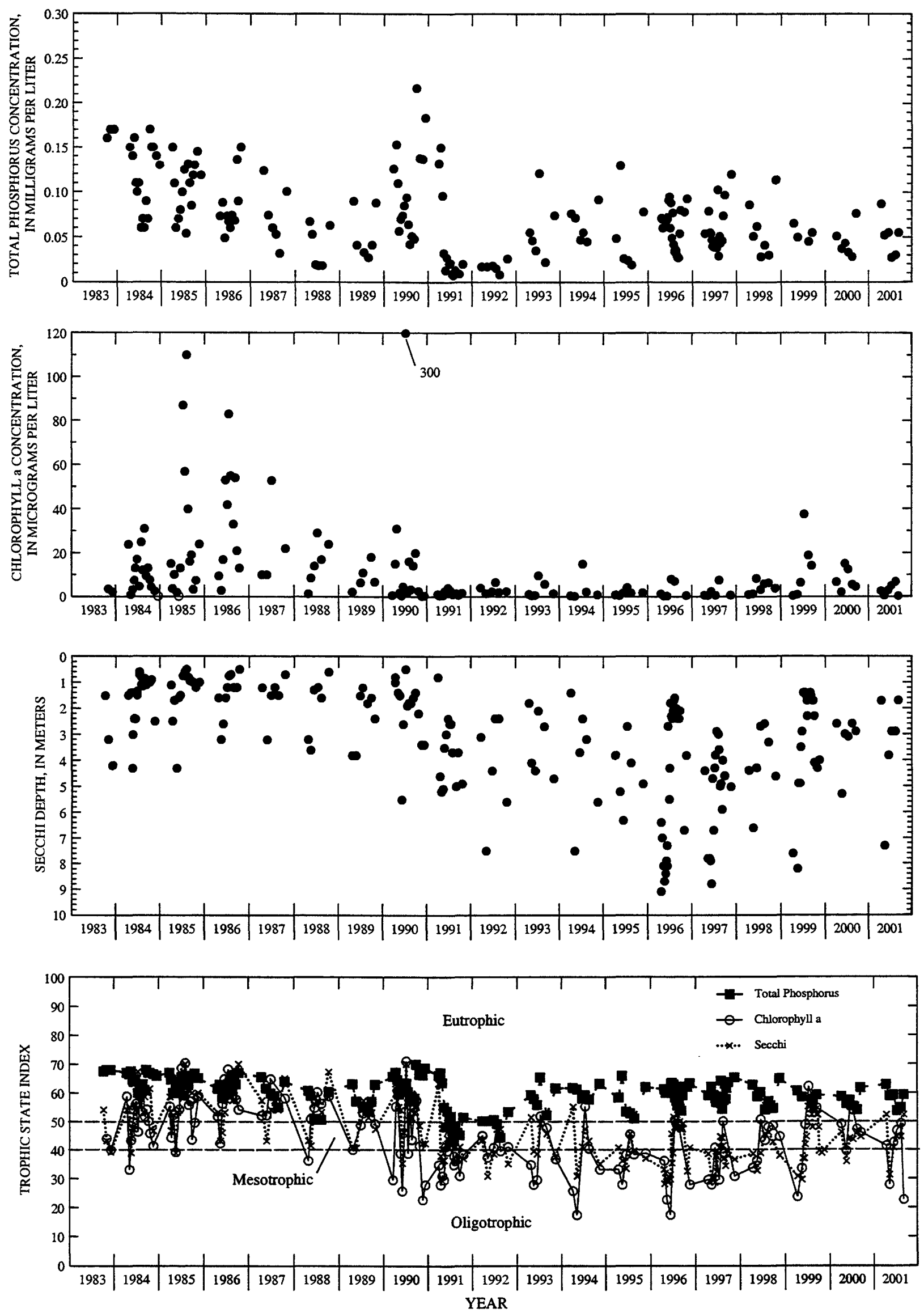

Surface total phosphorus, chlorophyll a concentrations, Secchi depths, and TSI data for Delavan Lake at Center near Delavan, Wisconsin. 
Lake stage $(\mathrm{ft})$

Secchi-depth (meters)

Depth of sample (meters)

water temperature $\left({ }^{\circ} \mathrm{C}\right)$

Phosphorus, total (as P)

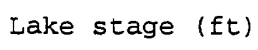

Phosphorus, total (as P)

ADDITIONAL WATER-QUALITY DATA, OCTOBER 11, 2000 TO SEPTEMBER 27, 2001 (Milligrams per liter unless otherwise indicated)

\begin{tabular}{cccccc} 
Oct. 11 & May 2 & May 31 & June 2 & June 26 & July 9 \\
\cline { 3 - 5 } 4.99 & 4.98 & 5.05 & 5.19 & 4.96 & 4.85 \\
9.1 & 9.0 & 4.1 & 4.7 & 2.4 & 2.9 \\
0.5 & 0.5 & 0.5 & 0.5 & 0.5 & 0.5 \\
14.0 & 14.5 & 16.1 & 11.5 & 24.0 & 24.0 \\
0.129 & 0.052 & 0.066 & 0.075 & 0.051 & 0.035
\end{tabular}

Julv 19
4.79
2.7
0.5
25.2
0.026

Ju2 24
4.87
2.9
0.5
26.0
0.210

Aug. 1

2.9

0.5

25.0

0.022

Aug. 8
4.88
2.9
0.5
27.0
0.022

Aug. 29
5.02
2.1
0.5
22.5
0.028

Sept. 27

5.02

2.3

0.5

15.8

0.113 
LOCATION.--Lat $42^{\circ} 36^{\prime} 59^{\prime \prime}$, long $88^{\circ} 35^{\prime} 44^{\prime \prime}$, in NW 1/4 SW 1/4, sec.22, T.2 N., R.16 E., Walworth County, Hydrologic Unit 07090001, $2.6 \mathrm{mi}$ southeast of Delavan.

DRAINAGE AREA.--41.4 $\mathrm{mi}^{2}$, of which $2.3 \mathrm{mi}^{2}$ is non-contributing.

PERIOD OF RECORD.--October 1983 to current year.

\section{WATER-QUALITY DATA, MAY 16 TO AUGUST 20, 2001}

\begin{tabular}{cccc} 
May 16 & June 20 & July 16 & Aug. 20 \\
\hdashline 8.1 & 4.5 & 2.3 & 2.3
\end{tabular}

\section{DELAVAN LAKE, AT SW END, NEAR DELAVAN LAKE, WI}

LOCATION.--Lat $42^{\circ} 35^{\prime} 26^{\prime \prime}$, long $88^{\circ} 38^{\prime} 01^{\prime \prime}$, in SE $1 / 4 \mathrm{NW} 1 / 4$, sec.32, T.2 N., R.16 E., Walworth County, Hydrologic Unit 07090001, $2.6 \mathrm{mi}$ southeast of Delavan.

DRAINAGE AREA.--41.4 $\mathrm{mi}^{2}$, of which $2.3 \mathrm{mi}^{2}$ is non-contributing.

PERIOD OF RECORD.--October 1983 to current year.

WATER-QUALITY DATA, MAY 16 TO AUGUST 20, 2001

\begin{tabular}{cccc} 
May 16 & June 20 & July 16 & Aug. 20 \\
\hdashline 7.5 & 4.1 & 3.0 & 2.4
\end{tabular}

Secchi-depth (meters)

7.5

4.1

2.4 


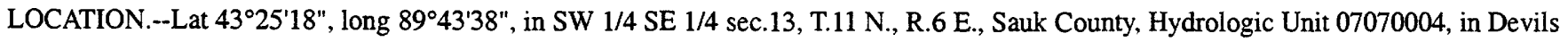
Lake State Park, $3.5 \mathrm{mi}$ south of Baraboo.

DRAINAGE AREA.--4.79 $\mathrm{mi}^{2}$. Area of Devils Lake, 361 acres.

PERIOD OF RECORD.--June 1922 to August 1930, June to August 1932, June 1934 to September 1981, October 1984 to June 1991 (fragmentary), July 1991 to current year. Unpublished daily stage records from October 1981 to September 1984 in District files.

REVISED RECORDS.--WDR WI-78-1: Drainage area.

GAGE.--Water-stage recorder installed July 17, 1991. Datum of gage is $955.00 \mathrm{ft}$, above sea level.

REMARKS.--Lake has no surface outlet.

EXTREMES FOR PERIOD OF RECORD.--Maximum gage height observed, $14.13 \mathrm{ft}$, July 18, 1993; minimum observed, $1.49 \mathrm{ft}$, Feb. 8 , 1965.

EXTREMES FOR CURRENT YEAR.--Maximum recorded gage height, $11.64 \mathrm{ft}$, June 22; minimum recorded, $9.73 \mathrm{ft}$, Jan. 27.

GAGE HEIGHT, FEET, WATER YEAR OCTOBER 2000 TO SEPTEMBER 2001

DAILY MEAN VALUES

\begin{tabular}{|c|c|c|c|c|c|c|c|c|c|c|c|c|}
\hline DAY & OCT & Nov & $\mathrm{DEC}$ & JAN & FEB & MAR & APR & MAY & JON & JUL & AUG & SEP \\
\hline $\begin{array}{l}1 \\
2 \\
3 \\
4 \\
5\end{array}$ & $\begin{array}{l}10.61 \\
10.59 \\
10.58 \\
10.57 \\
10.55\end{array}$ & $\begin{array}{l}10.15 \\
10.15 \\
10.13 \\
10.11 \\
10.09\end{array}$ & $\begin{array}{l}9.88 \\
9.87 \\
9.86 \\
9.84 \\
9.82\end{array}$ & $\begin{array}{l}9.87 \\
9.86 \\
9.85 \\
9.85 \\
9.85\end{array}$ & $\begin{array}{l}9.85 \\
9.84 \\
9.84 \\
9.83 \\
9.82\end{array}$ & $\begin{array}{l}9.89 \\
9.88 \\
9.88 \\
9.87 \\
9.87\end{array}$ & $\begin{array}{l}10.11 \\
10.13 \\
10.15 \\
10.16 \\
10.19\end{array}$ & $\begin{array}{l}11.03 \\
11.04 \\
11.12 \\
11.22 \\
11.25\end{array}$ & $\begin{array}{l}11.17 \\
11.20 \\
11.18 \\
11.16 \\
11.20\end{array}$ & $\begin{array}{l}11.47 \\
11.43 \\
11.40 \\
11.37 \\
11.34\end{array}$ & $\begin{array}{l}10.90 \\
11.39 \\
11.46 \\
11.44 \\
11.42\end{array}$ & $\begin{array}{l}10.97 \\
10.95 \\
10.92 \\
10.90 \\
10.88\end{array}$ \\
\hline $\begin{array}{r}6 \\
7 \\
8 \\
9 \\
10\end{array}$ & $\begin{array}{l}10.53 \\
10.50 \\
10.47 \\
10.44 \\
10.42\end{array}$ & $\begin{array}{l}10.09 \\
10.13 \\
10.11 \\
10.10 \\
10.08\end{array}$ & $\begin{array}{l}9.81 \\
9.81 \\
9.80 \\
9.79 \\
9.79\end{array}$ & $\begin{array}{l}9.85 \\
9.84 \\
9.83 \\
9.83 \\
9.82\end{array}$ & $\begin{array}{l}9.82 \\
9.82 \\
9.84 \\
9.93 \\
9.97\end{array}$ & $\begin{array}{l}9.86 \\
9.85 \\
9.84 \\
9.83 \\
9.83\end{array}$ & $\begin{array}{l}10.24 \\
10.34 \\
10.38 \\
10.53 \\
10.60\end{array}$ & $\begin{array}{l}11.28 \\
11.31 \\
11.33 \\
11.33 \\
11.34\end{array}$ & $\begin{array}{l}11.21 \\
11.21 \\
11.21 \\
11.20 \\
11.20\end{array}$ & $\begin{array}{l}11.31 \\
11.28 \\
11.27 \\
11.25 \\
11.22\end{array}$ & $\begin{array}{l}11.40 \\
11.38 \\
11.36 \\
11.33 \\
11.29\end{array}$ & $\begin{array}{l}10.87 \\
10.91 \\
11.01 \\
11.02 \\
11.03\end{array}$ \\
\hline $\begin{array}{l}11 \\
12 \\
13 \\
14 \\
15\end{array}$ & $\begin{array}{l}10.40 \\
10.39 \\
10.37 \\
10.36 \\
10.34\end{array}$ & $\begin{array}{l}10.07 \\
10.06 \\
10.05 \\
10.03 \\
10.02\end{array}$ & $\begin{array}{l}9.81 \\
9.82 \\
9.82 \\
9.82 \\
9.81\end{array}$ & $\begin{array}{l}9.82 \\
9.82 \\
9.82 \\
9.84 \\
9.84\end{array}$ & $\begin{array}{l}9.97 \\
9.96 \\
9.95 \\
9.96 \\
9.96\end{array}$ & $\begin{array}{l}9.84 \\
9.86 \\
9.86 \\
9.87 \\
9.86\end{array}$ & $\begin{array}{l}10.73 \\
10.89 \\
10.94 \\
10.97 \\
10.99\end{array}$ & $\begin{array}{l}11.38 \\
11.38 \\
11.37 \\
11.36 \\
11.35\end{array}$ & $\begin{array}{l}11.20 \\
11.37 \\
11.40 \\
11.42 \\
11.47\end{array}$ & $\begin{array}{l}11.19 \\
11.16 \\
11.13 \\
11.11 \\
11.08\end{array}$ & $\begin{array}{l}11.26 \\
11.23 \\
11.19 \\
11.16 \\
11.15\end{array}$ & $\begin{array}{l}11.01 \\
10.98 \\
10.96 \\
10.94 \\
10.91\end{array}$ \\
\hline $\begin{array}{l}16 \\
17 \\
18 \\
19 \\
20\end{array}$ & $\begin{array}{l}10.33 \\
10.32 \\
10.31 \\
10.30 \\
10.29\end{array}$ & $\begin{array}{r}10.02 \\
10.02 \\
10.01 \\
9.99 \\
9.97\end{array}$ & $\begin{array}{l}9.83 \\
9.82 \\
9.83 \\
9.85 \\
9.86\end{array}$ & $\begin{array}{l}9.83 \\
9.84 \\
9.83 \\
9.83 \\
9.82\end{array}$ & $\begin{array}{l}9.96 \\
9.96 \\
9.95 \\
9.92 \\
9.90\end{array}$ & $\begin{array}{l}9.85 \\
9.84 \\
9.84 \\
9.84 \\
9.84\end{array}$ & $\begin{array}{l}11.00 \\
11.00 \\
11.00 \\
11.00 \\
11.04\end{array}$ & $\begin{array}{l}11.35 \\
11.33 \\
11.32 \\
11.30 \\
11.28\end{array}$ & $\begin{array}{l}11.46 \\
11.45 \\
11.50 \\
11.48 \\
11.46\end{array}$ & $\begin{array}{l}11.05 \\
11.05 \\
11.10 \\
11.10 \\
11.08\end{array}$ & $\begin{array}{l}11.13 \\
11.11 \\
11.08 \\
11.05 \\
11.03\end{array}$ & $\begin{array}{l}10.89 \\
10.91 \\
10.91 \\
10.96 \\
10.96\end{array}$ \\
\hline $\begin{array}{l}21 \\
22 \\
23 \\
24 \\
25\end{array}$ & $\begin{array}{l}10.27 \\
10.25 \\
10.27 \\
10.27 \\
10.27\end{array}$ & $\begin{array}{l}9.96 \\
9.94 \\
9.93 \\
9.92 \\
9.90\end{array}$ & $\begin{array}{l}9.87 \\
9.87 \\
9.87 \\
9.86 \\
9.86\end{array}$ & $\begin{array}{l}9.81 \\
9.80 \\
9.80 \\
9.79 \\
9.79\end{array}$ & $\begin{array}{l}9.90 \\
9.90 \\
9.89 \\
9.89 \\
9.91\end{array}$ & $\begin{array}{l}9.86 \\
9.89 \\
9.92 \\
9.96 \\
9.97\end{array}$ & $\begin{array}{l}11.07 \\
11.08 \\
11.08 \\
11.08 \\
11.08\end{array}$ & $\begin{array}{l}11.28 \\
11.26 \\
11.26 \\
11.26 \\
11.26\end{array}$ & $\begin{array}{l}11.49 \\
11.63 \\
11.63 \\
11.62 \\
11.60\end{array}$ & $\begin{array}{l}11.06 \\
11.05 \\
11.05 \\
11.03 \\
11.00\end{array}$ & $\begin{array}{l}11.00 \\
11.06 \\
11.06 \\
11.05 \\
11.07\end{array}$ & $\begin{array}{l}10.95 \\
10.94 \\
11.02 \\
11.03 \\
11.00\end{array}$ \\
\hline $\begin{array}{l}26 \\
27 \\
28 \\
29 \\
30 \\
31\end{array}$ & $\begin{array}{l}10.26 \\
10.24 \\
10.23 \\
10.21 \\
10.19 \\
10.16\end{array}$ & $\begin{array}{r}9.89 \\
9.88 \\
9.88 \\
9.89 \\
9.88 \\
---\end{array}$ & $\begin{array}{l}9.85 \\
9.85 \\
9.85 \\
9.88 \\
9.88 \\
9.87\end{array}$ & $\begin{array}{l}9.78 \\
9.77 \\
9.77 \\
9.78 \\
9.85 \\
9.85\end{array}$ & $\begin{array}{r}9.92 \\
9.91 \\
9.91 \\
--- \\
--- \\
---\end{array}$ & $\begin{array}{r}9.97 \\
9.98 \\
9.99 \\
10.02 \\
10.03 \\
10.07\end{array}$ & $\begin{array}{r}11.07 \\
11.07 \\
11.06 \\
11.04 \\
11.03 \\
-\cdots\end{array}$ & $\begin{array}{l}11.25 \\
11.23 \\
11.21 \\
11.19 \\
11.17 \\
11.15\end{array}$ & $\begin{array}{r}11.58 \\
11.56 \\
11.55 \\
11.53 \\
11.50 \\
-.-\end{array}$ & $\begin{array}{l}10.97 \\
10.94 \\
10.92 \\
10.91 \\
10.89 \\
10.88\end{array}$ & $\begin{array}{l}11.10 \\
11.10 \\
11.08 \\
11.06 \\
11.03 \\
11.00\end{array}$ & $\begin{array}{l}10.97 \\
10.96 \\
10.94 \\
10.92 \\
10.91\end{array}$ \\
\hline $\begin{array}{l}\text { MEAN } \\
\text { MAX } \\
\text { MIN }\end{array}$ & $\begin{array}{l}10.36 \\
10.61 \\
10.16\end{array}$ & $\begin{array}{r}10.02 \\
10.15 \\
9.88\end{array}$ & $\begin{array}{l}9.84 \\
9.88 \\
9.79\end{array}$ & $\begin{array}{l}9.82 \\
9.87 \\
9.77\end{array}$ & $\begin{array}{l}9.90 \\
9.97 \\
9.82\end{array}$ & $\begin{array}{r}9.90 \\
10.07 \\
9.83\end{array}$ & $\begin{array}{l}10.77 \\
11.08 \\
10.11\end{array}$ & $\begin{array}{l}11.26 \\
11.38 \\
11.03\end{array}$ & $\begin{array}{l}11.39 \\
11.63 \\
11.16\end{array}$ & $\begin{array}{l}11.13 \\
11.47 \\
10.88\end{array}$ & $\begin{array}{l}11.17 \\
11.46 \\
10.90\end{array}$ & $\begin{array}{l}10.95 \\
11.03 \\
10.87\end{array}$ \\
\hline
\end{tabular}

ANNUAL MINIMUM AND MAXIMUM WATER LEVELS, 1936 - 2001

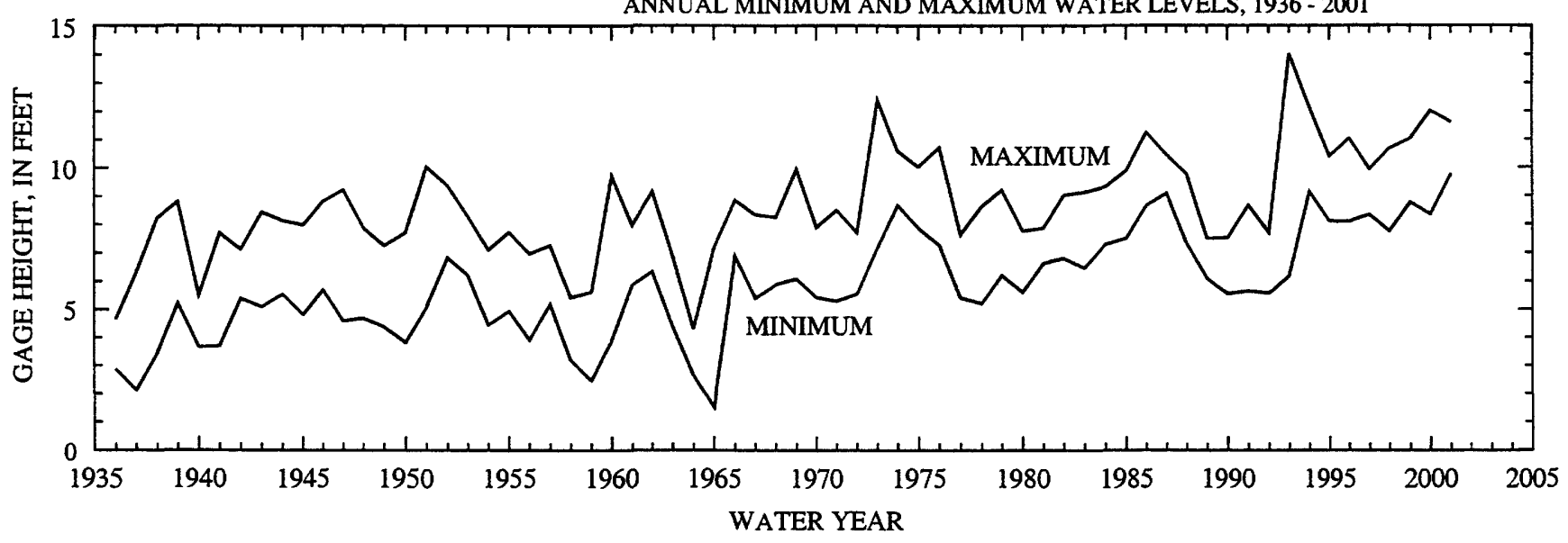




\section{EAGLE SPRING LAKE AT EAGLEVILLE, WI}

LOCATION.--Lat $42^{\circ} 51^{\prime} 03^{\prime \prime}$, long $88^{\circ} 26^{\prime} 15^{\prime \prime}$, in SE 1/4 NW 1/4 sec.36, T.5 N., R.17 E., Waukesha County, Hydrologic Unit 07120006, at Eagleville.

DRAINAGE AREA.--33.2 $\mathrm{mi}^{2}$.

PERIOD OF RECORD.--April 1991 to current year.

REMARKS.--Lake sampled near southeast end at a lake depth of about $3 \mathrm{~m}$ : Lake ice-covered during February sampling. Water-quality analyses done by Wisconsin State Laboratory of Hygiene. Lake-stage readings from 1991 to 1993 (except 2/4/93 and 4/19/93) were previously reported $1 \mathrm{ft}$ too high.

\section{WATER-QUALITY DATA, FEBRUARY 12 TO AUGUST 14, 2001 \\ (Milligrams per liter unless otherwise indicated)}

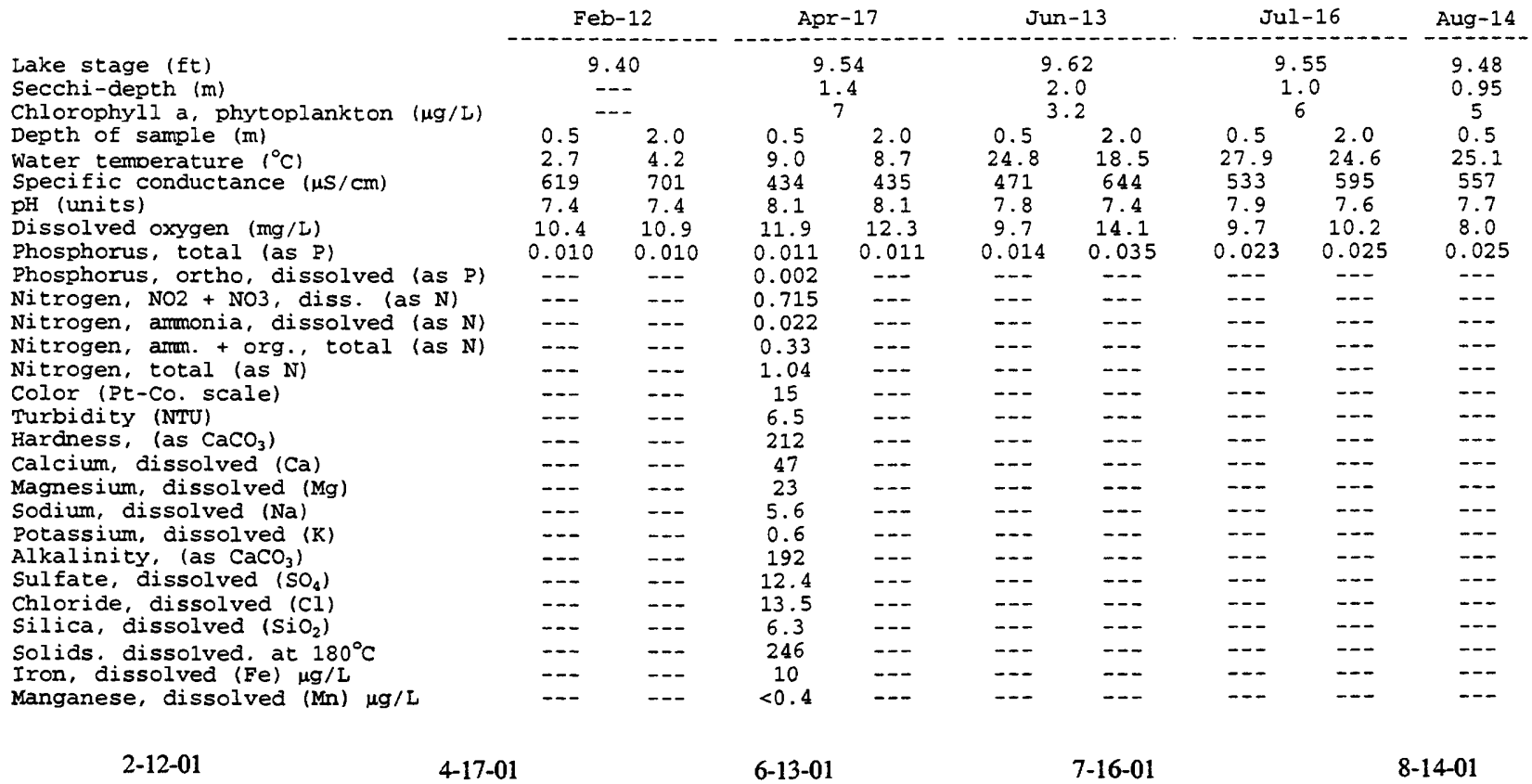

DISSOLVED OXYGEN (D.O.), IN MILLIGRAMS PER LITER
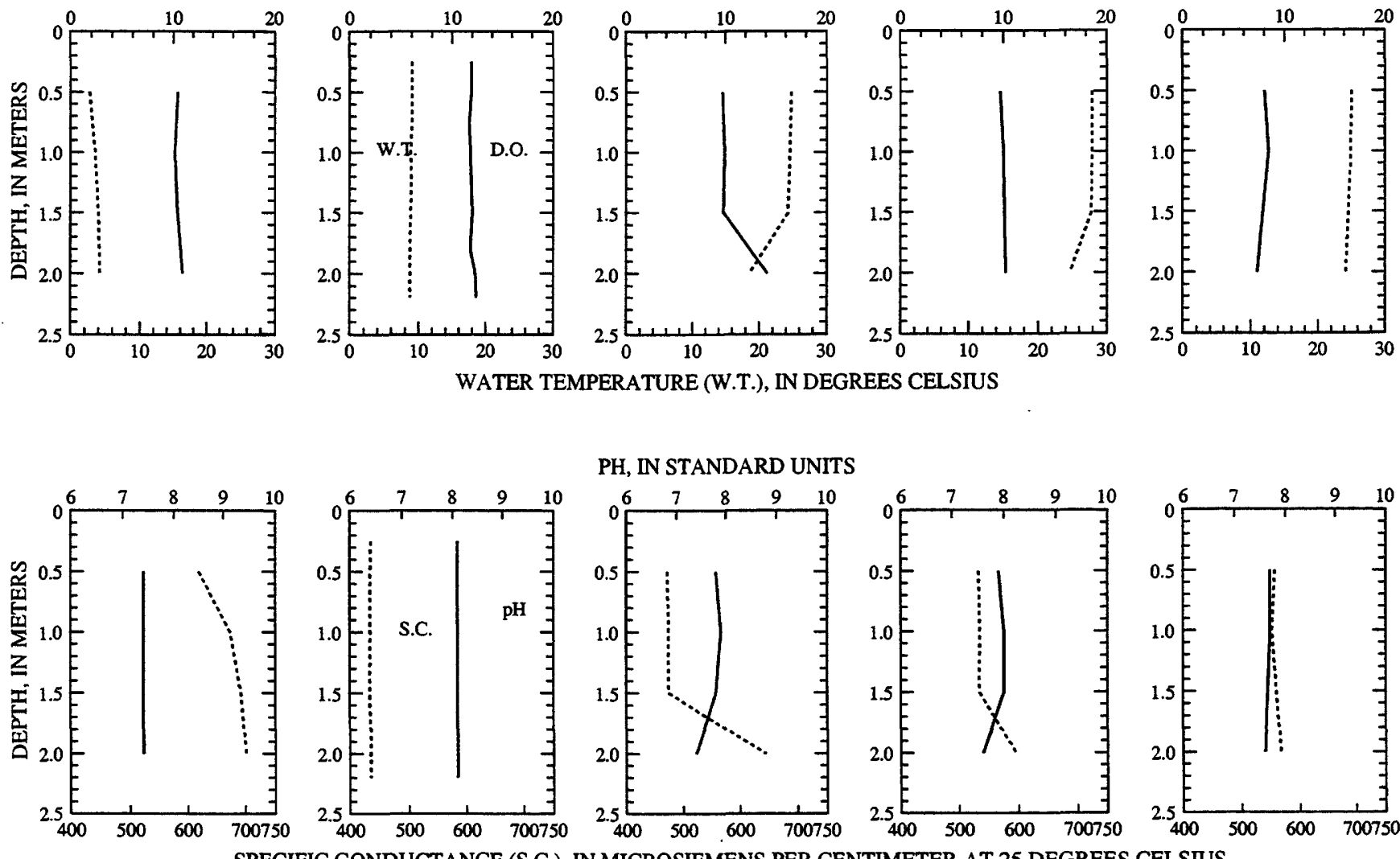

PH, IN STANDARD UNITS
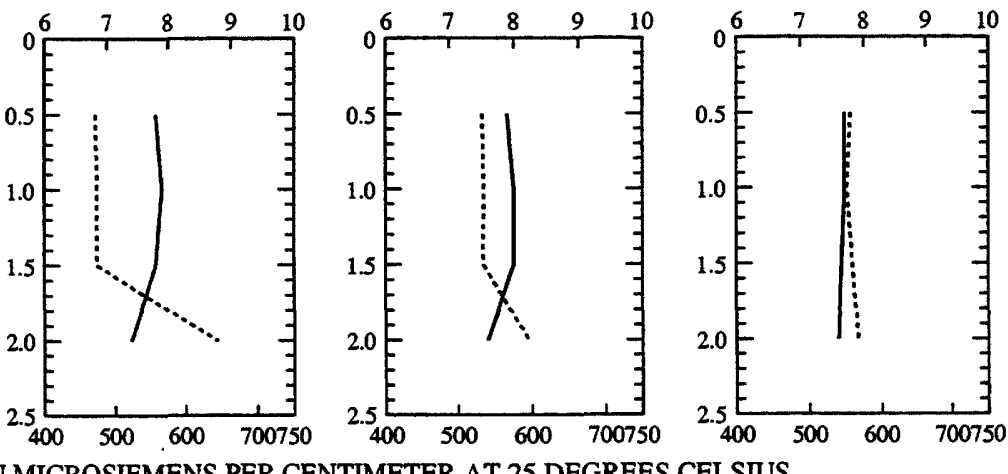

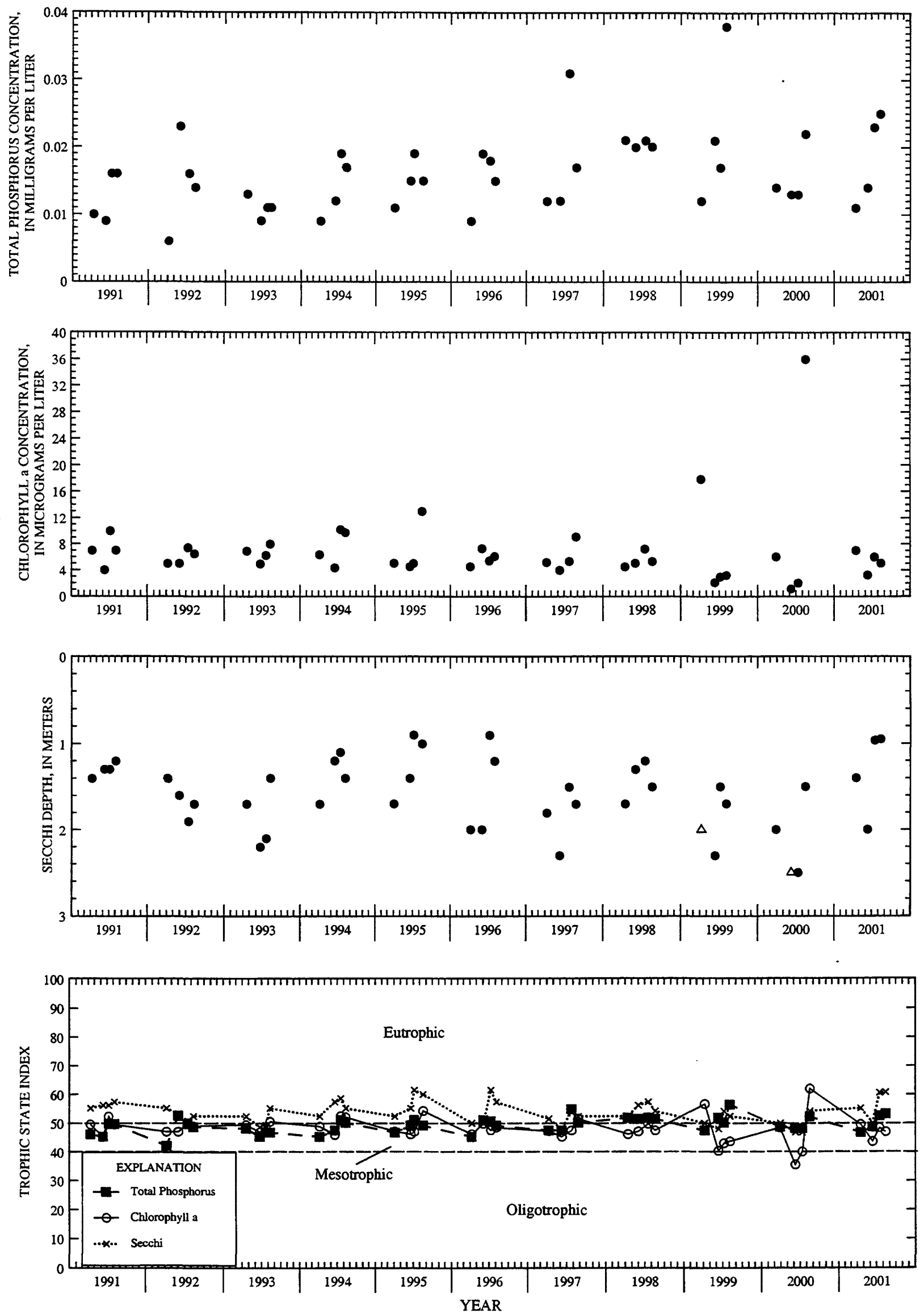

Surface total phosphorus, chlorophyll a concentrations, Secchi depths, and TSI data for Eagle Spring Lake at Eagleville, Wisconsin.

(Triangles indicate maximum depth at sampling site. Actual secchi depth on these days was greater than plotted triangles.) 
LOCATION.--Lat $43^{\circ} 17^{\prime} 27^{\prime \prime}$, long $89^{\circ} 39^{\prime} 09^{\prime \prime}$ in NE 1/4 SW 1/4 sec.3, T.9 N., R.7 E., Dane County, Hydrologic Unit 07070005, on north side of lake, $0.4 \mathrm{mi}$ southwest of Crystal Lake, and $3.1 \mathrm{mi}$ east of Sauk City.

DRAINAGE AREA.--2.23 $\mathrm{mi}^{2}$. Area of Fish Lake, 252 acres.

PERIOD OF RECORD.--November 1966 to September 1981, April 1985 to May 1987, May 1988, April 1989 to October 1990 (fragmentary); continuous record from October 1990 to November 1996; nonrecording gage November 1996 to current year.

REVISED RECORDS.--WDR WI-92-1: Drainage area. WDR WI-87-1: All published values for the 1987 water year are invalid. Two valid values for water years 1987 and 1988 are available: May 7, 1987, water surface $10.52 \mathrm{ft}$, and May 16, 1988, water surface $10.83 \mathrm{ft}$.

GAGE.--Nonrecording gage. Datum of gage is $848.07 \mathrm{ft}$ above sea level. Prior to Oct. 23, 1990, nonrecording gage. Local observer, Richard Lillie, reads staff gage when lake is ice-free.

REMARKS.--Lake has no surface outlet.

EXTREMES FOR PERIOD OF RECORD.--Maximum gage height observed, $12.83 \mathrm{ft}$, Sept. 23, 2001; minimum observed, $3.02 \mathrm{ft}$, Aug. 29 , 1970.

EXTREMES FOR CURRENT YEAR.--Maximum gage height observed, $12.83 \mathrm{ft}$, Sept. 23; minimum observed, 11.73 ft, Nov. 30.

GAGE HEIGHT, FEET, WATER YEAR OCTOBER 2000 TO SEPTEMBER 2001 DAILY MEAN VALUES

\begin{tabular}{|c|c|c|c|c|c|c|c|c|c|c|c|c|}
\hline DAY & OCT & Nov & DEC & JAN & FEB & MAR & A.PR & MAY & JUN & JUL & AUG & SEP \\
\hline 1 & 12.01 & -- & $\ldots$ & -- & -- & -.. & 12.09 & 12.24 & 12.31 & 12.30 & 12.06 & 12.58 \\
\hline 2 & -- & 11.81 & --- & --- & --- & --- & -- & 12.24 & -- & 12.28 & 12.75 & -- \\
\hline 3 & -- & --- & --- & -- & --- & --- & -- & 12.27 & 12.30 & 12.26 & 12.76 & 12.57 \\
\hline 4 & -- & 11.78 & -- & --- & --- & -- & -.- & 12.29 & -- & 12.24 & 12.75 & --- \\
\hline 5 & --- & 11.77 & -- & -- & -- & -- & -- & 12.29 & 12.33 & 12.20 & 12.74 & 12.54 \\
\hline 6 & -.. & -.. & $\ldots$ & -.. & -.- & -.- & -.. & 12.28 & 12.32 & 12.18 & 12.73 & 12.54 \\
\hline 7 & --- & -- & -- & --- & 11.98 & -.- & --- & 12.30 & -- & 12.16 & 12.72 & 12.59 \\
\hline 8 & -- & --- & --- & -- & -- & --- & --- & 12.28 & 12.33 & 12.16 & 12.71 & 12.71 \\
\hline 9 & -.- & --- & --- & --- & $-\ldots$ & -- & -- & 12.27 & - & 12.15 & 12.69 & 12.75 \\
\hline 10 & -- & 11.79 & -- & --- & -- & --- & --- & 12.27 & 12.31 & -- & 12.66 & 12.73 \\
\hline 11 & --- & -- & -- & -- &.-- & $\cdots$ & -.- & 12.38 & 12.30 & 12.11 & 12.63 & 12.71 \\
\hline 12 & 11.90 & -- & -- & --- & --- & -- & 12.27 & --- & 12.44 & 12.08 & 12.61 & --- \\
\hline 13 & 11.88 & -- & -- & $\ldots$ & --- & 12.11 & 12.29 & 12.36 & 12.42 & -.. & 12.58 & 12.68 \\
\hline 14 & -- & $\cdots$ & -- & $-\cdots$ & --- & $\ldots$ & - & - & 12.44 & 12.05 & - & 12.66 \\
\hline 15 & 11.88 & --- & --- & --- & $-\cdots$ & --- & 12.27 & -- & 12.45 & $\cdots$ & -- & -- \\
\hline 16 & 11.88 & 11.80 & --- & $\ldots$ & -.- & $-\cdots$ &.- & 12.34 & --- & -- & 12.58 & $\cdots$ \\
\hline 17 & --- & $\cdots$ & -- & --- & 12.08 & -- & 12.26 & -- & 12.42 & 12.12 &.-- & 12.68 \\
\hline 18 & 11.88 & -- & --- & $\cdots$ & -- & $-\cdot$ & --- & 12.30 & 12.45 & 12.13 & 12.54 & 12.67 \\
\hline 19 & 11.88 & 11.78 & -- & -- & 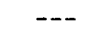 & -- & 12.24 & -- & 12.41 & -- & 12.52 & 12.75 \\
\hline 20 & --- & --- & --- & --- & -- & -- & 12.29 & --- & 12.40 & 12.15 & 12.50 & 12.74 \\
\hline 21 & 11.86 & -- & $-\cdot$ & $\cdots$ & $\ldots$ & -- & -- & 12.34 & 12.46 & 12.14 & 12.50 & 12.75 \\
\hline 22 & -- & -- & --- & --- & --- & --- & 12.30 &.-- & 12.44 & -- & 12.52 & --- \\
\hline 23 & 11.88 & -- & -- & -- & --- & -- & --- & --- & 12.42 & 12.22 & --- & 12.83 \\
\hline 24 & -.- & 11.74 & -- & -- & --- & -- & --- & 12.31 & 12.41 & 12.20 & 12.48 & 12.81 \\
\hline 25 & -- & --- & --- & $-\cdot$ & --- & --- & --- & --- & 12.38 & 12.18 & 12.66 & $\cdots$ \\
\hline 26 & --- & -- &.-- & -- & --- & -- & 12.28 & 12.30 & 12.39 & --- & 12.68 & 12.78 \\
\hline 27 & 11.87 & $\cdots$ & -- & -- & --- & $\ldots$ & -- & 12.30 & 12.38 & -- & 12.67 & 12.76 \\
\hline 28 & --- & -- & 11.88 & $\cdots$ & -- & -- & 12.26 & 12.29 & 12.36 & 12.11 & 12.65 & --- \\
\hline 29 & 11.85 & -- & -- & -- & -- & -- & 12.25 & -- & 12.34 & 12.10 & 12.64 & -- \\
\hline 30 & --- & 11.73 & -- & -- & --- & -- & --- & 12.27 & 12.32 & 12.08 & 12.63 & 12.74 \\
\hline 31 & -- & $\cdots$ & --- & -- & $\cdots$ & --- & -- & --- & -- & --- & 12.61 & $\cdots$ \\
\hline
\end{tabular}

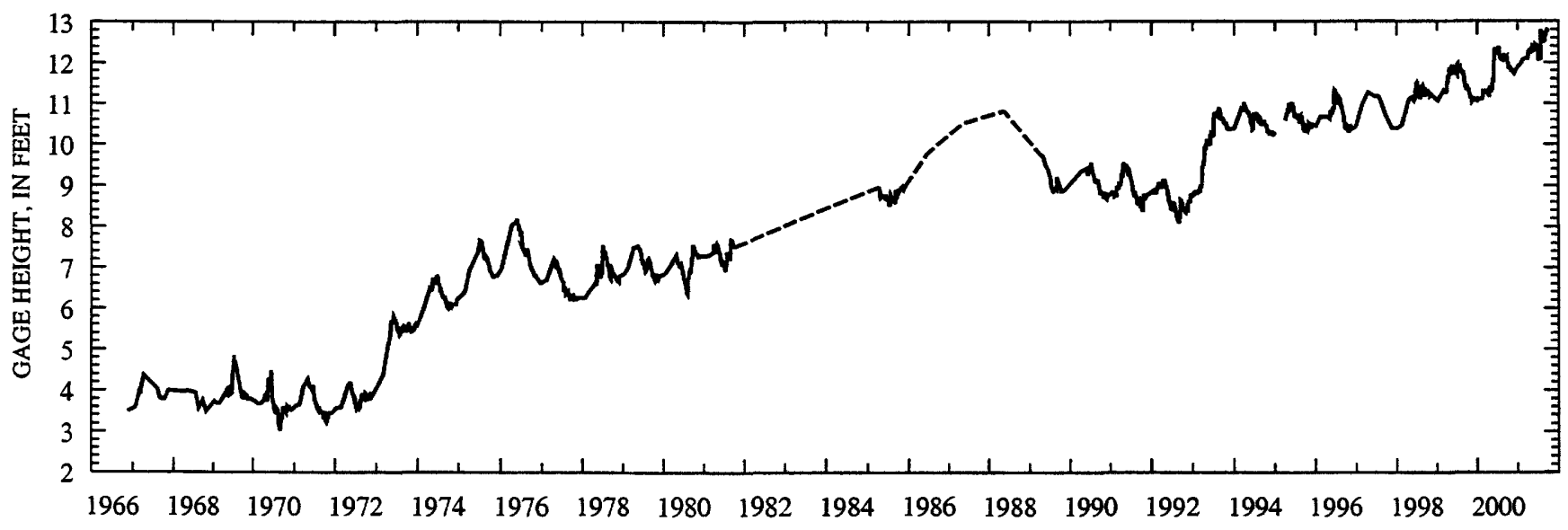


LOCATION.--Lat $42^{\circ} 35^{\prime} 25^{\prime \prime}$, long $88^{\circ} 26^{\prime} 04^{\prime \prime}$ in SE 1/4 NW 1/4 sec.36, T.2 N., R.17 E., Walworth County, Hydrologic Unit 07120006, at Geneva Lake dam at Center Street at Lake Geneva.

DRAINAGE AREA.--28.7 $\mathrm{mi}^{2}$. Area of Geneva Lake, 5,262 acres.

PERIOD OF RECORD.--October 1997 to current year.

GAGE.--Water-stage recorder. Datum of gage is $862.08 \mathrm{ft}$ above sea level.

REMARKS.--Recording rain gage and gage-height telemeter at station.

EXTREMES FOR PERIOD OF RECORD.--Maximum gage height, $3.29 \mathrm{ft}$, June 13, 2000; minimum gage height, 1.66 ft, Apr. 9, 2001. EXTREMES FOR CURRENT YEAR.--Maximum gage height, $3.05 \mathrm{ft}$, June 12; minimum gage height, $1.66 \mathrm{ft}$, Apr. 9.

GAGE HEIGHT, FEET, WATER YEAR OCTOBER 2000 TO SEPTEMBER 2001

DAILY MEAN VALUES

\begin{tabular}{|c|c|c|c|c|c|c|c|c|c|c|c|c|}
\hline DAY & OCT & NOV & $\mathrm{DEC}$ & JAN & FEB & MAR & APR & MAY & JUN & JUL & AUG & SEP \\
\hline $\begin{array}{l}1 \\
2 \\
3 \\
4 \\
5\end{array}$ & $\begin{array}{l}2.45 \\
2.44 \\
2.42 \\
2.45 \\
2.44\end{array}$ & $\begin{array}{l}2.39 \\
2.41 \\
2.41 \\
2.39 \\
2.38\end{array}$ & $\begin{array}{l}2.40 \\
2.39 \\
2.38 \\
2.39 \\
2.36\end{array}$ & $\begin{array}{c}-- \\
\cdots- \\
\overline{-} \\
2.37\end{array}$ & $\begin{array}{l}2.10 \\
2.09 \\
2.07 \\
2.06 \\
2.05\end{array}$ & $\begin{array}{l}2.22 \\
2.20 \\
2.19 \\
2.18 \\
2.17\end{array}$ & $\begin{array}{l}1.97 \\
1.95 \\
1.95 \\
1.95 \\
1.97\end{array}$ & $\begin{array}{l}2.47 \\
2.47 \\
2.46 \\
2.46 \\
2.45\end{array}$ & $\begin{array}{l}2.61 \\
2.66 \\
2.64 \\
2.62 \\
2.67\end{array}$ & $\begin{array}{l}2.58 \\
2.55 \\
2.55 \\
2.54 \\
2.51\end{array}$ & $\begin{array}{l}2.49 \\
2.53 \\
2.53 \\
2.52 \\
2.52\end{array}$ & $\begin{array}{l}2.40 \\
2.39 \\
2.41 \\
2.43 \\
2.42\end{array}$ \\
\hline $\begin{array}{r}6 \\
7 \\
8 \\
9 \\
10\end{array}$ & $\begin{array}{l}2.45 \\
2.42 \\
2.40 \\
2.39 \\
2.38\end{array}$ & $\begin{array}{l}2.37 \\
2.48 \\
2.46 \\
2.49 \\
2.50\end{array}$ & $\begin{array}{l}2.35 \\
2.34 \\
2.34 \\
2.33 \\
2.33\end{array}$ & $\begin{array}{l}2.37 \\
2.36 \\
2.36 \\
2.35 \\
2.34\end{array}$ & $\begin{array}{l}2.04 \\
2.02 \\
2.02 \\
2.23 \\
2.29\end{array}$ & $\begin{array}{l}2.16 \\
2.14 \\
2.13 \\
2.11 \\
2.10\end{array}$ & $\begin{array}{l}2.00 \\
2.07 \\
2.05 \\
2.06 \\
2.08\end{array}$ & $\begin{array}{l}2.45 \\
2.50 \\
2.53 \\
2.51 \\
2.53\end{array}$ & $\begin{array}{l}2.71 \\
2.71 \\
2.71 \\
2.70 \\
2.71\end{array}$ & $\begin{array}{l}2.50 \\
2.50 \\
2.50 \\
2.50 \\
2.50\end{array}$ & $\begin{array}{l}2.51 \\
2.50 \\
2.49 \\
2.49 \\
2.47\end{array}$ & $\begin{array}{l}2.42 \\
2.51 \\
2.68 \\
2.69 \\
2.71\end{array}$ \\
\hline $\begin{array}{l}11 \\
12 \\
13 \\
14 \\
15\end{array}$ & $\begin{array}{l}2.37 \\
2.37 \\
2.37 \\
2.36 \\
2.35\end{array}$ & $\begin{array}{l}2.49 \\
2.49 \\
2.50 \\
2.48 \\
2.46\end{array}$ & $\begin{array}{r}2.35 \\
2.38 \\
2.37 \\
\ldots- \\
\ldots-\end{array}$ & $\begin{array}{l}2.32 \\
2.30 \\
2.27 \\
2.27 \\
2.27\end{array}$ & $\begin{array}{l}2.28 \\
2.28 \\
2.27 \\
2.27 \\
2.26\end{array}$ & $\begin{array}{l}2.11 \\
2.11 \\
2.12 \\
2.11 \\
2.12\end{array}$ & $\begin{array}{l}2.13 \\
2.27 \\
2.23 \\
2.24 \\
2.26\end{array}$ & $\begin{array}{l}2.61 \\
2.61 \\
2.61 \\
2.60 \\
2.61\end{array}$ & $\begin{array}{l}2.68 \\
2.90 \\
2.92 \\
2.90 \\
2.89\end{array}$ & $\begin{array}{l}2.48 \\
2.46 \\
2.44 \\
2.44 \\
2.43\end{array}$ & $\begin{array}{l}2.45 \\
2.44 \\
2.40 \\
2.39 \\
2.37\end{array}$ & $\begin{array}{l}2.67 \\
2.66 \\
2.62 \\
2.59 \\
2.57\end{array}$ \\
\hline $\begin{array}{l}16 \\
17 \\
18 \\
19 \\
20\end{array}$ & $\begin{array}{l}2.36 \\
2.36 \\
2.37 \\
2.37 \\
2.37\end{array}$ & $\begin{array}{l}2.46 \\
2.45 \\
2.45 \\
2.44 \\
2.43\end{array}$ & $\begin{array}{r}2.38 \\
2.38 \\
2.37 \\
--- \\
--\end{array}$ & $\begin{array}{l}2.25 \\
2.24 \\
2.22 \\
2.20 \\
2.19\end{array}$ & $\begin{array}{l}2.25 \\
2.24 \\
2.22 \\
2.21 \\
2.19\end{array}$ & $\begin{array}{l}2.12 \\
2.12 \\
2.10 \\
2.09 \\
2.08\end{array}$ & $\begin{array}{l}2.27 \\
2.26 \\
2.27 \\
2.28 \\
2.33\end{array}$ & $\begin{array}{l}2.60 \\
2.59 \\
2.57 \\
2.56 \\
2.56\end{array}$ & $\begin{array}{l}2.87 \\
2.83 \\
2.83 \\
2.80 \\
2.76\end{array}$ & $\begin{array}{l}2.42 \\
2.44 \\
2.45 \\
2.45 \\
2.46\end{array}$ & $\begin{array}{l}2.47 \\
2.46 \\
2.44 \\
2.43 \\
2.42\end{array}$ & $\begin{array}{l}2.56 \\
2.54 \\
2.53 \\
2.60 \\
2.61\end{array}$ \\
\hline $\begin{array}{l}21 \\
22 \\
23 \\
24 \\
25\end{array}$ & $\begin{array}{l}2.36 \\
2.36 \\
2.37 \\
2.39 \\
2.39\end{array}$ & $\begin{array}{l}2.41 \\
2.39 \\
2.38 \\
2.38 \\
2.38\end{array}$ & $\begin{array}{l}--- \\
--- \\
--- \\
---\end{array}$ & $\begin{array}{l}2.17 \\
2.15 \\
2.13 \\
2.12 \\
2.10\end{array}$ & $\begin{array}{l}2.17 \\
2.16 \\
2.14 \\
2.16 \\
2.26\end{array}$ & $\begin{array}{l}2.07 \\
2.06 \\
2.06 \\
2.04 \\
2.03\end{array}$ & $\begin{array}{l}2.39 \\
2.40 \\
2.48 \\
2.44 \\
2.45\end{array}$ & $\begin{array}{l}2.59 \\
2.61 \\
2.58 \\
2.61 \\
2.66\end{array}$ & $\begin{array}{l}2.73 \\
2.71 \\
2.69 \\
2.68 \\
2.66\end{array}$ & $\begin{array}{l}2.46 \\
2.50 \\
2.57 \\
2.58 \\
2.55\end{array}$ & $\begin{array}{l}2.42 \\
2.44 \\
2.44 \\
2.44 \\
2.46\end{array}$ & $\begin{array}{l}2.63 \\
2.63 \\
2.64 \\
2.63 \\
2.60\end{array}$ \\
\hline $\begin{array}{l}26 \\
27 \\
28 \\
29 \\
30 \\
31\end{array}$ & $\begin{array}{l}2.40 \\
2.41 \\
2.39 \\
2.39 \\
2.39 \\
2.39\end{array}$ & $\begin{array}{c}2.40 \\
2.41 \\
2.40 \\
2.41 \\
2.41 \\
\ldots\end{array}$ & $\begin{array}{l}--- \\
--- \\
--- \\
--- \\
-\cdots\end{array}$ & $\begin{array}{l}2.08 \\
2.07 \\
2.05 \\
2.05 \\
2.11 \\
2.11\end{array}$ & $\begin{array}{r}2.25 \\
2.24 \\
2.23 \\
--- \\
--- \\
---\end{array}$ & $\begin{array}{l}2.02 \\
2.00 \\
1.99 \\
1.98 \\
1.97 \\
1.96\end{array}$ & $\begin{array}{l}2.46 \\
2.44 \\
2.44 \\
2.44 \\
2.45\end{array}$ & $\begin{array}{l}2.65 \\
2.64 \\
2.63 \\
2.62 \\
2.59 \\
2.58\end{array}$ & $\begin{array}{r}2.65 \\
2.64 \\
2.63 \\
2.62 \\
2.62 \\
---\end{array}$ & $\begin{array}{l}2.52 \\
2.49 \\
2.49 \\
2.48 \\
2.48 \\
2.48\end{array}$ & $\begin{array}{l}2.46 \\
2.46 \\
2.45 \\
2.45 \\
2.44 \\
2.42\end{array}$ & $\begin{array}{r}2.57 \\
2.55 \\
2.54 \\
2.53 \\
2.53 \\
---\end{array}$ \\
\hline $\begin{array}{l}\text { MEAN } \\
\text { MAXX } \\
\text { MIN }\end{array}$ & $\begin{array}{l}2.39 \\
2.45 \\
2.35\end{array}$ & $\begin{array}{l}2.43 \\
2.50 \\
2.37\end{array}$ & $\begin{array}{l}--- \\
--- \\
--\end{array}$ & $\begin{array}{l}--- \\
---\end{array}$ & $\begin{array}{l}2.18 \\
2.29 \\
2.02\end{array}$ & $\begin{array}{l}2.09 \\
2.22 \\
1.96\end{array}$ & $\begin{array}{l}2.23 \\
2.48 \\
1.95\end{array}$ & $\begin{array}{l}2.56 \\
2.66 \\
2.45\end{array}$ & $\begin{array}{l}2.72 \\
2.92 \\
2.61\end{array}$ & $\begin{array}{l}2.49 \\
2.58 \\
2.42\end{array}$ & $\begin{array}{l}2.46 \\
2.53 \\
2.37\end{array}$ & $\begin{array}{l}2.56 \\
2.71 \\
2.39\end{array}$ \\
\hline
\end{tabular}

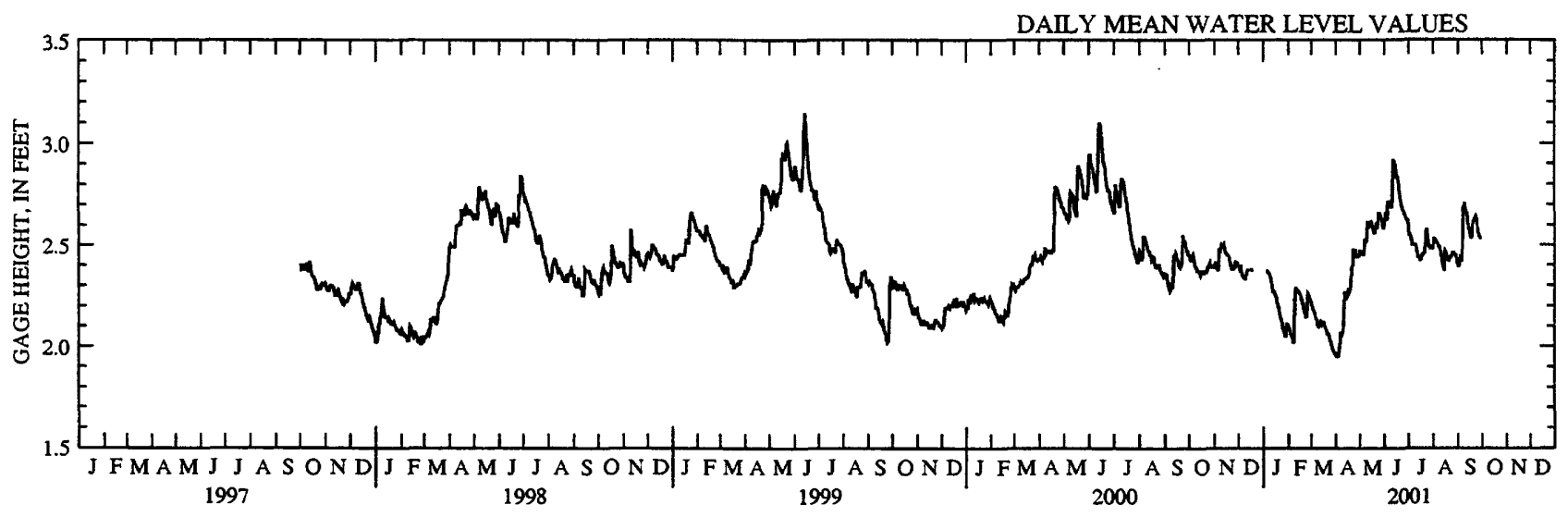




\section{GENEVA LAKE AT WEST END NEAR WILLIAMS BAY, WI}

LOCATION.--Lat $42^{\circ} 33^{\prime} 29^{\prime \prime}$, long $88^{\circ} 32^{\prime} 33^{\prime \prime}$, in NE 1/4 SE 1/4, sec.12, T.1 N., R.16 E., Walworth County, Hydrologic Unit 07120006, 1.3 mi south of Williams Bay.

DRAINAGE AREA.--28.7 $\mathrm{mi}^{2}$.

PERIOD OF RECORD.--April 1997 to current year.

REMARKS.--Lake sampled at deep hole at a depth of about $43 \mathrm{~m}$. Water-quality analyses done by Wisconsin State Laboratory of Hygiene. Samples for determination of chlorophyll-a concentration are collected from the top $1.5 \mathrm{ft}$ of the lake.

WATER-QUALITY DATA, OCTOBER 16, 2000 TO APRIL 18, 2001

(Milligrams per liter unless otherwise indicated)

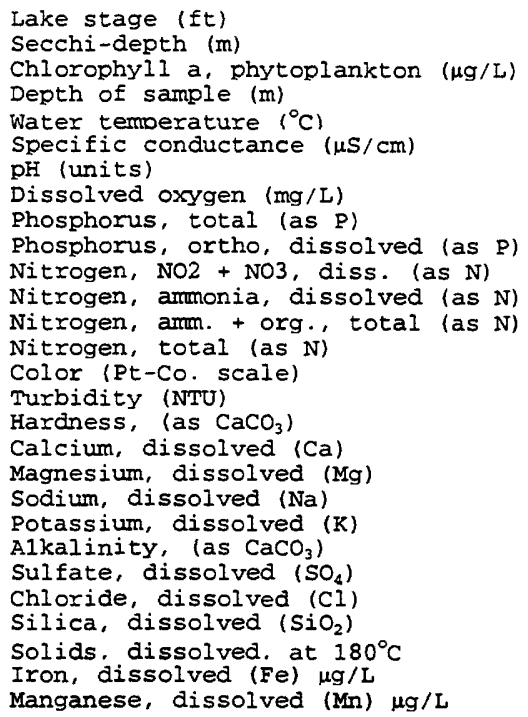

$10-16-00$
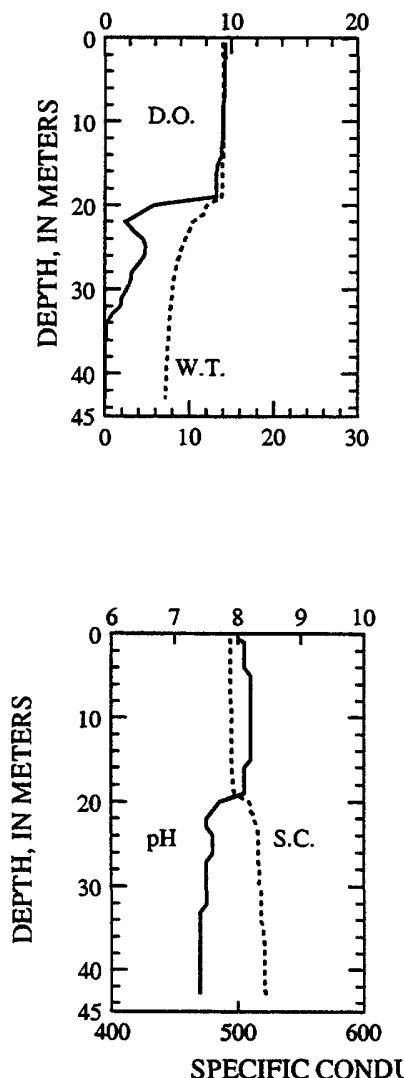

$11-15-00$

DISSOLVED OXYGEN (D.O.), IN MILLIGRAMS PER LITER
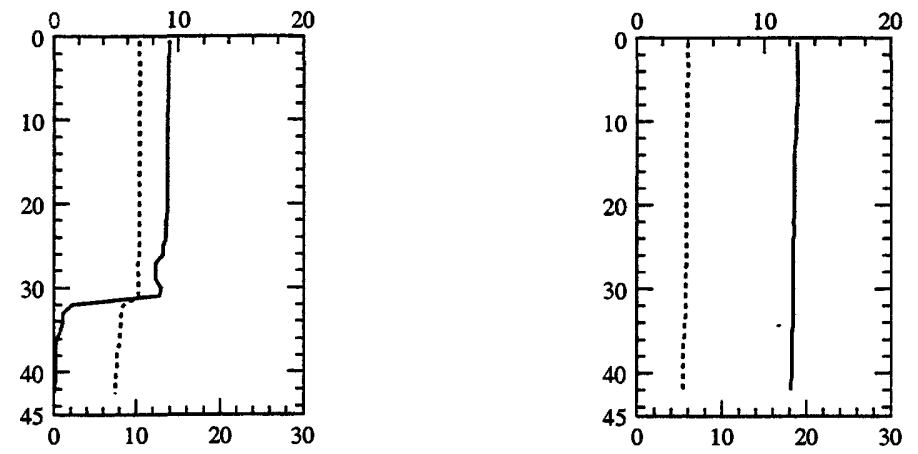

WATER TEMPERATURE (W.T.), IN DEGREES CELSIUS

PH, IN STANDARD UNITS
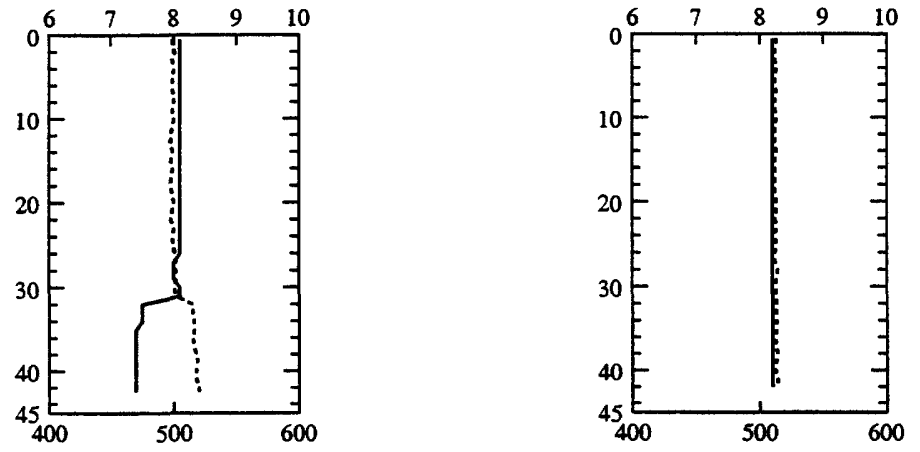

Apr -18

2.27

5.9

$<1.0$

$0.5 \quad 41.0$

$6.0 \quad 5.4$

513514

8.28 .2

$12.6 \quad 12.1$

$0.002<0.002$

$\begin{array}{ll}0.02 & 0.045\end{array}$

$0.35 \quad 0.37$

$0.46 \quad 0.493$

5

1.5

227

34
17

1.7

180

30.

35

280

$<10$

$<0.4$ 
WATER-QUALITY DATA, JUNE 20 TO JULY 16, 2001

(Milligrams per liter unless otherwise indicated)

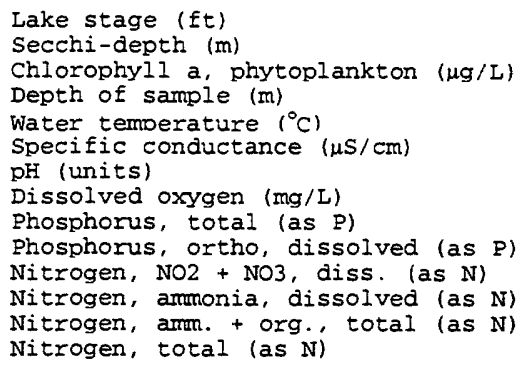

\begin{tabular}{|c|c|c|c|c|c|c|c|c|c|c|}
\hline \multicolumn{6}{|c|}{ Jun-20 } & \multicolumn{5}{|c|}{ JuI-16 } \\
\hline \multicolumn{6}{|c|}{$\begin{array}{c}2.76 \\
9.4 \\
<1.0\end{array}$} & \multicolumn{5}{|c|}{$\begin{array}{c}2.42 \\
4.3 \\
<1.0\end{array}$} \\
\hline $\begin{array}{c}0.5 \\
20.9 \\
494 \\
8.3 \\
9.2 \\
0.009\end{array}$ & $\begin{array}{c}7.0 \\
18.6 \\
500 \\
8.3 \\
9.5 \\
0.015\end{array}$ & $\begin{array}{c}20.0 \\
9.9 \\
511 \\
8.4 \\
9.5 \\
0.012\end{array}$ & $\begin{array}{l}33.0 \\
7.6 \\
514 \\
8.1 \\
8.6 \\
0.013\end{array}$ & $\begin{array}{c}38.0 \\
7.5 \\
514 \\
7.9 \\
7.8 \\
0.012\end{array}$ & $\begin{array}{c}42.0 \\
7.3 \\
516 \\
7.7 \\
6.4 \\
0.026\end{array}$ & $\begin{array}{c}0.5 \\
25.2 \\
488 \\
8.4 \\
9.5 \\
0.005\end{array}$ & $\begin{array}{c}25.0 \\
8.8 \\
507 \\
8.6 \\
8.6 \\
<0.005\end{array}$ & $\begin{array}{c}33.0 \\
7.7 \\
509 \\
8.2 \\
6.4 \\
<0.005\end{array}$ & $\begin{array}{c}38.0 \\
7.5 \\
511 \\
7.9 \\
4.5 \\
0.007\end{array}$ & $\begin{array}{c}42.0 \\
7.4 \\
512 \\
7.7 \\
3.1 \\
0.017\end{array}$ \\
\hline 0.004 & 2.0 & $\begin{array}{l}.0+2 \\
-. .-\end{array}$ & $\begin{array}{c}0.013 \\
-.-2\end{array}$ & $\begin{array}{l}.012 \\
-.-\end{array}$ & . & 0.003 & - & - & --- & 0. \\
\hline 0.053 & $-\cdots$ & --- & --- & -. & -- & 0.012 & --- & $\ldots$ & $\ldots$ & -- \\
\hline 0.027 & -- & -- & --- & -- & $-\ldots$ & 0.026 & -- & $-\infty$ & - - & --- \\
\hline 0.36 & $-\ldots$ & $\ldots$ & $-\ldots$ & -- & --- & 0.51 & --- & -- & --- & $\ldots$ \\
\hline 0.413 & --- & -- & -- & -- & --- & 0.525 & -- & $\ldots$ & -- & $\ldots$ \\
\hline
\end{tabular}

DISSOLVED OXYGEN (D.O.), IN MILLIGRAMS PER LITER
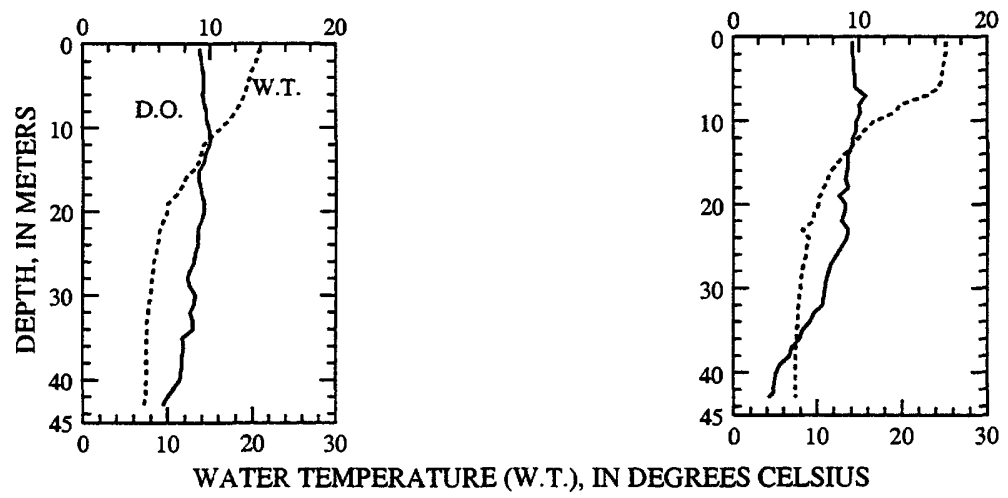

PH, IN STANDARD UNITS
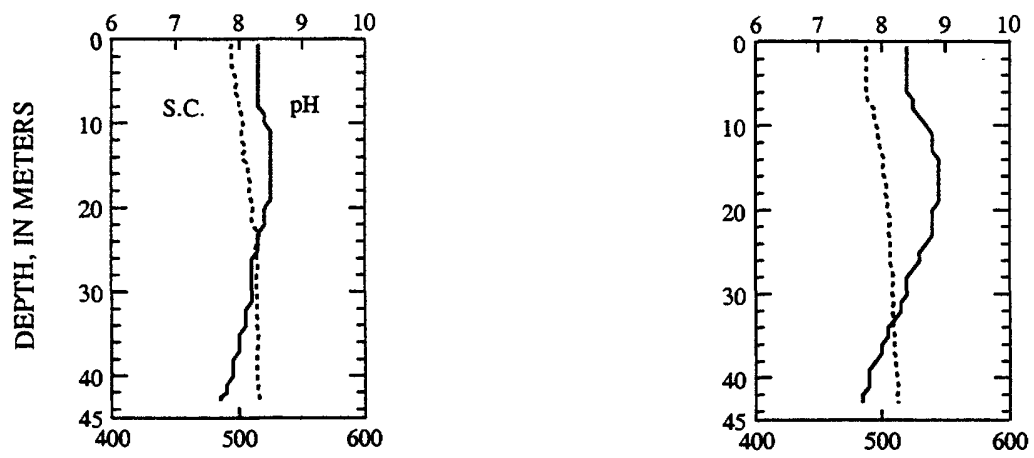

SPECIFIC CONDUCTANCE (S.C.), IN MICROSIEMENS PER CENTIMETER AT 25 DEGREES CELSIUS 
WATER-QUALITY DATA, AUGUST 20 TO SEPTEMBER 11, 2001

(Milligrams per liter unless otherwise indicated)

Lake stage ( $f t)$

Secchi-depth (m)

Chlorophyll a, phytoplankton ( $\mu \mathrm{g} / \mathrm{L}$ )

Depth of sample (m)

water temoerature $\left({ }^{\circ} \mathrm{C}\right)$

Specific conductance ( $\mu \mathrm{S} / \mathrm{cm})$

pH (units)

Dissolved oxygen (mg/L)

Phosphorus, total (as P)

Phosphorus, ortho, dissolved (as P)

Nitrogen, $\mathrm{NO} 2+\mathrm{NO} 3$, diss. (as N)

Nitrogen, ammonia, dissolved (as N)

Nitrogen, amm. + org.. total (as N)

Nitrogen, total (as N)

Aug -20

\begin{tabular}{|c|c|c|c|c|c|c|c|c|c|c|c|}
\hline \multicolumn{6}{|c|}{ Aug -20} & \multicolumn{6}{|c|}{ Sep-11 } \\
\hline \multicolumn{6}{|c|}{$\begin{array}{l}2.42 \\
3.2 \\
2.3\end{array}$} & \multicolumn{6}{|c|}{$\begin{array}{c}2.67 \\
5.20 \\
4\end{array}$} \\
\hline $\begin{array}{c}0.5 \\
23.9 \\
492 \\
8.4 \\
8.6 \\
0.008 \\
0.003 \\
<0.010 \\
0.033 \\
0.48\end{array}$ & $\begin{array}{c}9.0 \\
23.4 \\
493 \\
8.3 \\
8.0 \\
0.008 \\
\ldots- \\
\ldots- \\
\ldots- \\
\ldots-\end{array}$ & $\begin{array}{c}21.0 \\
10.1 \\
517 \\
8.4 \\
4.3 \\
0.009 \\
--- \\
--- \\
--- \\
---\end{array}$ & $\begin{array}{c}33.0 \\
7.8 \\
521 \\
7.9 \\
2.9 \\
0.011 \\
--- \\
\cdots- \\
\ldots-\end{array}$ & $\begin{array}{c}38.0 \\
7.5 \\
523 \\
7.7 \\
0.8 \\
0.021 \\
--- \\
--- \\
--- \\
---\end{array}$ & $\begin{array}{c}42.0 \\
7.4 \\
527 \\
7.6 \\
0.5 \\
0.051 \\
--- \\
-\ldots \\
-.- \\
\ldots-\end{array}$ & $\begin{array}{c}0.5 \\
21.9 \\
491 \\
8.3 \\
8.7 \\
0.008 \\
0.003 \\
<0.010 \\
<0.013 \\
0.53\end{array}$ & $\begin{array}{c}11.0 \\
21.8 \\
493 \\
8.1 \\
8.2 \\
0.011 \\
0.003 \\
<0.010 \\
0.016 \\
0.53\end{array}$ & $\begin{array}{c}29.0 \\
8.1 \\
520 \\
7.7 \\
3.0 \\
0.008 \\
0.004 \\
0.27 \\
0.034 \\
0.46\end{array}$ & $\begin{array}{c}32.0 \\
7.9 \\
526 \\
7.6 \\
2.3 \\
0.011 \\
0.005 \\
0.285 \\
0.048 \\
0.52\end{array}$ & $\begin{array}{l}37.0 \\
7.6 \\
527 \\
7.5 \\
0.7 \\
0.016 \\
0.009 \\
0.17 \\
0.125 \\
0.59\end{array}$ & $\begin{array}{c}41.0 \\
7.5 \\
527 \\
7.5 \\
0.7 \\
0.025 \\
0.015 \\
0.096 \\
0.182 \\
0.65\end{array}$ \\
\hline$\ldots$ & $\ldots$ & --- & -- & $\ldots$ & $-\cdots$ & & -- & 0.73 & 0.805 & 0.76 & 0.746 \\
\hline
\end{tabular}

DISSOLVED OXYGEN (D.O.), IN MILLIGRAMS PER LITER

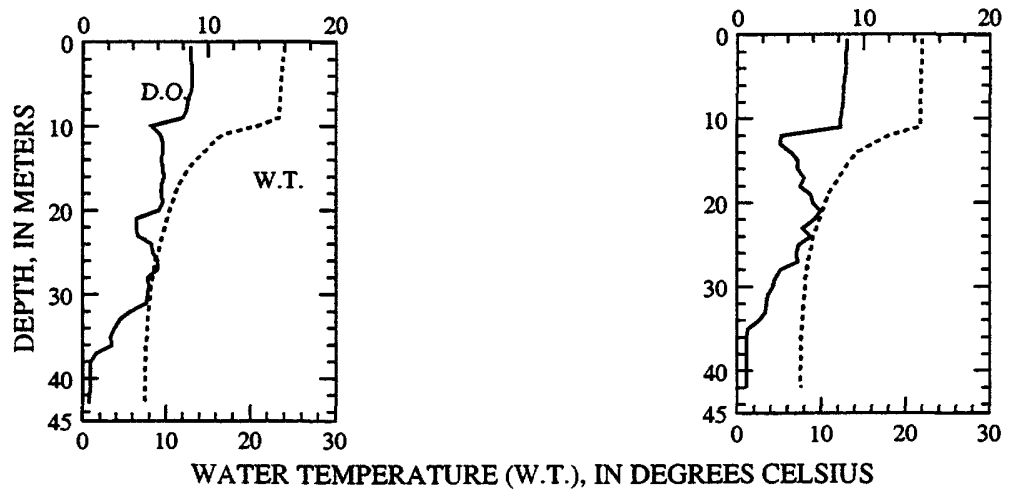

PH, IN STANDARD UNITS
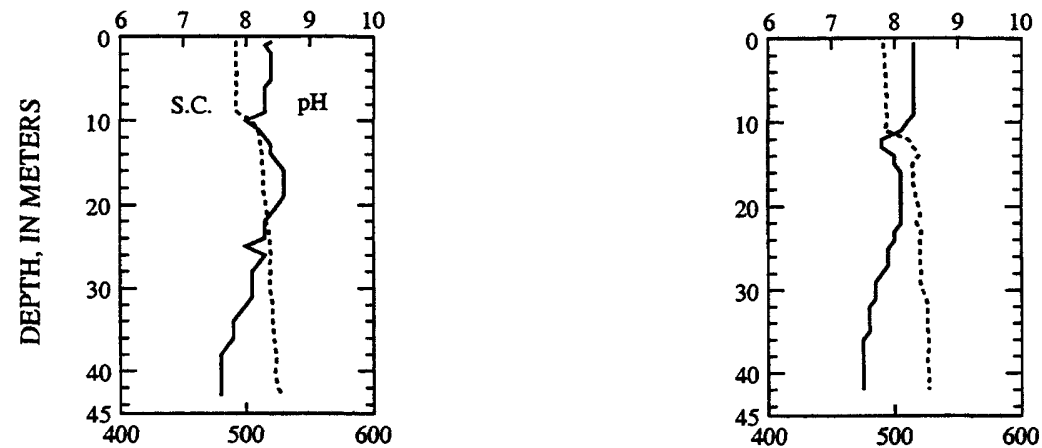

SPECIFIC CONDUCTANCE (S.C.), IN MICROSIEMENS PER CENTIMETER AT 25 DEGREES CELSIUS 

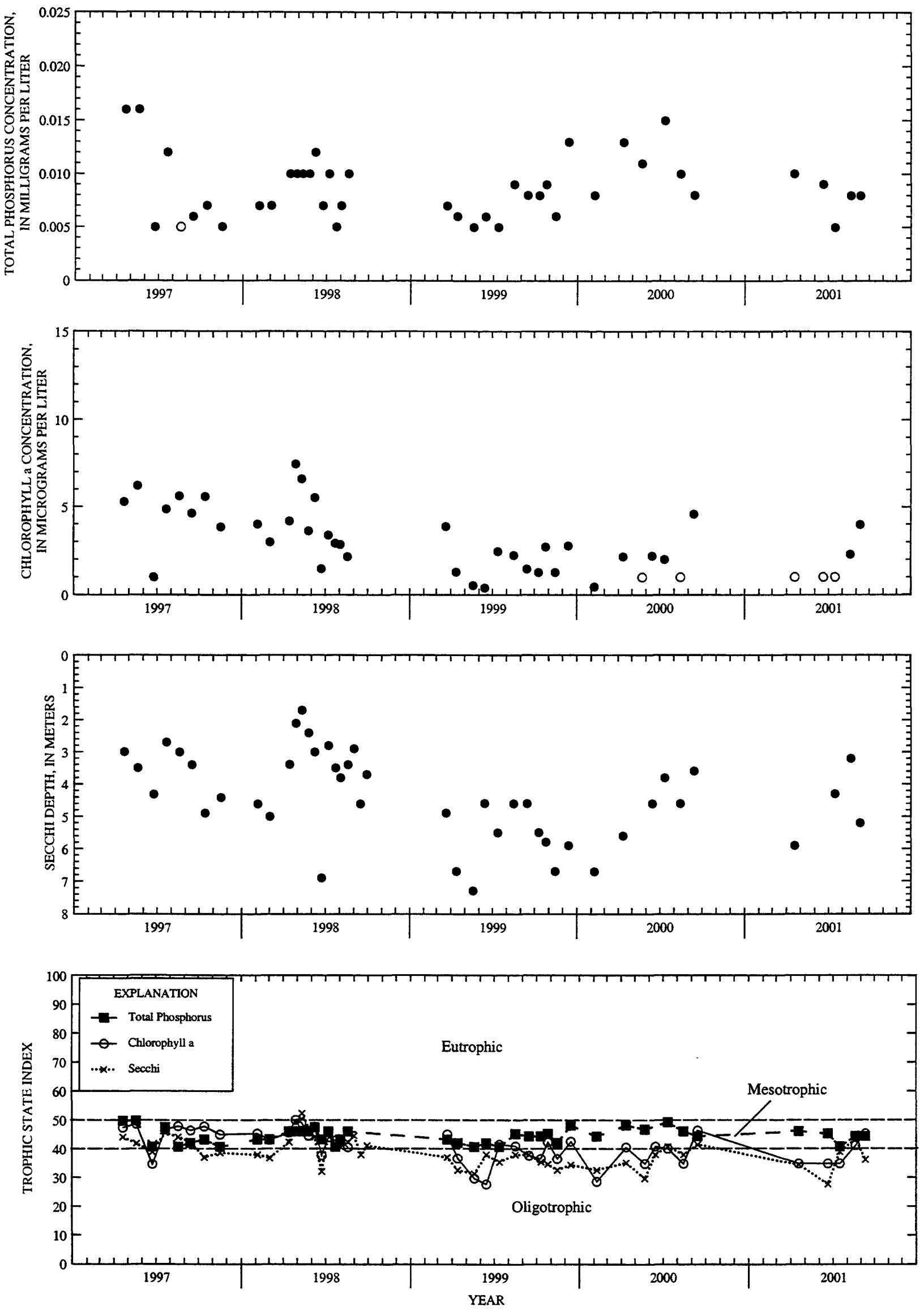

Surface total phosphorus, chlorophyll a concentrations, Secchi depths, and TSI data for Lake Geneva, West End, near Williams Bay, Wisconsin.

(Actual concentrations for these particular analyses are less than the plotted circles.

Circles on the first three plots indicate laboratory detection limit for selected analyses.) 
LOCATION.--Lat $43^{\circ} 49^{\prime} 28^{\prime \prime}$, long $88^{\circ} 55^{\prime} 36^{\prime \prime}$ in NE 1/4 SE 1/4 SE 1/4 sec.27, T.16 N., R.13 E., Green Lake County, Hydrologic Unit 04030201, on left bank at downstream side of County Trunk Highway A, 2.3 mi southeast of Green Lake.

DRAINAGE AREA.--103 $\mathrm{mi}^{2}$.

PERIOD OF RECORD.--October 1993 to current year.

GAGE.--Water-stage recorder. Datum of gage is $790.00 \mathrm{ft}$ above sea level.

REMARKS.--Lake level regulated by dam at outlet at Green Lake. Gage-height telemeter at station.

EXTREMES FOR PERIOD OF RECORD.--Maximum recorded gage height, $7.05 \mathrm{ft}$, Apr. 12, 2001; minimum recorded, $5.41 \mathrm{ft}$, Jan. 17, 1995.

EXTREMES FOR CURRENT YEAR.--Maximum recorded gage height, $7.05 \mathrm{ft}$, Apr. 12; minimum recorded, $6.04 \mathrm{ft}$, Dec. 9.

GAGE HEIGHT, FEET, WATER YEAR OCTOBER 2000 TO SEPTEMBER 2001

DAILY MEAN VALUES

\begin{tabular}{|c|c|c|c|c|c|c|c|c|c|c|c|c|}
\hline DAY & OCT & NOV & DEC & JAN & FEB & MAR & APR & MAY & JUN & JUL & AUG & SEP \\
\hline $\begin{array}{l}1 \\
2 \\
3 \\
4 \\
5\end{array}$ & $\begin{array}{l}6.41 \\
6.40 \\
6.38 \\
6.38 \\
6.37\end{array}$ & $\begin{array}{l}6.19 \\
6.18 \\
6.17 \\
6.14 \\
6.13\end{array}$ & $\begin{array}{l}6.12 \\
6.12 \\
6.12 \\
6.13 \\
6.11\end{array}$ & $\begin{array}{l}6.20 \\
6.20 \\
6.20 \\
6.20 \\
6.20\end{array}$ & $\begin{array}{l}6.29 \\
6.29 \\
6.29 \\
6.30 \\
6.30\end{array}$ & $\begin{array}{l}6.36 \\
6.36 \\
6.36 \\
6.36 \\
6.36\end{array}$ & $\begin{array}{l}6.63 \\
6.63 \\
6.65 \\
6.65 \\
6.66\end{array}$ & $\begin{array}{l}6.44 \\
6.44 \\
6.49 \\
6.59 \\
6.61\end{array}$ & $\begin{array}{l}6.56 \\
6.60 \\
6.60 \\
6.59 \\
6.61\end{array}$ & $\begin{array}{l}6.53 \\
6.48 \\
6.46 \\
6.46 \\
6.42\end{array}$ & $\begin{array}{l}6.32 \\
6.42 \\
6.43 \\
6.43 \\
6.43\end{array}$ & $\begin{array}{l}6.37 \\
6.36 \\
6.35 \\
6.33 \\
6.32\end{array}$ \\
\hline $\begin{array}{r}6 \\
7 \\
8 \\
9 \\
10\end{array}$ & $\begin{array}{l}6.36 \\
6.34 \\
6.31 \\
6.30 \\
6.28\end{array}$ & $\begin{array}{l}6.10 \\
6.16 \\
6.16 \\
6.15 \\
6.16\end{array}$ & $\begin{array}{l}6.09 \\
6.08 \\
6.09 \\
6.07 \\
6.08\end{array}$ & $\begin{array}{r}\text { e6. } \\
\text { e6.20 } \\
6.20 \\
6.20 \\
6.20\end{array}$ & $\begin{array}{l}6.29 \\
6.29 \\
6.30 \\
6.36 \\
6.38\end{array}$ & $\begin{array}{l}6.35 \\
6.35 \\
6.35 \\
6.35 \\
6.35\end{array}$ & $\begin{array}{l}6.71 \\
6.82 \\
6.85 \\
6.85 \\
6.85\end{array}$ & $\begin{array}{l}6.62 \\
6.65 \\
6.67 \\
6.65 \\
6.66\end{array}$ & $\begin{array}{l}6.63 \\
6.64 \\
6.64 \\
6.64 \\
6.65\end{array}$ & $\begin{array}{l}6.40 \\
6.37 \\
6.36 \\
6.36 \\
6.36\end{array}$ & $\begin{array}{l}6.43 \\
6.42 \\
6.42 \\
6.41 \\
6.35\end{array}$ & $\begin{array}{l}6.32 \\
6.42 \\
6.54 \\
6.55 \\
6.58\end{array}$ \\
\hline $\begin{array}{l}11 \\
12 \\
13 \\
14 \\
15\end{array}$ & $\begin{array}{l}6.27 \\
6.26 \\
6.26 \\
6.26 \\
6.25\end{array}$ & $\begin{array}{l}6.15 \\
6.14 \\
6.17 \\
6.18 \\
6.15\end{array}$ & $\begin{array}{l}6.09 \\
6.10 \\
6.09 \\
6.09 \\
6.10\end{array}$ & $\begin{array}{l}6.20 \\
6.20 \\
6.20 \\
6.22 \\
6.24\end{array}$ & $\begin{array}{l}6.38 \\
6.37 \\
6.37 \\
6.37 \\
6.38\end{array}$ & $\begin{array}{l}6.35 \\
6.36 \\
6.37 \\
6.37 \\
6.38\end{array}$ & $\begin{array}{l}6.89 \\
6.96 \\
6.90 \\
6.87 \\
6.84\end{array}$ & $\begin{array}{l}6.73 \\
6.73 \\
6.72 \\
6.75 \\
6.80\end{array}$ & $\begin{array}{l}6.65 \\
6.79 \\
6.81 \\
6.82 \\
6.88\end{array}$ & $\begin{array}{l}6.33 \\
6.32 \\
6.31 \\
6.30 \\
6.29\end{array}$ & $\begin{array}{l}6.33 \\
6.32 \\
6.30 \\
6.29 \\
6.28\end{array}$ & $\begin{array}{l}6.56 \\
6.55 \\
6.51 \\
6.48 \\
6.47\end{array}$ \\
\hline $\begin{array}{l}16 \\
17 \\
18 \\
19 \\
20\end{array}$ & $\begin{array}{l}6.25 \\
6.25 \\
6.24 \\
6.24 \\
6.24\end{array}$ & $\begin{array}{l}6.15 \\
6.17 \\
6.17 \\
6.15 \\
6.16\end{array}$ & $\begin{array}{l}6.15 \\
6.15 \\
6.15 \\
6.17 \\
6.17\end{array}$ & $\begin{array}{l}6.24 \\
6.24 \\
6.24 \\
6.23 \\
6.23\end{array}$ & $\begin{array}{l}6.37 \\
6.37 \\
6.36 \\
6.36 \\
6.36\end{array}$ & $\begin{array}{l}6.38 \\
6.38 \\
6.39 \\
6.40 \\
6.42\end{array}$ & $\begin{array}{l}6.81 \\
6.75 \\
6.72 \\
6.68 \\
6.67\end{array}$ & $\begin{array}{l}6.80 \\
6.79 \\
6.76 \\
6.73 \\
6.70\end{array}$ & $\begin{array}{l}6.88 \\
6.85 \\
6.90 \\
6.90 \\
6.84\end{array}$ & $\begin{array}{l}6.28 \\
6.28 \\
6.30 \\
6.31 \\
6.31\end{array}$ & $\begin{array}{l}6.30 \\
6.29 \\
6.27 \\
6.26 \\
6.26\end{array}$ & $\begin{array}{l}6.46 \\
6.46 \\
6.46 \\
6.46 \\
6.48\end{array}$ \\
\hline $\begin{array}{l}21 \\
22 \\
23 \\
24 \\
25\end{array}$ & $\begin{array}{l}6.23 \\
6.22 \\
6.22 \\
6.23 \\
6.23\end{array}$ & $\begin{array}{l}6.14 \\
6.11 \\
6.10 \\
6.09 \\
6.09\end{array}$ & $\begin{array}{l}6.19 \\
6.18 \\
6.17 \\
6.18 \\
6.17\end{array}$ & $\begin{array}{l}6.23 \\
6.23 \\
6.23 \\
6.23 \\
6.22\end{array}$ & $\begin{array}{l}6.35 \\
6.35 \\
6.34 \\
6.34 \\
6.37\end{array}$ & $\begin{array}{l}6.44 \\
6.46 \\
6.48 \\
6.50 \\
6.51\end{array}$ & $\begin{array}{l}6.68 \\
6.63 \\
6.64 \\
6.59 \\
6.56\end{array}$ & $\begin{array}{l}6.71 \\
6.73 \\
6.70 \\
6.68 \\
6.67\end{array}$ & $\begin{array}{l}6.82 \\
6.82 \\
6.79 \\
6.75 \\
6.72\end{array}$ & $\begin{array}{l}6.31 \\
6.32 \\
6.36 \\
6.37 \\
6.34\end{array}$ & $\begin{array}{l}6.25 \\
6.34 \\
6.39 \\
6.38 \\
6.42\end{array}$ & $\begin{array}{l}6.47 \\
6.46 \\
6.46 \\
6.46 \\
6.44\end{array}$ \\
\hline $\begin{array}{l}26 \\
27 \\
28 \\
29 \\
30 \\
31\end{array}$ & $\begin{array}{l}6.24 \\
6.25 \\
6.23 \\
6.22 \\
6.21 \\
6.21\end{array}$ & $\begin{array}{l}6.09 \\
6.10 \\
6.09 \\
6.11 \\
6.12 \\
.--\end{array}$ & $\begin{array}{l}6.17 \\
6.17 \\
6.17 \\
6.20 \\
6.20 \\
6.20\end{array}$ & $\begin{array}{l}6.23 \\
6.23 \\
6.23 \\
6.23 \\
6.29 \\
6.30\end{array}$ & $\begin{array}{r}6.37 \\
6.37 \\
6.37 \\
--- \\
- \\
-\end{array}$ & $\begin{array}{l}6.53 \\
6.54 \\
6.55 \\
6.57 \\
6.59 \\
6.60\end{array}$ & $\begin{array}{l}6.53 \\
6.49 \\
6.47 \\
6.45 \\
6.45 \\
.-\end{array}$ & $\begin{array}{l}6.66 \\
6.64 \\
6.62 \\
6.59 \\
6.57 \\
6.54\end{array}$ & $\begin{array}{l}6.69 \\
6.66 \\
6.63 \\
6.60 \\
6.58 \\
\ldots\end{array}$ & $\begin{array}{l}6.32 \\
6.30 \\
6.29 \\
6.29 \\
6.29 \\
6.29\end{array}$ & $\begin{array}{l}6.44 \\
6.45 \\
6.44 \\
6.43 \\
6.42 \\
6.39\end{array}$ & $\begin{array}{l}6.43 \\
6.41 \\
6.40 \\
6.38 \\
6.38 \\
\end{array}$ \\
\hline $\begin{array}{l}\text { MEAN } \\
\text { MAX } \\
\text { MIN }\end{array}$ & $\begin{array}{l}6.28 \\
6.41 \\
6.21\end{array}$ & $\begin{array}{l}6.14 \\
6.19 \\
6.09\end{array}$ & $\begin{array}{l}6.14 \\
6.20 \\
6.07\end{array}$ & $\begin{array}{l}6.22 \\
6.30 \\
6.20\end{array}$ & $\begin{array}{l}6.34 \\
6.38 \\
6.29\end{array}$ & $\begin{array}{l}6.42 \\
6.60 \\
6.35\end{array}$ & $\begin{array}{l}6.70 \\
6.96 \\
6.45\end{array}$ & $\begin{array}{l}6.66 \\
6.80 \\
6.44\end{array}$ & $\begin{array}{l}6.72 \\
6.90 \\
6.56\end{array}$ & $\begin{array}{l}6.35 \\
6.53 \\
6.28\end{array}$ & $\begin{array}{l}6.36 \\
6.45 \\
6.25\end{array}$ & $\begin{array}{l}6.44 \\
6.58 \\
6.32\end{array}$ \\
\hline
\end{tabular}

e Estimated 
LOCATION.--Lat 45 34'21", long 91 $33^{\prime} 37^{\prime \prime}$, in SE 1/4 SE 1/4 sec.26, T.36 N., R.10 W., Barron County, Hydrologic Unit 07050007, 2.5 mi southeast of Mikana.

PERIOD OF RECORD.--March 1993 to August 1994, March 1996 to August 1997, and March to September 2001.

REMARKS.--Lake sampled at deep hole near center of lake. Lake ice-covered during March sampling. Water-quality analyses by Wisconsin State Laboratory of Hygiene.

WATER-QUALITY DATA, MARCH 01 TO JUNE 12, 2001

(Milligrams per liter unless otherwise indicated
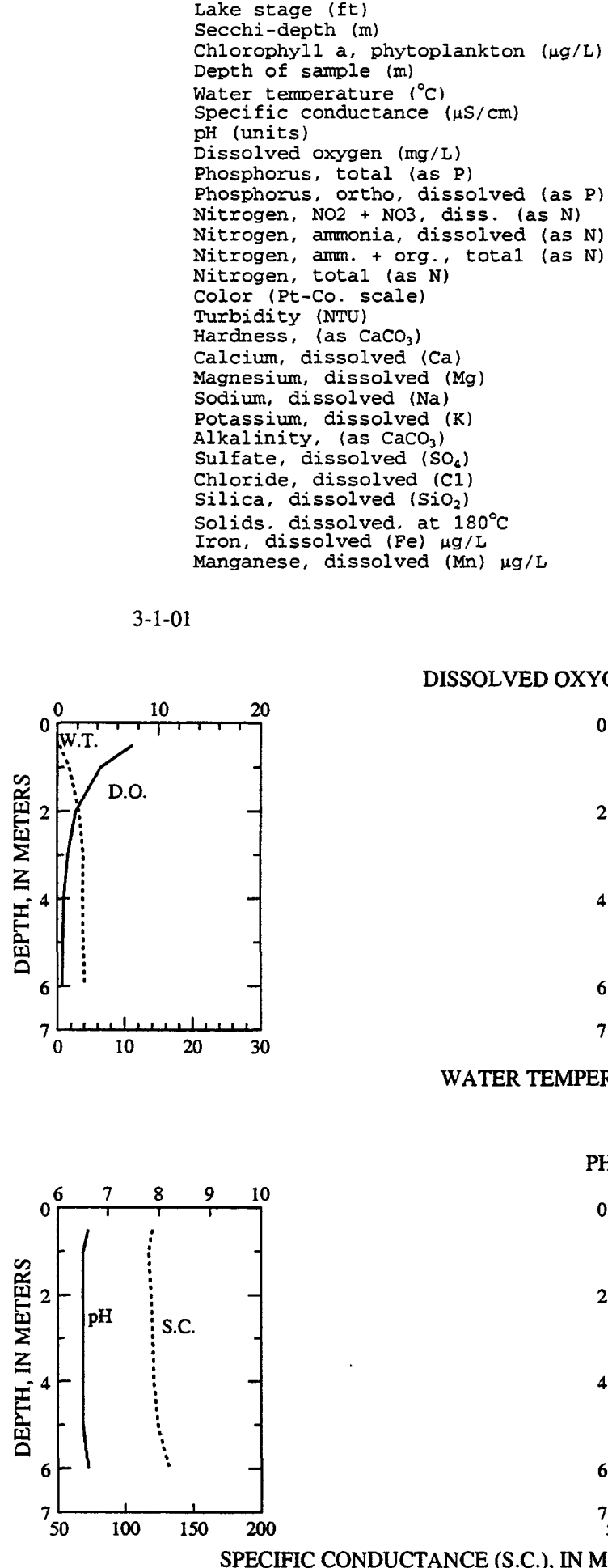

\section{DISSOLVED OXYGEN (D.O.), IN MILLIGRAMS PER LITER}

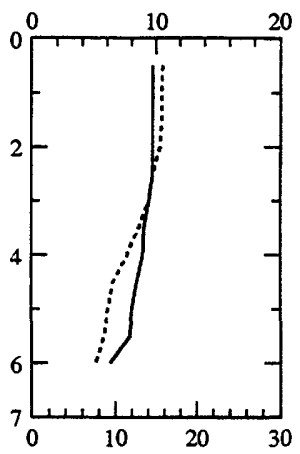

WATER TEMPERATURE (W.T.), IN DEGREES CELSIUS

\section{PH, IN STANDARD UNITS}
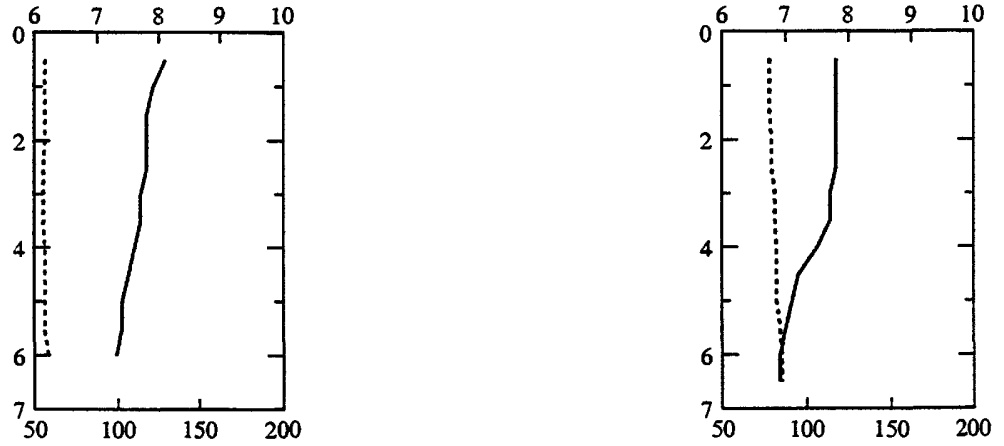

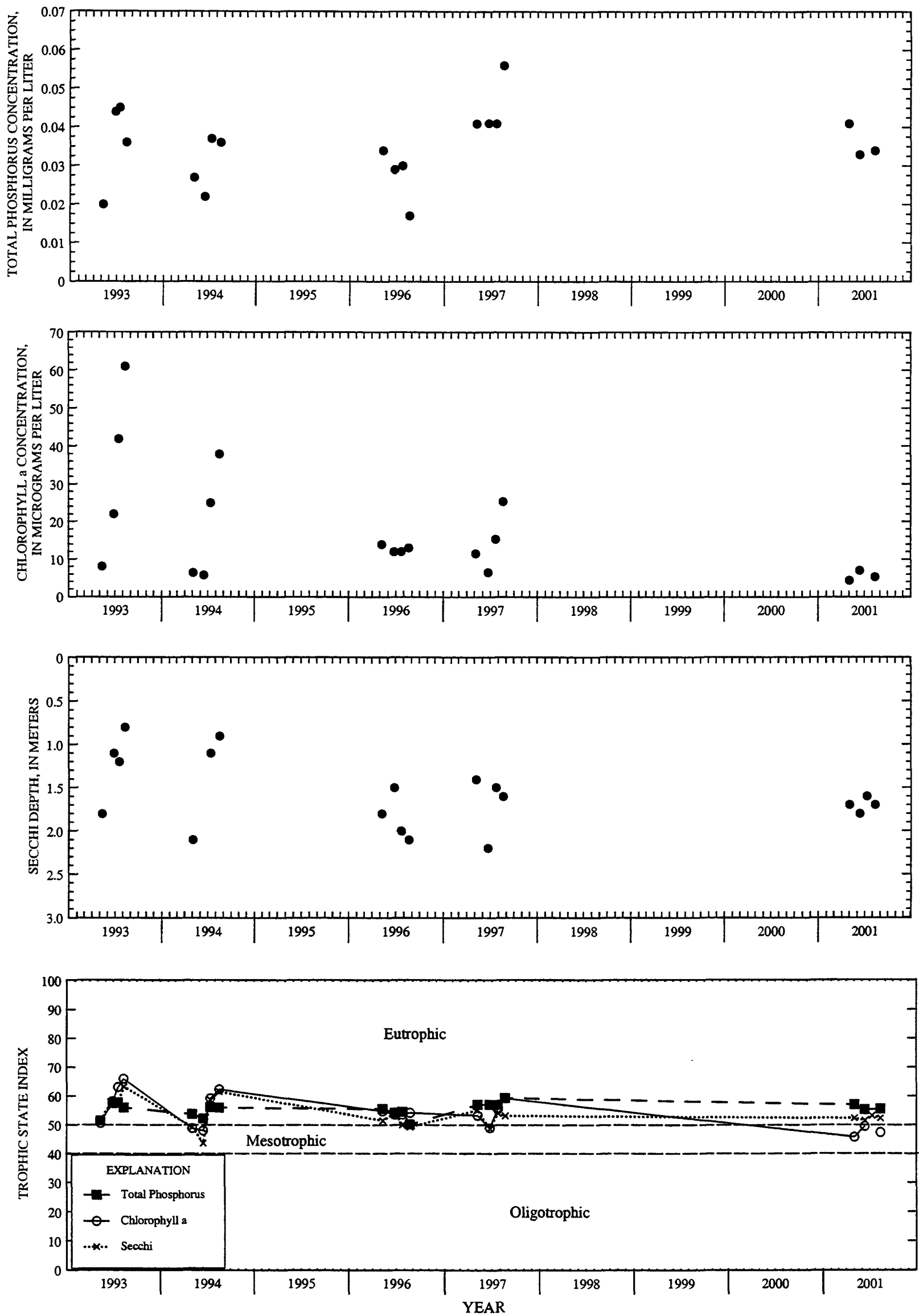

Surface total phosphorus, chlorophyll a concentrations, Secchi depths, and TSI data for Hemlock Lake, near Mikana, Wisconsin. 


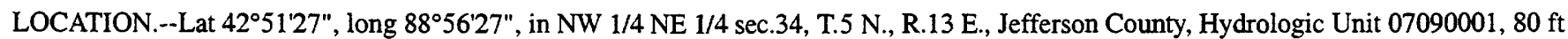
east of Pottawatomi Trail Bridge at Bingham Point Estates, and $4.5 \mathrm{mi}$ northeast of Newville.

DRAINAGE AREA.--2,560 $\mathrm{mi}^{2}$, at lake outlet. Area of Lake Koshkonong, $16.3 \mathrm{mi}^{2}$.

PERIOD OF RECORD.--July 1987 to current year.

GAGE.--Water-stage recorder. Datum of gage is $770.00 \mathrm{ft}$ above sea level.

REMARKS.--Lake level regulated by dam at Indianford. Gage-height telemeter at station.

EXTREMES FOR PERIOD OF RECORD.--Maximum recorded gage height, $12.23 \mathrm{ft}$, Apr. 25, 1993; minimum recorded, $5.10 \mathrm{ft}$, Dec. 28 , 29, 1999.

EXTREMES FOR CURRENT YEAR.--Maximum recorded gage height, 8.98 ft, Apr. 16, 17; minimum recorded, $5.51 \mathrm{ft}$, Nov. 1.

GAGE HEIGHT, FEET, WATER YEAR OCTOBER 2000 TO SEPTEMBER 2001

DAILY MEAN VALUES

\begin{tabular}{|c|c|c|c|c|c|c|c|c|c|c|c|c|}
\hline DAY & OCT & NOV & $D E C$ & JAN & $F E B$ & MAR & $A P R$ & MAY & JUN & JUL & AUG & SEP \\
\hline $\begin{array}{l}1 \\
2 \\
3 \\
4 \\
5\end{array}$ & $\begin{array}{l}6.51 \\
6.48 \\
6.42 \\
6.34 \\
6.25\end{array}$ & $\begin{array}{l}5.59 \\
5.62 \\
5.66 \\
5.67 \\
5.68\end{array}$ & $\begin{array}{l}5.67 \\
5.70 \\
5.71 \\
5.70 \\
5.68\end{array}$ & $\begin{array}{l}5.86 \\
5.85 \\
5.85 \\
5.82 \\
5.79\end{array}$ & $\begin{array}{l}5.79 \\
5.83 \\
5.86 \\
5.88 \\
5.90\end{array}$ & $\begin{array}{l}6.75 \\
6.76 \\
6.77 \\
6.80 \\
6.86\end{array}$ & $\begin{array}{l}8.20 \\
8.17 \\
8.14 \\
8.11 \\
8.08\end{array}$ & $\begin{array}{l}8.09 \\
8.01 \\
7.97 \\
8.04 \\
8.00\end{array}$ & $\begin{array}{l}7.22 \\
7.22 \\
7.16 \\
7.11 \\
7.10\end{array}$ & $\begin{array}{l}8.21 \\
8.09 \\
7.98 \\
7.89 \\
7.76\end{array}$ & $\begin{array}{l}6.23 \\
6.51 \\
6.54 \\
6.48 \\
6.41\end{array}$ & $\begin{array}{l}6.27 \\
6.29 \\
6.30 \\
6.30 \\
6.24\end{array}$ \\
\hline $\begin{array}{r}6 \\
7 \\
8 \\
9 \\
10\end{array}$ & $\begin{array}{l}6.27 \\
6.25 \\
6.22 \\
6.21 \\
6.21\end{array}$ & $\begin{array}{l}5.67 \\
5.78 \\
5.81 \\
5.86 \\
5.87\end{array}$ & $\begin{array}{l}5.63 \\
5.60 \\
5.57 \\
5.56 \\
5.57\end{array}$ & $\begin{array}{l}5.76 \\
5.74 \\
5.72 \\
5.70 \\
5.69\end{array}$ & $\begin{array}{l}5.89 \\
5.88 \\
5.87 \\
6.01 \\
6.10\end{array}$ & $\begin{array}{l}6.92 \\
6.96 \\
7.00 \\
7.03 \\
7.06\end{array}$ & $\begin{array}{l}8.08 \\
8.05 \\
8.07 \\
8.21 \\
8.28\end{array}$ & $\begin{array}{l}7.97 \\
7.95 \\
7.92 \\
7.86 \\
7.82\end{array}$ & $\begin{array}{l}7.11 \\
7.11 \\
7.12 \\
7.12 \\
7.10\end{array}$ & $\begin{array}{l}7.61 \\
7.48 \\
7.35 \\
7.21 \\
7.07\end{array}$ & $\begin{array}{l}6.36 \\
6.30 \\
6.23 \\
6.19 \\
6.15\end{array}$ & $\begin{array}{l}6.20 \\
6.29 \\
6.49 \\
6.64 \\
6.84\end{array}$ \\
\hline $\begin{array}{l}11 \\
12 \\
13 \\
14 \\
15\end{array}$ & $\begin{array}{l}6.20 \\
6.21 \\
6.24 \\
6.26 \\
6.26\end{array}$ & $\begin{array}{l}5.84 \\
5.82 \\
5.84 \\
5.85 \\
5.79\end{array}$ & $\begin{array}{l}5.60 \\
5.63 \\
5.63 \\
5.62 \\
5.62\end{array}$ & $\begin{array}{l}5.67 \\
5.65 \\
5.64 \\
5.65 \\
5.65\end{array}$ & $\begin{array}{l}6.20 \\
6.29 \\
6.40 \\
6.51 \\
6.60\end{array}$ & $\begin{array}{l}7.10 \\
7.16 \\
7.25 \\
7.34 \\
7.45\end{array}$ & $\begin{array}{l}8.35 \\
8.52 \\
8.70 \\
8.82 \\
8.88\end{array}$ & $\begin{array}{l}7.89 \\
7.85 \\
7.79 \\
7.73 \\
7.69\end{array}$ & $\begin{array}{l}7.08 \\
7.21 \\
7.37 \\
7.59 \\
7.81\end{array}$ & $\begin{array}{l}6.89 \\
6.72 \\
6.55 \\
6.41 \\
6.28\end{array}$ & $\begin{array}{l}6.09 \\
6.05 \\
6.02 \\
6.03 \\
6.05\end{array}$ & $\begin{array}{l}6.91 \\
6.94 \\
6.92 \\
6.89 \\
6.85\end{array}$ \\
\hline $\begin{array}{l}16 \\
17 \\
18 \\
19 \\
20\end{array}$ & $\begin{array}{l}6.26 \\
6.21 \\
6.17 \\
6.11 \\
6.06\end{array}$ & $\begin{array}{l}5.80 \\
5.86 \\
5.87 \\
5.88 \\
5.90\end{array}$ & $\begin{array}{l}5.65 \\
5.69 \\
5.73 \\
5.77 \\
5.79\end{array}$ & $\begin{array}{l}5.65 \\
5.65 \\
5.67 \\
5.68 \\
5.70\end{array}$ & $\begin{array}{l}6.67 \\
6.72 \\
6.75 \\
6.76 \\
6.74\end{array}$ & $\begin{array}{l}7.56 \\
7.66 \\
7.76 \\
7.86 \\
8.01\end{array}$ & $\begin{array}{l}8.93 \\
8.92 \\
8.83 \\
8.75 \\
8.73\end{array}$ & $\begin{array}{l}7.65 \\
7.59 \\
7.52 \\
7.45 \\
7.36\end{array}$ & $\begin{array}{l}7.97 \\
8.06 \\
8.13 \\
8.21 \\
8.28\end{array}$ & $\begin{array}{l}6.17 \\
6.19 \\
6.22 \\
6.22 \\
6.22\end{array}$ & $\begin{array}{l}6.15 \\
6.17 \\
6.19 \\
6.22 \\
6.21\end{array}$ & $\begin{array}{l}6.80 \\
6.76 \\
6.73 \\
6.80 \\
6.77\end{array}$ \\
\hline $\begin{array}{l}21 \\
22 \\
23 \\
24 \\
25\end{array}$ & $\begin{array}{l}6.03 \\
6.01 \\
6.01 \\
6.02 \\
5.95\end{array}$ & $\begin{array}{l}5.84 \\
5.77 \\
5.72 \\
5.67 \\
5.62\end{array}$ & $\begin{array}{l}5.81 \\
5.82 \\
5.83 \\
5.84 \\
5.84\end{array}$ & $\begin{array}{l}5.71 \\
5.71 \\
5.71 \\
5.70 \\
5.68\end{array}$ & $\begin{array}{l}6.72 \\
6.68 \\
6.63 \\
6.59 \\
6.61\end{array}$ & $\begin{array}{l}8.09 \\
8.16 \\
8.24 \\
8.31 \\
8.37\end{array}$ & $\begin{array}{l}8.70 \\
8.68 \\
8.63 \\
8.63 \\
8.56\end{array}$ & $\begin{array}{l}7.35 \\
7.32 \\
7.30 \\
7.29 \\
7.30\end{array}$ & $\begin{array}{l}8.34 \\
8.41 \\
8.44 \\
8.47 \\
8.47\end{array}$ & $\begin{array}{l}6.22 \\
6.20 \\
6.19 \\
6.16 \\
6.13\end{array}$ & $\begin{array}{l}6.22 \\
6.28 \\
6.27 \\
6.23 \\
6.23\end{array}$ & $\begin{array}{l}6.81 \\
6.83 \\
6.95 \\
7.06 \\
7.12\end{array}$ \\
\hline $\begin{array}{l}26 \\
27 \\
28 \\
29 \\
30 \\
31\end{array}$ & $\begin{array}{l}5.88 \\
5.84 \\
5.79 \\
5.73 \\
5.68 \\
5.65\end{array}$ & $\begin{array}{l}5.60 \\
5.58 \\
5.59 \\
5.62 \\
5.64 \\
---\end{array}$ & $\begin{array}{l}5.84 \\
5.84 \\
5.85 \\
5.87 \\
5.88 \\
5.87\end{array}$ & $\begin{array}{l}5.67 \\
5.65 \\
5.63 \\
5.63 \\
5.70 \\
5.74\end{array}$ & $\begin{array}{r}6.63 \\
6.67 \\
6.72 \\
- \\
- \\
-\end{array}$ & $\begin{array}{l}8.39 \\
8.39 \\
8.37 \\
8.32 \\
8.27 \\
8.22\end{array}$ & $\begin{array}{l}8.50 \\
8.45 \\
8.36 \\
8.25 \\
8.16 \\
-\end{array}$ & $\begin{array}{l}7.31 \\
7.32 \\
7.32 \\
7.30 \\
7.27 \\
7.22\end{array}$ & $\begin{array}{l}8.47 \\
8.44 \\
8.40 \\
8.34 \\
8.27 \\
-.-\end{array}$ & $\begin{array}{l}6.10 \\
6.07 \\
6.10 \\
6.16 \\
6.19 \\
6.21\end{array}$ & $\begin{array}{l}6.27 \\
6.28 \\
6.30 \\
6.28 \\
6.26 \\
6.27\end{array}$ & $\begin{array}{r}7.18 \\
7.22 \\
7.25 \\
7.26 \\
7.24 \\
-\end{array}$ \\
\hline $\begin{array}{l}\text { MEAN } \\
\text { MAX } \\
\text { MIN }\end{array}$ & $\begin{array}{l}6.12 \\
6.51 \\
5.65\end{array}$ & $\begin{array}{l}5.74 \\
5.90 \\
5.58\end{array}$ & $\begin{array}{l}5.72 \\
5.88 \\
5.56\end{array}$ & $\begin{array}{l}5.71 \\
5.86 \\
5.63\end{array}$ & $\begin{array}{l}6.35 \\
6.76 \\
5.79\end{array}$ & $\begin{array}{l}7.59 \\
8.39 \\
6.75\end{array}$ & $\begin{array}{l}8.46 \\
8.93 \\
8.05\end{array}$ & $\begin{array}{l}7.63 \\
8.09 \\
7.22\end{array}$ & $\begin{array}{l}7.77 \\
8.47 \\
7.08\end{array}$ & $\begin{array}{l}6.72 \\
8.21 \\
6.07\end{array}$ & $\begin{array}{l}6.24 \\
6.54 \\
6.02\end{array}$ & $\begin{array}{l}6.77 \\
7.26 \\
6.20\end{array}$ \\
\hline
\end{tabular}


LOCATION.--Lat $43^{\circ} 07^{\prime} 33^{\prime \prime}$, long $88^{\circ} 30^{\prime} 59^{\prime \prime}$, in NW 1/4 SW 1/4 sec.29, T.8 N., R.17 E., Waukesha County, Hydrologic Unit 07090001, at Oconomowoc.

DRAINAGE AREA.--99.6 $\mathrm{mi}^{2}$.

PERIOD OF RECORD.--February 1984 to August 1985, April to August 1991, and February to September, 2001.

REMARKS.--Lake sampled near center of lake at a depth of $13 \mathrm{~m}$. Water-quality analyses by Wisconsin State Laboratory of Hygiene. WATER-QUALITY DATA, FEBRUARY 14 TO AUGUST 15, 2001

(Milligrams per liter unless otherwise indicated)

Secchi-depth (m)

Chlorophyll a, phytoplankton $(\mu g / L)$

Depth of sample (m)

water temperature $\left({ }^{\circ} \mathrm{C}\right)$

Specific conductance $(\mu \mathrm{S} / \mathrm{cm})$

$\mathrm{pH}$ (units)

Dissolved oxygen (mg/L)

Phosphorus, total (as P)

Phosphorus, ortho, dissolved (as P)

Nitrogen, NO2 + NO3, diss. (as N)

Nitrogen, ammonia, dissolved (as $N$ )

Nitrogen, amm. + org., total (as N)

Nitrogen, total (as N)

Color (Pt-Co. scale)

Turbidity (NTU)

Hardness, (as $\mathrm{CaCO}_{3}$ )

Calcium, dissolved (Ca)

Magnesium, dissolved (Mg)

Sodium, dissolved (Na)

Potassium, dissolved (K)

Alkalinity, (as $\mathrm{CaCO}_{3}$ )

Sulfate, dissolved $\left(\mathrm{SO}_{4}\right)$

Chloride, dissolved (Cl)

Silica, dissolved $\left(\mathrm{SiO}_{2}\right)$

Solids, dissolved, at $180^{\circ} \mathrm{C}$

Iron, dissolved (Fe) $\mu \mathrm{g} / \mathrm{L}$

Manganese, dissolved (Mn) $\mu \mathrm{g} / \mathrm{L}$

\begin{tabular}{|c|c|}
\hline \multicolumn{2}{|c|}{ Feb-14 } \\
\hline \multicolumn{2}{|c|}{--} \\
\hline $\begin{array}{c}0.5 \\
0.7 \\
575 \\
7.9 \\
13.2 \\
0.017\end{array}$ & $\begin{array}{c}13.0 \\
4.3 \\
751 \\
7.4 \\
1.2 \\
0.042\end{array}$ \\
\hline--- & -- \\
\hline--- & --- \\
\hline--- & --- \\
\hline--- & --- \\
\hline--- & -- \\
\hline--- & --- \\
\hline--- & $-\cdots$ \\
\hline$-\cdots$ & --- \\
\hline--- & --- \\
\hline--- & $--\infty$ \\
\hline$\cdots$ & -- \\
\hline--- & -- \\
\hline-- & --- \\
\hline$\ldots$ & --- \\
\hline--- & $\cdots$ \\
\hline--- & --- \\
\hline-- & --- \\
\hline-- & -- \\
\hline
\end{tabular}

\begin{tabular}{cc}
\multicolumn{2}{c}{ Apr-26 } \\
\multicolumn{2}{c}{4.4} \\
0.5 & 13.0 \\
12.9 & 11.4 \\
556 & 570 \\
8.2 & 7.9 \\
10.5 & 7.3 \\
0.015 & 0.017 \\
$<0.002$ & -- \\
0.755 & -- \\
0.024 & -- \\
0.63 & --- \\
1.39 & -- \\
15 & --- \\
3.1 & -- \\
243 & -- \\
48 & -- \\
30 & --- \\
18 & -- \\
1.4 & --- \\
206 & -- \\
24.9 & -- \\
40.8 & -- \\
5.2 & -- \\
332 & -- \\
$<10$ & -- \\
$<0.4$ & -- \\
&
\end{tabular}

\begin{tabular}{|c|c|}
\hline \multicolumn{2}{|c|}{$\begin{array}{l}2.1 \\
5.6\end{array}$} \\
\hline $\begin{array}{c}0.5 \\
22.2 \\
525 \\
8.1 \\
8.7 \\
0.013\end{array}$ & $\begin{array}{c}12.0 \\
14.5 \\
560 \\
7.6 \\
-- \\
0.023\end{array}$ \\
\hline-- & -- \\
\hline--- & --- \\
\hline--- & --- \\
\hline$\cdots$ & --- \\
\hline$\cdots$ & --- \\
\hline--- & $\cdots$ \\
\hline$\cdots$ & --- \\
\hline--- & --- \\
\hline--- & -- \\
\hline$-\infty$ & $-x$ \\
\hline--- & -- \\
\hline--- & -- \\
\hline--- & $\cdots$ \\
\hline$m$ & $-\cdots$ \\
\hline-- & --- \\
\hline$\ldots$ & --- \\
\hline$-\cdots$ & -- \\
\hline$-\cdots$ & --- \\
\hline
\end{tabular}

\begin{tabular}{|c|c|c|c|}
\hline & & & \\
\hline & & & \\
\hline $\begin{array}{c}0.5 \\
26.1 \\
543 \\
7.8 \\
8.1\end{array}$ & $\begin{array}{c}12.5 \\
14.0 \\
587 \\
7.4 \\
0.2\end{array}$ & $\begin{array}{c}0.5 \\
25.2 \\
530 \\
8.1 \\
8.7\end{array}$ & $\begin{array}{l}13.0 \\
13.8 \\
612 \\
7.2 \\
0.2 \\
0.32\end{array}$ \\
\hline $\begin{array}{l}0.011 \\
0.004\end{array}$ & $\begin{array}{c}0.028 \\
-\end{array}$ & $\begin{array}{l}0.015 \\
0.003\end{array}$ & $\begin{array}{c}0.032 \\
---\end{array}$ \\
\hline 0.281 & --- & 0.208 & -- \\
\hline 0.043 & --- & 0.035 & -- \\
\hline-- & -- & $\ldots$ & $\cdots$ \\
\hline$\ldots$ & --- & -- & -- \\
\hline-- & -- & $\cdots$ & --- \\
\hline-- & $-\cdots$ & -- & --- \\
\hline$-\cdots$ & -- & $\cdots$ & $\cdots$ \\
\hline$\cdots$ & $\ldots-$ & $\ldots$ & -- \\
\hline$\ldots$ & $\cdots$ & $\cdots$ & -- \\
\hline$-\cdots$ & $-\cdots$ & -- & --- \\
\hline$\cdots$ & $-\infty$ & -- & $\cdots$ \\
\hline-- & --- & --- & $-\cdots$ \\
\hline--- & $\cdots$ & $\cdots$ & $\cdots$ \\
\hline--- & $-\cdots$ & -- & $\cdots$ \\
\hline$-\infty$ & --- & --- & $\cdots$ \\
\hline-- & -- & -- & -- \\
\hline$-\infty$ & -- & $-\infty$ & --- \\
\hline-- & --- & $--\infty$ & -- \\
\hline
\end{tabular}

2-14-01

4-26-01

6-20-01

7-18-01

8-15-01

DISSOLVED OXYGEN (D.O.), IN MILLIGRAMS PER LITER
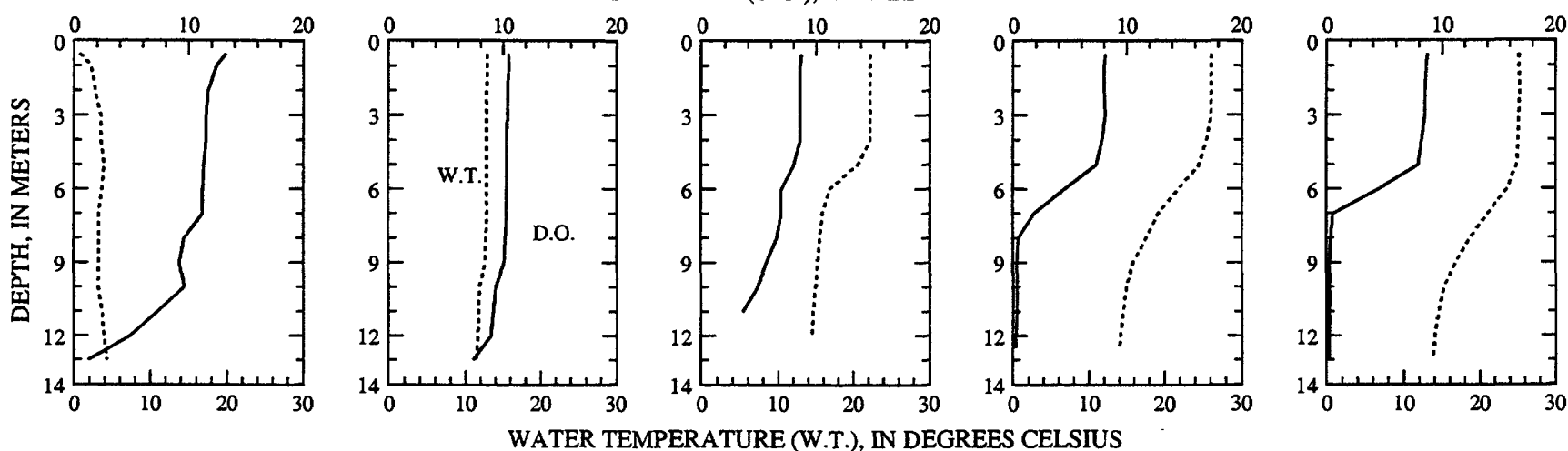

PH, IN STANDARD UNITS
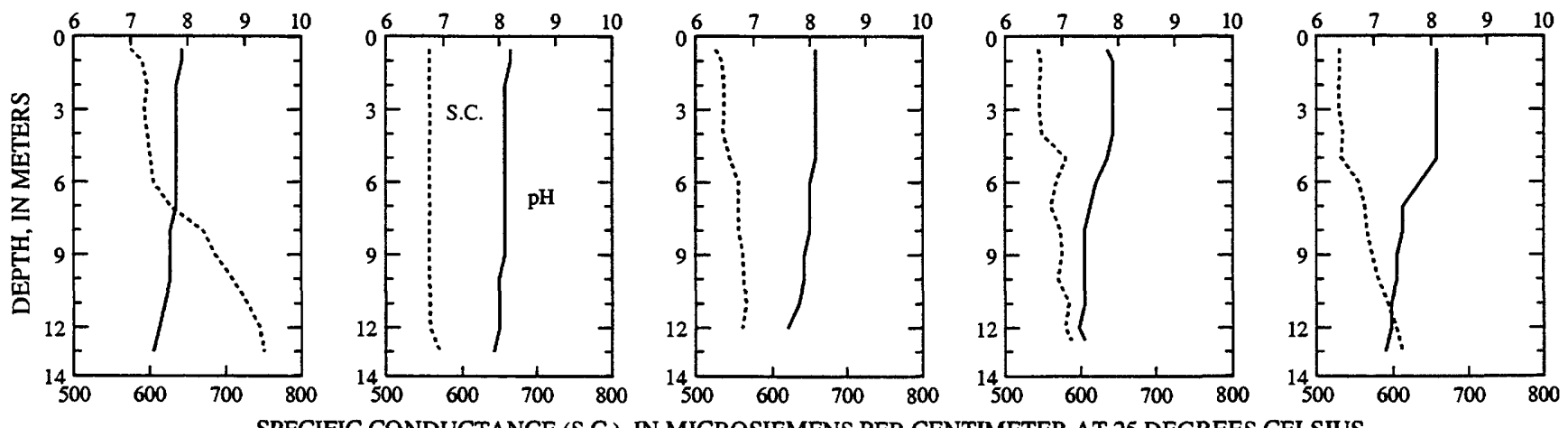

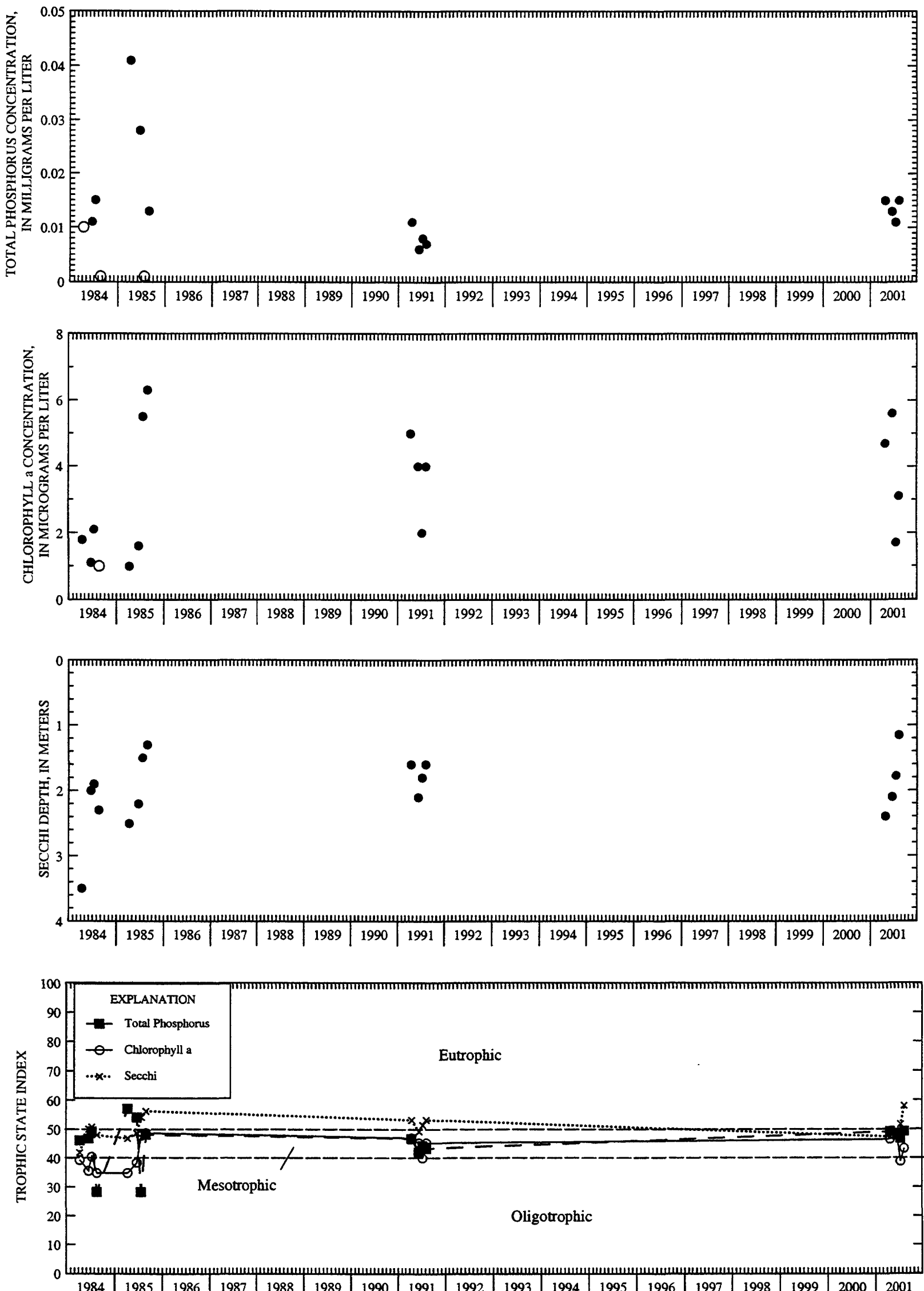

YEAR

Surface total phosphorus, chlorophyll a concentrations, Secchi depths, and TSI data for Lac La Belle, at Oconomowoc, Wisconsin.

Circles on the first three plots indicate laboratory detection limit for selected analysis. Actual concentrations for these particular analyses are less than the plotted circles. 


\section{MIDDE LAKE AT LAUDERDALE, WI}

LOCATION.--Lat $42^{\circ} 46^{\prime} 21^{\prime \prime}$, long 88 $33^{\prime} 55^{\prime \prime}$, in SE 1/4 SE 1/4 sec.26, T.4 N., R.16 E., Walworth County, Hydrologic Unit 07120006, at Lauderdale. PERIOD OF RECORD.--November 1993 to November 1994, February 1999 to August 2000, and February to August 2001.

REMARKS.--Lake sampled near east end of lake at a depth of about $52 \mathrm{ft}$. Lake ice-covered during February sampling. Water-quality analyses done by Wisconsin State Laboratory of Hygiene.

WATER-QUALITY DATA, FEBRUARY 13 TO AUGUST 20, 2001

(Milligrams per liter unless otherwise indicated)

Secchi-depth (m)

Chlorophyll a, phytoplankton ( $\mu \mathrm{g} / \mathrm{L}$ )

Depth of sample (m)

Water temoerature $\left({ }^{\circ} \mathrm{C}\right)$

Specific conductance ( $(\mu \mathrm{S} / \mathrm{cm})$

pH (units)

Dissolved oxygen (mg/L)

Phosphorus, total (as P)

Phosphorus, ortho, dissolved (as P)

Nitrogen, $\mathrm{NO2}+\mathrm{NO3}$, diss. (as N)

Nitrogen, ammonia, dissolved (as N)

Nitrogen, amm. + org.., total (as N)

Color (Pt-Co. scale)

Turbidity (NTU)

Hardness, (as $\mathrm{CaCO}_{3}$ )

Calcium, dissolved ( $\mathrm{Ca}$ )

Magnesium, dissolved (Mg)

Sodium, dissolved (Na)

Potassium, dissolved (K)

Alkalinity, (as $\mathrm{CaCO}_{3}$ )

Sulfate, dissolved $\left(\mathrm{SO}_{4}\right)$

Chloride, dissolved (Cl)

Silica, dissolved $\left(\mathrm{SiO}_{2}\right)$

Solids. dissolved, at $180^{\circ} \mathrm{C}$

Iron, dissolved ( $\mathrm{Fe}$ ) $\mu \mathrm{g} / \mathrm{L}$

Manganese, dissolved $(\mathrm{Mn}) \mu \mathrm{g} / \mathrm{L}$

\begin{tabular}{|c|c|c|c|}
\hline \multicolumn{2}{|c|}{ Feb-13 } & \multicolumn{2}{|c|}{ Apr -10} \\
\hline \multicolumn{2}{|c|}{--} & \multicolumn{2}{|c|}{$\begin{array}{l}3.5 \\
1.3\end{array}$} \\
\hline $\begin{array}{l}0.5 \\
2.2 \\
487 \\
7.9 \\
8.9 \\
0.006\end{array}$ & $\begin{array}{c}14.0 \\
4.3 \\
660 \\
7.3 \\
1.2 \\
0.025\end{array}$ & $\begin{array}{c}0.5 \\
8.8 \\
557 \\
7.8 \\
11.0 \\
0.009\end{array}$ & $\begin{array}{c}14.0 \\
5.4 \\
635 \\
7.4 \\
7.2 \\
0.012\end{array}$ \\
\hline$\ldots$ & -- & $<0.002$ & --- \\
\hline-- & --- & 1.61 & -- \\
\hline$\cdots$ &.- & 0.25 & $\cdots$ \\
\hline-- & -- & 0.67 & $-\cdots$ \\
\hline$-\cdots$ & --- & 2.28 & --- \\
\hline$\ldots$ & -- & 5 & --- \\
\hline-- & $\cdots$ & 1.7 & $-\cdots$ \\
\hline$\cdots$ & --- & 264 & $--\infty$ \\
\hline$-\cdots$ & --- & 53 & --- \\
\hline-- & --- & 32 & --- \\
\hline-- & --- & 7.5 & -- \\
\hline-- & --- & 1 & -- \\
\hline-- & -- & 214 & $-\cdots$ \\
\hline-- & --- & 30.7 & $-\infty$ \\
\hline-- & $-\infty$ & 21.2 & -- \\
\hline$-\infty$ & $--\infty$ & 8.0 & --- \\
\hline-- & $-\cdots$ & 304 & $\cdots$ \\
\hline-- & $-\cdots$ & $<10$ & -- \\
\hline$\cdots$ & $\cdots$ & 0.7 & -- \\
\hline
\end{tabular}

\begin{tabular}{|c|c|}
\hline \multicolumn{2}{|c|}{$2^{5.5}$} \\
\hline $\begin{array}{c}0.5 \\
23.8 \\
491 \\
8.0 \\
8.7 \\
0.011\end{array}$ & $\begin{array}{c}14.0 \\
8.9 \\
566 \\
7.4 \\
1.9 \\
0.018\end{array}$ \\
\hline--- & --- \\
\hline--- & $\cdots$ \\
\hline-- & --- \\
\hline$-\cdots$ & $-\cdots$ \\
\hline-- & --- \\
\hline--- & --- \\
\hline--- & --- \\
\hline$-\cdots$ & --- \\
\hline-- & -- \\
\hline-- & --- \\
\hline-- & --- \\
\hline- & --- \\
\hline- & -- \\
\hline--- & --- \\
\hline--- & -- \\
\hline- & --- \\
\hline- & - \\
\hline & \\
\hline
\end{tabular}

\begin{tabular}{|c|c|}
\hline \multicolumn{2}{|c|}{ Jul-17 } \\
\hline \multicolumn{2}{|c|}{$\begin{array}{c}1.6 \\
6\end{array}$} \\
\hline $\begin{array}{c}0.5 \\
27.7 \\
492 \\
7.9 \\
8.4 \\
0.017\end{array}$ & $\begin{array}{c}14.0 \\
9.2 \\
587 \\
7.3 \\
0.3 \\
0.041\end{array}$ \\
\hline--- & --- \\
\hline-- & -- \\
\hline$\cdots$ & -- \\
\hline--- & -- \\
\hline$\cdots$ & --- \\
\hline$-\cdots$ & --- \\
\hline$\cdots$ & -- \\
\hline$\cdots$ & $-\cdots$ \\
\hline$\cdots$ & -- \\
\hline--- & --- \\
\hline$\cdots$ & --- \\
\hline-- & -- \\
\hline-- & --- \\
\hline--- & --- \\
\hline-- & -- \\
\hline--- & --- \\
\hline$--\infty$ & --- \\
\hline--- & --- \\
\hline$-\cdots$ & --- \\
\hline
\end{tabular}

\begin{tabular}{|c|c|c|}
\hline \multicolumn{3}{|c|}{ Aug -20} \\
\hline & $\begin{array}{c}0.95 \\
6\end{array}$ & \\
\hline $\begin{array}{c}0.5 \\
23.2 \\
490 \\
7.8 \\
8.2 \\
0.016\end{array}$ & $\begin{array}{c}7.0 \\
18.6 \\
514 \\
7.2 \\
0.3 \\
0.019\end{array}$ & $\begin{array}{c}13.5 \\
9.7 \\
585 \\
7.2 \\
0.2 \\
0.036\end{array}$ \\
\hline-- & $\cdots$ & -- \\
\hline--- & --- & $\ldots$ \\
\hline$\ldots$ & --- & -- \\
\hline--- & -- & -- \\
\hline-- & $\cdots$ & -- \\
\hline--- & $-\cdots$ & $\cdots$ \\
\hline$-\cdots$ & --- & $-\cdots$ \\
\hline--- & --- & -- \\
\hline$-\infty$ & $\ldots$ & --- \\
\hline-- & $\cdots$ & -- \\
\hline-- & --- & $\cdots$ \\
\hline$\cdots$ & -- & -- \\
\hline$-\cdots$ & $-\cdots$ & --- \\
\hline$\cdots$ & $\cdots$ & --- \\
\hline$\cdots$ & $\cdots$ & -- \\
\hline--- & --- & --- \\
\hline--- & --- & $-\cdots$ \\
\hline--- & --- & -- \\
\hline--- & --- & --- \\
\hline
\end{tabular}

2-13-01

4-10-01

6-18-01

$7-17-01$

8-20-01

DISSOLVED OXYGEN (D.O.), IN MILLIGRAMS PER LITER
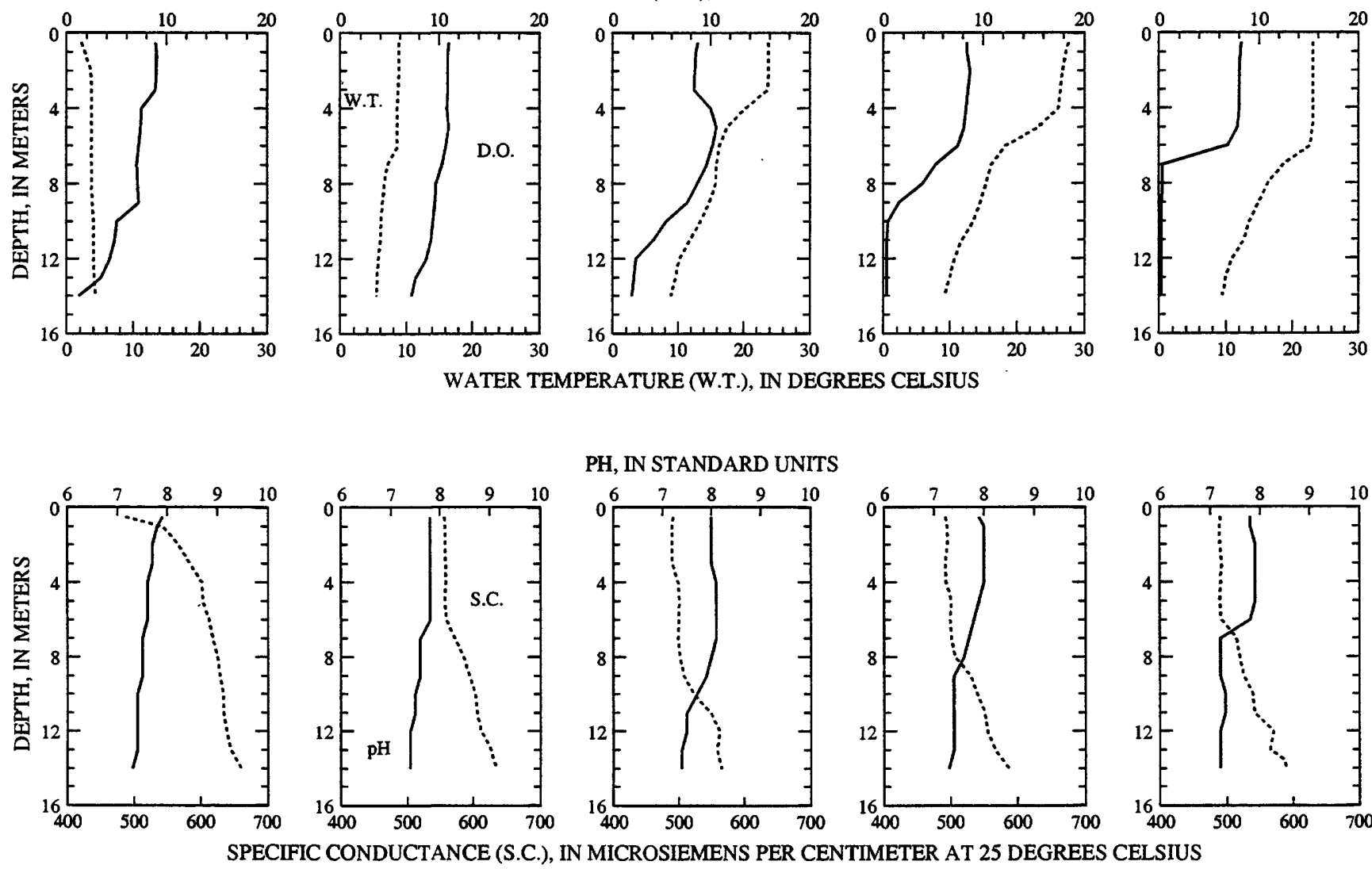

PH, IN STANDARD UNITS
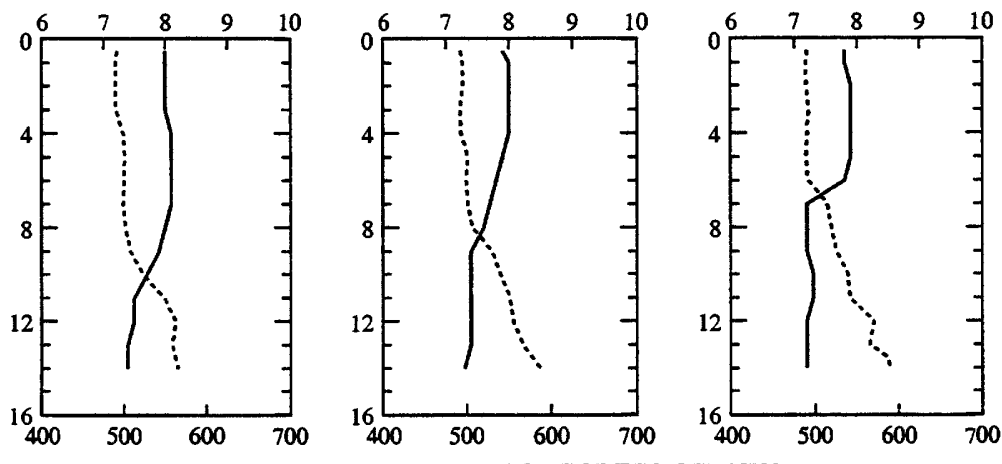

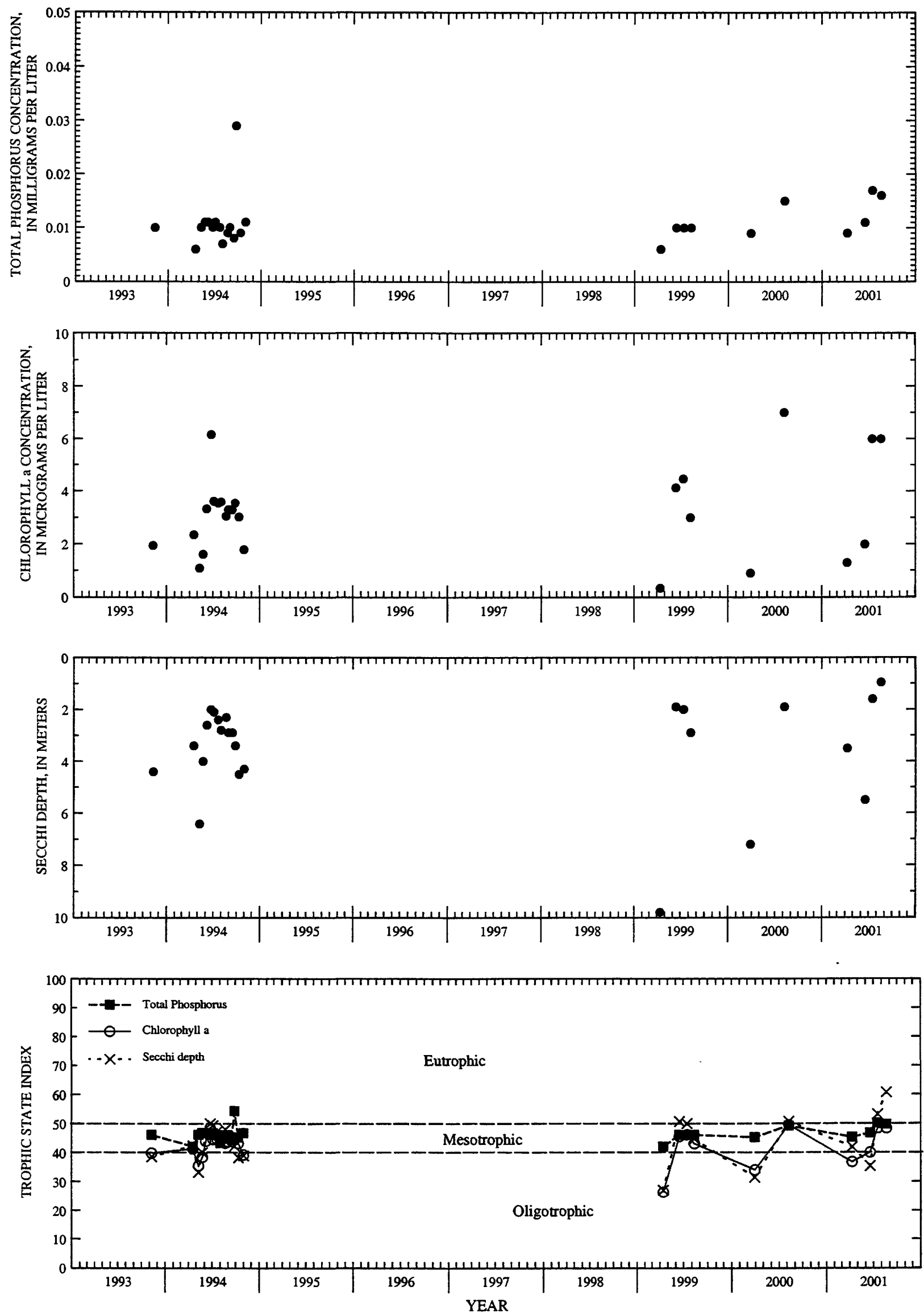

Surface total phosphorus, chlorophyll a concentrations, Secchi depths, and TSI data for Lauderdale Lake (Middle) near Lauderdale, Wisconsin. 
LOCATION.--Lat 45 $54^{\prime} 46^{\prime \prime}$ long $89^{\circ} 37^{\prime 0} 03^{\prime \prime}$, in SW 1/4 SE 1/4 sec.28, T.40 N., R.7 E., Vilas County, Hydrologic Unit 07070001, 4 mi northeast of Woodruff.

PERIOD OF RECORD.--February 1991 to current year.

GAGE.--Staff gage read by Glyn A. Roberts.

EXTREMES FOR PERIOD OF RECORD.--Maximum gage height observed, $8.76 \mathrm{ft}$, July 13, 2000; minimum observed, $7.63 \mathrm{ft}$, Aug. 23 , 2001.

EXTREMES FOR CURRENT YEAR.--Maximum gage height observed, $7.90 \mathrm{ft}$, July 31; minimum observed, $7.63 \mathrm{ft}$, Aug. 23.

GAGE HEIGHT, FEET, WATER YEAR OCTOBER 2000 TO SEPTEMBER 2001

DAILY MEAN VALUES

\begin{tabular}{|c|c|c|c|c|c|c|c|c|c|c|c|c|}
\hline DAY & OCT & Nov & DEC & JAN & FEB & MAR & APR & MAY & JUN & JUL & AUG & SEP \\
\hline$\frac{1}{2}$ & $\because--$ & 7.72 & --- & $\begin{array}{l}--- \\
---\end{array}$ & $=-$ & $\begin{array}{r}7.77 \\
-\end{array}$ & $\begin{array}{r}7.75 \\
-0\end{array}$ & $\begin{array}{l}--- \\
---\end{array}$ & $\begin{array}{r}7.77 \\
-\end{array}$ & $\begin{array}{l}--- \\
---\end{array}$ & 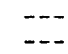 & 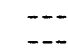 \\
\hline 3 & 7.76 & $\cdots$ & --- & - & --- & -.- & -- & -- & --- & 7.72 & -- & \\
\hline$\frac{4}{5}$ & $\ldots$ & $-\overline{-}$ & - & $\begin{array}{r}7.76 \\
\end{array}$ & $\overline{77}$ & 7.78 & -- & 782 & -- & --- & -84 & $\begin{array}{r}7.68 \\
\end{array}$ \\
\hline 6 & 7.74 & $-\ldots$ & -- & 7.76 & -.. & -.. & -- & $\ldots$ & $\ldots$ & --. & -.- & --- \\
\hline 7 & $-\cdots$ & -- & --- & $\cdots$ & -- & -- & 7.78 & -- & 7.76 & 7.70 & -- & --- \\
\hline 9 & -.. & -.. & - & $\cdots$ & -- & $\cdots$ & -- & 7.82 & -- & -.. & -.- & -- \\
\hline & --- & -- & 7.78 & --- & --- & 7.76 & $\ldots$ & --- & --- & -.. & -.. & 7.88 \\
\hline 11 & 7.75 & 7.74 & --- & -- & 7.76 & 7.76 & -- & -- & - & $-\overline{-}$ & 7.75 & -- \\
\hline 12 & 7.75 & --- & 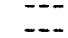 & 7.76 & $\overline{-}$ & $\overline{--}$ & 780 & 7.82 & $\begin{array}{r}7.76 \\
---\end{array}$ & $\begin{array}{r}7.74 \\
-\end{array}$ & 7.80 & -- \\
\hline 14 & 7.76 & -- & -- & -- & -- & -- & - & $\cdots$ & --- & -- & $\cdots$ & 7.76 \\
\hline & 7.76 & -- & $\cdots$ & -- & 7.76 & $-\cdots$ & -- & -- & - & $\cdots$ & $\cdots$ & $\cdots$ \\
\hline 16 & $\cdots$ & 7.78 & - & $\cdots$ & $\cdots$ & - & $\cdots$ & -- & $-\overline{-}$ & 7.80 & -- & --- \\
\hline & $\cdots$ & -- & 7.78 & -- & $-\overline{78}$ & 7.76 & --- & -- & 7.76 & $\cdots$ & $7---$ & -- \\
\hline $\begin{array}{l}10 \\
19\end{array}$ & $-\cdots$ & $\cdots$ & -- & 7.76 & 7.78 & $\cdots$ & - & $\ldots$ & -- & -.. & -.. & 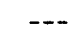 \\
\hline 20 & --- & --- & -- & & & -- & 7.84 & 7.78 & --- & 7.80 & -- & 7.78 \\
\hline 21 & $\cdots$ & 6 & --- & -- & --- & $=$ & $\cdots$ & $\cdots$ & -- & $-\cdot$ & -- & -- \\
\hline $\begin{array}{l}22 \\
23\end{array}$ & $\begin{array}{r}7.75 \\
---\end{array}$ & $\begin{array}{r}7.76 \\
--.\end{array}$ & 7.78 & --- & - & $\begin{array}{l}7.76 \\
-\ldots-\end{array}$ & -- & --- & 7.80 & -- & 7.63 & $\cdots$ \\
\hline $\begin{array}{l}24 \\
25\end{array}$ &.-- & $\begin{array}{l}--- \\
---\end{array}$ & $=-$ & $\overline{7.77}$ & $=-$ & $=-$ & -- & --- & --. & 7.82 & --- & 7.80 \\
\hline 26 & -.. & --- & --- & -- & 7.80 & $\ldots$ & 7.86 & 7.78 & -- & $\cdots$ & $\cdots$ & $\cdots$ \\
\hline 27 & $7-75$ & $7-\overline{7}$ & 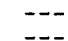 & 7 & $-\cdots$ & 7.78 & -- & $\ldots$ & --- & $=-$ & $=-$ & $\because$ \\
\hline 29 & - & - & 7.76 & - & -- & $\cdots$ & $\cdots$ & -- & 7.74 & -- & 7.65 & 7.75 \\
\hline $\begin{array}{l}30 \\
31\end{array}$ & $\cdots$ & -- & -- & 7.78 & $\cdots$ & 7.76 & $\begin{array}{l}7.82 \\
-.-1\end{array}$ & $\cdots$ & $\cdots$ & 7.90 & -- & -- \\
\hline
\end{tabular}




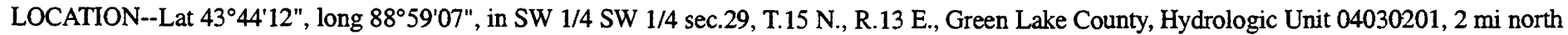
of Markesan.

PERIOD OF RECORD.--February 1991 to current year.

REMARKS.--Lake sampled near center at the deep hole. Lake ice-covered during February sampling. Water-quality analyses done by Wisconsin State Laboratory of Hygiene.

WATER-QUALITY DATA, FEBRUARY 15 TO AUGUST 22, 2001

(Milligrams per liter unless otherwise indicated)

\begin{tabular}{|c|c|c|c|c|c|c|c|c|c|c|c|c|c|}
\hline & \multicolumn{2}{|c|}{ Feb-15 } & \multicolumn{2}{|c|}{ Apr -25} & \multicolumn{2}{|c|}{ Jun-21 } & \multicolumn{4}{|c|}{ Ju1-24 } & \multicolumn{3}{|c|}{ Aug - 22} \\
\hline $\begin{array}{l}\text { Lake stage (ft) } \\
\text { Secchi-depth (m) } \\
\text { Chlorophyll a, phytoplankton (ug, }\end{array}$ & \multicolumn{2}{|c|}{--} & \multicolumn{2}{|c|}{$\begin{array}{l}96.84 \\
0.6 \\
67\end{array}$} & \multicolumn{2}{|c|}{$\begin{array}{l}97.63 \\
0.7 \\
19\end{array}$} & \multicolumn{4}{|c|}{$\begin{array}{l}97.01 \\
0.5 \\
23\end{array}$} & \multicolumn{3}{|c|}{$\begin{array}{l}96.01 \\
0.6 \\
66\end{array}$} \\
\hline Depth of sample (m) & 0.5 & 7.5 & 0.5 & 7.5 & 0.5 & 7.0 & 0.5 & 3.0 & 6.0 & 7.5 & 0.5 & 6.0 & 7.0 \\
\hline $\begin{array}{l}\text { Water temberature }\left({ }^{\circ} \mathrm{C}\right) \\
\text { Specific conductance (uS/cm) } \\
\text { pH (units) } \\
\text { Dissolved oxygen (mg/L) } \\
\text { Phosphorus, total (as P) }\end{array}$ & $\begin{array}{c}1.0 \\
391 \\
8.0 \\
11.3 \\
0.057\end{array}$ & $\begin{array}{c}4.5 \\
.398 \\
7.6 \\
2.8 \\
0.071\end{array}$ & $\begin{array}{l}12.0 \\
350 \\
8.1 \\
9.6 \\
0.105\end{array}$ & $\begin{array}{l}11.3 \\
356 \\
7.6 \\
5.4 \\
0.127\end{array}$ & $\begin{array}{c}21.7 \\
344 \\
8.2 \\
9.8 \\
0.070\end{array}$ & $\begin{array}{l}16.6 \\
375 \\
7.4 \\
0.3 \\
0.207\end{array}$ & $\begin{array}{l}28.5 \\
303 \\
8.4 \\
10.5 \\
0.069\end{array}$ & $\begin{array}{l}26.4 \\
319 \\
7.9 \\
3.0 \\
0.077\end{array}$ & $\begin{array}{l}20.1 \\
390 \\
7.1 \\
0.2 \\
0.649\end{array}$ & $\begin{array}{l}19.0 \\
400 \\
7.0 \\
0.2 \\
0.395\end{array}$ & $\begin{array}{l}22.6 \\
324 \\
7.7 \\
8.1 \\
0.126\end{array}$ & $\begin{array}{c}22.1 \\
332 \\
7.4 \\
3.5 \\
0.111\end{array}$ & $\begin{array}{c}21.5 \\
371 \\
7.0 \\
0.3 \\
0.393\end{array}$ \\
\hline Phosphorus, ortho, dissolved (as & -- & --- & 0.003 & --- & -- & $-\infty$ & -- & -- & -- & -- & 0.006 & -- & -- \\
\hline Nitrogen, $\mathrm{NO} 2$ + NO3, diss. (as N: & --- & --- & 0.017 & --- & -- & --- & -- & -- & --- & --- & 0.012 & --- & -- \\
\hline Nitrogen, ammonia, dissolved (as & --- & -- & 0.013 & --- & --- & -- & --- & --- & -- & --- & 0.023 & -- & --- \\
\hline Nitrogen, amm. + org., total (as & --- & --- & 1.7 & --- & --- & --- & $\cdots$ & -- & --- & --- & --- & --- & --- \\
\hline Nitrogen, total (as N) & -- & --- & 1.73 & --- & -- & --- & --- & --- & --- & $-\infty$ & $-\cdots$ & -- & --- \\
\hline Color (Pt-Co. scale) & $-\infty$ & $-\cdots$ & 15 & --- & -- & --- & --- & --- & --- & $-\infty$ & -- & --- & -- \\
\hline Turbidity (NTU) & --- & -- & 14.0 & -- & -- & --- & -- & -- & --- & --- & --- & --- & $-\infty$ \\
\hline Hardness, (as $\mathrm{CaCO}_{3}$ ) & --- & -- & 164 & --- & --- & --- & -- & --- & --- & -- & --- & --- & $-\cdots$ \\
\hline Calcium, dissolved (Ca) & -- & --- & 31 & --- & --- & --- & --- & --- & --- & --- & --- & --- & $-\infty$ \\
\hline Magnesium, dissolved (Mg) & -- & $-\cdots$ & 21 & --- & -- & --- & --- & -- & -- & -- & -- & -- & $-\cdots$ \\
\hline Sodium, dissolved (Na) & --- & -- & 8.1 & -- & --- & --- & --- & --- & --- & --- & --- & -- & -- \\
\hline Potassium, dissolved (K) & --- & --- & 3.4 & --- & -- & -- & --- & -- & -- & $-\infty$ & -- & -- & -- \\
\hline Alkalinity, (as $\mathrm{CaCO}_{3}$ ) & -- & --- & 147 & -- & --- & -- & -- & --- & --- & --- & --- & -- & --- \\
\hline Sulfate, dissolved $\left(\mathrm{SO}_{4}\right)$ & --- & -- & 5.6 & -- & --- & -- & $--\infty$ & --- & --- & --- & --- & $-\infty$ & --- \\
\hline Chloride, dissolved (C1) & -- & -- & 17.3 & --- & -- & -- & --- & -- & -- & $\ldots$ & $-\ldots$ & -- & $-\infty$ \\
\hline Silica, dissolved $\left(\mathrm{SiO}_{2}\right)$ & -- & --- & 0.0 & --- & -- & $--\infty$ & --- & --- & -- & -- & --- & $-\infty$ & -- \\
\hline $\begin{array}{l}\text { Solids dissolved, at } 180^{\circ} \mathrm{C} \\
\text { Iron, dissolved (Fe) } \mu \mathrm{g} / \mathrm{L}\end{array}$ & $m$ & --- & $\begin{array}{c}206 \\
10\end{array}$ & -- & --- & $-\cdots$ & --- & --- & -- & --- & --- & $m$ & $=-$ \\
\hline Manganese, dissolved (Mn) $\mu \mathrm{g} / \mathrm{L}$ & $-\infty$ & -- & $<0.4$ & -- & -- & $-\cdots$ & --- & -- & -- & -- & -- & -- & -- \\
\hline
\end{tabular}

2-15-01

4-25-01

6-21-01

7-24-01

8-22-01

DISSOLVED OXYGEN (D.O.), IN MILLIGRAMS PER LITER
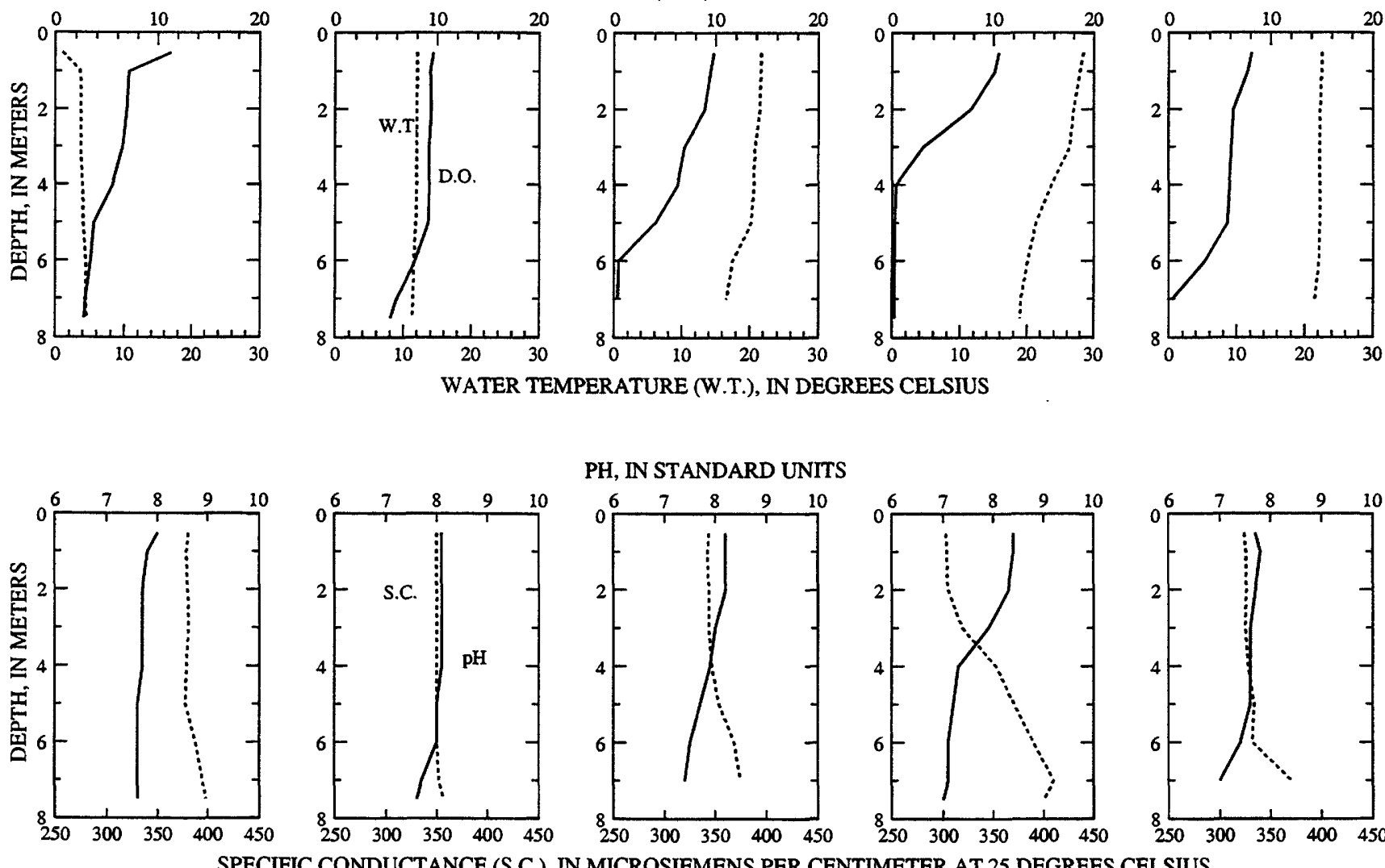

PH, IN STANDARD UNITS
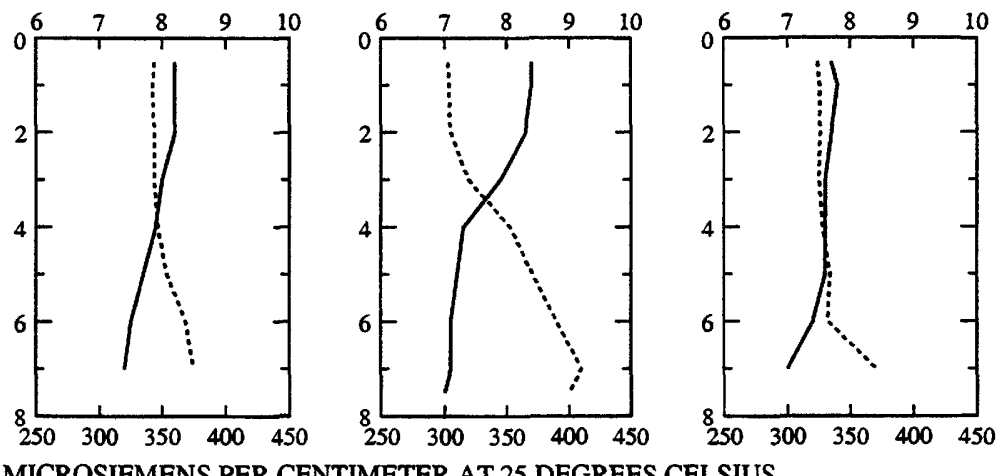

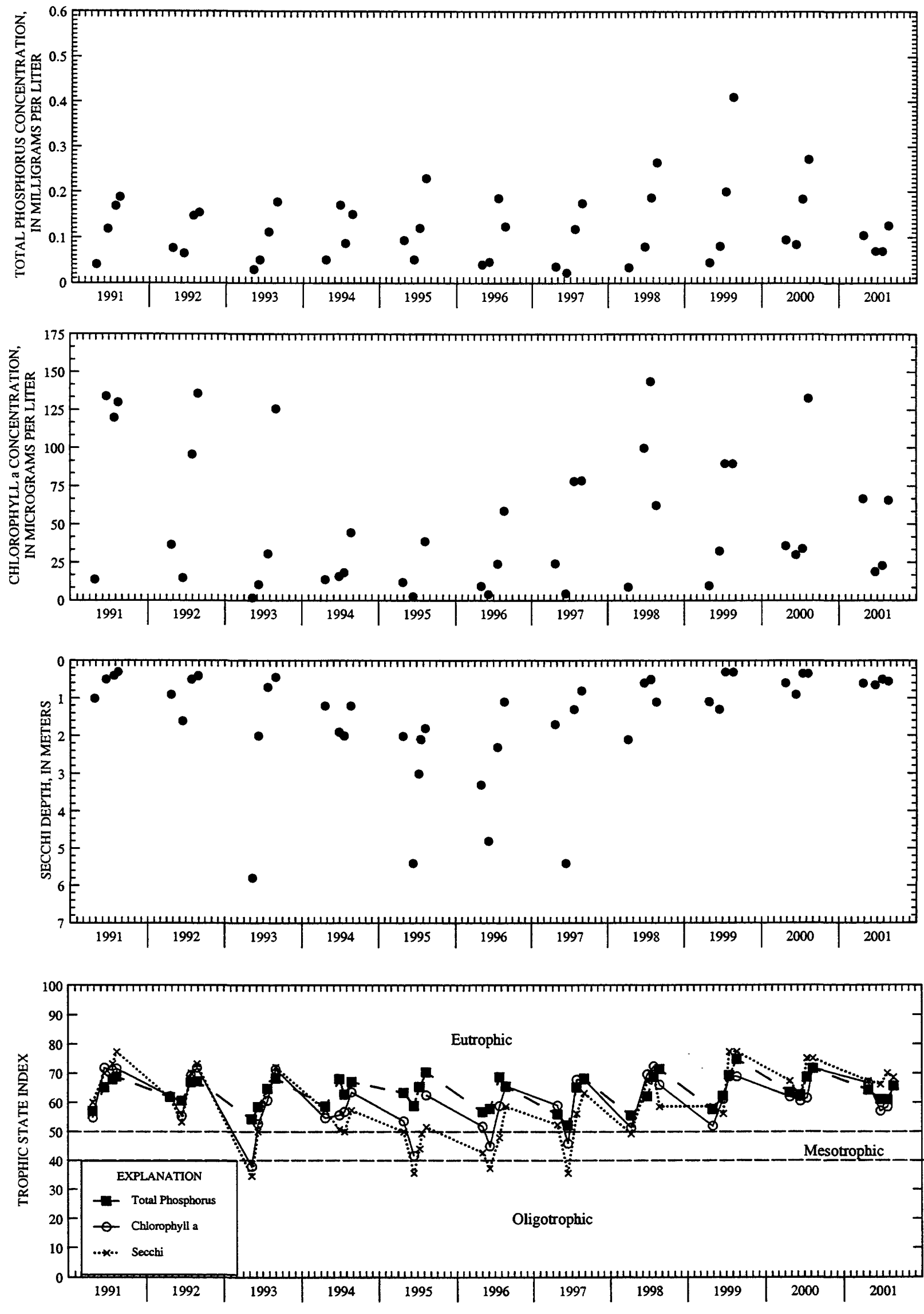

YEAR

Surface total phosphorus, chlorophyll a concentrations, Secchi depths, and TSI data for Little Green Lake, near Markensan, Wisconsin. 


\section{LITTLE MUSKEGO LAKE AT MUSKEGO, WI}

LOCATION.--Lat $42^{\circ} 54^{\prime} 25^{\prime \prime}$, long $88^{\circ} 08^{\prime} 35^{\prime \prime}$, in SE 1/4 NW 1/4 sec.9, T.5 N., R.20 E., Waukesha County, Hydrologic Unit 07120006, at Muskego.

DRAINAGE AREA.--11.6 $\mathrm{mi}^{2}$.

PERIOD OF RECORD.--October 1986 to current year.

LAKE-STAGE GAGE.--Datum of gage is $693.40 \mathrm{ft}$ above sea level.

REMARKS.--Lake sampled at the deep hole about 1,000 ft north-northwest of dam at outlet. Lake ice-covered during February sampling. An aeration system was operated from April to November for the years 1987-91. Water-quality analyses done by Wisconsin State Laboratory of Hygiene. Prior to October 1987, published under station number 425450088083500 .

WATER-QUALITY DATA, FEBRUARY 13 TO AUGUST 20, 2001 (Milligrams per liter unless otherwise indicated)

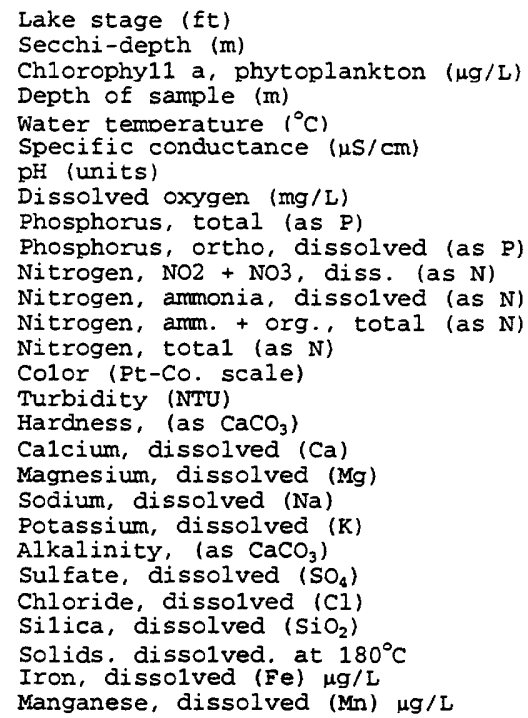

\begin{tabular}{|c|c|c|c|c|c|c|c|}
\hline \multicolumn{2}{|c|}{ Feb-13 } & \multicolumn{2}{|c|}{ Apr-18 } & \multicolumn{2}{|c|}{ Jun-19 } & \multicolumn{2}{|c|}{ Ju1-17 } \\
\hline \multicolumn{2}{|c|}{$\begin{array}{c}97.65 \\
--- \\
---\end{array}$} & \multicolumn{2}{|c|}{$\begin{array}{c}98.46 \\
1.3 \\
20\end{array}$} & \multicolumn{2}{|c|}{$\begin{array}{c}98.87 \\
4.8 \\
15\end{array}$} & \multicolumn{2}{|c|}{$\begin{array}{c}98.66 \\
4.3 \\
<1\end{array}$} \\
\hline $\begin{array}{c}0.5 \\
0.7 \\
696 \\
7.7 \\
13.6 \\
0.135\end{array}$ & $\begin{array}{c}19.0 \\
2.3 \\
1040 \\
7.6 \\
7.8 \\
0.075\end{array}$ & $\begin{array}{c}0.5 \\
8.6 \\
811 \\
8.0 \\
11.8 \\
0.023\end{array}$ & $\begin{array}{c}19.0 \\
3.0 \\
1040 \\
7.3 \\
3.9 \\
0.082\end{array}$ & $\begin{array}{c}0.5 \\
22.8 \\
738 \\
8.3 \\
10.4 \\
0.023\end{array}$ & $\begin{array}{c}20.0 \\
5.9 \\
915 \\
7.4 \\
0.3 \\
0.174\end{array}$ & $\begin{array}{c}0.5 \\
26.2 \\
724 \\
8.2 \\
8.7 \\
0.012\end{array}$ & $\begin{array}{c}20.0 \\
6.3 \\
924 \\
7.3 \\
0.3 \\
0.244\end{array}$ \\
\hline--- & -- & $<0.002$ & $\ldots$ & -- & $\ldots$ & -- & -- \\
\hline$-\cdots$ & -- & 0.403 & -- & --- & --- & -- & -- \\
\hline-- & $\cdots$ & 0.032 & -- & -- & --- & --- & -- \\
\hline--- & --- & 0.66 & $\ldots$ & -- & -- & $-\infty$ & -- \\
\hline$\ldots$ & -- & 1.06 & $\ldots$ & --- & --- & $-\infty$ & --- \\
\hline--- & --- & 20 & -- & --- & --- & -- & -- \\
\hline-- & -- & 4.7 & -- & $-\cdots$ & -- & -- & --- \\
\hline-- & --- & 261 & --- & --- & --- & -- & --- \\
\hline$\ldots$ & --- & 55 & -- & --- & -- & --- & --- \\
\hline-- & --- & 30 & -- & -- & --- & --- & --- \\
\hline--- & --- & 61 & --- & $\cdots$ & -- & -- & --- \\
\hline--- & --- & 2.4 & --- & --- & -- & -- & --- \\
\hline-- & -- & 193 & --- & --- & -- & - - & -- \\
\hline-- & $-\cdots$ & 36.9 & -- & --- & $-\infty$ & -- & --- \\
\hline$\cdots$ & $\cdots$ & 120 & --- & --- & -- & $-\infty$ & $\cdots$ \\
\hline$-\cdots$ & --- & 3.4 & --- & -- & --- & -- & --- \\
\hline--- & --- & 458 & --- & --- & $--\infty$ & -- & --- \\
\hline$-\infty$ & --- & $<10$ & --- & -- & --- & -- & --- \\
\hline-- & --- & $<0.4$ & --- & -- & --- & $-\cdots$ & --- \\
\hline
\end{tabular}

\begin{tabular}{|c|c|c|c|c|}
\hline & & Aug - 20 & & \\
\hline & & $\begin{array}{c}93.97 \\
3.9 \\
2.2\end{array}$ & & \\
\hline $\begin{array}{c}0.5 \\
23.4 \\
715 \\
8.3 \\
8.9 \\
0.016\end{array}$ & $\begin{array}{c}6.0 \\
22.2 \\
714 \\
8.3 \\
7.9 \\
0.017\end{array}$ & $\begin{array}{c}8.0 \\
15.6 \\
796 \\
7.5 \\
1.6 \\
0.018\end{array}$ & $\begin{array}{c}19.0 \\
6.9 \\
918 \\
7.3 \\
0.2 \\
0.254\end{array}$ & $\begin{array}{c}20.0 \\
6.8 \\
920 \\
7.3 \\
0.2 \\
0.282\end{array}$ \\
\hline--- & --- & $\ldots$ & --- & $\ldots$ \\
\hline--- & -- & -- & --- & $\cdots$ \\
\hline--- & --- & --- & --- & $\cdots$ \\
\hline--- & --- & --- & --- & -- \\
\hline--- & -- & --- & -- & -- \\
\hline--- & --- & --- & --- & --- \\
\hline-- & -- & -- & $-\cdots$ & -- \\
\hline--- & $\ldots$ & --- & --- & -- \\
\hline--- & --- & $-\infty$ & --- & -- \\
\hline-- & --- & $-\infty$ & $-\cdots$ & -- \\
\hline--- & --- & --- & -- & -- \\
\hline--- & --- & --- & --- & --- \\
\hline--- & -- & -- & -- & -- \\
\hline--- & -- & --- & --- & --- \\
\hline$\cdots$ & --- & $\cdots$ & -- & -- \\
\hline$-\infty-$ & $--\infty$ & --- & -- & --- \\
\hline--- & -- & --- & --- & --- \\
\hline$\cdots$ & $\cdots$ & --- & -- & -- \\
\hline-- & $-\cdots$ & & --- & \\
\hline
\end{tabular}

DISSOLVED OXYGEN (D.O.), IN MILLIGRAMS PER LITER
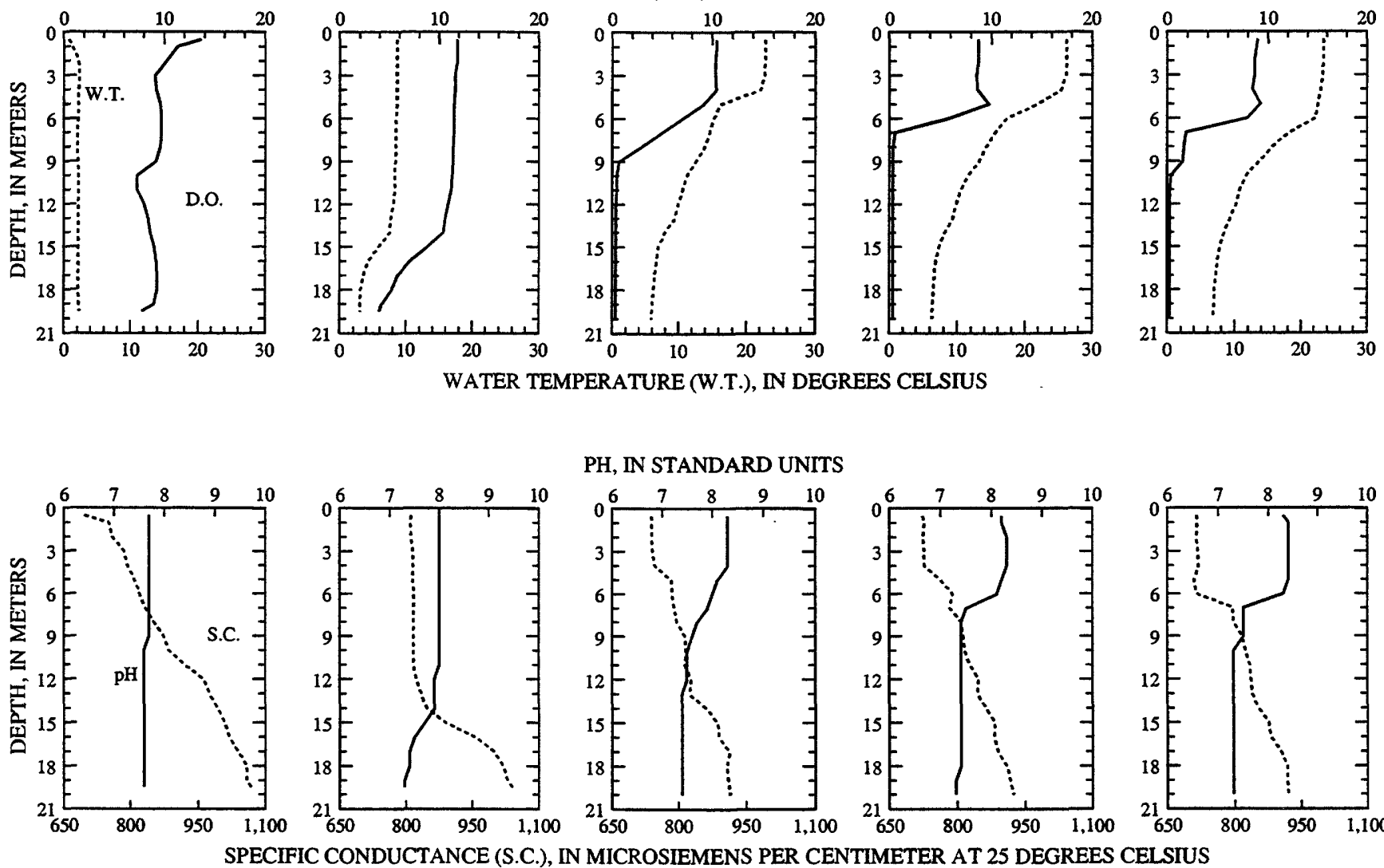

PH, IN STANDARD UNITS
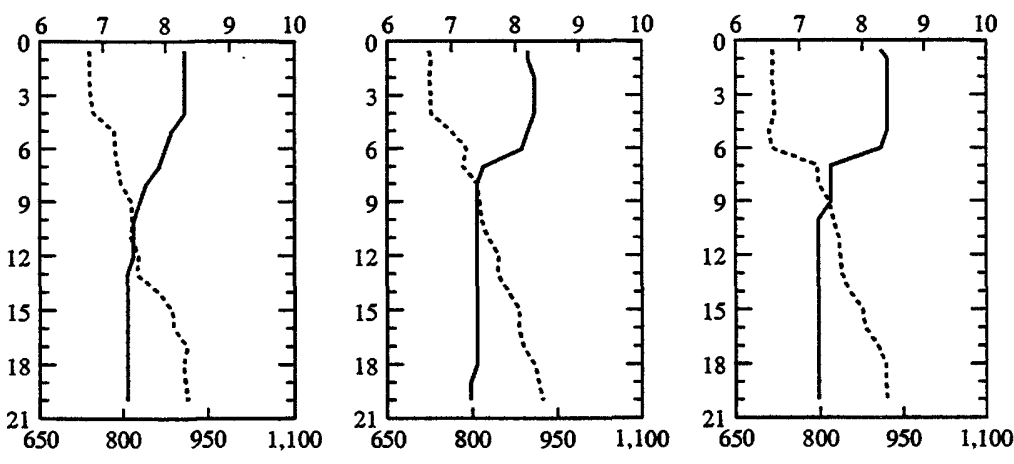

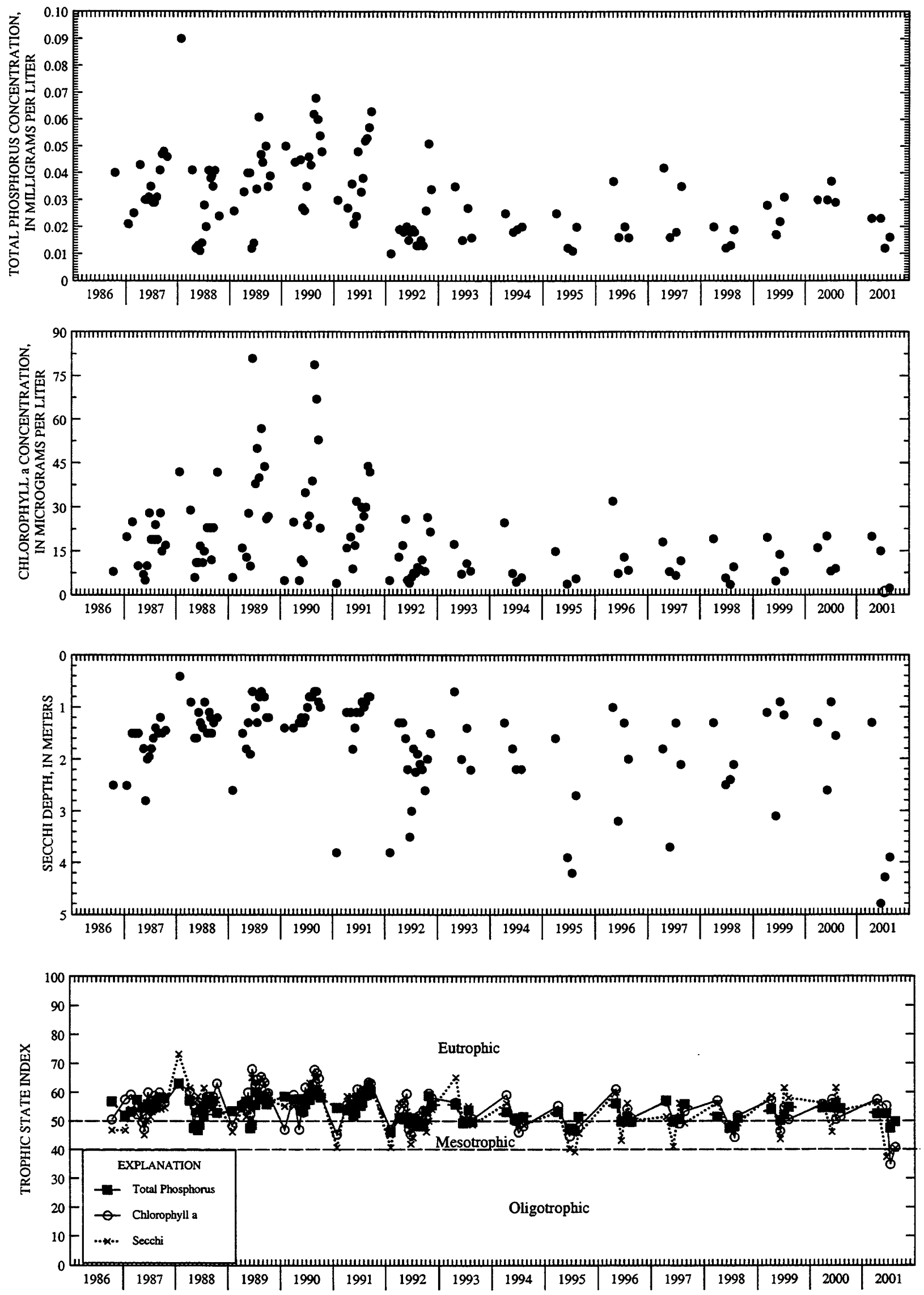

YEAR

Surface total phosphorus, chlorophyll a concentrations, Secchi depths, and TSI data for Little Muskego Lake, at Muskego, Wisconsin.

(Circles on the first three plots indicate laboratory detection limit for selected analyses. Actual concentrations for thse particular analyses are less than the plotted circles.) 
LOCATION--Lat 45 53'55", long 89²7'10", in SW 1/4 SE 1/4 sec.35, T.40 N., R.8 E., Vilas County, Hydrologic Unit 07070001 , 9.6 mi west of Eagle River.

DRAINAGE AREA.--19.0 $\mathrm{mi}^{2}$.

PERIOD OF RECORD.--October 1990 to current year.

GAGE.--Staff gage mounted on the dam wall at lake outlet. Datum of gage is $1,600 \mathrm{ft}$, above sea level.

REMARKS.--Lake level regulated by dam at outlet.

COOPERATION.--Gage readings furnished by Wisconsin Valley Improvement Company.

EXTREMES FOR PERIOD OF RECORD.--Maximum gage height observed, $14.00 \mathrm{ft}$, June 6, 1997; minimum observed, $12.00 \mathrm{ft}$, Jan. 3 and Feb. 3, 1992.

EXTREMES FOR CURRENT YEAR.--Maximum gage height observed, 13.86, June 19; minimum observed, $11.90 \mathrm{ft}$, Jan. 7. GAGE HEIGHT, FEET, WATER YEAR OCTOBER 2000 TO SEPTEMBER 2001

DAILY MEAN VALUE

\begin{tabular}{|c|c|c|c|c|c|c|c|c|c|c|c|c|}
\hline DAY & OCT & NOV & DEC & JAN & $F E B$ & MAR & $A P R$ & MAY & UNN & JUL & AUG & SEP \\
\hline $\begin{array}{l}1 \\
2 \\
3 \\
4 \\
5\end{array}$ & $\begin{array}{l}13.50 \\
13.50 \\
13.50 \\
13.50 \\
13.48\end{array}$ & $\begin{array}{r}--- \\
13.24 \\
13.24 \\
13.24\end{array}$ & $\begin{array}{l}13.00 \\
12.98 \\
12.96 \\
12.86 \\
12.84\end{array}$ & $\begin{array}{r}12.10 \\
12.00 \\
---\end{array}$ & $\begin{array}{r}12.18 \\
12.14 \\
-\end{array}$ & $\begin{array}{r}11.96 \\
11.96 \\
--- \\
12.16 \\
---\end{array}$ & $\begin{array}{l}12.42 \\
12.40 \\
12.44 \\
12.46 \\
12.46\end{array}$ & $\begin{array}{l}13.64 \\
13.68 \\
13.68 \\
13.70 \\
13.68\end{array}$ & $\begin{array}{l}13.78 \\
13.78 \\
13.76 \\
13.72 \\
13.70\end{array}$ & $\begin{array}{l}13.70 \\
13.66 \\
13.62 \\
13.60 \\
13.60\end{array}$ & $\begin{array}{l}13.52 \\
13.60 \\
13.60 \\
13.58 \\
13.58\end{array}$ & $\begin{array}{l}13.30 \\
13.28 \\
13.28 \\
13.24 \\
13.18\end{array}$ \\
\hline $\begin{array}{r}6 \\
7 \\
8 \\
9 \\
10\end{array}$ & $\begin{array}{r}13.48 \\
13.48 \\
13.48 \\
--- \\
13.42\end{array}$ & $\begin{array}{r}13.30 \\
--- \\
13.24\end{array}$ & $\begin{array}{r}--- \\
12.76 \\
12.72\end{array}$ & $\begin{array}{r}11.90 \\
12.16 \\
--1 .-\end{array}$ & $\begin{array}{r}12.10 \\
- \\
12.16 \\
-\end{array}$ & $\begin{array}{r}12.14 \\
\ldots- \\
12.14 \\
\ldots\end{array}$ & $\begin{array}{l}12.50 \\
12.54 \\
12.62 \\
12.64 \\
12.66\end{array}$ & $\begin{array}{l}13.66 \\
13.68 \\
13.68 \\
13.68 \\
13.74\end{array}$ & $\begin{array}{l}13.70 \\
13.70 \\
13.70 \\
13.70 \\
13.70\end{array}$ & $\begin{array}{l}13 \cdot 60 \\
13.58 \\
13.58 \\
13.58 \\
13.56\end{array}$ & $\begin{array}{l}13.58 \\
13.58 \\
13.58 \\
13.56 \\
13.56\end{array}$ & $\begin{array}{l}13.18 \\
13.36 \\
13.40 \\
13.42 \\
13.42\end{array}$ \\
\hline $\begin{array}{l}11 \\
12 \\
13 \\
14 \\
15\end{array}$ & $\begin{array}{r}--- \\
13.38 \\
13.36\end{array}$ & $\begin{array}{r}13.20 \\
--- \\
13.28 \\
-\end{array}$ & $\begin{array}{r}12.72 \\
--- \\
12.56\end{array}$ & $\begin{array}{r}12.20 \\
12.26 \\
----\end{array}$ & $\begin{array}{r}12.16 \\
-- \\
12.12 \\
=- \\
=-\end{array}$ & $\begin{array}{r}12.12 \\
-- \\
12.18 \\
\\
=-\end{array}$ & $\begin{array}{l}12.70 \\
12.80 \\
12.88 \\
12.92 \\
12.96\end{array}$ & $\begin{array}{l}13.74 \\
13.74 \\
13.74 \\
13.76 \\
13.76\end{array}$ & $\begin{array}{l}13.76 \\
13.82 \\
13.78 \\
13.74 \\
13.74\end{array}$ & $\begin{array}{l}13.54 \\
13.52 \\
13.52 \\
13.48 \\
13.48\end{array}$ & $\begin{array}{l}13.52 \\
13.48 \\
13.48 \\
13.46 \\
13.42\end{array}$ & $\begin{array}{l}13.38 \\
13.38 \\
13.36 \\
13.36 \\
13.36\end{array}$ \\
\hline $\begin{array}{l}16 \\
17 \\
18 \\
19 \\
20\end{array}$ & $\begin{array}{r}13.32 \\
---- \\
13.26 \\
13.28\end{array}$ & $\begin{array}{r}13.24 \\
13.22 \\
13.22 \\
13.20 \\
-\end{array}$ & $\begin{array}{r}12.54 \\
12.50 \\
---\end{array}$ & $\begin{array}{r}12.24 \\
12.22 \\
--- \\
12.16 \\
---\end{array}$ & $\begin{array}{r}12.08 \\
-.- \\
12.06\end{array}$ & $\begin{array}{r}12.26 \\
12.28 \\
12.32\end{array}$ & $\begin{array}{l}13.00 \\
13.04 \\
13.04 \\
13.06 \\
13.08\end{array}$ & $\begin{array}{l}13.76 \\
13.76 \\
13.76 \\
13.76 \\
13.74\end{array}$ & $\begin{array}{l}13.70 \\
13.70 \\
13.72 \\
13.86 \\
13.80\end{array}$ & $\begin{array}{l}13.50 \\
13.48 \\
13.48 \\
13.48 \\
13.54\end{array}$ & $\begin{array}{l}13.44 \\
13.44 \\
13.42 \\
13.42 \\
13.40\end{array}$ & $\begin{array}{l}13 \cdot 36 \\
13 \cdot 34 \\
13 \cdot 34 \\
13 \cdot 34 \\
13 \cdot 34\end{array}$ \\
\hline $\begin{array}{l}21 \\
22 \\
23 \\
24 \\
25\end{array}$ & $\begin{array}{r}13.26 \\
--- \\
13.28 \\
---\end{array}$ & $\begin{array}{r}13.18 \\
--- \\
13.14 \\
13.12\end{array}$ & $\begin{array}{r}12.31 \\
12.36 \\
----\end{array}$ & $\begin{array}{r}12.14 \\
12.14 \\
- \\
-\end{array}$ & 12.04 & $\begin{array}{l}12.34 \\
12.34 \\
12.36 \\
12.36\end{array}$ & $\begin{array}{l}13.12 \\
13.22 \\
13.30 \\
13.44 \\
13.48\end{array}$ & $\begin{array}{l}13.76 \\
13.78 \\
13.78 \\
13.84 \\
13.82\end{array}$ & $\begin{array}{l}13.78 \\
13.76 \\
13.74 \\
13.72 \\
13.72\end{array}$ & $\begin{array}{l}13.58 \\
13.56 \\
13.56 \\
13.56 \\
13.54\end{array}$ & $\begin{array}{l}13.38 \\
13.36 \\
13.36 \\
13.36 \\
13.34\end{array}$ & $\begin{array}{l}13.32 \\
13.32 \\
13.40 \\
13.40 \\
13.38\end{array}$ \\
\hline $\begin{array}{l}26 \\
27 \\
28 \\
29 \\
30 \\
31\end{array}$ & $\begin{array}{r}--- \\
--- \\
13.24 \\
13.22\end{array}$ & $\begin{array}{r}13.12 \\
13.04 \\
- \\
- \\
-\end{array}$ & $\begin{array}{r}12.28 \\
- \\
12.22 \\
12.15\end{array}$ & $\begin{array}{r}12.12 \\
12.12 \\
--\overline{1} \\
12.16 \\
12.20\end{array}$ & 12.00 & $\begin{array}{l}12.38 \\
12.38 \\
12.38 \\
12.38 \\
12.38 \\
12.40\end{array}$ & $\begin{array}{c}13.50 \\
13.54 \\
13.56 \\
13.50 \\
13.56 \\
---\end{array}$ & $\begin{array}{l}13.84 \\
13.84 \\
13.82 \\
13.82 \\
13.80 \\
13.78\end{array}$ & $\begin{array}{r}13.70 \\
13.70 \\
13.72 \\
13.70 \\
13.70 \\
\ldots\end{array}$ & $\begin{array}{l}13.52 \\
13.52 \\
13.50 \\
13.51 \\
13.52 \\
13.52\end{array}$ & $\begin{array}{l}13.34 \\
13.32 \\
13.30 \\
13.30 \\
13.30 \\
13.30\end{array}$ & $\begin{array}{r}13.38 \\
13.36 \\
13.34 \\
13.32 \\
13.30 \\
-\end{array}$ \\
\hline $\begin{array}{l}\text { MEAN } \\
\text { MAX } \\
\text { MIN }\end{array}$ & $\begin{array}{l}--- \\
--- \\
---\end{array}$ & --- & $\begin{array}{l}--- \\
--- \\
---\end{array}$ & --- & -- & -- & $\begin{array}{l}12.96 \\
13.56 \\
12.40\end{array}$ & $\begin{array}{l}13.75 \\
13.84 \\
13.64\end{array}$ & $\begin{array}{l}13.74 \\
13.86 \\
13.70\end{array}$ & $\begin{array}{l}13.55 \\
13.70 \\
13.48\end{array}$ & $\begin{array}{l}13.45 \\
13.60 \\
13.30\end{array}$ & $\begin{array}{l}13.34 \\
13.42 \\
13.18\end{array}$ \\
\hline
\end{tabular}

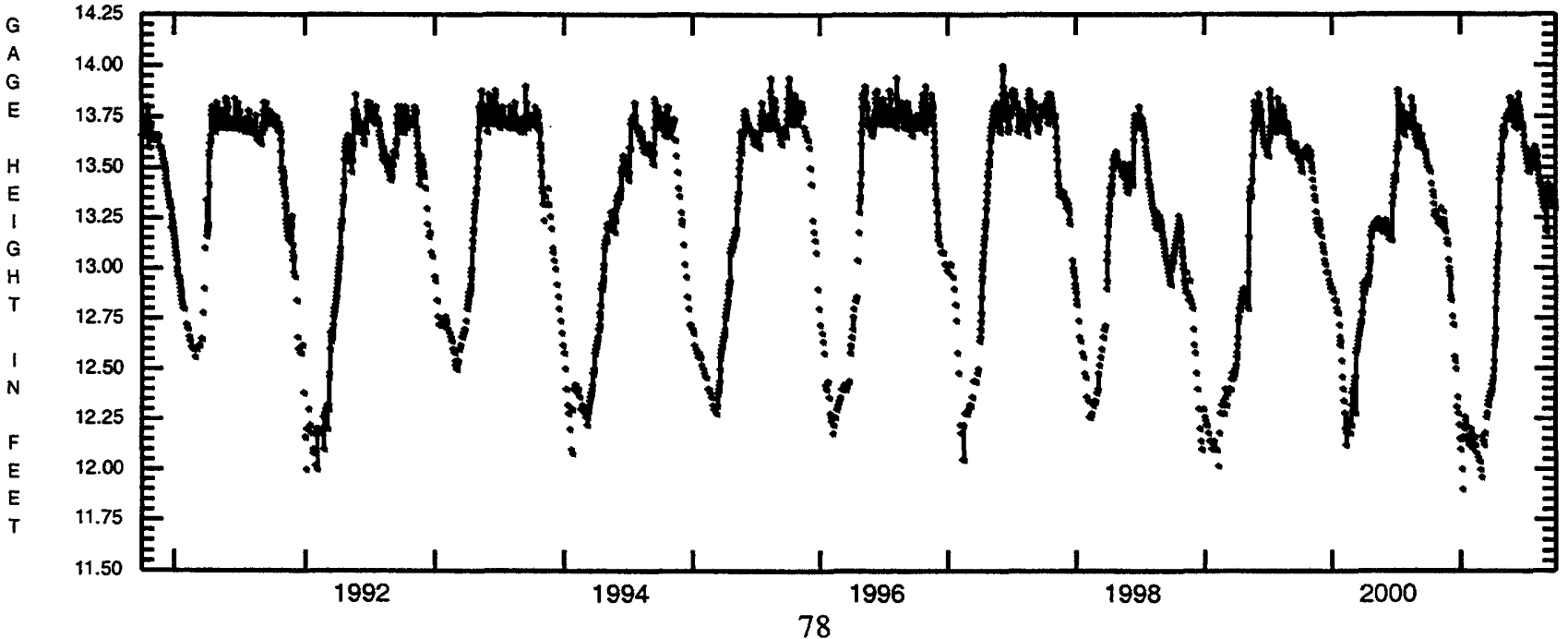




\section{LITTLE ST. GERMAIN LAKE, UPPER EAST BAY, AT ST. GERMAIN, WI}

LOCATION.--Lat 4555'32", long 89²5'39", in NE 1/4 NW 1/4 sec.25, T.40 N., R.8 E., Vilas County, Hydrologic Unit 07070001, near St. Germain.

PERIOD OF RECORD.--December 1996, January-March 1997, March 1999, and March 2000 to current year.

REMARKS.--Water-quality analyses by Wisconsin State Laboratory of Hygiene.

WATER-QUALITY DATA, JANUARY 17 TO MAY 01, 2001

(Milligrams per liter unless otherwise indicated)

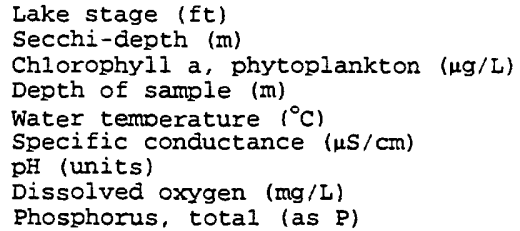

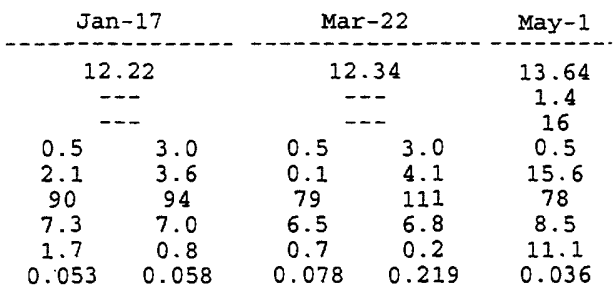

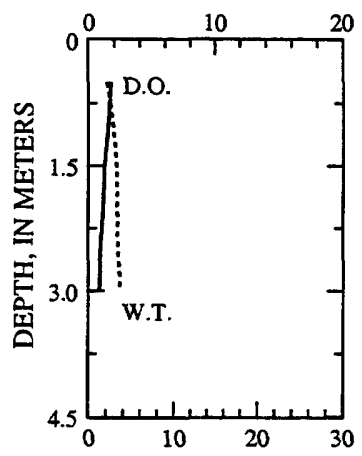

DISSOLVED OXYGEN (D.O.), IN MILLIGRAMS PER LITER
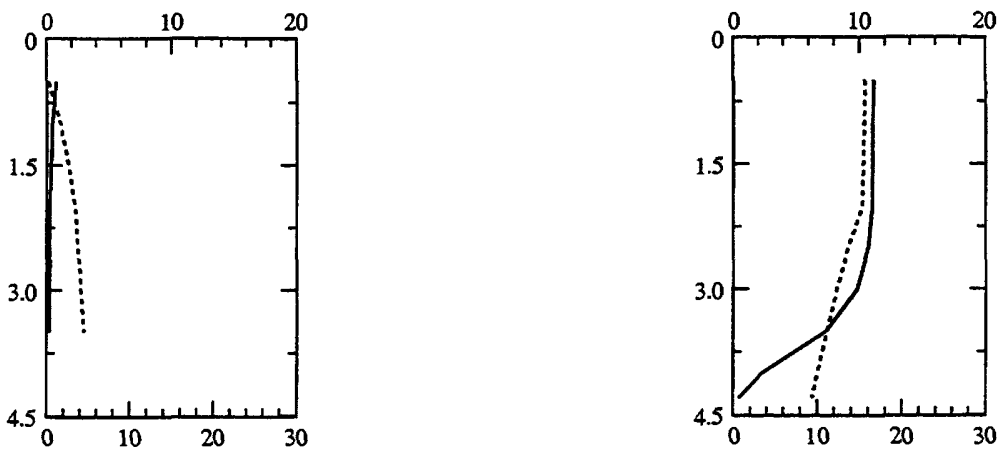

WATER TEMPERATURE (W.T.), IN DEGREES CELSIUS

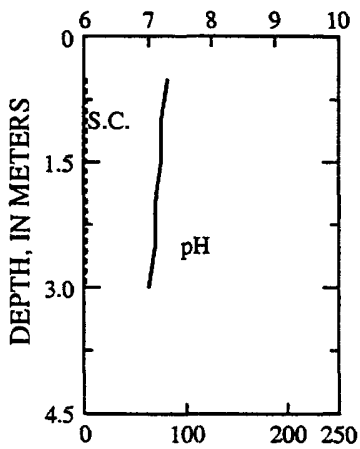

PH, IN STANDARD UNITS
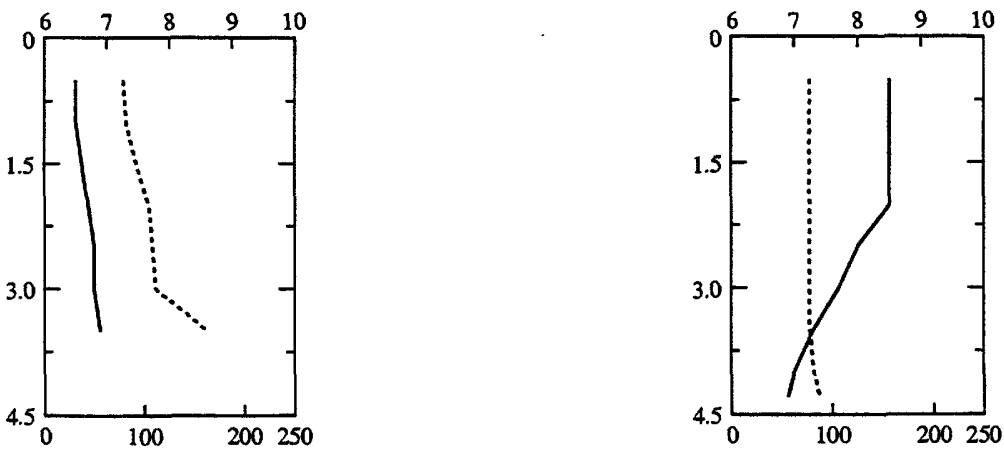

SPECIFIC CONDUCTANCE (S.C.), IN MICROSIEMENS PER CENTIMETER AT 25 DEGREES CELSIUS 
WATER-QUALITY DATA, JUNE 26 TO AUGUST 28, 2001

(Milligrams per liter unless otherwise indicated)

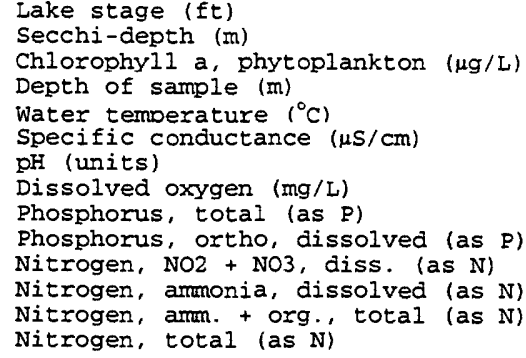

\begin{tabular}{|c|c|}
\hline \multicolumn{2}{|c|}{ Jun-26 } \\
\hline \multicolumn{2}{|c|}{$\begin{array}{c}13.70 \\
1.5 \\
12\end{array}$} \\
\hline $\begin{array}{c}0.5 \\
24.4 \\
76 \\
8.5 \\
10.6 \\
0.030\end{array}$ & $\begin{array}{c}4.0 \\
15.3 \\
82 \\
6.7 \\
1.3 \\
0.061\end{array}$ \\
\hline$\cdots$ & -- \\
\hline$\cdots$ & $\cdots$ \\
\hline-- & $m$ \\
\hline--- & --- \\
\hline
\end{tabular}

\begin{tabular}{|c|c|c|c|}
\hline \multicolumn{2}{|c|}{ Jul-26 } & \multicolumn{2}{|c|}{ Aug -28 } \\
\hline \multicolumn{2}{|c|}{$\begin{array}{c}13.52 \\
1.0 \\
26\end{array}$} & \multicolumn{2}{|c|}{$\begin{array}{c}13.30 \\
0.9 \\
34\end{array}$} \\
\hline $\begin{array}{c}0.5 \\
24.2 \\
82 \\
8.6 \\
8.6\end{array}$ & $\begin{array}{c}4.0 \\
17.4 \\
117 \\
6.4 \\
0.2\end{array}$ & $\begin{array}{c}0.5 \\
21.8 \\
86 \\
8.4 \\
9.8 \\
0.061\end{array}$ & $\begin{array}{l}3.0 \\
19.5 \\
103 \\
6.7 \\
0.4 \\
0.076\end{array}$ \\
\hline $\begin{array}{l}0.049 \\
0.004\end{array}$ & $\begin{array}{c}0.154 \\
\ldots-\end{array}$ & 0.061 & $\begin{array}{c}0.076 \\
---\end{array}$ \\
\hline 0.015 & --- & -- & $\cdots$ \\
\hline 0.018 & -- & -- & -- \\
\hline 0.93 & -- & $-\cdots$ & $-\cdots$ \\
\hline 0.945 & - - & -- & --- \\
\hline
\end{tabular}

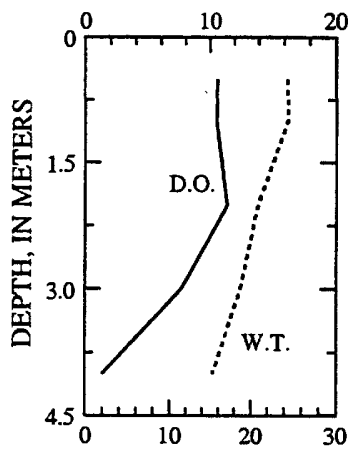

DISSOLVED OXYGEN (D.O.), IN MILLIGRAMS PER LITER
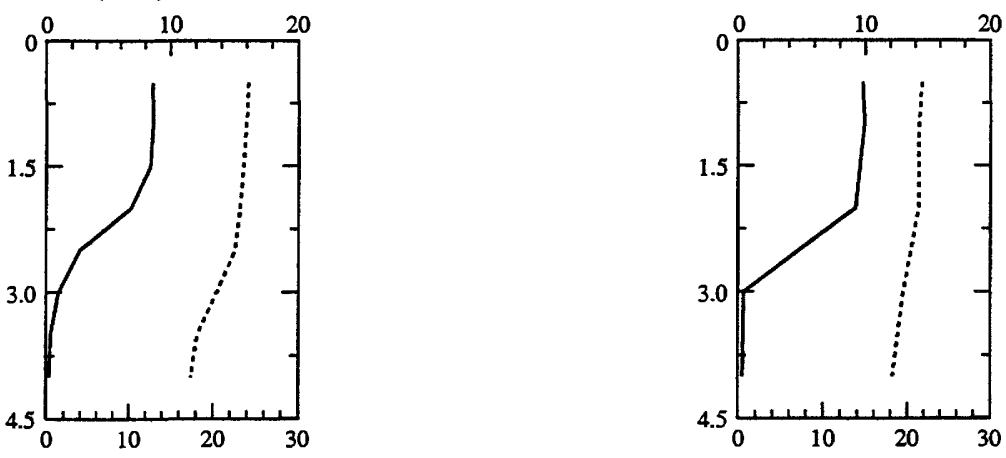

WATER TEMPERATURE (W.T.), IN DEGREES CELSIUS

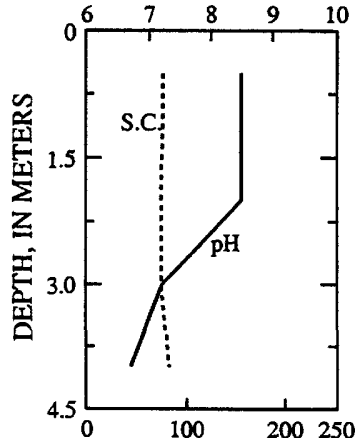

PH, IN STANDARD UNITS
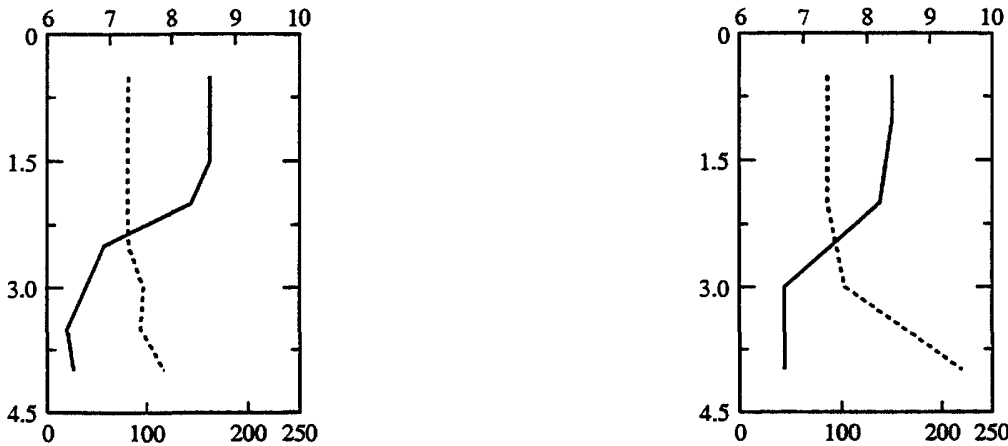

SPECIFIC CONDUCTANCE (S.C.), IN MICROSIEMENS PER CENTIMETER AT 25 DEGREES CELSIUS 

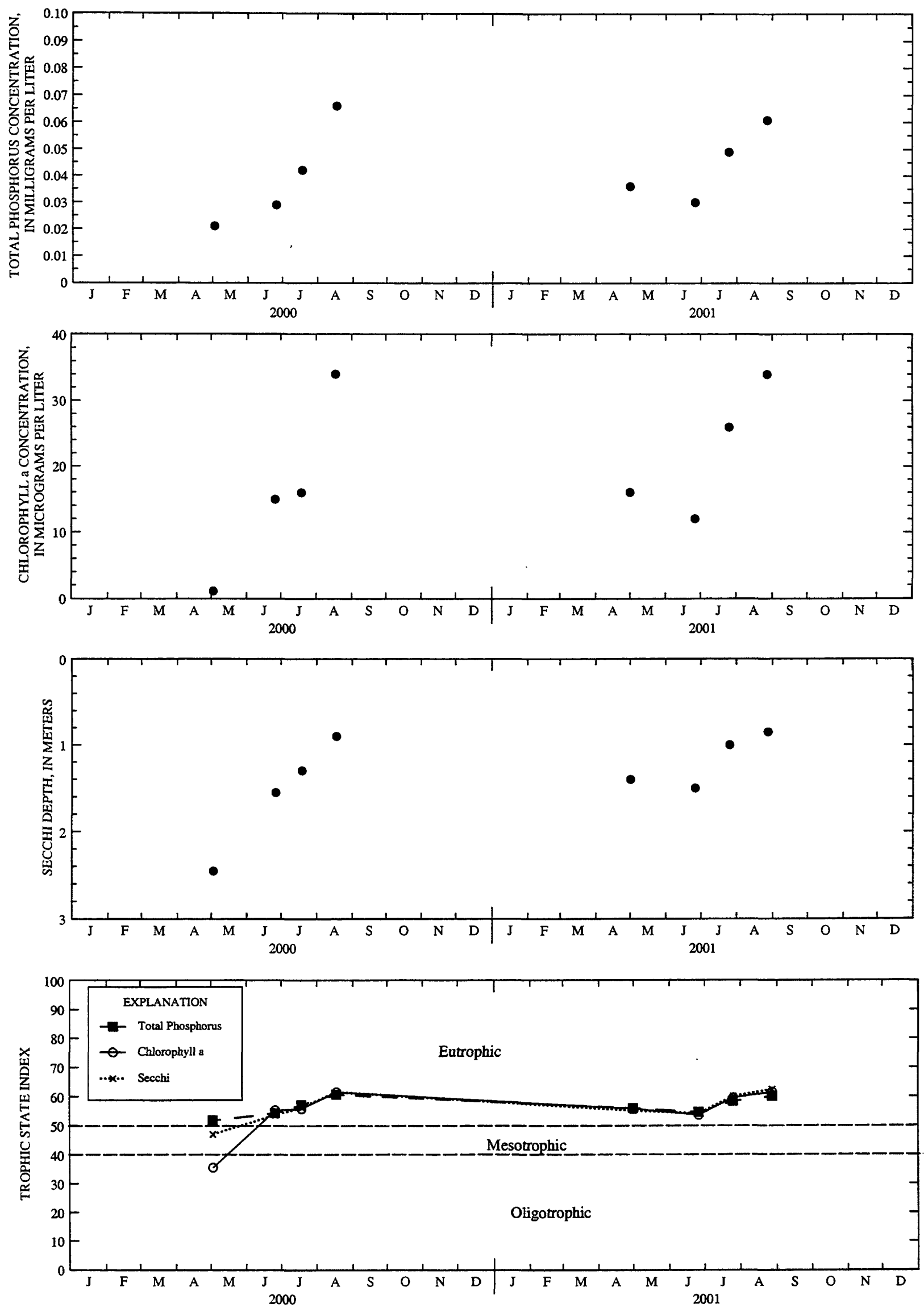

Surface total phosphorus, chlorophyll a concentrations, Secchi depths, and TSI data for Little St. Germain Lake, Upper East Bay, at St. Germain, Wisconsin. 
LOCATION.--Lat $45^{\circ} 55^{\prime} 45^{\prime \prime}$, long $89^{\circ} 26^{\prime} 25^{\prime \prime}$, in SW 1/4 SE 1/4 sec.24, T.40 N., R.8 E., Vilas County, Hydrologic Unit 07070001, near St. Germain.

PERIOD OF RECORD.--April 1991 to August 1994, August 1996 to August 1997, March 1999 to current year.

REMARKS.--Lake sampled in northeast bay at a lake depth of about $4 \mathrm{~m}$. Lake ice-covered during January and March sampling. Waterquality analyses by Wisconsin State Laboratory of Hygiene.

WATER-QUALITY DATA, JANUARY 17 TO MAY 01, 2001

(Milligrams per liter unless otherwise indicated)

Lake stage (ft)

Secchi-depth (m)

Chlorophyl $a$, phytoplankton $(\mu g / L)$

Depth of sample (m)

Water temperature $\left({ }^{\circ} \mathrm{C}\right.$

Specific conductance $(\mu \mathrm{S} / \mathrm{cm})$

pH (units)

Dissolved oxygen (mg/L)

Phosphorus, total (as P)

Nitrogen, NO2 + NO3, diss. (as N)

Nitrogen, anmonia, dissolved (as N)

Nitrogen, amm. + org. , total (as N)

Nitrogen, total (as N)
Phosphorus, ortho, dissolved (as P)

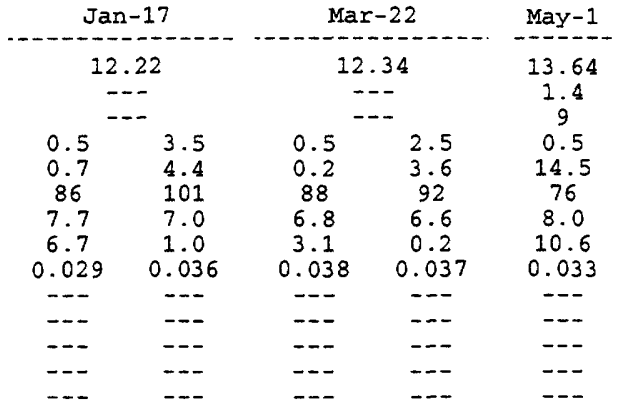

$3-22-01$

5-1-01

DISSOLVED OXYGEN (D.O.), IN MILLIGRAMS PER LITER
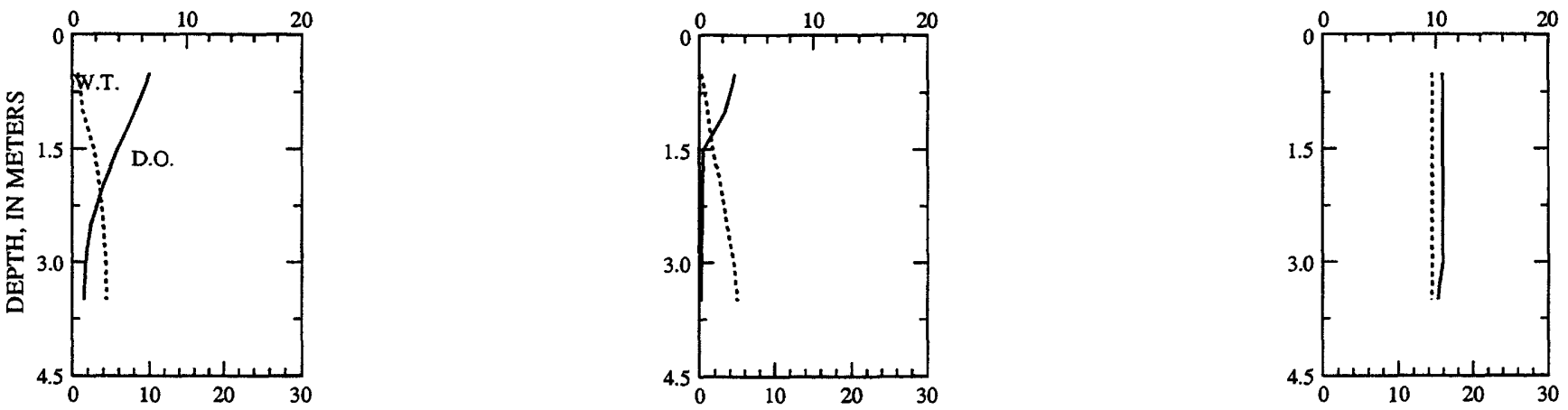

WATER TEMPERATURE (W.T.), IN DEGREES CELSIUS

PH, IN STANDARD UNITS
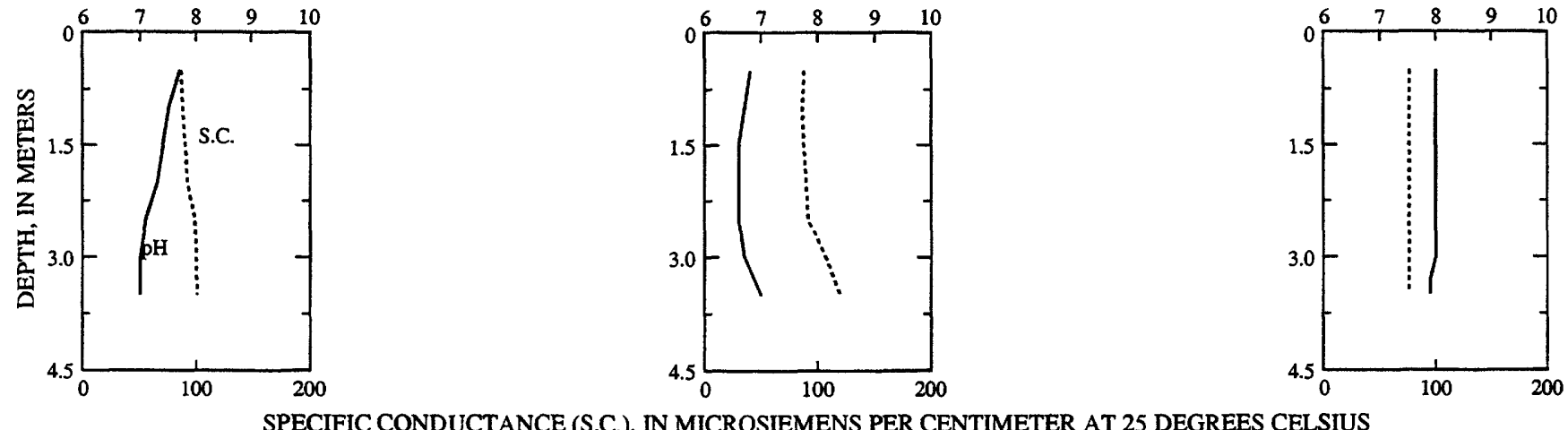
WATER-QUALITY DATA, JUNE 26 TO AUGUST 28, 2001

(Milligrams per liter unless otherwise indicated)

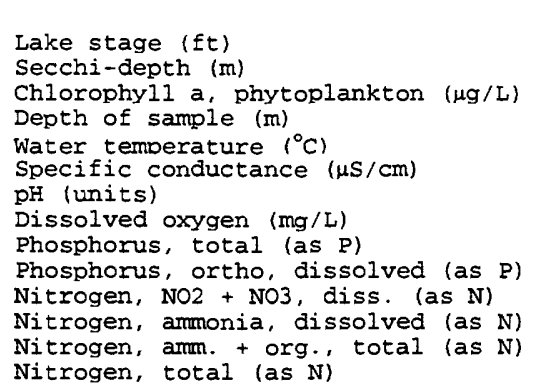

\begin{tabular}{|c|c|c|c|c|c|}
\hline \multicolumn{2}{|c|}{ Jun -26} & \multicolumn{2}{|c|}{ Jul-26 } & \multicolumn{2}{|c|}{ Aug-28 } \\
\hline \multicolumn{2}{|c|}{$\begin{array}{c}13.70 \\
1.1 \\
16\end{array}$} & \multicolumn{2}{|c|}{$\begin{array}{c}13.52 \\
0.7 \\
57\end{array}$} & \multicolumn{2}{|c|}{$\begin{array}{c}13.30 \\
0.8 \\
55\end{array}$} \\
\hline $\begin{array}{c}0.5 \\
23.6 \\
76 \\
8.1 \\
10.5 \\
0.040\end{array}$ & $\begin{array}{c}3.5 \\
22.4 \\
77 \\
7.2 \\
8.2 \\
0.040\end{array}$ & $\begin{array}{c}0.5 \\
23.8 \\
83 \\
8.4 \\
7.8 \\
0.059\end{array}$ & $\begin{array}{c}4.0 \\
20.3 \\
134 \\
7.0 \\
0.3 \\
\ldots .-\end{array}$ & $\begin{array}{c}0.5 \\
21.9 \\
86 \\
8.4 \\
10.4 \\
0.069\end{array}$ & $\begin{array}{c}4.0 \\
20.9 \\
98 \\
6.8 \\
0.4 \\
0.095\end{array}$ \\
\hline--- & $\ldots$ & 0.004 & $-\infty$ & -- & -- \\
\hline--- & --- & 0.014 & --- & --- & --- \\
\hline--- & $--\infty$ & 0.036 & -- & --- & --- \\
\hline$\cdots$ & -- & 1.2 & --- & -- & --- \\
\hline & & & - - & & \\
\hline
\end{tabular}

6-26-01

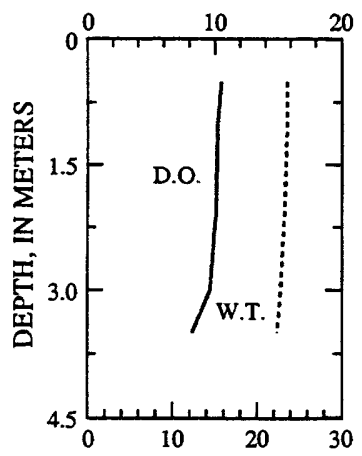

DISSOLVED OXYGEN (D.O.), IN MILLIGRAMS PER LITER
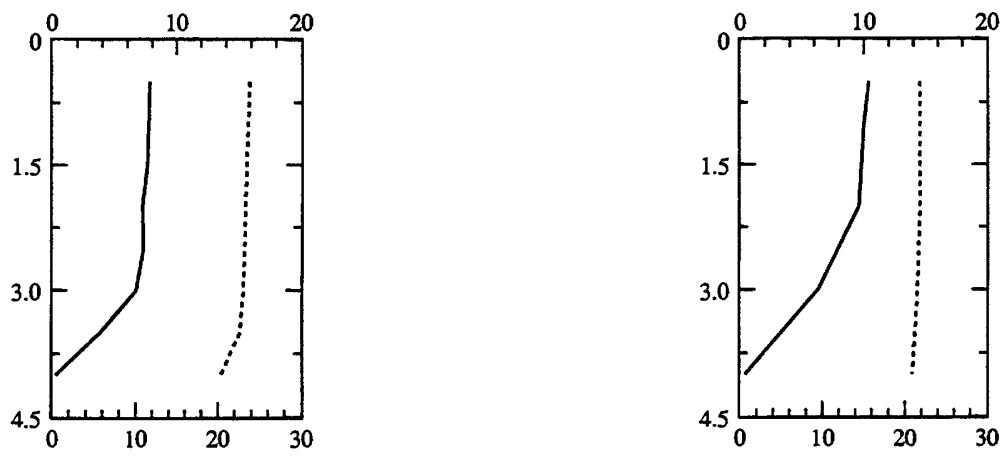

WATER TEMPERATURE (W.T.), IN DEGREES CELSIUS

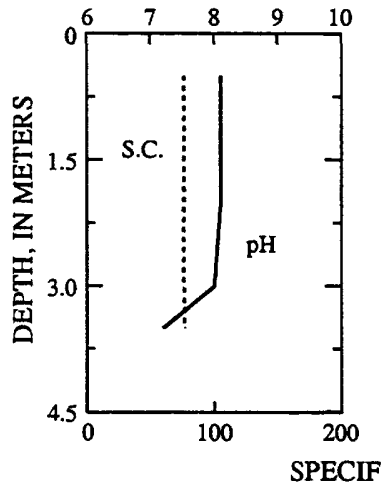

PH, IN STANDARD UNITS
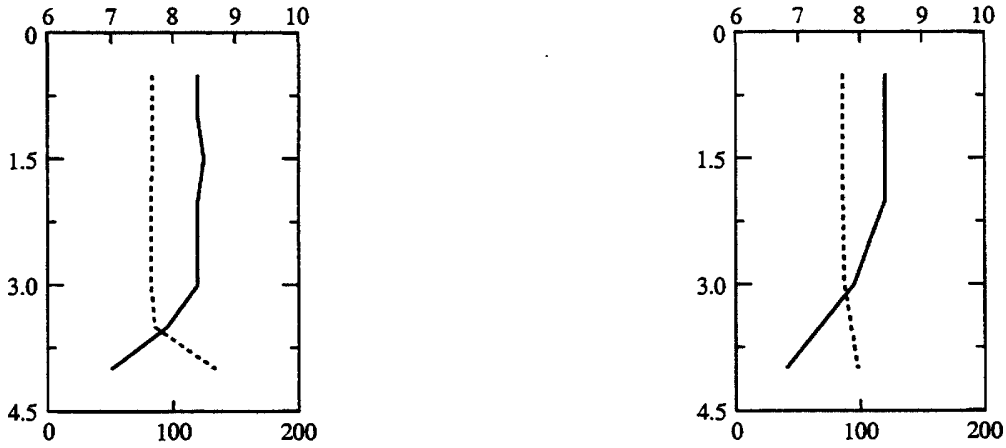

SPECIFIC CONDUCTANCE (S.C.), IN MICROSIEMENS PER CENTIMETER AT 25 DEGREES CELSIUS 

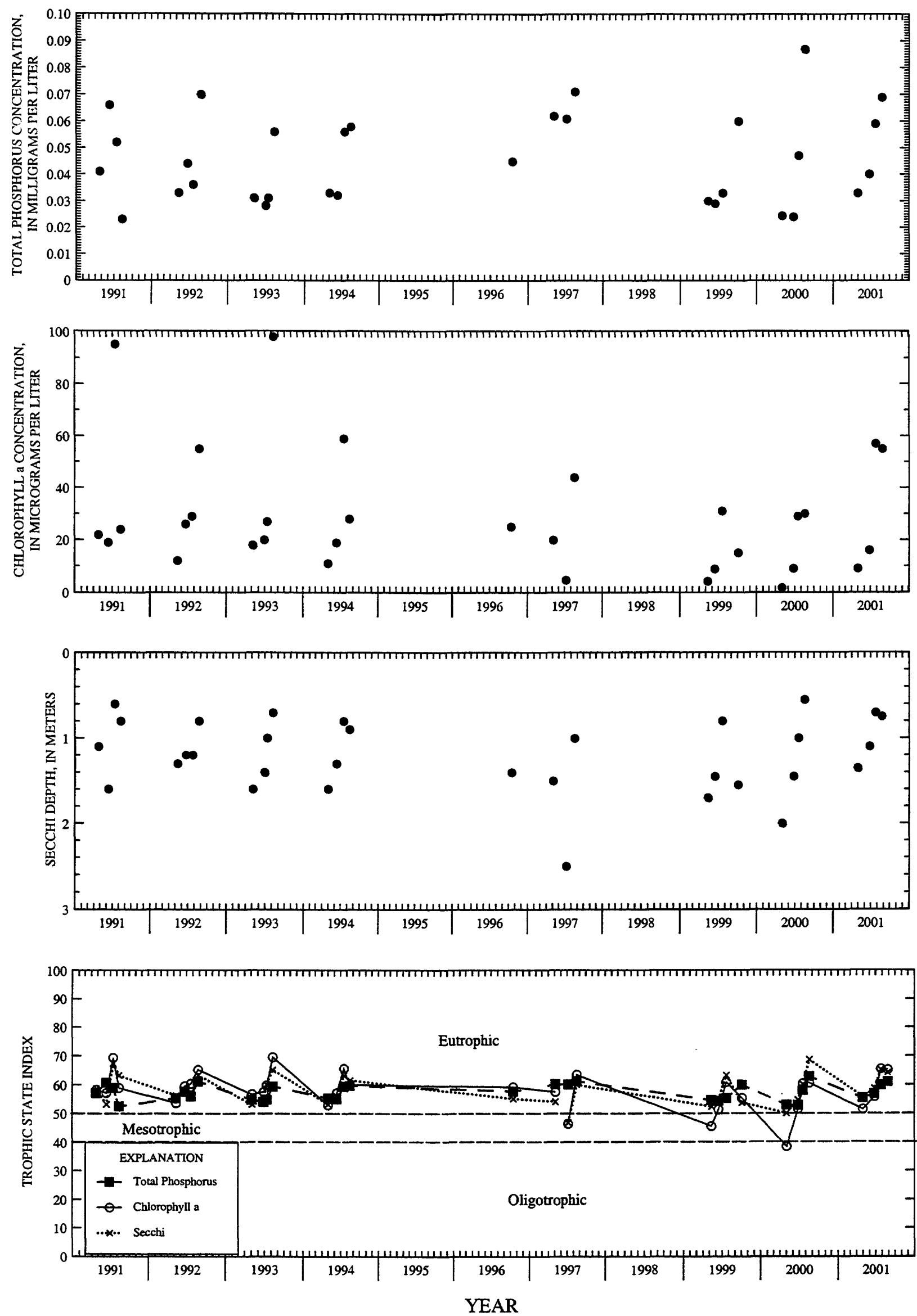

Surface total phosphorus, chlorophyll a concentrations, Secchi depths, and TSI data for Little St. Germain Lake, Northeast Bay, at St. Germain, Wisconsin. 
LOCATION.--Lat 4554'37", long 89²7'08", in NW 1/4 NE 1/4 sec.35, T.40 N., R.8 E., Vilas County, Hydrologic Unit 07070001, $1.7 \mathrm{mi}$ east of St. Germain.

PERIOD OF RECORD.--April 1991 to August 1994, August 1996 to August 1997, March 1999 to current year.

REMARKS.--Lake sampled in south bay at a lake depth of about $7 \mathrm{~m}$. Lake ice-covered during January and March sampling. Water-quality analyses by Wisconsin State Laboratory of Hygiene.

WATER-QUALITY DATA, JANUARY 17 TO MAY 01, 2001

(Milligrams per liter unless otherwise indicated

\begin{tabular}{|c|c|c|c|c|c|}
\hline & \multicolumn{2}{|c|}{$\mathrm{Jan}-17$} & \multicolumn{2}{|c|}{$\operatorname{Mar}-22$} & May-1 \\
\hline Lake stage (ft) & \multicolumn{2}{|c|}{12.22} & \multicolumn{2}{|c|}{12.34} & 13.64 \\
\hline Secchi-depth (m) & \multirow{2}{*}{\multicolumn{2}{|c|}{--}} & \multicolumn{2}{|c|}{$-\ldots$} & 1.3 \\
\hline Chlorophyll a, phytoplankton $(\mu \mathrm{g} / \mathrm{L})$ & & & & & 13 \\
\hline Depth of sample (m) & 0.5 & 6.5 & 0.5 & 6.0 & 0.5 \\
\hline Water temperature $\left({ }^{\circ} \mathrm{C}\right)$ & 1.8 & 4.2 & 0.2 & 5.0 & 13.9 \\
\hline Specific conductance ( $\mu \mathrm{S} / \mathrm{cm})$ & 75 & 98 & 98 & 146 & 83 \\
\hline $\begin{array}{l}\text { pH (units) } \\
\text { Dissolved oxygen (mg/L) }\end{array}$ & $\begin{array}{l}7.4 \\
4.5\end{array}$ & $\begin{array}{l}6.6 \\
0.8\end{array}$ & $\begin{array}{l}7.0 \\
0.9\end{array}$ & $\begin{array}{l}7.1 \\
0.1\end{array}$ & $\begin{array}{c}8.2 \\
11.1\end{array}$ \\
\hline Phosphorus, total (as P) & 0.030 & 0.029 & 0.039 & 0.209 & 0.052 \\
\hline Phosphorus, ortho, dissolved (as P) & $-\infty$ & $\ldots$ & -- & $\ldots$ & 0.007 \\
\hline Nitrogen, $\mathrm{NO} 2+\mathrm{NO} 3$, diss. (as N) & -- & -- & -- & -- & $<0.010$ \\
\hline Nitrogen, ammonia, dissolved (as N) & -- & -- & --- & -- & 0.014 \\
\hline Nitrogen, amm. + org., total (as N) & --- & --- & -- & -- & 0.68 \\
\hline Nitrogen, total (as N) & --- & --- & --- & --- & 0.68 \\
\hline Color (Pt-Co. scale) & --- & --- & -- & $\ldots$ & 40 \\
\hline Turbidity (NTU) & $\ldots$ & -.- & --- & $-\ldots$ & 4.6 \\
\hline Hardness, (as $\mathrm{CaCO}_{3}$ ) & $\ldots$ & $-\ldots$ & -- & --- & 36.9 \\
\hline Calcium, dissolved (Ca) & -- & --- & --- & --- & 10 \\
\hline Magnesium, dissolved (Mg) & -- & -- & -- & -- & 2.9 \\
\hline Sodium, dissolved (Na) & -- & --- & --- & --- & 2.1 \\
\hline Potassium, dissolved (K) & -- & -- & -- & -- & 0.5 \\
\hline Alkalinity, (as $\left.\mathrm{CaCO}_{3}\right)$ & -- & -- & -- & $\ldots$ & 36 \\
\hline Sulfate, dissolved $\left(\mathrm{SO}_{4}\right)$ & --- & --- & -- & -- & $<4.5$ \\
\hline Chloride, dissolved (CI) & --- & -- & -- & -- & 2 \\
\hline Silica, dissolved $\left(\mathrm{SiO}_{2}\right)$ & $-\infty$ & --- & --- & -- & 12.2 \\
\hline Solids, dissolved at $180^{\circ} \mathrm{C}$ & -- & -- & --- & --- & 62 \\
\hline Iron, dissolved (Fe) $\mu \mathrm{g} / \mathrm{L}$ & $\ldots$ & $\ldots$ & $\ldots$ & 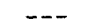 & 430 \\
\hline Manganese, dissolved (Mn) $\mu \mathrm{g} / \mathrm{L}$ & --- & $-\ldots$ & $\ldots$ & $\ldots$ & 35 \\
\hline
\end{tabular}

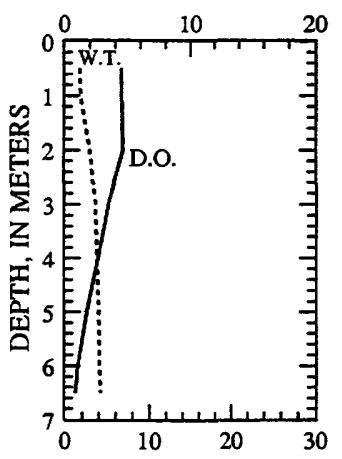

DISSOLVED OXYGEN (D.O.), IN MILLIGRAMS PER LITER
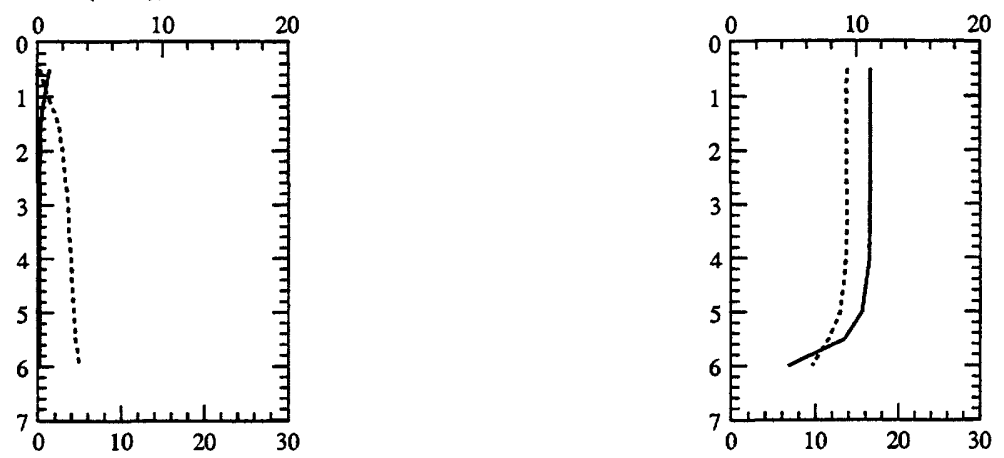

WATER TEMPERATURE (W.T.), IN DEGREES CELSIUS

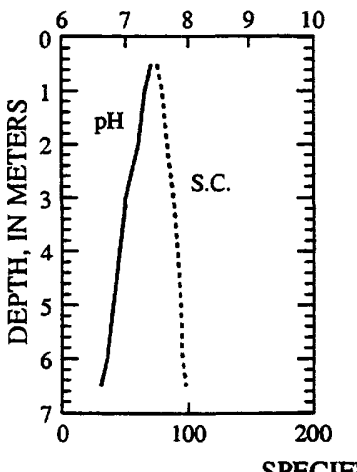

PH, IN STANDARD UNITS

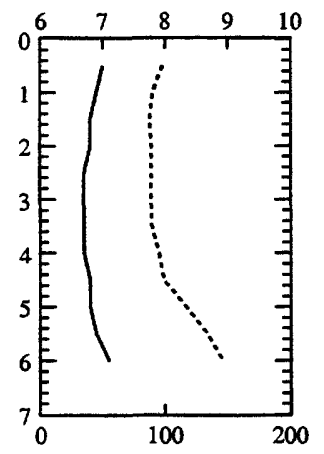

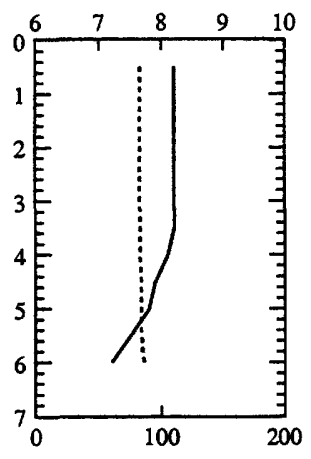


WATER-QUALITY DATA, JUNE 26 TO AUGUST 28, 2001

(Milligrams per liter unless otherwise indicated)

lake stage (ft)

Secchi-depth (m)

Chlorophyll a, phytoplankton $(\mu \mathrm{g} / \mathrm{L})$

Depth of sample (m)

Water temoerature $\left({ }^{\circ} \mathrm{C}\right)$

Specific conductance $(\mu \mathrm{S} / \mathrm{cm})$

$\mathrm{pH}$ (units)

Dissolved oxygen (mg/L)

Phosphorus, total (as P)

\begin{tabular}{|c|c|c|c|c|c|c|}
\hline \multicolumn{2}{|c|}{ Jun-26 } & \multicolumn{2}{|c|}{ Jul-26 } & \multicolumn{3}{|c|}{ Aug-28 } \\
\hline \multicolumn{2}{|c|}{$\begin{array}{c}13.70 \\
2.9 \\
5.5\end{array}$} & \multicolumn{2}{|c|}{$\begin{array}{c}13.52 \\
1.5 \\
3.6\end{array}$} & & $\begin{array}{c}13.30 \\
2.1 \\
10\end{array}$ & \\
\hline $\begin{array}{c}0.5 \\
23.1 \\
75 \\
8.0 \\
9.8 \\
0.025\end{array}$ & $\begin{array}{c}5.5 \\
14.6 \\
84 \\
6.6 \\
0.7 \\
0.054\end{array}$ & $\begin{array}{c}0.5 \\
24.2 \\
80 \\
8.5 \\
8.9 \\
0.012\end{array}$ & $\begin{array}{c}6.0 \\
15.5 \\
145 \\
6.9 \\
0.3 \\
0.108\end{array}$ & $\begin{array}{c}0.5 \\
22.0 \\
84 \\
8.1 \\
9.4 \\
0.030\end{array}$ & $\begin{array}{c}5.0 \\
19.1 \\
95 \\
6.5 \\
0.3 \\
0.042\end{array}$ & $\begin{array}{c}7.0 \\
17.2 \\
155 \\
7.0 \\
0.2 \\
0.087\end{array}$ \\
\hline
\end{tabular}

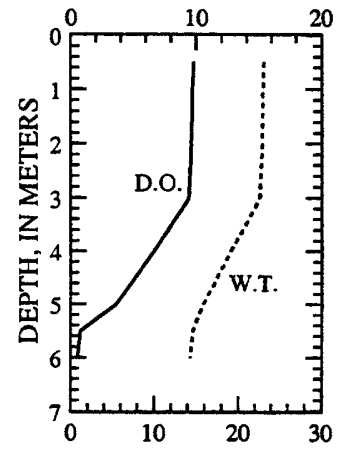

DISSOLVED OXYGEN (D.O.), IN MILLIGRAMS PER LITER
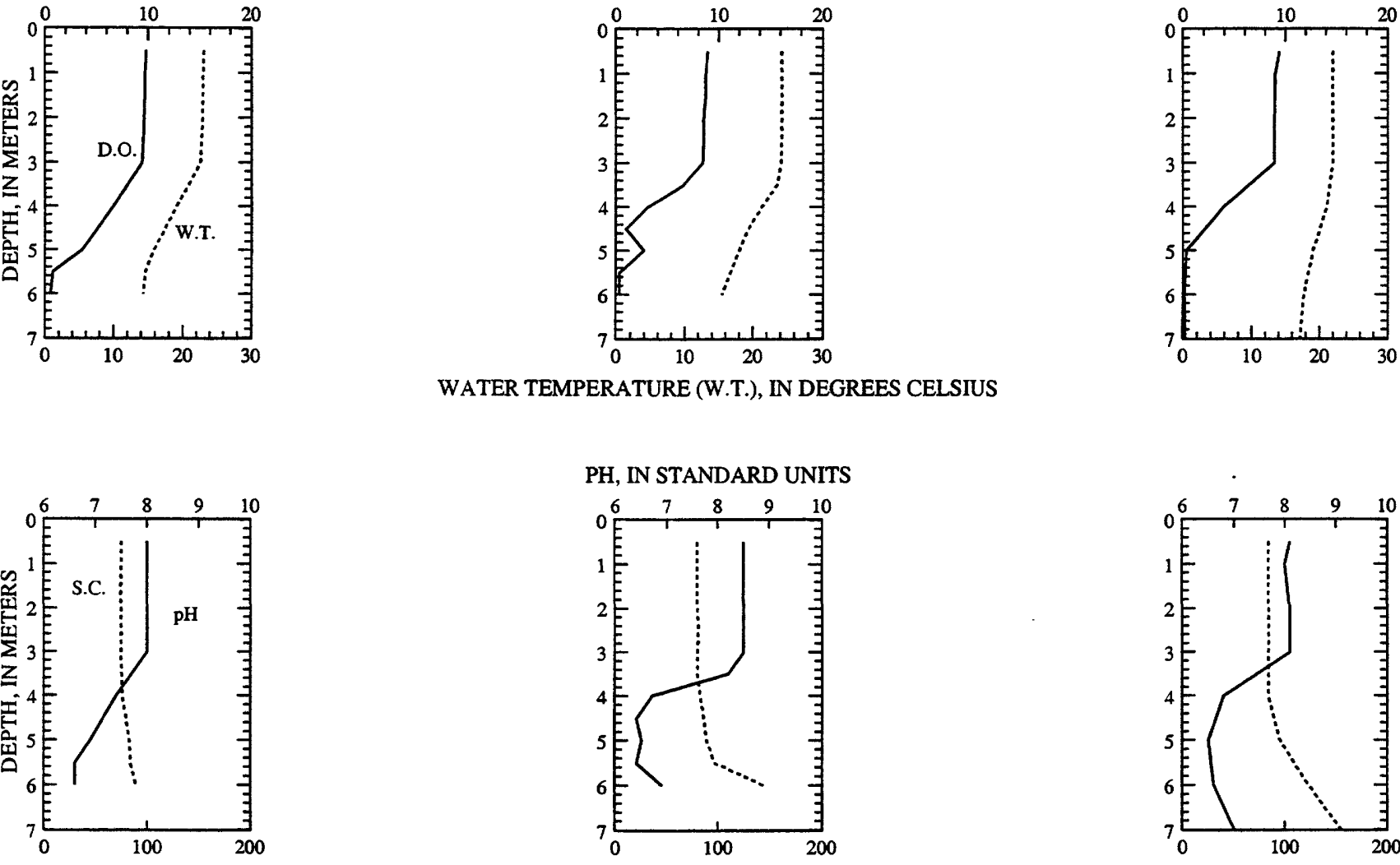

WATER TEMPERATURE (W.T.), IN DEGREES CELSIUS

PH, IN STANDARD UNITS

SPECIFIC CONDUCTANCE (S.C.), IN MICROSIEMENS PER CENTIMETER AT 25 DEGREES CELSIUS

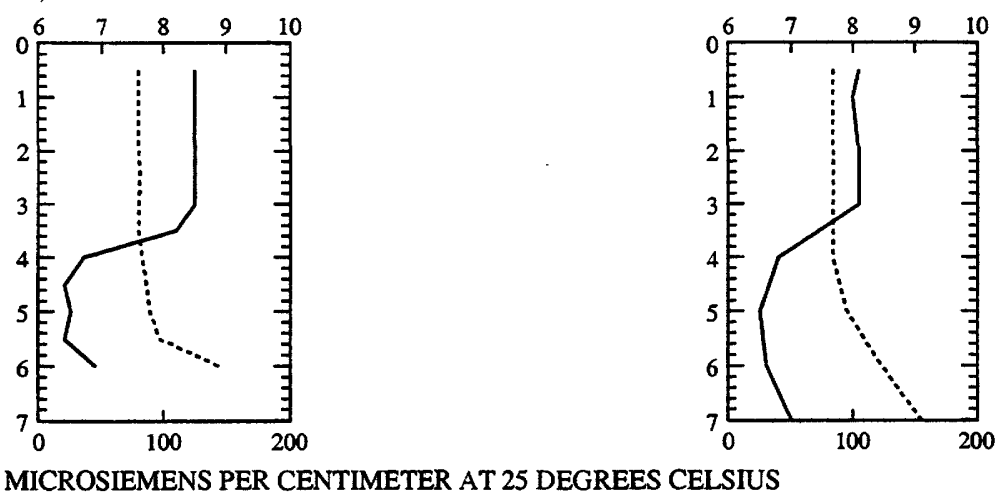



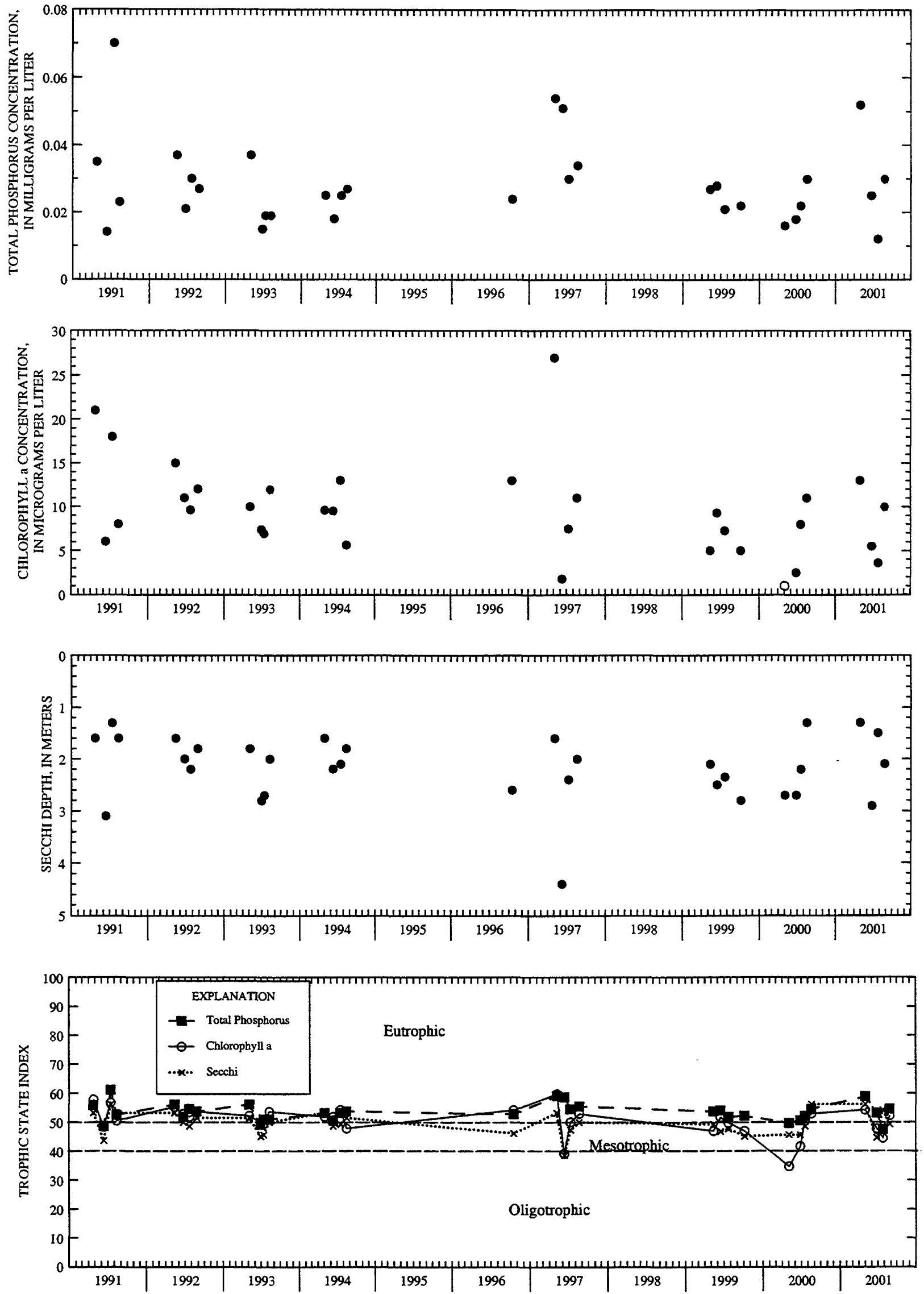

Surface total phosphorus, chlorophyll a concentrations, Secchi depths, and TSI data for Little St. Germain Lake, South Bay, at St. Germain, Wisconsin.

(Circles on the first three plots indicate laboratory detection limit for selected analyses.

Actual concentrations for these particular analyses are less than the plotted circles.) 
LOCATION.--Lat 45 54'28", long $89^{\circ} 28^{\prime} 24^{\prime \prime}$, in SW 1/4 NE 1/4 sec.34, T.40 N., R.8 E., Vilas County, Hydrologic Unit 07070001, at St. Germain.

PERIOD OF RECORD.--April 1991 to August 1994, August 1996 to August 1997, March 1999 to current year.

REMARKS.--Lake sampled in west bay at a lake depth of about $18 \mathrm{~m}$. Lake ice-covered during January and March sampling. Water-quality analyses by Wisconsin State Laboratory of Hygiene.

WATER-QUALITY DATA, JANUARY 17 TO MAY 01, 2001

(Milligrams per liter unless otherwise indicated)

Lake stage ( $f t$ )

Secchi-depth (m)

Chlorophy1l a, phytoplankton ( $\mu \mathrm{g} / \mathrm{L}$ )

Depth of sample (m)

water temberature $\left({ }^{\circ} \mathrm{C}\right)$

Specific conductance $(\mu \mathrm{s} / \mathrm{cm})$

pH (units)

Dissolved oxygen (mg/L)

Phosphorus, total (as P)

Phosphorus, ortho, dissolved (as P)

Nitrogen, NO2 + NO3, diss. (as N)

Nitrogen, ammonia, dissolved (as N)

Nitrogen, amm. + org.. total (as N)

Nitrogen, total (as N)

Color (Pt-Co. scale)

Turbidity (NTU)

Hardness, (as $\mathrm{CaCO}_{3}$ )

Calcium, dissolved (Ca)

Magnesium, dissolved (Mg)

Sodium, dissolved (Na)

Potassium, dissolved (K)

Alkalinity, (as $\mathrm{CaCO}_{3}$ )

Sulfate, dissolved $\left(\mathrm{SO}_{4}\right)$

Chloride, dissolved (CI)

Silica, dissolved $\left(\mathrm{SiO}_{2}\right)$

Solids. dissolved. at $180^{\circ} \mathrm{C}$

Iron, dissolved (Fe) $\mu \mathrm{g} / \mathrm{I}$

Manganese, dissolved (Mn) $\mu \mathrm{g} / \mathrm{L}$

\begin{tabular}{|c|c|c|c|c|}
\hline \multicolumn{2}{|c|}{ Jan-17 } & \multicolumn{2}{|c|}{$\operatorname{Mar}-22$} & May - 1 \\
\hline \multicolumn{2}{|c|}{12.22} & \multicolumn{2}{|c|}{12.34} & 13.64 \\
\hline \multirow{2}{*}{\multicolumn{2}{|c|}{---}} & \multicolumn{2}{|c|}{---} & 1.9 \\
\hline & --- & \multicolumn{2}{|c|}{---} & 10 \\
\hline 0.5 & 15.0 & 0.5 & 12.5 & 0.5 \\
\hline 0.4 & 4.1 & 0.1 & 4.1 & 11.1 \\
\hline 82 & 80 & 78 & 73 & 75 \\
\hline 7.8 & 6.9 & 7.0 & 6.4 & 7.8 \\
\hline 12.0 & 0.7 & 9.5 & 0.2 & 11.4 \\
\hline 0.008 & 0.044 & 0.014 & 0.017 & 0.015 \\
\hline--- & $-\infty$ & -- & -- & 0.002 \\
\hline--- & $-\cdots$ & -- & --- & 0.102 \\
\hline-- & $-\infty$ & -- & -- & $<0.013$ \\
\hline-- & $-\infty$ & --- & -- & 0.46 \\
\hline--- & -- & -- & -- & 0.562 \\
\hline-- & -- & -- & --- & 20 \\
\hline--- & --- & $-\cdots$ & --- & 1.9 \\
\hline--- & $\cdots$ & --- & -- & 32.8 \\
\hline-- & $-\cdots$ & -- & -- & 8.5 \\
\hline--- & -- & $\cdots$ & --- & 2.8 \\
\hline--- & $\cdots$ & --- & -- & 2.2 \\
\hline-- & $-\cdots$ & -- & -- & $<0.30$ \\
\hline--- & $-\infty$ & --- & -- & 32 \\
\hline--- & $-\infty$ & -- & -- & $<4.5$ \\
\hline-- & $-\cdots$ & -- & -- & 2.1 \\
\hline--- & -- & -- & -- & 8.7 \\
\hline--- & --- & -- & --- & 52 \\
\hline--- & -- & --- & --- & 50 \\
\hline-- & -- & --- & --- & 39 \\
\hline
\end{tabular}

$3-22-01$
5-1-01
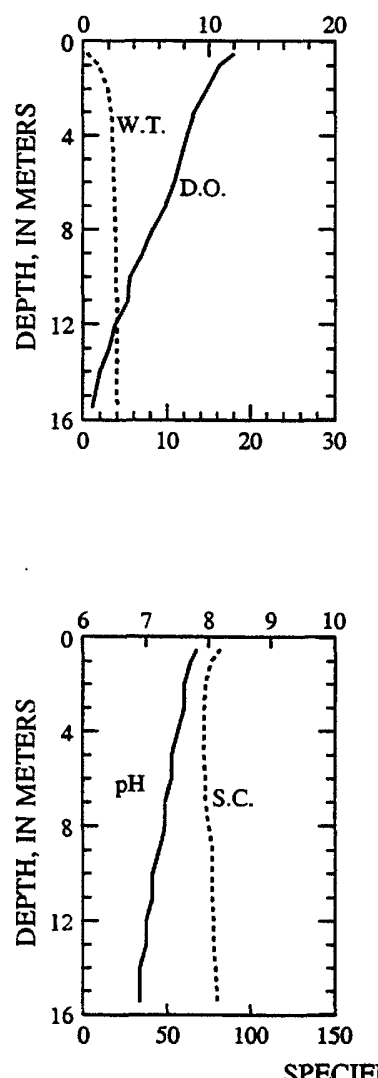

DISSOLVED OXYGEN (D.O.), IN MILLIGRAMS PER LITER

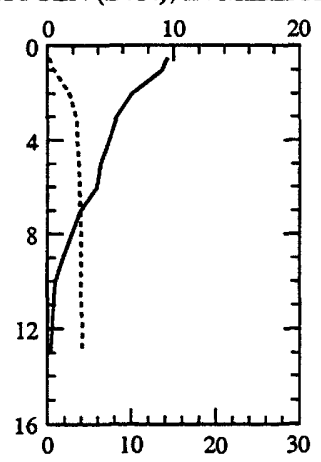

WATER TEMPERATURE (W.T.), IN DEGREES CELSIUS

PH, IN STANDARD UNITS
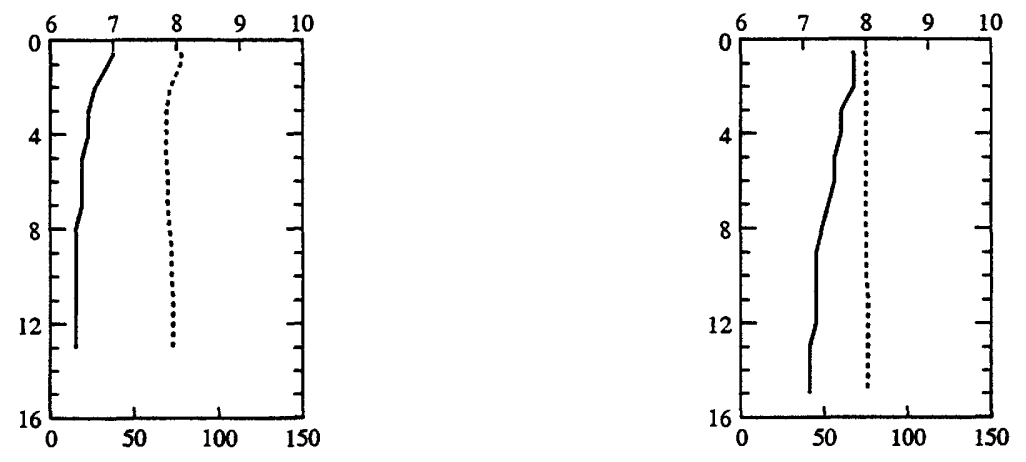
WATER-QUALITY DATA, JUNE 26 TO AUGUST 28, 2001

(Milligrams per liter unless otherwise indicated)

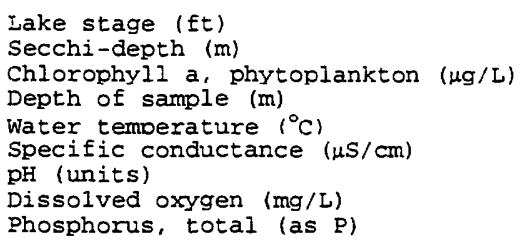

Aug -28

\begin{tabular}{|c|c|c|c|c|c|c|}
\hline \multicolumn{2}{|c|}{ Jun -26} & \multicolumn{2}{|c|}{ Jul-26 } & \multicolumn{3}{|c|}{ Aug -28} \\
\hline & & & & & $\begin{array}{c}13.30 \\
4.1 \\
3.2\end{array}$ & \\
\hline $\begin{array}{c}0.5 \\
21.8 \\
71 \\
7.3 \\
9.6 \\
0.015\end{array}$ & $\begin{array}{c}15.0 \\
6.4 \\
84 \\
6.5 \\
0.3 \\
0.074\end{array}$ & $\begin{array}{c}0.5 \\
23.7 \\
75 \\
7.6 \\
8.2 \\
0.026\end{array}$ & $\begin{array}{c}15.0 \\
6.7 \\
97 \\
6.6 \\
0.3 \\
0.088\end{array}$ & $\begin{array}{c}0.5 \\
22.0 \\
76 \\
7.3 \\
9.2 \\
0.009\end{array}$ & $\begin{array}{c}8.0 \\
11.3 \\
74 \\
6.3 \\
0.2 \\
0.023\end{array}$ & $\begin{array}{l}14.0 \\
6.8 \\
115 \\
6.7 \\
0.2 \\
0.211\end{array}$ \\
\hline
\end{tabular}

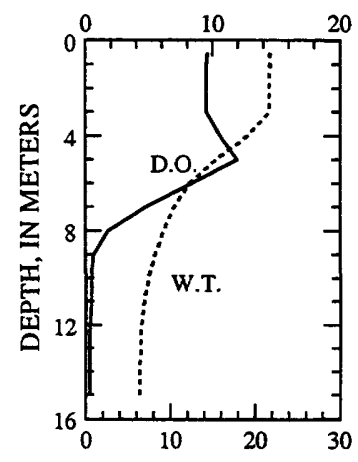

DISSOLVED OXYGEN (D.O.), IN MILLIGRAMS PER LITER
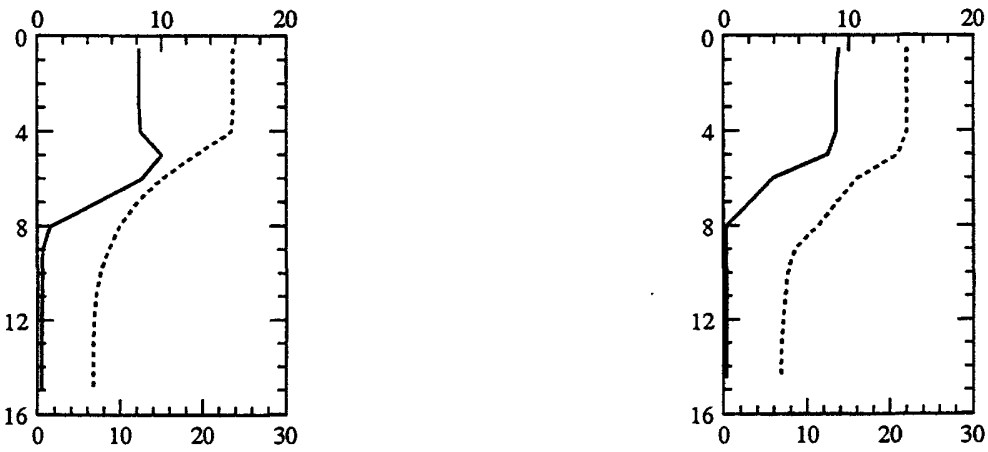

WATER TEMPERATURE (W.T.), IN DEGREES CELSIUS

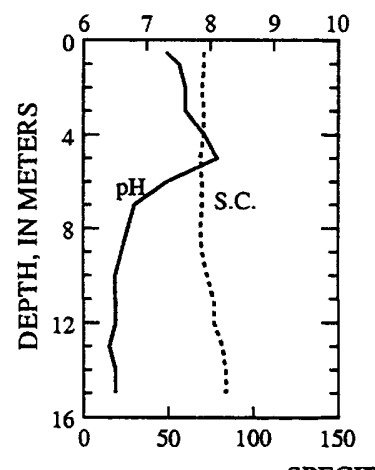

PH, IN STANDARD UNITS
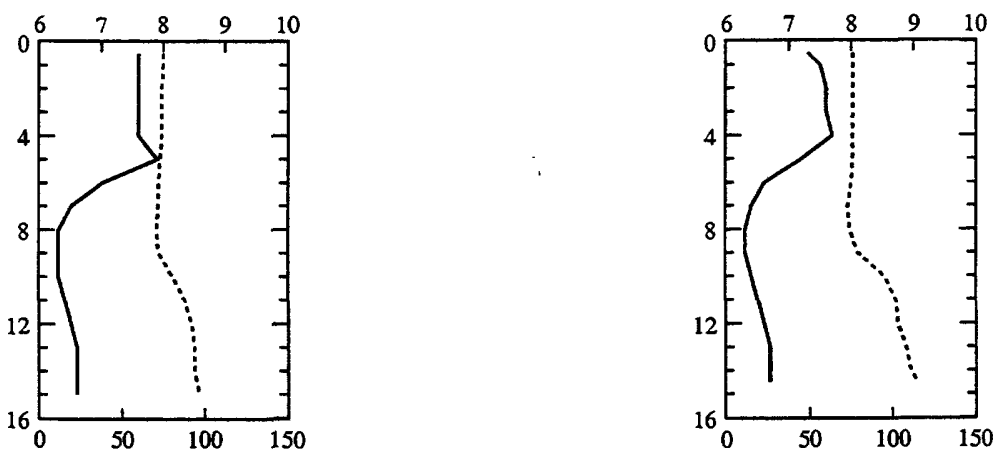

SPECIFIC CONDUCTANCE (S.C.), IN MICROSIEMENS PER CENTIMETER AT 25 DEGREES CELSIUS 

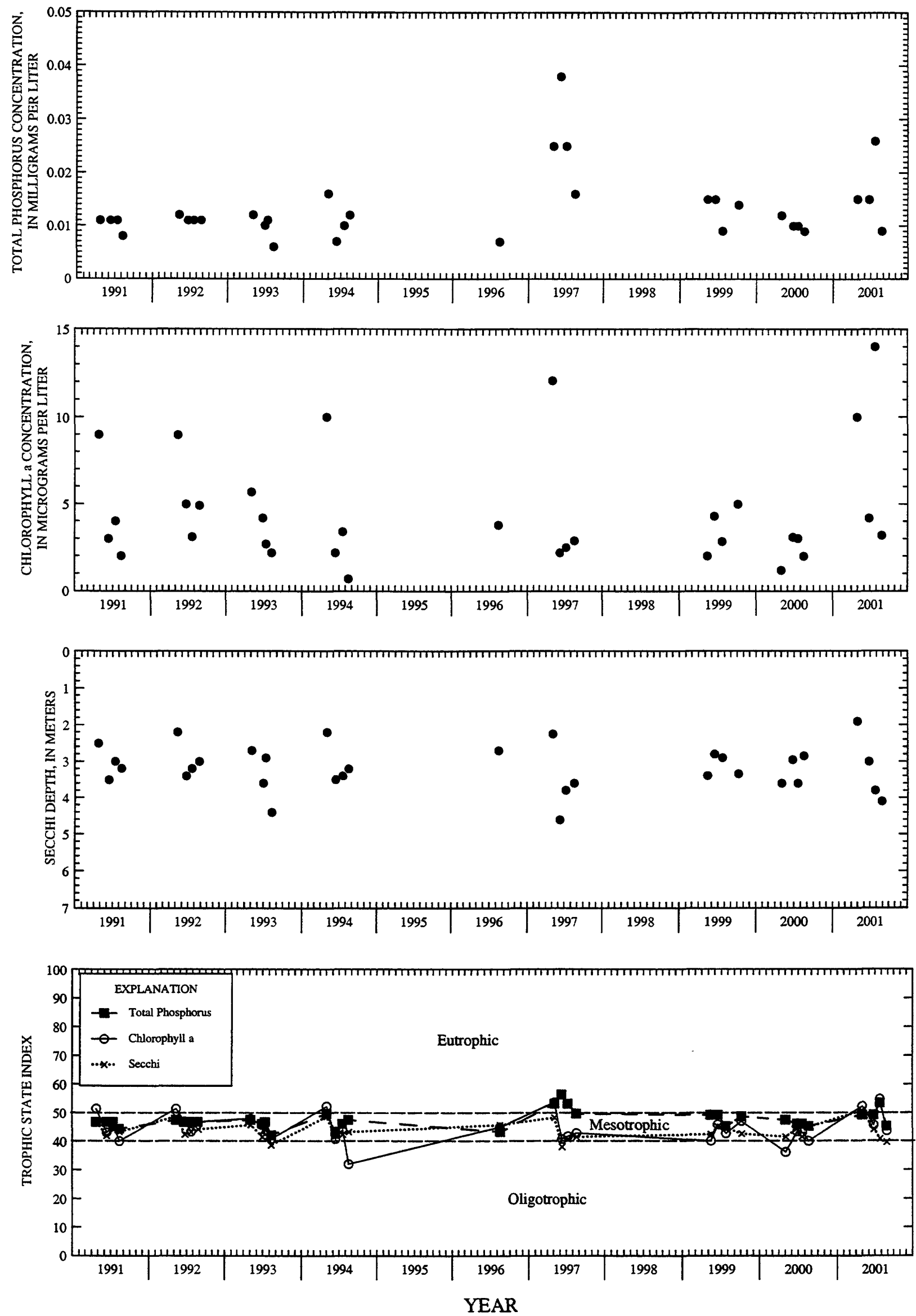

Surface total phosphorus, chlorophyll a concentrations, Secchi depths, and TSI data for Little St. Germain Lake, West Bay, at St. Germain, Wisconsin. 
LOCATION.--Lat 45²8'26" long 88 $54^{\prime} 41^{\prime \prime}$, in SW 1/4 NE 1/4 sec.31, T.35 N., R.13 E., Forest County, Hydrologic Unit 04030202, on left bank $1 \mathrm{mi}$ upstream of outlet, $3 \mathrm{mi}$ southeast of Mole Lake.

PERIOD OF RECORD.--May 1996 to current year. Prior to October 2000 published as "Little Sand Lake near Mole Lake, WI" under station number 04074651 .

GAGE.--Water-stage recorder. Datum of gage is $1,587.32 \mathrm{ft}$ above sea level.

REMARKS.--Recorder removed during period of ice, Nov. 11, 2000 to May 1, 2001.

EXTREMES FOR PERIOD OF RECORD.--Maximum gage height observed, $5.97 \mathrm{ft}$, May 25, 1997; minimum observed, $3.78 \mathrm{ft}$, Nov. 7-9, 1999.

EXTREMES FOR CURRENT YEAR.-- Maximum gage height observed, $5.05 \mathrm{ft}$, Oct. 3; minimum observed, $3.84 \mathrm{ft}$, Sept. 6.

GAGE HEIGHT, FEET, WATER YEAR OCTOBER 2000 TO SEPTEMBER 2001

DAILY MEAN VALUES

\begin{tabular}{|c|c|c|c|c|c|c|c|c|c|c|c|c|}
\hline DAY & OCT & NOV & $D E C$ & JAN & $F E B$ & MAR & APR & MAY & JUN & JUL & AUG & SEP \\
\hline $\begin{array}{l}1 \\
2 \\
3 \\
4 \\
5\end{array}$ & $\begin{array}{l}5.05 \\
5.05 \\
5.05 \\
5.03 \\
5.01\end{array}$ & $\begin{array}{l}4.70 \\
4.69 \\
4.67 \\
4.65 \\
4.64\end{array}$ & $\begin{array}{l}\cdots- \\
\cdots- \\
-\cdots \\
-\cdots\end{array}$ & $\begin{array}{l}--- \\
--- \\
--- \\
--- \\
--\end{array}$ & $\begin{array}{l}--- \\
-- \\
--- \\
--- \\
---\end{array}$ & $\begin{array}{l}--- \\
--- \\
--- \\
---\end{array}$ & $\begin{array}{l}--- \\
-- \\
--- \\
---\end{array}$ & $\begin{array}{l}--7 \\
4.73 \\
4.74 \\
4.73 \\
4.70\end{array}$ & $\begin{array}{l}4.60 \\
4.60 \\
4.59 \\
4.57 \\
4.55\end{array}$ & $\begin{array}{l}4.39 \\
4.36 \\
4.34 \\
4.33 \\
4.30\end{array}$ & $\begin{array}{l}4.19 \\
4.20 \\
4.19 \\
4.17 \\
4.17\end{array}$ & $\begin{array}{l}3.92 \\
3.90 \\
3.88 \\
3.86 \\
3.85\end{array}$ \\
\hline $\begin{array}{r}6 \\
7 \\
8 \\
9 \\
10\end{array}$ & $\begin{array}{l}5.01 \\
5.00 \\
4.98 \\
4.96 \\
4.95\end{array}$ & $\begin{array}{l}4.63 \\
4.67 \\
4.67 \\
4.67 \\
4.66\end{array}$ & $\begin{array}{l}--- \\
--- \\
-- \\
--\end{array}$ & 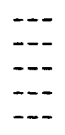 & 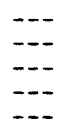 & $\begin{array}{l}--- \\
--- \\
--- \\
---\end{array}$ & $\begin{array}{l}--- \\
--- \\
--- \\
--\end{array}$ & $\begin{array}{l}4.68 \\
4.70 \\
4.72 \\
4.68 \\
4.69\end{array}$ & $\begin{array}{l}4.53 \\
4.53 \\
4.52 \\
4.49 \\
4.51\end{array}$ & $\begin{array}{l}4.28 \\
4.26 \\
4.24 \\
4.23 \\
4.22\end{array}$ & $\begin{array}{l}4.16 \\
4.16 \\
4.13 \\
4.11 \\
4.10\end{array}$ & $\begin{array}{l}3.84 \\
3.96 \\
4.02 \\
4.03 \\
4.01\end{array}$ \\
\hline $\begin{array}{l}11 \\
12 \\
13 \\
14 \\
15\end{array}$ & $\begin{array}{l}4.95 \\
4.94 \\
4.94 \\
4.96 \\
4.95\end{array}$ & $\begin{array}{l}--- \\
-- \\
--- \\
---\end{array}$ & $\begin{array}{l}--- \\
-\cdots \\
--- \\
--\end{array}$ & $\begin{array}{l}--- \\
\cdots- \\
-\cdots \\
--\end{array}$ & $\begin{array}{l}--- \\
--- \\
--- \\
---\end{array}$ & $\begin{array}{l}--- \\
--- \\
-- \\
--\end{array}$ & $\begin{array}{l}-- \\
-- \\
-- \\
---\end{array}$ & $\begin{array}{l}4.69 \\
4.66 \\
4.64 \\
4.64 \\
4.62\end{array}$ & $\begin{array}{l}4.52 \\
4.54 \\
4.53 \\
4.52 \\
4.53\end{array}$ & $\begin{array}{l}4.22 \\
4.20 \\
4.17 \\
4.15 \\
4.15\end{array}$ & $\begin{array}{l}4.07 \\
4.06 \\
4.10 \\
4.07 \\
4.04\end{array}$ & $\begin{array}{l}3.99 \\
3.97 \\
3.96 \\
3.95 \\
3.94\end{array}$ \\
\hline $\begin{array}{l}16 \\
17 \\
18 \\
19 \\
20\end{array}$ & $\begin{array}{l}4.95 \\
4.95 \\
4.92 \\
4.89 \\
4.86\end{array}$ & $\begin{array}{l}--- \\
-- \\
--- \\
--- \\
--\end{array}$ & $\begin{array}{l}--- \\
-- \\
--- \\
--\end{array}$ & $\begin{array}{l}--- \\
--- \\
--- \\
---\end{array}$ & $\begin{array}{l}--- \\
--- \\
--- \\
---\end{array}$ & $\begin{array}{l}--- \\
--- \\
--- \\
--\end{array}$ & $\begin{array}{l}--- \\
--- \\
-- \\
---\end{array}$ & $\begin{array}{l}4.63 \\
4.61 \\
4.58 \\
4.55 \\
4.53\end{array}$ & $\begin{array}{l}4.52 \\
4.50 \\
4.52 \\
4.53 \\
4.51\end{array}$ & $\begin{array}{l}4.13 \\
4.11 \\
4.14 \\
4.15 \\
4.23\end{array}$ & $\begin{array}{l}4.04 \\
4.04 \\
4.03 \\
4.02 \\
4.01\end{array}$ & $\begin{array}{l}3.92 \\
3.91 \\
3.91 \\
3.92 \\
3.93\end{array}$ \\
\hline $\begin{array}{l}21 \\
22 \\
23 \\
24 \\
25\end{array}$ & $\begin{array}{l}4.84 \\
4.83 \\
4.82 \\
4.82 \\
4.80\end{array}$ & $\begin{array}{l}--- \\
--- \\
--- \\
---\end{array}$ & $\begin{array}{l}--- \\
--- \\
--- \\
---\end{array}$ & $\begin{array}{l}--- \\
\overline{--} \\
\overline{--} \\
-\cdots\end{array}$ & $\begin{array}{l}--- \\
-\cdots \\
-- \\
--\end{array}$ & $\begin{array}{l}--- \\
-- \\
--\end{array}$ & $\begin{array}{l}--- \\
-- \\
--- \\
---\end{array}$ & $\begin{array}{l}4.55 \\
4.58 \\
4.57 \\
4.59 \\
4.61\end{array}$ & $\begin{array}{l}4.54 \\
4.55 \\
4.53 \\
4.51 \\
4.49\end{array}$ & $\begin{array}{l}4.25 \\
4.24 \\
4.24 \\
4.22 \\
4.20\end{array}$ & $\begin{array}{l}4.00 \\
3.99 \\
3.99 \\
3.98 \\
3.97\end{array}$ & $\begin{array}{l}3.92 \\
3.92 \\
3.97 \\
3.97 \\
3.95\end{array}$ \\
\hline $\begin{array}{l}26 \\
27 \\
28 \\
29 \\
30 \\
31\end{array}$ & $\begin{array}{l}4.78 \\
4.77 \\
4.76 \\
4.75 \\
4.73 \\
4.71\end{array}$ & $\begin{array}{l}--- \\
--- \\
--- \\
--- \\
---\end{array}$ & $\begin{array}{l}--- \\
--- \\
--- \\
--- \\
--\end{array}$ & $\begin{array}{l}--- \\
--- \\
--- \\
--- \\
--\end{array}$ & $\begin{array}{l}--- \\
--- \\
-\cdots \\
-\cdots \\
-\cdots\end{array}$ & $\begin{array}{l}--- \\
-- \\
--- \\
-- \\
--\end{array}$ & $\begin{array}{l}-- \\
-- \\
--- \\
--- \\
---\end{array}$ & $\begin{array}{l}4.62 \\
4.63 \\
4.61 \\
4.61 \\
4.60 \\
4.58\end{array}$ & $\begin{array}{r}4.48 \\
4.47 \\
4.47 \\
4.46 \\
4.44 \\
---\end{array}$ & $\begin{array}{l}4.17 \\
4.14 \\
4.16 \\
4.18 \\
4.18 \\
4.18\end{array}$ & $\begin{array}{l}3.97 \\
3.97 \\
3.95 \\
3.94 \\
3.95 \\
3.94\end{array}$ & $\begin{array}{l}3.92 \\
3.91 \\
3.91 \\
3.91 \\
3.90\end{array}$ \\
\hline $\begin{array}{l}\text { MEAN } \\
\text { MAX } \\
\text { MIN }\end{array}$ & $\begin{array}{l}4.90 \\
5.05 \\
4.71\end{array}$ & $\begin{array}{l}-- \\
-- \\
--\end{array}$ & $\begin{array}{l}--- \\
-\cdots \\
---\end{array}$ & $\overline{---}$ & $\begin{array}{l}--- \\
---\end{array}$ & $\begin{array}{l}--- \\
---\end{array}$ & $\begin{array}{l}--- \\
--- \\
---\end{array}$ & --- & $\begin{array}{l}4.52 \\
4.60 \\
4.44\end{array}$ & $\begin{array}{l}4.22 \\
4.39 \\
4.11\end{array}$ & $\begin{array}{l}4.06 \\
4.20 \\
3.94\end{array}$ & $\begin{array}{l}3.93 \\
4.03 \\
3.84\end{array}$ \\
\hline
\end{tabular}


LOCATION.--Lat $43^{\circ} 05^{\prime} 42^{\prime \prime}$, long $89^{\circ} 22^{\prime} 12^{\prime \prime}$, in SE $1 / 4 \mathrm{sec} .12$, T.7 N., R.9 E., Dane County, Hydrologic Unit 07090001 , in county boat house at dam at outlet, in Madison.

DRAINAGE AREA.--233 $\mathrm{mi}^{2}$. Area of Lake Mendota, $15.2 \mathrm{mi}^{2}$.

PERIOD OF RECORD.--January 1916 to current year (incomplete).

REVISED RECORDS.--WDR WI-73-1: Drainage area.

GAGE.--Water-stage recorder. Datum of gage is $840.00 \mathrm{ft}$ above sea level, or $5.60 \mathrm{ft}$ below City of Madison datum. Prior to Oct. 1, 1979, at datum $7.82 \mathrm{ft}$ higher; prior to Nov. 15, 1971, nonrecording gage at same site.

REMARKS.--Lake level regulated by concrete dam with two 12-foot gates and 20-foot lock at outlet. Gage-height telemeter at station.

EXTREMES FOR PERIOD OF RECORD.--Maximum gage height observed, $12.75 \mathrm{ft}$, June 5, 2000; minimum observed, $8.02 \mathrm{ft}$, Feb. 24 to Mar. 10, 1920, current datum.

EXTREMES FOR CURRENT YEAR.--Maximum recorded gage height, $11.66 \mathrm{ft}$, Aug. 9; minimum recorded, $8.09 \mathrm{ft}$, Feb. 2, 3.

GAGE HEIGHT, FEET, WATER YEAR OCTOBER 2000 TO SEPTEMBER 2001

DAILY MEAN VALUES

\begin{tabular}{|c|c|c|c|c|c|c|c|c|c|c|c|c|}
\hline DAY & OCT & NOV & DEC & JAN & FEB & MAR & APR & MAY & JUN & JUL & AUG & SEP \\
\hline $\begin{array}{l}1 \\
2 \\
3 \\
4 \\
5\end{array}$ & $\begin{array}{l}10.12 \\
10.12 \\
10.11 \\
10.10 \\
10.09\end{array}$ & $\begin{array}{l}9.65 \\
9.65 \\
9.62 \\
9.58 \\
9.53\end{array}$ & $\begin{array}{l}9.29 \\
9.29 \\
9.27 \\
9.27 \\
9.27\end{array}$ & $\begin{array}{l}9.10 \\
9.08 \\
9.05 \\
9.02 \\
8.98\end{array}$ & $\begin{array}{l}8.14 \\
8.12 \\
8.12 \\
8.14 \\
8.14\end{array}$ & $\begin{array}{l}8.66 \\
8.67 \\
8.68 \\
8.69 \\
8.69\end{array}$ & $\begin{array}{l}9.28 \\
9.30 \\
9.32 \\
9.33 \\
9.36\end{array}$ & $\begin{array}{l}10.01 \\
10.01 \\
10.04 \\
10.06 \\
10.03\end{array}$ & $\begin{array}{l}9.88 \\
9.93 \\
9.92 \\
9.92 \\
9.96\end{array}$ & $\begin{array}{l}10.41 \\
10.34 \\
10.31 \\
10.28 \\
10.22\end{array}$ & $\begin{array}{r}9.95 \\
10.74 \\
11.26 \\
11.38 \\
11.41\end{array}$ & $\begin{array}{l}11.18 \\
11.15 \\
11.13 \\
11.11 \\
11.07\end{array}$ \\
\hline $\begin{array}{r}6 \\
7 \\
8 \\
9 \\
10\end{array}$ & $\begin{array}{r}10.09 \\
10.04 \\
9.99 \\
9.97 \\
9.95\end{array}$ & $\begin{array}{l}9.50 \\
9.56 \\
9.54 \\
9.55 \\
9.53\end{array}$ & $\begin{array}{l}9.24 \\
9.22 \\
9.22 \\
9.20 \\
9.21\end{array}$ & $\begin{array}{l}8.94 \\
8.91 \\
8.88 \\
8.85 \\
8.81\end{array}$ & $\begin{array}{l}8.15 \\
8.15 \\
8.19 \\
8.35 \\
8.41\end{array}$ & $\begin{array}{l}8.70 \\
8.70 \\
8.71 \\
8.72 \\
8.73\end{array}$ & $\begin{array}{l}9.41 \\
9.44 \\
9.45 \\
9.56 \\
9.61\end{array}$ & $\begin{array}{r}10.01 \\
10.02 \\
10.02 \\
9.99 \\
9.99\end{array}$ & $\begin{array}{l}10.02 \\
10.04 \\
10.06 \\
10.08 \\
10.10\end{array}$ & $\begin{array}{l}10.18 \\
10.16 \\
10.16 \\
10.16 \\
10.14\end{array}$ & $\begin{array}{l}11.43 \\
11.43 \\
11.43 \\
11.42 \\
11.38\end{array}$ & $\begin{array}{l}11.04 \\
11.05 \\
11.16 \\
11.21 \\
11.23\end{array}$ \\
\hline $\begin{array}{l}11 \\
12 \\
13 \\
14 \\
15\end{array}$ & $\begin{array}{l}9.93 \\
9.90 \\
9.89 \\
9.88 \\
9.87\end{array}$ & $\begin{array}{l}9.51 \\
9.49 \\
9.49 \\
9.48 \\
9.44\end{array}$ & $\begin{array}{l}9.22 \\
9.22 \\
9.21 \\
9.21 \\
9.21\end{array}$ & $\begin{array}{l}8.77 \\
8.73 \\
8.69 \\
8.68 \\
8.65\end{array}$ & $\begin{array}{l}8.44 \\
8.46 \\
8.48 \\
8.50 \\
8.51\end{array}$ & $\begin{array}{l}8.75 \\
8.77 \\
8.79 \\
8.82 \\
8.86\end{array}$ & $\begin{array}{l}9.67 \\
9.74 \\
9.76 \\
9.78 \\
9.80\end{array}$ & $\begin{array}{r}10.04 \\
10.02 \\
9.99 \\
9.95 \\
9.93\end{array}$ & $\begin{array}{l}10.11 \\
10.34 \\
10.41 \\
10.47 \\
10.65\end{array}$ & $\begin{array}{l}10.10 \\
10.08 \\
10.05 \\
10.03 \\
10.00\end{array}$ & $\begin{array}{l}11.33 \\
11.31 \\
11.29 \\
11.25 \\
11.21\end{array}$ & $\begin{array}{l}11.21 \\
11.20 \\
11.18 \\
11.14 \\
11.12\end{array}$ \\
\hline $\begin{array}{l}16 \\
17 \\
18 \\
19 \\
20\end{array}$ & $\begin{array}{l}9.86 \\
9.84 \\
9.84 \\
9.83 \\
9.81\end{array}$ & $\begin{array}{l}9.46 \\
9.46 \\
9.43 \\
9.41 \\
9.42\end{array}$ & $\begin{array}{l}9.21 \\
9.21 \\
9.21 \\
9.21 \\
9.21\end{array}$ & $\begin{array}{l}8.62 \\
8.58 \\
8.54 \\
8.51 \\
8.46\end{array}$ & $\begin{array}{l}8.52 \\
8.52 \\
8.52 \\
8.53 \\
8.53\end{array}$ & $\begin{array}{l}8.88 \\
8.90 \\
8.92 \\
8.95 \\
8.98\end{array}$ & $\begin{array}{l}9.84 \\
9.84 \\
9.83 \\
9.83 \\
9.89\end{array}$ & $\begin{array}{l}9.92 \\
9.90 \\
9.88 \\
9.84 \\
9.80\end{array}$ & $\begin{array}{l}10.69 \\
10.69 \\
10.71 \\
10.72 \\
10.70\end{array}$ & $\begin{array}{r}9.97 \\
10.01 \\
10.04 \\
10.05 \\
10.05\end{array}$ & $\begin{array}{l}11.21 \\
11.17 \\
11.13 \\
11.11 \\
11.08\end{array}$ & $\begin{array}{l}11.10 \\
11.10 \\
11.11 \\
11.15 \\
11.15\end{array}$ \\
\hline $\begin{array}{l}21 \\
22 \\
23 \\
24 \\
25\end{array}$ & $\begin{array}{l}9.80 \\
9.78 \\
9.77 \\
9.78 \\
9.77\end{array}$ & $\begin{array}{l}9.37 \\
9.34 \\
9.33 \\
9.32 \\
9.31\end{array}$ & $\begin{array}{l}9.21 \\
9.21 \\
9.21 \\
9.21 \\
9.21\end{array}$ & $\begin{array}{l}8.42 \\
8.38 \\
8.34 \\
8.31 \\
8.27\end{array}$ & $\begin{array}{l}8.53 \\
8.54 \\
8.54 \\
8.57 \\
8.61\end{array}$ & $\begin{array}{l}9.02 \\
9.06 \\
9.10 \\
9.13 \\
9.15\end{array}$ & $\begin{array}{r}9.97 \\
10.00 \\
10.03 \\
10.03 \\
10.04\end{array}$ & $\begin{array}{l}9.89 \\
9.92 \\
9.92 \\
9.93 \\
9.92\end{array}$ & $\begin{array}{l}10.69 \\
10.68 \\
10.65 \\
10.62 \\
10.59\end{array}$ & $\begin{array}{l}10.05 \\
10.04 \\
10.04 \\
10.03 \\
10.02\end{array}$ & $\begin{array}{l}11.05 \\
11.05 \\
11.06 \\
11.04 \\
11.15\end{array}$ & $\begin{array}{l}11.17 \\
11.15 \\
11.30 \\
11.38 \\
11.38\end{array}$ \\
\hline $\begin{array}{l}26 \\
27 \\
28 \\
29 \\
30 \\
31\end{array}$ & $\begin{array}{l}9.77 \\
9.77 \\
9.75 \\
9.72 \\
9.68 \\
9.67\end{array}$ & $\begin{array}{l}9.31 \\
9.30 \\
9.29 \\
9.30 \\
9.30 \\
-.-\end{array}$ & $\begin{array}{l}9.21 \\
9.21 \\
9.19 \\
9.17 \\
9.15 \\
9.13\end{array}$ & $\begin{array}{l}8.24 \\
8.20 \\
8.16 \\
8.15 \\
8.19 \\
8.17\end{array}$ & $\begin{array}{r}8.63 \\
8.64 \\
8.65 \\
-.- \\
\ldots- \\
\ldots-\end{array}$ & $\begin{array}{l}9.16 \\
9.18 \\
9.19 \\
9.21 \\
9.23 \\
9.25\end{array}$ & $\begin{array}{r}10.05 \\
10.05 \\
10.03 \\
10.01 \\
10.00 \\
\end{array}$ & $\begin{array}{l}9.90 \\
9.89 \\
9.87 \\
9.85 \\
9.84 \\
9.83\end{array}$ & $\begin{array}{c}10.57 \\
10.54 \\
10.51 \\
10.48 \\
10.45 \\
\\
.--\end{array}$ & $\begin{array}{l}9.99 \\
9.96 \\
9.94 \\
9.95 \\
9.94 \\
9.93\end{array}$ & $\begin{array}{l}11.30 \\
11.31 \\
11.30 \\
11.27 \\
11.25 \\
11.22\end{array}$ & $\begin{array}{r}11.36 \\
11.33 \\
11.30 \\
11.28 \\
11.25 \\
-\end{array}$ \\
\hline $\begin{array}{l}\text { MEAN } \\
\text { MAX } \\
\text { MIN }\end{array}$ & $\begin{array}{r}9.89 \\
10.12 \\
9.67\end{array}$ & $\begin{array}{l}9.45 \\
9.65 \\
9.29\end{array}$ & $\begin{array}{l}9.22 \\
9.29 \\
9.13\end{array}$ & $\begin{array}{l}8.60 \\
9.10 \\
8.15\end{array}$ & $\begin{array}{l}8.42 \\
8.65 \\
8.12\end{array}$ & $\begin{array}{l}8.91 \\
9.25 \\
8.66\end{array}$ & $\begin{array}{r}9.74 \\
10.05 \\
9.28\end{array}$ & $\begin{array}{r}9.94 \\
10.06 \\
9.80\end{array}$ & $\begin{array}{r}10.37 \\
10.72 \\
9.88\end{array}$ & $\begin{array}{r}10.08 \\
10.41 \\
9.93\end{array}$ & $\begin{array}{r}11.19 \\
11.43 \\
9.95\end{array}$ & $\begin{array}{l}11.19 \\
11.38 \\
11.04\end{array}$ \\
\hline
\end{tabular}


LOCATION.--Lat $43^{\circ} 03^{\prime} 09^{\prime \prime}$, long $88^{\circ} 28^{\prime} 48^{\prime \prime}$, in NW 1/4 SW 1/4 sec.22, T.7 N., R.17 E., Waukesha County, Hydrologic Unit 07090001 , 1.8 mi south of Oconomowoc.

PERIOD OF RECORD.--February 1996 to current year.

REMARKS.--Lake sampled near center at the deep hole. Lake ice-covered during February sampling. Water-quality analyses done by Wisconsin State Laboratory of Hygiene.

WATER-QUALITY DATA, FEBRUARY 12 TO AUGUST 15, 2001

(Milligrams per liter unless otherwise indicated)

Lake stage (ft)

Chlorophyll a, phytoplankton $(\mu \mathrm{g} / \mathrm{L})$

Depth of sample (m)

Water temoerature $\left({ }^{\circ} \mathrm{C}\right)$

Specific conductance $(\mu \mathrm{s} / \mathrm{cm})$

$\mathrm{pH}$ (units)

Dissolved oxygen (mg/L)

Phosphorus, total (as P)

Phosphorus, ortho, dissolved (as P)

Nitrogen, $\mathrm{NO} 2+\mathrm{NO} 3$, diss. (as N)

Nitrogen, anmonia, dissolved (as N)

Nitrogen, amm. + org. total (as N)

Nitrogen, total (as N)

Color (Pt-Co. scale)

Turbidity (NTU)

Hardness, (as $\mathrm{CaCO}_{3}$ )

Calcium, dissolved ( $\mathrm{Ca})$

Magnesium, dissolved (Mg)

Sodium, dissolved (Na)

Potassium, dissolved (K)

Alkalinity, (as $\mathrm{CaCO}_{3}$ )

Sulfate, dissolved $\left(\mathrm{SO}_{4}\right)$

Chloride, dissolved (CI)

Silica, dissolved $\left(\mathrm{SiO}_{2}\right)$

Solids. dissolved, at $180^{\circ} \mathrm{C}$

Iron, dissolved (Fe) $\mu \mathrm{g} / \mathrm{L}$

Manganese, dissolved (Mn) $\mu \mathrm{g} / \mathrm{L}$
Feb-12

\begin{tabular}{cc}
\multicolumn{2}{c}{ Feb-12 } \\
& -1 \\
0.5 & 12.0 \\
2.3 & 5.3 \\
405 & 506 \\
8.1 & 7.3 \\
12.9 & 0.5 \\
0.008 & 0.024 \\
& -
\end{tabular}

2-12-01

4-26-01

6-13-01

7-17-01

8-15-01

Jun -13

Jul-17

\begin{tabular}{|c|c|c|c|c|c|c|c|}
\hline \multicolumn{2}{|c|}{$\begin{array}{c}867.07 \\
2.7 \\
4.8\end{array}$} & \multicolumn{2}{|c|}{$\begin{array}{c}867.18 \\
3.1 \\
4.8\end{array}$} & \multicolumn{2}{|c|}{$\begin{array}{c}866.79 \\
2.3 \\
2.7\end{array}$} & \multicolumn{2}{|c|}{$\begin{array}{c}866.35 \\
3.0 \\
---\end{array}$} \\
\hline $\begin{array}{c}0.5 \\
12.9 \\
410 \\
8.2 \\
11.2 \\
0.008\end{array}$ & $\begin{array}{c}11.0 \\
10.3 \\
412 \\
8.0 \\
9.4 \\
0.009\end{array}$ & $\begin{array}{c}0.5 \\
22.1 \\
409 \\
7.9 \\
9.7 \\
0.012\end{array}$ & $\begin{array}{c}12.0 \\
11.7 \\
442 \\
7.3 \\
0.3 \\
0.031\end{array}$ & $\begin{array}{c}0.5 \\
26.9 \\
415 \\
8.1 \\
8.9 \\
0.011\end{array}$ & $\begin{array}{c}11.5 \\
12.0 \\
480 \\
7.3 \\
0.2 \\
0.050\end{array}$ & $\begin{array}{c}0.5 \\
25.9 \\
404 \\
8.2 \\
9.0 \\
0.010\end{array}$ & $\begin{array}{c}12.0 \\
12.2 \\
532 \\
7.1 \\
0.2 \\
0.068\end{array}$ \\
\hline$<0.002$ & -- & -- & --- & --- & -- & -- & --- \\
\hline 0.114 & --- & $\cdots$ & --- & $-\infty$ & --- & --- & --- \\
\hline 0.12 & -- & --- & --- & --- & --- & --- & --- \\
\hline 0.91 & --- & -- & --- & $\cdots$ & -- & --- & -- \\
\hline 1.02 & -- & --- & $-\cdots$ & --- & --- & -- & -- \\
\hline 10 & -- & --- & --- & -- & $\cdots$ & -- & --- \\
\hline 1.9 & -- & $\cdots$ & --- & -- & $\cdots$ & -- & -- \\
\hline 187 & $-\cdots$ & -- & --- & $\cdots$ & -- & $\ldots$ & $-\cdots$ \\
\hline 32 & --- & --- & $-\cdots$ & -- & $\cdots$ & $-\infty$ & -- \\
\hline 26 & $\cdots$ & $-\cdots$ & --- & -- & $\cdots$ & -- & -- \\
\hline 11 & $-\cdots$ & --- & --- & -- & $-\cdots$ & -- & --- \\
\hline 1.2 & --- & --- & $\cdots$ & --- & $\cdots$ & $\cdots$ & -- \\
\hline 160 & $\cdots$ & $\cdots$ & $-\cdots$ & --- & --- & $\cdots$ & $-\cdots$ \\
\hline 14.1 & --- & --- & -- & --- & -- & --- & -- \\
\hline 26 & $\cdots$ & -- & -- & $\cdots$ & --- & -- & -- \\
\hline 1.0 & -- & -- & --- & $-\cdots$ & --- & -- & --- \\
\hline 244 & $\rightarrow$ & --- & --- & -- & $\cdots$ & -- & -- \\
\hline$<10$ & -- & -- & $\cdots$ & -- & $\cdots$ & --- & -- \\
\hline$<0.4$ & --- & --- & --- & $\cdots$ & $\cdots$ & --- & --- \\
\hline
\end{tabular}
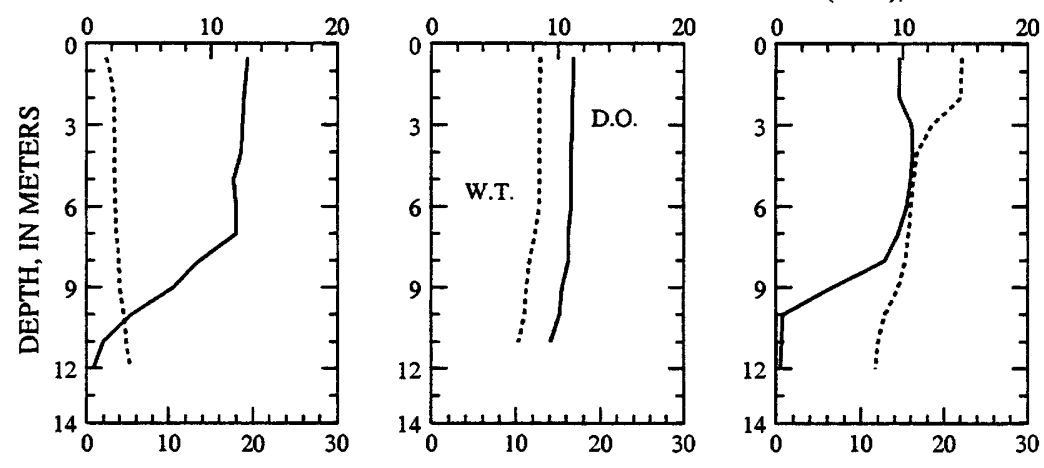

Aug-15

DISSOLVED OXYGEN (D.O.), IN MILLIGRAMS PER LITER

WATER TEMPERATURE (W.T.), IN DEGREES CELSIUS
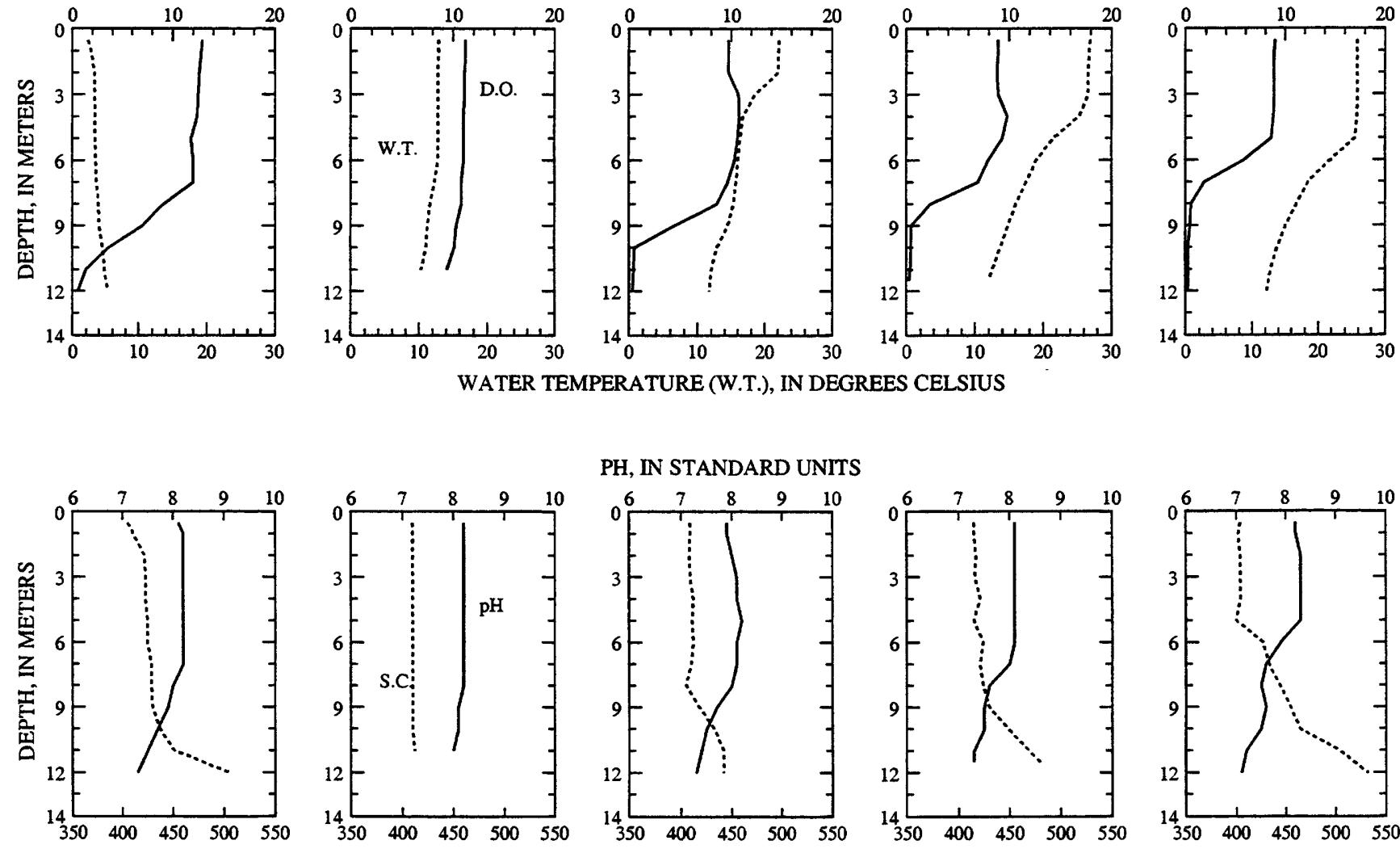

PH, IN STANDARD UNITS
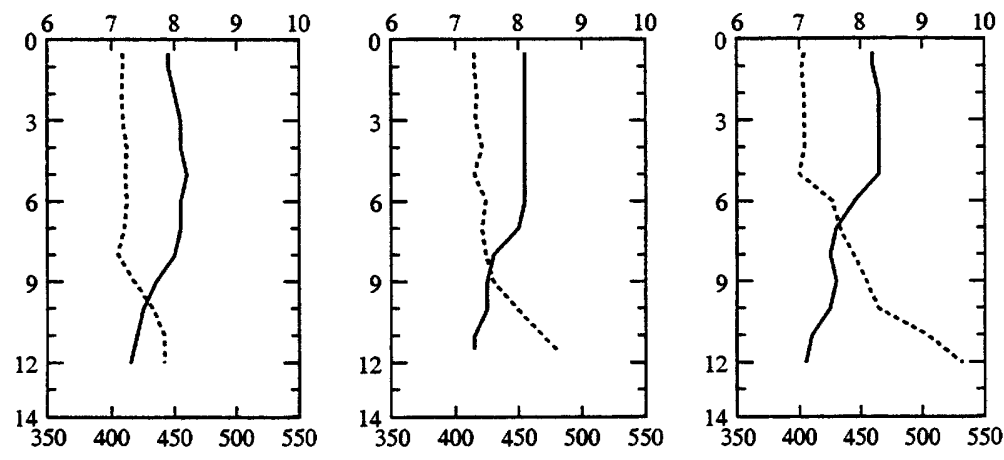

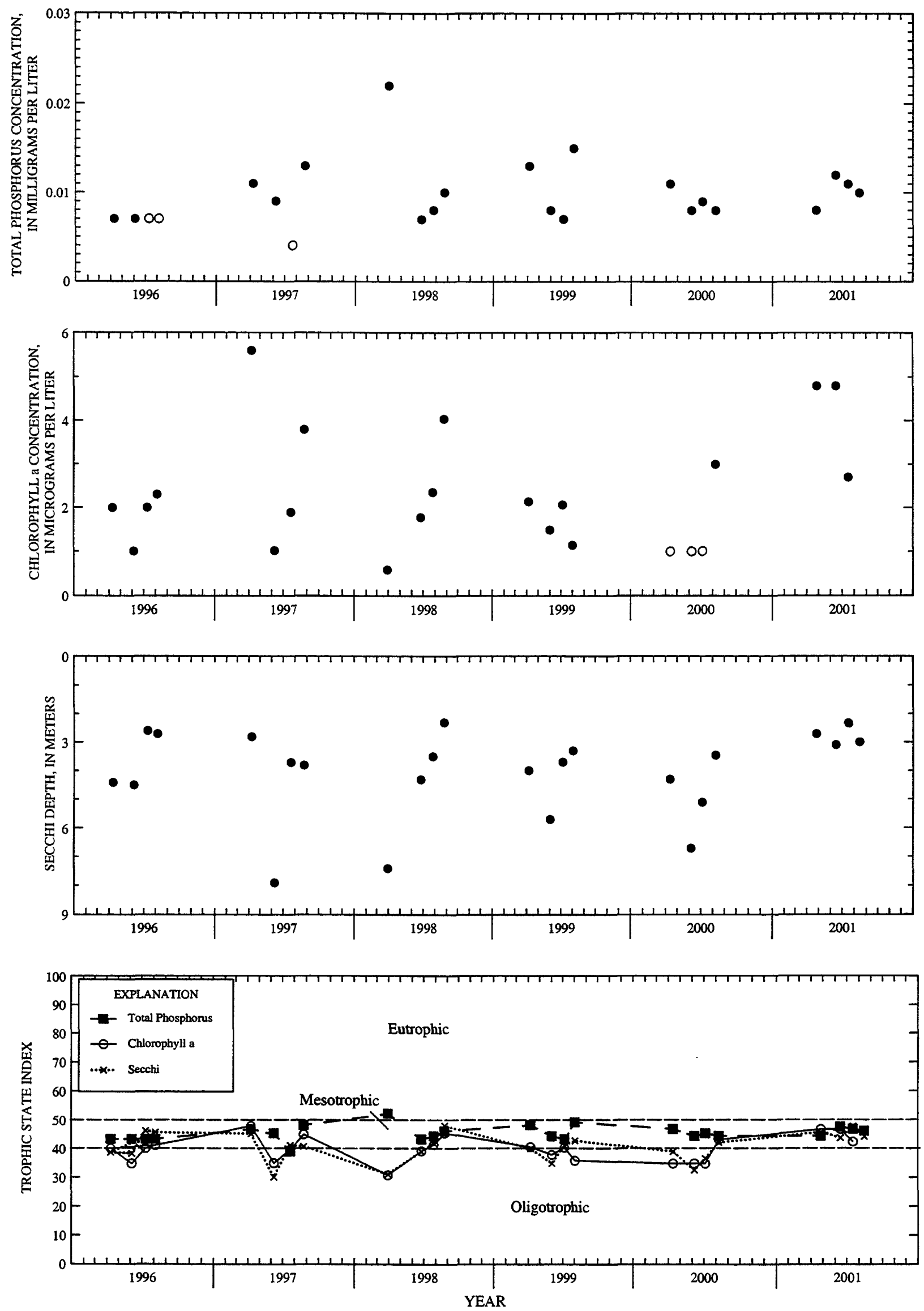

Surface total phosphorus, chlorophyll a concentrations, Secchi depths, and TSI data for Middle Genesee Lake, near Oconomowoc, Wisconsin.

(Circles on the first three plots indicate laboratory detection limit for selected analyses. Actual concentrations for these particular analyses are less than the plotted circles.) 
LOCATION.--Lat 4303'48", long 89²3'49', in SW 1/4 sec.23, T.7 N., R.9 E., Dane County, Hydrologic Unit 07090001, in Brittingham Park, in Madison.

DRAINAGE AREA.--279 $\mathrm{mi}^{2}$. Area of Lake Monona, $5.3 \mathrm{mi}^{2}$.

PERIOD OF RECORD.--September 1915 to current year (fragmentary) in reports of the Geological Survey. For 1856 to March 1917 in reports of Wisconsin Railroad Commission, volume 19.

REVISED RECORDS.--WSP 1338: Lake area. WDR WI-73-1: Drainage area.

GAGE.--Water-stage recorder. Datum of gage is $840.00 \mathrm{ft}$ above sea level, or $5.60 \mathrm{ft}$ below City of Madison datum. Prior to Oct. 1,1979 , datum $3.61 \mathrm{ft}$ higher; prior to Nov. 15, 1971, nonrecording gage at same site at the higher datum.

REMARKS.--Lake level regulated by concrete dam with four 12-foot stop-log sections and 12-foot lock at outlet of Lake Waubesa. Gageheight telemeter at station.

EXTREMES FOR PERIOD OF RECORD.--Maximum gage height observed, $7.48 \mathrm{ft}$, June 14, 15, 2000; minimum observed, $3.22 \mathrm{ft}$, Jan. 20 , 1965 , current datum.

EXTREMES FOR CURRENT YEAR.--Maximum recorded gage height, $6.58 \mathrm{ft}$, Aug. 9; minimum recorded, 4.08, Apr. 7.

GAGE HEIGHT, FEET, WATER YEAR OCTOBER 2000 TO SEPTEMBER 2001

DAILY MEAN VALUES

\begin{tabular}{|c|c|c|c|c|c|c|c|c|c|c|c|c|}
\hline DAY & OCT & NOV & $\mathrm{DEC}$ & JAN & EEB & MAR & APR & MAY & JUN & JUL & AUG & SEP \\
\hline $\begin{array}{l}1 \\
2 \\
3 \\
4 \\
5\end{array}$ & $\begin{array}{l}5.52 \\
5.49 \\
5.45 \\
5.42 \\
5.39\end{array}$ & $\begin{array}{l}4.55 \\
4.53 \\
4.53 \\
4.54 \\
4.55\end{array}$ & $\begin{array}{l}4.27 \\
4.25 \\
4.24 \\
4.23 \\
4.21\end{array}$ & $\begin{array}{l}4.51 \\
4.53 \\
4.55 \\
4.58 \\
4.63\end{array}$ & $\begin{array}{l}5.07 \\
5.04 \\
4.96 \\
4.88 \\
4.80\end{array}$ & $\begin{array}{l}4.29 \\
4.27 \\
4.27 \\
4.26 \\
4.24\end{array}$ & $\begin{array}{l}4.14 \\
4.13 \\
4.13 \\
4.13 \\
4.14\end{array}$ & $\begin{array}{l}4.59 \\
4.60 \\
4.64 \\
4.71 \\
4.76\end{array}$ & $\begin{array}{l}5.22 \\
5.22 \\
5.19 \\
5.17 \\
5.20\end{array}$ & $\begin{array}{l}5.89 \\
5.86 \\
5.84 \\
5.82 \\
5.80\end{array}$ & $\begin{array}{l}5.42 \\
6.21 \\
6.46 \\
6.52 \\
6.54\end{array}$ & $\begin{array}{l}6.28 \\
6.24 \\
6.21 \\
6.18 \\
6.15\end{array}$ \\
\hline $\begin{array}{r}6 \\
7 \\
8 \\
9 \\
10\end{array}$ & $\begin{array}{l}5.32 \\
5.26 \\
5.21 \\
5.16 \\
5.13\end{array}$ & $\begin{array}{l}4.62 \\
4.71 \\
4.70 \\
4.70 \\
4.64\end{array}$ & $\begin{array}{l}4.19 \\
4.20 \\
4.20 \\
4.20 \\
4.20\end{array}$ & $\begin{array}{l}4.68 \\
4.72 \\
4.75 \\
4.79 \\
4.81\end{array}$ & $\begin{array}{l}4.74 \\
4.68 \\
4.66 \\
4.82 \\
4.86\end{array}$ & $\begin{array}{l}4.22 \\
4.21 \\
4.20 \\
4.18 \\
4.18\end{array}$ & $\begin{array}{l}4.18 \\
4.17 \\
4.21 \\
4.39 \\
4.46\end{array}$ & $\begin{array}{l}4.79 \\
4.84 \\
4.87 \\
4.89 \\
4.95\end{array}$ & $\begin{array}{l}5.22 \\
5.20 \\
5.19 \\
5.18 \\
5.18\end{array}$ & $\begin{array}{l}5.77 \\
5.72 \\
5.69 \\
5.66 \\
5.62\end{array}$ & $\begin{array}{l}6.55 \\
6.56 \\
6.56 \\
6.55 \\
6.55\end{array}$ & $\begin{array}{l}6.11 \\
6.12 \\
6.26 \\
6.28 \\
6.30\end{array}$ \\
\hline $\begin{array}{l}11 \\
12 \\
13 \\
14 \\
15\end{array}$ & $\begin{array}{l}5.10 \\
5.07 \\
5.04 \\
5.01 \\
4.98\end{array}$ & $\begin{array}{l}4.61 \\
4.59 \\
4.55 \\
4.51 \\
4.48\end{array}$ & $\begin{array}{l}4.22 \\
4.23 \\
4.24 \\
4.26 \\
4.26\end{array}$ & $\begin{array}{l}4.84 \\
4.86 \\
4.87 \\
4.91 \\
4.93\end{array}$ & $\begin{array}{l}4.83 \\
4.78 \\
4.73 \\
4.69 \\
4.65\end{array}$ & $\begin{array}{l}4.18 \\
4.20 \\
4.21 \\
4.21 \\
4.22\end{array}$ & $\begin{array}{l}4.53 \\
4.56 \\
4.56 \\
4.55 \\
4.54\end{array}$ & $\begin{array}{l}5.06 \\
5.09 \\
5.09 \\
5.11 \\
5.12\end{array}$ & $\begin{array}{l}5.19 \\
5.45 \\
5.54 \\
5.62 \\
5.73\end{array}$ & $\begin{array}{l}5.58 \\
5.55 \\
5.52 \\
5.49 \\
5.46\end{array}$ & $\begin{array}{l}6.52 \\
6.49 \\
6.48 \\
6.43 \\
6.40\end{array}$ & $\begin{array}{l}6.28 \\
6.25 \\
6.22 \\
6.18 \\
6.13\end{array}$ \\
\hline $\begin{array}{l}16 \\
17 \\
18 \\
19 \\
20\end{array}$ & $\begin{array}{l}4.95 \\
4.92 \\
4.89 \\
4.85 \\
4.82\end{array}$ & $\begin{array}{l}4.48 \\
4.45 \\
4.42 \\
4.40 \\
4.35\end{array}$ & $\begin{array}{l}4.27 \\
4.26 \\
4.27 \\
4.29 \\
4.30\end{array}$ & $\begin{array}{l}4.94 \\
4.94 \\
4.95 \\
4.95 \\
4.95\end{array}$ & $\begin{array}{l}4.60 \\
4.56 \\
4.52 \\
4.48 \\
4.43\end{array}$ & $\begin{array}{l}4.22 \\
4.21 \\
4.21 \\
4.21 \\
4.21\end{array}$ & $\begin{array}{l}4.51 \\
4.49 \\
4.49 \\
4.49 \\
4.55\end{array}$ & $\begin{array}{l}5.13 \\
5.13 \\
5.14 \\
5.13 \\
5.14\end{array}$ & $\begin{array}{l}5.78 \\
5.81 \\
5.91 \\
5.93 \\
5.94\end{array}$ & $\begin{array}{l}5.44 \\
5.47 \\
5.49 \\
5.47 \\
5.46\end{array}$ & $\begin{array}{l}6.38 \\
6.34 \\
6.31 \\
6.28 \\
6.25\end{array}$ & $\begin{array}{l}6.10 \\
6.09 \\
6.09 \\
6.10 \\
6.08\end{array}$ \\
\hline $\begin{array}{l}21 \\
22 \\
23 \\
24 \\
25\end{array}$ & $\begin{array}{l}4.80 \\
4.76 \\
4.75 \\
4.74 \\
4.72\end{array}$ & $\begin{array}{l}4.34 \\
4.33 \\
4.32 \\
4.31 \\
4.30\end{array}$ & $\begin{array}{l}4.31 \\
4.31 \\
4.32 \\
4.32 \\
4.32\end{array}$ & $\begin{array}{l}4.95 \\
4.95 \\
4.95 \\
4.94 \\
4.94\end{array}$ & $\begin{array}{l}4.39 \\
4.37 \\
4.33 \\
4.33 \\
4.38\end{array}$ & $\begin{array}{l}4.21 \\
4.21 \\
4.20 \\
4.18 \\
4.16\end{array}$ & $\begin{array}{l}4.58 \\
4.62 \\
4.61 \\
4.58 \\
4.57\end{array}$ & $\begin{array}{l}5.24 \\
5.27 \\
5.32 \\
5.34 \\
5.34\end{array}$ & $\begin{array}{l}5.94 \\
5.94 \\
5.94 \\
5.94 \\
5.93\end{array}$ & $\begin{array}{l}5.45 \\
5.48 \\
5.52 \\
5.51 \\
5.51\end{array}$ & $\begin{array}{l}6.22 \\
6.22 \\
6.22 \\
6.19 \\
6.29\end{array}$ & $\begin{array}{l}6.08 \\
6.06 \\
6.23 \\
6.32 \\
6.29\end{array}$ \\
\hline $\begin{array}{l}26 \\
27 \\
28 \\
29 \\
30 \\
31\end{array}$ & $\begin{array}{l}4.70 \\
4.67 \\
4.65 \\
4.61 \\
4.59 \\
4.56\end{array}$ & $\begin{array}{c}4.29 \\
4.28 \\
4.27 \\
4.28 \\
4.28 \\
---\end{array}$ & $\begin{array}{l}4.31 \\
4.32 \\
4.36 \\
4.42 \\
4.46 \\
4.49\end{array}$ & $\begin{array}{l}4.94 \\
4.94 \\
4.93 \\
4.96 \\
5.07 \\
5.07\end{array}$ & $\begin{array}{r}4.37 \\
4.34 \\
4.32 \\
--- \\
--- \\
---\end{array}$ & $\begin{array}{l}4.14 \\
4.13 \\
4.13 \\
4.13 \\
4.13 \\
4.13\end{array}$ & $\begin{array}{r}4.56 \\
4.55 \\
4.57 \\
4.58 \\
4.58 \\
---\end{array}$ & $\begin{array}{l}5.35 \\
5.34 \\
5.34 \\
5.32 \\
5.30 \\
5.24\end{array}$ & $\begin{array}{l}5.93 \\
5.93 \\
5.92 \\
5.91 \\
5.90 \\
\ldots\end{array}$ & $\begin{array}{l}5.47 \\
5.44 \\
5.42 \\
5.42 \\
5.42 \\
5.41\end{array}$ & $\begin{array}{l}6.44 \\
6.43 \\
6.41 \\
6.37 \\
6.34 \\
6.31\end{array}$ & $\begin{array}{l}6.24 \\
6.21 \\
6.19 \\
6.17 \\
6.15 \\
\end{array}$ \\
\hline $\begin{array}{l}\text { MEAN } \\
\text { MAX } \\
\text { MIN }\end{array}$ & $\begin{array}{l}4.98 \\
5.52 \\
4.56\end{array}$ & $\begin{array}{l}4.46 \\
4.71 \\
4.27\end{array}$ & $\begin{array}{l}4.28 \\
4.49 \\
4.19\end{array}$ & $\begin{array}{l}4.85 \\
5.07 \\
4.51\end{array}$ & $\begin{array}{l}4.63 \\
5.07 \\
4.32\end{array}$ & $\begin{array}{l}4.20 \\
4.29 \\
4.13\end{array}$ & $\begin{array}{l}4.44 \\
4.62 \\
4.13\end{array}$ & $\begin{array}{l}5.07 \\
5.35 \\
4.59\end{array}$ & $\begin{array}{l}5.61 \\
5.94 \\
5.17\end{array}$ & $\begin{array}{l}5.57 \\
5.89 \\
5.41\end{array}$ & $\begin{array}{l}6.36 \\
6.56 \\
5.42\end{array}$ & $\begin{array}{l}6.19 \\
6.32 \\
6.06\end{array}$ \\
\hline
\end{tabular}


LOCATION.--Lat $42^{\circ} 53^{\prime} 44^{\prime \prime}$, long $88^{\circ} 07^{\prime} 01^{\prime \prime}$, in SW 1/4 NE 1/4 sec.15, T.5 N., R.20 E., Waukesha County, Hydrologic Unit 07120006, 1.3 mi southeast of Muskego.

PERIOD OF RECORD.--February 1988 to current year. Prior to October 2000, publsihed as "Big Muskego Lake".

REMARKS.--Lake sampled near center at the deep hole. Lake ice-covered during January and February sampling. Water-quality analyses done by Wisconsin State Laboratory of Hygiene.

WATER-QUALITY DATA, JANUARY 10 TO APRIL 19, 2001

(Milligrams per liter unless otherwise indicated)

\begin{tabular}{|c|c|c|c|c|c|c|}
\hline & \multicolumn{2}{|c|}{$\operatorname{Jan}-10$} & \multicolumn{2}{|c|}{ Feb-13 } & \multicolumn{2}{|c|}{ Apr-19 } \\
\hline $\begin{array}{l}\text { Lake stage ( } \mathrm{ft}) \\
\text { Secchi-depth }(\mathrm{m}) \\
\text { Chlorophyll a, phytoplankton }(\mu \mathrm{g} / \mathrm{L})\end{array}$ & & & & & & \\
\hline Depth of sample (m) & 0.5 & 7.2 & 0.5 & 7.0 & 0.5 & 7.0 \\
\hline Water temoerature $\left({ }^{\circ} \mathrm{C}\right)$ & 1.1 & 3.6 & 1.3 & 3.2 & 10.0 & 9.1 \\
\hline $\begin{array}{l}\text { Specific conductance ( } \mu \mathrm{S} / \mathrm{cm}) \\
\mathrm{pH} \text { (units) }\end{array}$ & 7.9 & --- & $\begin{array}{l}501 \\
7.8\end{array}$ & $\begin{array}{l}940 \\
7.3\end{array}$ & $\begin{array}{l}577 \\
8.1\end{array}$ & 579 \\
\hline Dissolved oxygen (mg/L) & 10.9 & 0.7 & 9.9 & 2.6 & 11.7 & 8.2 \\
\hline Phosphorus, total (as P) & -- & --- & 0.296 & 0.051 & 0.031 & 0.032 \\
\hline Phosphorus, ortho, dissolved (as P: & -- & -- & -- & - & 0.002 & -- \\
\hline Nitrogen, $\mathrm{NO} 2+\mathrm{NO} 3$, diss. (as N) & -- & -- & --- & -- & 0.376 & --- \\
\hline Nitrogen, anmonia, dissolved (as N; & -- & --- & --- & -- & $<0.013$ & --- \\
\hline Nitrogen, anm. + org., total (as N; & --- & -- & --- & $-\infty$ & 0.78 & -- \\
\hline Nitrogen, total (as N) & -- & -- & $-\ldots$ & -- & 1.16 & --- \\
\hline Color (Pt-Co. scale) & --- & -- & -- & -- & 25 & --- \\
\hline Turbidity (NTU) & --- & -.- & -- & $\ldots$ & 4.2 & -- \\
\hline Hardness, (as $\mathrm{CaCO}_{3}$ ) & --- & $-\ldots$ & -- & --- & 234 & --- \\
\hline Calcium, dissolved (Ca) & -- & -- & -- & -- & 51 & -- \\
\hline Magnesium, dissolved (Mg) & --- & -- & -- & -- & 26 & -- \\
\hline Sodium, dissolved (Na) & -.- & --- & -.. & -.- & 26 & -- \\
\hline Potassium, dissolved (K) & -- & -- & -- & -- & 2.1 & -- \\
\hline Alkalinity, (as $\mathrm{CaCO}_{3}$ ) & $\ldots$ & --- & -- & - - & 172 & $\ldots$ \\
\hline Sulfate, dissolved $\left(\mathrm{SO}_{4}\right)$ & --- & -- & --- & -- & 36.5 & --- \\
\hline Chloride, dissolved (Cl) & --- & -- & --- & --- & 57.7 & --- \\
\hline Silica, dissolved $\left(\mathrm{SiO}_{2}\right)$ & --- & -- & --- & --- & 0.8 & -- \\
\hline Solids, dissolved, at $180^{\circ} \mathrm{C}$ & --- & -- & -- & --- & 340 & -- \\
\hline dissolved (Fe) $\mu \mathrm{g} / \mathrm{L}$ & -- & -- & -- & -- & $<10$ & -- \\
\hline Manganese, dissolved (Mn) $\mu \mathrm{g} / \mathrm{L}$ & --- & $\ldots$ & $\ldots$ & -- & 0.4 & -- \\
\hline
\end{tabular}
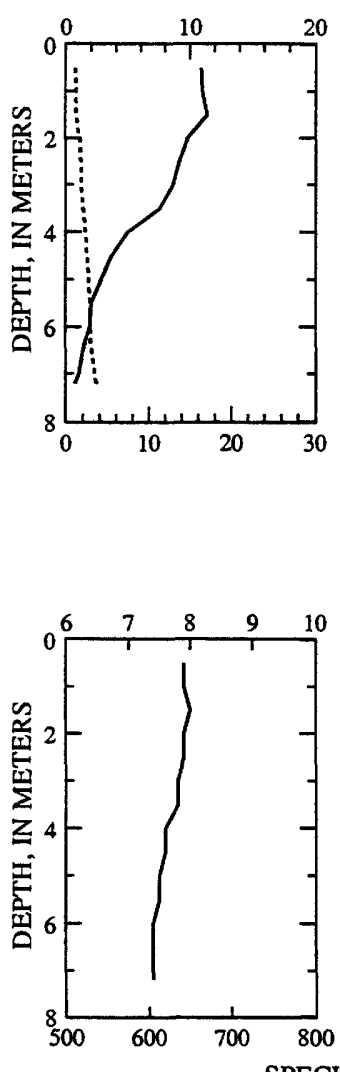

DISSOLVED OXYGEN (D.O.), IN MLLIGRAMS PER LITER
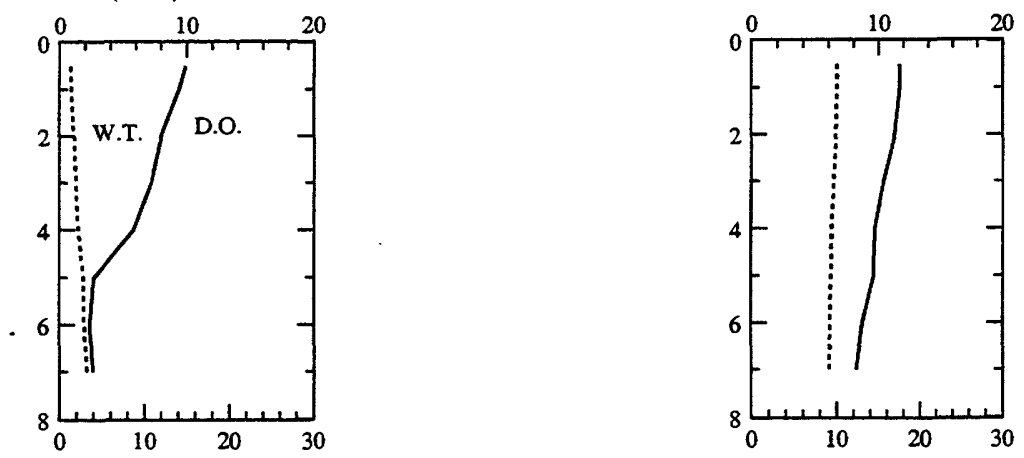

WATER TEMPERATURE (W.T.), IN DEGREES CELSIUS

PH, IN STANDARD UNITS
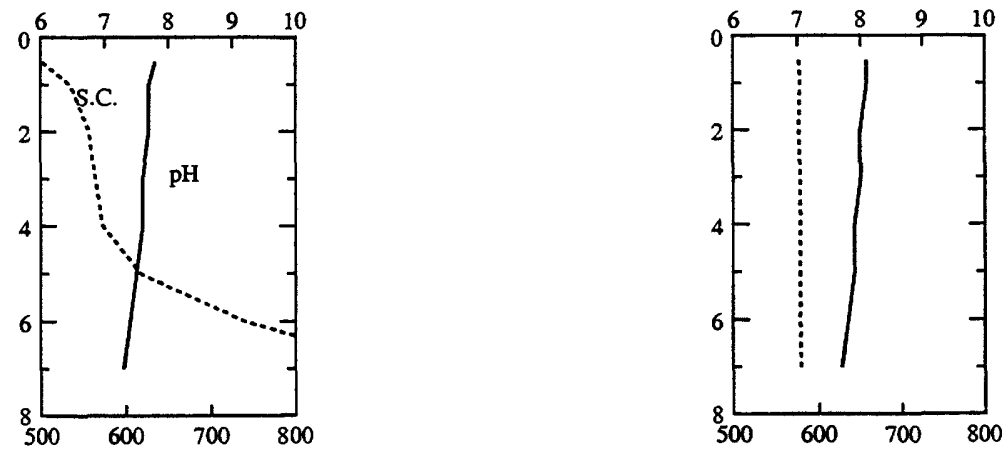

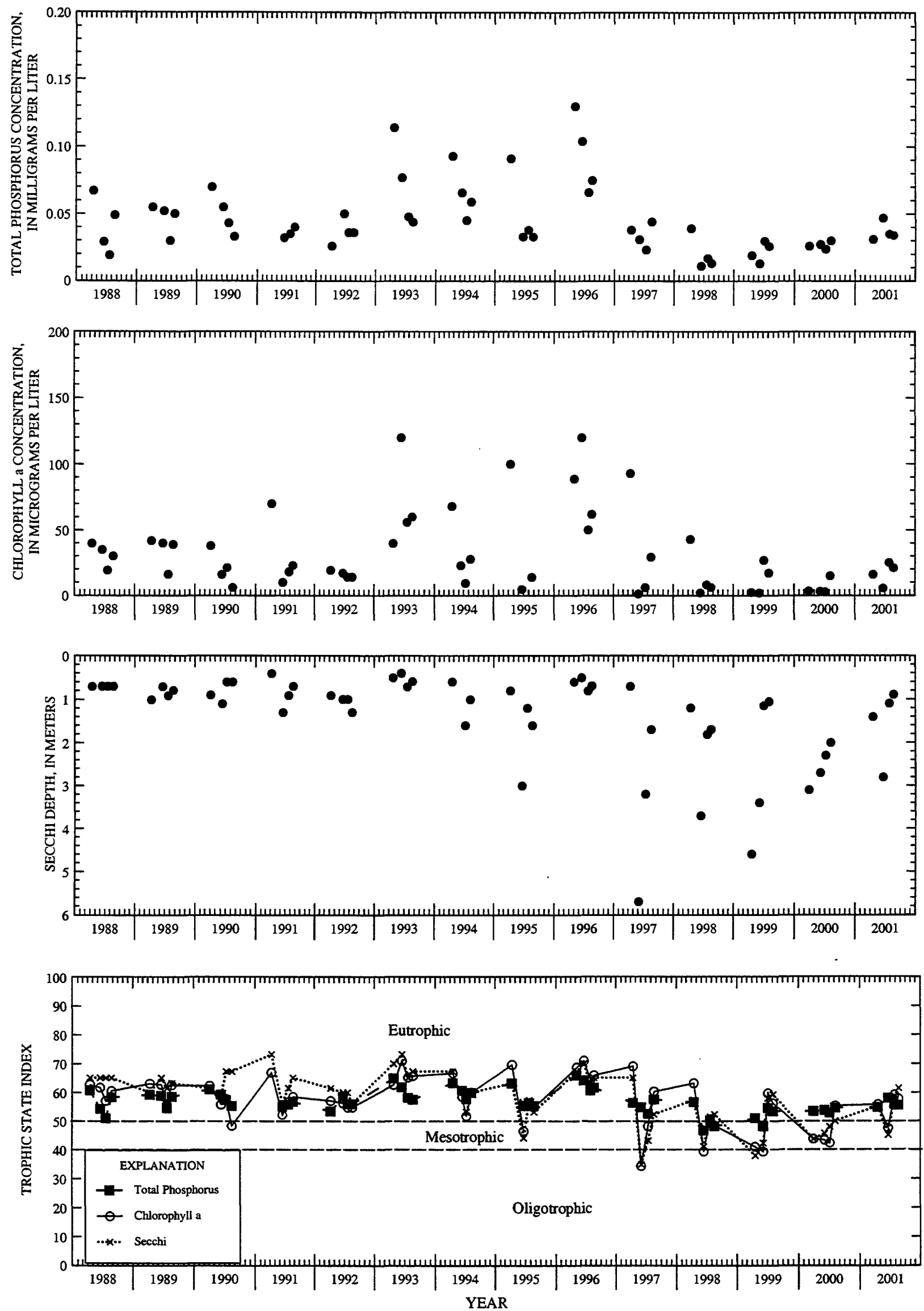

Surface total phosphorus, chlorophyll a concentrations, Secchi depths, and TSI data for Muskego (Big Muskego) Lake, Bass Bay, near Muskego, Wisconsin. 


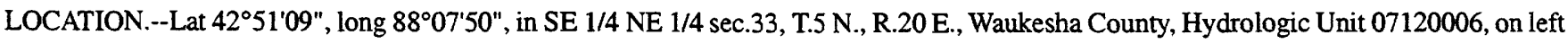
bank $8 \mathrm{ft}$ upstream of dam outlet of Muskego Lake, $700 \mathrm{ft}$ north of Muskego Dam Drive, 2 mi northeast of Wind Lake.

DRAINAGE AREA.--33.9 $\mathrm{mi}^{2}$ (revised).

PERIOD OF RECORD.--October 1987 to September 1989, January 1991 to current year. Prior to October 1993, published as Muskego Lake Outlet near Wind Lake, WI. October 1993 to September 2000, published as "Big Muskego Lake".

GAGE.--Water-stage recorder. Datum of gage is $760.00 \mathrm{ft}$ above sea level. October to December 1987 and January 1991 to September 1995 , nonrecording gage at the same datum. December 1987 through September 1989, data collected using water-stage recorder at the same datum.

REMARKS.--Lake levels regulated by concrete dam with one 5-ft lift gate.

EXTREMES FOR PERIOD OF RECORD.--Maximum observed gage height, $12.60 \mathrm{ft}$, Oct. 7, 1991 and Aug. 8, 1994; minimum instantaneous, less than $8.72 \mathrm{ft}$, July 12, 1996 to Feb. 18, 1997, due to drawdown of lake.

EXTREMES FOR CURRENT YEAR.--Maximum observed gage-height, $12.18 \mathrm{ft}$, Feb. 11; minimum observed, $10.73 \mathrm{ft}$, Aug. 15.

GAGE HEIGHT, FEET, WATER YEAR OCTOBER 2000 TO SEPTEMBER 2001

DAILY MEAN VALUES

\begin{tabular}{|c|c|c|c|c|c|c|c|c|c|c|c|c|}
\hline DAY & OCT & NOV & $\mathrm{DEC}$ & JAN & FEB & MAR & APR & MAY & JUN & JUL & AUG & SEP \\
\hline $\begin{array}{l}1 \\
2 \\
3 \\
4 \\
5\end{array}$ & $\begin{array}{l}11.45 \\
11.42 \\
11.40 \\
11.38 \\
11.34\end{array}$ & $\begin{array}{l}11.26 \\
11.25 \\
11.22 \\
11.22 \\
11.20\end{array}$ & $\begin{array}{l}11.31 \\
11.32 \\
11.33 \\
11.33 \\
11.35\end{array}$ & $\begin{array}{l}11.65 \\
11.65 \\
11.65 \\
11.66 \\
11.66\end{array}$ & $\begin{array}{l}11.84 \\
11.84 \\
11.84 \\
11.84 \\
11.83\end{array}$ & $\begin{array}{l}11.87 \\
11.86 \\
11.89 \\
11.92 \\
11.93\end{array}$ & $\begin{array}{l}11.72 \\
11.71 \\
11.73 \\
11.68 \\
11.67\end{array}$ & $\begin{array}{l}11.42 \\
11.38 \\
11.40 \\
11.41 \\
11.33\end{array}$ & $\begin{array}{l}11.40 \\
11.46 \\
11.49 \\
11.51 \\
11.56\end{array}$ & $\begin{array}{l}11.43 \\
11.37 \\
11.34 \\
11.36 \\
11.35\end{array}$ & $\begin{array}{l}10.95 \\
11.00 \\
11.02 \\
11.00 \\
10.96\end{array}$ & $\begin{array}{l}10.91 \\
10.88 \\
10.86 \\
10.97 \\
11.04\end{array}$ \\
\hline $\begin{array}{r}6 \\
7 \\
8 \\
9 \\
10\end{array}$ & $\begin{array}{l}11.28 \\
11.23 \\
11.21 \\
11.19 \\
11.19\end{array}$ & $\begin{array}{l}11.21 \\
11.25 \\
11.28 \\
11.38 \\
11.38\end{array}$ & $\begin{array}{l}11.35 \\
11.37 \\
11.38 \\
11.38 \\
11.40\end{array}$ & $\begin{array}{l}11.67 \\
11.68 \\
11.68 \\
11.69 \\
11.70\end{array}$ & $\begin{array}{l}11.82 \\
11.81 \\
11.84 \\
12.06 \\
12.14\end{array}$ & $\begin{array}{l}11.92 \\
11.90 \\
11.88 \\
11.86 \\
11.85\end{array}$ & $\begin{array}{l}11.69 \\
11.54 \\
11.66 \\
11.84 \\
11.84\end{array}$ & $\begin{array}{l}11.26 \\
11.26 \\
11.24 \\
11.27 \\
11.26\end{array}$ & $\begin{array}{l}11.62 \\
11.61 \\
11.59 \\
11.56 \\
11.51\end{array}$ & $\begin{array}{r}11.29 \\
11.29 \\
\mathrm{e} 11.27 \\
\mathrm{e} 11.25 \\
\mathrm{e} 11.22\end{array}$ & $\begin{array}{l}10.92 \\
10.92 \\
10.89 \\
10.83 \\
10.90\end{array}$ & $\begin{array}{l}10.90 \\
10.88 \\
10.92 \\
11.04 \\
11.05\end{array}$ \\
\hline $\begin{array}{l}11 \\
12 \\
13 \\
14 \\
15\end{array}$ & $\begin{array}{l}11.19 \\
11.20 \\
11.19 \\
11.18 \\
11.21\end{array}$ & $\begin{array}{l}11.38 \\
11.38 \\
11.34 \\
11.34 \\
11.34\end{array}$ & $\begin{array}{l}11.42 \\
11.44 \\
11.45 \\
11.47 \\
11.47\end{array}$ & $\begin{array}{l}11.70 \\
11.70 \\
11.71 \\
11.73 \\
11.75\end{array}$ & $\begin{array}{l}12.17 \\
12.16 \\
12.12 \\
12.09 \\
12.04\end{array}$ & $\begin{array}{l}11.85 \\
11.85 \\
11.85 \\
11.85 \\
11.93\end{array}$ & $\begin{array}{l}11.80 \\
11.73 \\
11.89 \\
11.86 \\
11.82\end{array}$ & $\begin{array}{l}11.46 \\
11.48 \\
11.45 \\
11.51 \\
11.67\end{array}$ & $\begin{array}{l}11.50 \\
11.66 \\
11.78 \\
11.82 \\
11.78\end{array}$ & $\begin{array}{l}\text { e11.18 } \\
\text { e11.16 } \\
\text { e11.12 } \\
\text { e11.08 } \\
\text { e11.05 }\end{array}$ & $\begin{array}{l}10.86 \\
10.82 \\
10.88 \\
10.80 \\
10.79\end{array}$ & $\begin{array}{l}11.07 \\
11.08 \\
11.19 \\
11.14 \\
11.11\end{array}$ \\
\hline $\begin{array}{l}16 \\
17 \\
18 \\
19 \\
20\end{array}$ & $\begin{array}{l}11.19 \\
11.19 \\
11.18 \\
11.19 \\
11.19\end{array}$ & $\begin{array}{l}11.35 \\
11.35 \\
11.33 \\
11.33 \\
11.32\end{array}$ & $\begin{array}{l}11.49 \\
11.50 \\
11.51 \\
11.53 \\
11.54\end{array}$ & $\begin{array}{l}11.76 \\
11.76 \\
11.76 \\
11.76 \\
11.76\end{array}$ & $\begin{array}{l}11.98 \\
11.92 \\
11.86 \\
11.79 \\
11.72\end{array}$ & $\begin{array}{l}11.97 \\
11.95 \\
11.94 \\
11.93 \\
11.92\end{array}$ & $\begin{array}{l}11.78 \\
11.78 \\
11.69 \\
11.64 \\
11.65\end{array}$ & $\begin{array}{l}11.74 \\
11.73 \\
11.74 \\
11.67 \\
11.61\end{array}$ & $\begin{array}{l}11.76 \\
11.71 \\
11.60 \\
11.55 \\
11.54\end{array}$ & $\begin{array}{l}\text { e11.01 } \\
\text { e10.98 } \\
\text { e10.99 } \\
\text { e10.97 } \\
\text { e10.96 }\end{array}$ & $\begin{array}{l}10.89 \\
10.88 \\
10.88 \\
11.01 \\
10.95\end{array}$ & $\begin{array}{l}11.11 \\
11.11 \\
11.13 \\
11.29 \\
11.20\end{array}$ \\
\hline $\begin{array}{l}21 \\
22 \\
23 \\
24 \\
25\end{array}$ & $\begin{array}{l}11.26 \\
11.25 \\
11.26 \\
11.31 \\
11.31\end{array}$ & $\begin{array}{l}11.31 \\
11.29 \\
11.27 \\
11.24 \\
11.23\end{array}$ & $\begin{array}{l}11.57 \\
11.57 \\
11.58 \\
11.58 \\
11.58\end{array}$ & $\begin{array}{l}11.76 \\
11.76 \\
11.76 \\
11.75 \\
11.74\end{array}$ & $\begin{array}{l}11.67 \\
11.60 \\
11.57 \\
11.60 \\
11.75\end{array}$ & $\begin{array}{l}11.91 \\
11.90 \\
11.88 \\
11.86 \\
11.84\end{array}$ & $\begin{array}{l}11.64 \\
11.76 \\
11.59 \\
11.71 \\
11.67\end{array}$ & $\begin{array}{l}11.57 \\
11.51 \\
11.51 \\
11.53 \\
11.50\end{array}$ & $\begin{array}{l}11.49 \\
11.50 \\
11.52 \\
11.47 \\
11.44\end{array}$ & $\begin{array}{r}\text { e10.98 } \\
\text { e11.00 } \\
11.09 \\
11.10 \\
11.17\end{array}$ & $\begin{array}{l}10.92 \\
10.94 \\
10.98 \\
10.98 \\
10.93\end{array}$ & $\begin{array}{l}11.25 \\
11.24 \\
11.35 \\
11.45 \\
11.47\end{array}$ \\
\hline $\begin{array}{l}26 \\
27 \\
28 \\
29 \\
30 \\
31\end{array}$ & $\begin{array}{r}11.30 \\
11.34 \\
11.35 \\
11.31 \\
11.31 \\
\mathrm{e} 11.28\end{array}$ & $\begin{array}{r}11.24 \\
11.23 \\
11.24 \\
11.27 \\
11.29 \\
---\end{array}$ & $\begin{array}{l}11.59 \\
11.59 \\
11.60 \\
11.63 \\
11.65 \\
11.65\end{array}$ & $\begin{array}{l}11.74 \\
11.74 \\
11.73 \\
11.75 \\
11.82 \\
11.84\end{array}$ & $\begin{array}{r}11.83 \\
11.87 \\
11.88 \\
-- \\
-- \\
--\end{array}$ & $\begin{array}{l}11.82 \\
11.79 \\
11.77 \\
11.76 \\
11.74 \\
11.73\end{array}$ & $\begin{array}{r}11.59 \\
11.66 \\
11.59 \\
11.54 \\
11.49 \\
-.-\end{array}$ & $\begin{array}{l}11.48 \\
11.48 \\
11.45 \\
11.45 \\
11.40 \\
11.39\end{array}$ & $\begin{array}{r}11.42 \\
11.39 \\
11.39 \\
11.40 \\
11.38 \\
---\end{array}$ & $\begin{array}{l}11.14 \\
11.08 \\
11.03 \\
11.03 \\
11.03 \\
10.99\end{array}$ & $\begin{array}{l}10.96 \\
10.94 \\
10.96 \\
10.94 \\
10.89 \\
10.95\end{array}$ & $\begin{array}{l}11.53 \\
11.54 \\
11.55 \\
11.54 \\
11.53\end{array}$ \\
\hline $\begin{array}{l}\text { MEAN } \\
\text { MAX } \\
\text { MIN }\end{array}$ & $\begin{array}{l}11.27 \\
11.45 \\
11.18\end{array}$ & $\begin{array}{l}11.29 \\
11.38 \\
11.20\end{array}$ & $\begin{array}{l}11.48 \\
11.65 \\
11.31\end{array}$ & $\begin{array}{l}11.72 \\
11.84 \\
11.65\end{array}$ & $\begin{array}{l}11.87 \\
12.17 \\
11.57\end{array}$ & $\begin{array}{l}11.87 \\
11.97 \\
11.73\end{array}$ & $\begin{array}{l}11.70 \\
11.89 \\
11.49\end{array}$ & $\begin{array}{l}11.47 \\
11.74 \\
11.24\end{array}$ & $\begin{array}{l}11.55 \\
11.82 \\
11.38\end{array}$ & $\begin{array}{l}11.14 \\
11.43 \\
10.96\end{array}$ & $\begin{array}{l}10.92 \\
11.02 \\
10.79\end{array}$ & $\begin{array}{l}11.18 \\
11.55 \\
10.86\end{array}$ \\
\hline
\end{tabular}

e Estimated 
LOCATION.--Lat $42^{\circ} 52^{\prime} 12^{\prime \prime}$, long $88^{\circ} 07^{\prime} 28^{\prime \prime}$, in NW 1/4 NW 1/4 sec.27, T.5 N., R.20 E., Waukesha County, Hydrologic Unit 07120006, near Muskego.

DRAINAGE AREA.--33.9 $\mathrm{mi}^{2}$.

PERIOD OF RECORD.--February 1988 to current year. Prior to October 2000, published as "Big Muskego Lake".

REMARKS.--Lake sampled at south end of lake at a depth of about $1 \mathrm{~m}$. Lake ice-covered during February sampling. Water-quality analyses done by Wisconsin State Laboratory of Hygiene.

WATER-QUALITY DATA, FEBRUARY 13 TO AUGUST 21, 2001 (Milligrams per liter unless otherwise indicated)

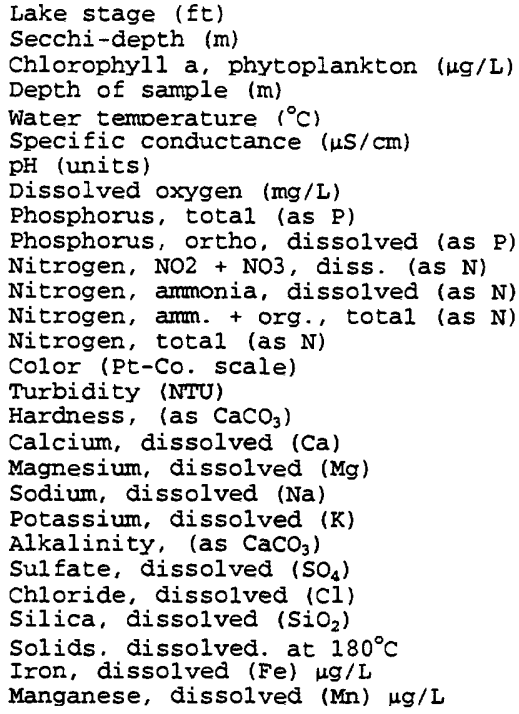

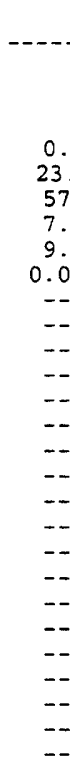

Jun
11.
$>1$.

$0.5^{6} 1.0$

$23.8 \quad 23.7$

$576 \quad 576$

$7.8 \quad 7.9$

$9.5 \quad 9.8$

.0510 .039

---

$---$

$-2 \quad-2$

-

$\cdots$

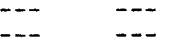

-

-

$\cdots$

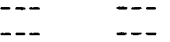

-

$-2$

$-2$

$\begin{array}{ll}--- \\ -- & --\end{array}$

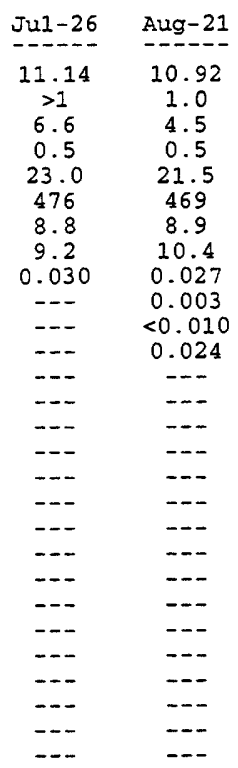

7-26-01
8-21-01

DISSOLVED OXYGEN (D.O.), IN MILLIGRAMS PER LITER
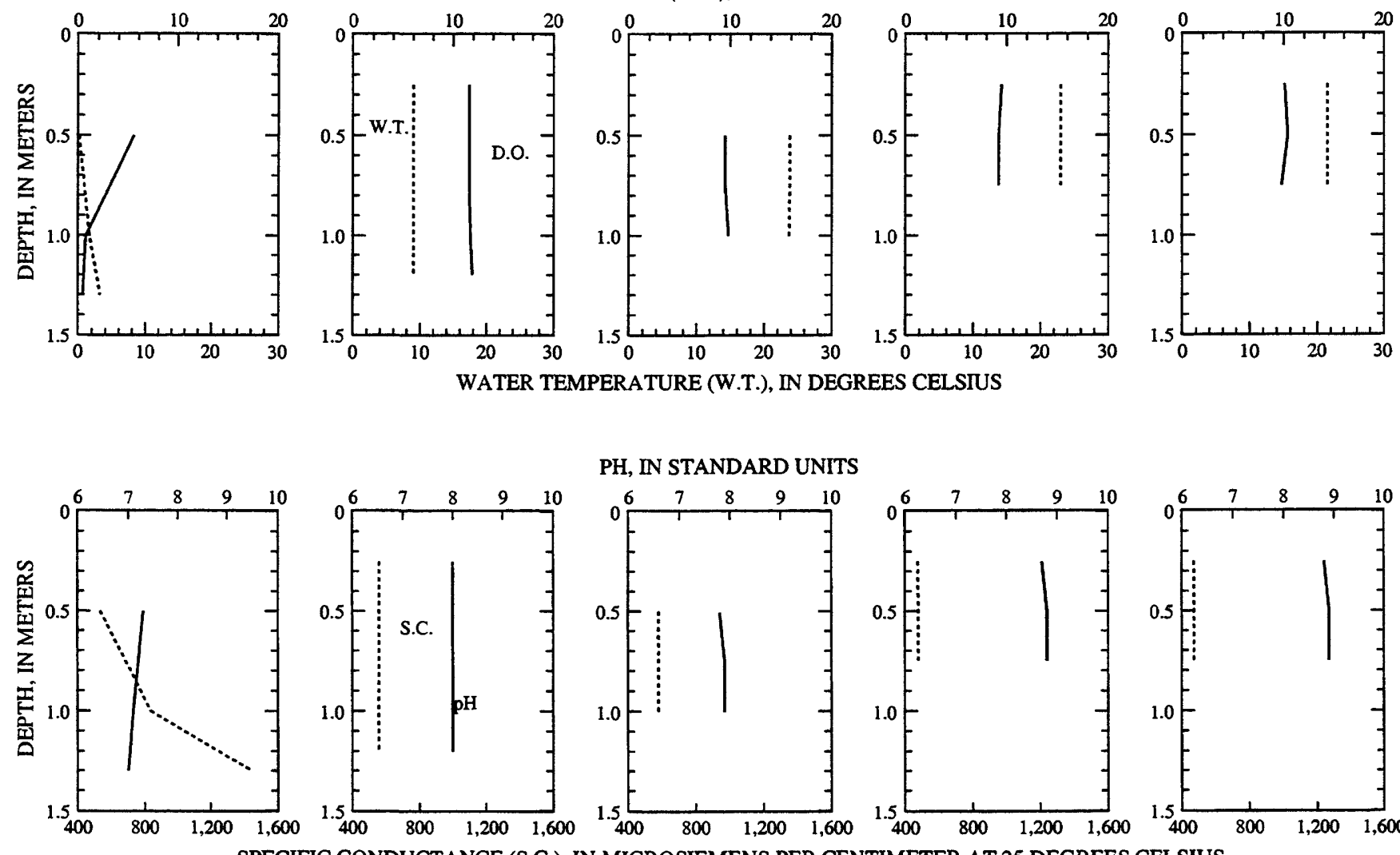

PH, IN STANDARD UNITS
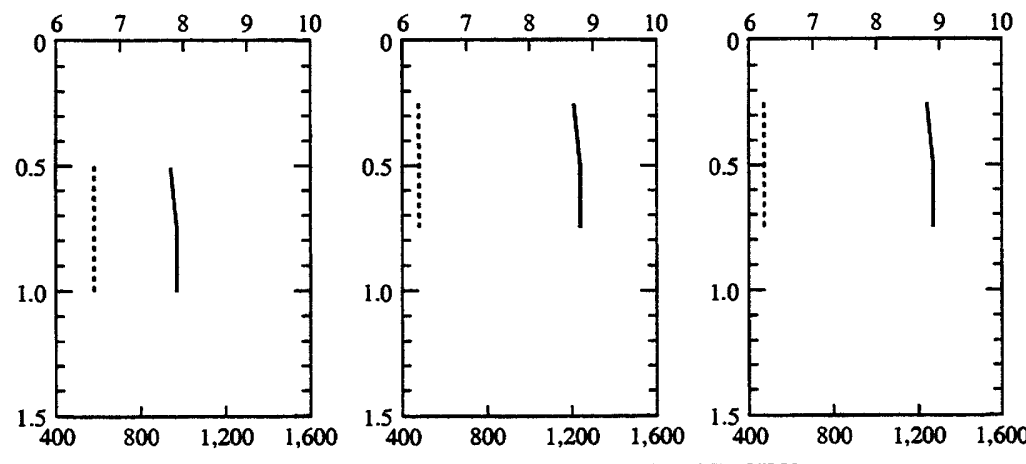

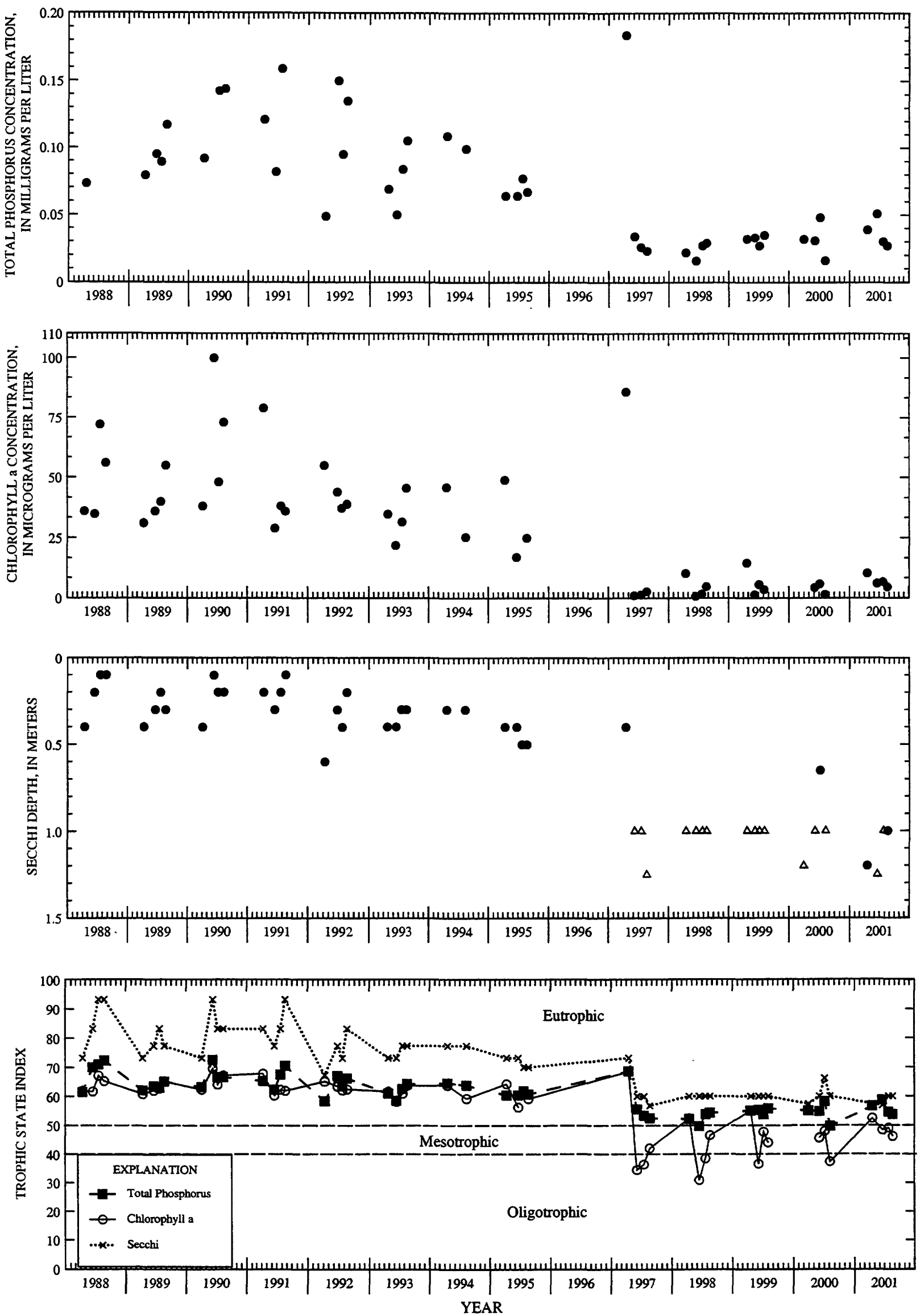

Surface total phosphorus, chlorophyll a concentrations, Secchi depths, and TSI data for Muskego (Big Muskego) Lake, South Site, near Muskego, Wisconsin.

(Triangles indicate maximum depth at sampling site. Actual Secchi depth on these days was greater than plotted triangles.) 


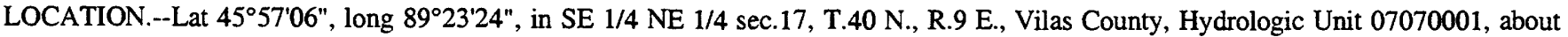
$12 \mathrm{mi}$ northwest of Eagle River.

DRAINAGE AREA.--4.49 $\mathrm{mi}^{2}$.

PERIOD OF RECORD.--November 2000 to October 2001 (discontinued).

GAGE.--Water-stage recorder. Elevation of gage is about 1,612 ft above sea level.

EXTREMES FOR CURRENT YEAR.--Maximum gage height observed, $10.56 \mathrm{ft}$, Apr. 24; minimum observed, $9.93 \mathrm{ft}$, Sept. 5 and 6.

GAGE HEIGHT, FEET, YEAR NOVEMBER 2000 TO OCTOBER 2001 DAILY MEAN VALUES

\begin{tabular}{|c|c|c|c|c|c|c|c|c|c|c|c|c|}
\hline DAY & NOV & $\mathrm{DEC}$ & JAN & FEB & MAR & APR & MAY & JUN & JUL & AUG & SEP & OCT \\
\hline $\begin{array}{l}1 \\
2 \\
3 \\
4 \\
5\end{array}$ & $\begin{array}{l}10.11 \\
10.12 \\
10.10 \\
10.10 \\
10.10\end{array}$ & $\begin{array}{l}\mathrm{e} 10.18 \\
\mathrm{e} 10.18 \\
\mathrm{e} 10.18 \\
\mathrm{e} 10.18 \\
\mathrm{e} 10.17\end{array}$ & $\begin{array}{l}10.20 \\
10.20 \\
10.20 \\
10.20 \\
10.19\end{array}$ & $\begin{array}{l}10.18 \\
10.18 \\
10.18 \\
10.18 \\
10.18\end{array}$ & $\begin{array}{l}10.19 \\
10.18 \\
10.18 \\
10.17 \\
10.17\end{array}$ & $\begin{array}{l}10.18 \\
10.18 \\
10.19 \\
10.19 \\
10.19\end{array}$ & $\begin{array}{l}10.36 \\
10.36 \\
10.35 \\
10.34 \\
10.32\end{array}$ & $\begin{array}{l}10.28 \\
10.27 \\
10.25 \\
10.24 \\
10.23\end{array}$ & $\begin{array}{l}10.18 \\
10.16 \\
10.16 \\
10.16 \\
10.14\end{array}$ & $\begin{array}{l}10.19 \\
10.22 \\
10.21 \\
10.20 \\
10.19\end{array}$ & $\begin{array}{l}9.9 E \\
9.9= \\
9.9 \\
9.9 \\
9.94\end{array}$ & $\begin{array}{r}.06 \\
98 \\
.1 \\
-0 \\
39\end{array}$ \\
\hline $\begin{array}{r}6 \\
7 \\
8 \\
9 \\
10\end{array}$ & $\begin{array}{l}10.11 \\
10.13 \\
10.13 \\
10.12 \\
10.12\end{array}$ & $\begin{array}{r}\text { e10.17 } \\
10.17 \\
10.17 \\
10.17 \\
10.17\end{array}$ & $\begin{array}{l}10.19 \\
10.19 \\
10.19 \\
10.19 \\
10.19\end{array}$ & $\begin{array}{l}10.18 \\
10.17 \\
10.17 \\
10.19 \\
10.20\end{array}$ & $\begin{array}{l}10.17 \\
10.17 \\
10.17 \\
10.17 \\
10.17\end{array}$ & $\begin{array}{l}10.20 \\
10.26 \\
10.31 \\
10.32 \\
10.34\end{array}$ & $\begin{array}{l}10.30 \\
10.30 \\
10.30 \\
10.29 \\
10.35\end{array}$ & $\begin{array}{l}10.23 \\
10.22 \\
10.22 \\
10.21 \\
10.24\end{array}$ & $\begin{array}{l}10.12 \\
10.12 \\
10.11 \\
10.11 \\
10.10\end{array}$ & $\begin{array}{l}10.17 \\
10.16 \\
10.15 \\
10.12 \\
10.09\end{array}$ & $\begin{array}{r}9.96 \\
10.09 \\
10.12 \\
10.12 \\
10.12\end{array}$ & $\begin{array}{l}10.08 \\
10.07 \\
10.07 \\
10.08 \\
10.14\end{array}$ \\
\hline $\begin{array}{l}11 \\
12 \\
13 \\
14 \\
15\end{array}$ & $\begin{array}{l}10.12 \\
10.14 \\
10.18 \\
10.21 \\
10.21\end{array}$ & $\begin{array}{l}10.17 \\
10.17 \\
10.17 \\
10.17 \\
10.17\end{array}$ & $\begin{array}{l}10.19 \\
10.17 \\
10.17 \\
10.17 \\
10.18\end{array}$ & $\begin{array}{l}10.20 \\
10.20 \\
10.20 \\
10.19 \\
10.19\end{array}$ & $\begin{array}{l}10.17 \\
10.17 \\
10.19 \\
10.20 \\
10.19\end{array}$ & $\begin{array}{l}10.38 \\
10.45 \\
10.47 \\
10.46 \\
10.44\end{array}$ & $\begin{array}{l}10.34 \\
10.32 \\
10.31 \\
10.30 \\
10.29\end{array}$ & $\begin{array}{l}10.27 \\
10.30 \\
10.30 \\
10.28 \\
10.26\end{array}$ & $\begin{array}{l}10.09 \\
10.08 \\
10.07 \\
10.06 \\
10.07\end{array}$ & $\begin{array}{l}10.07 \\
10.07 \\
10.07 \\
10.05 \\
10.05\end{array}$ & $\begin{array}{l}10.11 \\
10.11 \\
10.10 \\
10.08 \\
10.08\end{array}$ & $\begin{array}{l}10.15 \\
10.14 \\
10.15 \\
10.17 \\
10.17\end{array}$ \\
\hline $\begin{array}{l}16 \\
17 \\
18 \\
19 \\
20\end{array}$ & $\begin{array}{r}10.21 \\
\mathrm{e} 10.21 \\
\mathrm{e} 10.21 \\
\mathrm{e} 10.20 \\
\mathrm{e} 10.20\end{array}$ & $\begin{array}{l}10.19 \\
10.21 \\
10.20 \\
10.20 \\
10.20\end{array}$ & $\begin{array}{r}10.18 \\
\mathrm{e} 10.18 \\
\mathrm{e} 10.18 \\
\mathrm{e} 10.17 \\
10.17\end{array}$ & $\begin{array}{l}10.19 \\
10.19 \\
10.19 \\
10.19 \\
10.19\end{array}$ & $\begin{array}{l}10.19 \\
10.18 \\
10.18 \\
10.18 \\
10.17\end{array}$ & $\begin{array}{l}10.42 \\
10.39 \\
10.37 \\
10.35 \\
10.35\end{array}$ & $\begin{array}{l}10.28 \\
10.27 \\
10.25 \\
10.24 \\
10.23\end{array}$ & $\begin{array}{l}10.27 \\
10.31 \\
10.33 \\
10.40 \\
10.38\end{array}$ & $\begin{array}{l}10.07 \\
10.07 \\
10.07 \\
10.11 \\
10.20\end{array}$ & $\begin{array}{l}10.05 \\
10.05 \\
10.05 \\
10.04 \\
10.03\end{array}$ & $\begin{array}{l}10.07 \\
10.07 \\
10.07 \\
10.07 \\
10.07\end{array}$ & $\begin{array}{l}10.16 \\
10.15 \\
10.15 \\
10.15 \\
10.14\end{array}$ \\
\hline $\begin{array}{l}21 \\
22 \\
23 \\
24 \\
25\end{array}$ & $\begin{array}{l}\text { e10.20 } \\
\text { e10.20 } \\
\text { e10.20 } \\
\text { e10.19 } \\
\text { e10.19 }\end{array}$ & $\begin{array}{l}10.20 \\
10.20 \\
10.20 \\
10.20 \\
10.20\end{array}$ & $\begin{array}{l}10.17 \\
10.17 \\
10.17 \\
10.17 \\
10.16\end{array}$ & $\begin{array}{l}10.19 \\
10.19 \\
10.18 \\
10.18 \\
10.19\end{array}$ & $\begin{array}{l}10.18 \\
10.18 \\
10.18 \\
10.18 \\
10.18\end{array}$ & $\begin{array}{l}10.40 \\
10.47 \\
10.52 \\
10.55 \\
10.52\end{array}$ & $\begin{array}{l}10.24 \\
10.28 \\
10.29 \\
10.30 \\
10.32\end{array}$ & $\begin{array}{l}10.37 \\
10.34 \\
10.32 \\
10.30 \\
10.28\end{array}$ & $\begin{array}{l}10.20 \\
10.19 \\
10.18 \\
10.16 \\
10.14\end{array}$ & $\begin{array}{l}10.03 \\
10.02 \\
10.02 \\
10.01 \\
10.01\end{array}$ & $\begin{array}{l}10.06 \\
10.06 \\
10.11 \\
10.10 \\
10.09\end{array}$ & $\begin{array}{l}10.14 \\
10.13 \\
10.14 \\
10.15 \\
10.15\end{array}$ \\
\hline $\begin{array}{l}26 \\
27 \\
28 \\
29 \\
30 \\
31\end{array}$ & $\begin{array}{r}\text { e10.19 } \\
\text { e10.19 } \\
\text { e10.19 } \\
\text { e10.19 } \\
\text { e10.18 } \\
\end{array}$ & $\begin{array}{l}10.20 \\
10.20 \\
10.20 \\
10.20 \\
10.20 \\
10.20\end{array}$ & $\begin{array}{l}10.16 \\
10.16 \\
10.16 \\
10.16 \\
10.18 \\
10.18\end{array}$ & $\begin{array}{r}10.19 \\
10.19 \\
10.19 \\
.-- \\
-.--\end{array}$ & $\begin{array}{l}10.18 \\
10.18 \\
10.18 \\
10.17 \\
10.17 \\
10.16\end{array}$ & $\begin{array}{r}10.48 \\
10.44 \\
10.41 \\
10.38 \\
10.36 \\
\end{array}$ & $\begin{array}{l}10.34 \\
10.35 \\
10.34 \\
10.32 \\
10.30 \\
10.28\end{array}$ & $\begin{array}{r}10.27 \\
10.26 \\
10.25 \\
10.23 \\
10.21 \\
\end{array}$ & $\begin{array}{r}\mathrm{e} 10.14 \\
\mathrm{e} 10.13 \\
\mathrm{e} 10.13 \\
10.12 \\
10.13 \\
10.14\end{array}$ & $\begin{array}{r}10.00 \\
9.99 \\
9.98 \\
9.98 \\
9.97 \\
9.97\end{array}$ & $\begin{array}{r}10.08 \\
10.07 \\
10.07 \\
10.07 \\
10.06 \\
\end{array}$ & $\begin{array}{l}10.20 \\
10.20 \\
10.20 \\
10.19 \\
10.19 \\
10.21\end{array}$ \\
\hline $\begin{array}{l}\text { MEAN } \\
\text { MAX } \\
\text { MIN }\end{array}$ & $\begin{array}{l}10.16 \\
10.21 \\
10.10\end{array}$ & $\begin{array}{l}10.19 \\
10.21 \\
10.17\end{array}$ & $\begin{array}{l}10.18 \\
10.20 \\
10.16\end{array}$ & $\begin{array}{l}10.19 \\
10.20 \\
10.17\end{array}$ & $\begin{array}{l}10.18 \\
10.20 \\
10.16\end{array}$ & $\begin{array}{l}10.37 \\
10.55 \\
10.18\end{array}$ & $\begin{array}{l}10.31 \\
10.36 \\
10.23\end{array}$ & $\begin{array}{l}10.28 \\
10.40 \\
10.21\end{array}$ & $\begin{array}{l}10.13 \\
10.20 \\
10.06\end{array}$ & $\begin{array}{r}10.07 \\
10.22 \\
9.97\end{array}$ & $\begin{array}{r}10.06 \\
10.12 \\
9.94\end{array}$ & $\begin{array}{l}10.14 \\
10.21 \\
10.06\end{array}$ \\
\hline
\end{tabular}

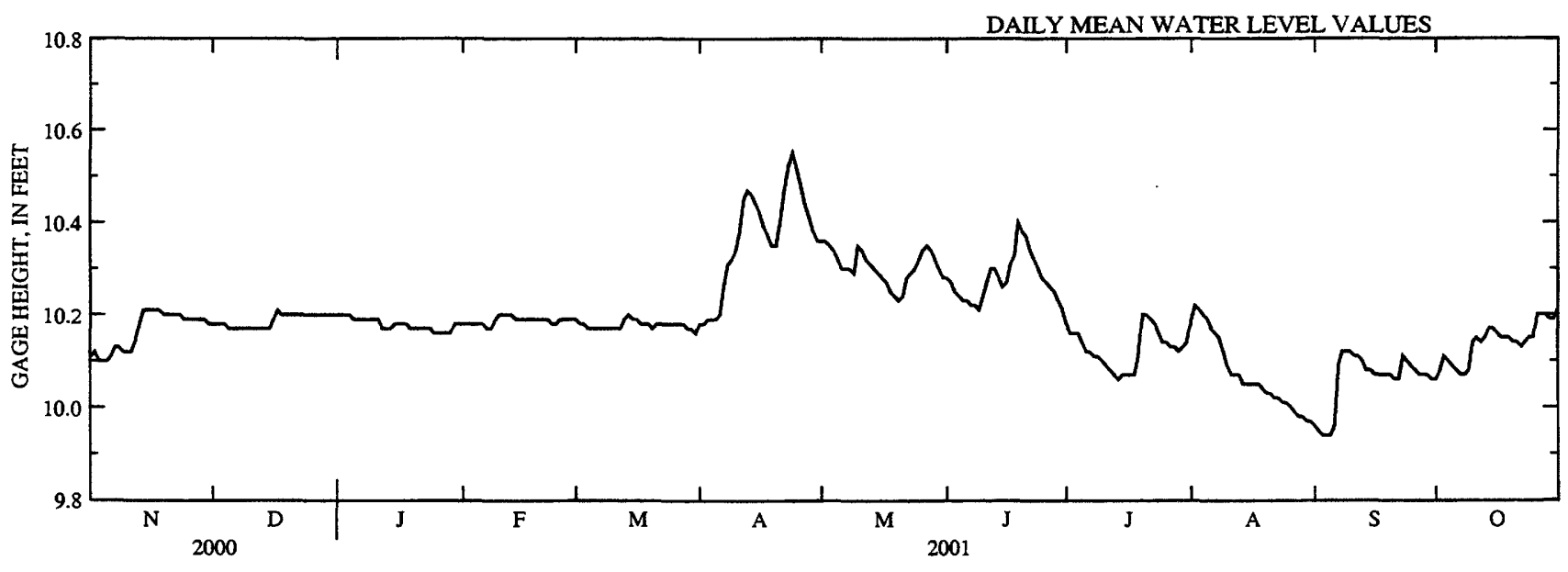




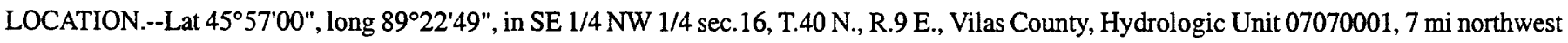
of Eagle River.

PERIOD OF RECORD.--June 2000 to August 2001 (discontinued).

REMARKS.--Lake ice-covered during March sampling. Water-quality analyses by Wisconsin State Laboratory of Hygiene.

WATER-QUALITY DATA, MARCH 22 TO AUGUST 27, 2001

(Milligrams per liter unless otherwise indicated)

Lake stage (ft)

Secchi-depth (m)

Chlorophyll a, phytoplankton ( $\mu \mathrm{g} / \mathrm{L}$ )

Depth of sample (m)

Water temperature $\left({ }^{\circ} \mathrm{C}\right\}$

Specific conductance $(\mu \mathrm{S} / \mathrm{cm})$

$\mathrm{pH}$ (units)

Dissolved oxygen (mg/L)

Phosphorus, total (as P)

Phosphorus, ortho, dissolved (as P)

Nitrogen, NO2 + NO3, diss. (as N)

Nitrogen, ammonia, dissolved (as N)

Nitrogen, amm. + org., total (as $N$ )

Nitrogen, total (as N)

Color (Pt-Co. scale)

Turbidity (NTU)

Hardness, (as $\mathrm{CaCO}_{3}$ )

Calcium, dissolved (Ca)

Magnesium, dissolved (Mg)

Sodium, dissolved (Na)

Potassium, dissolved (K)

Alkalinity, (as $\mathrm{CaCO}_{3}$ )

Sulfate, dissolved $\left(\mathrm{SO}_{4}\right)$

Chloride, dissolved (Cl)

Silica, dissolved $\left(\mathrm{SiO}_{2}\right)$

Solids, dissolved, at $180^{\circ} \mathrm{C}$

Iron, dissolved (Fe) $\mu \mathrm{g} / \mathrm{L}$

Manganese, dissolved (Mn) $\mu \mathrm{g} / \mathrm{L}$
$\operatorname{Mar}-22$

$\operatorname{Mar}-22$
10.18
-

-..

May-1

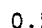

$\begin{array}{ll}0.5 & 3.9 \\ 100 & 1.9\end{array}$

$6.7 \quad 6.6$

$12.9 \quad 9.3$

$0.027 \quad 0.036$

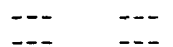

$\begin{array}{ll}--2 & --- \\ --- & --1\end{array}$

$\cdots$

-

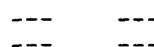

$\cdots$

$\cdots+-$

$\begin{array}{ll}--- \\ -\cdots & ---\end{array}$

---

-

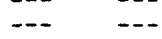

--

-

-

--

0.59

0.674

40
4.8

4.8
38.2

10

3.2
2.2

0.4

$\begin{aligned} & 36 \\ &<4.5\end{aligned}$

$<4.5$
2.7

14.6

66

40 -..

\begin{tabular}{cc} 
Jun -26 \\
\hline 10.27 \\
1.5 \\
\multicolumn{2}{c}{10} \\
$0.5^{10}$ & 4.0 \\
23.6 & 17.3 \\
80 & 87 \\
7.8 & 6.7 \\
10.0 & 0.6 \\
0.033 & 0.049
\end{tabular}

-..

--

$---$

5.6

DISSOLVED OXYGEN (D.O.), IN MILLIGRAMS PER LITER
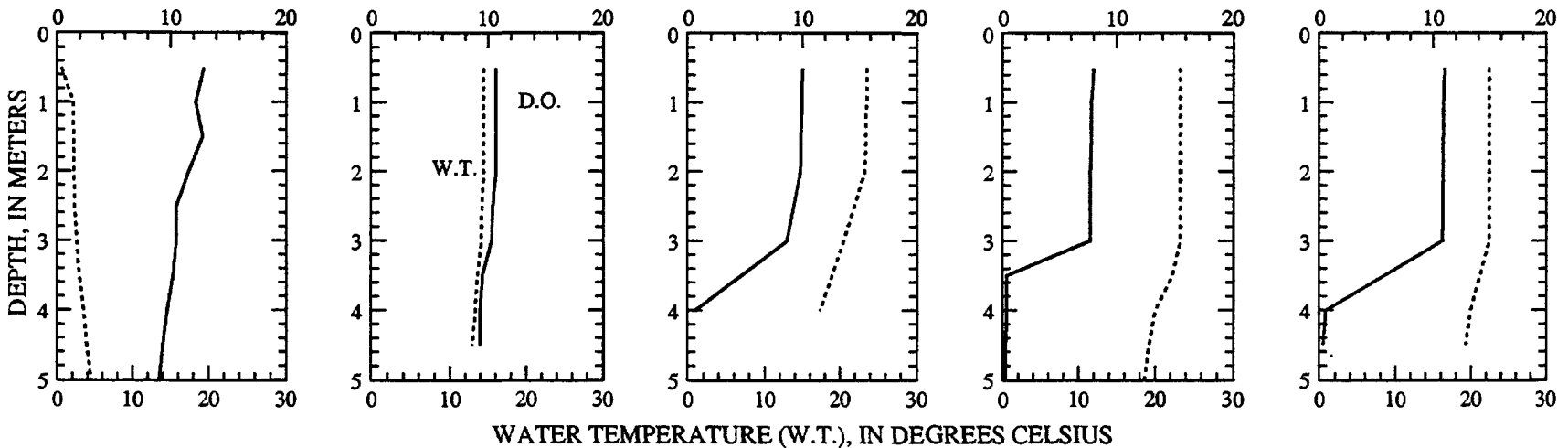

WATER TEMPERATURE (W.T.), IN DEGREES CELSIUS
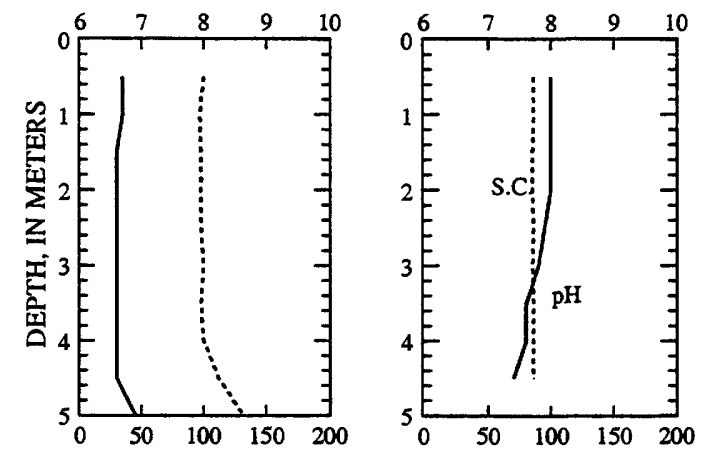

PH, IN STANDARD UNITS
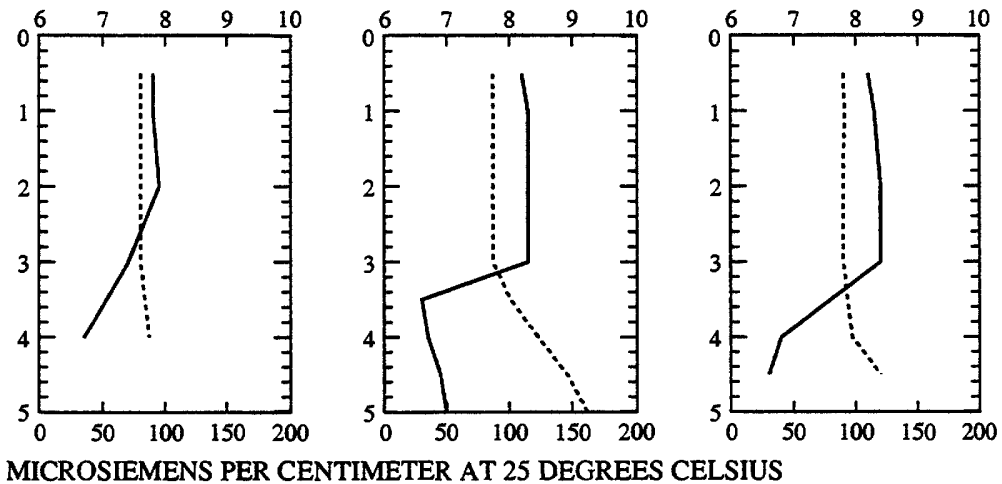

SPECIFIC CONDUCTANCE (S.C.), IN MICROSIEMENS PER CENTIMETER AT 25 DEGREES CELSIUS 

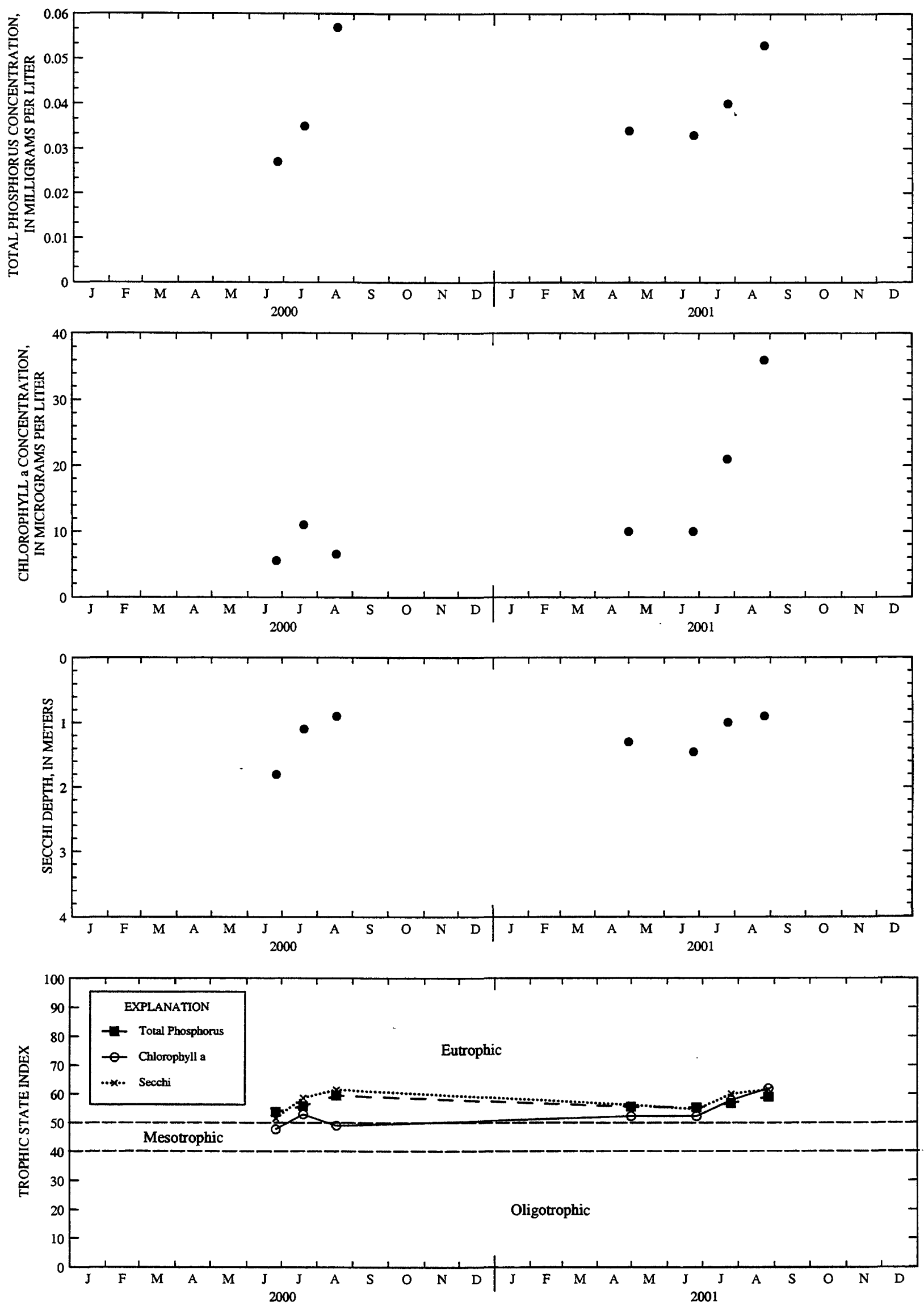

YEAR

Surface total phosphorus, chlorophyll a concentrations, Secchi depths, and TSI data for Muskellunge Lake, near Eagle River, Wisconsin. 


\section{OCONOMOWOC LAKE NO. 1 (CENTER) AT OCONOMOWOC, WI}

LOCATION.--Lat $43^{\circ} 05^{\prime} 51^{\prime \prime}$, long $88^{\circ} 27^{\prime} 35^{\prime \prime}$, in NW 1/4 SE $1 / 4$ sec.2, T.7 N., R.17 E., Waukesha County, Hydrologic Unit 07090001, at Oconomowoc.

PERIOD OF RECORD.--March 1986 to current year.

REMARKS.--Lake sampled near center at the deep hole. Lake ice-covered during February sampling. Water-quality analyses done by Wisconsin State Laboratory of Hygiene.

WATER-QUALITY DATA, FEBRUARY 14 TO AUGUST 15, 2001

(Milligrams per liter unless otherwise indicated)

Lake stage ( $f t)$

Secchi-depth (m)

Chlorophyll a phytoplankton (ug/L)

Depth of sample (m)

water temoerature $\left({ }^{\circ} \mathrm{C}\right)$

Specific conductance $(\mu \mathrm{S} / \mathrm{cm})$

$\mathrm{pH}$ (units)

Dissolved oxygen ( $\mathrm{mg} / \mathrm{L}$ )

Phosphorus, total (as P)

Phosphorus, ortho, dissolved (as P)

Nitrogen, $\mathrm{NO} 2$ + NO3, diss. (as N)

Nitrogen, ammonia, dissolved (as N)

Nitrogen, amm. + org., total (as N)

Nitrogen, total (as N)

Color (Pt-Co. scale

Turbidity (NTU)

Hardness, (as $\mathrm{CaCO}_{3}$ )

Calcium, dissolved ( $\mathrm{Ca})$

Magnesium, dissolved (Mg)

Sodium, dissolved (Na)

Potassium, dissolved ( $\mathrm{K}$ )

Alkalinity, (as $\mathrm{CaCO}_{3}$ )

Sulfate, dissolved $\left(\mathrm{SO}_{4}\right)$

Chloride, dissolved ( $\mathrm{Cl}$ )

Silica, dissolved $\left(\mathrm{SiO}_{2}\right)$

Solids, dissolved. at $180^{\circ} \mathrm{C}$

Iron, dissolved (Fe) $\mu \mathrm{g} / \mathrm{L}$

Manganese, dissolved (Mn) $\mu \mathrm{g} / \mathrm{L}$

\begin{tabular}{|c|c|c|c|c|c|c|c|c|c|c|}
\hline $\mathrm{Fe}$ & 14 & $\mathrm{Ap}$ & & & & & & & Aug -15 & \\
\hline & & & & & & & & & $\begin{array}{l}8.05 \\
1.9 \\
2.5\end{array}$ & \\
\hline $\begin{array}{c}0.5 \\
1.3 \\
564 \\
8.1 \\
11.9 \\
<0.005\end{array}$ & $\begin{array}{c}17.0 \\
3.6 \\
602 \\
7.6 \\
3.2 \\
0.018\end{array}$ & $\begin{array}{c}0.5 \\
9.3 \\
550 \\
8.0 \\
10.9 \\
0.011\end{array}$ & $\begin{array}{c}18.0 \\
7.5 \\
553 \\
8.0 \\
10.5 \\
0.009\end{array}$ & $\begin{array}{c}0.5 \\
23.0 \\
535 \\
8.0 \\
9.2 \\
0.012\end{array}$ & $\begin{array}{c}18.0 \\
8.5 \\
544 \\
7.5 \\
1.9 \\
0.033\end{array}$ & $\begin{array}{c}0.5 \\
26.1 \\
540 \\
8.0 \\
8.9 \\
0.010\end{array}$ & $\begin{array}{c}17.5 \\
8.6 \\
546 \\
7.4 \\
0.3 \\
0.040\end{array}$ & $\begin{array}{c}0.5 \\
25.8 \\
517 \\
8.1 \\
9.4 \\
0.013\end{array}$ & $\begin{array}{l}12.0 \\
10.5 \\
550 \\
7.5 \\
0.3 \\
0.046\end{array}$ & $\begin{array}{c}17.5 \\
8.7 \\
562 \\
7.5 \\
0.2 \\
0.029\end{array}$ \\
\hline--- & -- & 0.002 & $\ldots$ & $\ldots$ & $\ldots$ & -- & -- & --- & --- & --- \\
\hline--- & --- & 0.37 & --- & $\cdots$ & -- & -- & $-\cdots$ & $-\cdots$ & $\cdots$ & -- \\
\hline--- & --- & 0.027 & --- & --- & --- & --- & -- & -- & --- & -- \\
\hline$-\cdots$ & --- & 0.49 & --- & $-\infty$ & --- & -- & --- & --- & --- & $\cdots$ \\
\hline--- & --- & 0.86 & - - & -- & -- & - & - & --- & -- & -- \\
\hline - - & $\ldots$ & 15 & --- & --- & $\ldots$ & -- & --- & --- & -- & -- \\
\hline-- & -- & 1.2 & -- & $\ldots$ & $\ldots$ & -- & $\ldots$ & $\ldots$ & $-\cdots$ & -- \\
\hline$\ldots$ & -- & 257 & --- & -- & -- & -- & --- & --- & -- &.- \\
\hline-- & -- & 50 & --- & -- & -- & $-\cdots$ & -- & --- & --- & $\cdots$ \\
\hline-- & -- & 32 & --- & -- & --- & -- & --- & -- & -- & -- \\
\hline$\ldots$ & -- & 16 & -- & -- & --- & $\ldots$ & --- & --- & -- & $-\cdots$ \\
\hline$\ldots$ & $-\cdots$ & 1.6 & -- & - - & --- & -- & -- & --- & -- & -- \\
\hline-- & -- & 213 & --- & -- & --- & -- & --- & -- & -- & --- \\
\hline$\cdots$ & -- & 25.3 & --- & --- & --- & --- & --- & --- & --- & -- \\
\hline--- & --- & 36.4 & --- & --- & --- & -- & --- & --- & -- & -- \\
\hline-- & --- & 5.2 & -- & --- & -- & -- & -- & $-\cdots$ & $\cdots$ & --- \\
\hline$-\cdots$ & $-\cdots$ & 320 & -- & $\cdots$ & -- & -- & -- & -- & -- & $-\cdots$ \\
\hline$-\cdots$ & --- & $<10$ & -- & -- & $-\cdots$ & --- & -- & --- & -- & $\cdots$ \\
\hline--- & -- & $<0.4$ & $-\cdots$ & --- & -- & -- & --- & --- & -- & $\cdots$ \\
\hline
\end{tabular}
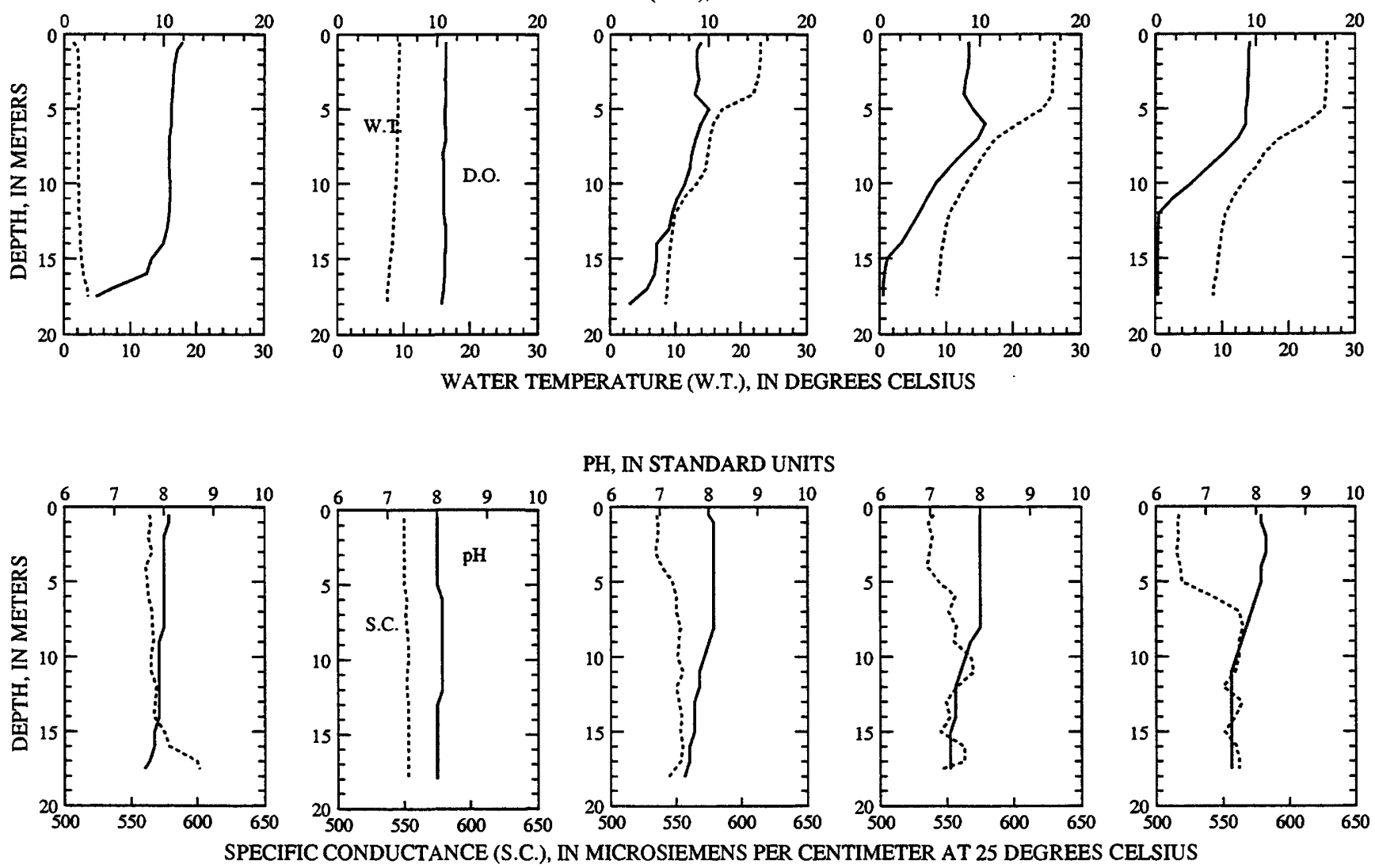

PH, IN STANDARD UNITS
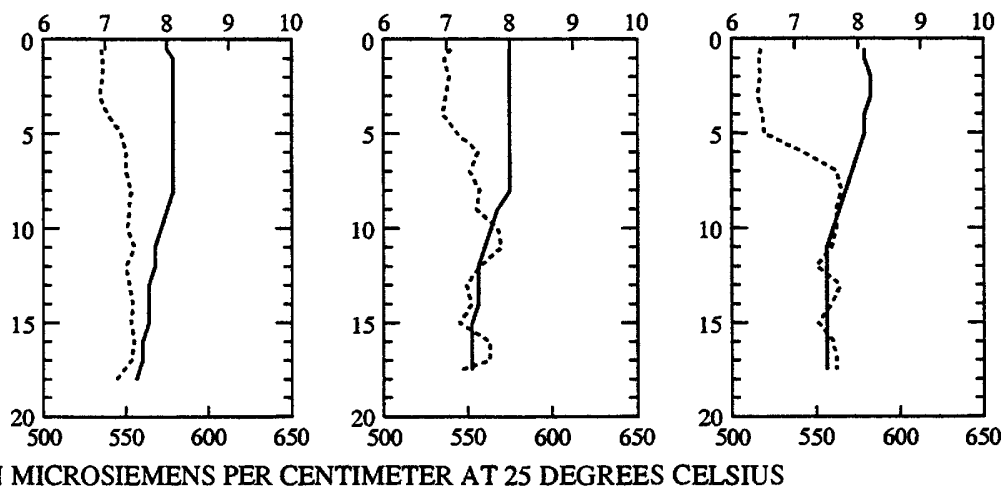

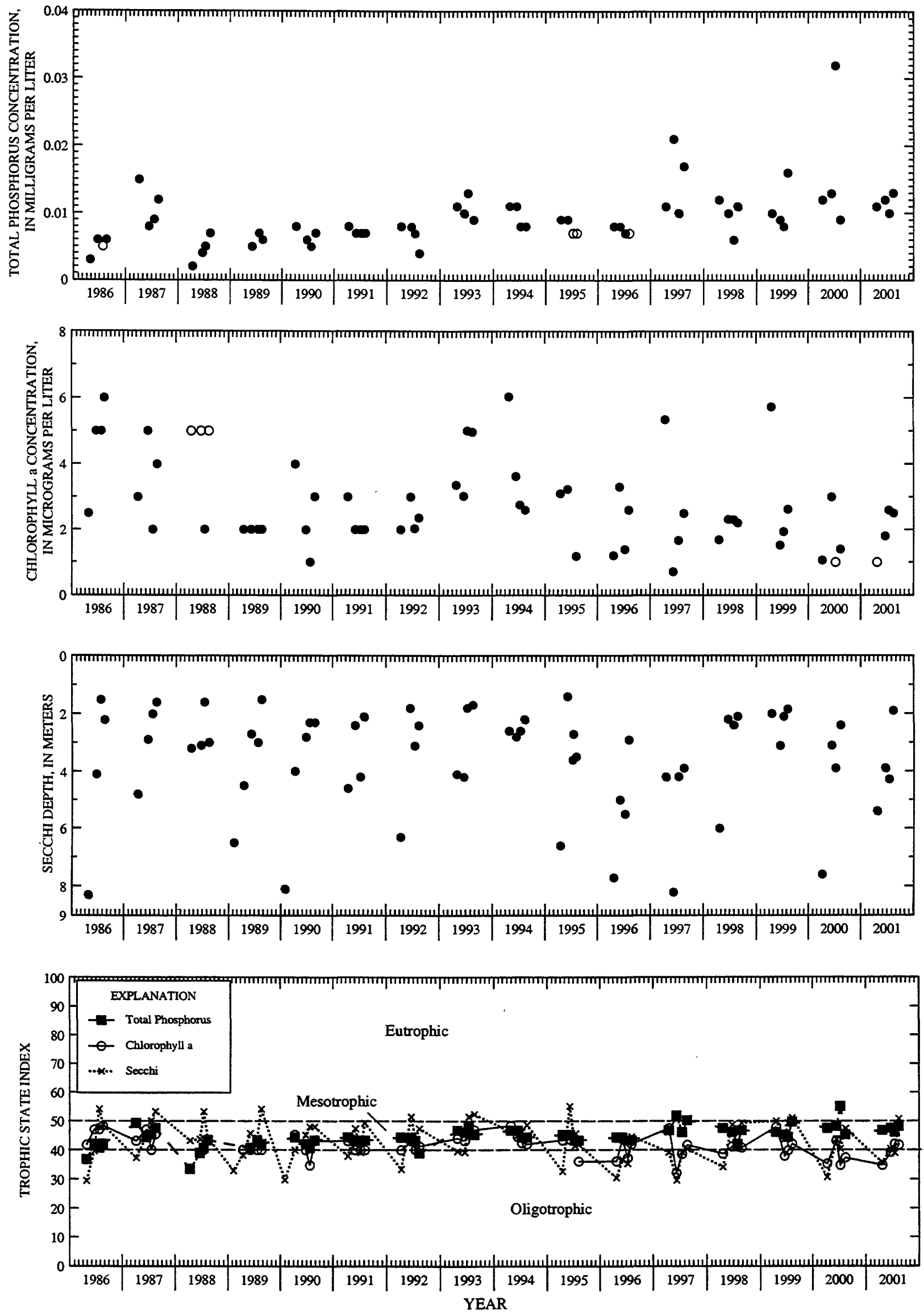

Surface total phosphorus, chlorophyll a concentrations, Secchi depths, and TSI data for Oconomowoc Lake, Center Site, at Oconomowoc, Wisconsin.

(Circles on the first three plots indicate laboratory detection limit for selected analyses. Actual concentrations for these particular analyses are less than the plotted circles.) 
LOCATION.--Lat $43^{\circ} 06^{\prime} 09^{\prime \prime}$, long $88^{\circ} 26^{\prime 2} 22^{\prime \prime}$, in NW 1/4 NW 1/4 sec.1, T.7 N., R.17 E., Waukesha County, Hydrologic Unit 07090001, at Oconomowoc.

PERIOD OF RECORD.--March 1986 to current year.

REMARKS.--Lake sampled at the deepest point in northeast bay near Hewitt Point. Lake ice-covered during February sampling. Water-quality analyses done by Wisconsin State Laboratory of Hygiene.

WATER-QUALITY DATA, FEBRUARY 14 TO AUGUST 15, 2001

(Milligrams per liter unless otherwise indicated)

Lake stage (ft)

Secchi-depth $(m)$

Chlorophyll a, phytoplankton (ug/I)

Depth of sample (m)

water temperature $\left({ }^{\circ} \mathrm{C}\right)$

Specific conductance $(\mu \mathrm{S} / \mathrm{cm})$

$\mathrm{pH}$ (units)

Dissolved oxygen (mg/L)

Phosphorus, total (as P)

\begin{tabular}{|c|c|c|c|}
\hline \multicolumn{2}{|c|}{ Feb-14 } & \multicolumn{2}{|c|}{ Apr -24} \\
\hline & & & \\
\hline $\begin{array}{c}0.5 \\
1.9 \\
594 \\
8.1 \\
11.4 \\
0.009\end{array}$ & $\begin{array}{l}14.0 \\
3.9 \\
687 \\
7.5 \\
4.4 \\
0.012\end{array}$ & $\begin{array}{c}0.5 \\
10.3 \\
595 \\
8.0 \\
10.5 \\
---\end{array}$ & $\begin{array}{c}14.0 \\
7.6 \\
598 \\
7.8 \\
10.0 \\
0.012\end{array}$ \\
\hline
\end{tabular}

Jun-20

Jul-18

Aug -15

\begin{tabular}{|c|c|c|c|c|c|c|}
\hline \multicolumn{2}{|c|}{$\begin{array}{l}8.14 \\
7.1 \\
---\end{array}$} & \multicolumn{2}{|c|}{$\begin{array}{l}8.15 \\
3.9 \\
1.2\end{array}$} & \multicolumn{3}{|c|}{$\begin{array}{c}8.05 \\
1.70 \\
3.4\end{array}$} \\
\hline $\begin{array}{l}5 \\
.0 \\
72 \\
.1 \\
0\end{array}$ & $\begin{array}{c}14.5 \\
9.8 \\
600 \\
7.5 \\
1.1 \\
0.018\end{array}$ & $\begin{array}{c}0.5 \\
25.9 \\
582 \\
8.1 \\
8.7 \\
0.009\end{array}$ & $\begin{array}{c}14.5 \\
9.8 \\
621 \\
7.4 \\
0.3 \\
0.097\end{array}$ & $\begin{array}{c}0.5 \\
25.7 \\
560 \\
8.2 \\
9.5 \\
0.013\end{array}$ & $\begin{array}{c}11.0 \\
11.7 \\
617 \\
7.5 \\
0.4 \\
0.028\end{array}$ & $\begin{array}{c}14.5 \\
9.8 \\
645 \\
7.4 \\
0.2 \\
0.046\end{array}$ \\
\hline
\end{tabular}

2-14-01

4-24-01

6-20-01

7-18-01

8-15-01

DISSOLVED OXYGEN (D.O.), IN MILLIGRAMS PER LITER
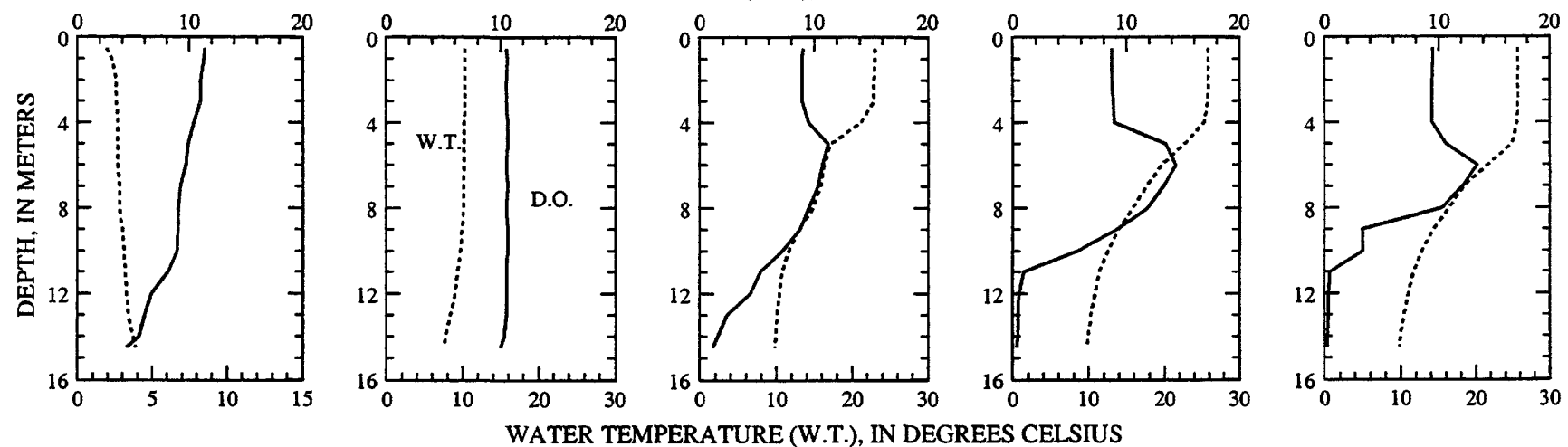

WATER TEMPERATURE (W.T.), IN DEGREES CELSIUS
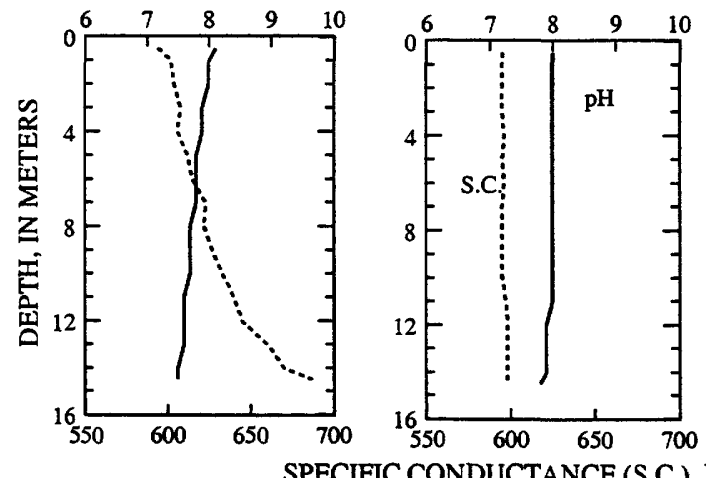

PH, IN STANDARD UNITS
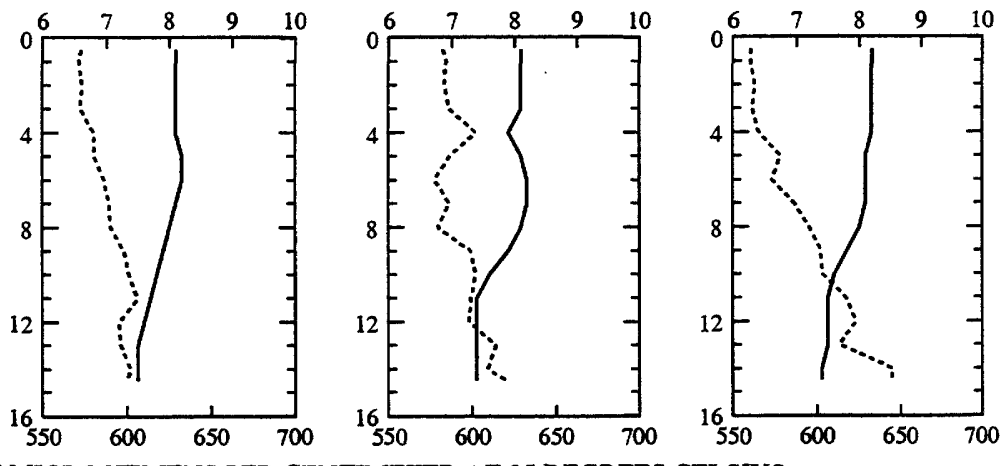

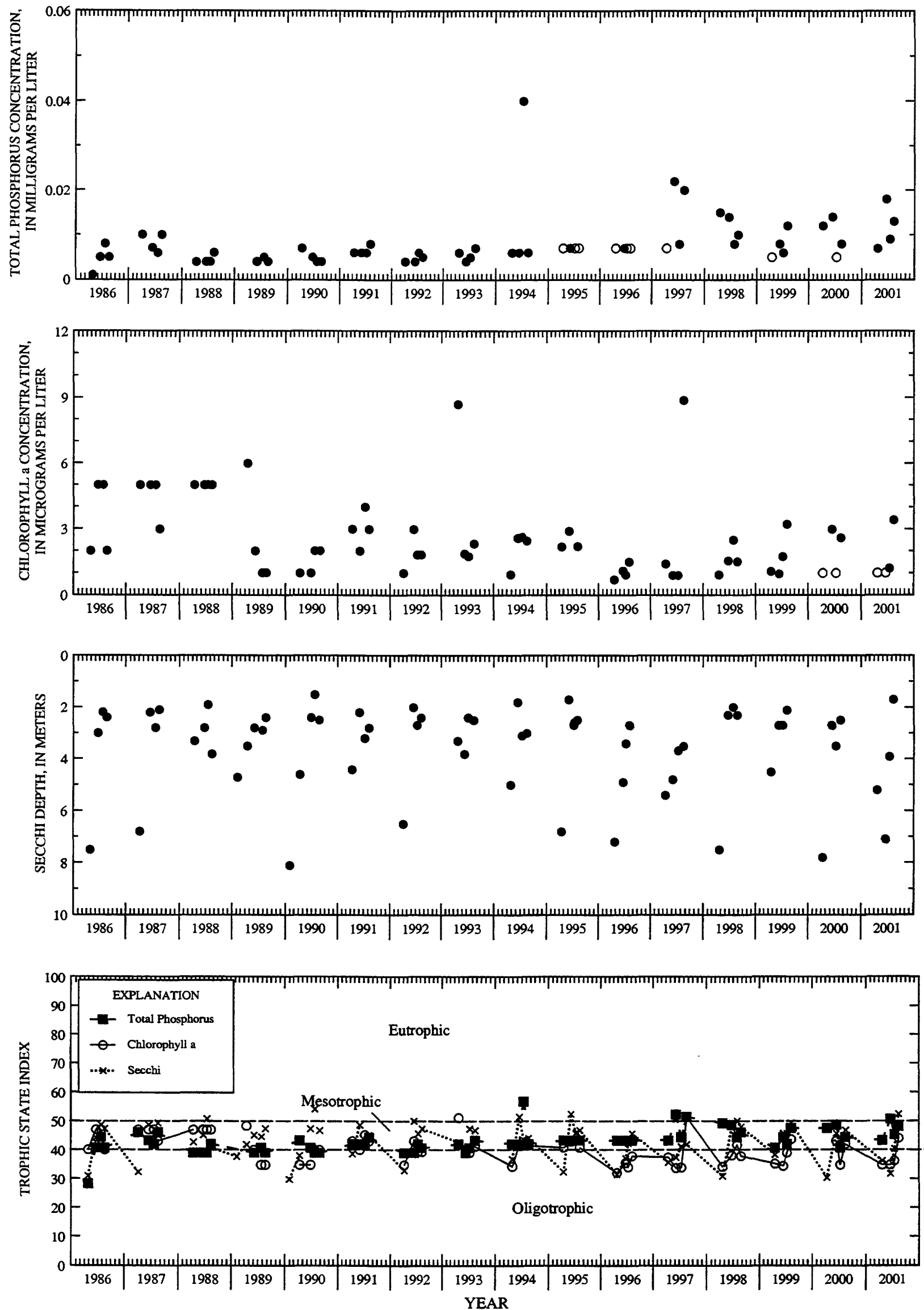

Surface total phosphorus, chlorophyll a concentrations, Secchi depths, and TSI data for Oconomowoc Lake, Hewitt Point, at Ocononomowoc, Wisconsin.

(Circles on the first three plots indicate laboratory detection limit for selected analyses. Actual concentrations for these particular analyses are less than the plotted circles.) 
LOCATION.--Lat $43^{\circ} 07^{\prime} 23^{\prime \prime}$, long $88^{\circ} 25^{\prime} 21^{\prime \prime}$, in SE 1/4 SE 1/4, sec.25, T.8 N., R.17 E., Waukesha County, Hydrologic Unit 07090001, at Okauchee. DRAINAGE AREA.--80.7 $\mathrm{mi}^{2}$.

PERIOD OF RECORD.--February 1984 to current year.

LAKE-STAGE GAGE.--Datum of gage is $869.00 \mathrm{ft}$ above sea level.

REMARKS.--Lake sampled near center at the deep hole. Lake ice-covered during February sampling. Water-quality analyses done by Wisconsin State Laboratory of Hygiene.

WATER-QUALITY DATA, FEBRUARY 14 TO AUGUST 21, 2001

(Milligrams per liter unless otherwise indicatedd

Lake stage (ft)

Secchi-depth (m)

Chlorophyll a, phytoplankton ( $\mu \mathrm{g} / \mathrm{L})$

Depth of sample (m)

water temoerature $\left({ }^{\circ} \mathrm{C}\right)$

Specific conductance $(\mu \mathrm{s} / \mathrm{cm})$

$\mathrm{pH}$ (units)

Dissolved oxygen (mg/L)

Phosphorus, total (as P)

Phosphorus, ortho, dissolved (as P)

Nitrogen, NO2 + NO3, diss. (as N)

Nitrogen, ammonia, dissolved (as N)

Nitrogen, amm. + org., total (as N)

Nitrogen, total (as N)

Color (Pt-Co. scale)

Turbidity (NTU)

Hardness, (as $\mathrm{CaCO}_{3}$ )

Calcium, dissolved ( $\mathrm{Ca}$ )

Magnesium, dissolved (Mg)

Sodium, dissolved (Na)

Potassium, dissolved (K)

Alkalinity, (as $\mathrm{CaCO}_{3}$ )

Sulfate, dissolved ( $\left.\mathrm{SO}_{4}\right)$

Chloride, dissolved (Cl)

Silica, dissolved $\left(\mathrm{SiO}_{2}\right)$

Solids, dissolved, at $180^{\circ} \mathrm{C}$

Iron, dissolved (Fe) $\mu \mathrm{g} / \mathrm{L}$

Manganese, dissolved (Mn) $\mu \mathrm{g} / \mathrm{L}$

2-14-01
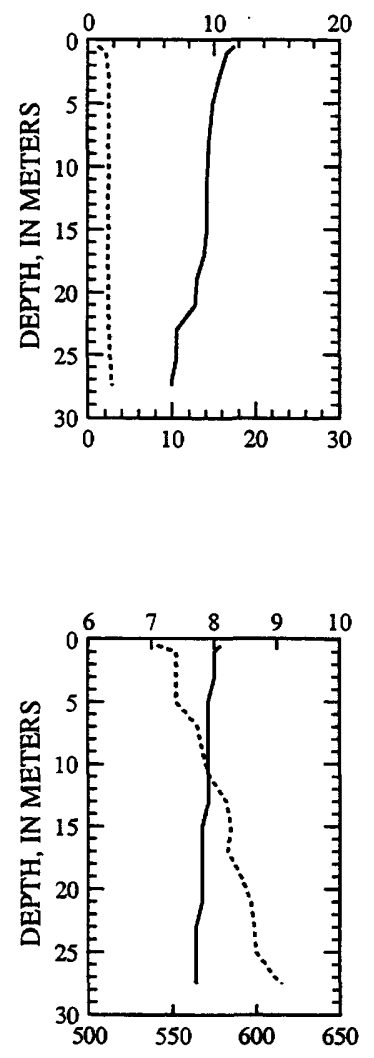

Apr -24

Jun -20

Ju1 -20

Aug - 21

\begin{tabular}{|c|c|c|c|c|c|c|c|c|c|c|}
\hline \multicolumn{2}{|c|}{ Feb-14 } & \multicolumn{2}{|c|}{ Apr -24} & \multicolumn{2}{|c|}{ Jun-20 } & \multicolumn{2}{|c|}{ Jul-20 } & \multicolumn{3}{|c|}{ Aug-21 } \\
\hline & & & & & & & & & $\begin{array}{c}3.81 \\
1.5 \\
8\end{array}$ & \\
\hline $\begin{array}{c}0.5 \\
1.2 \\
541 \\
8.1 \\
11.6 \\
0.015\end{array}$ & $\begin{array}{c}27.0 \\
2.8 \\
615 \\
7.7 \\
6.6 \\
0.014\end{array}$ & $\begin{array}{c}0.5 \\
8.5 \\
565 \\
8.0 \\
11.3 \\
0.024\end{array}$ & $\begin{array}{c}27.0 \\
6.2 \\
565 \\
8.0 \\
10.3 \\
0.041\end{array}$ & $\begin{array}{c}0.5 \\
22.4 \\
541 \\
8.0 \\
9.2 \\
0.024\end{array}$ & $\begin{array}{c}27.5 \\
6.7 \\
570 \\
7.4 \\
0.4 \\
0.024\end{array}$ & $\begin{array}{c}0.5 \\
-\cdots \\
--- \\
--- \\
--- \\
0.016\end{array}$ & $\begin{array}{c}27.0 \\
--- \\
--- \\
--- \\
--- \\
0.092\end{array}$ & $\begin{array}{c}0.5 \\
23.2 \\
507 \\
8.0 \\
8.5 \\
0.013\end{array}$ & $\begin{array}{l}7.0 \\
17.4 \\
574 \\
7.5 \\
0.6 \\
0.015\end{array}$ & $\begin{array}{c}27.5 \\
6.6 \\
575 \\
7.4 \\
0.2 \\
0.103\end{array}$ \\
\hline--- & --- & 0.002 & --- & -- & --- & -- & -- & 0.003 & --- & --- \\
\hline--- & --- & 0.618 & --- & --- & --- & --- & -- & 0.051 & $-\infty$ & -- \\
\hline--- & --- & $<0.013$ & -- & --- & --- & -- & --- & 0.023 & --- & --- \\
\hline-- & $\ldots$ & 0.59 & --- & $-\cdots$ & -- & $-\cdots$ & --- & --- & -- & --- \\
\hline--- & -- & 1.21 & -- & -- & -- & -- & -- & --- & --- & -- \\
\hline--- & --- & 20 & $-\infty$ & -- & $-\infty$ & --- & -- & --- & -- & -- \\
\hline--- & -- & 2.7 & --- & --- & -- & --- & $-\infty$ & --- & --- & --- \\
\hline-- & -- & 278 & --- & -- & --- & --- & -- & --- & --- & -- \\
\hline$-\cdots$ & $\ldots$ & 57 & --- & -- & $-\ldots$ & $-\infty$ & -- & $-\cdots$ & -- & --- \\
\hline--- & --- & 33 & -- & --- & --- & $-\infty$ & --- & --- & --- & -- \\
\hline-- & -- & 14 & --- & -- & --- & --- & -- & -- & --- & --- \\
\hline--- & $\ldots$ & 1.8 & -- & $\ldots$ & -- & --- & -- & --- & --- & $-\infty$ \\
\hline-- & $-\cdots$ & 230 & -- & --- & -- & -- & -- & -- & --- & --- \\
\hline--- & -- & 24.6 & $-\infty$ & -- & --- & -- & $\cdots$ & --- & -- & -- \\
\hline--- & $-\cdots$ & 32.3 & --- & --- & $-\infty$ & $-\infty$ & --- & -- & --- & -- \\
\hline--- & --- & 3.8 & --- & --- & $m$ & $-\infty$ & --- & --- & --- & -- \\
\hline--- & -- & 338 & --- & -- & -- & -- & --- & --- & --- & --- \\
\hline--- & -- & $<10$ & --- & --- & --- & --- & $m$ & --- & $-\cdots$ & --- \\
\hline--- & --- & $<0.4$ & --- & --- & --- & $-\cdots$ & $--\infty$ & --- & --- & --- \\
\hline
\end{tabular}

4-24-01

6-20-01

8-21-01

DISSOLVED OXYGEN (D.O.), IN MILLIGRAMS PER LITER
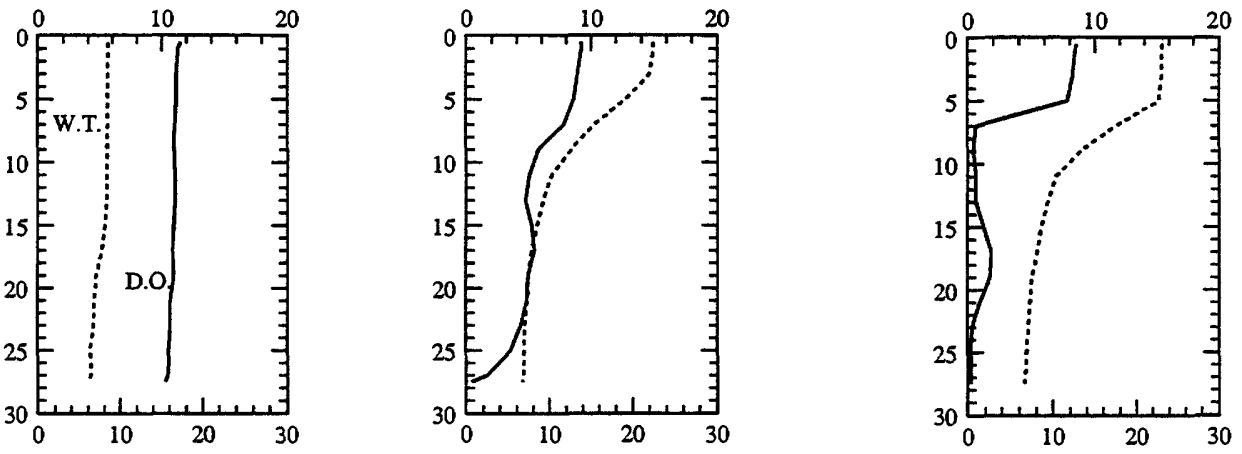

WATER TEMPERATURE (W.T.), IN DEGREES CELSIUS

PH, IN STANDARD UNITS
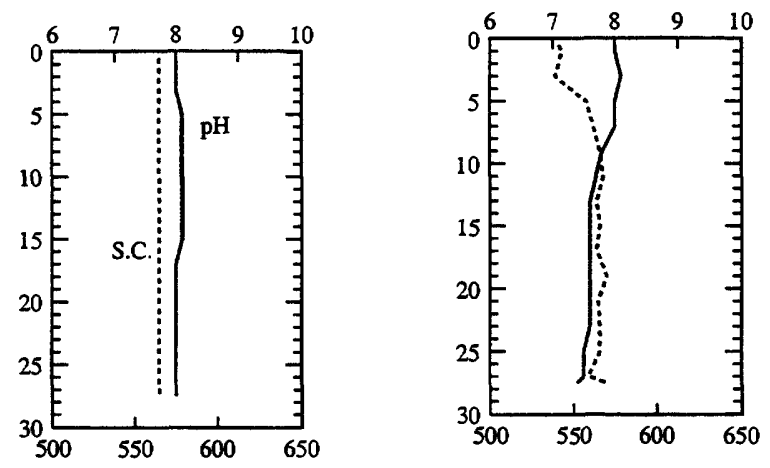

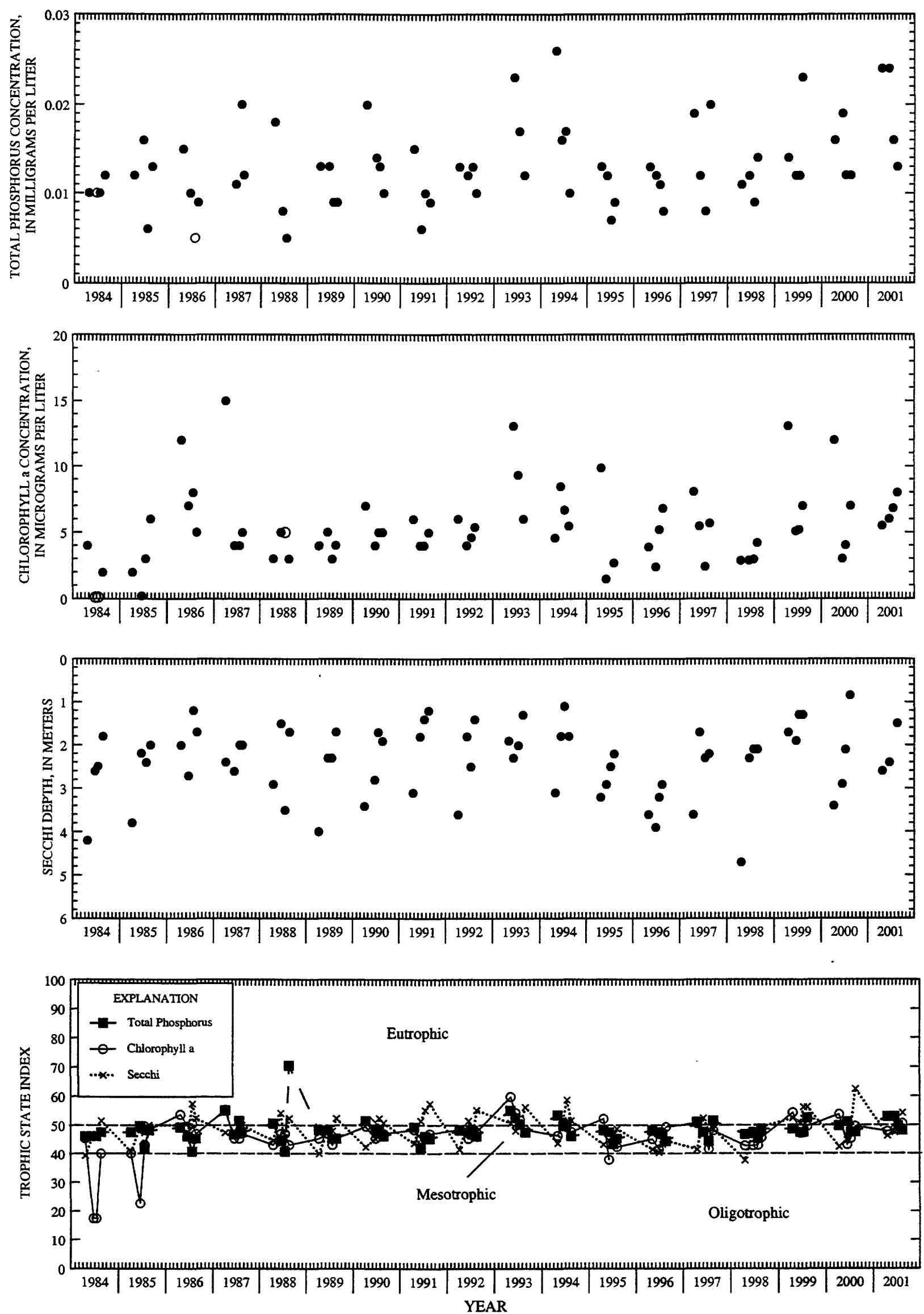

Surface total phosphorus, chlorophyll a concentrations, Secchi depths, and TSI data for Okauchee Lake, near Okauchee, Wisconsin.

(Circles on the first three plots indicate laboratory detection limit for selected analyses.

Actual concentrations for these particular analyses are less than the plotted circles.) 
LOCATION.--Lat $43^{\circ} 07^{\prime} 59^{\prime \prime}$, long 88²4'42", in NE 1/4 NW 1/4 sec.30, T.8 N., R.18 E., Waukesha County, Hydrologic Unit 07090001, near Okauchee.

PERIOD OF RECORD.--April 1986 to current year.

LAKE-STAGE GAGE.--Datum of gage is $869.00 \mathrm{ft}$ above sea level.

REMARKS.--Lake sampled in Crane's Nest Bay, in the northeast part of the lake, at an approximate depth of $2 \mathrm{~m}$. Water-quality analyses done by Wisconsin State Laboratory of Hygiene.

WATER-QUALITY DATA, APRIL 24 TO AUGUST 21, 2001

(Milligrams per liter unless otherwise indicated)

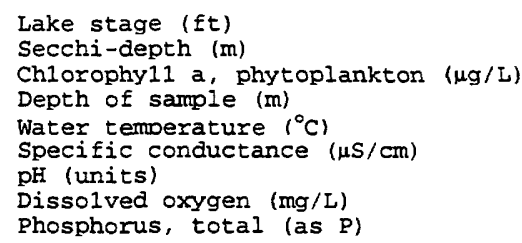

\begin{tabular}{cccc} 
Apr-24 & Jun-20 & Jul-20 & Aug-21 \\
\hline 3.76 & 3.90 & -- & 3.81 \\
2.7 & 1.3 & --- & 1.7 \\
7 & 6 & 6 & 7 \\
0.5 & 0.5 & 0.5 & 0.5 \\
8.6 & 21.9 & --- & 23.3 \\
566 & 576 & --- & 525 \\
8.1 & 8.0 & --- & 8.2 \\
11.6 & 8.4 & --5 & 8.8 \\
0.022 & 0.038 & 0.019 & 0.021
\end{tabular}



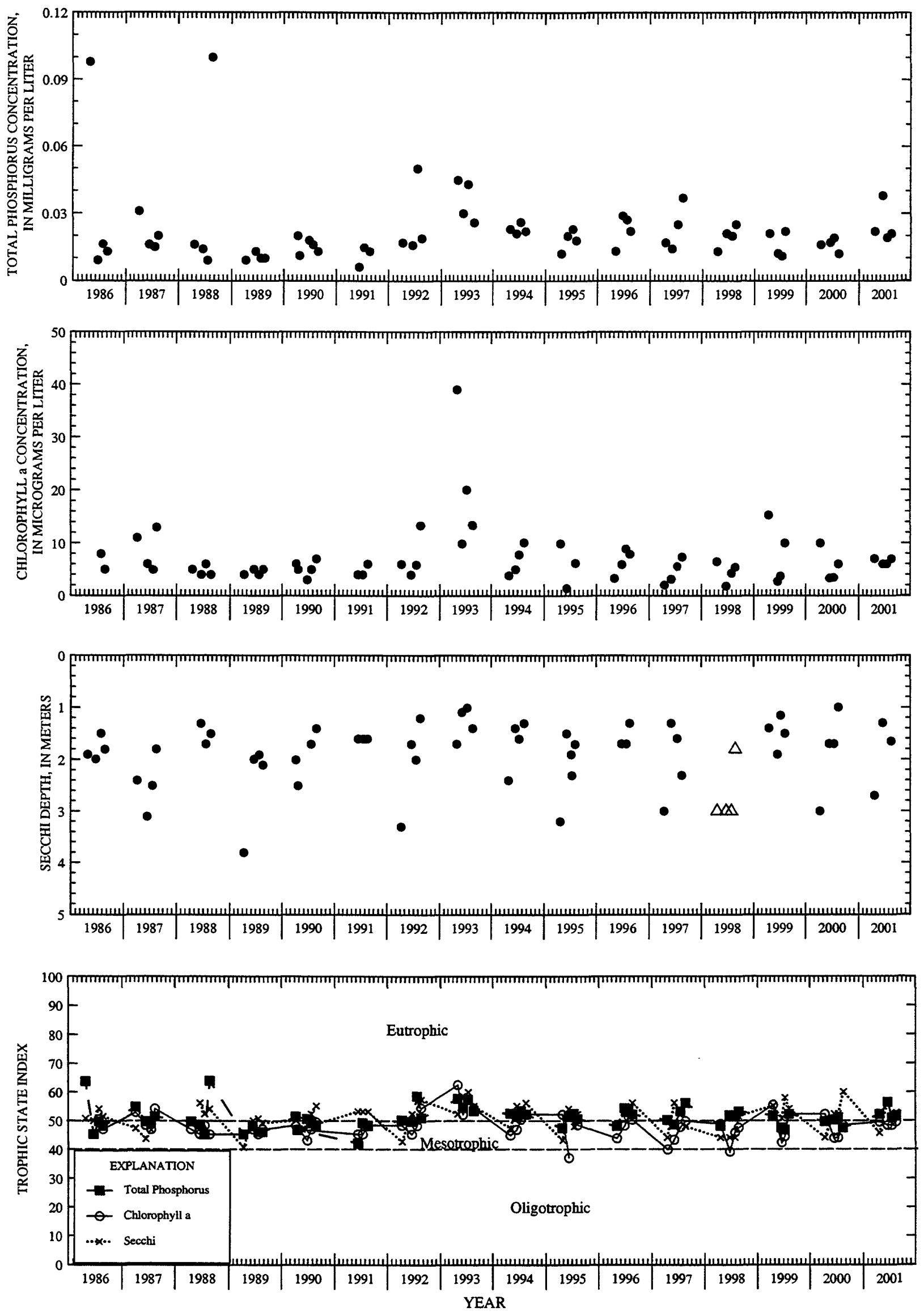

Surface total phosphorus, chlorophyll a concentrations, Secchi depths, and TSI data for Okauchee Lake, No. 1, near Okauchee, Wisconsin.

(Circles on the first three plots indicate laboratory detection limit for selected analyses. Actual concentrations for these particular analyses are less than the plotted circles.) 
LOCATION.--Lat $43^{\circ} 06^{\prime} 45^{\prime \prime}$, long $88^{\circ} 26^{\prime} 45^{\prime \prime}$, in SE 1/4 NE 1/4 sec.35, T.8 N., R.17 E., Waukesha County, Hydrologic Unit 07090001, at Okauchee.

PERIOD OF RECORD.--April 1986 to current year.

LAKE-STAGE GAGE.--Datum of gage is $869.00 \mathrm{ft}$ above sea level.

REMARKS.--Lake sampled in Lower Okauchee Lake, at an approximate depth of $5 \mathrm{~m}$. Water-quality analyses done by Wisconsin State Laboratory of Hygiene.

WATER-QUALITY DATA, APRIL 24 TO AUGUST 21, 2001

(Milligrams per liter unless otherwise indicated)

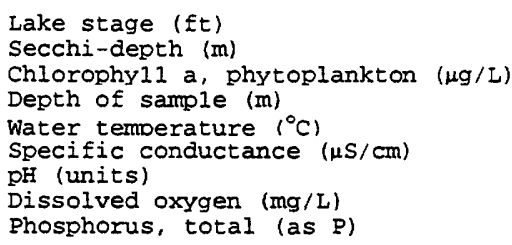

\begin{tabular}{c} 
Apr-24 \\
\hline 3.76 \\
2.6 \\
4.5 \\
0.5 \\
11.0 \\
562 \\
8.2 \\
10.4 \\
0.020
\end{tabular}

\begin{tabular}{c} 
Jun-20 \\
\hline 3.90 \\
2.5 \\
4.4 \\
0.5 \\
23.7 \\
530 \\
8.1 \\
8.9 \\
0.024
\end{tabular}

Jul-20
---
---
4.7
0.5
---
---
---
.--
0.020

Aug -21

3.81

2.4

4.7

0.5

23.9

474

8.3

9.8 

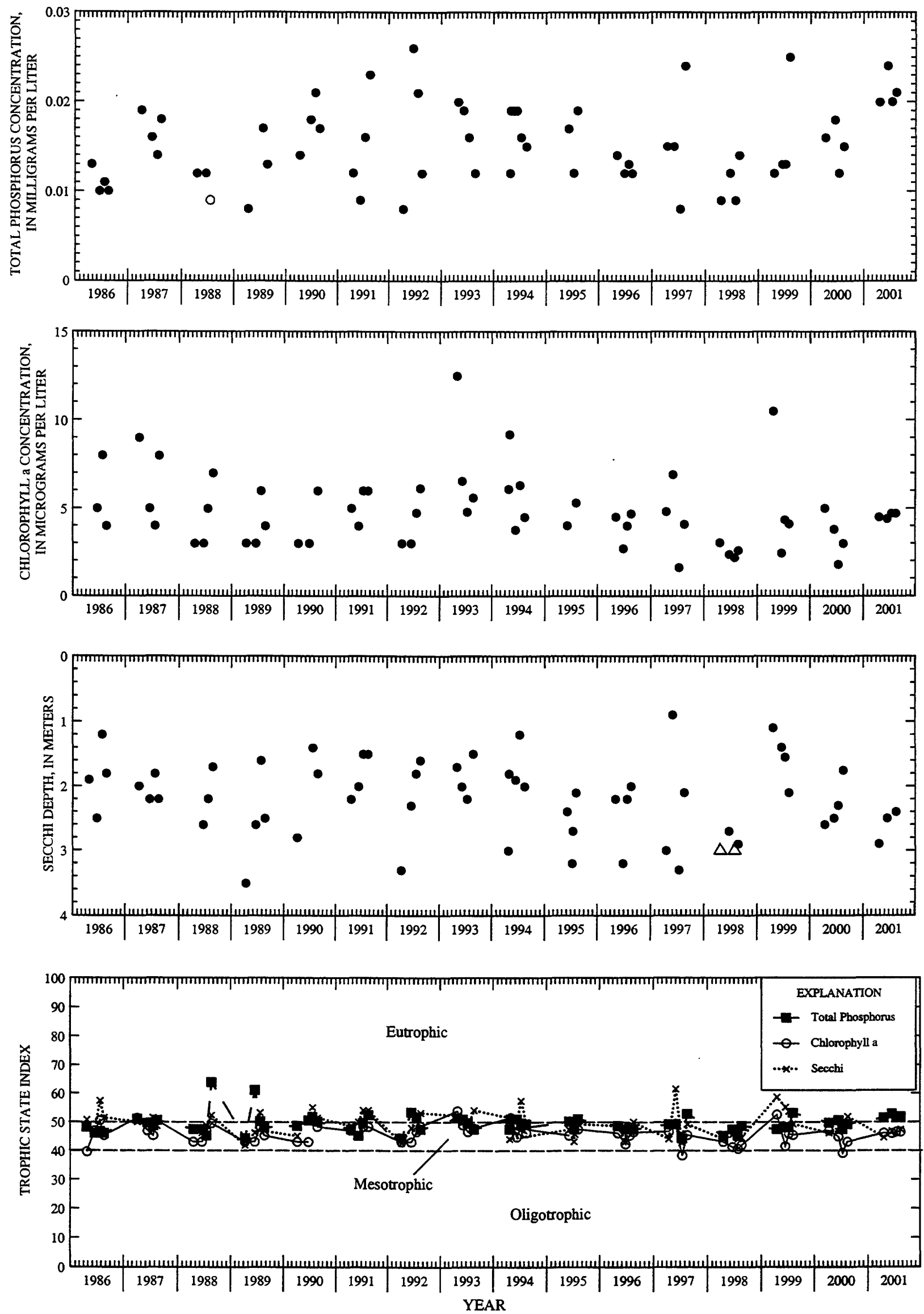

Surface total phosphorus, chlorophyll a concentrations, Secchi depths, and TSI data for Okauchee Lake, No. 2, near Okauchee, Wisconsin. (Circles on the first three plots indicate laboratory detection limit for selected analyses. Actual concentrations for these particular analyses are less than the plotted circles. Triangles Indicate maximum depth at sampling site. Actual secchi depth on these days was greater than plotted triangles.) 
LOCATION.--Lat $43^{\circ} 06^{\prime} 42^{\prime \prime}$, long $88^{\circ} 25^{\prime} 24^{\prime \prime}$, in NE 1/4 SE 1/4 sec.36, T.8 N., R.17 E., Waukesha County, Hydrologic Unit 07090001, at Okauchee.

PERIOD OF RECORD.--April 1986 to current year.

LAKE-STAGE GAGE.--Datum of gage is $869.00 \mathrm{ft}$ above sea level.

REMARKS.--Lake sampled in Ice House Bay, in the southern part of the lake, at an approximate depth of $4 \mathrm{~m}$. Water-quality analyses done by Wisconsin State Laboratory of Hygiene.

WATER-QUALITY DATA, APRIL 24 TO AUGUST 21, 2001

(Milligrams per liter unless otherwise indicated)

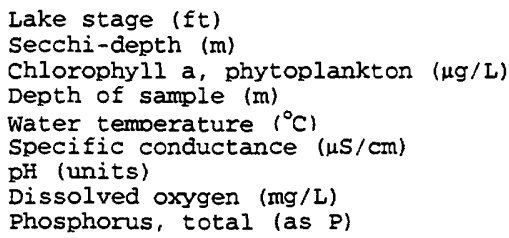

\begin{tabular}{c} 
Apr-24 \\
\hdashline 3.76 \\
2.2 \\
3.5 \\
0.5 \\
9.8 \\
562 \\
8.2 \\
11.1 \\
0.019
\end{tabular}

\begin{tabular}{c} 
Jun -20 \\
\hline 3.90 \\
1.7 \\
8 \\
0.5 \\
23.4 \\
521 \\
8.2 \\
10.3 \\
0.020
\end{tabular}

\begin{tabular}{c} 
Jul-20 \\
\hdashline-- \\
-- \\
4 \\
0.5 \\
$\cdots-$ \\
$\cdots-$ \\
$-\cdots$ \\
0.020
\end{tabular}

Aug-21 

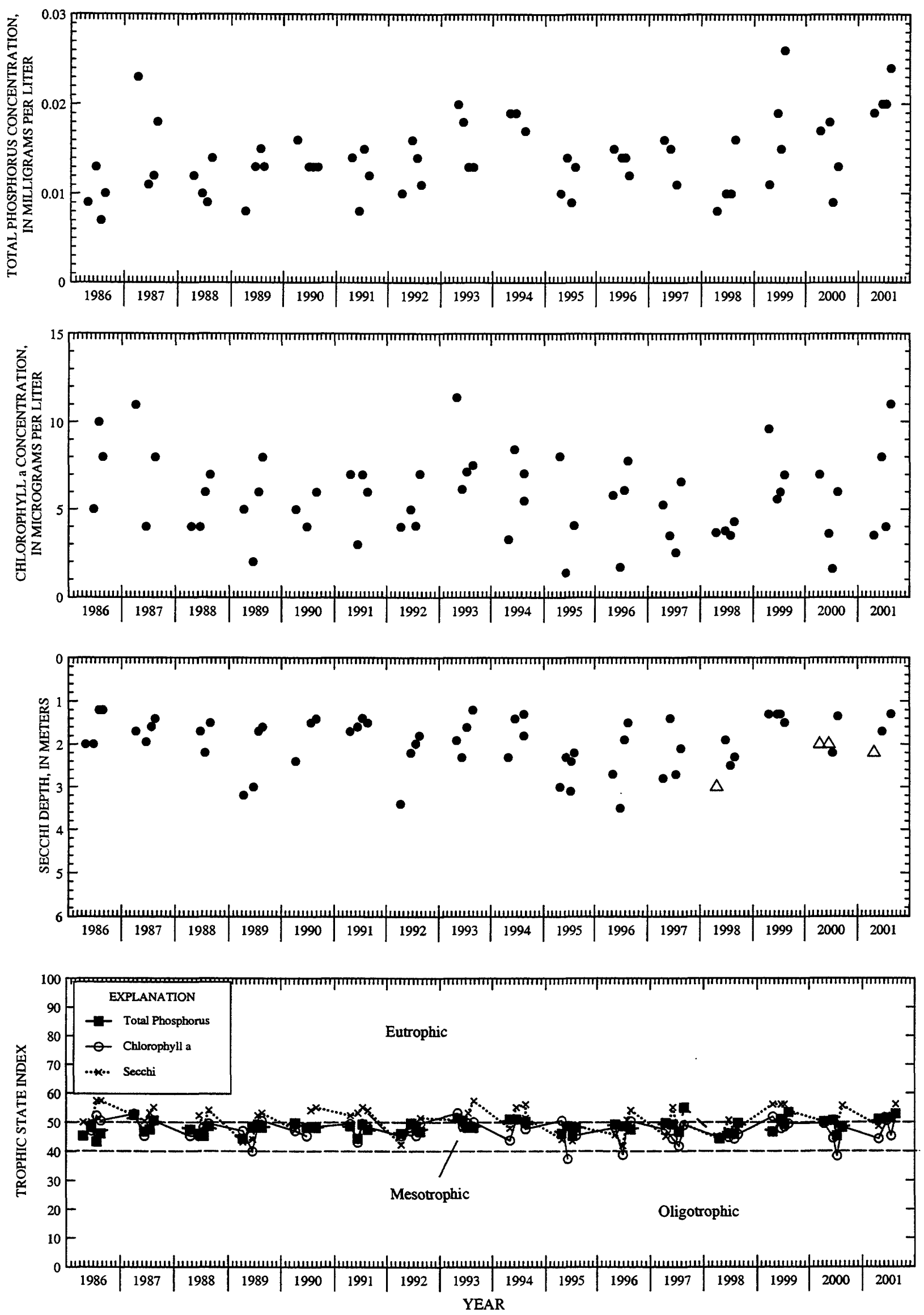

Surface total phosphorus, chlorophyll a concentrations, Secchi depths, and TSI data for Okauchee Lake, No. 3, near Okauchee, Wisconsin.

(Triangles indicate maximum depth at sampling site. Actual Secchi depth on these days was greater thank the plotted triangles.) 
LOCATION.--Lat $43^{\circ} 07^{\prime} 57^{\prime \prime}$, long $88^{\circ} 26^{\prime} 17^{\prime \prime}$, in NW 1/4 NW 1/4 sec.25, T.8 N., R.17 E., Waukesha County, Hydrologic Unit 07090001, at Okauchee.

PERIOD OF RECORD.--June 1986 to current year.

LAKE-STAGE GAGE.--Datum of gage is $869.00 \mathrm{ft}$ above sea level.

REMARKS.--Lake sampled near McDowell (Crazyman's) Island, in the northwest bay of the lake, at an approximate depth of $2 \mathrm{~m}$. Waterquality analyses done by Wisconsin State Laboratory of Hygiene.

WATER-QUALITY DATA, APRIL 24 TO AUGUST 21, 2001

(Milligrams per liter unless otherwise indicated)

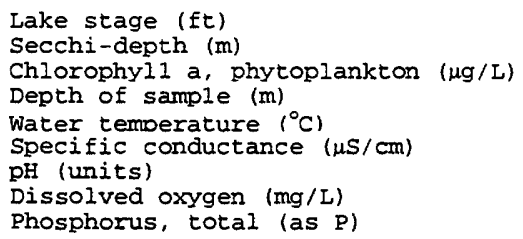

\begin{tabular}{cccc} 
Apr-24 & Jun-20 & Jul-20 & Aug-21 \\
\hdashline 3.76 & 3.90 & --- & 3.81 \\
2.9 & 1.5 & -- & 1.3 \\
5 & 8 & 3.4 & 7 \\
0.5 & 0.5 & 0.5 & 0.5 \\
8.7 & 23.2 & -- & 23.4 \\
564 & 510 & -- & 496 \\
8.1 & 8.2 & -- & 8.2 \\
11.4 & 10.7 & $-\cdots$ & 9.7 \\
0.023 & 0.018 & 0.017 & 0.023
\end{tabular}



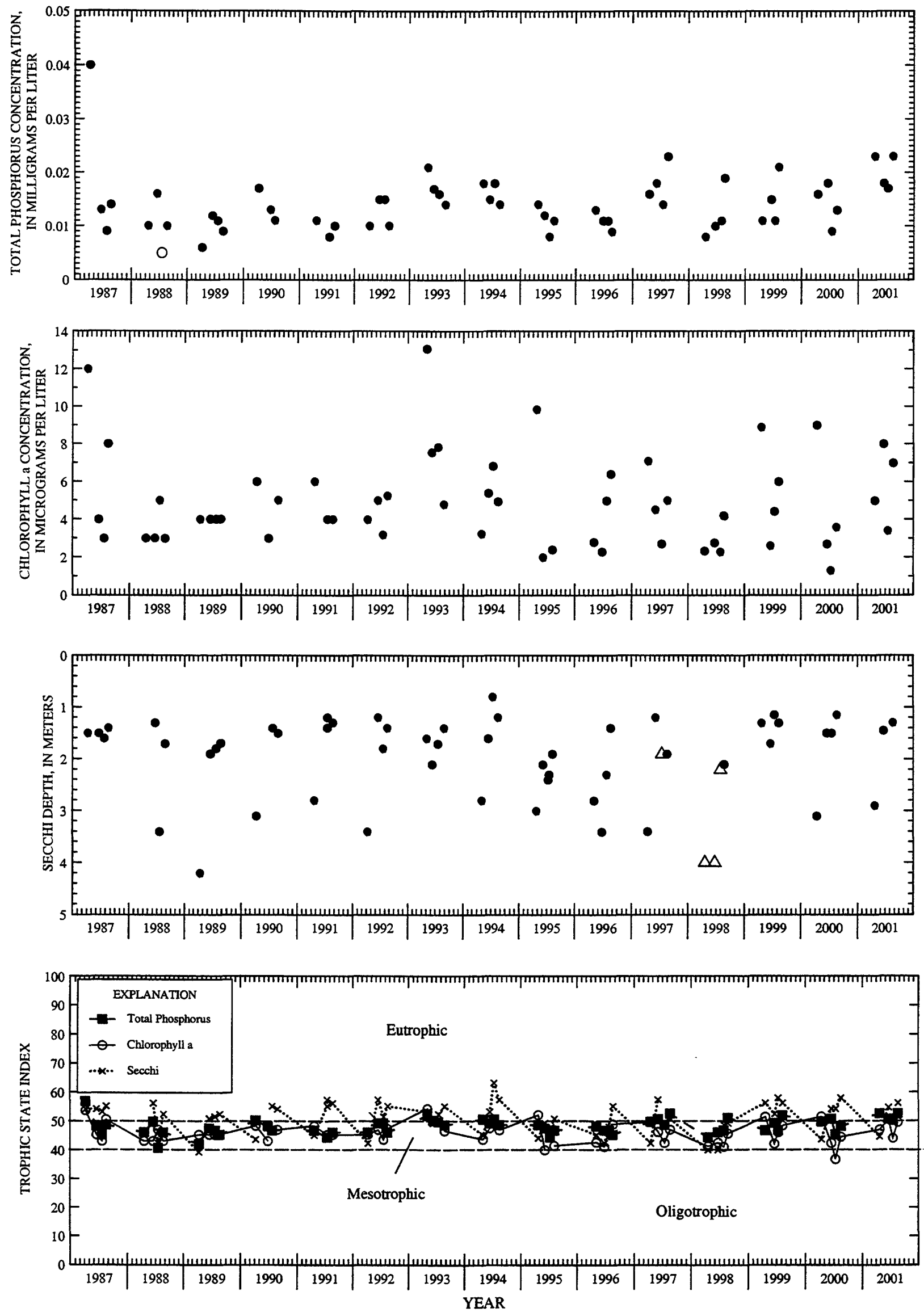

Surface total phosphorus, chlorophyll a concentrations, Secchi depths, and TSI data for Okauchee Lake, No. 4, near Okauchee, Wisconsin. (Circles on the first three plots indicate laboratory detection limit for selected analyses. Actual concentrations for these particular analyses are less than the plotted circles. Triangles indicate maximum depth at sampling site. Actual Secchi depth on these days was greater than the plotted triangles.) 
CATION.--Lat $42^{\circ} 49^{\prime} 05^{\prime \prime}$, long $88^{\circ} 20^{\prime} 40^{\prime \prime}$, in NW 1/4 SW 1/4 sec.11, T.4 N., R.18 E., Walworth County, Hydrologic Unit 07120006, 3.3 mi south of Mukwonago.

RIOD OF RECORD.--February 1993 to current year.

MARKS.--Lake sampled at the deep hole. Lake ice-covered during February sampling. Water-quality analyses done by Wisconsin State Laboratory of Hygiene.

WATER-QUALITY DATA, FEBRUARY 12 TO AUGUST 14, 2001

(Milligrams per liter unless otherwise indicated)

ce stage (ft)

chi-depth (m)

lorophy11 a, phytoplankton ( $\mu \mathrm{g} / \mathrm{L}$ )

th of sample (m)

cer temperature $\left({ }^{\circ} \mathrm{C}\right)$

ecific conductance ( $\mu \mathrm{S} / \mathrm{cm})$

(units)

solved oxygen (mg/L)

osphorus, total (as P)

sphorus, ortho, dissolved (as P)

crogen, $\mathrm{NO} 2$ + NO3, diss. (as N)

crogen, ammonia, dissolved (as N)

Erogen, arm. + org., total (as N)

rogen, total (as N)

or (Pt-Co. scale)

bidity (NTU)

idness, (as $\mathrm{CaCO}_{3}$ )

cium, dissolved (Ca)

mesium, dissolved ( $\mathrm{Mg})$

ium, dissolved (Na)

:assium, dissolved (K)

calinity, (as $\mathrm{CaCO}_{3}$ )

fate, dissolved $\left(\mathrm{SO}_{4}\right)$

oride, dissolved (C1)

ica, dissolved $\left(\mathrm{SiO}_{2}\right)$

ids. dissolved, at $180^{\circ} \mathrm{C}$

on, dissolved (Fe) $\mu \mathrm{g} / \mathrm{I}$

uganese, dissolved (Mn) $\mu \mathrm{g} / \mathrm{L}$

\begin{tabular}{|c|c|c|c|}
\hline \multicolumn{2}{|c|}{ Feb-12 } & \multicolumn{2}{|c|}{ Apr -18} \\
\hline \multicolumn{2}{|c|}{$\begin{array}{c}8.16 \\
--\end{array}$} & \multicolumn{2}{|c|}{$\begin{array}{c}8.54 \\
1.1 \\
20\end{array}$} \\
\hline 0.5 & 7.0 & 0.5 & 7.0 \\
\hline 2.9 & 4.5 & 10.3 & 10.0 \\
\hline 495 & 530 & 457 & 459 \\
\hline 7.4 & 7.4 & 8.3 & 8.3 \\
\hline 7.5 & 3.3 & 11.3 & 11.1 \\
\hline 0.016 & 0.029 & 0.029 & 0.025 \\
\hline--- & --- & 0.01 & -- \\
\hline--- & -- & 0.115 & -- \\
\hline-- & --- & 0.177 & --- \\
\hline-- & --- & 1.3 & --- \\
\hline-- & --- & 1.44 & -- \\
\hline--- & --- & 15 & --- \\
\hline--- & -- & 4.1 & $\ldots$ \\
\hline$-\infty$ & --- & 182 & --- \\
\hline--- & -- & 40 & -- \\
\hline--- & -- & 20 & --- \\
\hline-- & -- & 20 & --- \\
\hline-- & -- & 2.1 & -- \\
\hline-- & -- & 158 & --- \\
\hline--- & --- & $<4.5$ & --- \\
\hline$-\cdots$ & -- & 44.9 & --- \\
\hline--- & --- & 1.5 & --- \\
\hline--- & --- & 264 & $\cdots$ \\
\hline-- & -- & $<10$ & -- \\
\hline$\cdots$ & -- & $<0.4$ & -- \\
\hline
\end{tabular}

4-18-01

6-13-01
Jun-13

8.58

1.1

$0.5^{13.9} 7.0$

$24.2 \quad 14.8$

$431 \quad 457$

$\begin{array}{cl}8.2 & 7.7 \\ 12.2 & 1.8\end{array}$

$0.033 \quad 0.052$

$---$

$---$

$\overline{---}$

$---$

$--$

-

ㅍ.

$--$

$--$

--

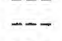

$--$

---

$---$

$---$

$--$

---

---

$---$

---
---
---
--
Ju1-16

0

0.7
5.7

$\begin{array}{cc}0.5 & 7.0 \\ 27.7 & 14.7 \\ 445 & 507 \\ 8.1 & 7.0 \\ 9.1 & 0.2\end{array}$

$0.032 \quad 0.064$

-. -

---

$---$

$--$

$\ldots$

$\cdots$

-..

2-12-01

7-16-01

8-14-01

DISSOLVED OXYGEN (D.O.), IN MILLIGRAMS PER LITER
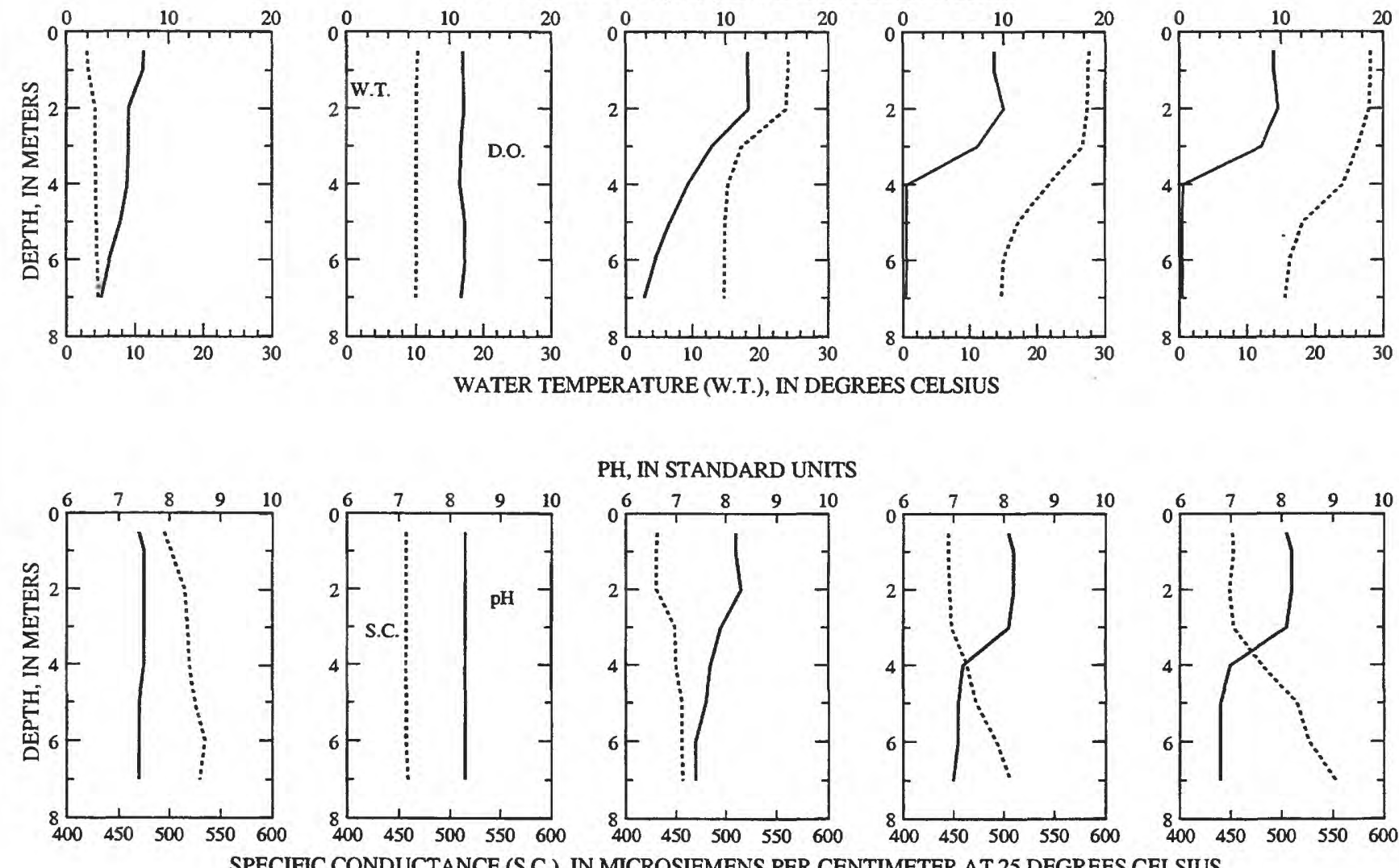

PH, IN STANDARD UNITS
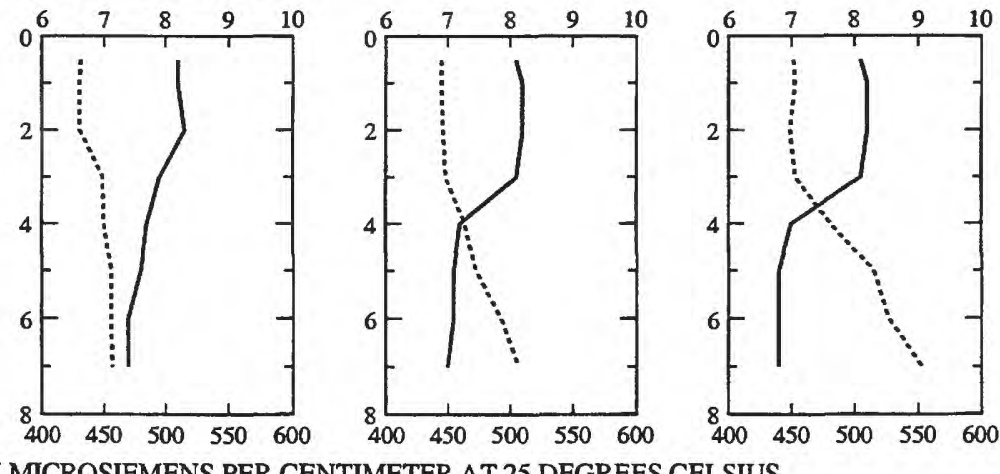

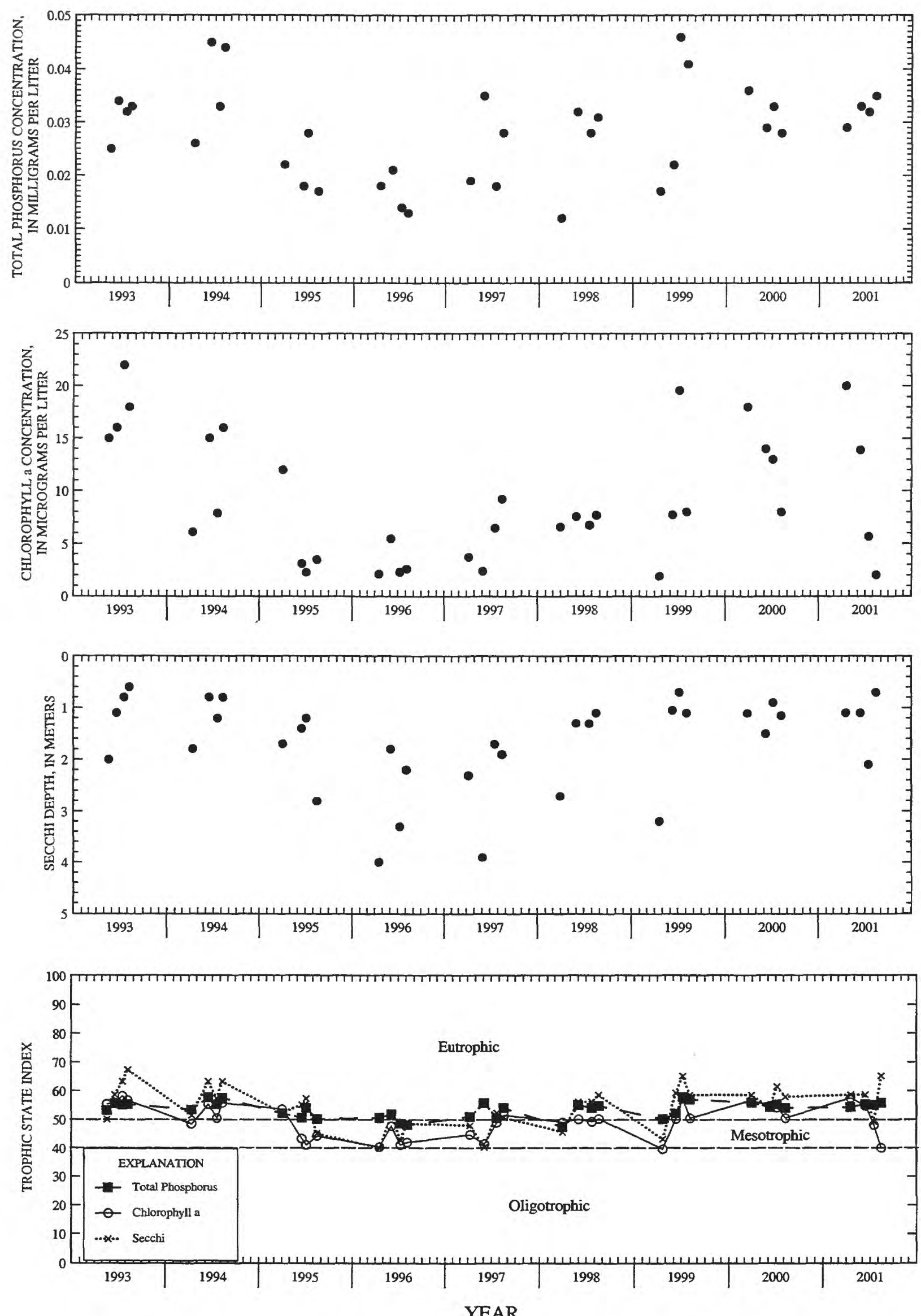

Surface total phosphorus, chlorophyll a concentrations, Secchi depths, and TSI data for Potter Lake near Mukwonago, Wisconsin. 
LOCATION.--Lat 42³2'46", long 88¹7'58", in NW 1/4 SE 1/4 sec.13, T.1 N., R.18 E., Walworth County, Hydrologic Unit 07120006, at Powers Lake. DRAINAGE AREA.--3.42 $\mathrm{mi}^{2}$.

PERIOD OF RECORD.--March 1986 to August 1996, and April 1998 to current year.

REMARKS.--Lake sampled near center at the deep hole. Lake ice-covered during February sampling. Water-quality analyses done by Wisconsin State Laboratory of Hygiene.

WATER-QUALITY DATA, FEBRUARY 12 TO AUGUST 20, 2001

(Milligrams per liter unless otherwise indicated)

Lake stage ( $f t)$

Secchi-depth (m)

Chlorophy11 a, phytoplankton ( $\mu \mathrm{g} / \mathrm{L}$ )

Depth of sample (m)

Water temperature $\left({ }^{\circ} \mathrm{C}\right)$

specific conductance ( $\mu \mathrm{s} / \mathrm{cm}$ )

pH (units)

Dissolved oxygen (mg/L)

Phosphorus, total (as P)

Phosphorus, ortho, dissolved (as P)

Nitrogen, $\mathrm{NO} 2$ + NO3, diss. (as N)

Nitrogen, ammonia, dissolved (as N)

Nitrogen, amm. + org.. total (as N)

Nitrogen, total (as $N$ )

Color (Pt-Co. scale)

Turbidity (NTU)

Hardness, (as $\mathrm{CaCO}_{3}$ )

Calcium, dissolved (Ca)

Magnesium, dissolved (Mg)

Sodium, dissolved (Na)

Potassium, dissolved (K)

Alkalinity, (as $\mathrm{CaCO}_{3}$ )

Sulfate, dissolved $\left(\mathrm{SO}_{4}\right)$

Chloride, dissolved (CI)

Silica, dissolved $\left(\mathrm{SiO}_{2}\right)$

Solids, dissolved. at $180^{\circ} \mathrm{C}$

Iron, dissolved ( $\mathrm{Fe}) \mu \mathrm{g} / \mathrm{L}$

Manganese, dissolved (Mn) $\mu \mathrm{g} / \mathrm{L}$

\begin{tabular}{|c|c|c|c|}
\hline \multicolumn{2}{|c|}{ Feb-12 } & \multicolumn{2}{|c|}{ Apr -10} \\
\hline & & \multicolumn{2}{|c|}{$\begin{array}{c}3.9 \\
6\end{array}$} \\
\hline 0.5 & 9.5 & 0.5 & 9.5 \\
\hline 1.7 & 4.5 & 8.6 & 8.5 \\
\hline 484 & 569 & 511 & 514 \\
\hline 7.9 & 7.4 & 8.0 & 8.1 \\
\hline $\begin{array}{l}14.0 \\
0.017\end{array}$ & $\begin{array}{c}4.1 \\
0.009\end{array}$ & $\begin{array}{l}11.8 \\
0.023\end{array}$ & $\begin{array}{l}11.5 \\
0.012\end{array}$ \\
\hline$\ldots$ & $\ldots$ & 0.003 & -- \\
\hline--- & $\ldots$ & 0.06 & --- \\
\hline-- & --- & 0.041 & $\ldots$ \\
\hline-- & -- & 0.51 & $-\cdots$ \\
\hline--- & -- & 0.57 & $-\cdots$ \\
\hline-- & --- & 10 & $\ldots$ \\
\hline--- & --- & 1.2 & --- \\
\hline--- & $\ldots$ & 217 & --- \\
\hline--- & --- & 39 & --- \\
\hline-- & -- & 29 & --- \\
\hline-- & --- & 16 & $\cdots$ \\
\hline-- & --- & 2.5 & --- \\
\hline-- & -- & 177 & --- \\
\hline-- & --- & 28.2 & --- \\
\hline--- & --- & 35.2 & --- \\
\hline--- & --- & 9.4 & --- \\
\hline--- & --- & 278 & -- \\
\hline-- & --- & $<10$ & --- \\
\hline--- & $-\cdots$ & 4.2 & -- \\
\hline
\end{tabular}

12.37

Jul-26

Aug-20

$\begin{array}{cc} & 6 \\ 0.5 & 10.0 \\ 22.8 & 15.3 \\ 480 & 499 \\ 8.0 & 7.4 \\ 8.7 & 1.1 \\ 0.021 & 0.019\end{array}$

10.03

2.6

$0.5^{4.8} 9.5$

26.2

6.215 .3

475

$8.1 \quad 7.6$

$8.0 \quad 0.2$

$0.013 \quad 0.013$

n.

---

--

-.

---

-

--

$--$

$--$

---

$---$

---

‥

$--$

DISSOLVED OXYGEN (D.O.), IN MILLIGRAMS PER LITER
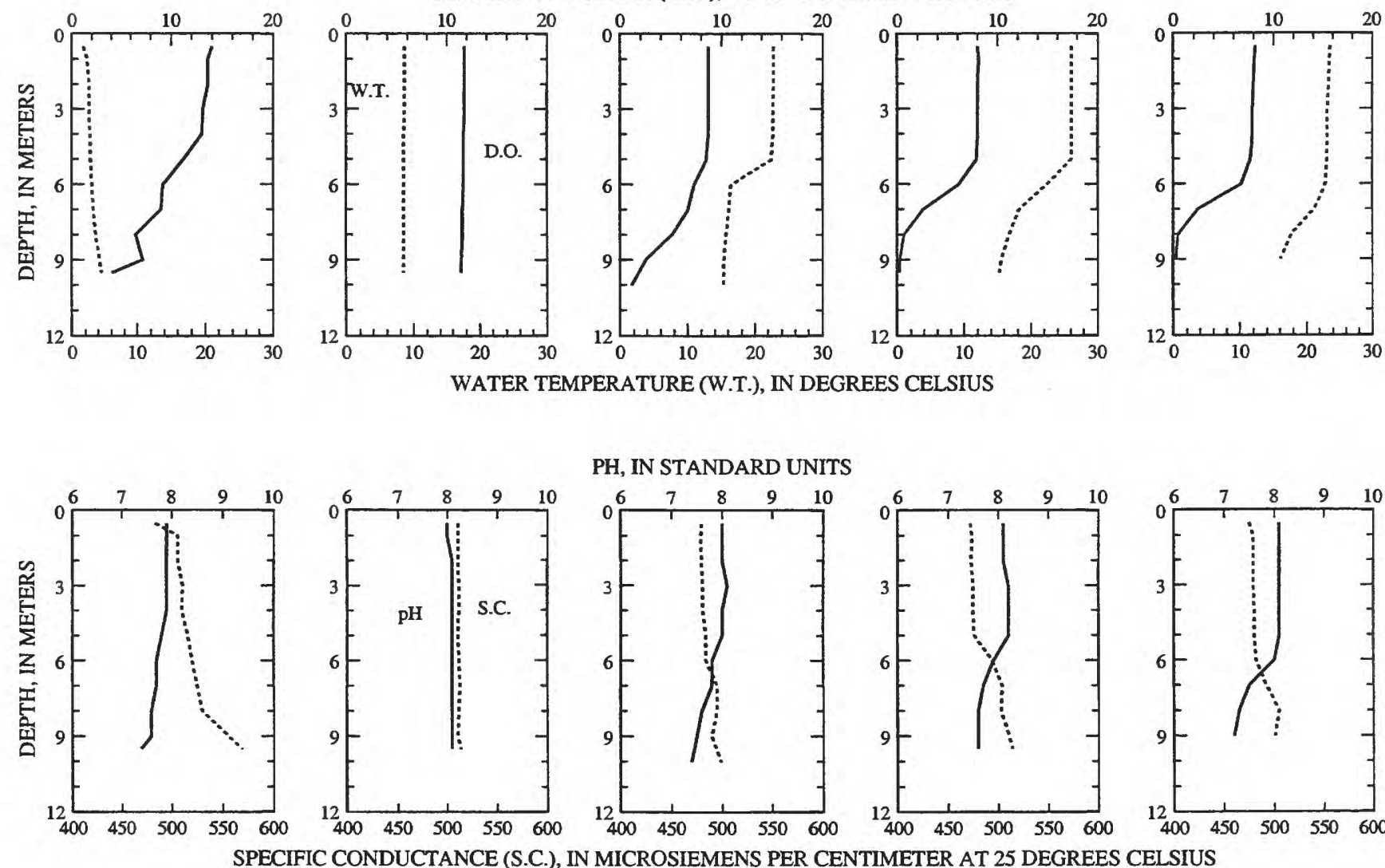

PH, IN STANDARD UNITS
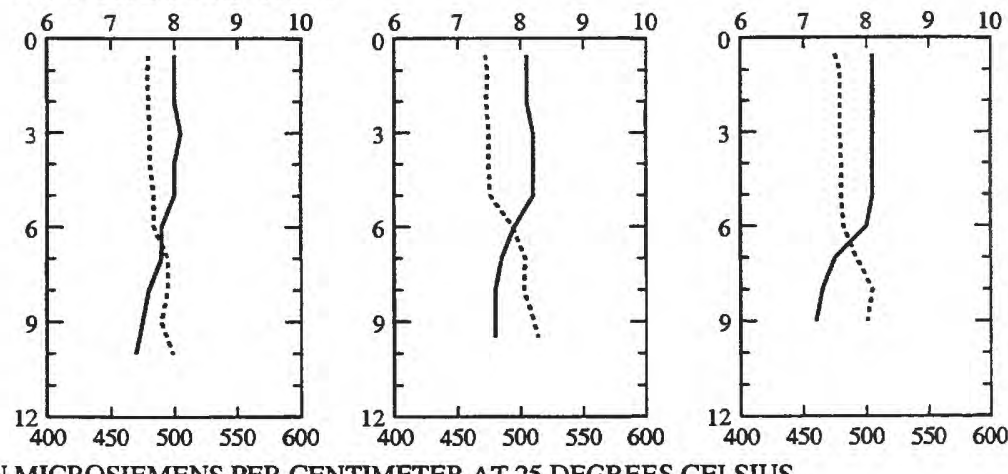

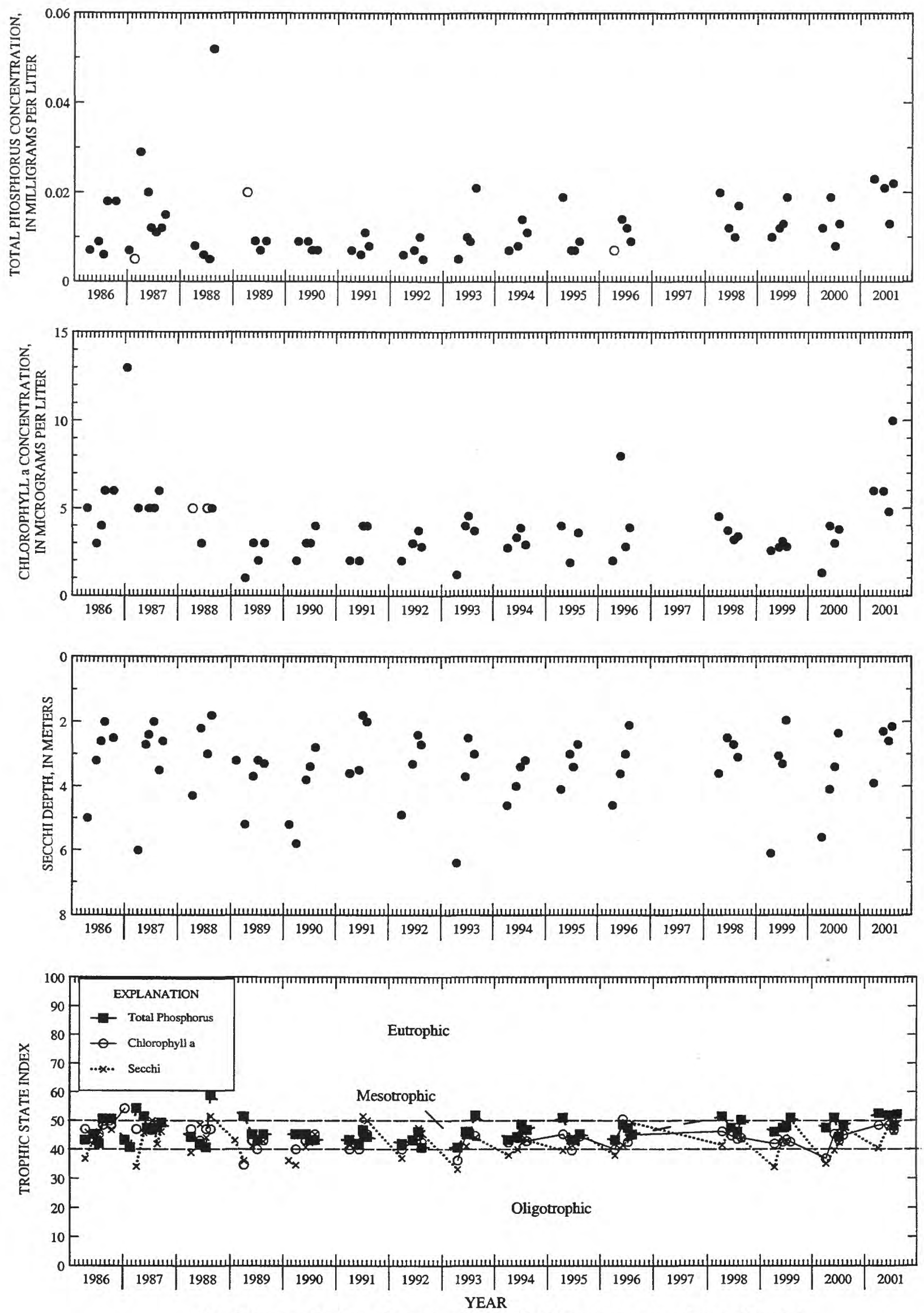

Surface total phosphorus, chlorophyll a concentrations, Secchi depths, and TSI data for Powers Lake, at Powers Lake, Wisconsin.

(Circles on the first three plots indicate laboratory detection limit for selected analyses. Actual concentrations for these particular analyses are less than the plotted circles.) 
LOCATION.--Lat 45 $35^{\prime} 22^{\prime \prime}$, long 91 $36^{\circ} 06^{\prime \prime}$, in SE 1/4 SE 1/4 NE 1/4 sec. 21, T.36 N., R.10 W., Barron County, Hydrologic Unit 07050007, on end of left wingwall of dam at outlet to the Red Cedar River.

DRAINAGE AREA.--151 $\mathrm{mi}^{2}$. Area of Red Cedar Lake, 1,841 acres.

PERIOD OF RECORD.--March 1993 to August 1994, March 1996 to August 1997, and October 2000 to September 2001.

GAGE.--Water-stage recorder. Elevation of gage is $1,170 \mathrm{ft}$ above sea level, from topographic map.

REMARKS.--Gage-height telemeter at station.

EXTREMES FOR PERIOD OF RECORD.--Maximum recorded gage height, 11.38 ft, Apr. 12, 2001; minimum recorded, $9.69 \mathrm{ft}$, Mar. 8, 1993.

EXTREMES FOR CURRENT YEAR.--Maximum recorded gage height, $11.38 \mathrm{ft}$, Apr. 12; minimum recorded, $9.84 \mathrm{ft}$, June 14.

GAGE HEIGHT, FEET, WATER YEAR OCTOBER 2000 TO SEPTEMBER 2001

DAILY MEAN VALUES

\begin{tabular}{|c|c|c|c|c|c|c|c|c|c|c|c|c|}
\hline DAY & OCT & NOV & DEC & JAN & FEB & MAR & APR & MAY & JUN & $\pi \pi$ & AUG & SEP \\
\hline $\begin{array}{l}1 \\
2 \\
3 \\
4 \\
5\end{array}$ & $\begin{array}{l}10.62 \\
10.63 \\
10.63 \\
10.63 \\
10.65\end{array}$ & $\begin{array}{l}10.48 \\
10.65 \\
10.71 \\
10.79 \\
10.86\end{array}$ & $\begin{array}{l}10.20 \\
10.20 \\
10.21 \\
10.22 \\
10.22\end{array}$ & $\begin{array}{l}10.24 \\
10.23 \\
10.23 \\
10.23 \\
10.22\end{array}$ & $\begin{array}{l}10.21 \\
10.21 \\
10.21 \\
10.21 \\
10.21\end{array}$ & $\begin{array}{l}10.19 \\
10.18 \\
10.18 \\
10.17 \\
10.16\end{array}$ & $\begin{array}{l}10.22 \\
10.24 \\
10.26 \\
10.29 \\
10.34\end{array}$ & $\begin{array}{l}10.66 \\
10.67 \\
10.67 \\
10.71 \\
10.77\end{array}$ & $\begin{array}{l}10.72 \\
10.68 \\
10.63 \\
10.62 \\
10.62\end{array}$ & $\begin{array}{l}10.60 \\
10.56 \\
10.56 \\
10.55 \\
10.55\end{array}$ & $\begin{array}{l}10.88 \\
10.75 \\
10.69 \\
10.77 \\
10.85\end{array}$ & $\begin{array}{l}10.61 \\
10.60 \\
10.61 \\
10.60 \\
10.62\end{array}$ \\
\hline $\begin{array}{r}6 \\
7 \\
8 \\
9 \\
10\end{array}$ & $\begin{array}{l}10.65 \\
10.65 \\
10.64 \\
10.64 \\
10.65\end{array}$ & $\begin{array}{l}10.93 \\
10.94 \\
10.95 \\
10.96 \\
10.94\end{array}$ & $\begin{array}{l}10.23 \\
10.25 \\
10.25 \\
10.25 \\
10.27\end{array}$ & $\begin{array}{l}10.22 \\
10.21 \\
10.21 \\
10.20 \\
10.20\end{array}$ & $\begin{array}{l}10.21 \\
10.21 \\
10.23 \\
10.24 \\
10.23\end{array}$ & $\begin{array}{l}10.16 \\
10.15 \\
10.15 \\
10.15 \\
10.15\end{array}$ & $\begin{array}{l}10.43 \\
10.69 \\
11.03 \\
11.16 \\
11.08\end{array}$ & $\begin{array}{l}10.82 \\
10.83 \\
10.83 \\
10.81 \\
10.78\end{array}$ & $\begin{array}{l}10.63 \\
10.67 \\
10.70 \\
10.72 \\
10.79\end{array}$ & $\begin{array}{l}10.57 \\
10.61 \\
10.65 \\
10.68 \\
10.71\end{array}$ & $\begin{array}{l}10.86 \\
10.84 \\
10.80 \\
10.75 \\
10.69\end{array}$ & $\begin{array}{l}10.64 \\
10.71 \\
10.76 \\
10.75 \\
10.71\end{array}$ \\
\hline $\begin{array}{l}11 \\
12 \\
13 \\
14 \\
15\end{array}$ & $\begin{array}{l}10.65 \\
10.66 \\
10.68 \\
10.70 \\
10.71\end{array}$ & $\begin{array}{l}10.90 \\
10.87 \\
10.85 \\
10.82 \\
10.79\end{array}$ & $\begin{array}{l}10.27 \\
10.26 \\
10.26 \\
10.27 \\
10.27\end{array}$ & $\begin{array}{l}10.20 \\
10.19 \\
10.19 \\
10.20 \\
10.21\end{array}$ & $\begin{array}{l}10.22 \\
10.22 \\
10.22 \\
10.22 \\
10.21\end{array}$ & $\begin{array}{l}10.14 \\
10.16 \\
10.17 \\
10.16 \\
10.16\end{array}$ & $\begin{array}{l}11.10 \\
11.09 \\
10.91 \\
10.92 \\
10.99\end{array}$ & $\begin{array}{l}10.74 \\
10.75 \\
10.76 \\
10.77 \\
10.80\end{array}$ & $\begin{array}{l}10.86 \\
10.72 \\
10.57 \\
10.41 \\
10.74\end{array}$ & $\begin{array}{l}10.73 \\
10.75 \\
10.78 \\
10.80 \\
10.82\end{array}$ & $\begin{array}{l}10.65 \\
10.65 \\
10.66 \\
10.65 \\
10.65\end{array}$ & $\begin{array}{l}10.67 \\
10.67 \\
10.66 \\
10.66 \\
10.68\end{array}$ \\
\hline $\begin{array}{l}16 \\
17 \\
18 \\
19 \\
20\end{array}$ & $\begin{array}{l}10.72 \\
10.70 \\
10.69 \\
10.66 \\
10.63\end{array}$ & $\begin{array}{l}10.73 \\
10.66 \\
10.60 \\
10.54 \\
10.48\end{array}$ & $\begin{array}{l}10.28 \\
10.28 \\
10.28 \\
10.30 \\
10.29\end{array}$ & $\begin{array}{l}10.21 \\
10.20 \\
10.20 \\
10.20 \\
10.19\end{array}$ & $\begin{array}{l}10.21 \\
10.20 \\
10.19 \\
10.19 \\
10.18\end{array}$ & $\begin{array}{l}10.16 \\
10.15 \\
10.14 \\
10.14 \\
10.14\end{array}$ & $\begin{array}{l}10.85 \\
10.67 \\
10.58 \\
10.67 \\
10.66\end{array}$ & $\begin{array}{l}10.86 \\
10.91 \\
10.92 \\
10.85 \\
10.78\end{array}$ & $\begin{array}{l}10.78 \\
10.75 \\
10.61 \\
10.63 \\
10.68\end{array}$ & $\begin{array}{l}10.84 \\
11.06 \\
10.92 \\
10.75 \\
10.66\end{array}$ & $\begin{array}{l}10.70 \\
10.70 \\
10.73 \\
10.76 \\
10.76\end{array}$ & $\begin{array}{l}10.69 \\
10.70 \\
10.72 \\
10.73 \\
10.74\end{array}$ \\
\hline $\begin{array}{l}21 \\
22 \\
23 \\
24 \\
25\end{array}$ & $\begin{array}{l}10.57 \\
10.54 \\
10.52 \\
10.49 \\
10.46\end{array}$ & $\begin{array}{l}10.42 \\
10.35 \\
10.28 \\
10.23 \\
10.19\end{array}$ & $\begin{array}{l}10.28 \\
10.28 \\
10.28 \\
10.27 \\
10.26\end{array}$ & $\begin{array}{l}10.19 \\
10.19 \\
10.18 \\
10.17 \\
10.17\end{array}$ & $\begin{array}{l}10.17 \\
10.17 \\
10.16 \\
10.18 \\
10.21\end{array}$ & $\begin{array}{l}10.15 \\
10.15 \\
10.15 \\
10.15 \\
10.15\end{array}$ & $\begin{array}{l}10.55 \\
10.44 \\
10.58 \\
10.80 \\
10.90\end{array}$ & $\begin{array}{l}10.77 \\
10.76 \\
10.78 \\
10.79 \\
10.83\end{array}$ & $\begin{array}{l}10.72 \\
10.72 \\
10.70 \\
10.67 \\
10.63\end{array}$ & $\begin{array}{l}10.70 \\
10.72 \\
10.73 \\
10.74 \\
10.74\end{array}$ & $\begin{array}{l}10.76 \\
10.76 \\
10.76 \\
10.72 \\
10.69\end{array}$ & $\begin{array}{l}10.74 \\
10.71 \\
10.68 \\
10.64 \\
10.61\end{array}$ \\
\hline $\begin{array}{l}26 \\
27 \\
28 \\
29 \\
30 \\
31\end{array}$ & $\begin{array}{l}10.44 \\
10.42 \\
10.40 \\
10.38 \\
10.37 \\
10.38\end{array}$ & $\begin{array}{c}10.14 \\
10.10 \\
10.13 \\
10.15 \\
10.18 \\
\ldots .-\end{array}$ & $\begin{array}{l}10.26 \\
10.25 \\
10.25 \\
10.26 \\
10.25 \\
10.25\end{array}$ & $\begin{array}{l}10.17 \\
10.16 \\
10.16 \\
10.16 \\
10.21 \\
10.22\end{array}$ & $\begin{array}{r}10.20 \\
10.19 \\
10.19 \\
.- \\
.-\end{array}$ & $\begin{array}{l}10.16 \\
10.17 \\
10.18 \\
10.18 \\
10.19 \\
10.21\end{array}$ & $\begin{array}{c}10.89 \\
10.86 \\
10.85 \\
10.75 \\
10.67 \\
\ldots--\end{array}$ & $\begin{array}{l}10.86 \\
10.85 \\
10.81 \\
10.77 \\
10.75 \\
10.75\end{array}$ & $\begin{array}{c}10.59 \\
10.65 \\
10.66 \\
10.63 \\
10.62 \\
-\end{array}$ & $\begin{array}{l}10.73 \\
10.71 \\
10.76 \\
10.77 \\
10.77 \\
10.77\end{array}$ & $\begin{array}{l}10.66 \\
10.65 \\
10.63 \\
10.61 \\
10.64 \\
10.63\end{array}$ & $\begin{array}{r}10.59 \\
10.60 \\
10.60 \\
10.61 \\
10.62 \\
.\end{array}$ \\
\hline $\begin{array}{l}\text { MEAN } \\
\text { MAX } \\
\text { MIN }\end{array}$ & $\begin{array}{l}10.59 \\
10.72 \\
10.37\end{array}$ & $\begin{array}{l}10.59 \\
10.96 \\
10.10\end{array}$ & $\begin{array}{l}10.26 \\
10.30 \\
10.20\end{array}$ & $\begin{array}{l}10.20 \\
10.24 \\
10.16\end{array}$ & $\begin{array}{l}10.20 \\
10.24 \\
10.16\end{array}$ & $\begin{array}{l}10.16 \\
10.21 \\
10.14\end{array}$ & $\begin{array}{l}10.72 \\
11.16 \\
10.22\end{array}$ & $\begin{array}{l}10.79 \\
10.92 \\
10.66\end{array}$ & $\begin{array}{l}10.67 \\
10.86 \\
10.41\end{array}$ & $\begin{array}{l}10.72 \\
11.06 \\
10.55\end{array}$ & $\begin{array}{l}10.72 \\
10.88 \\
10.61\end{array}$ & $\begin{array}{l}10.66 \\
10.76 \\
10.59\end{array}$ \\
\hline
\end{tabular}


LOCATION.--Lat $45^{\circ} 37^{\prime} 25^{\prime \prime}$, long 91 $34^{\circ} 51^{\prime \prime}$, in NW 1/4 NW 1/4 sec.11, T.36 N., R.10 W., Barron County, Hydrologic Unit 07050007, $2.4 \mathrm{mi}$ northeast of Mikana.

PERIOD OF RECORD.--March 1993 to August 1994, March 1996 to August 1997, and March to September 2001.

REMARKS.--Lake sampled in northern part of lake at deep hole. Lake ice-covered during March sampling. Water-quality analyses by Wisconsin State Laboratory of Hygiene.

WATER-QUALITY DATA, MARCH 01 TO JUNE 12, 2001

(Milligrams per liter unless otherwise indicated)
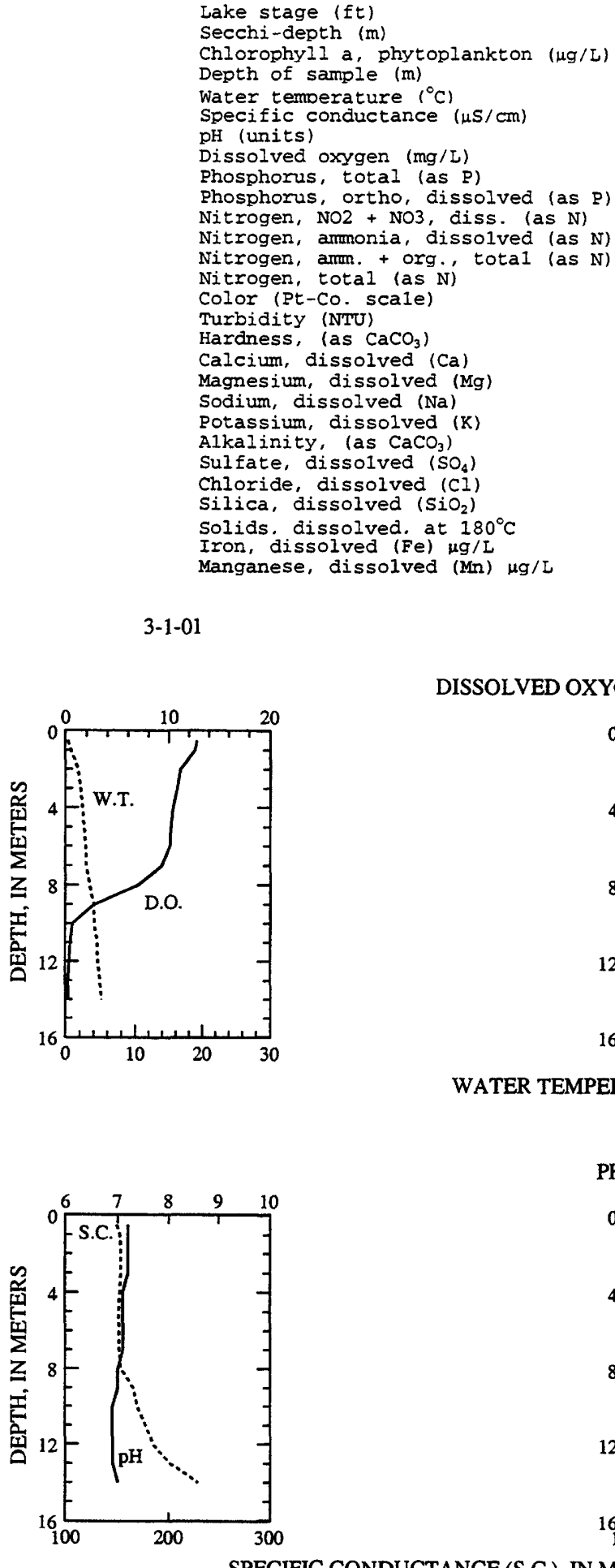

DISSOLVED OXYGEN (D.O.), IN MILLIGRAMS PER LITER

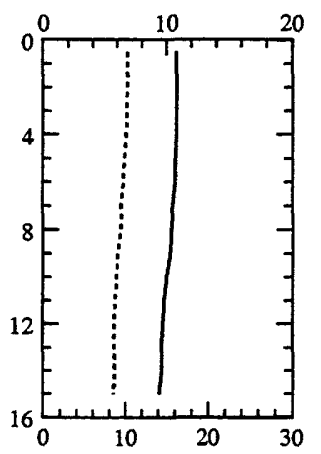

WATER TEMPERATURE (W.T.), IN DEGREES CELSIUS
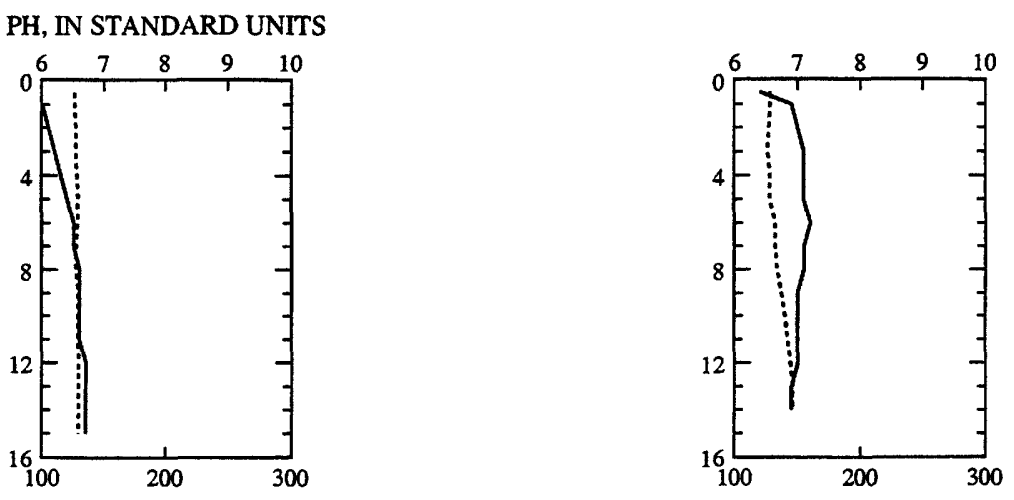

6-12-01

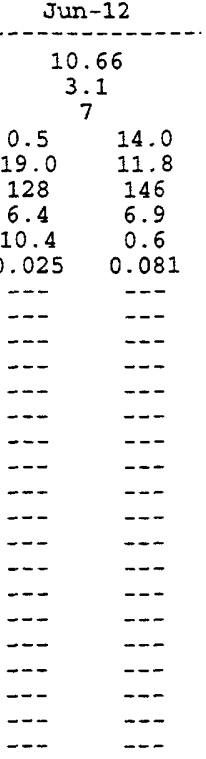

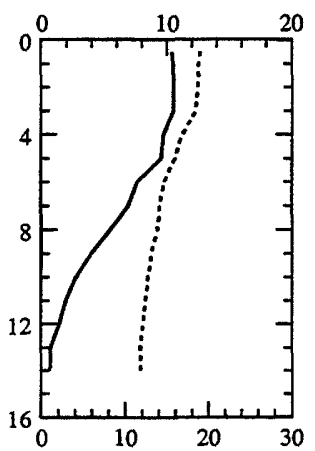

SPECIFIC CONDUCTANCE (S.C.), IN MICROSIEMENS PER CENTIMETER AT 25 DEGREES CELSIUS 
WATER-QUALITY DATA, JULY 10 TO SEPTEMBER 20, 2001

(Milligrams per liter unless otherwise indicated)

\begin{tabular}{|c|c|c|c|c|c|c|c|c|}
\hline & \multicolumn{2}{|c|}{ Jul-10 } & \multicolumn{3}{|c|}{ Aug - 13} & \multicolumn{3}{|c|}{ Sep-20 } \\
\hline $\begin{array}{l}\text { Lake stage (ft) } \\
\text { Secchi-depth (m) } \\
\text { Chlorophyll a, phytoplankton ( } \mu \mathrm{g} / \mathrm{L})\end{array}$ & & & & $\begin{array}{c}10.67 \\
3.20 \\
7\end{array}$ & & & $\begin{array}{c}10.74 \\
1.90 \\
18\end{array}$ & \\
\hline $\begin{array}{l}\text { Depth of sample (m) } \\
\text { Water temoerature }\left({ }^{\circ} \mathrm{C}\right) \\
\text { Specific conductance ( } \mathrm{sS} / \mathrm{cm}) \\
\text { pH (units) } \\
\text { Dissolved oxygen (mg/L) } \\
\text { Phosphorus, total (as P) }\end{array}$ & $\begin{array}{l}0.5 \\
24.0 \\
123 \\
8.6 \\
9.8 \\
0.021\end{array}$ & $\begin{array}{l}14.0 \\
12.1 \\
174 \\
7.2 \\
0.2 \\
0.187\end{array}$ & $\begin{array}{l}0.5 \\
24.7 \\
121 \\
7.9 \\
7.7 \\
0.021\end{array}$ & $\begin{array}{l}8.0 \\
17.3 \\
143 \\
7.1 \\
0.5 \\
0.060\end{array}$ & $\begin{array}{l}14.0 \\
12.4 \\
181 \\
7.1 \\
0.2 \\
0.457\end{array}$ & $\begin{array}{l}0.5 \\
18.5 \\
131 \\
7.2 \\
8.4 \\
0.022\end{array}$ & $\begin{array}{l}10.0 \\
15.2 \\
194 \\
7.0 \\
0.6 \\
0.079\end{array}$ & $\begin{array}{l}14.0 \\
12.3 \\
226 \\
7.0 \\
0.3 \\
0.719\end{array}$ \\
\hline
\end{tabular}

DISSOLVED OXYGEN (D.O.), IN MILLIGRAMS PER LITER
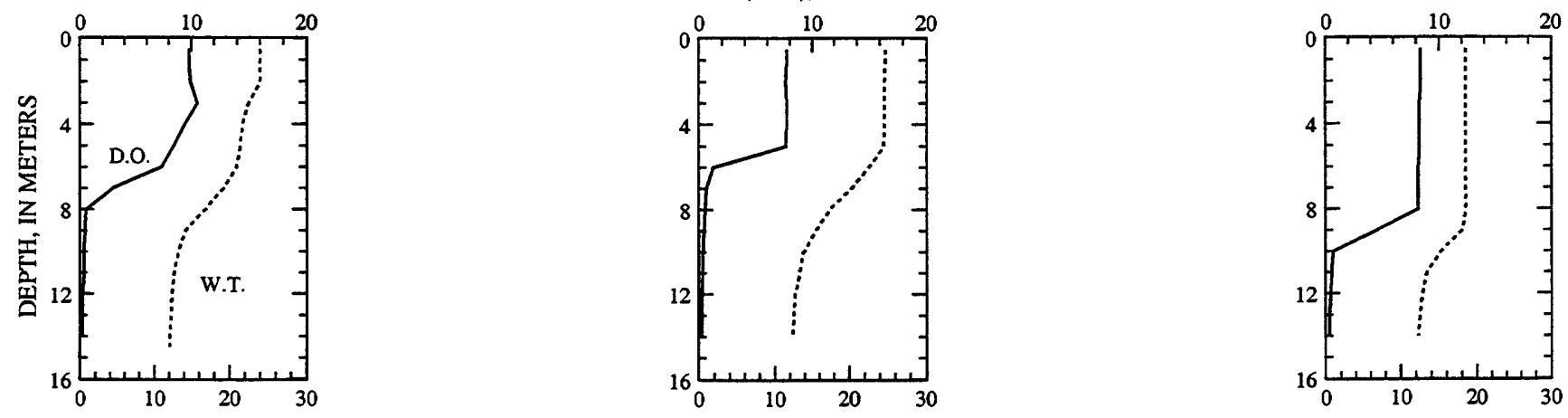

WATER TEMPERATURE (W.T.), IN DEGREES CELSIUS

PH, IN STANDARD UNITS
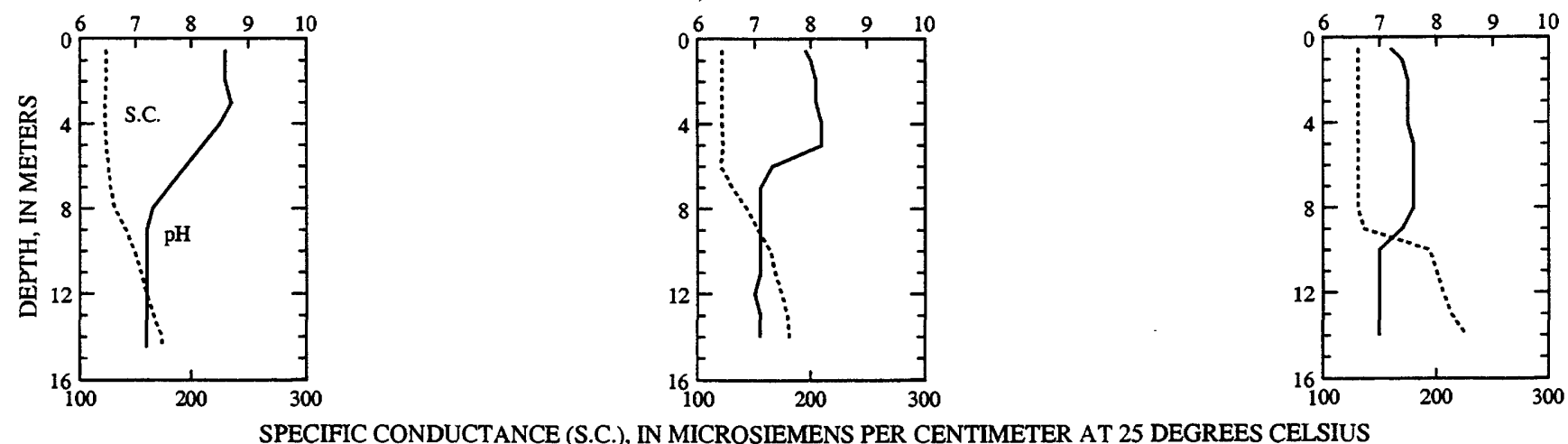

SPECIFIC CONDUCTANCE (S.C.), IN MICROSIEMENS PER CENTIMETER AT 25 DEGREES CELSIUS 

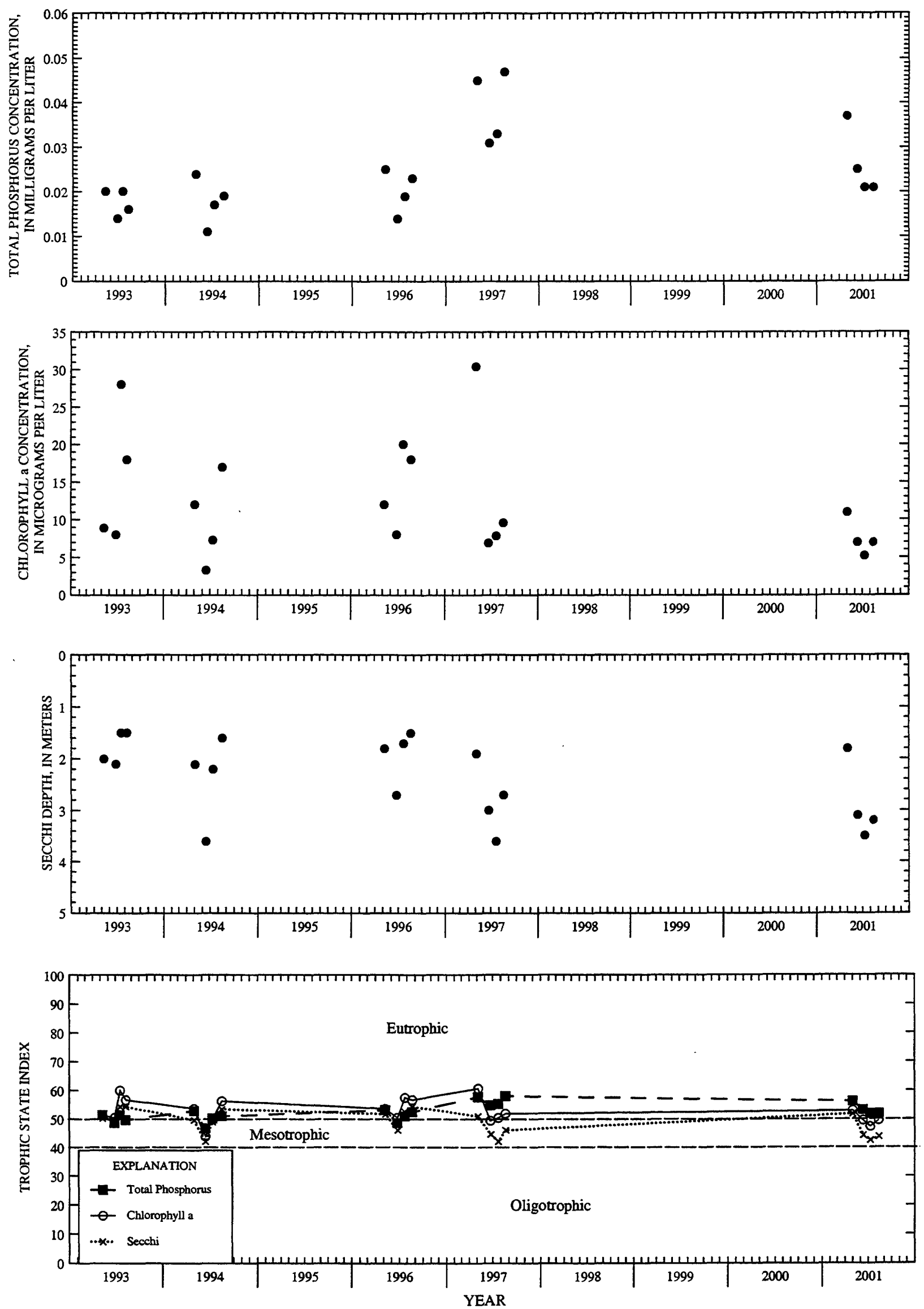

Surface total phosphorus, chlorophyll a concentrations, Secchi depths, and TSI data for Red Cedar Lake, Deep Hole, near Mikana, Wisconsin. 


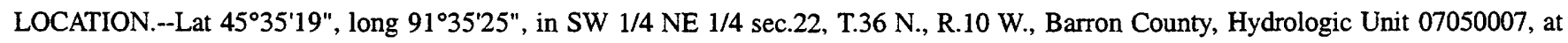
Mikana.

PERIOD OF RECORD.--March 1993 to August 1994, March 1996 to August 1997, and March to September 2001.

REMARKS.--Lake sampled $0.2 \mathrm{mi}$ northwest of Honeymoon Island. Lake ice-covered during March sampling. Water-quality analyses by Wisconsin State Laboratory of Hygiene.

WATER-QUALITY DATA, MARCH 01 TO JUNE 12, 2001

(Milligrams per liter unless otherwise indicated)

Lake stage (ft)

Secchi-depth (m)

Chlorophyll a, phytoplankt on $(\mu \mathrm{g} / \mathrm{L})$

Depth of sample (m)

Water temberature $\left({ }^{\circ} \mathrm{C}\right)$

Specific conductance $(\mu \mathrm{S} / \mathrm{cm})$

$\mathrm{pH}$ (units)

Dissolved oxygen (mg/L)

Phosphorus, total (as P)

\begin{tabular}{ccc} 
Mar-1 & May-2 & Jun-12 \\
\hdashline 10.19 & 10.67 & 10.66 \\
--- & 1.9 & 3.6 \\
--- & 9 & 1.7 \\
0.5 & 0.5 & 0.5 \\
0.0 & 10.0 & 19.0 \\
135 & 118 & 125 \\
7.5 & 7.7 & 8.1 \\
12.5 & 9.8 & 10.7 \\
0.024 & 0.028 & 0.024
\end{tabular}

$5-2-01$

6-12-01

DISSOLVED OXYGEN (D.O.), IN MILLIGRAMS PER LITER
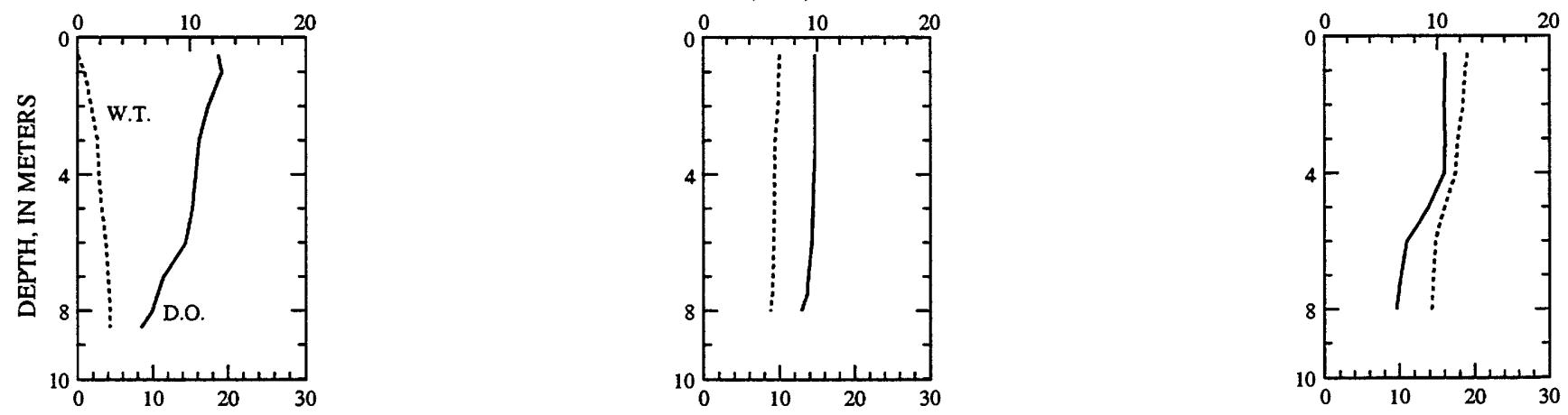

WATER TEMPERATURE (W.T.), IN DEGREES CELSIUS

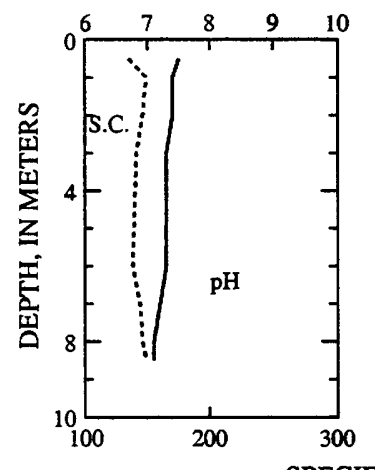

PH, IN STANDARD UNITS
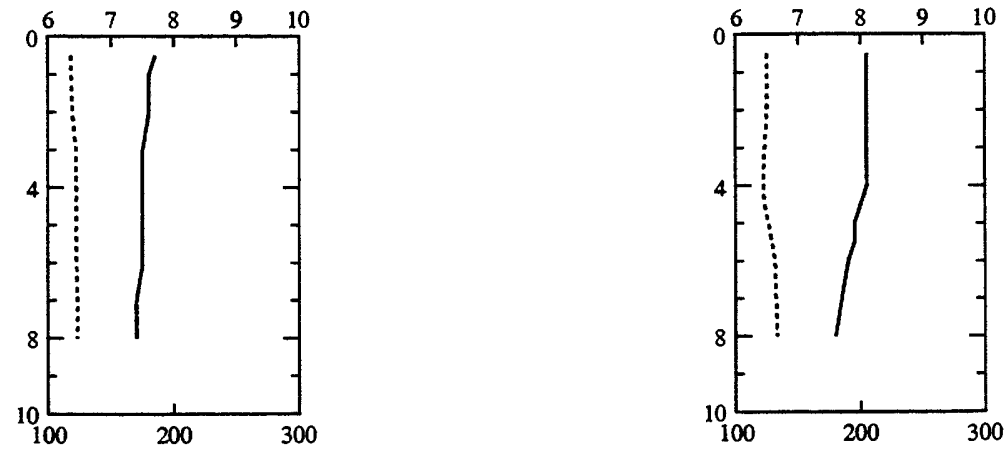
WATER-QUALITY DATA, JULY 10 TO SEPTEMBER 20, 2001

(Milligrams per liter unless otherwise indicated)
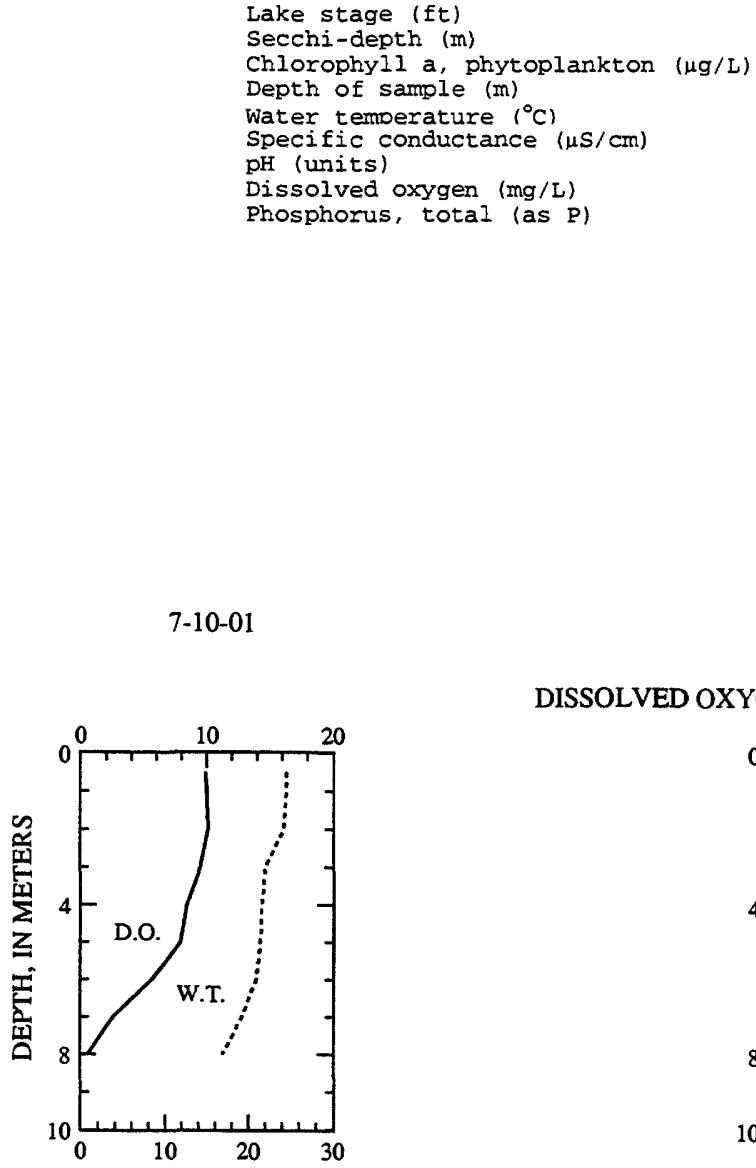

DISSOLVED OXYGEN (D.O.), IN MILLIGRAMS PER LITER
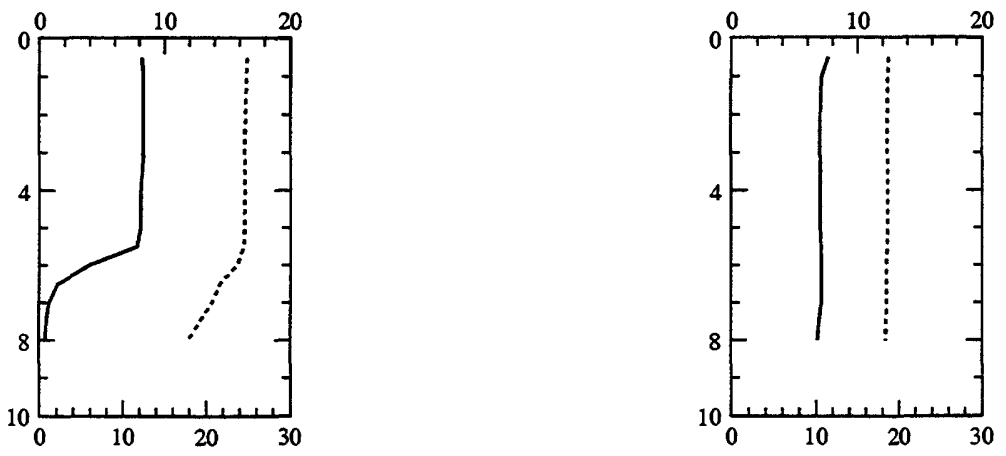

WATER TEMPERATURE (W.T.), IN DEGREES CELSIUS

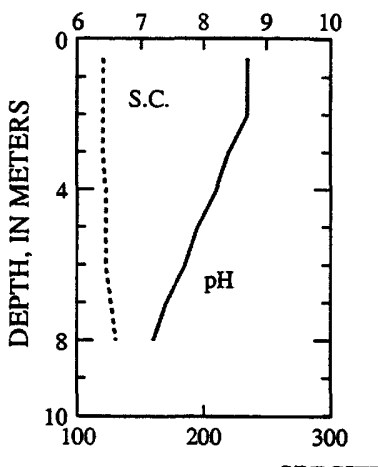

PH, IN STANDARD UNITS
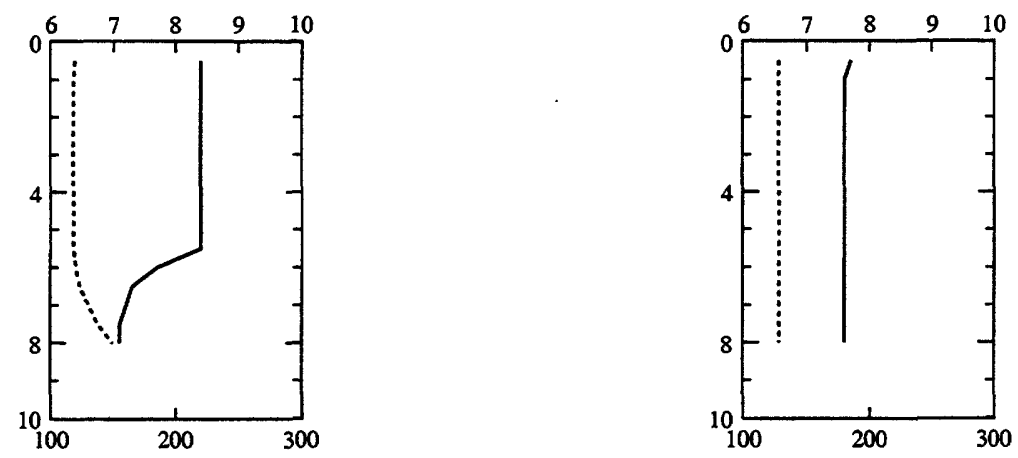

SPECIFIC CONDUCTANCE (S.C.), IN MICROSIEMENS PER CENTIMETER AT 25 DEGREES CELSIUS 

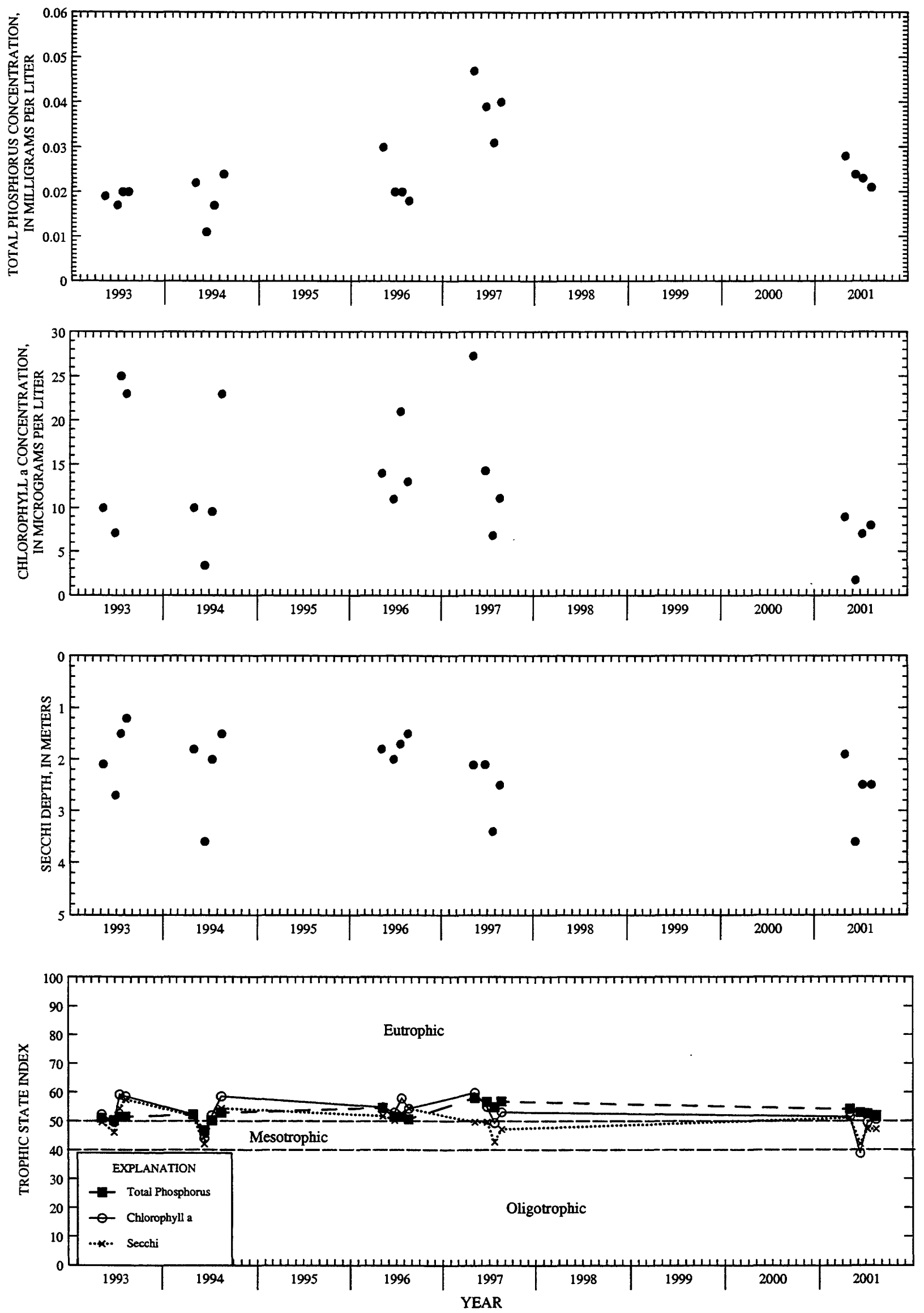

Surface total phosphorus, chlorophyll a concentrations, Secchi depths, and TSI data for Red Cedar Lake, South End, near Mikana, Wisconsin. 
LOCATION.--Lat 46¹3'21", long 91'52'09", in NW 1/4 SE 1/4 sec.9, T.43 N., R.12 W., Douglas County, Hydrologic Unit 07030002, near Gordon.

PERIOD OF RECORD.--March 1998 to current year.

REMARKS.--Lake sampled at deepest part of northern basin. Lake ice-covered during March sampling. Water-quality analyses done by Wisconsin State Laboratory of Hygiene.

WATER-QUALITY DATA, MARCH 02 TO AUGUST 14, 2001

(Milligrams per liter unless otherwise indicated)

Lake stage ( $f t$ )

Secchi-depth (m)

Chlorophyll a, phytoplankton ( $\mu \mathrm{g} / \mathrm{L}$ )

Depth of sample (m)

Water temberature $\left({ }^{\circ} \mathrm{C}\right)$

Specific conductance ( $\mu \mathrm{S} / \mathrm{cm}$ )

$\mathrm{pH}$ (units)

Dissolved oxygen (mg/L)

Phosphorus, total (as P)

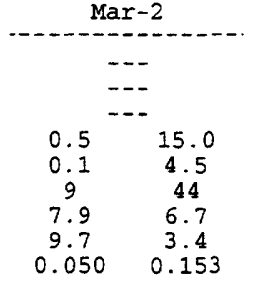

\begin{tabular}{l} 
May -3 \\
\hline 5.27 \\
3.7 \\
3.3 \\
0.5 \\
10.1 \\
35 \\
7.6 \\
11.8 \\
$<0.005$
\end{tabular}

\begin{tabular}{ccc} 
Jul-12 & \multicolumn{2}{c}{ Aug-14 } \\
\hline 5.10 & \multicolumn{2}{c}{5.09} \\
9.1 & \multicolumn{2}{c}{6.5} \\
$<1$ & \multicolumn{2}{c}{1.2} \\
0.5 & 0.5 & 15.0 \\
23.2 & 24.4 & 12.8 \\
38 & 38 & 63 \\
8.0 & 8.1 & 6.7 \\
8.5 & 8.9 & 0.4 \\
$<0.005$ & 0.006 & 0.040
\end{tabular}

3-2-01

5-3-01

6-13-01

7-12-01

8-14-01
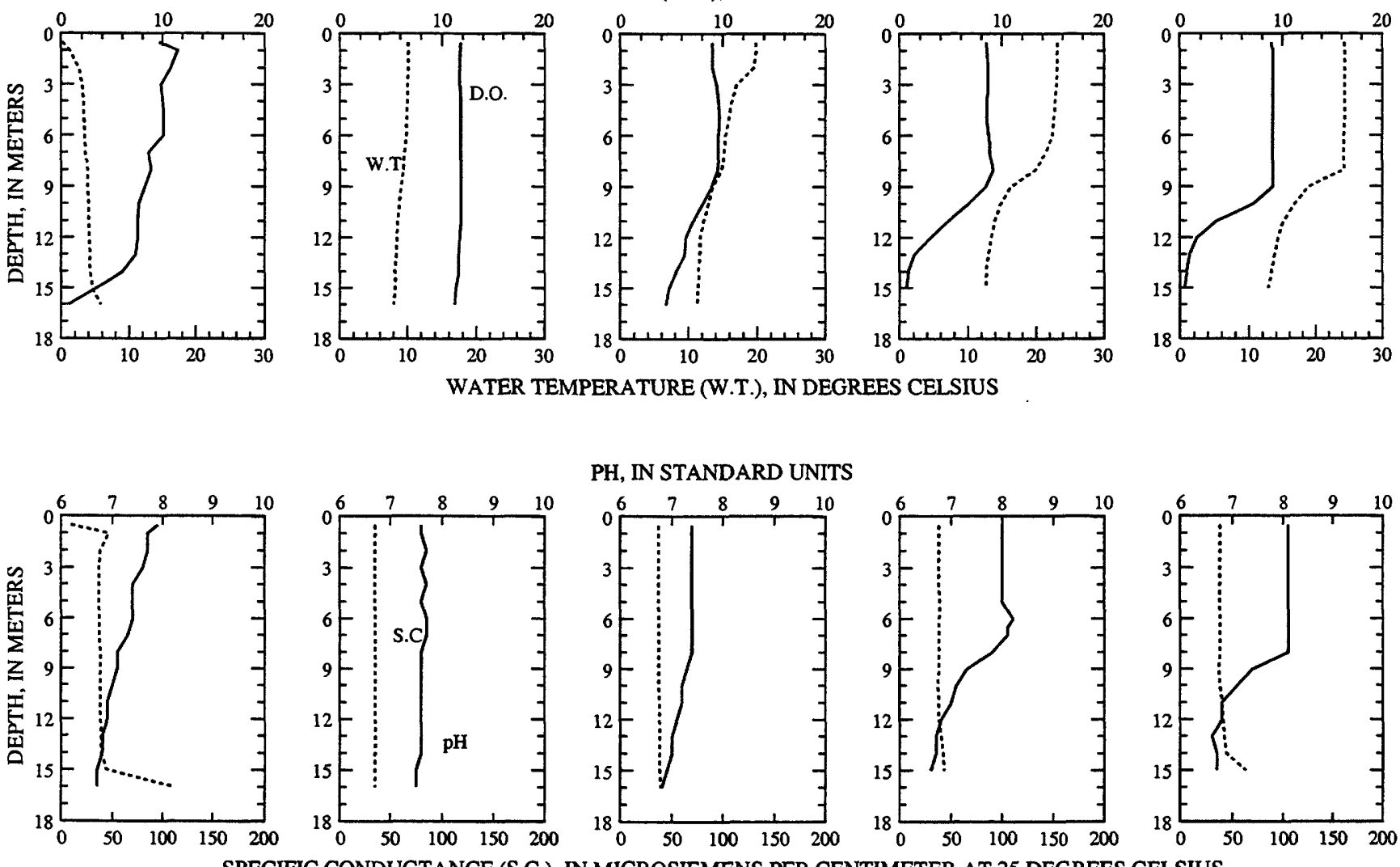

PH, IN STANDARD UNITS
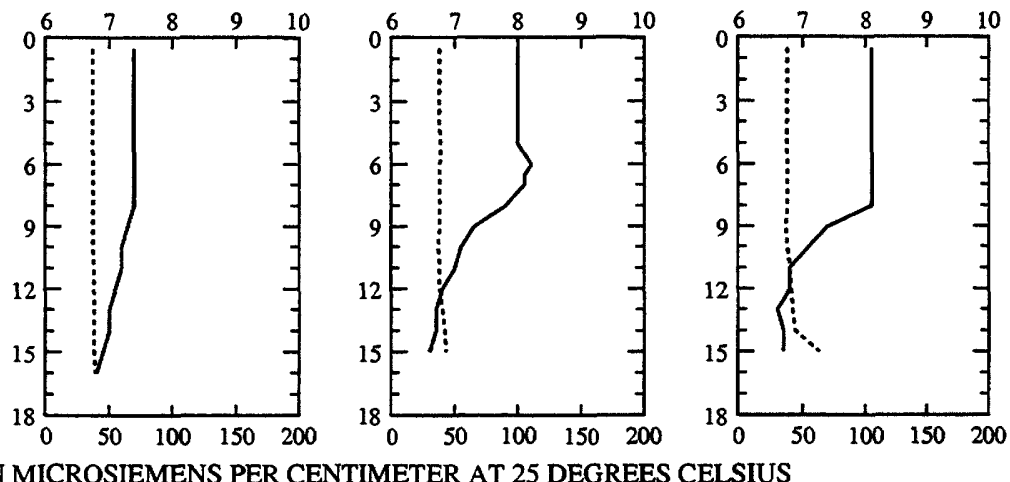

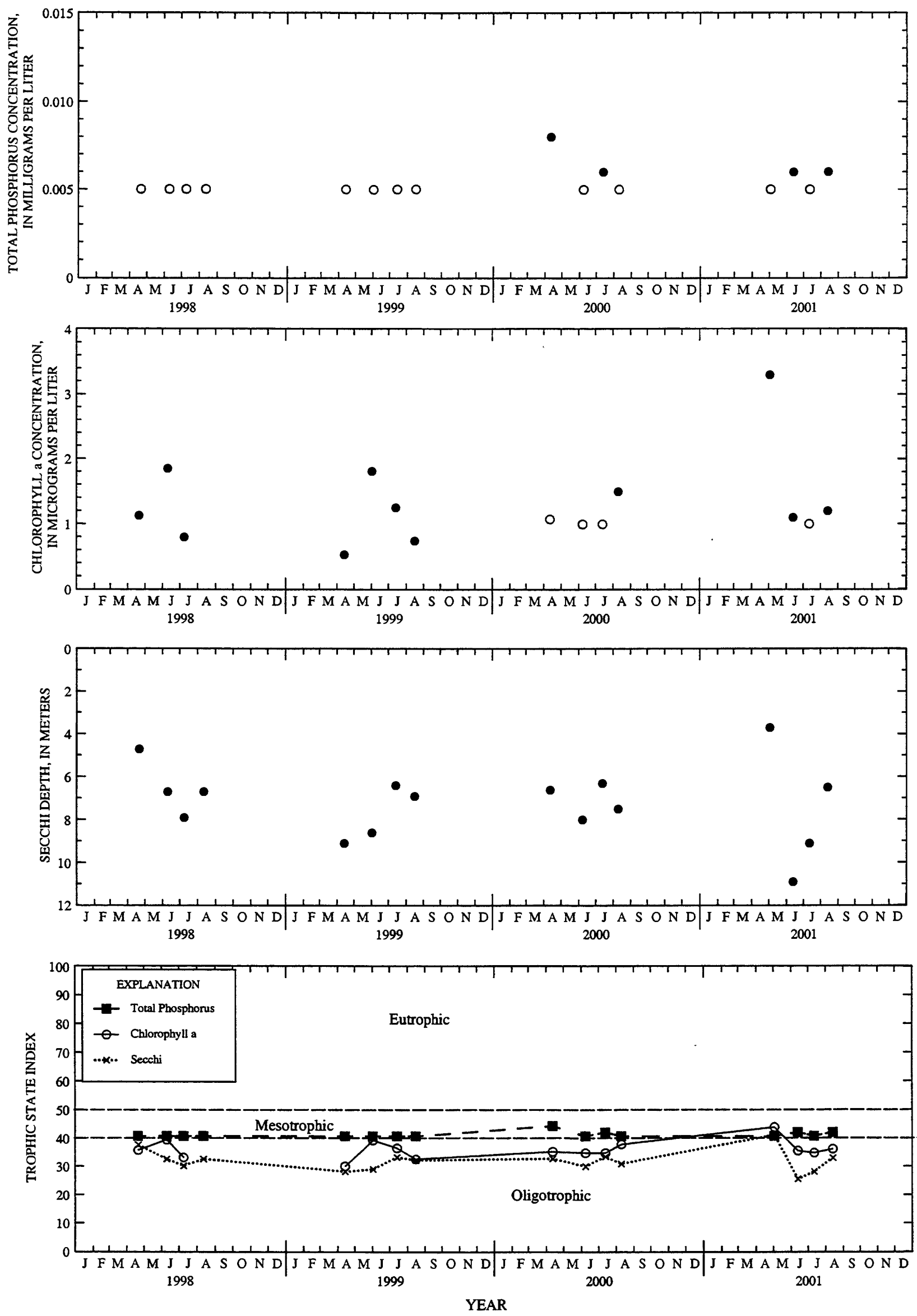

Surface total phosphorus, chlorophyll a concentrations, Secchi depths, and TSI data for Whitefish Lake, North Basin, near Gordon, Wisconsin.

(Circles on the first three plots indicate laboratory detection limit for selected analyses.

Actual concentrations for these particular analyses are less than the plotted circles.) 
LOCATION.--Lat 46¹2'12", long 91'52'32", in SE 1/4 SW 1/4 sec.16, T.43 N., R.12 W., Douglas County, Hydrologic Unit 07030002, near Gordon.

PERIOD OF RECORD.--March 1998 to current year.

REMARKS.--Lake sampled at deepest part of southern basin. Lake ice-covered during March sampling. Water-quality analyses done by Wisconsin State Laboratory of Hygiene.

WATER-QUALITY DATA, MARCH 02 TO AUGUST 14, 2001

(Milligrams per liter unless otherwise indicated)

Lake stage (Et)

Secchi-depth (m)

Chlorophyll a, phytoplankton $(\mu \mathrm{g} / \mathrm{L})$

Depth of sample (m)

Water temoerature $\left({ }^{\circ} \mathrm{C}\right)$

Specific conductance $(\mu \mathrm{S} / \mathrm{cm})$

$\mathrm{pH}$ (units)

Dissolved oxygen (mg/L)

Phosphorus, total (as P)

Phosphorus, ortho, dissolved (as P)

Nitrogen, NO2 + NO3, diss, (as N)

Nitrogen, ammonia, dissolved (as N)

Nitrogen, amm. + org., total (as N)

Nitrogen, total (as $N$ )

Color (Pt-Co. scale)

Turbidity (NTU)

Hardness, (as $\mathrm{CaCO}_{3}$ )

Calcium, dissolved (Ca)

Magnesium, dissolved (Mg)

Sodium, dissolved (Na)

Potassium, dissolved (K)

Alkalinity, (as $\mathrm{CaCO}_{3}$ )

Sulfate, dissolved $\left(\mathrm{SO}_{4}\right)$

Chloride, dissolved (CI)

Silica, dissolved $\left(\mathrm{SiO}_{2}\right)$

Solids, dissolved, at $180^{\circ} \mathrm{C}$

Iron, dissolved ( $\mathrm{Fe}) \mu \mathrm{g} / \mathrm{L}$

Manganese, dissolved (Mn) $\mu \mathrm{g} / \mathrm{L}$

\begin{tabular}{|c|c|c|}
\hline \multicolumn{2}{|c|}{ Mar-2 } & May -3 \\
\hline \multicolumn{2}{|c|}{---} & 5.27 \\
\hline \multicolumn{2}{|c|}{$-\cdots$} & 3.7 \\
\hline \multicolumn{2}{|c|}{--} & 1.8 \\
\hline 0.5 & 27.0 & 0.5 \\
\hline 0.5 & 4.1 & 9.2 \\
\hline 50 & 59 & 35 \\
\hline 7.5 & 6.5 & 8.1 \\
\hline$<0.12$ & 0.8 & 12.1 \\
\hline - & 0.200 & $\begin{array}{l}0.006 \\
<0.002\end{array}$ \\
\hline-- & --- & L. \\
\hline-- & -- & 0.039 \\
\hline--- & --- & 0.014 \\
\hline--- & $--\infty$ & 0.24 \\
\hline--- & -- & 0.279 \\
\hline-- & -- & 20 \\
\hline-- & --- & 2.1 \\
\hline-- & --- & 17.6 \\
\hline--- & -- & 4.9 \\
\hline--- & -- & 1.3 \\
\hline-- & $\cdots$ & 0.9 \\
\hline--- & -- & 0.4 \\
\hline--- & -- & 17 \\
\hline--- & -- & $<4.5$ \\
\hline--- & --- & 0.9 \\
\hline-- & -- & 0.5 \\
\hline$\ldots$ & -- & 26 \\
\hline-- & -- & $<10$ \\
\hline & --- & 0.8 \\
\hline
\end{tabular}

\begin{tabular}{|c|c|}
\hline \multicolumn{2}{|c|}{$\begin{array}{l}5.39 \\
11.6 \\
<1\end{array}$} \\
\hline $\begin{array}{c}0.5 \\
19.5 \\
38 \\
7.4 \\
9.4 \\
0.006\end{array}$ & $\begin{array}{c}28.0 \\
6.2 \\
37 \\
7.0 \\
7.9 \\
0.013\end{array}$ \\
\hline-- & --- \\
\hline--- & --- \\
\hline$\cdots$ & --- \\
\hline--- & --- \\
\hline-- & $\cdots$ \\
\hline--- & -- \\
\hline--- & --- \\
\hline--- & -- \\
\hline--- & --- \\
\hline-- & -- \\
\hline$-\cdots$ & $-\infty$ \\
\hline--- & --- \\
\hline--- & -- \\
\hline-- & -- \\
\hline--- & -- \\
\hline-- & --- \\
\hline-- & -- \\
\hline-- & -- \\
\hline
\end{tabular}

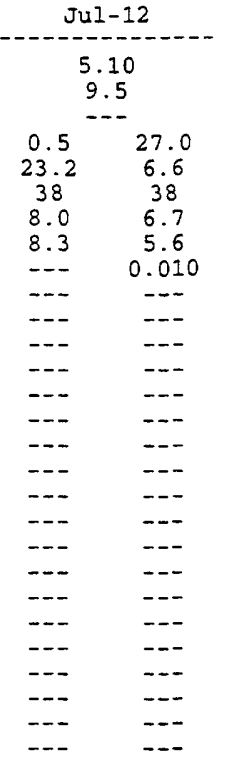

\begin{tabular}{|c|c|c|}
\hline \multicolumn{3}{|c|}{ Aug-14 } \\
\hline & $\begin{array}{l}5.09 \\
6.90 \\
<1\end{array}$ & \\
\hline $\begin{array}{c}0.5 \\
24.2 \\
38 \\
8.1 \\
9.0 \\
0.007\end{array}$ & $\begin{array}{c}11.0 \\
15.4 \\
37 \\
7.6 \\
10.8 \\
0.009\end{array}$ & $\begin{array}{c}27.0 \\
6.6 \\
40 \\
6.6 \\
1.4 \\
0.041\end{array}$ \\
\hline-- & -- & --- \\
\hline-- & --- & -- \\
\hline--- & -- & --- \\
\hline-- & --- & --- \\
\hline-- & $m$ & $m$ \\
\hline$\cdots$ & --- & $\cdots$ \\
\hline$\cdots$ & -- & -- \\
\hline$\cdots$ & --- & $-\cdots$ \\
\hline-- & --- & --- \\
\hline--- & --- & -- \\
\hline--- & --- & --- \\
\hline--- & --- & -- \\
\hline--- & -- & -- \\
\hline-- & --- & --- \\
\hline--- & --- & --- \\
\hline--- & -- & --- \\
\hline-- & $-\infty$ & -- \\
\hline--- & -- & $-\overline{-}$ \\
\hline
\end{tabular}

3-2-01

5-3-01

6-13-01

7-12-01

8-14-01

DISSOLVED OXYGEN (D.O.), IN MILLIGRAMS PER LITER
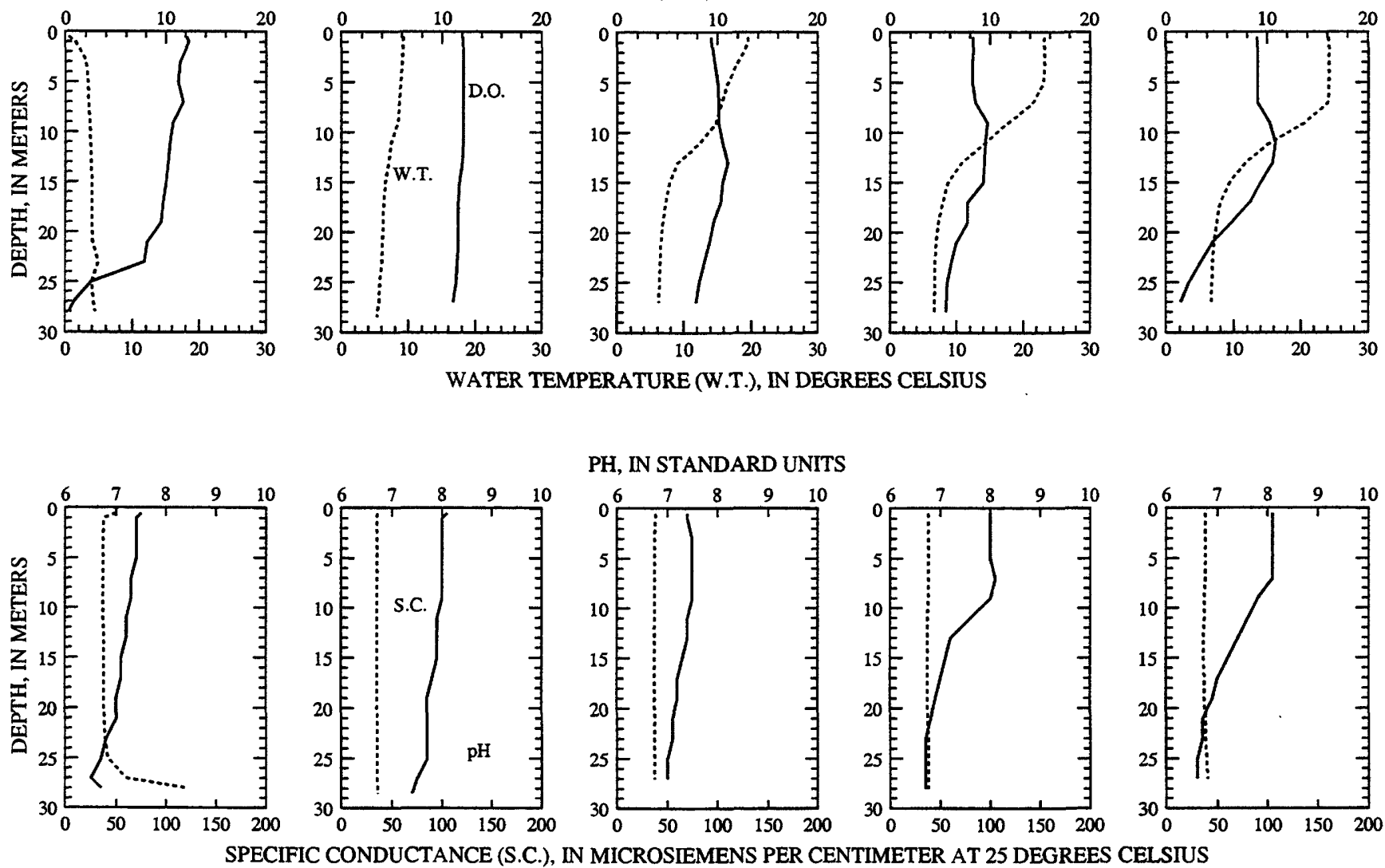

PH, IN STANDARD UNITS
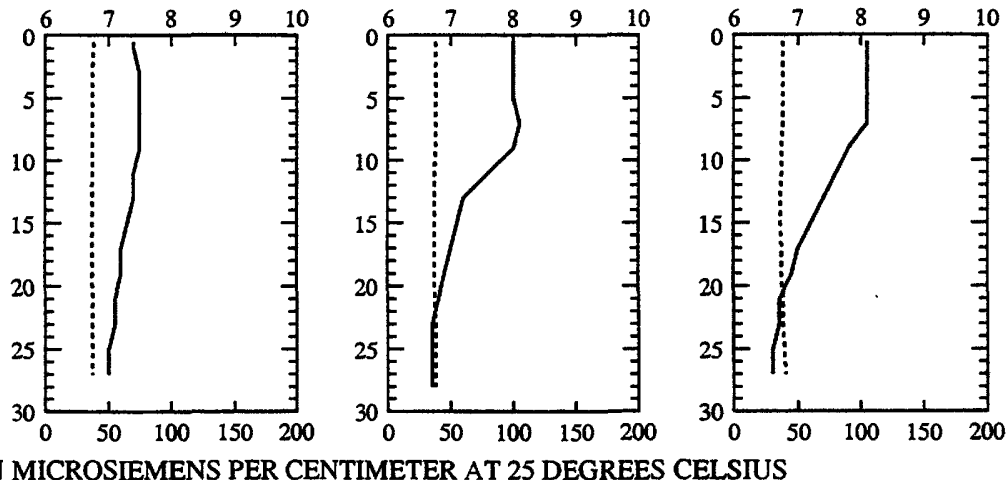

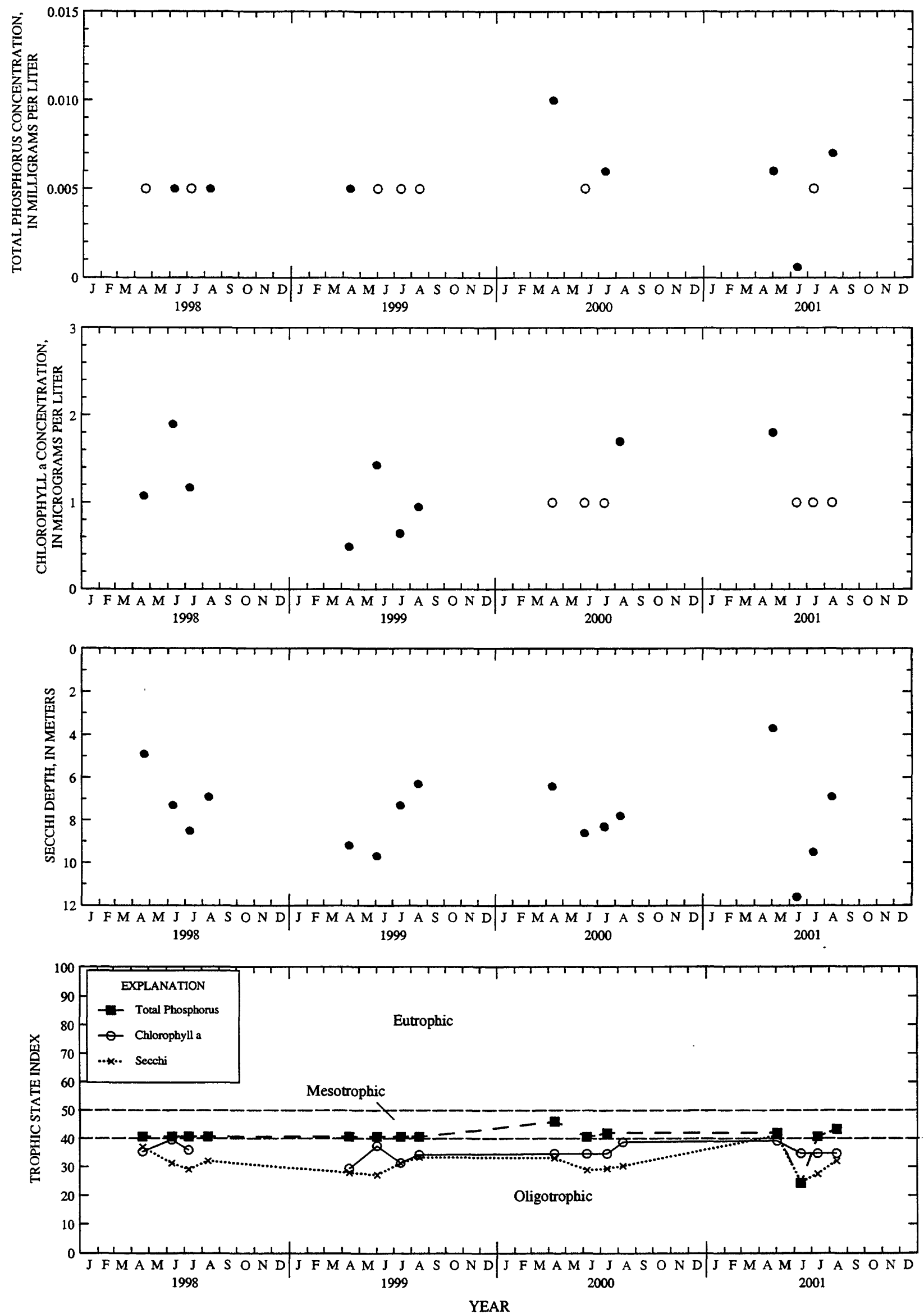

Surface total phosphorus, chlorophyll a concentrations, Secchi depths, and TSI data for Whitefish Lake, South Basin, near Gordon, Wisconsin.

(Circles on the first three plots indicate laboratory detection limit for selected analyses. Actual concentrations for these particular analyses are less than the plotted circles.) 
LOCATION.--Lat $42^{\circ} 46^{\prime} 08^{\prime \prime}$, long $88^{\circ} 41^{\prime} 48^{\prime \prime}$, in NW 1/4 NW 1/4 sec.35, T.4 N., R.15 E., Walworth County, Hydrologic Unit 07090001, at outlet, $5.0 \mathrm{mi}$ southeast of Whitewater and $10.0 \mathrm{mi}$ north of Delavan.

DRAINAGE AREA.-- $10.9 \mathrm{mi}^{2}$, of which $8.5 \mathrm{mi}^{2}$ is non-contributing.

PERIOD OF RECORD.--November 1990 to current year.

GAGE.--Water-stage recorder. Datum of gage is $861.00 \mathrm{ft}$ above sea level (Wisconsin Department of Natural Resources).

REMARKS.--Point of zero flow of dam crest is $10.97 \mathrm{ft}$. Rainfall data published in 1991 under this station number are now stored under station number 424559088420300 .

EXTREMES FOR PERIOD OF RECORD.--Maximum gage height, $11.61 \mathrm{ft}$, Feb. 25, 2001; minimum daily gage height, $8.89 \mathrm{ft}$, Oct. 2,3 , 1991.

EXTREMES FOR CURRENT YEAR.--Maximum recorded gage height, $11.61 \mathrm{ft}$, Feb. 25; minimum recorded, $10.79 \mathrm{ft}$, Aug. 15.

GAGE HEIGHT, FEET, WATER YEAR OCTOBER 2000 TO SEPTEMBER 2001

DAILY MEAN VALUES

\begin{tabular}{|c|c|c|c|c|c|c|c|c|c|c|c|c|}
\hline DAY & $O C T$ & NOV & DEC & JAN & $F E B$ & MAR & APR & MAY & JUN & JUL & AUG & SEP \\
\hline $\begin{array}{l}1 \\
2 \\
3 \\
4 \\
5\end{array}$ & $\begin{array}{l}11.02 \\
11.01 \\
11.00 \\
11.01 \\
11.01\end{array}$ & $\begin{array}{l}10.91 \\
10.92 \\
10.91 \\
10.90 \\
10.90\end{array}$ & $\begin{array}{l}11.02 \\
11.02 \\
11.02 \\
11.03 \\
11.03\end{array}$ & $\begin{array}{l}11.23 \\
11.23 \\
11.24 \\
11.24 \\
11.24\end{array}$ & $\begin{array}{l}11.32 \\
11 \cdot 32 \\
11 \cdot 32 \\
11.32 \\
11.32\end{array}$ & $\begin{array}{l}11.55 \\
11.52 \\
11.50 \\
11.47 \\
11.44\end{array}$ & $\begin{array}{l}11.24 \\
11.24 \\
11.23 \\
11.22 \\
11.22\end{array}$ & $\begin{array}{l}11.16 \\
11.15 \\
11.21 \\
11.31 \\
11.29\end{array}$ & $\begin{array}{l}11.22 \\
11.26 \\
11.26 \\
11.25 \\
11.27\end{array}$ & $\begin{array}{l}11.12 \\
11.09 \\
11.07 \\
11.05 \\
11.03\end{array}$ & $\begin{array}{l}10.84 \\
10.89 \\
10.90 \\
10.90 \\
10.90\end{array}$ & $\begin{array}{l}10.87 \\
10.86 \\
10.85 \\
10.85 \\
10.84\end{array}$ \\
\hline $\begin{array}{r}6 \\
7 \\
8 \\
9 \\
10\end{array}$ & $\begin{array}{l}11.01 \\
10.99 \\
10.99 \\
10.98 \\
10.98\end{array}$ & $\begin{array}{l}10.90 \\
10.96 \\
10.95 \\
10.97 \\
10.98\end{array}$ & $\begin{array}{l}11.04 \\
11.04 \\
11.04 \\
11.04 \\
11.04\end{array}$ & $\begin{array}{l}11.24 \\
11.24 \\
11.24 \\
11.24 \\
11.23\end{array}$ & $\begin{array}{l}11.32 \\
11.32 \\
11.34 \\
11.51 \\
11.56\end{array}$ & $\begin{array}{l}11.41 \\
11.39 \\
11.37 \\
11.36 \\
11.36\end{array}$ & $\begin{array}{l}11.24 \\
11.25 \\
11.23 \\
11.28 \\
11.28\end{array}$ & $\begin{array}{l}11.27 \\
11.27 \\
11.26 \\
11.25 \\
11.26\end{array}$ & $\begin{array}{l}11.29 \\
11.29 \\
11.28 \\
11.28 \\
11.27\end{array}$ & $\begin{array}{l}11.02 \\
11.00 \\
11.00 \\
11.00 \\
10.99\end{array}$ & $\begin{array}{l}10.89 \\
10.88 \\
10.88 \\
10.88 \\
10.89\end{array}$ & $\begin{array}{l}10.84 \\
10.90 \\
10.95 \\
10.99 \\
11.05\end{array}$ \\
\hline $\begin{array}{l}11 \\
12 \\
13 \\
14 \\
15\end{array}$ & $\begin{array}{l}10.97 \\
10.97 \\
10.96 \\
10.96 \\
10.95\end{array}$ & $\begin{array}{l}10.98 \\
10.99 \\
10.99 \\
10.98 \\
10.98\end{array}$ & $\begin{array}{l}11.08 \\
11.14 \\
11.14 \\
11.14 \\
11.15\end{array}$ & $\begin{array}{l}11.23 \\
11.24 \\
11.24 \\
11.25 \\
11.26\end{array}$ & $\begin{array}{l}11.56 \\
11.55 \\
11.55 \\
11.55 \\
11.55\end{array}$ & $\begin{array}{l}11.36 \\
11.35 \\
11.36 \\
11.34 \\
11.35\end{array}$ & $\begin{array}{l}11.31 \\
11.34 \\
11.31 \\
11.29 \\
11.29\end{array}$ & $\begin{array}{l}11.33 \\
11.34 \\
11.32 \\
11.30 \\
11.28\end{array}$ & $\begin{array}{l}11.27 \\
11.40 \\
11.41 \\
11.39 \\
11.37\end{array}$ & $\begin{array}{l}10.97 \\
10.95 \\
10.93 \\
10.92 \\
10.91\end{array}$ & $\begin{array}{l}10.86 \\
10.85 \\
10.84 \\
10.84 \\
10.81\end{array}$ & $\begin{array}{l}11.05 \\
11.04 \\
11.02 \\
11.01 \\
11.00\end{array}$ \\
\hline $\begin{array}{l}16 \\
17 \\
18 \\
19 \\
20\end{array}$ & $\begin{array}{l}10.95 \\
10.94 \\
10.94 \\
10.94 \\
10.94\end{array}$ & $\begin{array}{l}10.98 \\
10.98 \\
10.99 \\
10.99 \\
10.98\end{array}$ & $\begin{array}{l}11.16 \\
11.16 \\
11.16 \\
11.18 \\
11.18\end{array}$ & $\begin{array}{l}11.26 \\
11.26 \\
11.26 \\
11.26 \\
11.26\end{array}$ & $\begin{array}{l}11.55 \\
11.55 \\
11.55 \\
11.54 \\
11.54\end{array}$ & $\begin{array}{l}11.36 \\
11.35 \\
11.33 \\
11.31 \\
11.30\end{array}$ & $\begin{array}{l}11.26 \\
11.24 \\
11.23 \\
11.23 \\
11.25\end{array}$ & $\begin{array}{l}11.27 \\
11.27 \\
11.25 \\
11.24 \\
11.23\end{array}$ & $\begin{array}{l}11.36 \\
11.35 \\
11.35 \\
11.32 \\
11.30\end{array}$ & $\begin{array}{l}10.90 \\
10.92 \\
10.94 \\
10.94 \\
10.94\end{array}$ & $\begin{array}{l}10.89 \\
10.90 \\
10.90 \\
10.89 \\
10.89\end{array}$ & $\begin{array}{l}11.00 \\
10.99 \\
10.99 \\
11.04 \\
11.05\end{array}$ \\
\hline $\begin{array}{l}21 \\
22 \\
23 \\
24 \\
25\end{array}$ & $\begin{array}{l}10.93 \\
10.92 \\
10.92 \\
10.93 \\
10.94\end{array}$ & $\begin{array}{l}10.98 \\
10.97 \\
10.97 \\
10.97 \\
10.97\end{array}$ & $\begin{array}{l}11.20 \\
11.20 \\
11.20 \\
11.20 \\
11.20\end{array}$ & $\begin{array}{l}11.25 \\
11.25 \\
11.25 \\
11.25 \\
11.25\end{array}$ & $\begin{array}{l}11.53 \\
11.53 \\
11.53 \\
11.54 \\
11.60\end{array}$ & $\begin{array}{l}11.29 \\
11.28 \\
11.28 \\
11.27 \\
11.27\end{array}$ & $\begin{array}{l}11.28 \\
11.27 \\
11.28 \\
11.26 \\
11.25\end{array}$ & $\begin{array}{l}11.24 \\
11.24 \\
11.24 \\
11.25 \\
11.27\end{array}$ & $\begin{array}{l}11.28 \\
11.27 \\
11.26 \\
11.25 \\
11.24\end{array}$ & $\begin{array}{l}10.93 \\
10.93 \\
10.92 \\
10.91 \\
10.90\end{array}$ & $\begin{array}{l}10.88 \\
10.89 \\
10.89 \\
10.89 \\
10.90\end{array}$ & $\begin{array}{l}11.07 \\
11.08 \\
11.13 \\
11.15 \\
11.14\end{array}$ \\
\hline $\begin{array}{l}26 \\
27 \\
28 \\
29 \\
30 \\
31\end{array}$ & $\begin{array}{l}10.95 \\
10.94 \\
10.93 \\
10.92 \\
10.92 \\
10.91\end{array}$ & $\begin{array}{l}10.98 \\
10.99 \\
10.99 \\
11.01 \\
11.02\end{array}$ & $\begin{array}{l}11.20 \\
11.19 \\
11.19 \\
11.22 \\
11.24 \\
11.24\end{array}$ & $\begin{array}{l}11.25 \\
11.25 \\
11.25 \\
11.27 \\
11.31 \\
11.32\end{array}$ & $\begin{array}{r}11.60 \\
11.60 \\
11.58 \\
-\ldots \\
-.-\end{array}$ & $\begin{array}{l}11.26 \\
11.25 \\
11.25 \\
11.24 \\
11.23 \\
11.23\end{array}$ & $\begin{array}{r}11.24 \\
11.23 \\
11.22 \\
11.19 \\
11.17 \\
-.-\end{array}$ & $\begin{array}{l}11.27 \\
11.25 \\
11.24 \\
11.24 \\
11.22 \\
11.21\end{array}$ & $\begin{array}{r}11.22 \\
11.19 \\
11.17 \\
11.15 \\
11.14 \\
-\end{array}$ & $\begin{array}{l}10.89 \\
10.87 \\
10.85 \\
10.85 \\
10.85 \\
10.84\end{array}$ & $\begin{array}{l}10.91 \\
10.91 \\
10.91 \\
10.90 \\
10.89 \\
10.88\end{array}$ & $\begin{array}{l}11.12 \\
11.11 \\
11.10 \\
11.09 \\
11.09\end{array}$ \\
\hline $\begin{array}{l}\text { MEAN } \\
\text { MAX } \\
\text { MIN }\end{array}$ & $\begin{array}{l}10.96 \\
11.02 \\
10.91\end{array}$ & $\begin{array}{l}10.97 \\
11.02 \\
10.90\end{array}$ & $\begin{array}{l}11.13 \\
11.24 \\
11.02\end{array}$ & $\begin{array}{l}11.25 \\
11.32 \\
11.23\end{array}$ & $\begin{array}{l}11.49 \\
11.60 \\
11.32\end{array}$ & $\begin{array}{l}11.34 \\
11.55 \\
11.23\end{array}$ & $\begin{array}{l}11.25 \\
11.34 \\
11.17\end{array}$ & $\begin{array}{l}11.26 \\
11.34 \\
11.15\end{array}$ & $\begin{array}{l}11.28 \\
11.41 \\
11.14\end{array}$ & $\begin{array}{l}10.95 \\
11.12 \\
10.84\end{array}$ & $\begin{array}{l}10.88 \\
10.91 \\
10.81\end{array}$ & $\begin{array}{l}11.01 \\
11.15 \\
10.84\end{array}$ \\
\hline
\end{tabular}

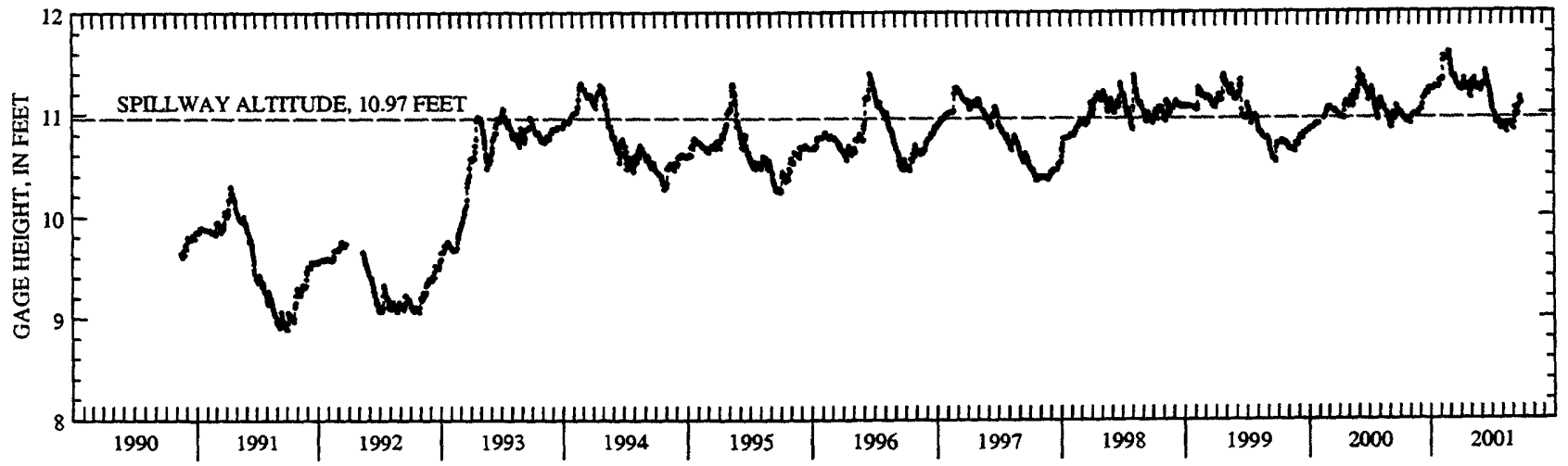


LOCATION.--Lat $42^{\circ} 48^{\prime} 48^{\prime \prime}$ long $88^{\circ} 08^{\prime} 31^{\prime \prime}$, in NE 1/4 NW 1/4 sec.16, T.4 N., R.20 E., Racine County, Hydrologic Unit 07120006, at Wind Lake.

DRAINAGE AREA.--39.6 $\mathrm{mi}^{2}$.

PERIOD OF RECORD.--March 1985 to current year. Prior to October 2000, published as "Wind Lake Outlet".

REVISED RECORDS.--WDR WI-91-1: 1988(m).

GAGE.--Water-stage recorder and concrete dam. Datum of gage is $760.30 \mathrm{ft}$ above sea level. Prior to Oct. 2, 1987, nonrecording gage at same site and datum.

REMARKS.--Lake level regulated by dam with two 10-foot gates at outlet. Lake ice-covered Dec. 5 to Apr. 5 . Prior to October 1987 , published as Wind Lake at Wind Lake, Wis. Gage-height telemeter at station.

EXTREMES FOR PERIOD OF RECORD.--Maximum gage height, $8.93 \mathrm{ft}$, June 15, 1999; minimum recorded, $5.95 \mathrm{ft}$, Jan. $2,1996$.

EXTREMES FOR CURRENT YEAR.--Maximum recorded gage height, $8.46 \mathrm{ft}$, June 12; minimum recorded, $7.13 \mathrm{ft}$, Feb. 24.

GAGE HEIGHT, FEET, WATER YEAR OCTOBER 2000 TO SEPTEMBER 2001

DAILY MEAN VALUES

\begin{tabular}{|c|c|c|c|c|c|c|c|c|c|c|c|c|}
\hline DAY & OCT & NOV & $D E C$ & JAN & $F E B$ & MAR & APR & MAY & JUN & JUL & AUG & SEP \\
\hline $\begin{array}{l}1 \\
2 \\
3 \\
4 \\
5\end{array}$ & $\begin{array}{l}8.23 \\
8.23 \\
8.26 \\
8.31 \\
8.32\end{array}$ & $\begin{array}{l}7.37 \\
7.38 \\
7.37 \\
7.36 \\
7.34\end{array}$ & $\begin{array}{l}7.30 \\
7.30 \\
7.30 \\
7.29 \\
7.31\end{array}$ & $\begin{array}{l}7.57 \\
7.58 \\
7.59 \\
7.60 \\
7.60\end{array}$ & $\begin{array}{l}7.36 \\
7.34 \\
7.31 \\
7.29 \\
7.27\end{array}$ & $\begin{array}{l}7.26 \\
7.20 \\
7.22 \\
7.29 \\
7.31\end{array}$ & $\begin{array}{l}7.28 \\
7.27 \\
7.26 \\
7.26 \\
7.25\end{array}$ & $\begin{array}{l}8.24 \\
8.21 \\
8.20 \\
8.19 \\
8.16\end{array}$ & $\begin{array}{l}8.24 \\
8.25 \\
8.19 \\
8.20 \\
8.24\end{array}$ & $\begin{array}{l}8.17 \\
8.14 \\
8.14 \\
8.14 \\
8.11\end{array}$ & $\begin{array}{l}7.79 \\
7.82 \\
7.82 \\
7.80 \\
7.78\end{array}$ & $\begin{array}{l}7.81 \\
7.79 \\
7.78 \\
7.78 \\
7.76\end{array}$ \\
\hline $\begin{array}{r}6 \\
7 \\
8 \\
9 \\
10\end{array}$ & $\begin{array}{l}8.32 \\
8.27 \\
8.18 \\
8.01 \\
7.84\end{array}$ & $\begin{array}{l}7.33 \\
7.38 \\
7.34 \\
7.36 \\
7.41\end{array}$ & $\begin{array}{l}7.29 \\
7.29 \\
7.30 \\
7.30 \\
7.31\end{array}$ & $\begin{array}{l}7.60 \\
7.60 \\
7.58 \\
7.53 \\
7.48\end{array}$ & $\begin{array}{l}7.24 \\
7.21 \\
7.20 \\
7.54 \\
7.88\end{array}$ & $\begin{array}{l}7.30 \\
7.28 \\
7.28 \\
7.27 \\
7.26\end{array}$ & $\begin{array}{l}7.29 \\
7.35 \\
7.45 \\
7.63 \\
7.86\end{array}$ & $\begin{array}{l}8.14 \\
8.19 \\
8.19 \\
8.18 \\
8.19\end{array}$ & $\begin{array}{l}8.23 \\
8.25 \\
8.24 \\
8.20 \\
8.21\end{array}$ & $\begin{array}{l}8.08 \\
8.08 \\
8.07 \\
8.06 \\
8.05\end{array}$ & $\begin{array}{l}7.76 \\
7.75 \\
7.74 \\
7.74 \\
7.82\end{array}$ & $\begin{array}{l}7.75 \\
7.79 \\
7.84 \\
7.89 \\
7.94\end{array}$ \\
\hline $\begin{array}{l}11 \\
12 \\
13 \\
14 \\
15\end{array}$ & $\begin{array}{l}7.68 \\
7.53 \\
7.40 \\
7.31 \\
7.27\end{array}$ & $\begin{array}{l}7.41 \\
7.40 \\
7.39 \\
7.38 \\
7.36\end{array}$ & $\begin{array}{l}7.33 \\
7.37 \\
7.37 \\
7.39 \\
7.39\end{array}$ & $\begin{array}{l}7.43 \\
7.40 \\
7.36 \\
7.34 \\
7.34\end{array}$ & $\begin{array}{l}8.01 \\
8.00 \\
7.93 \\
7.85 \\
7.75\end{array}$ & $\begin{array}{l}7.28 \\
7.27 \\
7.29 \\
7.29 \\
7.37\end{array}$ & $\begin{array}{l}8.07 \\
8.22 \\
8.24 \\
8.19 \\
8.16\end{array}$ & $\begin{array}{l}8.22 \\
8.23 \\
8.20 \\
8.22 \\
8.27\end{array}$ & $\begin{array}{l}8.22 \\
8.41 \\
8.38 \\
8.22 \\
8.14\end{array}$ & $\begin{array}{l}8.01 \\
7.99 \\
7.98 \\
7.96 \\
7.93\end{array}$ & $\begin{array}{l}7.79 \\
7.77 \\
7.78 \\
7.76 \\
7.74\end{array}$ & $\begin{array}{l}7.92 \\
7.91 \\
7.90 \\
7.88 \\
7.87\end{array}$ \\
\hline $\begin{array}{l}16 \\
17 \\
18 \\
19 \\
20\end{array}$ & $\begin{array}{l}7.27 \\
7.27 \\
7.27 \\
7.27 \\
7.23\end{array}$ & $\begin{array}{l}7.34 \\
7.33 \\
7.30 \\
7.28 \\
7.28\end{array}$ & $\begin{array}{l}7.40 \\
7.40 \\
7.41 \\
7.43 \\
7.43\end{array}$ & $\begin{array}{l}7.32 \\
7.30 \\
7.28 \\
7.28 \\
7.28\end{array}$ & $\begin{array}{l}7.64 \\
7.56 \\
7.49 \\
7.38 \\
7.29\end{array}$ & $\begin{array}{l}7.42 \\
7.37 \\
7.31 \\
7.27 \\
7.27\end{array}$ & $\begin{array}{l}8.20 \\
8.23 \\
8.22 \\
8.20 \\
8.21\end{array}$ & $\begin{array}{l}8.24 \\
8.21 \\
8.21 \\
8.21 \\
8.22\end{array}$ & $\begin{array}{l}8.16 \\
8.17 \\
8.19 \\
8.20 \\
8.24\end{array}$ & $\begin{array}{l}7.91 \\
7.94 \\
7.96 \\
7.95 \\
7.94\end{array}$ & $\begin{array}{l}7.83 \\
7.82 \\
7.81 \\
7.91 \\
7.91\end{array}$ & $\begin{array}{l}7.86 \\
7.85 \\
7.85 \\
7.94 \\
7.93\end{array}$ \\
\hline $\begin{array}{l}21 \\
22 \\
23 \\
24 \\
25\end{array}$ & $\begin{array}{l}7.23 \\
7.22 \\
7.21 \\
7.23 \\
7.26\end{array}$ & $\begin{array}{l}7.26 \\
7.32 \\
7.35 \\
7.38 \\
7.41\end{array}$ & $\begin{array}{l}7.45 \\
7.45 \\
7.46 \\
7.47 \\
7.47\end{array}$ & $\begin{array}{l}7.28 \\
7.28 \\
7.28 \\
7.28 \\
7.28\end{array}$ & $\begin{array}{l}7.25 \\
7.23 \\
7.22 \\
7.16 \\
7.32\end{array}$ & $\begin{array}{l}7.27 \\
7.27 \\
7.27 \\
7.26 \\
7.23\end{array}$ & $\begin{array}{l}8.24 \\
8.23 \\
8.16 \\
8.14 \\
8.16\end{array}$ & $\begin{array}{l}8.22 \\
8.21 \\
8.19 \\
8.23 \\
8.22\end{array}$ & $\begin{array}{l}8.25 \\
8.18 \\
8.14 \\
8.17 \\
8.20\end{array}$ & $\begin{array}{l}7.93 \\
7.92 \\
7.91 \\
7.90 \\
7.91\end{array}$ & $\begin{array}{l}7.89 \\
7.89 \\
7.90 \\
7.89 \\
7.89\end{array}$ & $\begin{array}{l}7.95 \\
7.95 \\
7.99 \\
8.02 \\
7.99\end{array}$ \\
\hline $\begin{array}{l}26 \\
27 \\
28 \\
29 \\
30 \\
31\end{array}$ & $\begin{array}{l}7.29 \\
7.33 \\
7.33 \\
7.33 \\
7.35 \\
7.36\end{array}$ & $\begin{array}{r}7.44 \\
7.45 \\
7.41 \\
7.38 \\
7.34 \\
----\end{array}$ & $\begin{array}{l}7.47 \\
7.47 \\
7.47 \\
7.50 \\
7.53 \\
7.55\end{array}$ & $\begin{array}{l}7.27 \\
7.27 \\
7.26 \\
7.26 \\
7.33 \\
7.36\end{array}$ & $\begin{array}{r}7.44 \\
7.45 \\
7.37 \\
--- \\
--- \\
--\end{array}$ & $\begin{array}{l}7.23 \\
7.22 \\
7.23 \\
7.26 \\
7.28 \\
7.28\end{array}$ & $\begin{array}{l}8.21 \\
8.28 \\
8.30 \\
8.28 \\
8.26 \\
---\end{array}$ & $\begin{array}{l}8.20 \\
8.24 \\
8.26 \\
8.26 \\
8.23 \\
8.19\end{array}$ & $\begin{array}{l}8.23 \\
8.24 \\
8.21 \\
8.19 \\
8.18 \\
-\end{array}$ & $\begin{array}{l}7.88 \\
7.85 \\
7.83 \\
7.83 \\
7.82 \\
7.80\end{array}$ & $\begin{array}{l}7.90 \\
7.89 \\
7.88 \\
7.86 \\
7.84 \\
7.83\end{array}$ & $\begin{array}{r}7.97 \\
7.95 \\
7.95 \\
7.94 \\
7.93 \\
-\end{array}$ \\
\hline $\begin{array}{l}\text { MEAN } \\
\text { MAX } \\
\text { MIN }\end{array}$ & $\begin{array}{l}7.60 \\
8.32 \\
7.21\end{array}$ & $\begin{array}{l}7.36 \\
7.45 \\
7.26\end{array}$ & $\begin{array}{l}7.39 \\
7.55 \\
7.29\end{array}$ & $\begin{array}{l}7.39 \\
7.60 \\
7.26\end{array}$ & $\begin{array}{l}7.46 \\
8.01 \\
7.16\end{array}$ & $\begin{array}{l}7.28 \\
7.42 \\
7.20\end{array}$ & $\begin{array}{l}7.94 \\
8.30 \\
7.25\end{array}$ & $\begin{array}{l}8.21 \\
8.27 \\
8.14\end{array}$ & $\begin{array}{l}8.22 \\
8.41 \\
8.14\end{array}$ & $\begin{array}{l}7.97 \\
8.17 \\
7.80\end{array}$ & $\begin{array}{l}7.83 \\
7.91 \\
7.74\end{array}$ & $\begin{array}{l}7.89 \\
8.02 \\
7.75\end{array}$ \\
\hline
\end{tabular}

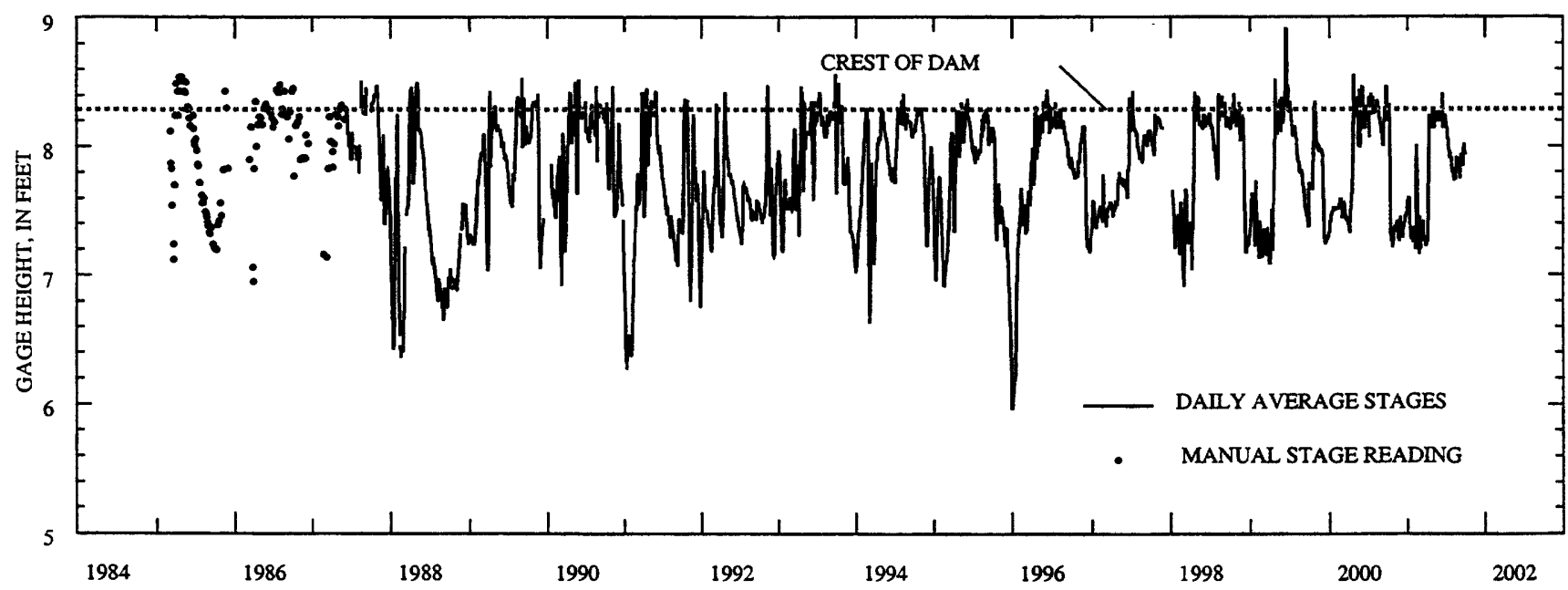




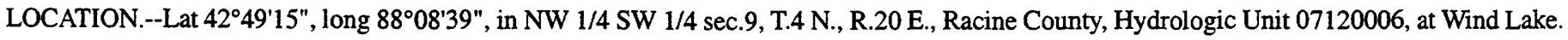
PERIOD OF RECORD.--February 1985 to current year.

REMARKS.--Lake sampled near center at the deep hole. Lake ice-covered during February sampling. Water-quality analyses done by Wisconsin State Laboratory of Hygiene.

WATER-QUALITY DATA, FEBRUARY 13 TO JUNE 18, 2001

(Milligrams per liter unless otherwise indicated)

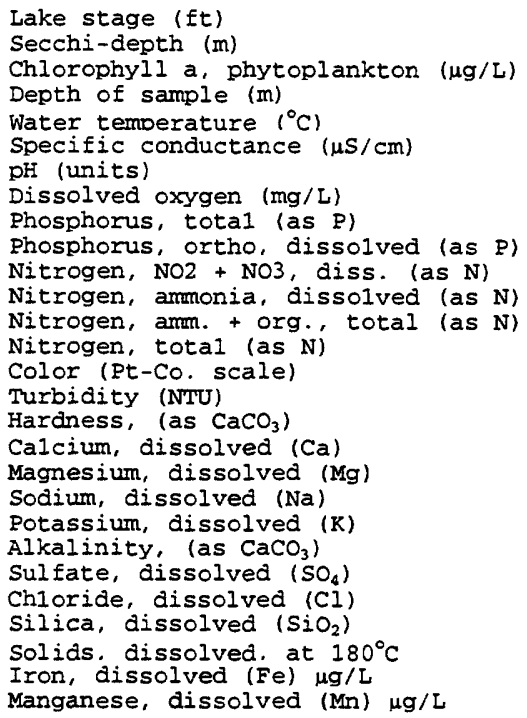

2-13-01

\begin{tabular}{|c|c|c|c|c|c|}
\hline \multicolumn{2}{|c|}{ Feb-13 } & \multicolumn{2}{|c|}{ Apr -18} & \multicolumn{2}{|c|}{ Jun-18 } \\
\hline \multicolumn{2}{|c|}{$\begin{array}{l}7.93 \\
---\end{array}$} & \multicolumn{2}{|c|}{$\begin{array}{c}8.22 \\
1.0 \\
42\end{array}$} & \multicolumn{2}{|c|}{$\begin{array}{c}8.19 \\
1.9 \\
13\end{array}$} \\
\hline $\begin{array}{c}0.5 \\
1.6 \\
620 \\
8.0 \\
13.8 \\
0.034\end{array}$ & $\begin{array}{c}15.0 \\
2.3 \\
916 \\
7.2 \\
0.1 \\
0.051\end{array}$ & $\begin{array}{c}0.5 \\
9.3 \\
637 \\
8.1 \\
11.1 \\
0.042\end{array}$ & $\begin{array}{c}14.0 \\
9.0 \\
640 \\
8.1 \\
10.8 \\
0.036\end{array}$ & $\begin{array}{c}0.5 \\
23.1 \\
582 \\
7.9 \\
8.9 \\
0.029\end{array}$ & $\begin{array}{c}15.0 \\
14.4 \\
609 \\
7.5 \\
1.2 \\
0.144\end{array}$ \\
\hline-- & $\cdots$ & $<0.002$ & $\ldots$ & -- & -- \\
\hline$--\cdot$ & - & 0.078 & $-\cdots$ & --- & -- \\
\hline$-\cdots$ & -- & $\begin{array}{c}0.133 \\
1.4\end{array}$ & $\ldots$ & $\ldots$ & $\ldots$ \\
\hline$\ldots$ & $\ldots$ & 1.45 & $\ldots$ & --- & -- \\
\hline--- & -- & 40 & -- & -- & -.. \\
\hline--- & --- & 8.0 & -- & $\cdots$ & -- \\
\hline--- & -- & 235 & $\cdots$ & -- & -- \\
\hline-- & -- & 53 & $\cdots$ & -- & $\cdots$ \\
\hline$\cdots$ & -- & 25 & -- & --- & $\cdots$ \\
\hline$\cdots$ & -- & 36 & -- & -.- & $\cdots$ \\
\hline-- & -- & 3.2 & $\cdots$ & $\cdots$ & -- \\
\hline-- & $-\cdots$ & 185 & $\cdots$ & $\cdots$ & $\cdots$ \\
\hline-- & -- & 32.1 & -- & -- & --- \\
\hline-- & -- & 70.6 & -- & -- & $-\cdots$ \\
\hline-- & -- & 0.0 & -- & -- & -- \\
\hline-- & --- & 370 & -- & $\ldots$ & $\ldots$ \\
\hline-- & --- & 10 & $-\cdots$ & --- & -- \\
\hline -. & -- & 0.7 & -- & -- & $\cdots$ \\
\hline
\end{tabular}

4-18-01

DISSOLVED OXYGEN (D.O.), IN MILLIGRAMS PER LITER
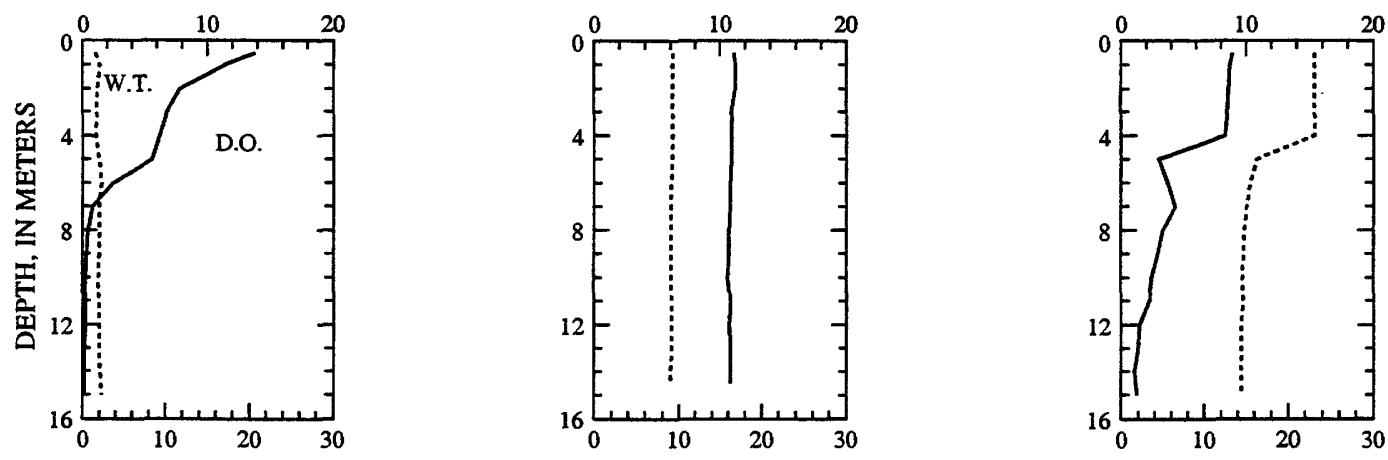

WATER TEMPERATURE (W.T.), IN DEGREES CELSIUS

PH, IN STANDARD UNITS
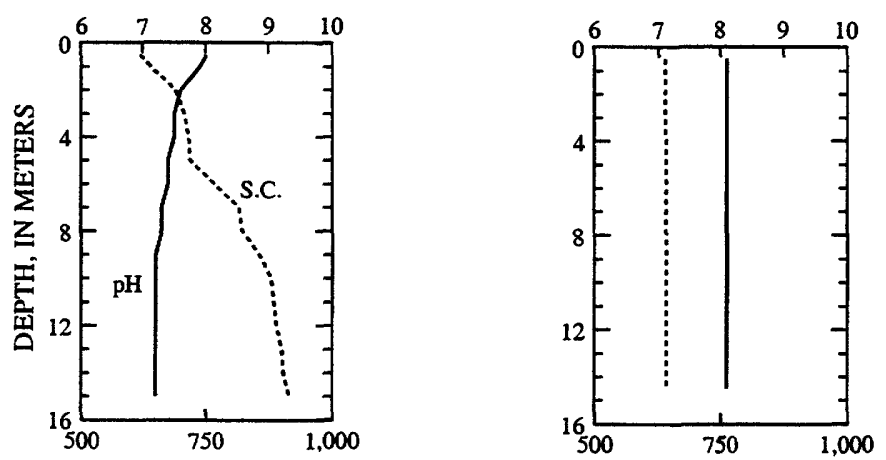

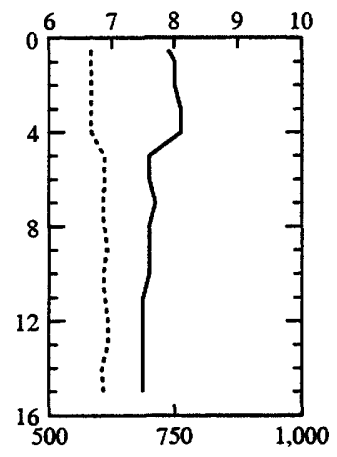

SPECIFIC CONDUCTANCE (S.C.), IN MICROSIEMENS PER CENTIMETER AT 25 DEGREES CELSIUS 
WATER-QUALITY DATA, JULY 18 TO AUGUST 20, 2001

(Milligrams per liter unless otherwise indicated)

Lake stage (ft)

Secchi-depth (m)

Chlorophyll a, phytoplankton ( $\mu \mathrm{g} / \mathrm{L})$

Depth of sample (m)

water temberature $\left({ }^{\circ} \mathrm{C}\right)$

Specific conductance ( $\mu \mathrm{S} / \mathrm{cm})$

$\mathrm{pH}$ (units)

Dissolved oxygen ( $\mathrm{mg} / \mathrm{L}$ )

Phosphorus, total (as P)

Phosphorus, ortho, dissolved (as P)

Nitrogen, $\mathrm{NO} 2$ + $\mathrm{NO} 3$, diss. (as N)

Nitrogen, ammonia, dissolved (as N)

\begin{tabular}{|c|c|c|c|c|c|c|c|c|c|}
\hline \multicolumn{5}{|c|}{ Jul-18 } & \multicolumn{5}{|c|}{ Aug -20 } \\
\hline & & $\begin{array}{c}7.96 \\
1.0 \\
19\end{array}$ & & & & & $\begin{array}{c}7.91 \\
0.75 \\
8\end{array}$ & & \\
\hline $\begin{array}{c}0.5 \\
25.0 \\
578 \\
7.9 \\
6.9 \\
0.035\end{array}$ & $\begin{array}{c}5.0 \\
23.7 \\
590 \\
7.9 \\
4.7 \\
0.028\end{array}$ & $\begin{array}{c}9.0 \\
14.7 \\
638 \\
7.7 \\
0.2 \\
0.078\end{array}$ & $\begin{array}{c}12.0 \\
14.2 \\
640 \\
7.7 \\
0.2 \\
0.148\end{array}$ & $\begin{array}{c}14.0 \\
14.1 \\
647 \\
7.6 \\
0.2 \\
0.162\end{array}$ & $\begin{array}{c}0.5 \\
22.8 \\
563 \\
8.1 \\
8.5 \\
0.032\end{array}$ & $\begin{array}{c}7.0 \\
20.9 \\
573 \\
7.8 \\
4.2 \\
0.037\end{array}$ & $\begin{array}{c}8.0 \\
17.1 \\
624 \\
7.1 \\
0.3 \\
0.032\end{array}$ & $\begin{array}{c}9.0 \\
15.1 \\
640 \\
7.1 \\
0.2 \\
0.055\end{array}$ & $\begin{array}{c}15.0 \\
14.1 \\
649 \\
7.0 \\
0.2 \\
0.116\end{array}$ \\
\hline - & -0 & - & $\begin{array}{l}.140 \\
---\end{array}$ & -10 & $<0.002$ & $\ldots$ & - & - & $-1+$ \\
\hline--- & --- & -- & -- & --- & $<0.010$ & -- & --- & -- & $-\infty$ \\
\hline-- & --- & --- & $-\ldots$ & $\ldots$ & $<0.013$ & --- & -- & $\cdots$ & $-\ldots$ \\
\hline
\end{tabular}

DISSOLVED OXYGEN (D.O.), IN MILLIGRAMS PER LITER
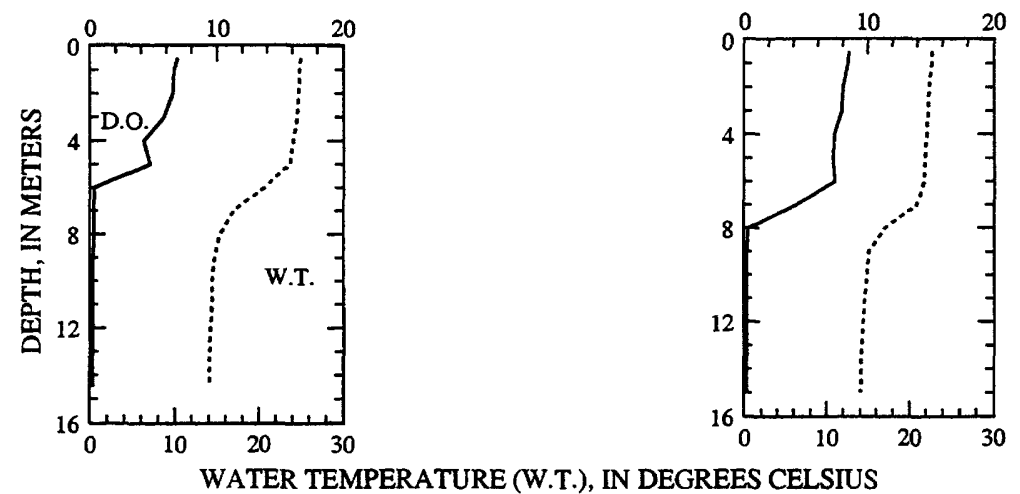

PH, IN STANDARD UNITS
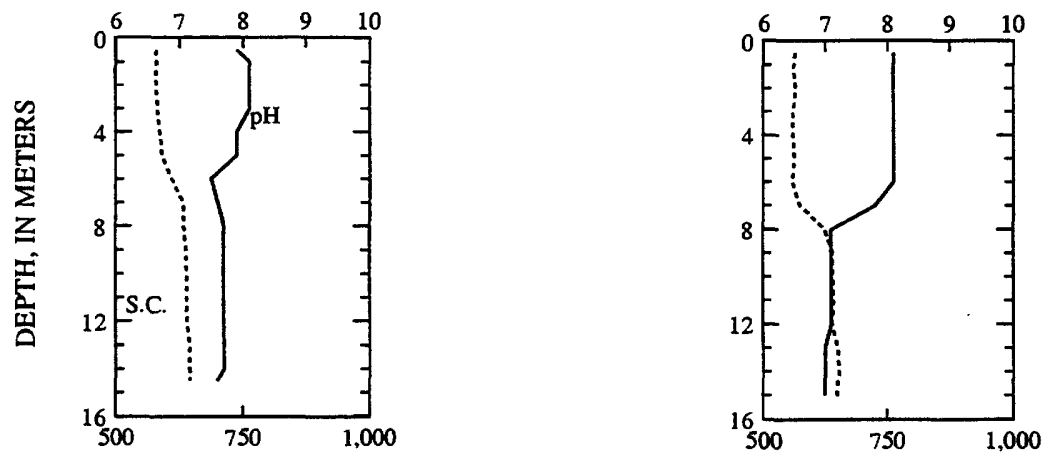

SPECIFIC CONDUCTANCE (S.C.), IN MICROSIEMENS PER CENTIMETER AT 25 DEGREES CELSIUS 

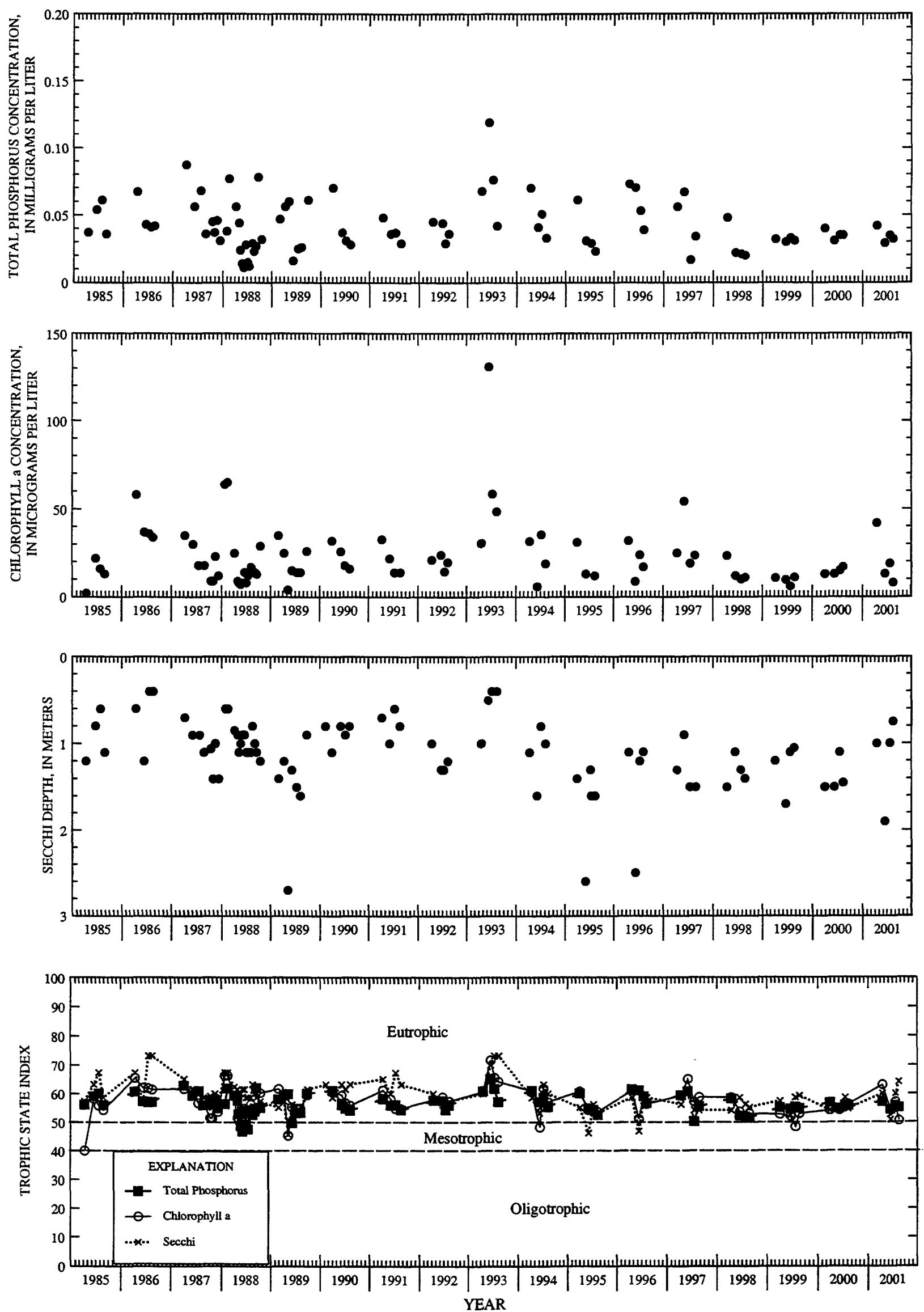

Surface total phosphorus, chlorophyll a concentrations, Secchi depths, and TSI data for Wind Lake, Deep Hole, at Wind Lake, Wisconsin. 
LOCATION.--Lat $44^{\circ} 00^{\prime} 35^{\prime \prime}$, long $88^{\circ} 31^{\prime} 38^{\prime \prime}$, in NE 1/4 NE 1/4 sec.25, T.18 N., R.16 E., Winnebago County, Hydrologic Unit 04030203 , at 905 Bay Shore Drive, $800 \mathrm{ft}$ east of mouth of the upper Fox River.

DRAINAGE AREA.--5,880 $\mathrm{mi}^{2}$, at lake outlet at Menasha Dam. Area of Lake Winnebago, $215 \mathrm{mi}^{2}$.

PERIOD OF RECORD.--October 1938 to current year in reports of Geological Survey. Records from July 1882 to September 1938 in files of Geological Survey and U.S. Army Corps of Engineers. A report on Fox River by U.S. Army Corps of Engineers, published as House Document No. 146, 67th Congress, 2nd session, contains semi-monthly records of inflow of Lake Winnebago for the period $1896-1917$.

REVISED RECORD.--WDR WI-83-1: Drainage area.

GAGE.--Water-stage recorder. Nonrecording gage read once daily October 1938 to October 1978. Datum of gage is $745.05 \mathrm{ft}$ above mean tide at New York City (levels by U.S. Army Corps of Engineers). Datum of Deuchman gage is $745.00 \mathrm{ft}$ above mean tide at New York City.

REMARKS.--Lake elevations controlled by dams at Menasha and Neenah, which are operated in the interest of navigation. Crests of both dams are at elevation $746.73 \mathrm{ft}$. Present limits of regulation are from 21 1/4 in. above the crest of Menasha dam to crest during navigation season, plus additional 18 in. below crest during winter. Oshkosh staff gage gives true level of lake, while Deuchman gage readings are affected by loss of head in the channel between lake and dam. Data-collection platform and gage-height telemeter at station.

EXTREMES FOR PERIOD OF RECORD.--Maximum gage height observed, $5.33 \mathrm{ft}$ (Deuchman gage) Nov. 8, 1881; minimum observed, $-2.00 \mathrm{ft}$ (Deuchman gage) Nov. 28, 1891.

EXTREMES FOR CURRENT YEAR.--Maximum daily mean gage height, $3.18 \mathrm{ft}$, June 4; minimum recorded, $1.45 \mathrm{ft}$, June 11.

GAGE HEIGHT, FEET, WATER YEAR OCTOBER 2000 TO SEPTEMBER 2001

DAILY MEAN VALUES

\begin{tabular}{|c|c|c|c|c|c|c|c|c|c|c|c|c|}
\hline DAY & OCT & NOV & $D E C$ & JAN & FEB & MAR & $A P R$ & MAY & JUN & JUL & AUG & SEP \\
\hline $\begin{array}{l}1 \\
2 \\
3 \\
4 \\
5\end{array}$ & $\begin{array}{l}2.86 \\
2.86 \\
2.89 \\
2.90 \\
2.90\end{array}$ & $\begin{array}{l}2.47 \\
2.40 \\
2.39 \\
2.40 \\
2.39\end{array}$ & $\begin{array}{l}2.39 \\
2.34 \\
2.31 \\
2.24 \\
2.22\end{array}$ & $\begin{array}{l}2.05 \\
2.05 \\
2.03 \\
2.01 \\
1.98\end{array}$ & $\begin{array}{l}1.67 \\
1.64 \\
1.62 \\
1.60 \\
1.59\end{array}$ & $\begin{array}{l}1.59 \\
1.59 \\
1.59 \\
1.59 \\
1.59\end{array}$ & $\begin{array}{l}2.00 \\
2.04 \\
2.08 \\
2.11 \\
2.15\end{array}$ & $\begin{array}{l}2.59 \\
2.65 \\
2.77 \\
2.89 \\
2.93\end{array}$ & $\begin{array}{l}3.10 \\
3.11 \\
3.16 \\
3.18 \\
3.15\end{array}$ & $\begin{array}{l}3.06 \\
3.02 \\
3.00 \\
2.99 \\
3.00\end{array}$ & $\begin{array}{l}2.86 \\
2.93 \\
2.92 \\
2.91 \\
2.89\end{array}$ & $\begin{array}{l}2.91 \\
2.87 \\
2.87 \\
2.89 \\
2.86\end{array}$ \\
\hline $\begin{array}{r}6 \\
7 \\
8 \\
9 \\
10\end{array}$ & $\begin{array}{l}2.84 \\
2.86 \\
2.84 \\
2.82 \\
2.81\end{array}$ & $\begin{array}{l}2.43 \\
2.26 \\
2.32 \\
2.37 \\
2.37\end{array}$ & $\begin{array}{l}2.26 \\
2.23 \\
2.21 \\
2.18 \\
2.17\end{array}$ & $\begin{array}{l}1.97 \\
1.95 \\
1.93 \\
1.91 \\
1.89\end{array}$ & $\begin{array}{l}1.58 \\
1.56 \\
1.56 \\
1.60 \\
1.61\end{array}$ & $\begin{array}{l}1.60 \\
1.60 \\
1.60 \\
1.60 \\
1.61\end{array}$ & $\begin{array}{l}2.19 \\
2.28 \\
2.37 \\
2.46 \\
2.47\end{array}$ & $\begin{array}{l}2.94 \\
2.92 \\
2.95 \\
3.03 \\
3.00\end{array}$ & $\begin{array}{l}3.10 \\
3.07 \\
3.06 \\
3.03 \\
3.04\end{array}$ & $\begin{array}{l}2.96 \\
2.94 \\
2.95 \\
2.94 \\
2.93\end{array}$ & $\begin{array}{l}2.88 \\
2.89 \\
2.87 \\
2.83 \\
2.83\end{array}$ & $\begin{array}{l}2.85 \\
2.86 \\
2.93 \\
2.99 \\
2.99\end{array}$ \\
\hline $\begin{array}{l}11 \\
12 \\
13 \\
14 \\
15\end{array}$ & $\begin{array}{l}2.80 \\
2.79 \\
2.79 \\
2.78 \\
2.79\end{array}$ & $\begin{array}{l}2.41 \\
2.39 \\
2.34 \\
2.36 \\
2.39\end{array}$ & $\begin{array}{l}2.16 \\
2.15 \\
2.13 \\
2.12 \\
2.10\end{array}$ & $\begin{array}{l}1.85 \\
1.83 \\
1.80 \\
1.79 \\
1.78\end{array}$ & $\begin{array}{l}1.59 \\
1.57 \\
1.56 \\
1.56 \\
1.56\end{array}$ & $\begin{array}{l}1.62 \\
1.62 \\
1.64 \\
1.65 \\
1.65\end{array}$ & $\begin{array}{l}2.57 \\
2.54 \\
2.68 \\
2.68 \\
2.71\end{array}$ & $\begin{array}{l}3.11 \\
3.08 \\
3.05 \\
3.05 \\
3.00\end{array}$ & $\begin{array}{l}3.05 \\
3.13 \\
3.15 \\
3.10 \\
3.08\end{array}$ & $\begin{array}{l}2.90 \\
2.89 \\
2.87 \\
2.88 \\
2.88\end{array}$ & $\begin{array}{l}2.79 \\
2.78 \\
2.81 \\
2.77 \\
2.75\end{array}$ & $\begin{array}{l}3.03 \\
3.03 \\
3.06 \\
3.05 \\
3.04\end{array}$ \\
\hline $\begin{array}{l}16 \\
17 \\
18 \\
19 \\
20\end{array}$ & $\begin{array}{l}2.78 \\
2.76 \\
2.75 \\
2.73 \\
2.69\end{array}$ & $\begin{array}{l}2.38 \\
2.39 \\
2.38 \\
2.37 \\
2.31\end{array}$ & $\begin{array}{l}2.09 \\
2.08 \\
2.07 \\
2.10 \\
2.10\end{array}$ & $\begin{array}{l}1.76 \\
1.74 \\
1.72 \\
1.70 \\
1.70\end{array}$ & $\begin{array}{l}1.56 \\
1.56 \\
1.56 \\
1.56 \\
1.56\end{array}$ & $\begin{array}{l}1.64 \\
1.65 \\
1.67 \\
1.69 \\
1.71\end{array}$ & $\begin{array}{l}2.70 \\
2.78 \\
2.80 \\
2.80 \\
2.81\end{array}$ & $\begin{array}{l}2.98 \\
2.96 \\
2.96 \\
2.92 \\
2.90\end{array}$ & $\begin{array}{l}3.08 \\
3.08 \\
3.06 \\
2.99 \\
3.02\end{array}$ & $\begin{array}{l}2.87 \\
2.88 \\
2.89 \\
2.89 \\
2.90\end{array}$ & $\begin{array}{l}2.77 \\
2.78 \\
2.78 \\
2.83 \\
2.80\end{array}$ & $\begin{array}{l}3.05 \\
3.05 \\
3.08 \\
3.07 \\
3.07\end{array}$ \\
\hline $\begin{array}{l}21 \\
22 \\
23 \\
24 \\
25\end{array}$ & $\begin{array}{l}2.71 \\
2.66 \\
2.66 \\
2.66 \\
2.63\end{array}$ & $\begin{array}{l}2.34 \\
2.36 \\
2.37 \\
2.35 \\
2.35\end{array}$ & $\begin{array}{l}2.12 \\
2.11 \\
2.11 \\
2.11 \\
2.10\end{array}$ & $\begin{array}{l}1.69 \\
1.69 \\
1.69 \\
1.68 \\
1.68\end{array}$ & $\begin{array}{l}1.56 \\
1.56 \\
1.55 \\
1.55 \\
1.58\end{array}$ & $\begin{array}{l}1.74 \\
1.77 \\
1.79 \\
1.82 \\
1.85\end{array}$ & $\begin{array}{l}2.76 \\
2.86 \\
2.72 \\
2.78 \\
2.78\end{array}$ & $\begin{array}{l}2.80 \\
2.77 \\
2.85 \\
2.88 \\
2.91\end{array}$ & $\begin{array}{l}3.00 \\
2.99 \\
2.97 \\
2.94 \\
2.92\end{array}$ & $\begin{array}{l}2.90 \\
2.89 \\
2.92 \\
2.94 \\
2.97\end{array}$ & $\begin{array}{l}2.79 \\
2.82 \\
2.86 \\
2.85 \\
2.86\end{array}$ & $\begin{array}{l}3.06 \\
3.05 \\
3.05 \\
3.07 \\
3.01\end{array}$ \\
\hline $\begin{array}{l}26 \\
27 \\
28 \\
29 \\
30 \\
31\end{array}$ & $\begin{array}{l}2.60 \\
2.61 \\
2.58 \\
2.53 \\
2.48 \\
2.46\end{array}$ & $\begin{array}{r}2.35 \\
2.33 \\
2.34 \\
2.38 \\
2.38 \\
\end{array}$ & $\begin{array}{l}2.09 \\
2.08 \\
2.07 \\
2.08 \\
2.07 \\
2.06\end{array}$ & $\begin{array}{l}1.67 \\
1.68 \\
1.67 \\
1.67 \\
1.70 \\
1.68\end{array}$ & $\begin{array}{r}1.59 \\
1.59 \\
1.59 \\
--- \\
--- \\
---\end{array}$ & $\begin{array}{l}1.86 \\
1.87 \\
1.88 \\
1.90 \\
1.92 \\
1.95\end{array}$ & $\begin{array}{l}2.71 \\
2.73 \\
2.68 \\
2.60 \\
2.55 \\
\ldots\end{array}$ & $\begin{array}{l}2.96 \\
2.99 \\
3.04 \\
3.10 \\
3.08 \\
3.09\end{array}$ & $\begin{array}{l}2.94 \\
2.98 \\
3.02 \\
3.02 \\
3.03 \\
-\end{array}$ & $\begin{array}{l}2.93 \\
2.90 \\
2.84 \\
2.87 \\
2.86 \\
2.85\end{array}$ & $\begin{array}{l}2.92 \\
2.92 \\
2.94 \\
2.92 \\
2.89 \\
2.93\end{array}$ & $\begin{array}{r}2.99 \\
2.99 \\
2.97 \\
2.96 \\
2.95 \\
-\end{array}$ \\
\hline $\begin{array}{l}\text { MEAN } \\
\text { MAX } \\
\text { MIN }\end{array}$ & $\begin{array}{l}2.74 \\
2.90 \\
2.46\end{array}$ & $\begin{array}{l}2.37 \\
2.47 \\
2.26\end{array}$ & $\begin{array}{l}2.15 \\
2.39 \\
2.06\end{array}$ & $\begin{array}{l}1.80 \\
2.05 \\
1.67\end{array}$ & $\begin{array}{l}1.58 \\
1.67 \\
1.55\end{array}$ & $\begin{array}{l}1.70 \\
1.95 \\
1.59\end{array}$ & $\begin{array}{l}2.55 \\
2.86 \\
2.00\end{array}$ & $\begin{array}{l}2.94 \\
3.11 \\
2.59\end{array}$ & $\begin{array}{l}3.05 \\
3.18 \\
2.92\end{array}$ & $\begin{array}{l}2.92 \\
3.06 \\
2.84\end{array}$ & $\begin{array}{l}2.85 \\
2.94 \\
2.75\end{array}$ & $\begin{array}{l}2.99 \\
3.08 \\
2.85\end{array}$ \\
\hline
\end{tabular}


LOCATION.--Lat $44^{\circ} 04^{\prime} 17^{\prime \prime}$, long $88^{\circ} 19^{\prime} 52^{\prime \prime}$, Stockbridge Indian Reservation, Calumet County, Hydrologic Unit 04030203, on east shore of Lake Winnebago, $300 \mathrm{ft}$ south of County Highway E and $1.6 \mathrm{mi}$ west of Stockbridge.

DRAINAGE AREA.- $5,880 \mathrm{mi}^{2}$, at lake outlet at Menasha Dam. Area of Lake Winnebago, $215 \mathrm{mi}^{2}$.

PERIOD OF RECORD.--November 1982 to current year.

GAGE.--Water-stage recorder. Datum of gage is $745.05 \mathrm{ft}$ above mean tide of New York City (levels by U. S. Army Corps of Engineers).

REMARKS.--Lake elevations controlled by dams at Menasha and Neenah, which are operated in the interest of navigation. Crests of both dams are at elevation $746.73 \mathrm{ft}$. Present limits of regulation are from $21 \mathrm{1} / 4 \mathrm{in}$. above the crest of Menasha dam to crest during navigation season, plus additional $18 \mathrm{in}$. below crest during winter. Data-collection platform and gage-height telemeter at station.

EXTREMES FOR PERIOD OF RECORD.--Maximum daily mean gage height, $3.85 \mathrm{ft}$, July 9, 11, 1993; minimum observed, $0.30 \mathrm{ft}$, Mar. 1 , 1986.

EXTREMES FOR CURRENT YEAR.--Maximum daily mean gage height, $3.11 \mathrm{ft}$, June 3, 12, 13; minimum recorded, $1.46 \mathrm{ft}$, Feb. 24.

GA'GE HEIGHT, FEET, WATER YEAR OCTOBER 2000 TO SEPTEMBER 2001

DAILY MEAN VALUES

\begin{tabular}{|c|c|c|c|c|c|c|c|c|c|c|c|c|}
\hline DAY & OCT & NOV & $\mathrm{DEC}$ & JAN & $F E B$ & MAR & APR & MAY & JUN & JUL & AUG & SEP \\
\hline $\begin{array}{l}1 \\
2 \\
3 \\
4 \\
5\end{array}$ & $\begin{array}{l}2.87 \\
2.89 \\
2.87 \\
2.87 \\
2.84\end{array}$ & $\begin{array}{l}2.40 \\
2.41 \\
2.47 \\
2.41 \\
2.36\end{array}$ & $\begin{array}{l}2.27 \\
2.30 \\
2.31 \\
2.31 \\
2.28\end{array}$ & $\begin{array}{l}2.02 \\
2.01 \\
2.00 \\
1.97 \\
1.97\end{array}$ & $\begin{array}{l}1.64 \\
1.62 \\
1.59 \\
1.58 \\
1.56\end{array}$ & $\begin{array}{l}1.56 \\
1.56 \\
1.56 \\
1.57 \\
1.59\end{array}$ & $\begin{array}{l}1.99 \\
2.01 \\
2.05 \\
2.08 \\
2.11\end{array}$ & $\begin{array}{l}2.55 \\
2.61 \\
2.69 \\
2.79 \\
2.82\end{array}$ & $\begin{array}{l}3.04 \\
3.08 \\
3.11 \\
3.10 \\
3.06\end{array}$ & $\begin{array}{l}3.00 \\
3.00 \\
2.97 \\
3.02 \\
2.95\end{array}$ & $\begin{array}{l}2.86 \\
2.89 \\
2.85 \\
2.86 \\
2.87\end{array}$ & $\begin{array}{l}2.85 \\
2.87 \\
2.86 \\
2.81 \\
2.81\end{array}$ \\
\hline $\begin{array}{r}6 \\
7 \\
8 \\
9 \\
10\end{array}$ & $\begin{array}{l}2.92 \\
2.87 \\
2.87 \\
2.84 \\
2.81\end{array}$ & $\begin{array}{l}2.23 \\
2.29 \\
2.37 \\
2.34 \\
2.43\end{array}$ & $\begin{array}{l}2.23 \\
2.20 \\
2.18 \\
2.15 \\
2.14\end{array}$ & $\begin{array}{l}1.94 \\
1.92 \\
1.90 \\
1.88 \\
1.84\end{array}$ & $\begin{array}{l}1.55 \\
1.53 \\
1.53 \\
1.58 \\
1.59\end{array}$ & $\begin{array}{l}1.58 \\
1.58 \\
1.58 \\
1.58 \\
1.58\end{array}$ & $\begin{array}{l}2.15 \\
2.34 \\
2.39 \\
2.37 \\
2.42\end{array}$ & $\begin{array}{l}2.85 \\
2.92 \\
3.03 \\
2.98 \\
3.01\end{array}$ & $\begin{array}{l}3.05 \\
3.03 \\
3.01 \\
3.01 \\
3.01\end{array}$ & $\begin{array}{l}2.96 \\
2.92 \\
2.91 \\
2.90 \\
2.88\end{array}$ & $\begin{array}{l}2.88 \\
2.85 \\
2.87 \\
2.86 \\
2.77\end{array}$ & $\begin{array}{l}2.80 \\
2.87 \\
2.94 \\
2.97 \\
3.06\end{array}$ \\
\hline $\begin{array}{l}11 \\
12 \\
13 \\
14 \\
15\end{array}$ & $\begin{array}{l}2.79 \\
2.78 \\
2.76 \\
2.77 \\
2.74\end{array}$ & $\begin{array}{l}2.34 \\
2.31 \\
2.43 \\
2.48 \\
2.44\end{array}$ & $\begin{array}{l}2.14 \\
2.13 \\
2.10 \\
2.09 \\
2.06\end{array}$ & $\begin{array}{l}1.82 \\
1.79 \\
1.76 \\
1.75 \\
1.75\end{array}$ & $\begin{array}{l}1.56 \\
1.54 \\
1.53 \\
1.53 \\
1.53\end{array}$ & $\begin{array}{l}1.60 \\
1.60 \\
1.63 \\
1.62 \\
1.62\end{array}$ & $\begin{array}{l}2.49 \\
2.72 \\
2.67 \\
2.69 \\
2.71\end{array}$ & $\begin{array}{l}3.04 \\
3.03 \\
3.03 \\
2.99 \\
2.93\end{array}$ & $\begin{array}{l}2.99 \\
3.11 \\
3.11 \\
3.08 \\
3.10\end{array}$ & $\begin{array}{l}2.85 \\
2.83 \\
2.82 \\
2.84 \\
2.85\end{array}$ & $\begin{array}{l}2.76 \\
2.75 \\
2.74 \\
2.75 \\
2.75\end{array}$ & $\begin{array}{l}2.99 \\
3.00 \\
2.95 \\
2.95 \\
2.98\end{array}$ \\
\hline $\begin{array}{l}16 \\
17 \\
18 \\
19 \\
20\end{array}$ & $\begin{array}{l}2.75 \\
2.73 \\
2.72 \\
2.69 \\
2.69\end{array}$ & $\begin{array}{l}2.37 \\
2.45 \\
2.47 \\
2.43 \\
2.44\end{array}$ & $\begin{array}{l}2.07 \\
2.06 \\
2.05 \\
2.08 \\
2.08\end{array}$ & $\begin{array}{l}1.73 \\
1.70 \\
1.67 \\
1.67 \\
1.66\end{array}$ & $\begin{array}{l}1.54 \\
1.54 \\
1.53 \\
1.53 \\
1.54\end{array}$ & $\begin{array}{l}1.62 \\
1.64 \\
1.64 \\
1.66 \\
1.69\end{array}$ & $\begin{array}{l}2.79 \\
2.71 \\
2.77 \\
2.76 \\
2.77\end{array}$ & $\begin{array}{l}2.92 \\
2.93 \\
2.91 \\
2.88 \\
2.82\end{array}$ & $\begin{array}{l}3.09 \\
3.05 \\
3.02 \\
3.05 \\
2.99\end{array}$ & $\begin{array}{l}2.84 \\
2.84 \\
2.84 \\
2.84 \\
2.85\end{array}$ & $\begin{array}{l}2.76 \\
2.79 \\
2.72 \\
2.72 \\
2.76\end{array}$ & $\begin{array}{l}3.00 \\
3.01 \\
2.99 \\
2.95 \\
3.08\end{array}$ \\
\hline $\begin{array}{l}21 \\
22 \\
23 \\
24 \\
25\end{array}$ & $\begin{array}{l}2.62 \\
2.60 \\
2.62 \\
2.62 \\
2.59\end{array}$ & $\begin{array}{l}2.44 \\
2.37 \\
2.34 \\
2.33 \\
2.31\end{array}$ & $\begin{array}{l}2.10 \\
2.09 \\
2.08 \\
2.08 \\
2.07\end{array}$ & $\begin{array}{l}1.66 \\
1.65 \\
1.66 \\
1.65 \\
1.65\end{array}$ & $\begin{array}{l}1.53 \\
1.53 \\
1.53 \\
1.52 \\
1.57\end{array}$ & $\begin{array}{l}1.72 \\
1.74 \\
1.78 \\
1.82 \\
1.84\end{array}$ & $\begin{array}{l}2.83 \\
2.78 \\
2.90 \\
2.81 \\
2.77\end{array}$ & $\begin{array}{l}2.76 \\
2.88 \\
2.81 \\
2.75 \\
2.85\end{array}$ & $\begin{array}{l}2.96 \\
2.93 \\
2.93 \\
2.92 \\
2.90\end{array}$ & $\begin{array}{l}2.85 \\
2.88 \\
2.91 \\
2.92 \\
2.84\end{array}$ & $\begin{array}{l}2.76 \\
2.78 \\
2.79 \\
2.76 \\
2.80\end{array}$ & $\begin{array}{l}3.02 \\
3.03 \\
2.93 \\
2.92 \\
2.96\end{array}$ \\
\hline $\begin{array}{l}26 \\
27 \\
28 \\
29 \\
30 \\
31\end{array}$ & $\begin{array}{l}2.57 \\
2.51 \\
2.46 \\
2.44 \\
2.41 \\
2.42\end{array}$ & $\begin{array}{r}2.32 \\
2.35 \\
2.33 \\
2.34 \\
2.34 \\
---\end{array}$ & $\begin{array}{l}2.06 \\
2.05 \\
2.04 \\
2.05 \\
2.05 \\
2.03\end{array}$ & $\begin{array}{l}1.65 \\
1.65 \\
1.64 \\
1.64 \\
1.67 \\
1.66\end{array}$ & $\begin{array}{r}1.57 \\
1.57 \\
1.56 \\
-\ldots- \\
- \\
-\end{array}$ & $\begin{array}{l}1.84 \\
1.84 \\
1.85 \\
1.87 \\
1.90 \\
1.93\end{array}$ & $\begin{array}{r}2.75 \\
2.65 \\
2.59 \\
2.55 \\
2.53 \\
-\end{array}$ & $\begin{array}{l}2.89 \\
2.91 \\
2.99 \\
3.01 \\
3.03 \\
3.00\end{array}$ & $\begin{array}{l}2.91 \\
2.95 \\
2.96 \\
3.02 \\
3.05 \\
-\end{array}$ & $\begin{array}{l}2.81 \\
2.78 \\
2.78 \\
2.80 \\
2.80 \\
2.80\end{array}$ & $\begin{array}{l}2.88 \\
2.90 \\
2.90 \\
2.90 \\
2.91 \\
2.86\end{array}$ & $\begin{array}{r}3.01 \\
2.94 \\
2.92 \\
2.93 \\
2.92 \\
-.-\end{array}$ \\
\hline $\begin{array}{l}\text { MEAN } \\
\text { MAX } \\
\text { MIN }\end{array}$ & $\begin{array}{l}2.71 \\
2.92 \\
2.41\end{array}$ & $\begin{array}{l}2.38 \\
2.48 \\
2.23\end{array}$ & $\begin{array}{l}2.13 \\
2.31 \\
2.03\end{array}$ & $\begin{array}{l}1.77 \\
2.02 \\
1.64\end{array}$ & $\begin{array}{l}1.55 \\
1.64 \\
1.52\end{array}$ & $\begin{array}{l}1.68 \\
1.93 \\
1.56\end{array}$ & $\begin{array}{l}2.54 \\
2.90 \\
1.99\end{array}$ & $\begin{array}{l}2.89 \\
3.04 \\
2.55\end{array}$ & $\begin{array}{l}3.02 \\
3.11 \\
2.90\end{array}$ & $\begin{array}{l}2.87 \\
3.02 \\
2.78\end{array}$ & $\begin{array}{l}2.82 \\
2.91 \\
2.72\end{array}$ & $\begin{array}{l}2.94 \\
3.08 \\
2.80\end{array}$ \\
\hline
\end{tabular}




\section{WISCONSIN DISTRICT PUBLICATIONS PERTAINING TO LAKES}

The reports published in a U.S. Geological Survey series are for sale by the U.S. Geological Survey, Box 25425, Federal Center, Denver, CO 80225. Prepayment is required. Remittance should be sent by check or money order payable to the U.S. Geological Survey. Prices can be obtained by writing to the above address or by calling (303) 236-7476. Reprints of journal articles may be obtained by writing directly to the author at U.S. Geological Survey, 8505 Research Way, Middleton, WI 53562.

2000

Elder, J.F., Robertson, D.M., and Garrison, P.J., 2000, Chemical composition of surficial deposits in Geneva Lake, Wisconsin: U.S. Geological Survey Fact Sheet FS-121-00.

Elder J.F., Rybicki, N.B., Carter, V.P., and Weintraub, V., 2000, Sources and yields of dissolved carbon in northern Wisconsin stream catchments with differing amounts of peatland: Wetlands, Vol. 20, No. 1, p.113-125.

Hunt, R.J., Graczyk, D.J., and Rose, W.J., 2000, Water flows in the Necedah National Wildlife Refuge: U.S. Geological Survey Fact Sheet FS-068-00, 4 p.

Lathrop, R.C., Carpenter, S.R., and Robertson, D.M., 2000, Interacting factors causing exceptional water clarity in Lakes Mendota and Monona, Wisconsin, Proc. of the International Limnological Society, SIL, Dublin, Ireland, August 1998.

Magnuson, J.J., Robertson, D.M. Wynne, R.H., Benson, B.J., Livigstone, D.M., Arai, T., Assel, R.A., Barry, R.G., Card, V., Kuusisto, E., Granin, N.G., Prowse, T.D., Stewart, K.M., and Vuglinski, V.S., 2000, Historical Trends in Lake and River Ice Cover in the Northern Hemisphere, Science, Vol. 289, No. 5485, p. 1743-1746.

Magnuson, J.J., Wynne, R.H, Benson, B.J., and Robertson, D.M., 2000, Lake and river ice as a powerful indicator of past and present climates, Proc. of the International Limnological Society, SIL, Dublin, Ireland, August 1998.

Robertson, D.M., 2000, One-dimensional simulation of stratification and dissolved oxygen in McCook Reservoir, Illinois: U.S. Geological Survey Water Resources Investigations Report 00-4258, $17 \mathrm{p}$.

Robertson, D.M., Goddard, G.L., Helsel, D.R., and MacKinnon, K.L., 2000, Rehabilitation of Delavan Lake, Wisconsin, Lake and Reservoir Management, Vol. 20, No. 3, p. 155-176.

Robertson, D.M., and Rose, W.J., 2000, Hydrology, water quality, and phosphorus loading of Little St. Germain Lake, Vilas County, Wisconsin: U.S. Geological Survey Water Resources Investigations Report 00-4209, 8 p.

Robertson, D.M., Wynne, R.H., and Chang, W.Y.B., 2000, Variability in ice cover across the northern hemisphere during the 1900's associated with EI Nino events, Proceedings of the International Limnological Society, SIL, Dublin, Ireland, August 1998.

Hunt, R.J., Lin, J., Krohelski, J.T., and Juckem, P.F., 2000, Simulation of the shallow hydrologic system in the vicinity of Middle Genesee Lake, Wisconsin, using analytic elements and parameter estimation: U.S. Geological Survey Water Resources Investigations Report 00-4136, $16 \mathrm{p}$. 
Saad, D.A., and Robertson, D.M., 2000, Water-resources-related information for the St. Croix Reservation and vicinity, Wisconsin: U.S. Geological Survey Water Resources Investigations Report 00-4133, 65 p.

Johnson, G.P., Hornewer, N.J., Robertson, D.M., and Olson, D.T., 2000, Methodology, data collection, and data analysis for determination of water-mixing patterns induced by aerators and mixers, U.S. Geological Survey Water Resources Investigations Report 00-4101, 72 .

Grannemann, N.G., Hunt, R.J., Nicholas, J.R. Reilly, T.E., and Winter, T.C., 2000, The importance of ground water to the Great Lakes Region: U.S. Geological Survey Water Resources Investigations Report 00-4008, 12 p.

Lathrop, R.C., Carpenter, S.R., and Robertson, D.M., 1999, Summer water clarity responses to phosphorus, Daphnia grazing, and internal mixing in Lake Mendota, Limnology and Dceanography, Vol. 44 (1), p. 137-146.

Krohelski, J.T., Feinstein, D.T., and Lenz, B.N., 1999, Simulation of stage and hydrolog. pudget for Shell Lake, Washburn County, Wisconsin, U.S. Geological Survey Water-Resources Investigations Report 99-4209, 23 p.

Panuska, J.C., and Robertson, D.M., 1999, Estimating phosphorus concentrations following alum treatment using apparent settling velocities, Lakes and Reservoir Management, Vol. 15 (1), p. 28-38.

1998

Hunt, R.J, Anderson, M.P., and Kelson, V.A., 1998, Improving a complex finite-difference ground water flow model through the use of an analytic element screening model, Ground Water, Vol. 36, No. 6, p. 1011-1017.

Hunt, R.J, Anderson, M.P., and Kelson, V.A., 1998, Linking an analytic element flow code to MODFLOW - Implementation and benefits. p. 477-504 in MODFLOW'98: Proceedings of the 3rd International Conference of the International Ground Water Center. Golden, CO: Colorado School of Mines.

Krabbenhoft, D.P., Gilmour, C.C., Benoit, J.M., Babiarz, C.L., Andren, A.W., and Hurley, J.P., 1998, Methyl mercury dynamics in littoral sediments of a temperate lake, Canadian Journal of Fisheries and Aquatic Sciences, Vol. 55, p. 835-844.

Robertson, D.M., Elder, J.F., Goddard, G.L., and James, W.F., 1998, Dynamics in phosphorus retention in wetlands upstream of Delavan Lake, Wisconsin, Lakes and Reservoir Management, Vol. 14, No. 4, p. 466-477.

Rose, W.J., and Robertson, D.M., 1998, Hydrology, water quality, and phosphorus loading of Kirby Lake, Barron County, Wisconsin, U.S. Geological Survey Fact Sheet FS-066-98, 4 p.

Walker, J.F. and Krabbenhoft, D.P., 1998, Groundwater and surface-water interactions in riparian and lake-dominated systems, in Kendall, C. and McDonnell, J.J. eds., Isotope tracers in catchment hydrology, Elsevier Publishing, New York, 839 P.

Elder, J.F., Manion, B.J., and Goddard, G.L., 1997, Mesocosm experiments to assess factors affecting phosphorus retention and release in an extended Wisconsin wetland: U.S. Geological Survey Water-Resources Investigations Report 97-4272, 14 p. 
Goddard, G.L., and Elder, J.F., 1997, Retention of sediments and nutrients in Jackson Creek Wetland near Delavan Lake, Wisconsin, 1993-95, U.S. Geological Survey Water-Resources Investigations Report 97-4014, 22 p.

Hornewer, N.J., Johnson, G.P., Robertson, D.M. and Hondzo, M., 1997, Field-scale tests for determining mixing patterns associated with coarse-bubble air diffuser configurations, Egan Quarry, Illinois, In: Environmental and Coastal Hydraulics: Protecting the Aquatic Habitat, Proceedings of the International Association of Hydraulic Research, San Francisco, CA, USA, p. 57-63.

Robertson, D.M., 1997, Regionalized loads of sediment and phosphorus to Lakes Michigan and Superior - High flow and long-term average, Journal of Great Lakes Research, Vol. 23, p. 416439.

Anderson, W.L., Robertson, D.M., and Magnuson, J.J., 1996, Evidence of recent warming and EI Nino-related variation in ice breakup of Wisconsin lakes, Limnology and Oceanography, Vol. 41, p. $815-821$.

Elder, J.F. and Goddard, G.L., 1996, Sediment and nutrient trapping efficiency of a constructed wetland near Delavan Lake, Wisconsin, 1993-1995: U.S. Geological Survey Fact Sheet FS232-96.

Garn, H.S., Olson, D.L., Seidel, T.L., and Rose, W.J., 1996, Hydrology and water quality of Lauderdale Lakes, Walworth County, Wisconsin, 1993-94: U.S. Geological Survey Water-Resources Investigations Report 96-4235, 29 p.

Hunt, R.J. and Krohelski, J.T., 1996, The application of an analytical element model to investigate groundwater-lake interactions at Pretty Lake, Wisconsin, Lake and Reservoir Management, Vol. 12, p. 487-495.

Imberger, J., Robertson, D.M., and Boland, K., 1996, Lake Number: A quantitative indicator of mixing to be used in water quality management. Scientific Impeller, Solna, Sweden, No. 4, p. 9-15.

Kammerer, P.A., Jr., 1996, Hydrology and water quality of Park Lake, South-central Wisconsin: U.S. Geological Survey Fact Sheet FS-197-96.

Robertson, D.M., Field, S.J, Elder, J.F., Goddard, G.L., and James, W.F., 1996, Phosphorus dynamics of Delavan Lake Inlet in southeastern Wisconsin. U.S. Geological Survey Water-Resources Investigations Report 96-4160, 18 p.

\section{5}

Assel, R.A. and Robertson, D.M. 1995, Changes in winter air temperatures near Lake Michigan during 1851 - 1993, as determined from regional lake-ice records, Limnology and Oceanography, v. 40, p 165-176.

Assel, R.A., Robertson, D.M., Hoff, M.H., and Selgeby, J.H., 1995, Climatic-change implications from long-term (1823-1994) ice records near the Laurentian Great Lakes: Annals of Glaciology, v. 21 , p. $383-386$.

Krabbenhoft, D.P., and Webster, K.E., 1995, Transient hydrogeological controls on the chemistry of a seepage lake: Water Resources Research, v. 31, no. 9, p. 2295-2305.

Krohelski, J.T. and Batten, W.G., 1995, Simulation of stage and the hydrologic budget of Devils Lake, Sauk County, Wisconsin: U.S. Geological Survey Open-File Report 94-348, 22 p. 
Wentz, D.A., Rose, W.J., and Webster, K.E., 1995, Long-term hydrologic and biogeochemical responses of a soft water seepage lake in north central Wisconsin: Water Resources Research, v. 31 , no. 1, p 199-212.

1994

Elder, J.F., 1994, Distribution and grain-size partitioning of metals in bottom sediments of an experimentally acidified lake: Water Resources Bulletin, v. 30, no. 2, p. 251-259.

Goddard, G.L., and Field, S.J., 1994, Hydrology and water quality of Whitewater and Rice Lakes in southeastern Wisconsin, 1990-91: U.S. Geological Survey Water-Resources Investigations Report 94-4101, $36 \mathrm{p}$.

Greb, S.R., and Wentz, D.A., 1994, Chemical budgets, in Klepinger, K.E., ed., RILWAS 1983-86: Wisconsin Regional Integrated Lake Watershed Acidification Study, Volume 1: Madison, Wisconsin Department of Natural Resources, PUBL-RS-909-94, Chapter 7, 20 p.

Hurley, J.P., Krabbenhoft, D.P., Babiarz, C.L., and Andren, A.W., 1994, Cycling processes of mercury across sediment/water interfaces in seepage lakes, in Baker, L.A. ed., Environmental Chemistry of Lakes and Reservoirs: Advances in Chemistry Series, American Chemical Society, Washington, D.C., p. 426-449.

Krabbenhoft, D.P., Bowser, C.J., Kendall, C., and Gat, J.R., 1994, Use of oxygen-19 and deuterium to assess the hydrology of ground-water/lake systems, in Baker, L.A. ed., Environmental Chemistry of Lakes and Reservoirs: Advances in Chemistry Series, American Chemical Society, Washington, D.C., p. 67-90.

Robertson, D.M., Anderson, W., and Magnuson, J.J., 1994, Relations between El Nino/Southern Oscillation events and the climate and ice cover of lakes in Wisconsin. P 48-57, in Greenland, D. ed., El Nino and Long-Term Ecological Research (LTER) Sites: Publication No. 18. LTER Network Office: University of Washington, Seattle, WA, USA., $57 \mathrm{p}$.

Robertson, D.M. and Imberger, J. 1994, Lake Number, a quantitative indicator of mixing used to estimate changes in dissolved oxygen, Internationale Revue der gesamten Hydrobiologie, v. 79, p. 159-176.

Watras, C.J., Bloom, N.S., Hurley, J.P., Fitzgerald, W.F., Andren, A.W., Krabbenhoft, D.P., and Porcella, D.B., 1994, Sources and fates of mercury and methylmercury in Wisconsin lakes, in Watras and Huckabee eds., Mercury as a Global Pollutant: Intergration and Synthesis, Lewis Pub., Chelsea, Ml., p 153-177.

Wentz, D.A., 1994, Chemistry of snowpack and ground water, in Klepinger, K.E., ed., RILWAS 1983-86: Wisconsin Regional Integrated Lake Watershed Acidification Study, Volume 1: Madison, Wisconsin Department of Natural Resources, PUBL-RS-909-94, Chapter 6, 45 p.

Wentz, D.A., Krohelski, J.T., and Rose, W.J., 1994, Hydrology, in Klepinger, K.E., ed., RILWAS 1983-86: Wisconsin Regional Integrated Lake Watershed Acidification Study, Volume 1: Madison, Wisconsin Department of Natural Resources, PUBL-RS-909-94, Chapter 7, 74 p.

1993

Field, S.J., 1993, Hydrology and water quality of Powers Lake, southeastern Wisconsin: U.S. Geological Survey Water-Resources Investigations Report 90-4126, 36 p.

Field, S.J., 1993, Hydrology and water quality of Wind Lake in southeastern Wisconsin: U.S. Geological Survey Water-Resources Investigations Report 91-4107, $61 \mathrm{p.}$ 
House, L.B., 1993, Simulation of the effects of hypothetical residential development on water levels in Graber Pond, Middleton, Wisconsin: U.S. Geological Survey Water-Resources Investigations Report 92-4029, 10 p.

House, L.B., Waschbusch, R.J., and Hughes, P.E., 1993, Water quality of an urban wet detention pond in Madison, Wisconsin, 1987-88: U.S. Geological Survey Open-File Report 93-172, $57 \mathrm{p}$.

Hughes, P.E., 1993, Hydrology, water quality, trophic status, and aquatic plants of Fowler Lake, Wisconsin: U.S. Geological Survey Water-Resources Investigations Report 91-4076, 44 p.

Rose, W.J., 1993, Hydrology of Little Rock Lake in Vilas County, north-central Wisconsin: U.S. Geological Survey Water-Resources Investigations Report 93-4139, 22 p.

Rose, W.J., 1993, Water and phosphorus budgets and trophic state, Balsam Lake, northwestern Wisconsin, 1987-1989: U.S. Geological Survey Water-Resources Investigations Report $91-4125,28$ p.

\section{2}

Elder, J.F., Krabbenhoft, D.P, and Walker, J.F., 1992, Water, energy, and biogeochemical budgets (WEBB) program: Data availability and research at the northern temperate lakes site, Wisconsin: U.S. Geological Survey Open-File Report 92-48, 15 p.

Krabbenhoft, D.P., and Babiarz, C.L., 1992, Role of groundwater transport in aquatic mercury cycling: Water Resources Research, vol. 28, no. 12, p. 3119-3128.

Krabbenhoft, D.P., and Krohelski, J.T., 1992, Data on water quality, lake sediment, and lake-level fluctuation, St. Croix Indian Reservation, Wisconsin, 1981-87: U.S. Geological Survey OpenFile Report 92-26, $53 \mathrm{p}$.

Robertson, D.M., Ragotzkie, R.A., and Magnuson, J.J. 1992, Lake ice records used to detect historical and future climatic changes: Climatic Change, v. 21, p. 407-427.

1991

Wentz, D.A., and Rose, W.J., 1991, Hydrology of Lakes Clara and Vandercook in North-Central Wisconsin: U.S. Geological Survey Water-Resources Investigations Report 89-4204, 24 p.

Watras, C.J., Andre, A.W., Bloom, N.S., Fitzgerald, W.F., Hurley, J.P., Krabbenhoft, D.P., Rada, R.G., Wiener, J.G., 1991, Mercury in temperate lakes: a mechanistic field study: Verhandlungen Internat. Verein. Limnologie, 24, p. 2199-2202.

\section{Pre-1990}

Walker, J.F., Pickard, S.A., and Sonzogni, W.C., 1989 Spreadsheet watershed modeling for nonpoint-source pollution management in a Wisconsin basin: Water Resources Bulletin, v. 25, no. 1, p. $139-147$.

Wentz, D.A., Garrison, P.J., and Bockheim, J.G., 1989, Section 7-Chemical input-output budgets, in Knauer, D., and Brouwer, S.A., eds., The Wisconsin Regional Integrated Lake-Watershed Acidification Study (RILWAS): 1981-1983: Palo Alto, California, Electric Power Research Institute Report EA-6214, p. 7-1 to 7-30.

Wentz, D.A., and Rose, W.J., 1989, Interrelationships among hydrologic-budget components of a northern Wisconsin seepage lake and implications for acid-deposition modeling: Archives of Environmental Contamination and Toxicology, v. 18, p. 147-155. 
Wentz, D.A., Rose, W.J., and Krohelski, J.T., 1989, Section 5-Hydrologic component, in Knauer, D., and Brouwer, S.A., eds., The Wisconsin Regional Integrated Lake-Watershed Acidification Study (RILWAS): 1981-1983: Palo Alto, California, Electric Power Research Institute Report EA-6214, p. 5-1 to 5-77.

Field, S.J., and Duerk, M.D., 1988, Hydrology and water quality of Delavan Lake in southeastern Wisconsin: U.S. Geological Survey Water-Resources Investigations Report 87-4168, 61 p.

Krug, W.R., Ostenso, N.A., and Krohelski, J.T., 1988, Prediction of the effects of mine dewatering on four lakes near Crandon, Wisconsin, by use of a water-budget model: U.S. Geological Survey Open-File Report 87-471, 63 p.

Wentz, D.A., Krohelski, J.T., Rose, W.J., Bockheim, J.G., Garrison, P.J., Knauer, D.R., and Goldstein, R.A., 1987, Hydrologic and chemical budgets of Wisconsin lakes, in Perrry, R., Harrison, R.M., Bel, J.N.B., and Lester, J.N., eds., Acid Rain: Scientific and Technical Advances, Selper Ltd., London, p. 309-316.

House, L.B., 1986, Stage fluctuations of Wisconsin Lakes: Wisconsin Geological and Natural History Survey Information Circular No. 49, 84 p.

House, L.B., 1984, Effects of urbanization on three ponds in Middleton, Wisconsin: U.S. Geological Survey Water-Resources Investigations Report 84-4051, 17 p.

Krug, W.R., and House, L.B., 1984, Evaluation of alternative reservoir-management practices in the Rock River basin, Wisconsin: U.S. Geological Survey Water-Resources Investigations Report 83-4186, $21 \mathrm{p}$.

House, L.B., 1981, An assessment of streamflow, water quality, and the effects of construction on impoundment on Bridge Creek at Augusta, Wisconsin: U.S. Geological Survey WaterResources Investigations Open-File Report 81-1192, 25 p.

Krug, W.R., 1981, Hydrologic effects of proposed changes in management practices, Winnebago Pool, Wisconsin: U.S. Geological Survey Water-Resources Investigations 80-107, 19 p.

Batten, W.G., and Hindall, S.M., 1980, Sediment deposition in the White River Reservoir, northwestern Wisconsin: U.S. Geological Survey Water-Supply Paper 2069, 30 p.

Novitzki, R.P., and Holmstrom, B.K., 1979, Monthly and annual water budgets of Lake Wingra, Madison, Wisconsin, 1971-77: U.S. Geological Survey Water-Resources Investigations 79-100, $31 \mathrm{p}$.

Rose, W.J., 1977, Hydrologic considerations associated with dredging spring ponds in Wisconsin: U.S. Geological Survey Water-Resources Investigations 77-18, 35 p.

Oakes, E.L., Hendrickson, G.E., and Zuehls, E.E., 1975, Hydrology of the Lake Wingra basin, Dane County, Wisconsin: U.S. Geological Survey Water-Resources Investigations 17-75, $31 \mathrm{p}$.

Novitzki, R.P., 1971, Hydrologic investigations of Heart Lake, Green Lake County, Wisconsin: U.S. Geological Survey Administrative Report, 9 p. 


\section{APPENDIX \\ Wisconsin Lakes Team \\ Quality-Assurance/Quality-Control Plan}

Most lake studies and monitoring programs that are conducted by the USGS Wisconsin District entail water sampling and analysis to determine water quality and biological productivity. Because all sampling and analyses are subject to error and random variability, a certain proportion of the sampling effort should include quality-assurance/quality-control (QAQQC) samples. These samples are collected and/or prepared solely for the purpose of assessing the magnitude of error and random variability so that the accuracy and precision of all data can be evaluated. The plan for this quality-assurance sampling is described below.

Three types of QAVQ samples are collected:

1. Blanks - samples that provide information about accuracy and errors due to treatment or reagents.

2. Replicates - samples that provide information about precision (variability).

3. Standard additions (spikes) - samples that provide information about accuracy and matrix interferences.

\section{Blank Sampling}

At one randomly-chosen lake each month, a field blank is prepared. This consists of deionized water or inorganic blank water treated exactly the same as regular samples. During winter, the field blank is only analyzed for total phosphorus. During summer, it is analyzed for total phosphorus and chlorophyll a, and in the spring, it is analyzed for total and dissolved phosphorus, nitrate plus nitrite, total Kjeldahl nitrogen, and chlorophyll a.

\section{Replicate Sampling}

At selected lakes in the program, triplicate samples are taken near the water surface in summer for analysis of total phosphorus and chlorophyll a. At two of these lakes, a set of triplicate samples is also taken from near-bottom water, for analysis of total phosphorus.

At three selected lakes in the spring (different lakes each year), triplicate samples are taken near water surface for analysis of total and dissolved phosphorus, nitrate plus nitrite, total Kjeldahl nitrogen, and chlorophyll a.

\section{Standard Addition Testing}

At Delavan Lake and one other lake (to be determined each year), 5 replicate samples are taken in August for a standard addition (spike) test. The spike consists of addition of a prepared phosphorus solution (standard) of known volume and concentration, such that the expected result of analysis is the natural water total phosphorus concentration plus the known addition. One sample from each set will receive a low-concentration spike, one will receive a high-concentration spike, and three will receive no spike (the mean of these gives the natural water total phosphorus concentration).

Data and results of replicate sampling and field blank testing are shown in Tables A1, A2, and A3. 
Table Al. Analyses of replicate samples from Wisconsin lakes in water year 2001 . See text for procedures used. All data except chlorophyil in milligrams per liter. (Symbol "<" indicates less than given detection limit (DL); -, not measured or not computed; mean and standard deviation not calculated for datasets containing values less than DL)

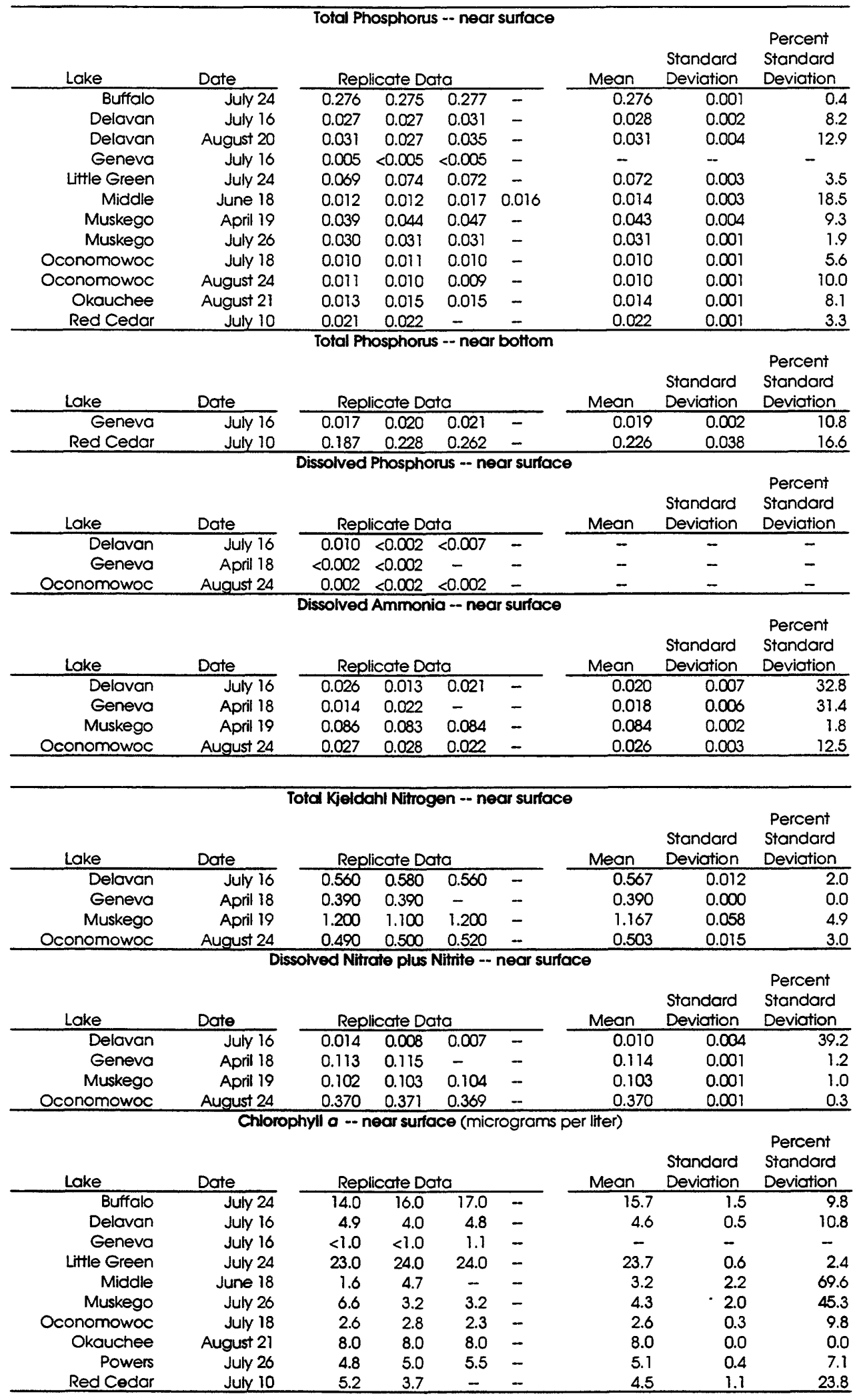


Table A2. Data from tests of blanks, Delavan Lake project, 2001. Analyses at U.S. Geological Survey National Water-Quality Laboratory, Lakewood, CO. All data in milligrams per liter. (<, less than given detection limit; $E$, estimated value; -, not measured)

\begin{tabular}{lccc}
\hline \multicolumn{1}{c}{ Constituent } & February 20 & April 18 & July 16 \\
\hline Total phosphorus & $\mathrm{E} 0.003$ & $<0.004$ & $<0.004$ \\
Dissolved orthophosphate & - & - & $<0.007$ \\
Chlorophyll a & $<0.1$ & $<0.1$ & - \\
Chlorophyll b & $<0.1$ & - & - \\
Total Kjeldahl nitrogen (as N) & - & - & $<0.08$ \\
Ammonia (as N) & - & - & $<0.02$ \\
Nitrate plus nitrite (as N) & - & - & $<0.05$ \\
\hline
\end{tabular}

Table A3. Data from standard addition tests using stock solution containing $5.00 \mathrm{mg} / \mathrm{L}$ phosphorus. See text for detail of procedures. All concentration data in milligrams per liter

\begin{tabular}{lccccc}
\hline & $\begin{array}{c}\text { Original } \\
\text { Sample } \\
\text { Concentration }\end{array}$ & $\begin{array}{c}\text { Stock } \\
\text { Solution } \\
\text { Volume }\end{array}$ & $\begin{array}{c}\text { Final } \\
\text { Expected } \\
\text { Concentration }\end{array}$ & $\begin{array}{c}\text { Actual } \\
\text { Detected } \\
\text { Concentration }\end{array}$ & $\begin{array}{c}\text { Percent } \\
\text { Recovery }\end{array}$ \\
\hline Delavan, 20 August 2001 & 0.031 & 0.125 & 0.036 & 0.046 & 128 \\
& 0.031 & 0.500 & 0.051 & 0.055 & 108 \\
& & & & & \\
Okauchee. 21 August 2001 & 0.014 & 0.125 & 0.017 & 0.025 & 147 \\
& 0.014 & 0.300 & 0.026 & 0.031 & 119 \\
\hline
\end{tabular}

